\title{
Palladium-Catalyzed Regioselective C-H Iodination of Unactivated Alkenes
}

\author{
Benedikt S. Schreib, Erick M. Carreira* \\ ETH Zürich, Vladimir-Prelog-Weg 3, HCI, 8093 Zürich, Switzerland
}

Supporting Information 


\section{Materials and Methods}

Unless otherwise noted, all reactions were carried out under ambient atmosphere, and all reagents were purchased from commercial suppliers (ABCR, ACROS, Sigma Aldrich, Fluka, TCI, Strem, Alfa, Combi-Blocks or Fluorochem) and used without further purification. Anhydrous solvents over molecular sieves were purchase from Acros and used as received. Analytical thin layer chromatography (TLC) was performed on Merck silica gel 60 F254 TLC glass plates and visualized with $254 \mathrm{~nm}$ light and potassium permanganate or ceric ammonium molybdate staining solutions followed by heating. Organic solutions were concentrated by rotary evaporation at $40{ }^{\circ} \mathrm{C}$. Purification of reaction products was carried out by flash chromatography using Brunschwig silica 32-63, $60 \AA$ under $0.3-0.5$ bar overpressure.

${ }^{1} \mathrm{H}$ NMR spectra were recorded on a Bruker AVIII $600 \mathrm{MHz}$ spectrometer with $\mathrm{He}$ or prodigy $\mathrm{N}_{2}$ cryo-probes, Bruker AVIII HD $500 \mathrm{MHz}$ and 400 spectrometers as well as Bruker Neo $500 \mathrm{MHz}$ and $400 \mathrm{MHz}$ spectrometers, and are reported in ppm with the solvent resonance as the reference unless noted otherwise $\left(\mathrm{CDCl}_{3}\right.$ at $7.26 \mathrm{ppm}, \mathrm{CD}_{2} \mathrm{Cl}_{2}$ at $5.32 \mathrm{ppm}$, DMSO-d6 at $2.50 \mathrm{ppm}, \mathrm{CD}_{3} \mathrm{CN}$ at 1.94 $\mathrm{ppm})$. Peaks are reported as $(\mathrm{s}=$ singlet, $\mathrm{d}=$ doublet, $\mathrm{t}=$ triplet, $\mathrm{q}=$ quartet, $\mathrm{m}=$ multiplet or unresolved, br $=$ broad signal, coupling constant(s) in $\mathrm{Hz}$, integration). ${ }^{13} \mathrm{C}$ NMR spectra were recorded with ${ }^{1} \mathrm{H}$-decoupling on Bruker AVIII $150 \mathrm{MHz}$ spectrometers with $\mathrm{He}$ or prodigy $\mathrm{N}_{2}$ cryoprobes, Bruker AVIII HD $125 \mathrm{MHz}$ and $100 \mathrm{MHz}$ spectrometers as well as Bruker Neo $125 \mathrm{MHz}$ and $100 \mathrm{MHz}$ spectrometers, and are reported in ppm with the solvent resonance as the reference unless noted otherwise $\left(\mathrm{CDCl}_{3}\right.$ at $77.16 \mathrm{ppm}, \mathrm{CD}_{2} \mathrm{Cl}_{2}$ at $54.00 \mathrm{ppm}$, DMSO- $d_{6}$ at $39.52 \mathrm{ppm}, \mathrm{CD}_{3} \mathrm{CN}$ at 1.32 ppm). ${ }^{19} \mathrm{~F}$-NMR spectra were referenced to $\mathrm{CFCl}_{3}(0.65 \mathrm{ppm})$ as internal standard according to a published procedure. ${ }^{1}$ Infrared spectra were recorded neat on a Perkin-Elmer Spectrum Two FT-IR spectrometer. The peaks are reported as absorption maxima $\left(\mathrm{cm}^{-1}\right)$. High resolution mass spectrometric data were obtained at the mass spectrometry service operated by the Laboratory of Organic Chemistry at the ETHZ on VG-TRIBRID for electron impact ionization (EI), Varian IonSpec Spectrometer for electrospray ionization (ESI), or IonSpec Ultima Fourier Transform Mass Spectrometer for matrix-assisted laser desorption/ionization (MALDI) and are reported as $(\mathrm{m} / \mathrm{z}$ ). Melting point ranges were determined on a Büchi B545 Melting Point Apparatus.

The geometry of disubstituted olefins was assigned based on the ${ }^{1} \mathrm{H}-\mathrm{NMR}{ }^{3} J$ coupling constant of the vinylic protons (ca $12 \mathrm{~Hz}$ for cis-olefins, ca $16 \mathrm{~Hz}$ for trans-olefins). The olefin geometry of $\mathbf{2 a}, \mathbf{2 c}$, $\mathbf{2 p}, \mathbf{5}$ and $\mathbf{6}$ was assigned by NOESY experiments. The geometry of the remaining alkenyl iodides was assigned based on analogy. 


\section{General Procedures}

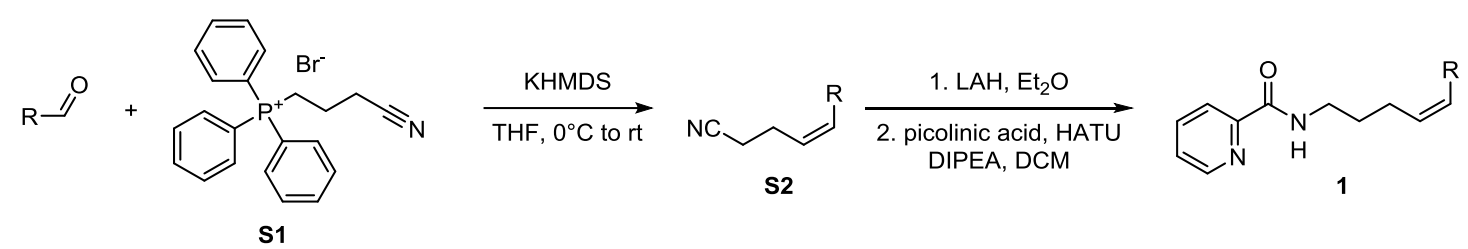

\section{General procedure for Wittig reactions (procedure 1)}

To a suspension of (3-cyanopropyl)triphenylphosphonium bromide ( $3.0 \mathrm{~g}, 7.3 \mathrm{mmol}, 1.1$ equiv) in $20 \mathrm{~mL}$ THF at $0{ }^{\circ} \mathrm{C}$ was added KHMDS (20 wt\% in THF, $9.4 \mathrm{~mL}, 8.3 \mathrm{mmol}, 1.25$ equiv) slowly over 2 minutes, which resulted in an orange suspension. The reaction was stirred at $0{ }^{\circ} \mathrm{C}$ for 15 minutes before the aldehyde ( $6.65 \mathrm{mmol}, 1.0$ equiv) was added in one portion. The reaction was stirred an additional $30 \mathrm{~min}$ at $0{ }^{\circ} \mathrm{C}, 90 \mathrm{~min}$ at room temperature, and then poured into $100 \mathrm{~mL}$ rapidly stirred hexanes. The resulting mixture was filtered through a silica plug (washed with the indicated eluent), concentrated and purified by flash column silica gel chromatography using the indicated solvent system.

\section{General procedure for nitrile reduction (procedure 2)}

To a suspension of lithium aluminium hydride (1.5 equiv) in diethyl ether $(0.3 \mathrm{M})$ under $\mathrm{N}_{2}$ at $0{ }^{\circ} \mathrm{C}$ was slowly added a solution of nitrile (1.0 equiv, dissolved in a small volume of diethyl ether). After 15 min the reaction was allowed to warm to room temperature and stirred for an additional $90 \mathrm{~min}$. The reaction was re-cooled to $0{ }^{\circ} \mathrm{C}$, diluted with wet diethyl ether (technical grade, ca 5-10 times the reaction volume), quenched by slow addition of aq $\mathrm{NaOH}\left(1 \mathrm{M}, 3 \mathrm{~mL} \mathrm{NaOH}\right.$ per g $\mathrm{LiAlH}_{4}$ used), stirred for an additional $30 \mathrm{~min}$, filtered through celite and concentrated under reduced pressure. The resulting crude product was used for the next step without further purification.

\section{General procedure for HATU coupling (procedure 3)}

To a solution of amine (1.0 equiv) in DCM $(0.2 \mathrm{M})$ was added picolinic acid (1.1 equiv), $\mathrm{N}, \mathrm{N}$ diisopropylethylamine (2.0 equiv), and HATU (1.1 equiv) in this order. The reaction was stirred at room temperature for $2 \mathrm{~h}$, quenched with a 1:1 mixture of brine and sat. aq. $\mathrm{NaHCO}_{3}$, extracted with DCM $(2 \mathrm{x})$, dried over $\mathrm{Na}_{2} \mathrm{SO}_{4}$ and concentrated. The crude residue was purified by flash column silica gel chromatography using the indicated solvent system.

\section{General procedure for the palladium-catalyzed alkenyl $\mathrm{C}-\mathrm{H}$ iodination $\left(\mathrm{PhI}(\mathrm{OPiv})_{2}\right.$, procedure}

4)

To a $7 \mathrm{~mL}$ microwave vial under ambient atmosphere was added starting material $(0.30 \mathrm{mmol}$, 1.0 equiv), pivalic acid $(31 \mathrm{mg}, 0.30 \mathrm{mmol}, 1.0$ equiv), tetrabutylammonium iodide (144 $\mathrm{mg}$, $0.39 \mathrm{mmol}, 1.3$ equiv), bis(tert-butylcarbonyloxy)iodobenzene (158 mg, $0.39 \mathrm{mmol}, 1.3$ equiv), 
$\mathrm{Pd}(\mathrm{OAc})_{2}(6.7 \mathrm{mg}, 0.030 \mathrm{mmol}, 0.10$ equiv), water $(0.6 \mathrm{~mL})$, and acetonitrile $(2.4 \mathrm{~mL})$. The vial was sealed, placed in a heating block pre-heated to $90{ }^{\circ} \mathrm{C}$, and stirred at this temperature for $8 \mathrm{~h}$. The reaction was allowed to cool to room temperature, quenched by addition of aq. $\mathrm{Na}_{2} \mathrm{~S}_{2} \mathrm{O}_{3}$ (5 wt $\%, 25$ $\mathrm{mL})$ and sat. aq. $\mathrm{NaHCO}_{3}(25 \mathrm{~mL})$, extracted with EtOAc $(2 \times 50 \mathrm{~mL}$, in case of poor phase separation $25 \mathrm{~mL}$ brine was added), dried over $\mathrm{Na}_{2} \mathrm{SO}_{4}$ and concentrated. The crude residue was purified by flash column silica gel chromatography using the indicated solvent system.

Note: For reactions on $0.1 \mathrm{mmol}$ scale a $1.6 \mathrm{~mL}$ screw-cap vial was used instead.

\section{General procedure for the palladium-catalyzed alkenyl C-H iodination (Oxone, procedure 5)}

To a $7 \mathrm{~mL}$ microwave vial under ambient atmosphere was added starting material $(0.30 \mathrm{mmol}$, 1.0 equiv), pivalic acid $(31 \mathrm{mg}, 0.30 \mathrm{mmol}, 1.0$ equiv), tetrabutylammonium iodide (144 mg, $0.39 \mathrm{mmol}, 1.3$ equiv), Oxone ( $\mathrm{KHSO}_{5} \cdot 0.5 \mathrm{KHSO}_{4} \cdot 0.5 \mathrm{~K}_{2} \mathrm{SO}_{4}, 120 \mathrm{mg}, 0.39 \mathrm{mmol}, 1.3$ equiv), $\mathrm{Pd}(\mathrm{OAc})_{2}(10.1 \mathrm{mg}, 0.045 \mathrm{mmol}, 0.15$ equiv), and anhydrous acetonitrile $(3 \mathrm{~mL})$. The vial was sealed, placed in a heating block pre-heated to $70{ }^{\circ} \mathrm{C}$, and stirred at this temperature for $8 \mathrm{~h}$. The reaction was allowed to cool to room temperature, quenched by addition of aq. $\mathrm{Na}_{2} \mathrm{~S}_{2} \mathrm{O}_{3}(5 \mathrm{wt} \%, 25 \mathrm{~mL})$ and sat. aq. $\mathrm{NaHCO}_{3}(25 \mathrm{~mL})$, extracted with EtOAc $(2 \times 50 \mathrm{~mL}$, in case of poor phase separation $25 \mathrm{~mL}$ brine was added), dried over $\mathrm{Na}_{2} \mathrm{SO}_{4}$ and concentrated. The crude residue was purified by flash column silica gel chromatography using the indicated solvent system.

Note: For reactions on 0.1 mmol scale a $1.6 \mathrm{~mL}$ screw-cap vial was used instead. 


\section{Starting Material Synthesis}

\section{(3-cyanopropyl)triphenylphosphonium bromide (S1)}

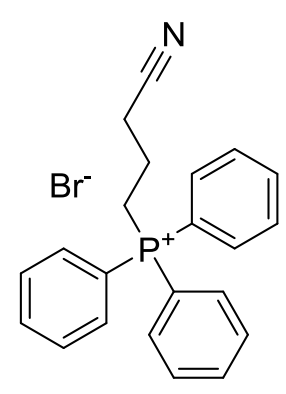

A solution of 4-bromobutanenitrile $(11.0 \mathrm{ml}, 111 \mathrm{mmol}, 1.0$ equiv) and triphenylphosphine $(29.1 \mathrm{~g}$, $111 \mathrm{mmol}, 1.0$ equiv) in toluene $(250 \mathrm{~mL})$ was heated to reflux for $20 \mathrm{~h}$. The reaction was allowed to cool to room temperature and the formed precipitate was collected by filtration, washed with $\mathrm{Et}_{2} \mathrm{O}$, and dried under reduced pressure to afford $\mathbf{S 1}(23.6 \mathrm{~g}, 58 \mathrm{mmol}, 52 \%)$ as a white powder.

${ }^{1} \mathbf{H}$ NMR $\left(400 \mathrm{MHz}, \mathrm{DMSO}-d_{6}, 25{ }^{\circ} \mathrm{C}\right) \delta 7.95-7.89(\mathrm{~m}, 1 \mathrm{H}), 7.87-7.74(\mathrm{~m}, 5 \mathrm{H}), 3.79-3.64(\mathrm{~m}$, $1 \mathrm{H}), 2.80-2.73(\mathrm{~m}, 1 \mathrm{H}), 1.92-1.82(\mathrm{~m}, 1 \mathrm{H}) ;{ }^{13} \mathbf{C}$ NMR $\left(100 \mathrm{MHz}\right.$, DMSO- $\left.d_{6}, 25{ }^{\circ} \mathrm{C}\right) \delta 135.1(\mathrm{~d}, J=$ $3.0 \mathrm{~Hz}), 133.7$ (d, $J=10.3 \mathrm{~Hz}), 130.3$ (d, $J=12.6 \mathrm{~Hz}), 119.6,118.0$ (d, $J=86.3 \mathrm{~Hz}), 19.9$ (d, $J=52.7$ $\mathrm{Hz}), 18.8,17.1(\mathrm{~d}, J=20.6 \mathrm{~Hz}) ;{ }^{31} \mathbf{P}$ NMR $\left(162 \mathrm{MHz}\right.$, DMSO- $\left.d_{6}, 25{ }^{\circ} \mathrm{C}\right) \delta 23.69$; FT-IR (neat) 3078 , $3057,3005,2924,2884,2803,2251,1586,1485,1437,1328,1142,1111,1019,998,877,754,746$, 690, 526, $499 \mathrm{~cm}^{-1}$; HRMS (ESI) $\mathrm{m} / z$ calculated for $\mathrm{C}_{22} \mathrm{H}_{21} \mathrm{NP}[\mathrm{M}]^{+}$330.1406, found 330.1416; Melting point $225-228^{\circ} \mathrm{C}$ (lit. $215-217^{\circ} \mathrm{C}$ ); Elem. anal. calcd for $\mathrm{C}_{22} \mathrm{H}_{21} \mathrm{NPBr}$ : C, 64.40: H, 5.16; $\mathrm{N}, 3.41$. Found C, 64.37; H, 5.19; N, 3.42. The analytical data are in agreement with the literature. ${ }^{2}$

\section{(Z)-5-phenylpent-4-enenitrile (S2a)}

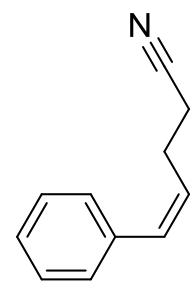

In a flame-dried round-bottom flask under $\mathrm{N}_{2}$ sodium hydride $(60 \%$ in mineral oil, $1.17 \mathrm{~g}, 29.2 \mathrm{mmol}$, 1.20 equiv) was washed with hexanes $(2 \times 10 \mathrm{~mL})$ and subsequently suspended in anhydrous THF $(100 \mathrm{~mL})$. The reaction was cooled to $0{ }^{\circ} \mathrm{C}$, (3-cyanopropyl)triphenylphosphonium bromide $(10.0 \mathrm{~g}$, $24.4 \mathrm{mmol}, 1.00$ equiv) was added in portions, the reaction was allowed to warm to room temperature and stirred for $1 \mathrm{~h}$. The reaction was re-cooled to $0{ }^{\circ} \mathrm{C}$, benzaldehyde $(2.96 \mathrm{~mL}, 29.2 \mathrm{mmol}$, 1.20 equiv) was added, and the reaction was allowed to warm to room temperature and stirred for $3 \mathrm{~h}$. The reaction was poured into $200 \mathrm{~mL}$ rapidly stirred hexanes, the resulting mixture was filtered 
through a silica plug (washed with 2:1 Hex/EtOAc), concentrated and purified by flash column silica gel chromatography (9:1 Hex/EtOAc) to afford a colorless oil (3.43 g, $21.8 \mathrm{mmol}, 90 \%)$.

${ }^{1} \mathbf{H}$ NMR $\left(400 \mathrm{MHz}, \mathrm{CDCl}_{3}, 25{ }^{\circ} \mathrm{C}\right) \delta 7.42-7.33(\mathrm{~m}, 2 \mathrm{H}), 7.30-7.21(\mathrm{~m}, 3 \mathrm{H}), 6.62(\mathrm{dt}, J=11.5,1.9$ $\mathrm{Hz}, 1 \mathrm{H}), 5.66(\mathrm{dt}, J=11.5,7.2 \mathrm{~Hz}, 1 \mathrm{H}), 2.68(\mathrm{qd}, J=7.2,1.8 \mathrm{~Hz}, 2 \mathrm{H}), 2.44(\mathrm{t}, J=7.3 \mathrm{~Hz}, 2 \mathrm{H})$; ${ }^{13} \mathbf{C ~ N M R}\left(100 \mathrm{MHz}, \mathrm{CDCl}_{3}, 25{ }^{\circ} \mathrm{C}\right) \delta 136.7,132.3,128.8,128.5,127.7,127.4,119.3,24.6,17.7$; FTIR (neat) 3056, 3016, 2245, 1599. 1493, 1424, 1446, 1235, 1078, 918, 794, 766, $699 \mathrm{~cm}^{-1}$; HRMS (EI) $\mathrm{m} / z$ calculated for $\mathrm{C}_{11} \mathrm{H}_{11} \mathrm{~N}[\mathrm{M}]^{+} 157.0886$, found 157.0887 . The analytical data are in agreement with the literature. ${ }^{3}$

\section{(Z)-N-(5-phenylpent-4-en-1-yl)picolinamide (1a)}<smiles>O=C(NCCC/C=C\c1ccccc1)c1ccccn1</smiles>

The title compound was prepared according to general procedures 2 and 3 from (Z)-5-phenylpent-4enenitrile S2a $(3.43 \mathrm{~g}, 21.8 \mathrm{mmol})$. The crude product was purified via flash column chromatography (3:1 Hex/EtOAc) to afford a viscous colorless oil (4.68 g, $17.6 \mathrm{mmol}, 81 \%)$.

${ }^{1} \mathbf{H}$ NMR $\left(400 \mathrm{MHz}, \mathrm{CDCl}_{3}, 25{ }^{\circ} \mathrm{C}\right) \delta 8.52(\mathrm{ddd}, J=4.8,1.7,0.9 \mathrm{~Hz}, 1 \mathrm{H}), 8.19(\mathrm{dt}, J=7.8,1.1 \mathrm{~Hz}$, $1 \mathrm{H}), 8.07$ (s, 1H), $7.83(\mathrm{td}, J=7.7,1.7 \mathrm{~Hz}, 1 \mathrm{H}), 7.41(\mathrm{ddd}, J=7.6,4.8,1.3 \mathrm{~Hz}, 1 \mathrm{H}), 7.34-7.25(\mathrm{~m}$, 4H), $7.24-7.16$ (m, 1H), 6.47 (d, $J=11.6 \mathrm{~Hz}, 1 \mathrm{H}), 5.69$ (dt, $J=11.6,7.3 \mathrm{~Hz}, 1 \mathrm{H}), 3.50$ (td, $J=7.2$, $6.2 \mathrm{~Hz}, 2 \mathrm{H}), 2.45(\mathrm{qd}, J=7.4,1.8 \mathrm{~Hz}, 2 \mathrm{H}), 1.86-1.75(\mathrm{~m}, 2 \mathrm{H}) ;{ }^{13} \mathbf{C ~ N M R}\left(100 \mathrm{MHz}, \mathrm{CDCl}_{3}, 25{ }^{\circ} \mathrm{C}\right)$ $\delta 164.4,150.1,148.1,137.6,137.4,131.7,129.9,128.8,128.3,126.7,126.2,126.1,122.3,39.1,29.9$, 26.1; FT-IR (neat) 3382, 3055, 3009, 2930, 2863, 1667, 1568, 1522, 1464, 1288, 1161, 997, 819, 789, 697, $620 \mathrm{~cm}^{-1}$; HRMS (ESI) $\mathrm{m} / z$ calculated for $\mathrm{C}_{17} \mathrm{H}_{19} \mathrm{~N}_{2} \mathrm{O}[\mathrm{M}+\mathrm{H}]^{+}$267.1492, found 267.1495. The analytical data are in agreement with the literature. ${ }^{4}$ 


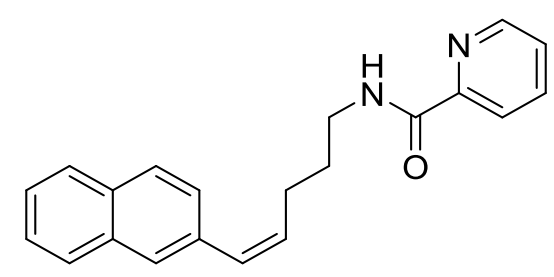

The title compound was prepared according to general procedures 2 and 3 from (Z)-5-(naphthalen-2yl)pent-4-enenitrile ${ }^{5}(600 \mathrm{mg}, 2.89 \mathrm{mmol})$. The crude product was purified via flash column chromatography (4:1 Hex/EtOAc) to afford a viscous faint yellow oil (592 mg, $1.87 \mathrm{mmol}, 65 \%$ ).

${ }^{1} \mathbf{H}$ NMR $\left(400 \mathrm{MHz}, \mathrm{CDCl}_{3}, 25{ }^{\circ} \mathrm{C}\right) \delta 8.41(\mathrm{~d}, J=4.7 \mathrm{~Hz}, 1 \mathrm{H}), 8.19-8.15(\mathrm{~m}, 1 \mathrm{H}), 8.05(\mathrm{~s}, 1 \mathrm{H}), 7.78$ $(\mathrm{m}, 4 \mathrm{H}), 7.71(\mathrm{~d}, J=1.7 \mathrm{~Hz}, 1 \mathrm{H}), 7.50-7.33(\mathrm{~m}, 4 \mathrm{H}), 6.63(\mathrm{~d}, J=11.6 \mathrm{~Hz}, 1 \mathrm{H}), 5.78(\mathrm{dt}, J=11.6$, $7.3 \mathrm{~Hz}, 2 \mathrm{H}), 3.51(\mathrm{td}, J=7.1,6.2 \mathrm{~Hz}, 2 \mathrm{H}), 2.54(\mathrm{qd}, J=7.4,1.8 \mathrm{~Hz}, 2 \mathrm{H}), 1.84(\mathrm{p}, J=7.3 \mathrm{~Hz}, 2 \mathrm{H}) ;{ }^{13} \mathbf{C}$ NMR $\left(100 \mathrm{MHz}, \mathrm{CDCl}_{3}, 25{ }^{\circ} \mathrm{C}\right) \delta 164.4,150.1,148.1,137.4,135.1,133.5,132.3,132.1,130.0$, 128.1, 127.8, 127.7, 127.5, 127.3, 126.14, 126.11, 125.8, 122.2, 39.0, 29.9, 26.1; FT-IR (neat) 3381, 3055, 3009, 2930, 2863, 1668, 1590, 1569, 1522, 1464, 1434, 1304, 1288, 1243, 1088, 1041, 998, 897, 860, 821, 749, $621 \mathrm{~cm}^{-1}$; HRMS (ESI) $\mathrm{m} / z$ calculated for $\mathrm{C}_{21} \mathrm{H}_{21} \mathrm{~N}_{2} \mathrm{O}[\mathrm{M}+\mathrm{H}]^{+} 317.1648$, found 317.1656; TLC: $R_{f}=0.28($ DCM/EtAOc 97:3).

\section{(Z)-5-(4-methoxyphenyl)pent-4-enenitrile (S2c)}

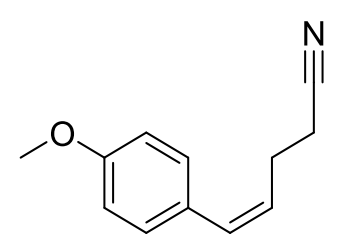

The title compound was prepared according to general procedure 1 from 4-methoxybenzaldehyde (905 mg, $6.65 \mathrm{mmol}$ ). The reaction was filtered through a silica plug using 3:1 Hex/EtOAc as eluent and the crude product was purified via flash column chromatography (4:1 Hex/EtOAc) to afford a pale yellow oil (1.02 g, $5.45 \mathrm{mmol}, 82 \%)$.

${ }^{1} \mathbf{H}$ NMR $\left(400 \mathrm{MHz}, \mathrm{CDCl}_{3}, 25{ }^{\circ} \mathrm{C}\right) \delta 7.20-7.15(\mathrm{~m}, 2 \mathrm{H}), 6.89(\mathrm{~d}, J=8.7 \mathrm{~Hz}, 2 \mathrm{H}), 6.56-6.51(\mathrm{~m}$, $1 \mathrm{H}), 5.60-5.52(\mathrm{~m}, 1 \mathrm{H}), 3.82(\mathrm{~s}, 3 \mathrm{H}), 2.73-2.63(\mathrm{~m}, 2 \mathrm{H}), 2.44(\mathrm{td}, J=7.3,0.6 \mathrm{~Hz}, 2 \mathrm{H}) ;{ }^{13} \mathbf{C} \mathbf{~ N M R}$ $\left(100 \mathrm{MHz}, \mathrm{CDCl}_{3}, 25^{\circ} \mathrm{C}\right) \delta 158.9,131.7,130.0,129.3,126.2,119.4,114.0,55.4,24.6,17.8$; FT-IR (neat) 3011, 2957, 2935, 2837, 2245, 1607, 1509, 1463, 1302, 1244, 1174, 1030, 838, 730, $527 \mathrm{~cm}^{-1}$; HRMS (EI) $\mathrm{m} / z$ calculated for $\mathrm{C}_{12} \mathrm{H}_{13} \mathrm{ON}[\mathrm{M}]^{+} 187.0992$, found 187.0986. The analytical data are in agreement with the literature. ${ }^{3}$ 


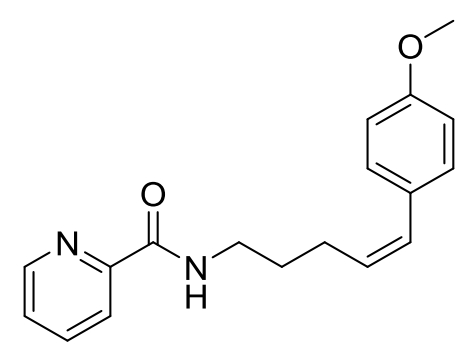

The title compound was prepared according to general procedures 2 and 3 from (Z)-5-(4methoxyphenyl)pent-4-enenitrile S2c (600 mg, $3.20 \mathrm{mmol})$. The crude product was purified via flash column chromatography (2:1 Hex/EtOAc) to afford a pale yellow oil (564 mg, $1.90 \mathrm{mmol}, 59 \%)$.

${ }^{1} \mathbf{H}$ NMR $\left(400 \mathrm{MHz}, \mathrm{CDCl}_{3}, 25{ }^{\circ} \mathrm{C}\right) \delta 8.52(\mathrm{ddd}, J=4.8,1.7,0.9 \mathrm{~Hz}, 1 \mathrm{H}), 8.18(\mathrm{dt}, J=7.8,1.1 \mathrm{~Hz}$, $1 \mathrm{H}), 8.06(\mathrm{~s}, 1 \mathrm{H}), 7.83(\mathrm{td}, J=7.7,1.7 \mathrm{~Hz}, 1 \mathrm{H}), 7.41(\mathrm{ddd}, J=7.6,4.8,1.3 \mathrm{~Hz}, 1 \mathrm{H}), 7.22-7.18(\mathrm{~m}$, $2 \mathrm{H}), 6.86-6.81(\mathrm{~m}, 2 \mathrm{H}), 6.40(\mathrm{dd}, J=11.6,1.9 \mathrm{~Hz}, 1 \mathrm{H}), 5.59(\mathrm{dt}, J=11.6,7.3 \mathrm{~Hz}, 1 \mathrm{H}), 3.79(\mathrm{~s}, 3 \mathrm{H})$, $3.50(\mathrm{td}, J=7.2,6.1 \mathrm{~Hz}, 2 \mathrm{H}), 2.43(\mathrm{qd}, J=7.3,1.8 \mathrm{~Hz}, 2 \mathrm{H}), 1.86-1.75(\mathrm{~m}, 2 \mathrm{H}) ;{ }^{13} \mathbf{C} \mathbf{N M R}(100$ $\left.\mathrm{MHz}, \mathrm{CDCl}_{3}, 25{ }^{\circ} \mathrm{C}\right) \delta 164.4,158.3,150.1,148.1,137.4,130.2,130.1,130.0,129.3,126.2,122.3$, 113.7, 55.3, 39.1, 30.0, 26.1; FT-IR (neat) 3384, 3007, 2934, 2836, 1673, 1607, 1591, 1570, 1525, 1510, 1465, 1246, 1175, 1034, 840,750, $621 \mathrm{~cm}^{-1}$; HRMS (ESI) $\mathrm{m} / z$ calculated for $\mathrm{C}_{18} \mathrm{H}_{21} \mathrm{~N}_{2} \mathrm{O}_{2}$ $[\mathrm{M}+\mathrm{H}]^{+}$297.1598, found 297.1958; TLC: $R_{f}=0.35(3: 1 \mathrm{Hex} / \mathrm{EtOAc})$.

(Z)- $N$-(5-(4-formylphenyl)pent-4-en-1-yl)picolinamide (1d)<smiles>O=Cc1ccc(/C=C\CCCNC(=O)c2ccccn2)cc1</smiles>

A flame-dried flask under $\mathrm{N}_{2}$ was charged with (Z)- $N$-(5-(4-bromophenyl)pent-4-en-1-yl)picolinamide 1h (290 mg, $0.84 \mathrm{mmol}, 1.0$ equiv), anhydrous THF ( $8.4 \mathrm{~mL})$, and cooled to $-78{ }^{\circ} \mathrm{C} . n \mathrm{BuLi}(1.6 \mathrm{M}$ in hexanes, $1.26 \mathrm{~mL}, 2.01 \mathrm{mmol}, 2.4$ equiv) was added dropwise, the reaction was stirred for $45 \mathrm{~min}$ at $-78{ }^{\circ} \mathrm{C}$, then DMF ( $0.33 \mathrm{~mL}, 4.20 \mathrm{mmol}, 5.0$ equiv) was added, the reaction was allowed to warm to room temperature and stirred for an additional $3 \mathrm{~h} .1 \mathrm{M}$ aq $\mathrm{HCl}(8 \mathrm{~mL})$ was added, the reaction was stirred for $10 \mathrm{~min}$ and subsequently quenched by addition of sat aq $\mathrm{NaHCO}_{3}(\mathrm{pH}>8)$ and extracted with EtOAc $(2 \times 40 \mathrm{~mL})$. The combined organic phases were washed with brine, dried over $\mathrm{Na}_{2} \mathrm{SO}_{4}$, concentrated, and purified by flash column chromatography (2:1 Hex/EA) to afford a pale yellow oil (55 mg, $0.19 \mathrm{mmol}, 22 \%)$. 
${ }^{1} \mathbf{H}$ NMR $\left(400 \mathrm{MHz}, \mathrm{CDCl}_{3}, 25{ }^{\circ} \mathrm{C}\right) \delta 9.95(\mathrm{~s}, 1 \mathrm{H}), 8.49(\mathrm{ddd}, J=4.8,1.7,0.9 \mathrm{~Hz}, 1 \mathrm{H}), 8.16(\mathrm{dt}, J=$ 7.8, 1.1 Hz, 1H), $8.04(\mathrm{~s}, 1 \mathrm{H}), 7.83(\mathrm{td}, J=7.7,1.7 \mathrm{~Hz}, 1 \mathrm{H}), 7.80-7.77(\mathrm{~m}, 2 \mathrm{H}), 7.43-7.36(\mathrm{~m}, 3 \mathrm{H})$, $6.50(\mathrm{dt}, J=11.7,1.9 \mathrm{~Hz}, 1 \mathrm{H}), 5.84(\mathrm{dt}, J=11.7,7.4 \mathrm{~Hz}, 1 \mathrm{H}), 3.49(\mathrm{td}, J=7.0,6.2 \mathrm{~Hz}, 2 \mathrm{H}), 2.45(\mathrm{qd}$, $J=7.5,1.8 \mathrm{~Hz}, 2 \mathrm{H}), 1.87-1.74(\mathrm{~m}, 2 \mathrm{H}) ;{ }^{13} \mathbf{C} \mathbf{~ N M R}\left(100 \mathrm{MHz}, \mathrm{CDCl}_{3}, 25{ }^{\circ} \mathrm{C}\right) \delta 191.9,164.4,149.9$, 148.1, 143.9, 137.5, 134.7, 134.6, 129.8, 129.4, 129.0, 126.3, 122.3, 38.9, 29.7, 26.2; FT-IR (neat) 3382, 3056, 3010, 2929, 2857, 2734, 1693, 1668, 1603, 1567, 1522, 1464, 1434,1306, 1213, 997, 839, 750, 691, $621 \mathrm{~cm}^{-1}$; HRMS (ESI) $\mathrm{m} / z$ calculated for $\mathrm{C}_{18} \mathrm{H}_{19} \mathrm{~N}_{2} \mathrm{O}_{2}[\mathrm{M}+\mathrm{H}]^{+}$295.1441, found 295.1443; TLC: $R_{f}=0.29(97: 3 \mathrm{DCM} / \mathrm{EtOAc})$.

\section{(Z)-5-(4-(trifluoromethyl)phenyl)pent-4-enenitrile (S2e)}<smiles>N#CCC/C=C\c1ccc(C(F)(F)F)cc1</smiles>

The title compound was prepared according to general procedure 1 from 4(trifluoromethyl)benzaldehyde $(1.16 \mathrm{~g}, 6.65 \mathrm{mmol})$. The reaction was filtered through a silica plug using 3:1 Hex/EtOAc as eluent and the crude product was purified via flash column chromatography (30:1 to 5:1 Hex/EtOAc) to afford a colorless oil $(885 \mathrm{mg}, 3.93 \mathrm{mmol}, 59 \%)$.

${ }^{1} \mathbf{H}$ NMR $\left(400 \mathrm{MHz}, \mathrm{CDCl}_{3}, 25{ }^{\circ} \mathrm{C}\right) \delta 7.61(\mathrm{~d}, J=8.1 \mathrm{~Hz}, 2 \mathrm{H}), 7.35(\mathrm{~d}, J=8.0 \mathrm{~Hz}, 2 \mathrm{H}), 6.65(\mathrm{~d}, J=$ $11.6 \mathrm{~Hz}, 1 \mathrm{H}), 5.77(\mathrm{dt}, J=11.6,7.2 \mathrm{~Hz}, 1 \mathrm{H}), 2.65(\mathrm{qd}, J=7.2,1.8 \mathrm{~Hz}, 2 \mathrm{H}), 2.46(\mathrm{t}, J=7.1 \mathrm{~Hz}, 2 \mathrm{H})$; ${ }^{13} \mathrm{C}$ NMR $\left(100 \mathrm{MHz}, \mathrm{CDCl}_{3}, 25{ }^{\circ} \mathrm{C}\right) \delta 140.2(\mathrm{~d}, J=1.5 \mathrm{~Hz}), 131.2,129.7,129.4(\mathrm{q}, J=32.6 \mathrm{~Hz})$, 129.0, 125.5 (q, $J=3.8 \mathrm{~Hz}), 124.2(\mathrm{q}, J=272.2 \mathrm{~Hz}), 119.0,24.5,17.6 ;{ }^{19} \mathbf{F} \mathbf{N M R}\left(376 \mathrm{MHz}, \mathrm{CDCl}_{3}\right.$, $\left.25{ }^{\circ} \mathrm{C}\right) \delta-62.43$; FT-IR (neat) 3021, 2935, 2247, 1617, 1428, 1403, 1321, 1162, 1109, 1066, 1016, 852, $597 \mathrm{~cm}^{-1}$; HRMS (ESI) $\mathrm{m} / z$ calculated for $\mathrm{C}_{12} \mathrm{H}_{20} \mathrm{~F}_{3} \mathrm{NNa}[\mathrm{M}+\mathrm{Na}]^{+} 248.0658$, found 248.0656; TLC: $R_{f}=0.26(5: 1 \mathrm{Hex} / \mathrm{EtOAc})$.

\section{(Z)-N-(5-(4-(trifluoromethyl)phenyl)pent-4-en-1-yl)picolinamide (1e)}<smiles>O=C(NCCC/C=C\c1ccc(C(F)(F)F)cc1)c1ccccn1</smiles> 
The title compound was prepared according to general procedures 2 and 3 from (Z)-5-(4(trifluoromethyl)phenyl)pent-4-enenitrile S2e $(490 \mathrm{mg}, 2.18 \mathrm{mmol})$. The crude product was purified via flash column chromatography (3:1 Hex/EtOAc) to afford an amorphous off-white solid (300 mg, $0.90 \mathrm{mmol}, 41 \%)$.

${ }^{1} \mathbf{H}$ NMR $\left(400 \mathrm{MHz}, \mathrm{CDCl}_{3}, 25{ }^{\circ} \mathrm{C}\right) \delta 8.50(\mathrm{ddd}, J=4.8,1.8,0.9 \mathrm{~Hz}, 1 \mathrm{H}), 8.17(\mathrm{dt}, J=7.8,1.1 \mathrm{~Hz}$, $1 \mathrm{H}), 8.04(\mathrm{~s}, 1 \mathrm{H}), 7.84(\mathrm{td}, J=7.7,1.7 \mathrm{~Hz}, 1 \mathrm{H}), 7.52(\mathrm{~d}, J=8.1 \mathrm{~Hz}, 2 \mathrm{H}), 7.41$ (ddd, $J=7.6,4.8,1.3$ $\mathrm{Hz}, 1 \mathrm{H}), 7.34(\mathrm{~d}, J=8.0 \mathrm{~Hz}, 2 \mathrm{H}), 6.48(\mathrm{~d}, J=11.6 \mathrm{~Hz}, 1 \mathrm{H}), 5.80(\mathrm{dt}, J=11.7,7.4 \mathrm{~Hz}, 1 \mathrm{H}), 3.49(\mathrm{td}, J$ $=7.0,6.2 \mathrm{~Hz}, 2 \mathrm{H}), 2.42(\mathrm{qd}, J=7.5,1.8 \mathrm{~Hz}, 2 \mathrm{H}), 1.81(\mathrm{p}, J=7.2 \mathrm{~Hz}, 2 \mathrm{H}) ;{ }^{13} \mathbf{C}$ NMR $(100 \mathrm{MHz}$, $\left.\mathrm{CDCl}_{3}, 25^{\circ} \mathrm{C}\right) \delta 164.4,150.0,148.1,141.1(\mathrm{~d}, J=1.5 \mathrm{~Hz}), 137.5,133.8,128.74,128.67(\mathrm{q}, J=32.4$ $\mathrm{Hz}), 126.3,125.2$ (q, $J=3.8 \mathrm{~Hz}), 124.4$ (q, $J=271.8 \mathrm{~Hz}), 122.3,38.9,29.8,26.0 ;{ }^{19} \mathbf{F}$ NMR $(376$ $\left.\mathrm{MHz}, \mathrm{CDCl}_{3}, 25{ }^{\circ} \mathrm{C}\right) \delta-62.29$; FT-IR (neat) 3391, 3059, 3015, 2935, 1673, 1525, 1325, 1163, 1119 , 1067, 856, 749, $621 \mathrm{~cm}^{-1}$; HRMS (ESI) $\mathrm{m} / z$ calculated for $\mathrm{C}_{18} \mathrm{H}_{18} \mathrm{~F}_{3} \mathrm{~N}_{2} \mathrm{O}[\mathrm{M}+\mathrm{H}]^{+}$335.1366, found 335.1369; Melting point $40.6-41.5^{\circ} \mathrm{C}$; TLC: $R_{f}=0.18$ (97:3 DCM/EtOAc).

\section{(Z)-5-(3,5-bis(trifluoromethyl)phenyl)pent-4-enenitrile (S2f)}

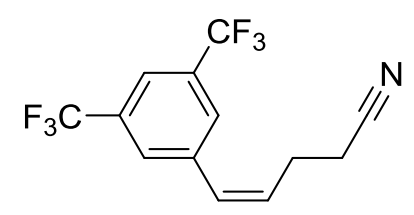

The title compound was prepared according to general procedure 1 from 3,5bis(trifluoromethyl)benzaldehyde $(1.61 \mathrm{~g}, 6.65 \mathrm{mmol})$. The reaction was filtered through a silica plug using 3:1 Hex/EtOAc as eluent and the crude product was purified via flash column chromatography (15:1 to 5:1 Hex/EtOAc) to afford a pale yellow oil (702 $\mathrm{mg}, 2.39 \mathrm{mmol}, 36 \%)$.

${ }^{1} \mathbf{H}$ NMR $\left(500 \mathrm{MHz}, \mathrm{CDCl}_{3}, 25{ }^{\circ} \mathrm{C}\right) \delta 7.79(\mathrm{~s}, 1 \mathrm{H}), 7.67(\mathrm{~s}, 2 \mathrm{H}), 6.68(\mathrm{dt}, J=11.7,2.0 \mathrm{~Hz}, 1 \mathrm{H}), 5.88$ (dt, $J=11.6,7.2 \mathrm{~Hz}, 1 \mathrm{H}), 2.63(\mathrm{qdd}, J=7.1,1.9,0.6 \mathrm{~Hz}, 2 \mathrm{H}), 2.49(\mathrm{td}, J=7.0,0.7 \mathrm{~Hz}, 2 \mathrm{H}) ; 138.5$, $132.0(\mathrm{q}, J=33.3 \mathrm{~Hz}), 131.2,129.8,129.0-128.2(\mathrm{~m}), 123.3(\mathrm{q}, J=273.0 \mathrm{~Hz}), 121.2(\mathrm{p}, J=3.8 \mathrm{~Hz})$, 118.7, 24.4, 17.6; ${ }^{19} \mathbf{F}$ NMR (471 MHz, $\left.\mathrm{CDCl}_{3}, 25{ }^{\circ} \mathrm{C}\right) \delta-62.81$; FT-IR (neat) 3028, 2934, 2249, $1616,1364,1275,1166,1123,897,847,706,680 \mathrm{~cm}^{-1} ;$ HRMS (EI) $\mathrm{m} / z$ calculated for $\mathrm{C}_{13} \mathrm{H}_{9} \mathrm{~F}_{6} \mathrm{~N}[\mathrm{M}]^{+}$ 293.0634, found 293.0630; TLC: $R_{f}=0.40$ (5:1 Hex/EtOAc). 


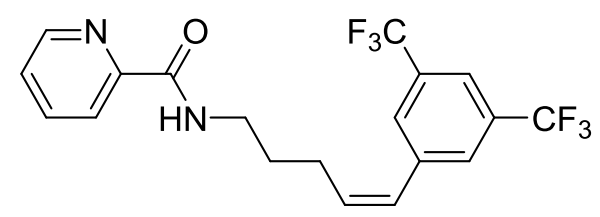

The title compound was prepared according to general procedures 2 (an additional $40 \mathrm{mg}, 1.0$ equiv, lithium aluminium hydride were added after 90 min, stirring was continued for an additional hour) and 3 from (Z)-5-(3,5-bis(trifluoromethyl)phenyl)pent-4-enenitrile S2f (310 mg, $1.06 \mathrm{mmol})$. The crude product was purified via flash column chromatography (3:1 to 2:1 Hex/EtOAc) to afford a viscous orange oil (207 mg, $0.51 \mathrm{mmol}, 49 \%)$.

${ }^{1} \mathbf{H}$ NMR $\left(500 \mathrm{MHz}, \mathrm{CDCl}_{3}, 25{ }^{\circ} \mathrm{C}\right) \delta 8.50(\mathrm{ddd}, J=4.8,1.7,0.9 \mathrm{~Hz}, 1 \mathrm{H}), 8.19-8.14(\mathrm{~m}, 1 \mathrm{H}), 8.06$ (s, 1H), $7.84(\mathrm{td}, J=7.7,1.7 \mathrm{~Hz}, 1 \mathrm{H}), 7.70(\mathrm{~s}, 1 \mathrm{H}), 7.66(\mathrm{~s}, 2 \mathrm{H}), 7.42(\mathrm{ddd}, J=7.6,4.8,1.2 \mathrm{~Hz}, 1 \mathrm{H})$, $6.50(\mathrm{dt}, J=11.7,1.9 \mathrm{~Hz}, 1 \mathrm{H}), 5.91(\mathrm{dt}, J=11.6,7.4 \mathrm{~Hz}, 1 \mathrm{H}), 3.53-3.45(\mathrm{~m}, 2 \mathrm{H}), 2.41(\mathrm{qd}, J=7.4$, $1.9 \mathrm{~Hz}, 2 \mathrm{H}), 1.87-1.77(\mathrm{~m}, 2 \mathrm{H}) ;{ }^{13} \mathbf{C}$ NMR $\left(126 \mathrm{MHz}, \mathrm{CDCl}_{3}, 25{ }^{\circ} \mathrm{C}\right) \delta 164.4,149.9,148.0,139.4$, 137.6, 135.4, 131.6 (q, $J=33.1 \mathrm{~Hz}), 129.2-128.5(\mathrm{~m}), 127.3,126.3,123.5$ (q, $J=272.7 \mathrm{~Hz}), 122.4$, 120.5 - $120.3(\mathrm{~m}), 38.8,29.6,25.9 ;{ }^{19} \mathbf{F}$ NMR $\left(471 \mathrm{MHz}, \mathrm{CDCl}_{3}, 25{ }^{\circ} \mathrm{C}\right) \delta-62.80$; FT-IR (neat) 3392 , 3060, 2934, 1675, 1526, 1277, 1166, 1129, 896, $680 \mathrm{~cm}^{-1}$; HRMS (ESI) $\mathrm{m} / z$ calculated for $\mathrm{C}_{19} \mathrm{H}_{17} \mathrm{~F}_{6} \mathrm{~N}_{2} \mathrm{O}[\mathrm{M}+\mathrm{H}]^{+}$403.1240, found 403.1244; TLC: $R_{f}=0.26$ (97:3 DCM/EtOAc).

\section{(Z)-5-(4-fluorophenyl)pent-4-enenitrile (S2g)}

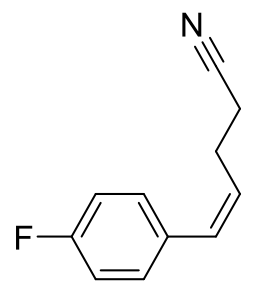

The title compound was prepared according to general procedure 1 using (3cyanopropyl)triphenylphosphonium bromide (3.00 g, $7.31 \mathrm{mmol}, 1.00$ equiv), 4-fluorobenzaldehyde (953 mg, $7.68 \mathrm{mmol}, 1.05$ equiv) and KHMDS (20 wt\% in THF, $10.0 \mathrm{~mL}, 8.77 \mathrm{mmol}, 1.20$ equiv). The reaction was filtered through a silica plug using 2:1 Hex/EtOAc as eluent and the crude product was purified via flash column chromatography $(8: 1 \mathrm{Hex} / \mathrm{EtOAc})$ to afford a pale yellow oil $(890 \mathrm{mg}$, $5.08 \mathrm{mmol}, 70 \%)$.

${ }^{1} \mathbf{H}$ NMR $\left(400 \mathrm{MHz}, \mathrm{CDCl}_{3}, 25{ }^{\circ} \mathrm{C}\right) \delta 7.24-7.16(\mathrm{~m}, 2 \mathrm{H}), 7.04(\mathrm{t}, J=8.7 \mathrm{~Hz}, 2 \mathrm{H}), 6.57(\mathrm{dtd}, J=$ $11.6,1.9,0.9 \mathrm{~Hz}, 1 \mathrm{H}), 5.64(\mathrm{dt}, J=11.5,7.1 \mathrm{~Hz}, 1 \mathrm{H}), 2.64(\mathrm{dtd}, J=7.4,6.7,1.5 \mathrm{~Hz}, 2 \mathrm{H}), 2.45(\mathrm{td}, J=$ 7.2, $0.6 \mathrm{~Hz}, 2 \mathrm{H}) ;{ }^{13} \mathbf{C} \mathbf{N M R}\left(100 \mathrm{MHz}, \mathrm{CDCl}_{3}, 25^{\circ} \mathrm{C}\right) \delta 162.0(\mathrm{~d}, J=247.0 \mathrm{~Hz}), 132.6(\mathrm{~d}, J=3.4 \mathrm{~Hz})$, 
131.3, $130.4(\mathrm{~d}, J=8.0 \mathrm{~Hz}), 127.7(\mathrm{~d}, J=1.4 \mathrm{~Hz}), 119.2,115.5(\mathrm{~d}, J=21.4 \mathrm{~Hz}), 24.4,17.7 ;{ }^{19} \mathbf{F}$ NMR (377 MHz, $\left.\mathrm{CDCl}_{3}\right) \delta$-114.40; FT-IR (neat) 3018, 2927, 2246, 1602, 1508, 1399, 1221, 1158, 1096, 1014, 841, 734, $520 \mathrm{~cm}^{-1}$; HRMS (EI) $\mathrm{m} / z$ calculated for $\mathrm{C}_{11} \mathrm{H}_{10} \mathrm{NF}[\mathrm{M}]^{+}$175.0792, found 175.0788; TLC: $R_{f}=0.28(5: 1 \mathrm{Hex} / \mathrm{EtOAc})$.

\section{(Z)-N-(5-(4-fluorophenyl)pent-4-en-1-yl)picolinamide (1g)}<smiles>O=C(NCCC/C=C\c1ccc(F)cc1)c1ccccn1</smiles>

The title compound was prepared according to general procedures 2 and 3 from (Z)-5-(4fluorophenyl)pent-4-enenitrile $\mathbf{S 2 g}(540 \mathrm{mg}, 3.08 \mathrm{mmol})$. The crude product was purified via flash column chromatography (3:2 Hex/EtOAc) to afford a faint yellow oil (571 mg, $2.00 \mathrm{mmol}, 65 \%)$.

${ }^{1} \mathrm{H}$ NMR $\left(400 \mathrm{MHz}, \mathrm{CDCl}_{3}, 25{ }^{\circ} \mathrm{C}\right) \delta 8.56-8.48(\mathrm{~m}, 1 \mathrm{H}), 8.18(\mathrm{dt}, J=7.9,1.1 \mathrm{~Hz}, 1 \mathrm{H}), 8.03(\mathrm{~s}, 1 \mathrm{H})$, $7.84(\mathrm{td}, J=7.7,1.7 \mathrm{~Hz}, 1 \mathrm{H}), 7.42(\mathrm{ddd}, J=7.6,4.8,1.2 \mathrm{~Hz}, 1 \mathrm{H}), 7.24-7.18(\mathrm{~m}, 2 \mathrm{H}), 6.97(\mathrm{t}, J=8.8$ $\mathrm{Hz}, 2 \mathrm{H}), 6.42$ (d, $J=11.6 \mathrm{~Hz}, 1 \mathrm{H}), 5.67(\mathrm{dt}, J=11.6,7.3 \mathrm{~Hz}, 1 \mathrm{H}), 3.49(\mathrm{td}, J=7.1,6.1 \mathrm{~Hz}, 2 \mathrm{H}), 2.40$ $(\mathrm{qd}, J=7.4,1.8 \mathrm{~Hz}, 2 \mathrm{H}), 1.85-1.74(\mathrm{~m}, 2 \mathrm{H}) ;{ }^{13} \mathbf{C} \mathbf{~ N M R}\left(100 \mathrm{MHz}, \mathrm{CDCl}_{3}, 25{ }^{\circ} \mathrm{C}\right) \delta 164.4,161.6(\mathrm{~d}$, $J=245.8 \mathrm{~Hz}), 150.1,148.1,137.5,133.6(\mathrm{~d}, J=3.3 \mathrm{~Hz}), 131.6,130.4(\mathrm{~d}, J=7.9 \mathrm{~Hz}), 128.9,126.2$, 122.3, $115.2(\mathrm{~d}, J=21.3 \mathrm{~Hz}), 39.0,29.8,25.9 ;{ }^{19} \mathbf{F}$ NMR $\left(377 \mathrm{MHz}, \mathrm{CDCl}_{3}\right) \delta-115.52$; FT-IR (neat) 3384, 3058, 3010, 2931, 1668, 1601, 1591, 1569, 1465, 1434, 1220, 1158, 997, 842, 821, 749, 691, 621, $522 \mathrm{~cm}^{-1}$; HRMS (ESI) $\mathrm{m} / z$ calculated for $\mathrm{C}_{17} \mathrm{H}_{18} \mathrm{FN}_{2} \mathrm{O}[\mathrm{M}+\mathrm{H}]^{+}$285.1398, found 285.1398; TLC: $R_{f}=0.36(3: 2 \mathrm{Hex} / \mathrm{EtOAc})$.

\section{(Z)-5-(4-bromophenyl)pent-4-enenitrile (S2h)}<smiles>N#CCC/C=C\c1ccc(Br)cc1</smiles>

A round bottom flask under an atmosphere of $\mathrm{N}_{2}$ was charged with sodium hydride $(60 \%$ in mineral oil, $1.73 \mathrm{~g}, 43.2 \mathrm{mmol}, 1.30$ equiv). The sodium hydride was washed with hexane ( $2 \times 10 \mathrm{~mL})$, suspended in $100 \mathrm{~mL}$ THF and cooled to $0{ }^{\circ} \mathrm{C}$. (3-cyanopropyl)triphenylphosphonium bromide $(15 \mathrm{~g}$, $36.6 \mathrm{mmol}, 1.10$ equiv) was added in portions, the reaction was allowed to warm to room temperature 
and stirred at this temperature for $1 \mathrm{~h}$. The reaction was re-cooled to $0{ }^{\circ} \mathrm{C}$ and 4-bromobenzaldehyde was added. After $15 \mathrm{~min}$ the reaction was allowed to warm to room temperature, stirred at this temperature for $3 \mathrm{~h}$ and poured into $250 \mathrm{~mL}$ rapidly stirred hexane. The reaction was filtered through a silica plug using 3:1 Hex/EtOAc as eluent and the crude product was purified via flash column chromatography (10:1 to 5:1 Hex/EtOAc) to afford a pale yellow oil (5.53 g, $23.4 \mathrm{mmol}, 71 \%)$.

${ }^{1} \mathbf{H}$ NMR $\left(400 \mathrm{MHz}, \mathrm{CDCl}_{3}, 25{ }^{\circ} \mathrm{C}\right) \delta 7.51-7.43(\mathrm{~m}, 2 \mathrm{H}), 7.13-7.05(\mathrm{~m}, 2 \mathrm{H}), 6.54(\mathrm{dt}, J=11.5,1.9$ $\mathrm{Hz}, 1 \mathrm{H}), 5.68(\mathrm{dt}, J=11.5,7.2 \mathrm{~Hz}, 1 \mathrm{H}), 2.67-2.59(\mathrm{~m}, 2 \mathrm{H}), 2.44(\mathrm{td}, J=7.1,0.7 \mathrm{~Hz}, 2 \mathrm{H}) ;{ }^{13} \mathbf{C} \mathbf{~ N M R}$ $\left(100 \mathrm{MHz}, \mathrm{CDCl}_{3}, 25^{\circ} \mathrm{C}\right) \delta 135.5,131.7,131.2,130.4,128.4,121.4,119.1,24.5,17.6$; FT-IR (neat) 3016, 2927, 2246, 1587, 1487, 1425, 1393, 1072, 1009, 838, 783, $513 \mathrm{~cm}^{-1}$; HRMS (EI) $\mathrm{m} / \mathrm{z}$ calculated for $\mathrm{C}_{11} \mathrm{H}_{10} \mathrm{BrN}[\mathrm{M}]^{+} 234.9991$, found 234.9989; TLC: $R_{f}=0.41$ (4:1 Hex/EtOAc).

(Z)- $N$-(5-(4-bromophenyl)pent-4-en-1-yl)picolinamide (1h)<smiles>O=C(NCCC/C=C\c1ccc(Br)cc1)c1ccccn1</smiles>

The title compound was prepared according to general procedures 2 (the reaction was quenched after $30 \mathrm{~min}$ at room temperature) and 3 from (Z)-5-(4-bromophenyl)pent-4-enenitrile $\mathbf{S 2 h}$ (4.96 g, $21.0 \mathrm{mmol}$ ). The crude product was purified via flash column chromatography (3:1 to 3:2 Hex/EtOAc) to afford a yellow oil $(3.04 \mathrm{~g}, 8.81 \mathrm{mmol}, 42 \%)$.

${ }^{1} \mathbf{H}$ NMR $\left(400 \mathrm{MHz}, \mathrm{CDCl}_{3}, 25{ }^{\circ} \mathrm{C}\right) \delta 8.53(\mathrm{ddd}, J=4.8,1.7,0.9 \mathrm{~Hz}, 1 \mathrm{H}), 8.18(\mathrm{dt}, J=7.8,1.1 \mathrm{~Hz}$, $1 \mathrm{H}), 8.03(\mathrm{~s}, 1 \mathrm{H}), 7.84(\mathrm{td}, J=7.7,1.7 \mathrm{~Hz}, 1 \mathrm{H}), 7.47-7.37(\mathrm{~m}, 3 \mathrm{H}), 7.17-7.07(\mathrm{~m}, 2 \mathrm{H}), 6.39$ (dt, $J=$ 11.6, $1.9 \mathrm{~Hz}, 1 \mathrm{H}), 5.71(\mathrm{dt}, J=11.6,7.4 \mathrm{~Hz}, 1 \mathrm{H}), 3.48(\mathrm{td}, J=7.0,6.2 \mathrm{~Hz}, 2 \mathrm{H}), 2.40(\mathrm{qd}, J=7.4,1.8$ $\mathrm{Hz}, 2 \mathrm{H}), 1.84-1.72(\mathrm{~m}, 2 \mathrm{H}) ;{ }^{13} \mathbf{C}$ NMR $\left(100 \mathrm{MHz}, \mathrm{CDCl}_{3}, 25{ }^{\circ} \mathrm{C}\right) \delta 164.4,150.0,148.1,137.5$, 136.4, 132.5, 131.4, 130.5, 128.8, 126.3, 122.3, 120.6, 39.0, 29.8, 26.0; FT-IR (neat) 3385, 3058, 3011, 2933, 1673, 1525, 1487, 1434, 1072, 1009, 841, 769, $621 \mathrm{~cm}^{-1}$; HRMS (ESI) $\mathrm{m} / \mathrm{z}$ calculated for $\mathrm{C}_{17} \mathrm{H}_{17} \mathrm{BrN}_{2} \mathrm{NaO}[\mathrm{M}+\mathrm{Na}]^{+}$367.0416, found 367.0418; TLC: $R_{f}=0.32$ (97:3 DCM/EtOAC). 


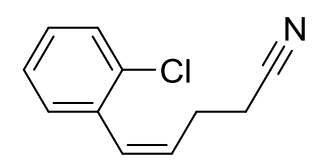

The title compound was prepared according to general procedure 1 from 2-chlorobenzaldehyde (943 mg, $6.65 \mathrm{mmol}$ ). The reaction was filtered through a silica plug using 3:1 Hex/EtOAc as eluent and the crude product was purified via flash column chromatography (20:1 Hex/EtOAc) to afford a colorless oil ( $820 \mathrm{mg}, 4.28 \mathrm{mmol}, 64 \%)$.

${ }^{1} \mathbf{H}$ NMR $\left(400 \mathrm{MHz}, \mathrm{CDCl}_{3}, 25{ }^{\circ} \mathrm{C}\right) \delta 7.41(\mathrm{ddd}, J=6.8,3.0,1.8 \mathrm{~Hz}, 1 \mathrm{H}), 7.26-7.19(\mathrm{~m}, 3 \mathrm{H}), 6.68$ (dt, $J=11.4,1.7 \mathrm{~Hz}, 1 \mathrm{H}), 5.80(\mathrm{dt}, J=11.4,7.2 \mathrm{~Hz}, 1 \mathrm{H}), 2.57-2.49(\mathrm{~m}, 2 \mathrm{H}), 2.45-2.39(\mathrm{~m}, 2 \mathrm{H})$; ${ }^{13} \mathrm{C}$ NMR $\left(100 \mathrm{MHz}, \mathrm{CDCl}_{3}, 25{ }^{\circ} \mathrm{C}\right) \delta 134.9,133.7,130.3,129.8,129.7,129.2,128.9,126.6,119.2$, 24.6, 17.5; FT-IR (neat) 3065, 3021, 2928, 2246, 1472, 1436, 1047, 1035, 762, 742, $714 \mathrm{~cm}^{-1}$; HRMS (ESI) $\mathrm{m} / z$ calculated for $\mathrm{C}_{11} \mathrm{H}_{10} \mathrm{ClNNa}[\mathrm{M}+\mathrm{Na}]^{+} 214.0394$, found 214.0395; TLC: $R_{f}=0.31(5: 1$ $\mathrm{Hex} / \mathrm{EtOAc})$.

\section{(Z)- $N$-(5-(2-chlorophenyl)pent-4-en-1-yl)picolinamide (1i)}

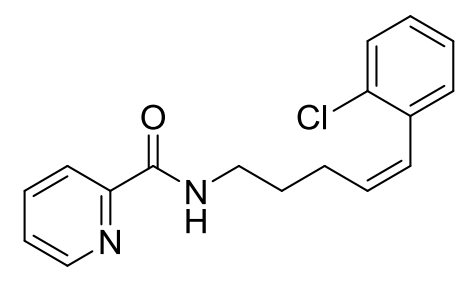

The title compound was prepared according to general procedures 2 and 3 from (Z)-5-(2chlorophenyl)pent-4-enenitrile S2i $(480 \mathrm{mg}, 2.50 \mathrm{mmol})$. The crude product was purified via flash column chromatography (3:1 Hex/EtOAc) to afford a colorless oil (517 mg, $1.72 \mathrm{mmol}, 69 \%)$.

${ }^{1} \mathbf{H}$ NMR $\left(500 \mathrm{MHz}, \mathrm{CDCl}_{3}, 25{ }^{\circ} \mathrm{C}\right) \delta 8.51(\mathrm{ddd}, J=4.8,1.7,0.9 \mathrm{~Hz}, 1 \mathrm{H}), 8.18(\mathrm{dt}, J=7.8,1.1 \mathrm{~Hz}$, $1 \mathrm{H}), 8.05(\mathrm{~s}, 1 \mathrm{H}), 7.84(\mathrm{td}, J=7.7,1.7 \mathrm{~Hz}, 1 \mathrm{H}), 7.41(\mathrm{ddd}, J=7.6,4.8,1.2 \mathrm{~Hz}, 1 \mathrm{H}), 7.38-7.34(\mathrm{~m}$, 1H), $7.28-7.24$ (m, 1H), $7.18-7.13(\mathrm{~m}, 2 \mathrm{H}), 6.55$ (dt, $J=11.7,1.8,1 \mathrm{H}), 5.82$ (dt, $J=11.5,7.5 \mathrm{~Hz}$, 1H), $3.50-3.43(\mathrm{~m}, 2 \mathrm{H}), 2.30$ (qd, $J=7.4,1.7 \mathrm{~Hz}, 2 \mathrm{H}), 1.82-1.73(\mathrm{~m}, 2 \mathrm{H}) .{ }^{13} \mathbf{C}$ NMR $(126 \mathrm{MHz}$, $\left.\mathrm{CDCl}_{3}, 25{ }^{\circ} \mathrm{C}\right) \delta 164.3,150.0,148.0,137.5,135.7,133.7,133.0,130.6,129.5,128.2,127.3,126.4$, 126.2, 122.3, 39.0, 29.7, 26.0; FT-IR (neat) 3384, 3058, 3015, 2933, 2863, 1669, 1591, 1569, 1523, 1466, 1435, 1288, 1050, 1036, 747, $621 \mathrm{~cm}^{-1}$; HRMS (ESI) $\mathrm{m} / z$ calculated for $\mathrm{C}_{17} \mathrm{H}_{17} \mathrm{ClN}_{2} \mathrm{NaO}$ $[\mathrm{M}+\mathrm{Na}]^{+}$323.0922, found 323.0921; TLC: $R_{f}=0.35(3: 2 \mathrm{Hex} / \mathrm{EtOAc})$. 


\section{(Z)-N-(5-(2,6-dimethylphenyl)pent-4-en-1-yl)picolinamide (1j)}<smiles>Cc1cccc(C)c1/C=C\CCCNC(=O)c1ccccn1</smiles>

To a suspension of (3-cyanopropyl)triphenylphosphonium bromide ( $3.0 \mathrm{~g}, 7.3 \mathrm{mmol}, 1.1$ equiv) in $20 \mathrm{~mL}$ THF at $0{ }^{\circ} \mathrm{C}$ was added KHMDS (20 wt\% in THF, $9.4 \mathrm{~mL}, 8.3 \mathrm{mmol}, 1.25$ equiv) slowly over 2 minutes, which resulted in an orange suspension. The reaction was stirred at $0{ }^{\circ} \mathrm{C}$ for 15 minutes before 2,6-dimethylbenzaldehyde ( $892 \mathrm{mg}, 6.65 \mathrm{mmol}, 1.0$ equiv) was added in one portion. The reaction was stirred an additional $30 \mathrm{~min}$ at $0{ }^{\circ} \mathrm{C}, 90 \mathrm{~min}$ at room temperature, and then poured into $100 \mathrm{~mL}$ rapidly stirred hexanes. The resulting mixture was filtered through a silica plug (washed with the indicated eluent), concentrated and purified by flash column silica gel chromatography (15:1 to 10:1 Hex/EtOAc) to afford a colorless oil (361 mg) containing (Z)-5-(2,6-dimethylphenyl)pent-4enenitrile and ca $10 \%$ of an unknown impurity.

The title compound was prepared according to general procedures 2 and 3 from the mixture prepared above $(290 \mathrm{mg})$. The crude product was purified via flash column chromatography (3:1 Hex/EtOAc) to afford a colorless oil (380 mg, $1.29 \mathrm{mmol}, 24 \%$ over 3 steps).

${ }^{1} \mathbf{H}$ NMR $\left(400 \mathrm{MHz}, \mathrm{CDCl}_{3}, 25{ }^{\circ} \mathrm{C}\right) \delta 8.51(\mathrm{ddd}, J=4.8,1.7,0.9 \mathrm{~Hz}, 1 \mathrm{H}), 8.17(\mathrm{dt}, J=7.9,1.1 \mathrm{~Hz}$, 1H), $7.98(\mathrm{~s}, 1 \mathrm{H}), 7.83(\mathrm{td}, J=7.7,1.7 \mathrm{~Hz}, 1 \mathrm{H}), 7.40(\mathrm{ddd}, J=7.6,4.8,1.2 \mathrm{~Hz}, 1 \mathrm{H}), 7.11-6.97(\mathrm{~m}$, $3 \mathrm{H}), 6.34(\mathrm{~d}, J=11.2 \mathrm{~Hz}, 1 \mathrm{H}), 5.78(\mathrm{dt}, J=11.1,7.2 \mathrm{~Hz}, 1 \mathrm{H}) ;{ }^{13} \mathbf{C ~ N M R}\left(100 \mathrm{MHz}, \mathrm{CDCl}_{3}, 25{ }^{\circ} \mathrm{C}\right) \delta$ 164.3, 150.1, 148.1, 137.4, 136.6, 136.1, 131.9, 128.5, 127.2, 126.6, 126.1, 122.3, 39.1, 29.2, 26.2, 20.5; FT-IR (neat) 3387, 3058, 3001, 2934, 2859, 1674, 1591, 1569, 1524, 1465, 1434,1289, 998, 770, 750, $621 \mathrm{~cm}^{-1}$; HRMS (ESI) $\mathrm{m} / z$ calculated for $\mathrm{C}_{19} \mathrm{H}_{22} \mathrm{~N}_{2} \mathrm{NaO}[\mathrm{M}+\mathrm{Na}]^{+} 317.1624$, found 317.1628; TLC: $R_{f}=0.63(1: 1 \mathrm{Hex} / \mathrm{EtOAc})$.

\section{(Z)-5-(2,6-dichlorophenyl)pent-4-enenitrile (S2k)}

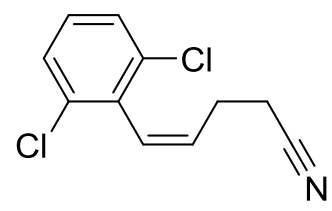

The title compound was prepared according to general procedure 1 from 2,6-dichlorobenzaldehyde $(1.16 \mathrm{~g}, 6.65 \mathrm{mmol})$. The reaction was filtered through a silica plug using 3:1 Hex/EtOAc as eluent and the crude product was purified via flash column chromatography (30:1 Hex/EtOAc) to afford an amorphous white solid (441 mg, $1.95 \mathrm{mmol}, 29 \%$ ). 
${ }^{1} \mathbf{H}$ NMR $\left(400 \mathrm{MHz}, \mathrm{CDCl}_{3}, 25{ }^{\circ} \mathrm{C}\right) \delta 7.34(\mathrm{~d}, J=8.0 \mathrm{~Hz}, 2 \mathrm{H}), 7.18(\mathrm{ddd}, J=8.5,7.5,0.8 \mathrm{~Hz}, 1 \mathrm{H})$, $6.39(\mathrm{dt}, J=10.9,1.6 \mathrm{~Hz}, 1 \mathrm{H}), 5.93(\mathrm{dt}, J=11.1,7.3 \mathrm{~Hz}, 1 \mathrm{H}), 2.44-2.37(\mathrm{~m}, 2 \mathrm{H}), 2.32-2.25(\mathrm{~m}$, 2H); ${ }^{13} \mathbf{C}$ NMR $\left(100 \mathrm{MHz}, \mathrm{CDCl}_{3}, 25{ }^{\circ} \mathrm{C}\right) \delta 134.7,134.2,131.4,129.2,128.1,126.8,119.2,25.4$, 16.7; FT-IR (neat) 3080, 3053, 3026, 2934, 2246, 1557, 1427, 1193, 1088, 785, 770, $747 \mathrm{~cm}^{-1}$; HRMS (EI) $\mathrm{m} / z$ calculated for $\mathrm{C}_{11} \mathrm{H}_{9} \mathrm{Cl}_{2} \mathrm{~N}[\mathrm{M}]^{+}$225.0107, found 225.0104; Melting point 38.0 $39.2{ }^{\circ} \mathrm{C}$; TLC: $R_{f}=0.60(3: 1 \mathrm{Hex} / \mathrm{EtOAc})$.

(Z)- $N$-(5-(2,6-dichlorophenyl)pent-4-en-1-yl)picolinamide (1k)

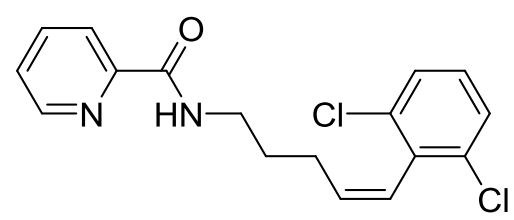

The title compound was prepared according to general procedures 2 and 3 from (Z)-5-(2,6dichlorophenyl)pent-4-enenitrile S2k (300 mg, $1.33 \mathrm{mmol}$ ). The crude product was purified via flash column chromatography ( $3: 1$ to $3: 2 \mathrm{Hex} / \mathrm{EtOAc}$ ) to afford an off-white solid (327 $\mathrm{mg}, 0.98 \mathrm{mmol}$, $73 \%)$.

${ }^{1} \mathbf{H}$ NMR $\left(400 \mathrm{MHz}, \mathrm{CDCl}_{3}, 25{ }^{\circ} \mathrm{C}\right) \delta 8.50(\mathrm{ddd}, J=4.8,1.8,0.9 \mathrm{~Hz}, 1 \mathrm{H}), 8.17(\mathrm{dt}, J=7.8,1.1 \mathrm{~Hz}$, $1 \mathrm{H}), 8.02(\mathrm{~s}, 1 \mathrm{H}), 7.83(\mathrm{td}, J=7.7,1.7 \mathrm{~Hz}, 1 \mathrm{H}), 7.40(\mathrm{ddd}, J=7.6,4.8,1.3 \mathrm{~Hz}, 1 \mathrm{H}), 7.29$ (d, $J=8.0$ $\mathrm{Hz}, 2 \mathrm{H}), 7.14-7.09$ (m, 1H), 6.27 (dt, $J=11.2,1.7 \mathrm{~Hz}, 1 \mathrm{H}), 5.92$ (dt, $J=11.2,7.4 \mathrm{~Hz}, 1 \mathrm{H}), 3.42$ (td, $J=7.2,6.2 \mathrm{~Hz}, 2 \mathrm{H}), 2.03(\mathrm{qd}, J=7.4,1.6 \mathrm{~Hz}, 2 \mathrm{H}), 1.79-1.67(\mathrm{~m}, 2 \mathrm{H}) ;{ }^{13} \mathbf{C} \mathbf{~ N M R}\left(100 \mathrm{MHz}, \mathrm{CDCl}_{3}\right.$, $\left.25^{\circ} \mathrm{C}\right) \delta 164.3,150.1,148.0,137.5,135.2,135.2,134.9,128.6,127.9,126.1,124.6,122.3,39.0,28.7$, 26.9; FT-IR (neat) 3387, 3057, 3014, 2934, 2864, 1672, 1590, 1524, 1428, 1288, 1088, 998, 778, 748, $621 \mathrm{~cm}^{-1}$; HRMS (ESI) $\mathrm{m} / z$ calculated for $\mathrm{C}_{17} \mathrm{H}_{17} \mathrm{Cl}_{2} \mathrm{~N}_{2} \mathrm{O}[\mathrm{M}+\mathrm{H}]^{+} 335.0712$, found 335.0712; Melting point $53.2-54.4{ }^{\circ} \mathrm{C}$; TLC: $R_{f}=0.42(3: 2 \mathrm{Hex} / \mathrm{EtOAc})$.

$1 \mathrm{k}-\mathrm{D}_{1}$<smiles>[2H]/C(=C/c1c(Cl)cccc1Cl)CCCNC(=O)c1ccccn1</smiles>

A $1.6 \mathrm{~mL}$ screw-cap vial under ambient atmosphere was charged with (Z)- $N$-(5-(2,6dichlorophenyl)pent-4-en-1-yl)picolinamide $1 \mathrm{k}$ (33 mg, $0.10 \mathrm{mmol}, 1.0$ equiv), pivalic acid (10 mg, $0.10 \mathrm{mmol}, 1.0$ equiv), $\mathrm{Pd}(\mathrm{OAc})_{2}\left(2.3 \mathrm{mg}, 0.010 \mathrm{mmol}, 0.10\right.$ equiv), $\mathrm{D}_{2} \mathrm{O}(0.20 \mathrm{~mL})$, and $\mathrm{CD}_{3} \mathrm{CN}$ 
$(0.80 \mathrm{~mL})$. The vial was sealed and stirred in a pre-heated heating block at $90{ }^{\circ} \mathrm{C}$ for $6 \mathrm{~h}$. The reaction was allowed to cool to room temperature, quenched by addition of aq. $\mathrm{Na}_{2} \mathrm{~S}_{2} \mathrm{O}_{3}(5 \mathrm{wt} \%, 10 \mathrm{~mL}$ ), brine $(10 \mathrm{~mL})$ and sat. aq. $\mathrm{NaHCO}_{3}(10 \mathrm{~mL})$, extracted with EtOAc $(2 \times 30 \mathrm{~mL})$, dried over $\mathrm{Na}_{2} \mathrm{SO}_{4}$ and concentrated. The crude residue was purified by flash column silica gel chromatography (97:3 DCM/EtOAc) to afford a colorless oil $(29 \mathrm{mg}, 0.086 \mathrm{mmol}, 86 \%)$. The amount of deuterium incorporation was determined to be greater than $95 \%$ by ${ }^{1} \mathrm{H}$ NMR spectroscpopy.

${ }^{1} \mathbf{H}$ NMR $\left(500 \mathrm{MHz}, \mathrm{CDCl}_{3}, 25{ }^{\circ} \mathrm{C}\right) \delta 8.50(\mathrm{ddd}, J=4.8,1.7,0.9 \mathrm{~Hz}, 1 \mathrm{H}), 8.17-8.13(\mathrm{~m}, 1 \mathrm{H}), 8.01$ (s, 1H), $7.82(\mathrm{td}, J=7.7,1.7 \mathrm{~Hz}, 1 \mathrm{H}), 7.40(\mathrm{ddd}, J=7.6,4.8,1.3 \mathrm{~Hz}, 1 \mathrm{H}), 7.30-7.27(\mathrm{~m}, 2 \mathrm{H}), 7.11$ (ddd, $J=8.4,7.6,0.7 \mathrm{~Hz}, 1 \mathrm{H}), 6.26(\mathrm{~s}, 1 \mathrm{H}), 3.46-3.36(\mathrm{~m}, 2 \mathrm{H}), 2.03(\mathrm{td}, J=7.4,1.6 \mathrm{~Hz}, 2 \mathrm{H}), 1.78-$ $1.70(\mathrm{~m}, 2 \mathrm{H}) ;{ }^{13} \mathbf{C}$ NMR $\left(126 \mathrm{MHz}, \mathrm{CDCl}_{3}, 25{ }^{\circ} \mathrm{C}\right.$, all peaks reported) $\delta 164.3,150.1,148.0,137.4$, 135.2, 135.0, 134.9, 134.8, 134.6, 128.6, 127.9, 126.1, 124.4, 122.3, 39.0, 28.7, 26.7; FT-IR (neat) 3387, 3057, 2933, 2861, 2228, 1671, 1524, 1428, 1288, 1091, 998, 775, 749, $621 \mathrm{~cm}^{-1}$; HRMS (ESI) $m / z$ calculated for $\mathrm{C}_{17} \mathrm{H}_{16} \mathrm{Cl}_{2} \mathrm{DN}_{2} \mathrm{O}[\mathrm{M}+\mathrm{H}]^{+} 336.0775$, found 336.0781 .

\section{1k-D}<smiles>[2H]/C(=C/c1c(Cl)cccc1Cl)CC([2H])([2H])CNC(=O)c1ccccn1</smiles>

Based on a literature protocol $^{6}$ sodium hydride (60\% in mineral oil, $238 \mathrm{mg}, 5.94 \mathrm{mmol}, 1.60$ equiv) was suspended in DMSO- $d_{6}(20 \mathrm{~g})$ and heated to $80^{\circ} \mathrm{C}$ for $1 \mathrm{~h}$ to obtain a yellow solution. The reaction was allowed to cool to room temperature, (3-cyanopropyl)triphenylphosphonium bromide $(1.67 \mathrm{~g}, 4.09 \mathrm{mmol}, 1.10$ equiv) was added and the reaction was stirred for $90 \mathrm{~min}$. 2,6Dichlorobenzaldehyde (650 mg, $3.71 \mathrm{mmol}, 1.00$ equiv) was added in one portion. The reaction was stirred at room temperature for $90 \mathrm{~min}$, quenched by addition of $\mathrm{D}_{2} \mathrm{O}(0.7 \mathrm{~mL})$ followed by addition of $\mathrm{H}_{2} \mathrm{O}(20 \mathrm{~mL})$. The reaction was diluted with EtOAc $(150 \mathrm{~mL})$ and water $(100 \mathrm{~mL})$, the phases were separated and the organic phase was washed with sat. aq. $\mathrm{NH}_{4} \mathrm{Cl}(1 \times 50 \mathrm{~mL})$, brine $(1 \times 50 \mathrm{~mL})$, dried over $\mathrm{MgSO}_{4}$ and concentrated to obtain a brown solid. The crude product was redissolved in a small amount of EtOAc, precipitated by addition to hexane $(100 \mathrm{~mL})$, filtered through a silica plug (washed with 5:1 Hex/EtOAc), concentrated and purified by flash column chromatography (40:1 to 20:1) to afford the crude nitrile as a colorless oil (324 mg).

The title compound was prepared according to general procedures 2 and 3 from the crude nitrile prepared above $(290 \mathrm{mg}, 1.27 \mathrm{mmol})$. The crude product was purified via flash column chromatography (3:1 Hex/EtOAc) to afford a white solid ( $180 \mathrm{mg}, 0.53 \mathrm{mmol}, 16 \%$ over 3 steps). The 
deuterium-content at the vinylic position was determined to be approximately $92 \%$, the deuteriumcontent at the aliphatic position was determined to be approximately $95 \%$ by ${ }^{1} \mathrm{H}-\mathrm{NMR}$ spectroscopy.

${ }^{1} \mathbf{H}$ NMR $\left(500 \mathrm{MHz}, \mathrm{CD}_{3} \mathrm{CN}, 25{ }^{\circ} \mathrm{C}\right) \delta 8.53(\mathrm{ddd}, J=4.8,1.7,0.9 \mathrm{~Hz}, 1 \mathrm{H}), 8.03(\mathrm{dt}, J=7.8,1.1 \mathrm{~Hz}$, 2H), $7.89(\mathrm{td}, J=7.7,1.7 \mathrm{~Hz}, 1 \mathrm{H}), 7.49(\mathrm{ddd}, J=7.6,4.8,1.3 \mathrm{~Hz}, 1 \mathrm{H}), 7.35(\mathrm{dt}, J=8.0,0.4 \mathrm{~Hz}, 2 \mathrm{H})$, $7.20(\mathrm{ddd}, J=8.5,7.6,0.7 \mathrm{~Hz}, 1 \mathrm{H}), 6.29-6.22(\mathrm{~m}, 1 \mathrm{H}), 3.30(\mathrm{~d}, J=6.3 \mathrm{~Hz}, 2 \mathrm{H}), 1.99-1.89(\mathrm{~m}, 4 \mathrm{H})$.

${ }^{13} \mathrm{C}$ NMR $\left(126 \mathrm{MHz}, \mathrm{CD}_{3} \mathrm{CN}, 25{ }^{\circ} \mathrm{C}\right.$, all peaks reported) $\delta 164.9,151.1,149.3,138.5,136.4,136.3$, 136.1, 135.9, 135.4, 130.1, 128.9, 127.2, 125.0, 124.8, 122.6, 39.3, 39.2, 28.5, 27.2, 27.1; HRMS (ESI) $\mathrm{m} / z$ calculated for $\mathrm{C}_{17} \mathrm{H}_{14} \mathrm{Cl}_{2} \mathrm{D}_{3} \mathrm{~N}_{2} \mathrm{O}[\mathrm{M}+\mathrm{H}]^{+} 338.0901$, found 338.0907.

\section{(Z)-5-(2-bromo-6-chlorophenyl)pent-4-enenitrile (S2I)}

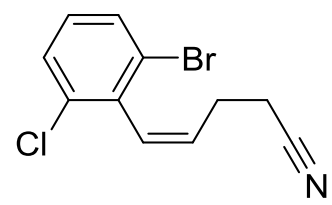

The title compound was prepared according to general procedure 1 from 2-bromo-6chlorobenzaldehyde $(1.46 \mathrm{~g}, 6.65 \mathrm{mmol})$. The reaction was filtered through a silica plug using 3:1 Hex/EtOAc as eluent and the crude product was purified via flash column chromatography (40:1 to 20:1 Hex/EtOAc) to afford a colorless oil (394 mg, $1.46 \mathrm{mmol}, 22 \%, 20: 1 \mathrm{Z} / \mathrm{E}$ mixture).

${ }^{1} \mathbf{H}$ NMR $\left(400 \mathrm{MHz}, \mathrm{CDCl}_{3}, 25{ }^{\circ} \mathrm{C}\right) \delta 7.52(\mathrm{ddd}, J=8.0,1.1,0.4 \mathrm{~Hz}, 1 \mathrm{H}), 7.38(\mathrm{ddd}, J=8.0,1.1,0.4$ Hz, 1H), 7.11 (td, $J=8.1,0.8 \mathrm{~Hz}, 1 \mathrm{H}), 6.41-6.34(\mathrm{~m}, 1 \mathrm{H}), 5.90(\mathrm{dt}, J=11.1,7.3 \mathrm{~Hz}, 1 \mathrm{H}), 2.44-$ $2.38(\mathrm{~m}, 2 \mathrm{H}), 2.28(\mathrm{qdd}, J=7.2,1.5,1.0 \mathrm{~Hz}, 2 \mathrm{H}) ;{ }^{13} \mathbf{C} \mathbf{~ N M R}\left(100 \mathrm{MHz}, \mathrm{CDCl}_{3}, 25{ }^{\circ} \mathrm{C}\right) \delta 136.0,134.4$, 131.3, 131.0, 129.6, 129.0, 128.7, 124.6, 119.2, 25.3, 16.6; FT-IR (neat) 3078, 3019, 2929, 2852, 2246, 1551, 1424, $765 \mathrm{~cm}^{-1}$; HRMS (ESI) $\mathrm{m} / z$ calculated for $\mathrm{C}_{11} \mathrm{H}_{9} \mathrm{BrClNNa}[\mathrm{M}+\mathrm{Na}]^{+} 291.9499$, found 291.9501; TLC: $R_{f}=0.47(5: 1 \mathrm{Hex} / \mathrm{EtOAc})$.

(Z)-N-(5-(2-bromo-6-chlorophenyl)pent-4-en-1-yl)picolinamide 11<smiles>O=C(NCCC/C=C\c1c(Cl)cccc1Br)c1ccccn1</smiles>

The title compound was prepared according to general procedures 2 and 3 from (Z)-5-(2-bromo-6chlorophenyl)pent-4-enenitrile S2l (310 mg, $1.15 \mathrm{mmol})$. The crude product was purified via flash 
column chromatography (4:1 to 2:1 Hex/EtOAc) to afford an off-white solid (306 mg, $0.81 \mathrm{mmol}$, $70 \%)$.

${ }^{1} \mathbf{H}$ NMR $\left(400 \mathrm{MHz}, \mathrm{CDCl}_{3}, 25{ }^{\circ} \mathrm{C}\right) \delta 8.50(\mathrm{ddd}, J=4.8,1.8,0.9 \mathrm{~Hz}, 1 \mathrm{H}), 8.17(\mathrm{dt}, J=7.8,1.1 \mathrm{~Hz}$, 1H), $8.02(\mathrm{~s}, 1 \mathrm{H}), 7.83(\mathrm{td}, J=7.7,1.7 \mathrm{~Hz}, 1 \mathrm{H}), 7.48(\mathrm{dd}, J=8.0,1.2 \mathrm{~Hz}, 1 \mathrm{H}), 7.40$ (ddd, $J=7.6,4.8$, $1.3 \mathrm{~Hz}, 1 \mathrm{H}), 7.34(\mathrm{dd}, J=8.0,1.2 \mathrm{~Hz}, 1 \mathrm{H}), 7.04(\mathrm{td}, J=8.0,0.8 \mathrm{~Hz}, 1 \mathrm{H}), 6.26(\mathrm{dt}, J=11.1,1.7 \mathrm{~Hz}$, $1 \mathrm{H}), 5.89(\mathrm{dt}, J=11.2,7.4 \mathrm{~Hz}, 1 \mathrm{H}), 3.50-3.34(\mathrm{~m}, 2 \mathrm{H}), 2.03(\mathrm{qd}, J=7.4,1.6 \mathrm{~Hz}, 2 \mathrm{H}), 1.81-1.66$ $(\mathrm{m}, 2 \mathrm{H}) ;{ }^{13} \mathrm{C}$ NMR $\left(100 \mathrm{MHz}, \mathrm{CDCl}_{3}, 25^{\circ} \mathrm{C}\right) \delta 164.3,150.1,148.0,137.4,136.9,134.8,134.6,131.1$, 129.0, 128.6, 126.8, 126.1, 124.9, 122.3, 39.1, 28.7, 26.8; FT-IR (neat) 3385, 3056, 3012, 2932, 2863 , 1669, 1522, 1464, 1424, 765, $621 \mathrm{~cm}^{-1}$; HRMS (ESI) $\mathrm{m} / z$ calculated for $\mathrm{C}_{17} \mathrm{H}_{17} \mathrm{BrClN}_{2} \mathrm{O}[\mathrm{M}+\mathrm{H}]^{+}$ 379.0207, found 379.0210; TLC: $R_{f}=0.20$ (97:3 DCM/EtOAc).

\section{(Z)-2-methyl-5-phenylpent-4-enenitrile (S2m)}

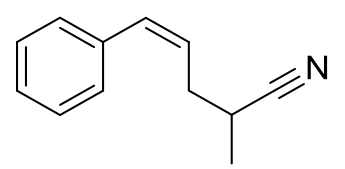

A flame dried flask under $\mathrm{N}_{2}$ was charged with anhydrous THF $(4.2 \mathrm{~mL})$, diisopropylamine $(0.21 \mathrm{~mL}$, $1.46 \mathrm{mmol}, 1.15$ equiv) and cooled to $0{ }^{\circ} \mathrm{C} . n$-BuLi $(1.6 \mathrm{M}$ in hexanes, $0.875 \mathrm{~mL}, 1.40 \mathrm{mmol}, 1.10$ equiv) was added dropwise and the reaction was stirred for $15 \mathrm{~min}$ at $0{ }^{\circ} \mathrm{C}$, then cooled to $-78{ }^{\circ} \mathrm{C}$. (Z)5-phenylpent-4-enenitrile 2a (dissolved in $3 \mathrm{~mL}$ anhydrous THF, $200 \mathrm{mg}, 1.27 \mathrm{mmol}, 1.0$ equiv) was added dropwise, the reaction was stirred for $1 \mathrm{~h}$ at $-78^{\circ} \mathrm{C}$, then methyl iodide $(0.084 \mathrm{~mL}, 1.34 \mathrm{mmol}$, 1.05 equiv) was added. The reaction was kept an additional $1 \mathrm{~h}$ at $-78^{\circ} \mathrm{C}$, allowed to warm to room temperature over $3 \mathrm{~h}$ and concentrated. The residue partitioned between water $(50 \mathrm{~mL})$ and EtOAc $(2 \times 50 \mathrm{~mL})$, the combined organic phases were washed with brine $(1 \times 50 \mathrm{~mL})$, dried over $\mathrm{MgSO}_{4}$, concentrated and purified by flash column chromatography (10:1 Hex/EtOAc) to afford a colorless oil (189 mg, $1.10 \mathrm{mmol}, 87 \%)$.

${ }^{1} \mathbf{H}$ NMR $\left(400 \mathrm{MHz}, \mathrm{CDCl}_{3}, 25{ }^{\circ} \mathrm{C}\right) \delta 7.39-7.33(\mathrm{~m}, 2 \mathrm{H}), 7.30-7.21(\mathrm{~m}, 3 \mathrm{H}), 6.65(\mathrm{dt}, J=11.5,1.8$ $\mathrm{Hz}, 1 \mathrm{H}), 5.70(\mathrm{dt}, J=11.5,7.0 \mathrm{~Hz}, 1 \mathrm{H}), 2.75-2.66(\mathrm{~m}, 1 \mathrm{H}), 2.62$ (tdd, $J=7.1,3.5,1.8 \mathrm{~Hz}, 2 \mathrm{H}), 1.32$ $(\mathrm{d}, J=6.9 \mathrm{~Hz}, 3 \mathrm{H}) ;{ }^{13} \mathbf{C} \mathbf{~ N M R}\left(100 \mathrm{MHz}, \mathrm{CDCl}_{3}, 2{ }^{\circ} \mathrm{C}\right) \delta 136.8,132.7,128.8,128.5,127.3,126.7$, 122.7, 32.6, 25.9, 17.7; FT-IR (neat) 3056, 3017, 2982, 2939, 2239, 1494, 1448, 1076, 807, 769, $699 \mathrm{~cm}^{-1}$; HRMS (EI) $\mathrm{m} / z$ calculated for $\mathrm{C}_{12} \mathrm{H}_{13} \mathrm{~N}[\mathrm{M}]^{+} 171.1043$, found 171.1043; TLC: $R_{f}=0.55$ (5:1 Hex/EtOAc). 


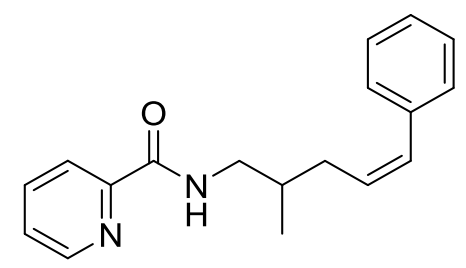

The title compound was prepared according to general procedures 2 and 3 from (Z)-2-methyl-5phenylpent-4-enenitrile $\mathbf{S 2 m}$ (130 $\mathrm{mg}, 0.76 \mathrm{mmol}$ ). The crude product was purified via flash column chromatography (3:1 Hex/EtOAc) to afford a colorless oil (153 mg, $0.55 \mathrm{mmol}, 72 \%)$.

${ }^{1} \mathbf{H}$ NMR $\left(400 \mathrm{MHz}, \mathrm{CDCl}_{3}, 25{ }^{\circ} \mathrm{C}\right) \delta 8.52(\mathrm{ddd}, J=4.8,1.7,0.9 \mathrm{~Hz}, 1 \mathrm{H}), 8.23-8.16(\mathrm{~m}, 1 \mathrm{H}), 8.14$ (s, 1H), $7.84(\mathrm{td}, J=7.7,1.7 \mathrm{~Hz}, 1 \mathrm{H}), 7.42(\mathrm{ddd}, J=7.6,4.8,1.3 \mathrm{~Hz}, 1 \mathrm{H}), 7.35-7.25(\mathrm{~m}, 4 \mathrm{H}), 7.23-$ $7.17(\mathrm{~m}, 1 \mathrm{H}), 6.51(\mathrm{dd}, J=11.7,1.9 \mathrm{~Hz}, 1 \mathrm{H}), 5.72(\mathrm{dt}, J=11.7,7.3 \mathrm{~Hz}, 1 \mathrm{H}), 3.44(\mathrm{dt}, J=13.5,6.3$ $\mathrm{Hz}, 1 \mathrm{H}), 3.32$ (ddd, $J=13.5,7.1,6.4 \mathrm{~Hz}, 1 \mathrm{H}), 2.47$ (dddd, $J=14.9,7.1,6.0,1.9 \mathrm{~Hz}, 1 \mathrm{H}), 2.28$ (dtd, $J$ $=15.0,7.6,1.8 \mathrm{~Hz}, 1 \mathrm{H}), 2.03-1.89(\mathrm{~m}, 1 \mathrm{H}), 1.01(\mathrm{~d}, J=6.7 \mathrm{~Hz}, 3 \mathrm{H}) ;{ }^{13} \mathbf{C} \mathbf{N M R}\left(100 \mathrm{MHz}, \mathrm{CDCl}_{3}\right.$, $\left.25^{\circ} \mathrm{C}\right) \delta 164.4,150.0,148.0,137.6,137.5,130.6,130.5,128.9,128.3,126.7,126.2,122.4,45.1,34.6$, 33.3, 17.9; FT-IR (neat) 3390, 3056, 3011, 2959, 2926, 2873, 1675, 1526, 1464, 1434, 1288, 998, 770, 750, 700, $621 \mathrm{~cm}^{-1}$; HRMS (ESI) $\mathrm{m} / z$ calculated for $\mathrm{C}_{18} \mathrm{H}_{20} \mathrm{~N}_{2} \mathrm{NaO}[\mathrm{M}+\mathrm{Na}]^{+} 303.1468$, found 303.1469 .

\section{(Z)-6-methylhept-4-enenitrile (S2n)}

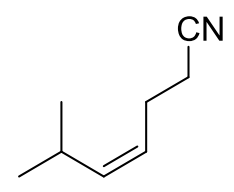

The title compound was prepared according to general procedure 1 using (3cyanopropyl)triphenylphosphonium bromide $(3.00 \mathrm{~g}, 7.31 \mathrm{mmol}, 1.00$ equiv), isobutyraldehyde ( $0.79 \mathrm{~mL}, 8.77 \mathrm{mmol}, 1.20$ equiv) and KHMDS (20 wt\% in THF, $10.0 \mathrm{~mL}, 8.77 \mathrm{mmol}, 1.20$ equiv). The reaction was filtered through a silica plug using 4:1 Hex/EtOAc as eluent and the crude product was purified via flash column chromatography (20:1 Hex/EtOAc) to afford a pale yellow oil (620 mg, $5.03 \mathrm{mmol}, 69 \%)$.

${ }^{1} \mathbf{H}$ NMR $\left(400 \mathrm{MHz}, \mathrm{CDCl}_{3}, 25{ }^{\circ} \mathrm{C}\right) \delta 5.39(\mathrm{ddt}, J=10.7,9.7,1.3 \mathrm{~Hz}, 1 \mathrm{H}), 5.29-5.17(\mathrm{~m}, 1 \mathrm{H}), 2.58$ $(\mathrm{m}, 1 \mathrm{H}), 2.45-2.32(\mathrm{~m}, 4 \mathrm{H}), 0.97(\mathrm{~d}, J=6.6 \mathrm{~Hz}, 6 \mathrm{H}) ;{ }^{13} \mathbf{C} \mathbf{N M R}\left(100 \mathrm{MHz}, \mathrm{CDCl}_{3}, 25{ }^{\circ} \mathrm{C}\right) \delta 141.2$, 122.6, 119.6, 26.8, 23.5, 23.2, 17.9; FT-IR (neat) 3008, 2959, 2929, 2870, 2246, 1732, 1466, 2559, 1363, 1301, 1103, 824, $745 \mathrm{~cm}^{-1}$; HRMS (EI) $\mathrm{m} / z$ calculated for $\mathrm{C}_{8} \mathrm{H}_{12} \mathrm{~N}[\mathrm{M}-\mathrm{H}]^{+} 122.0964$, found 122.0963; TLC: $R_{f}=0.46(5: 1 \mathrm{Hex} / \mathrm{EtOAc})$. 


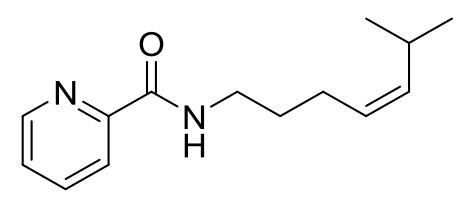

The title compound was prepared according to general procedures 2 and 3 from (Z)-6-methylhept-4enenitrile S2n (410 mg, $3.33 \mathrm{mmol}$ ). The crude product was purified via flash column chromatography (3:1 Hex/EtOAc) to afford a pale yellow oil (471 mg, $2.02 \mathrm{mmol}, 61 \%)$.

${ }^{1} \mathbf{H}$ NMR $\left(400 \mathrm{MHz}, \mathrm{CDCl}_{3}, 25{ }^{\circ} \mathrm{C}\right) \delta 8.53(\mathrm{ddd}, J=4.8,1.8,0.9 \mathrm{~Hz}, 1 \mathrm{H}), 8.19(\mathrm{dt}, J=7.8,1.1 \mathrm{~Hz}$, $1 \mathrm{H}), 8.08$ (s, 1H), $7.83(\mathrm{td}, J=7.7,1.8 \mathrm{~Hz}, 1 \mathrm{H}), 7.40$ (ddd, $J=7.6,4.8,1.3 \mathrm{~Hz}, 1 \mathrm{H}), 5.27-5.21$ (m, 2H), $3.52-3.40(\mathrm{~m}, 2 \mathrm{H}), 2.59$ (ddt, $J=13.2,8.6,6.6 \mathrm{~Hz}, 1 \mathrm{H}), 2.15(\mathrm{dtd}, J=7.7,5.8,1.1 \mathrm{~Hz}, 2 \mathrm{H}$ ), $1.76-1.60(\mathrm{~m}, 2 \mathrm{H}), 0.93(\mathrm{~d}, J=6.7 \mathrm{~Hz}, 6 \mathrm{H}) ;{ }^{13} \mathbf{C ~ N M R}\left(100 \mathrm{MHz}, \mathrm{CDCl}_{3}, 25{ }^{\circ} \mathrm{C}\right) \delta 164.3,150.2$, 148.1, 138.7, 137.5, 126.2, 126.1, 122.3, 39.2, 29.9, 26.6, 24.9, 23.3; FT-IR (neat) 3391, 3000, 2956, 2867, 1669, 1591, 1569, 1525, 1465, 1434, 1289, 1164, 997, 820, 749, 692, $621 \mathrm{~cm}^{-1}$; HRMS (ESI) $m / z$ calculated for $\mathrm{C}_{14} \mathrm{H}_{21} \mathrm{~N}_{2} \mathrm{O}[\mathrm{M}+\mathrm{H}]^{+} 233.1648$, found 233.1650; TLC: $R_{f}=0.36$ (97:3 DCM/ EtOAc).

\section{(Z)-6,6-dimethylhept-4-enenitrile (S20)}

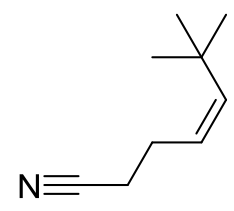

The title compound was prepared according to general procedure 1 using (3cyanopropyl)triphenylphosphonium bromide $\quad(3.00 \mathrm{~g}, \quad 7.31 \mathrm{mmol}, \quad 1.00$ equiv), pivalaldehyde (0.69 mL, $8.04 \mathrm{mmol}, 1.10$ equiv) and KHMDS (20 wt\% in THF, $10.3 \mathrm{~mL}, 9.14 \mathrm{mmol}, 1.25$ equiv). The reaction was filtered through a silica plug using 4:1 Hex/EtOAc as eluent and the crude product was purified via flash column chromatography $\left(20: 1 \mathrm{Hex} / \mathrm{Et}_{2} \mathrm{O}\right)$ to afford a pale yellow oil $(654 \mathrm{mg}$, $4.77 \mathrm{mmol}, 65 \%$ ).

${ }^{1} \mathbf{H}$ NMR $\left(400 \mathrm{MHz}, \mathrm{CDCl}_{3}, 25{ }^{\circ} \mathrm{C}\right) \delta 5.49(\mathrm{dt}, J=12.0,1.7 \mathrm{~Hz}, 1 \mathrm{H}), 5.13(\mathrm{dt}, J=12.0,7.4 \mathrm{~Hz}, 1 \mathrm{H})$, $2.56(\mathrm{ddd}, J=7.3,1.7,0.7 \mathrm{~Hz}, 2 \mathrm{H}), 2.38(\mathrm{td}, J=7.3,0.7 \mathrm{~Hz}, 2 \mathrm{H}), 1.12(\mathrm{~s}, 9 \mathrm{H}) ;{ }^{13} \mathbf{C}$ NMR $(100 \mathrm{MHz}$, $\left.\mathrm{CDCl}_{3}, 25{ }^{\circ} \mathrm{C}\right) \delta 143.4,123.8,119.5,33.6,31.2,24.3,18.0$; FT-IR (neat) 3007, 2957, 2869, 2246, 1653, 1478, 1466, 1428, 1363, 1204, 990, $728 \mathrm{~cm}^{-1}$; HRMS (EI) $\mathrm{m} / z$ calculated for $\mathrm{C}_{9} \mathrm{H}_{15} \mathrm{~N}[\mathrm{M}]^{+}$ 137.1199, found 137.1201; TLC: $R_{f}=0.49$ (5:1 Hex/EtOAc). 


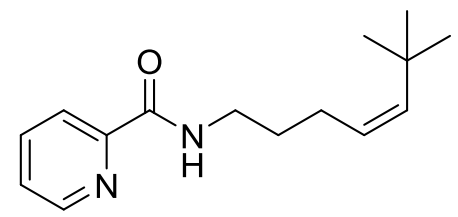

The title compound was prepared according to general procedures 2 and 3 from (Z)-6,6-dimethylhept4-enenitrile S2o $(350 \mathrm{mg}, 2.55 \mathrm{mmol})$. The crude product was purified via flash column chromatography (3:1 Hex/EtOAc) to afford a pale yellow oil (398 mg, $1.62 \mathrm{mmol}, 63 \%)$.

${ }^{1} \mathbf{H}$ NMR $\left(400 \mathrm{MHz}, \mathrm{CDCl}_{3}, 25{ }^{\circ} \mathrm{C}\right) \delta 8.54(\mathrm{ddd}, J=4.8,1.8,0.9 \mathrm{~Hz}, 1 \mathrm{H}), 8.19(\mathrm{dt}, J=7.9,1.1 \mathrm{~Hz}$, $1 \mathrm{H}), 8.09(\mathrm{~s}, 1 \mathrm{H}), 7.84(\mathrm{td}, J=7.7,1.7 \mathrm{~Hz}, 1 \mathrm{H}), 7.41(\mathrm{ddd}, J=7.6,4.8,1.3 \mathrm{~Hz}, 1 \mathrm{H}), 5.36(\mathrm{dt}, J=12.0$, $1.7 \mathrm{~Hz}, 1 \mathrm{H}), 5.21-5.11(\mathrm{~m}, 1 \mathrm{H}), 3.54-3.44(\mathrm{~m}, 2 \mathrm{H}), 2.29$ (qd, $J=7.4,1.7 \mathrm{~Hz}, 2 \mathrm{H}), 1.76-1.66(\mathrm{~m}$, 2H), $1.10(\mathrm{~s}, 9 \mathrm{H}) ;{ }^{13} \mathrm{C} \mathrm{NMR}\left(100 \mathrm{MHz}, \mathrm{CDCl}_{3}, 25{ }^{\circ} \mathrm{C}\right) \delta 164.4,150.2,148.1,140.8,137.5,127.6$, 126.2, 122.3, 39.2, 33.3, 31.3, 30.3, 25.9; FT-IR (neat) 3391, 3058, 2999, 2954, 2866, 1670, 1524, 1465, 1434, 1362, 1206, 998, $749 \mathrm{~cm}^{-1}$; HRMS (ESI) $\mathrm{m} / z$ calculated for $\mathrm{C}_{15} \mathrm{H}_{23} \mathrm{~N}_{2} \mathrm{O}[\mathrm{M}+\mathrm{H}]^{+}$ 247.1805, found 247.1801; TLC: $R_{f}=0.48$ (3:2 Hex/EtOAc).

\section{(Z)-5-cyclohexylpent-4-enenitrile (S2p)}

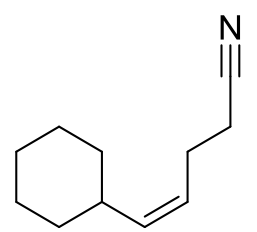

The title compound was prepared according to general procedure 1 using (3-

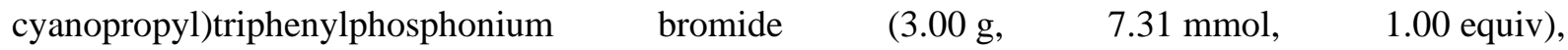
cyclohexanecarbaldehyde $(0.93 \mathrm{~mL}, 7.68 \mathrm{mmol}, 1.05$ equiv) and KHMDS (1M in THF, $8.04 \mathrm{~mL}$, $8.04 \mathrm{mmol}, 1.10$ equiv). The reaction was filtered through a silica plug using 2:1 Hex/EtOAc as eluent and the crude product was purified via flash column chromatography (97:3 Hex/EtOAc) to afford a colorless oil (820 mg, $5.02 \mathrm{mmol}$, 69\%).

${ }^{1} \mathbf{H}$ NMR $\left(400 \mathrm{MHz}, \mathrm{CDCl}_{3}, 25^{\circ} \mathrm{C}\right) \delta 5.39(\mathrm{ddt}, J=10.7,9.5,1.3 \mathrm{~Hz}, 1 \mathrm{H}), 5.29-5.20(\mathrm{~m}, 1 \mathrm{H}), 2.47-$ $2.32(\mathrm{~m}, 4 \mathrm{H}), 2.29-2.17(\mathrm{~m}, 1 \mathrm{H}), 1.76-1.54(\mathrm{~m}, 5 \mathrm{H}), 1.35-1.00(\mathrm{~m}, 5 \mathrm{H}) .{ }^{13} \mathbf{C}$ NMR $(100 \mathrm{MHz}$, $\left.\mathrm{CDCl}_{3}, 25{ }^{\circ} \mathrm{C}\right) \delta 139.7,123.2,119.5,36.6,33.3,26.0,25.9,23.6,18.0$; FT-IR (neat) 3006, 2922, 2849, 2245, 1448, 1427, 1327, 1259, 949, 890, $736 \mathrm{~cm}^{-1}$; HRMS (EI) $\mathrm{m} / z$ calculated for $\mathrm{C}_{11} \mathrm{H}_{17} \mathrm{~N}[\mathrm{M}]^{+}$ 163.1356, found 163.1351 . The analytical data are in agreement with the literature. ${ }^{7}$ 


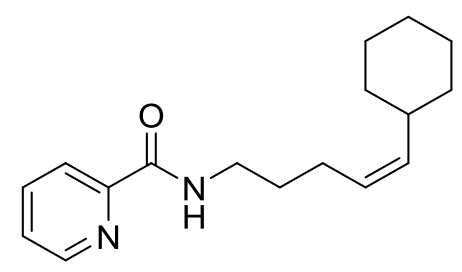

The title compound was prepared according to general procedures 2 and 3 from (Z)-5-cyclohexylpent4-enenitrile S2p (760 mg, $4.66 \mathrm{mmol})$. The crude product was purified via flash column chromatography (3:1 Hex/EtOAc) to afford a pale yellow oil (868 mg, $3.19 \mathrm{mmol}, 68 \%)$.

${ }^{1} \mathbf{H}$ NMR $\left(400 \mathrm{MHz}, \mathrm{CDCl}_{3}, 25{ }^{\circ} \mathrm{C}\right) \delta 8.54(\mathrm{ddd}, J=4.8,1.8,0.9 \mathrm{~Hz}, 1 \mathrm{H}), 8.20(\mathrm{~d}, J=7.9 \mathrm{~Hz}, 1 \mathrm{H})$, $8.08(\mathrm{~s}, 1 \mathrm{H}), 7.84(\mathrm{td}, J=7.7,1.7 \mathrm{~Hz}, 1 \mathrm{H}), 7.41(\mathrm{ddd}, J=7.6,4.8,1.3 \mathrm{~Hz}, 1 \mathrm{H}), 5.31-5.24(\mathrm{~m}, 2 \mathrm{H})$, $3.48(\mathrm{td}, J=7.2,6.1 \mathrm{~Hz}, 2 \mathrm{H}), 2.31-2.20(\mathrm{~m}, 1 \mathrm{H}), 2.20-2.13(\mathrm{~m}, 2 \mathrm{H}), 1.77-1.55(\mathrm{~m}, 7 \mathrm{H}), 1.32-$ $0.98(\mathrm{~m}, 5 \mathrm{H}) ;{ }^{13} \mathbf{C}$ NMR $\left(100 \mathrm{MHz}, \mathrm{CDCl}_{3}, 25{ }^{\circ} \mathrm{C}\right) \delta 164.4,150.2,148.1,137.5,137.2,126.6,126.2$, 122.3, 39.1, 36.5, 33.5, 29.9, 26.2, 26.1, 25.0; FT-IR (neat) 3391, 3057, 2999, 2992, 2848.1671, 1591, 1569, 1524, 1447, 1288, 1163, 997, 748, 692, $620 \mathrm{~cm}^{-1}$; HRMS (ESI) $\mathrm{m} / z$ calculated for $\mathrm{C}_{17} \mathrm{H}_{25} \mathrm{~N}_{2} \mathrm{O}$ $[\mathrm{M}+\mathrm{H}]^{+} 273.1961$, found 273.1963; TLC: $R_{f}=0.40(2: 1 \mathrm{Hex} / \mathrm{EtOAc})$.

\section{(Z)-tert-butyl 4-(4-cyanobut-1-en-1-yl)piperidine-1-carboxylate (S2q)}<smiles>CC(C)(C)OC(=O)N1CCC(/C=C\CCC#N)CC1</smiles>

The title compound was prepared according to general procedure 1 from tert-butyl 4-formylpiperidine1-carboxylate $(1.42 \mathrm{~g}, 6.65 \mathrm{mmol})$. The reaction was filtered through a silica plug using 3:1 $\mathrm{Hex} / \mathrm{EtOAc}$ as eluent and the crude product was purified via flash column chromatography (10:1 to 4:1 Hex/EtOAc) to afford a viscous colorless oil (1.51 g, $5.71 \mathrm{mmol}, 86 \%)$.

${ }^{1} \mathbf{H}$ NMR $\left(400 \mathrm{MHz}, \mathrm{CDCl}_{3}, 25{ }^{\circ} \mathrm{C}\right) \delta 5.43-5.27(\mathrm{~m}, 2 \mathrm{H}), 4.12-3.99(\mathrm{~m}, 2 \mathrm{H}), 2.81-2.68(\mathrm{~m}, 2 \mathrm{H})$, $2.46-2.32(\mathrm{~m}, 5 \mathrm{H}), 1.62-1.54(\mathrm{~m}, 2 \mathrm{H}), 1.45(\mathrm{~s}, 9 \mathrm{H}), 1.34-1.21(\mathrm{~m}, 2 \mathrm{H}) ;{ }^{13} \mathbf{C}$ NMR $(100 \mathrm{MHz}$, $\left.\mathrm{CDCl}_{3}, 25{ }^{\circ} \mathrm{C}\right) \delta 154.9,137.6,124.6,119.4,79.5,43.6,34.8,32.1,28.6,23.7,17.9$; FT-IR (neat) 3007, 2975, 2931, 2851, 2445, 1684, 1420, 1365, 1228, 1163, 1132, 956, 769, $742 \mathrm{~cm}^{-1}$; HRMS (ESI) $m / z$ calculated for $\mathrm{C}_{15} \mathrm{H}_{24} \mathrm{~N}_{2} \mathrm{NaO}_{2}[\mathrm{M}+\mathrm{Na}]^{+}$287.1730, found 287.1729; TLC: $R_{f}=0.71(1: 1$ Hex/EtOAc). 


\section{(Z)-tert-butyl 4-(5-(picolinamido)pent-1-en-1-yl)piperidine-1-carboxylate (1q)}

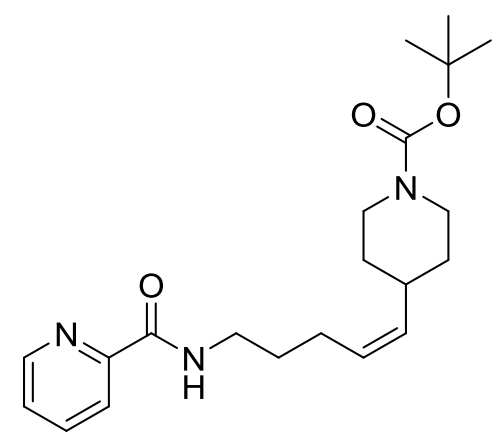

The title compound was prepared according to general procedures 2 and 3 from (Z)-tert-butyl 4-(4cyanobut-1-en-1-yl)piperidine-1-carboxylate $\mathbf{S 2 q}(860 \mathrm{mg}, 3.25 \mathrm{mmol})$. The crude product was purified via flash column chromatography (2:1 Hex/EtOAc) to afford a pale yellow oil (475 mg, $1.27 \mathrm{mmol}, 39 \%)$.

${ }^{1} \mathbf{H}$ NMR $\left(400 \mathrm{MHz}, \mathrm{CDCl}_{3}, 25{ }^{\circ} \mathrm{C}\right) \delta 8.54(\mathrm{ddt}, J=4.8,1.5,0.8 \mathrm{~Hz}, 1 \mathrm{H}), 8.20$ (ddt, $J=7.8,1.4,0.6$ $\mathrm{Hz}, 1 \mathrm{H}), 8.08(\mathrm{~s}, 1 \mathrm{H}), 7.91-7.78(\mathrm{~m}, 1 \mathrm{H}), 7.46-7.39(\mathrm{~m}, 1 \mathrm{H}), 5.38-5.30(\mathrm{~m}, 1 \mathrm{H}), 5.27-5.19(\mathrm{~m}$, 1H), $4.04(\mathrm{~s}, 2 \mathrm{H}), 3.49$ (qd, $J=6.7,6.2,1.1 \mathrm{~Hz}, 2 \mathrm{H}), 2.70(\mathrm{t}, J=12.7 \mathrm{~Hz}, 2 \mathrm{H}), 2.46-2.33(\mathrm{~m}, 1 \mathrm{H})$, $2.17(\mathrm{qd}, J=7.4,1.4 \mathrm{~Hz}, 2 \mathrm{H}), 1.75-1.65(\mathrm{~m}, 2 \mathrm{H}), 1.58-1.50(\mathrm{~m}, 2 \mathrm{H}), 1.45(\mathrm{~s}, 9 \mathrm{H}), 1.34-1.19(\mathrm{~m}$, 2H); ${ }^{13} \mathrm{C}$ NMR $\left(100 \mathrm{MHz}, \mathrm{CDCl}_{3}, 25{ }^{\circ} \mathrm{C}\right) \delta 164.4,155.0,150.1,148.1,137.6,135.0,128.1,126.3$, 122.3, 79.4, 43.4 (broad), 39.0, 34.6, 32.2, 29.8, 28.6, 25.0; FT-IR (neat) 3387, 3057, 3003, 2974, 2930, 2851, 1681, 1524, 1423, 1365, 1287, 1227, 1168, 998, 750, $621 \mathrm{~cm}^{-1}$; HRMS (ESI) $\mathrm{m} / \mathrm{z}$ calculated for $\mathrm{C}_{21} \mathrm{H}_{31} \mathrm{~N}_{3} \mathrm{NaO}_{3}[\mathrm{M}+\mathrm{Na}]^{+} 396.2258$, found 396.2256; TLC: $R_{f}=0.23(3: 2 \mathrm{Hex} / \mathrm{EtOAc})$.

\section{$N$-(2-(cyclohex-2-en-1-yl)ethyl)picolinamide (1r)}<smiles>O=C(NCCC1C=CCCC1)c1ccccn1</smiles>

Based on a literature procedure, ${ }^{8}$ a flame dried flask under $\mathrm{N}_{2}$ was charged with anhydrous THF $(10 \mathrm{~mL})$, diisopropylamine $\left(1.95 \mathrm{~mL}, 13.7 \mathrm{mmol}, 1.10\right.$ equiv) and cooled to $0{ }^{\circ} \mathrm{C} . n$-BuLi $(1.6 \mathrm{M}$ in hexanes, $8.54 \mathrm{~mL}, 13.7 \mathrm{mmol}, 1.10$ equiv) was added dropwise and the reaction was stirred for $15 \mathrm{~min}$ at $0{ }^{\circ} \mathrm{C}$, then cooled to $-78{ }^{\circ} \mathrm{C}$. Anhydrous acetonitrile $(0.65 \mathrm{~mL}, 12.4 \mathrm{mmol}, 1.00$ equiv) was added dropwise, the reaction was stirred for $10 \mathrm{~min}$ at $-78{ }^{\circ} \mathrm{C}$, placed in an ice bath and stirred for $1 \mathrm{~h}$ at $0{ }^{\circ} \mathrm{C}$. 3-Bromocyclohex-1-ene $(2.00 \mathrm{~g}, 12.4 \mathrm{mmol}, 1.00$ equiv $)$ was added dropwise, the reaction was allowed to warm to room temperature and stirred for $90 \mathrm{~min}$. The reaction was quenched by addition of water $(20 \mathrm{~mL})$, extracted with diethyl ether $(2 \times 25 \mathrm{~mL})$, dried over $\mathrm{MgSO}_{4}$ and concentrated. The crude 2-(cyclohex-2-en-1-yl)acetonitrile was used for the next step without further purification. 
The title compound was prepared according to general procedures 2 and 3 from crude 2-(cyclohex-2en-1-yl)acetonitrile prepared above (quantitative yield assumed, $12.4 \mathrm{mmol} \mathrm{scale}$ ). The crude product was purified via flash column chromatography (6:1 to $3: 1 \mathrm{Hex} / \mathrm{EtOAc})$ to afford an orange oil (695 mg, $3.02 \mathrm{mmol}, 25 \%$ over 3 steps).

${ }^{1} \mathbf{H}$ NMR $\left(400 \mathrm{MHz}, \mathrm{CDCl}_{3}, 25{ }^{\circ} \mathrm{C}\right) \delta 8.53(\mathrm{ddd}, J=4.8,1.8,0.9 \mathrm{~Hz}, 1 \mathrm{H}), 8.19(\mathrm{dt}, J=7.8,1.1 \mathrm{~Hz}$, $1 \mathrm{H}), 8.11-7.98(\mathrm{~m}, 1 \mathrm{H}), 7.83(\mathrm{td}, J=7.7,1.7 \mathrm{~Hz}, 1 \mathrm{H}), 7.40(\mathrm{ddd}, J=7.6,4.8,1.3 \mathrm{~Hz}, 1 \mathrm{H}), 5.73-$ $5.66(\mathrm{~m}, 1 \mathrm{H}), 5.63-5.57(\mathrm{~m}, 1 \mathrm{H}), 3.54(\mathrm{td}, J=7.4,6.0 \mathrm{~Hz}, 2 \mathrm{H}), 2.20(\mathrm{~m}, 1 \mathrm{H}), 1.97(\mathrm{~m}, 2 \mathrm{H}), 1.90-$ $1.80(\mathrm{~m}, 1 \mathrm{H}), 1.76-1.45(\mathrm{~m}, 4 \mathrm{H}), 1.29$ (dddd, $J=12.7,11.1,8.5,2.8 \mathrm{~Hz}, 1 \mathrm{H}) ;{ }^{13} \mathbf{C}$ NMR $(100 \mathrm{MHz}$, $\left.\mathrm{CDCl}_{3}, 25{ }^{\circ} \mathrm{C}\right) \delta 164.3,150.1,148.1,137.5,131.2,127.7,126.2,122.3,37.3,36.2,33.0,29.0,25.4$, 21.4; FT-IR (neat) 3385, 3322, 3059, 3015, 2934, 2857, 1665, 1523, 1464, 1433, 1285, 1243, 1143, 998, 819, 749, 692, $621 \mathrm{~cm}^{-1}$; HRMS (ESI) $\mathrm{m} / z$ calculated for $\mathrm{C}_{14} \mathrm{H}_{19} \mathrm{~N}_{2} \mathrm{O}[\mathrm{M}+\mathrm{H}]^{+} 231.1492$, found 231.1495; TLC: $R_{f}=0.39$ (2:1 Hex/EtOAc).

\section{(Z)-5-(cyclohex-3-en-1-yl)pent-4-enenitrile (S2s)}

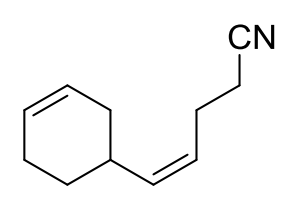

The title compound was prepared according to general procedure 1 from cyclohex-3-enecarbaldehyde $(1.42 \mathrm{~g}, 6.65 \mathrm{mmol})$. The reaction was filtered through a silica plug using 4:1 Hex/EtOAc as eluent and the crude product was purified via flash column chromatography (20:1 Hex/EtOAc) to afford a colorless oil (1.00 g, $6.20 \mathrm{mmol}, 93 \%)$.

${ }^{1} \mathbf{H}$ NMR $\left(400 \mathrm{MHz}, \mathrm{CDCl}_{3}, 25{ }^{\circ} \mathrm{C}\right) \delta 5.74-5.62(\mathrm{~m}, 2 \mathrm{H}), 5.52-5.43(\mathrm{~m}, 1 \mathrm{H}), 5.37-5.25(\mathrm{~m}, 1 \mathrm{H})$, $2.60-2.48(\mathrm{~m}, 1 \mathrm{H}), 2.47-2.34(\mathrm{~m}, 4 \mathrm{H}), 2.12-1.99(\mathrm{~m}, 3 \mathrm{H}), 1.87-1.75(\mathrm{~m}, 1 \mathrm{H}), 1.71-1.63(\mathrm{~m}$, $1 \mathrm{H}), 1.41$ (dddd, $J=12.9,11.1,8.9,7.2 \mathrm{~Hz}, 1 \mathrm{H}) ;{ }^{13} \mathbf{C} \mathbf{~ N M R}\left(100 \mathrm{MHz}, \mathrm{CDCl}_{3}, 25{ }^{\circ} \mathrm{C}\right) \delta 138.9,127.1$, 126.0, 123.9, 119.5, 32.4, 31.7, 29.0, 24.6, 23.6, 18.0; FT-IR (neat) 3021, 2913, 2835, 2245, 1652, 1435, 1326, 1139, 925, 735, $653 \mathrm{~cm}^{-1}$; HRMS (EI) $\mathrm{m} / z$ calculated for $\mathrm{C}_{11} \mathrm{H}_{14} \mathrm{~N}[\mathrm{M}]^{+} 160.1121$, found 160.1122; TLC: $R_{f}=0.59$ (3:1 Hex/EtOAc). 


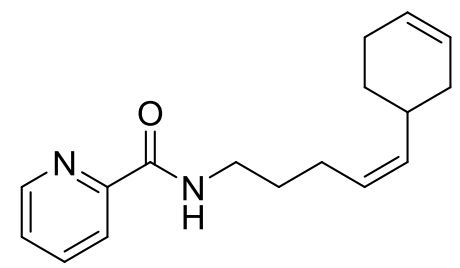

The title compound was prepared according to general procedures 2 and 3 from (Z)-5-(cyclohex-3-en1-yl)pent-4-enenitrile S2s (560 mg, $3.47 \mathrm{mmol}$ ). The crude product was purified via flash column chromatography (3:1 Hex/EtOAc) to afford a yellow oil $(181 \mathrm{mg}, 0.70 \mathrm{mmol}, 19 \%)$. Note: The compound slowly decomposed when stored under ambient conditions.

${ }^{1} \mathbf{H}$ NMR $\left(500 \mathrm{MHz}, \mathrm{CDCl}_{3}, 25{ }^{\circ} \mathrm{C}\right) \delta 8.53(\mathrm{ddd}, J=4.8,1.8,0.9 \mathrm{~Hz}, 1 \mathrm{H}), 8.19(\mathrm{dt}, J=7.8,1.1 \mathrm{~Hz}$, $1 \mathrm{H}), 8.08(\mathrm{~s}, 1 \mathrm{H}), 7.84(\mathrm{td}, J=7.7,1.7 \mathrm{~Hz}, 1 \mathrm{H}), 7.41(\mathrm{ddd}, J=7.5,4.8,1.3 \mathrm{~Hz}, 1 \mathrm{H}), 5.69-5.61(\mathrm{~m}$, $2 \mathrm{H}), 5.38-5.29(\mathrm{~m}, 2 \mathrm{H}), 3.52-3.45(\mathrm{~m}, 2 \mathrm{H}), 2.61-2.50(\mathrm{~m}, 1 \mathrm{H}), 2.18(\mathrm{dddd}, J=7.8,7.1,4.3,1.3$ $\mathrm{Hz}, 2 \mathrm{H}), 2.08-1.98(\mathrm{~m}, 3 \mathrm{H}), 1.82-1.61(\mathrm{~m}, 4 \mathrm{H}), 1.43-1.32(\mathrm{~m}, 1 \mathrm{H}) ;{ }^{13} \mathbf{C}$ NMR $\left(126 \mathrm{MHz}, \mathrm{CDCl}_{3}\right.$, $\left.25{ }^{\circ} \mathrm{C}\right) \delta 164.4,150.2,148.1,137.5,136.3,127.4,127.0,126.3,126.2,122.3,39.1,32.2,31.9,29.9$, 29.1, 25.0, 24.8; FT-IR (neat) 3390, 3058, 3020, 2913, 2834, 1670, 1591, 1569, 1523, 1465, 1434, 1288, 998, 748, 653, $621 \mathrm{~cm}^{-1}$; HRMS (ESI) $\mathrm{m} / z$ calculated for $\mathrm{C}_{17} \mathrm{H}_{23} \mathrm{~N}_{2} \mathrm{O}[\mathrm{M}+\mathrm{H}]^{+} 271.1805$, found 271.1808 .

(4Z,6E)-7-phenylhepta-4,6-dienenitrile (S2t)

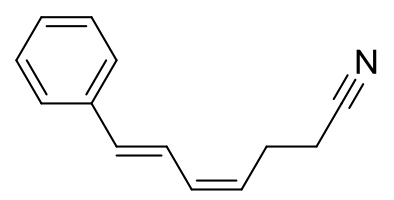

The title compound was prepared according to general procedure 1 from cinnamaldehyde $(0.88 \mathrm{~g}, 6.65$ mmol). The reaction was filtered through a silica plug using 3:1 Hex/EtOAc as eluent and the crude product was purified via flash column chromatography (10:1 Hex/EtOAc) to afford a faint yellow oil (0.98 g, $5.35 \mathrm{mmol}, 80 \%)$. The title compound is literature known; however, the reported analytical data is limited to HPLC retention times. ${ }^{9}$

${ }^{1} \mathbf{H}$ NMR $\left(400 \mathrm{MHz}, \mathrm{CD}_{2} \mathrm{Cl}_{2}, 25{ }^{\circ} \mathrm{C}\right) \delta 7.47-7.41(\mathrm{~m}, 2 \mathrm{H}), 7.38-7.31(\mathrm{~m}, 2 \mathrm{H}), 7.28-7.21(\mathrm{~m}, 1 \mathrm{H})$, $7.04(\mathrm{ddd}, J=15.5,11.2,1.2 \mathrm{~Hz}, 1 \mathrm{H}), 6.63(\mathrm{~d}, J=15.5 \mathrm{~Hz}, 1 \mathrm{H}), 6.32(\mathrm{tdd}, J=11.2,2.3,1.2 \mathrm{~Hz}, 1 \mathrm{H})$, $5.51(\mathrm{dtt}, J=10.7,7.6,1.0 \mathrm{~Hz}, 1 \mathrm{H}), 2.69-2.61(\mathrm{~m}, 2 \mathrm{H}), 2.47(\mathrm{td}, J=7.2,0.7 \mathrm{~Hz}, 2 \mathrm{H}) ;{ }^{13} \mathbf{C} \mathbf{~ N M R}(100$ $\left.\mathrm{MHz}, \mathrm{CD}_{2} \mathrm{Cl}_{2}, 25{ }^{\circ} \mathrm{C}\right) \delta 137.7,134.6,132.0,129.2,128.4,128.0,127.0,123.8,119.8,24.5,18.0 ;$ FT- 
IR (neat) 3027, 2927, 2245, 1491, 1449, 1425, 1329, 945, 759, 732, $691 \mathrm{~cm}^{-1}$; HRMS (EI) $\mathrm{m} / \mathrm{z}$ calculated for $\mathrm{C}_{13} \mathrm{H}_{13} \mathrm{~N}[\mathrm{M}]^{+} 183.1043$, found 183.1040; TLC: $R_{f}=0.35$ (5:1 Hex/EtOAc).

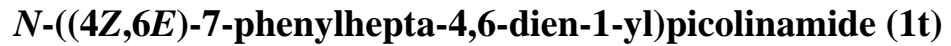

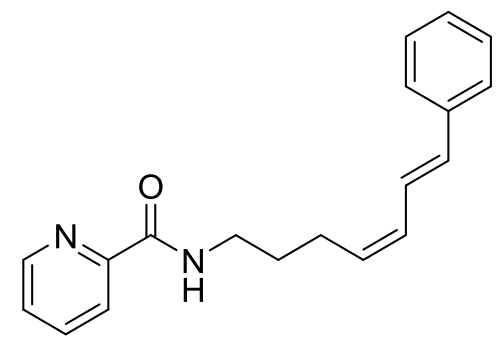

The title compound was prepared according to general procedures 2 and 3 from $(4 Z, 6 E)-7-$ phenylhepta-4,6-dienenitrile $(\mathbf{S 2 t})(620 \mathrm{mg}, 3.38 \mathrm{mmol})$. The crude product was purified via flash column chromatography (2:1 Hex/EtOAc) to afford a faint yellow (703 mg, $2.40 \mathrm{mmol}, 71 \%)$.

${ }^{1} \mathbf{H}$ NMR $\left(400 \mathrm{MHz}, \mathrm{CD}_{2} \mathrm{Cl}_{2}, 25{ }^{\circ} \mathrm{C}\right) \delta 8.55-8.50(\mathrm{~m}, 1 \mathrm{H}), 8.19-8.04(\mathrm{~m}, 2 \mathrm{H}), 7.85(\mathrm{td}, J=7.7,1.7$ $\mathrm{Hz}, 1 \mathrm{H}), 7.46-7.37$ (m, 3H), $7.34-7.26$ (m, 2H), $7.24-7.18$ (m, 1H), 7.09 (ddd, $J=15.5,11.1,1.2$ $\mathrm{Hz}, 1 \mathrm{H}), 6.55(\mathrm{~d}, J=15.6 \mathrm{~Hz}, 1 \mathrm{H}), 6.22(\mathrm{tq}, J=11.0,1.5 \mathrm{~Hz}, 1 \mathrm{H}), 5.63-5.52(\mathrm{~m}, 1 \mathrm{H}), 3.49$ (td, $J=$ 7.0, 6.1 Hz, 2H), $2.41(\mathrm{qd}, J=7.6,1.6 \mathrm{~Hz}, 2 \mathrm{H}), 1.83-1.73(\mathrm{~m}, 2 \mathrm{H}) ;{ }^{13} \mathbf{C} \mathbf{N M R}\left(100 \mathrm{MHz}, \mathrm{CD}_{2} \mathrm{Cl}_{2}, 25\right.$ $\left.{ }^{\circ} \mathrm{C}\right) \delta 164.5,150.8,148.6,138.1,137.9,132.9,132.4,130.0,129.1,128.0,126.9,126.6,124.7,122.5$, 39.3, 30.1, 25.9; FT-IR (neat) 3384, 3057, 3025, 3007, 2929, 1669, 1522, 1492, 1464, 1433, 1288, $1243,1124,997,748,733,691,620 \mathrm{~cm}^{-1}$; HRMS (ESI) $\mathrm{m} / z$ calculated for $\mathrm{C}_{19} \mathrm{H}_{20} \mathrm{~N}_{2} \mathrm{NaO}[\mathrm{M}+\mathrm{Na}]^{+}$ 315.1468, found 315.1473; TLC: $R_{f}=0.42$ (3:2 Hex/EtOAc).

(S,Z)-N-(5-(4-(prop-1-en-2-yl)cyclohex-1-en-1-yl)pent-4-en-1-yl)picolinamide (1u)<smiles>C=C(C)[C@H]1CC=C(/C=C\CCCNC(=O)c2ccccn2)CC1</smiles>

Crude (S,Z)-5-(4-(prop-1-en-2-yl)cyclohex-1-en-1-yl)pent-4-enenitrile S2u was prepared according to general procedure 1 from $(S)-(-)-P e r i l l a l d e h y d e ~(0.67 \mathrm{~g}, 4.10 \mathrm{mmol})$. The reaction was filtered through a silica plug using 4:1 Hex/EtOAc as eluent and the crude product was purified via flash column chromatography (20:1 Hex/EtOAc) to afford a colorless oil (565 mg, $2.61 \mathrm{mmol}, 64 \%)$ containing $7 \%$ aldehyde-impurities.

The title compound was prepared according to general procedures 2 and 3 from crude $(S, Z)-5$-(4(prop-1-en-2-yl)cyclohex-1-en-1-yl)pent-4-enenitrile S2u (565 mg, $2.61 \mathrm{mmol})$ prepared above. The 
crude product was purified via flash column chromatography (4:1 Hex/EtOAc) to afford a yellow oil (470 mg, $1.51 \mathrm{mmol}, 54 \%)$.

${ }^{1} \mathbf{H}$ NMR $\left(400 \mathrm{MHz}, \mathrm{CDCl}_{3}, 25{ }^{\circ} \mathrm{C}\right) \delta 8.53(\mathrm{ddd}, J=4.8,1.8,0.9 \mathrm{~Hz}, 1 \mathrm{H}), 8.24-8.10(\mathrm{~m}, 1 \mathrm{H}), 8.07$ (d, $J=7.6 \mathrm{~Hz}, 1 \mathrm{H}), 7.84$ (td, $J=7.7,1.7 \mathrm{~Hz}, 1 \mathrm{H}), 7.41$ (ddd, $J=7.6,4.8,1.3 \mathrm{~Hz}, 1 \mathrm{H}), 5.84-5.75(\mathrm{~m}$, $1 \mathrm{H}), 5.63(\mathrm{dq}, J=4.8,1.3 \mathrm{~Hz}, 1 \mathrm{H}), 5.31(\mathrm{dt}, J=11.7,7.4 \mathrm{~Hz}, 1 \mathrm{H}), 4.76-4.63(\mathrm{~m}, 2 \mathrm{H}), 3.52-3.43$ (m, 2H), 2.36 (dddd, $J=11.8,7.3,5.8,1.7 \mathrm{~Hz}, 2 \mathrm{H}), 2.27-2.08(\mathrm{~m}, 4 \mathrm{H}), 2.06-1.95(\mathrm{~m}, 1 \mathrm{H}), 1.85-$ $1.67(\mathrm{~m}, 6 \mathrm{H}), 1.45$ (dddd, $J=12.6,11.3,9.3,7.3 \mathrm{~Hz}, 1 \mathrm{H}) ;{ }^{13} \mathbf{C ~ N M R}\left(100 \mathrm{MHz}, \mathrm{CDCl}_{3}, 25{ }^{\circ} \mathrm{C}\right) \delta$ $164.4,150.2$, 150.0, 148.1, 137.5, 135.0, 132.3, 128.5, 126.9, 126.2, 122.3, 108.7, 40.8, 39.1, 31.2, 30.2, 29.6, 27.9, 26.3, 20.9; FT-IR (neat) 3392, 2923, 2858, 1673, 1645, 1591, 1569, 1524, 1434, 1288, 1243, 998, 888, 749, 692, $621 \mathrm{~cm}^{-1}$; HRMS (ESI) $\mathrm{m} / z$ calculated for $\mathrm{C}_{20} \mathrm{H}_{27} \mathrm{~N}_{2} \mathrm{O}[\mathrm{M}+\mathrm{H}]^{+}$ 311.2118 , found 311.2120 ; O.R. $[\alpha]_{\mathrm{D}}^{24}=-59.9\left(c 1.0, \mathrm{CHCl}_{3}\right)$; TLC: $R_{f}=0.45(2: 1 \mathrm{Hex} / \mathrm{EtOAc})$.

\section{$N$-((Z)-5-((1R,5S)-6,6-dimethylbicyclo[3.1.1]hept-2-en-2-yl)pent-4-en-1-yl)picolinamide (1v)}

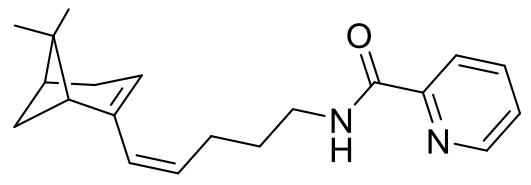

Crude (Z)-5-((1R,5S)-6,6-dimethylbicyclo[3.1.1]hept-2-en-2-yl)pent-4-enenitrile S2v was prepared according to general procedure 1 from $(R)-(-)$-myrtenal $(1.00 \mathrm{~g}, 6.65 \mathrm{mmol})$. The reaction was filtered through a silica plug using 4:1 Hex/EtOAc as eluent and the crude product was purified via flash column chromatography (pure Hexane to $20: 1 \mathrm{Hex} / \mathrm{EtOAc}$ ) to afford a colorless oil $(1.24 \mathrm{~g}$, $5.91 \mathrm{mmol}, 89 \%$ ) containing $4 \%$ aldehyde-impurities.

The title compound was prepared according to general procedures 2 and 3 from crude $(Z)-5-((1 \mathrm{R}, 5 \mathrm{~S})$ 6,6-dimethylbicyclo[3.1.1] hept-2-en-2-yl)pent-4-enenitrile $\mathbf{S 2 v}$ (750 mg, $3.73 \mathrm{mmol}$ ) prepared above. The crude product was purified via flash column chromatography (4:1 Hex/EtOAc) to afford an orange oil (763 mg, $2.46 \mathrm{mmol}, 66 \%)$.

${ }^{1} \mathbf{H}$ NMR $\left(400 \mathrm{MHz}, \mathrm{CDCl}_{3}, 25{ }^{\circ} \mathrm{C}\right) \delta 8.53(\mathrm{ddd}, J=4.8,1.7,0.9 \mathrm{~Hz}, 1 \mathrm{H}), 8.19(\mathrm{dt}, J=7.9,1.1 \mathrm{~Hz}$, $1 \mathrm{H}), 8.08(\mathrm{~d}, J=7.0 \mathrm{~Hz}, 1 \mathrm{H}), 7.83(\mathrm{td}, J=7.7,1.7 \mathrm{~Hz}, 1 \mathrm{H}), 7.41(\mathrm{ddd}, J=7.6,4.8,1.3 \mathrm{~Hz}, 1 \mathrm{H}), 5.75$ $(\mathrm{d}, J=11.5 \mathrm{~Hz}, 1 \mathrm{H}), 5.47-5.43(\mathrm{~m}, 1 \mathrm{H}), 5.31(\mathrm{dt}, J=11.6,7.3 \mathrm{~Hz}, 1 \mathrm{H}), 3.53-3.43(\mathrm{~m}, 2 \mathrm{H}), 2.40-$ $2.20(\mathrm{~m}, 6 \mathrm{H}), 2.10-2.00(\mathrm{~m}, 1 \mathrm{H}), 1.72(\mathrm{p}, J=7.3 \mathrm{~Hz}, 2 \mathrm{H}), 1.25$ (s, 3H), $1.18(\mathrm{~d}, J=8.6 \mathrm{~Hz}, 1 \mathrm{H}), 0.85$ $(\mathrm{s}, 3 \mathrm{H}) ;{ }^{13} \mathrm{C}$ NMR $\left(100 \mathrm{MHz}, \mathrm{CDCl}_{3}, 25{ }^{\circ} \mathrm{C}\right) \delta 164.3,150.2,148.1,145.2,137.4,130.8,128.7,126.1$, 122.4, 122.3, 46.6, 40.6, 39.2, 37.9, 32.0, 31.6, 30.2, 26.7, 26.5, 21.2; FT-IR (neat) 3391, 3056, 2984 , 2917, 2829, 1673, 1523, 1465, 1433, 1288, 998, 818, 749, $621 \mathrm{~cm}^{-1}$; HRMS (ESI) $m / z$ calculated for 
$\mathrm{C}_{20} \mathrm{H}_{26} \mathrm{~N}_{2} \mathrm{NaO}[\mathrm{M}+\mathrm{Na}]^{+} 333.1937$, found 333.1933; O.R. $[\alpha]_{\mathrm{D}}^{24}=-24.0\left(c\right.$ 1.0, $\left.\mathrm{CHCl}_{3}\right)$; TLC: $R_{f}=$ 0.45 (3:2 Hex/EtOAc). 


\section{(Z)-7-phenylhept-4-en-6-ynenitrile (S2w)}

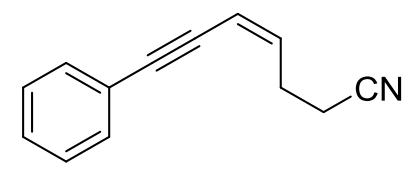

The title compound was prepared according to general procedure 1 from 3-phenylpropiolaldehyde $(0.58 \mathrm{~g}, 4.43 \mathrm{mmol})$. The reaction was filtered through a silica plug using 3:1 Hex/EtOAc as eluent and the crude product was purified via flash column chromatography (30:1 to 5:1 Hex/EtOAc) to afford a faint yellow oil $(0.46 \mathrm{~g}, 2.54 \mathrm{mmol}, 57 \%)$.

${ }^{1} \mathbf{H}$ NMR $\left(400 \mathrm{MHz}, \mathrm{CDCl}_{3}, 25{ }^{\circ} \mathrm{C}\right) \delta 7.49-7.41(\mathrm{~m}, 2 \mathrm{H}), 7.36-7.30(\mathrm{~m}, 3 \mathrm{H}), 6.00(\mathrm{dt}, J=10.7,7.2$ $\mathrm{Hz}, 1 \mathrm{H}), 5.87(\mathrm{dt}, J=10.7,1.2 \mathrm{~Hz}, 1 \mathrm{H}), 2.80-2.72(\mathrm{~m}, 2 \mathrm{H}), 2.51(\mathrm{t}, J=7.3 \mathrm{~Hz}, 2 \mathrm{H}) ;{ }^{13} \mathbf{C} \mathbf{~ N M R}(100$ $\left.\mathrm{MHz}, \mathrm{CDCl}_{3}, 2{ }^{\circ} \mathrm{C}\right) \delta 138.0,131.6,128.7,128.5,123.1,119.1,112.9,95.6,85.0,26.2,16.9$; FT-IR (neat) 3058, 3029, 2929, 2246, 2196, 1490, 1442, 156, $691 \mathrm{~cm}^{-1}$; HRMS (ESI) $\mathrm{m} / z$ calculated for $\mathrm{C}_{13} \mathrm{H}_{11} \mathrm{Na}[\mathrm{M}+\mathrm{Na}]^{+} 204.0784$, found 204.0784; TLC: $R_{f}=0.23$ (5:1 Hex/EtOAc).

(Z)- $N$-(7-phenylhept-4-en-6-yn-1-yl)picolinamide (1w)<smiles>O=C(NCCC/C=C\C#Cc1ccccc1)c1ccccn1</smiles>

The title compound was prepared according to general procedures 2 and 3 from (Z)-7-phenylhept-4en-6-ynenitrile (S2w) (400 mg, $2.21 \mathrm{mmol}$ ). The crude product was purified via flash column chromatography (2:1 Hex/EtOAc) to afford a faint yellow (556 mg, $1.92 \mathrm{mmol}, 87 \%)$.

${ }^{1} \mathbf{H}$ NMR $\left(400 \mathrm{MHz}, \mathrm{CDCl}_{3}, 25{ }^{\circ} \mathrm{C}\right) \delta 8.40(\mathrm{ddd}, J=4.8,1.8,0.9 \mathrm{~Hz}, 1 \mathrm{H}), 8.21-8.14(\mathrm{~m}, 2 \mathrm{H}), 7.82$ $(\mathrm{td}, J=7.7,1.7 \mathrm{~Hz}, 1 \mathrm{H}), 7.45-7.34(\mathrm{~m}, 3 \mathrm{H}), 7.30-7.23(\mathrm{~m}, 3 \mathrm{H}), 6.01(\mathrm{dt}, J=10.7,7.5 \mathrm{~Hz}, 1 \mathrm{H})$, $5.75(\mathrm{dt}, J=10.7,1.3 \mathrm{~Hz}, 1 \mathrm{H}), 3.54(\mathrm{q}, J=6.7 \mathrm{~Hz}, 2 \mathrm{H}), 2.53(\mathrm{qd}, J=7.4,1.3 \mathrm{~Hz}, 2 \mathrm{H}), 1.83(\mathrm{p}, J=7.1$ $\mathrm{Hz}, 2 \mathrm{H}) .{ }^{13} \mathrm{C} \mathbf{N M R}\left(100 \mathrm{MHz}, \mathrm{CDCl}_{3}, 25{ }^{\circ} \mathrm{C}\right) \delta 164.5,150.1,148.1,142.7,137.4,131.6,128.4,128.2$, 126.1, 123.6, 122.3, 110.4, 94.1, 86.2, 38.9, 28.9, 27.9; FT-IR (neat) 3385, 3056, 3020, 2930, 2196, 1668, 1521, 1242, 997, 749, 690, $620 \mathrm{~cm}^{-1}$; HRMS (ESI) $\mathrm{m} / z$ calculated for $\mathrm{C}_{19} \mathrm{H}_{19} \mathrm{~N}_{2} \mathrm{O}[\mathrm{M}+\mathrm{H}]^{+}$ 291.1492, found 291.1489; TLC: $R_{f}=0.17$ (97:3 DCM/EtOAc). 


\section{Synthesis of nopol derivative $1 \mathbf{y}$}

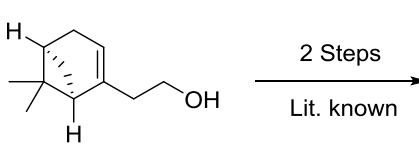

$(R)-(-)-$ Nopol

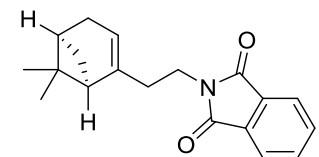

S3

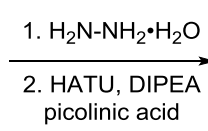

picolinic acid

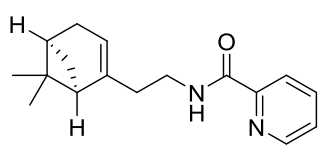

$1 y$

$N$-(2-((1R,5S)-6,6-dimethylbicyclo[3.1.1]hept-2-en-2-yl)ethyl)picolinamide (1y)<smiles>CC1CCC=C(CCNC(=O)c2ccccn2)C1</smiles>

To a solution 2-(2-((1R,5S)-6,6-dimethylbicyclo[3.1.1]hept-2-en-2-yl)ethyl)isoindoline-1,3-dione $\mathbf{S 3}^{10}$ $(2.00 \mathrm{~g}, 6.77 \mathrm{mmol}, 1.00$ equiv) in EtOH $(35 \mathrm{~mL})$ was added hydrazine monohydrate $(3.32 \mathrm{~mL}$, $67.7 \mathrm{mmol}, 10.0$ equiv). The reaction was heated to $70{ }^{\circ} \mathrm{C}$ for $90 \mathrm{~min}$ and a large amount of precipitate was formed. The reaction was cooled to $0{ }^{\circ} \mathrm{C}$, filtered, the precipitate was washed with $\mathrm{EtOH}$, and the residual solid was discarded. The filtrate was concentrated, suspended in DCM (100 mL), washed with $1 \mathrm{M}$ aq $\mathrm{NaOH}(100 \mathrm{~mL})$. The aqueous phase was extracted with DCM $(2 \times 100 \mathrm{~mL})$, the combined organic phases were dried over $\mathrm{Na}_{2} \mathrm{SO}_{4}$ and concentrated to afford the crude amine as a red oil. The material was used without further purification for the next step.

The title compound was prepared according to general procedure 3 from the crude amine prepared above (quant. yield assumed, $6.77 \mathrm{mmol}$ ). The crude product was purified via flash column chromatography (6:1 to 3:1 Hex/EtOAc) to afford a yellow oil (1.47 g, $5.43 \mathrm{mmol}, 80 \%)$.

${ }^{1} \mathbf{H}$ NMR $\left(400 \mathrm{MHz}, \mathrm{CDCl}_{3}, 25{ }^{\circ} \mathrm{C}\right) \delta 8.53(\mathrm{ddd}, J=4.8,1.7,0.9 \mathrm{~Hz}, 1 \mathrm{H}), 8.18(\mathrm{dt}, J=7.8,1.1 \mathrm{~Hz}$, $1 \mathrm{H}), 8.08(\mathrm{~s}, 1 \mathrm{H}), 7.83(\mathrm{td}, J=7.7,1.7 \mathrm{~Hz}, 1 \mathrm{H}), 7.40(\mathrm{ddd}, J=7.6,4.8,1.3 \mathrm{~Hz}, 1 \mathrm{H}), 5.43-5.33(\mathrm{~m}$, $1 \mathrm{H}), 3.58-3.39(\mathrm{~m}, 2 \mathrm{H}), 2.45-2.17(\mathrm{~m}, 5 \mathrm{H}), 2.14-2.04(\mathrm{~m}, 2 \mathrm{H}), 1.27$ (s, 3H), 1.17 (d, J = 8.5 Hz, 1H), 0.85 (s, 3H); ${ }^{13} \mathrm{C}$ NMR (100 MHz, $\left.\mathrm{CDCl}_{3}, 25^{\circ} \mathrm{C}\right) \delta 164.2,150.2,148.2,145.4,137.4,126.1$, 122.2, 118.8, 45.6, 40.9, 38.1, 37.4, 36.7, 31.9, 31.5, 26.4, 21.3; FT-IR (neat) 3390, 3056, 2984, 2915 , 2880, 2833, 1676, 1524, 1465, 1433, 1287, 1245, 820, 749, $621 \mathrm{~cm}^{-1}$; HRMS (ESI) $\mathrm{m} / z$ calculated for $\mathrm{C}_{17} \mathrm{H}_{23} \mathrm{~N}_{2} \mathrm{O}[\mathrm{M}+\mathrm{H}]^{+} 271.1805$, found 271.1809. O.R. $[\alpha]_{\mathrm{D}}^{24}=-28.6\left(c 1.0, \mathrm{CHCl}_{3}\right)$; TLC: $R_{f}=0.45$ (2:1 Hex/EtOAc). 


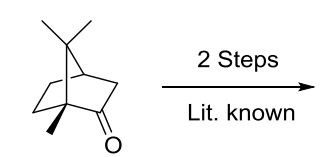

$(1 R)-(+)$-camphor

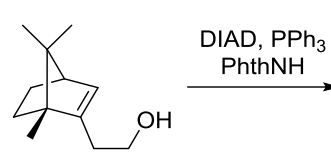

S4

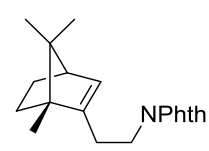

S5

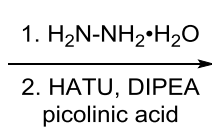

picolinic acid

$1 \mathrm{z}$

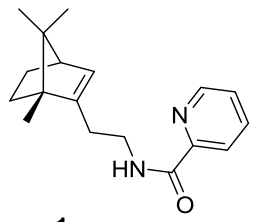

$1 z$

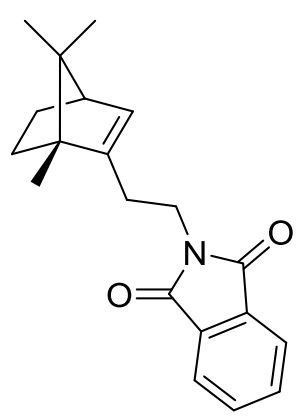

To a round bottom flask under $\mathrm{N}_{2}$ was added THF ( $8 \mathrm{~mL}$ ), triphenylphosphine $(873 \mathrm{mg}, 3.33 \mathrm{mmol}$, 2.00 equiv), isoindoline-1,3-dione ( $245 \mathrm{mg}, 1.66 \mathrm{mmol}, 1.00$ equiv), and a solution of 2-((1R,4R)1,7,7-trimethylbicyclo[2.2.1]hept-2-en-2-yl)ethanol $\mathbf{S 4}^{11}$ (300 $\mathrm{mg}, 1.66 \mathrm{mmol}, 1.00$ equiv) in THF (4 mL). The solution was cooled to $0{ }^{\circ} \mathrm{C}$ and diisopropyl azodicarboxylate $(0.65 \mathrm{~mL}, 3.33 \mathrm{mmol}$, 2.00 equiv) was added dropwise. The reaction was stirred for $2 \mathrm{~h}$ at $0{ }^{\circ} \mathrm{C}$, allowed to warm to room temperature and stirred for an additional $18 \mathrm{~h}$. The reaction was poured into rapidly stirred hexane $(80 \mathrm{~mL})$, filtered through a silica plug (washed with 2:1 Hex/EtOAc), concentrated, and purified by flash column chromatography (8:1 Hex/EtOAc) to afford an off-white solid (338 mg, $1.09 \mathrm{mmol}$, $66 \%)$.

${ }^{1} \mathbf{H}$ NMR $\left(500 \mathrm{MHz}, \mathrm{CDCl}_{3}, 25{ }^{\circ} \mathrm{C}\right) \delta 7.86-7.81(\mathrm{~m}, 2 \mathrm{H}), 7.74-7.67(\mathrm{~m}, 2 \mathrm{H}), 5.68(\mathrm{dt}, J=3.5,1.9$ $\mathrm{Hz}, 1 \mathrm{H}), 3.79(\mathrm{ddd}, J=8.7,6.9,1.7 \mathrm{~Hz}, 2 \mathrm{H}), 2.41-2.28(\mathrm{~m}, 2 \mathrm{H}), 2.23(\mathrm{t}, J=3.4 \mathrm{~Hz}, 1 \mathrm{H}), 1.85-1.75$ (m, 1H), $1.51-1.43(\mathrm{~m}, 1 \mathrm{H}), 0.98(\mathrm{~s}, 3 \mathrm{H}), 0.95-0.89(\mathrm{~m}, 2 \mathrm{H}), 0.75(\mathrm{~s}, 3 \mathrm{H}), 0.75(\mathrm{~s}, 3 \mathrm{H}) ;{ }^{13} \mathbf{C}$ NMR $\left(126 \mathrm{MHz}, \mathrm{CDCl}_{3}, 25{ }^{\circ} \mathrm{C}\right) \delta 168.5,145.8,134.0,132.3,128.3,123.3,56.4,54.4,51.5,36.7,31.5,26.7$, 25.9, 19.8, 19.8, 11.5; FT-IR (neat) 3048, 2949, 2870, 1773, 1709, 1467, 1395, 1377, 1187, 1101, 1007, $718 \mathrm{~cm}^{-1}$; HRMS (ESI) $\mathrm{m} / z$ calculated for $\mathrm{C}_{20} \mathrm{H}_{23} \mathrm{NNaO}_{2}[\mathrm{M}+\mathrm{Na}]^{+} 332.1621$, found 332.1620; Melting point $37.5-38.4{ }^{\circ} \mathrm{C}$; O.R. $[\alpha]_{\mathrm{D}}^{24}=-13.9\left(c 1.0, \mathrm{CHCl}_{3}\right)$; TLC: $R_{f}=0.46(3: 2 \mathrm{Hex} / \mathrm{EtOAc})$. 


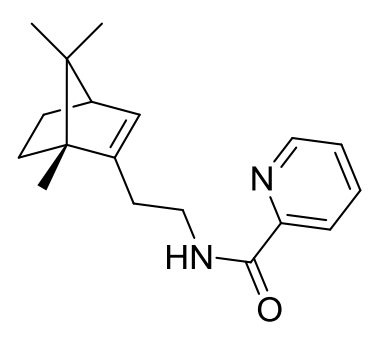

To a solution of 2-(2-((1R,4R)-1,7,7-trimethylbicyclo[2.2.1]hept-2-en-2-yl)ethyl)isoindoline-1,3-dione S5 (300 mg, $0.97 \mathrm{mmol}, 1.0$ equiv) in EtOH $(4 \mathrm{~mL})$ was added hydrazine monohydrate $(0.48 \mathrm{~mL}$, $9.70 \mathrm{mmol}, 10.0$ equiv). The reaction heated to $70{ }^{\circ} \mathrm{C}$ for $90 \mathrm{~min}$ and a large amount of precipitate was formed. The reaction was cooled to $0{ }^{\circ} \mathrm{C}$, filtered, the precipitate was washed with $\mathrm{EtOH}$, and the residual solid was discarded. The filtrate was concentrated, suspended in DCM (20 mL), washed with $1 \mathrm{M}$ aq $\mathrm{NaOH}(20 \mathrm{~mL})$. The aqueous phase was extracted with DCM $(2 \times 20 \mathrm{~mL})$, the combined organic phases were dried over $\mathrm{Na}_{2} \mathrm{SO}_{4}$ and concentrated to afford the crude amine as a colorless oil. The material was used without further purification for the next step.

The title compound was prepared according to general procedure 3 from the crude amine prepared above (quant. yield assumed, $0.97 \mathrm{mmol}$ ). The crude product was purified via flash column chromatography (4:1 Hex/EtOAc) to afford a colorless oil (181 mg, $0.64 \mathrm{mmol}, 66 \%)$.

${ }^{1} \mathbf{H}$ NMR $\left(500 \mathrm{MHz}, \mathrm{CDCl}_{3}, 25{ }^{\circ} \mathrm{C}\right) \delta 8.52(\mathrm{ddd}, J=4.8,1.7,0.9 \mathrm{~Hz}, 1 \mathrm{H}), 8.19(\mathrm{dt}, J=7.8,1.1 \mathrm{~Hz}$, $1 \mathrm{H}), 8.12(\mathrm{~s}, 1 \mathrm{H}), 7.83(\mathrm{td}, J=7.7,1.7 \mathrm{~Hz}, 1 \mathrm{H}), 7.40(\mathrm{ddd}, J=7.6,4.8,1.3 \mathrm{~Hz}, 1 \mathrm{H}), 5.71(\mathrm{dt}, J=3.6$, $1.8 \mathrm{~Hz}, 1 \mathrm{H}), 3.65-3.54(\mathrm{~m}, 2 \mathrm{H}), 2.37-2.24(\mathrm{~m}, 3 \mathrm{H}), 1.86-1.78(\mathrm{~m}, 1 \mathrm{H}), 1.53-1.47(\mathrm{~m}, 1 \mathrm{H}), 1.03$ $-0.90(\mathrm{~m}, 5 \mathrm{H}), 0.79$ (s, 3H), $0.76(\mathrm{~s}, 3 \mathrm{H}) ;{ }^{13} \mathbf{C ~ N M R}\left(126 \mathrm{MHz}, \mathrm{CDCl}_{3}, 25{ }^{\circ} \mathrm{C}\right) \delta 164.3,150.2,148.2$, 146.3, 137.4, 128.5, 126.1, 122.2, 56.5, 54.4, 51.5, 37.5, 31.5, 28.0, 26.0, 19.9, 19.7, 11.6; FT-IR (neat) 3388, 3055, 2949, 2871, 1676, 1523, 1465, 1434, 1286, 998, 749, $621 \mathrm{~cm}^{-1}$; HRMS (ESI) $\mathrm{m} / \mathrm{z}$ calculated for $\mathrm{C}_{18} \mathrm{H}_{24} \mathrm{~N}_{2} \mathrm{NaO}[\mathrm{M}+\mathrm{Na}]^{+} 307.1781$, found 307.1778; O.R. $[\alpha]_{\mathrm{D}}^{24}=-2.8\left(c 0.5, \mathrm{CHCl}_{3}\right)$; TLC: $R_{f}=0.27$ (97:3 DCM/EtOAc).

\section{(Z)-5-phenylpent-4-en-1-amine (S6)}

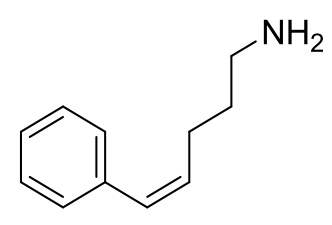

The title compound was prepared according to general procedure 2 from $(Z)-5$-phenylpent-4-enenitrile ( $1.00 \mathrm{~g}, 6.36 \mathrm{mmol}, 1.00$ equiv) to afford a colorless oil ( $0.94 \mathrm{~g}, 5.83 \mathrm{mmol}, 92 \%)$. 
${ }^{1} \mathbf{H}$ NMR $\left(400 \mathrm{MHz}, \mathrm{CDCl}_{3}, 25{ }^{\circ} \mathrm{C}\right) \delta 7.39-7.15(\mathrm{~m}, 5 \mathrm{H}), 6.44(\mathrm{~d}, J=11.6 \mathrm{~Hz}, 1 \mathrm{H}), 5.66(\mathrm{dt}, J=$ 11.7, 7.3 Hz, 1H), $2.75-2.69(\mathrm{~m}, 2 \mathrm{H}), 2.37$ (qd, $J=7.4,1.9 \mathrm{~Hz}, 2 \mathrm{H}), 1.65-1.56(\mathrm{~m}, 2 \mathrm{H}), 1.26$ (s, $2 \mathrm{H}) ;{ }^{13} \mathrm{C}$ NMR $\left(100 \mathrm{MHz}, \mathrm{CDCl}_{3}, 25{ }^{\circ} \mathrm{C}\right) \delta 137.7,132.5,129.4,128.9,128.3,126.7,42.0,34.1,26.1$; HRMS (ESI) $m / z$ calculated for $\mathrm{C}_{11} \mathrm{H}_{16} \mathrm{~N}[\mathrm{M}+\mathrm{H}]^{+} 162.1277$, found 162.1283 . The analytical data are in agreement with the literature. ${ }^{12}$

(Z)- $N$-(5-phenylpent-4-en-1-yl)quinoline-2-carboxamide (S7)

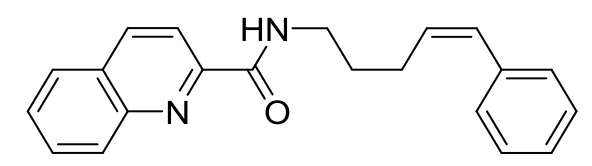

The title compound was prepared according to general procedure 3 from (Z)-5-phenylpent-4-en-1amine S6 (150 mg, $0.93 \mathrm{mmol}, 1.00$ equiv) and quinoline-2-carboxylic acid (177 mg, $1.02 \mathrm{mmol}$, 1.10 equiv). The crude product was purified via flash column chromatography (3:1 Hex/EtOAc) to afford a white solid (277 mg, $0.88 \mathrm{mmol}, 94 \%)$.

${ }^{1} \mathbf{H}$ NMR $\left(400 \mathrm{MHz}, \mathrm{CDCl}_{3}, 25{ }^{\circ} \mathrm{C}\right) \delta 8.31(\mathrm{~s}, 3 \mathrm{H}), 8.08(\mathrm{dt}, J=8.6,0.9 \mathrm{~Hz}, 1 \mathrm{H}), 7.88(\mathrm{ddd}, J=8.2$, 1.5, $0.7 \mathrm{~Hz}, 1 \mathrm{H}), 7.77(\mathrm{ddd}, J=8.5,6.9,1.4 \mathrm{~Hz}, 1 \mathrm{H}), 7.62(\mathrm{ddd}, J=8.1,6.9,1.2 \mathrm{~Hz}, 1 \mathrm{H}), 7.33-7.26$ (m, 4H), $7.22-7.16(\mathrm{~m}, 1 \mathrm{H}), 6.49(\mathrm{dt}, J=11.6,1.8 \mathrm{~Hz}, 1 \mathrm{H}), 5.73(\mathrm{dt}, J=11.6,7.3 \mathrm{~Hz}, 1 \mathrm{H}), 3.60-$ $3.51(\mathrm{~m}, 2 \mathrm{H}), 2.49(\mathrm{qd}, J=7.4,1.8 \mathrm{~Hz}, 2 \mathrm{H}), 1.94-1.80(\mathrm{~m}, 2 \mathrm{H}) ;{ }^{13} \mathbf{C ~ N M R}\left(100 \mathrm{MHz}, \mathrm{CDCl}_{3}, 25{ }^{\circ} \mathrm{C}\right)$ $\delta$ 164.6, 150.0, 146.6, 137.6, 137.6, 131.8, 130.2, 129.9, 129.8, 129.4, 128.8, 128.3, 128.0, 127.9, 126.7, 119.0, 39.3, 30.0, 26.2; FT-IR (neat) 3389, 3057, 3009, 2930, 2863, 1672, 1527, 1501, 1427, 1377, 1208, 847, 773, $700 \mathrm{~cm}^{-1}$; HRMS (ESI) $\mathrm{m} / z$ calculated for $\mathrm{C}_{21} \mathrm{H}_{21} \mathrm{~N}_{2} \mathrm{O}[\mathrm{M}+\mathrm{H}]^{+} 317.1648$, found 317.1650; Melting point $55.1-56.0{ }^{\circ} \mathrm{C}$; TLC: $R_{f}=0.41(3: 1 \mathrm{Hex} / \mathrm{EtOAc})$.

\section{(E)- $N$-(4-benzylidene-6-(trimethylsilyl)hex-5-yn-1-yl)picolinamide (S8)}<smiles>COc1cccc(C(=O)NCCC/C=C\c2ccccc2)n1</smiles>

The title compound was prepared according to general procedure 3 from (Z)-5-phenylpent-4-en-1amine S6 (125 mg, $0.78 \mathrm{mmol}, 1.00$ equiv) and 6-methoxypicolinic acid (131 mg, $0.85 \mathrm{mmol}$, 
1.10 equiv). The crude product was purified via flash column chromatography (4:1 to 2:1 Hex/EtOAc) to afford a colorless oil (205 $\mathrm{mg}, 0.69 \mathrm{mmol}, 89 \%)$.

${ }^{1} \mathbf{H}$ NMR $\left(400 \mathrm{MHz}, \mathrm{CDCl}_{3}, 25{ }^{\circ} \mathrm{C}\right) \delta 7.84(\mathrm{~s}, 1 \mathrm{H}), 7.79(\mathrm{dd}, J=7.3,1.0 \mathrm{~Hz}, 1 \mathrm{H}), 7.70(\mathrm{dd}, J=8.2$, $7.2 \mathrm{~Hz}, 1 \mathrm{H}), 7.33-7.24(\mathrm{~m}, 4 \mathrm{H}), 7.23-7.16(\mathrm{~m}, 1 \mathrm{H}), 6.87(\mathrm{dd}, J=8.2,1.0 \mathrm{~Hz}, 1 \mathrm{H}), 6.47$ (dt, $J=$ 11.6, $1.9 \mathrm{~Hz}, 1 \mathrm{H}), 5.70(\mathrm{dt}, J=11.6,7.3 \mathrm{~Hz}, 1 \mathrm{H}), 3.89$ (s, 3H), $3.50(\mathrm{td}, J=7.1,6.1 \mathrm{~Hz}, 2 \mathrm{H}), 2.45$ (qd, $J=7.4,1.8 \mathrm{~Hz}, 2 \mathrm{H}), 1.89-1.73(\mathrm{~m}, 2 \mathrm{H}) ;{ }^{13} \mathbf{C ~ N M R}\left(100 \mathrm{MHz}, \mathrm{CDCl}_{3}, 25{ }^{\circ} \mathrm{C}\right) \delta 164.3,162.9,147.5$, 139.8, 137.5, 131.7, 129.9, 128.8, 128.3, 126.7, 115.6, 114.2, 53.5, 39.1, 30.0, 26.2; FT-IR (neat) 3399, 3056, 3010, 2947, 2860, 1672, 1596, 1576, 1522, 1467, 1429, 1334, 1269, 1027, 769, $700 \mathrm{~cm}^{-1}$; HRMS (ESI) $m / z$ calculated for $\mathrm{C}_{18} \mathrm{H}_{21} \mathrm{~N}_{2} \mathrm{O}_{2}[\mathrm{M}+\mathrm{H}]^{+} 297.1598$, found 297.1599; TLC: $R_{f}=0.47$ (1:1 $\mathrm{Hex} / \mathrm{EtOAc})$.

(Z)- $N$-(5-phenylpent-4-en-1-yl)-6-(trifluoromethyl)picolinamide (S9)<smiles>O=C(NCCC/C=C\c1ccccc1)c1cccc(C(F)(F)F)n1</smiles>

The title compound was prepared according to general procedure 3 from (Z)-5-phenylpent-4-en-1amine S6 (125 mg, $0.78 \mathrm{mmol}, 1.00$ equiv) and 6-(trifluoromethyl)picolinic acid (163 mg, $0.85 \mathrm{mmol}$, 1.10 equiv). The crude product was purified via flash column chromatography (3:1 Hex/EtOAc) to afford a pale yellow oil (220 mg, $0.66 \mathrm{mmol}, 85 \%)$.

${ }^{1} \mathbf{H}$ NMR $\left(500 \mathrm{MHz}, \mathrm{CDCl}_{3}, 25{ }^{\circ} \mathrm{C}\right) \delta 8.39(\mathrm{ddd}, J=7.9,1.1,0.6 \mathrm{~Hz}, 1 \mathrm{H}), 8.04(\mathrm{tt}, J=7.8,0.7 \mathrm{~Hz}$, $1 \mathrm{H}), 7.96(\mathrm{~s}, 1 \mathrm{H}), 7.81(\mathrm{dd}, J=7.8,1.1 \mathrm{~Hz}, 1 \mathrm{H}), 7.32-7.24(\mathrm{~m}, 4 \mathrm{H}), 7.22-7.17(\mathrm{~m}, 1 \mathrm{H}), 6.48(\mathrm{dt}, J$ $=11.6,1.9 \mathrm{~Hz}, 1 \mathrm{H}), 5.70(\mathrm{dt}, J=11.6,7.3 \mathrm{~Hz}, 1 \mathrm{H}), 3.68-3.42(\mathrm{~m}, 2 \mathrm{H}), 2.45(\mathrm{qd}, J=7.4,1.8 \mathrm{~Hz}$, 2H), $1.97-1.73(\mathrm{~m}, 2 \mathrm{H}) ;{ }^{13} \mathbf{C} \mathbf{~ N M R}\left(126 \mathrm{MHz}, \mathrm{CDCl}_{3}, 25{ }^{\circ} \mathrm{C}\right) \delta 162.9,150.5,146.9$ (q, $\left.J=35.3 \mathrm{~Hz}\right)$, 139.2, 137.5, 131.6, 130.0, 128.8, 128.3, 126.8, 125.1, 122.7 (q, $J=2.7 \mathrm{~Hz}), 121.3$ (q, $J=274.4 \mathrm{~Hz}$ ), 39.4, 29.9, 26.2; ${ }^{19}$ F NMR $\left(471 \mathrm{MHz}, \mathrm{CDCl}_{3}, 25^{\circ} \mathrm{C}\right) \delta-67.69$; FT-IR (neat) 3406, 3012, 2935, 2865, 1679, 1527, 1342, 1246, 1189, 1141, 1113, 1081, 767, $700 \mathrm{~cm}^{-1}$; HRMS (ESI) $\mathrm{m} / z$ calculated for $\mathrm{C}_{18} \mathrm{H}_{18} \mathrm{~F}_{3} \mathrm{~N}_{2} \mathrm{O}[\mathrm{M}+\mathrm{H}]^{+} 335.1366$, found 335.1368; TLC: $R_{f}=0.59$ (1:1 Hex/EtOAc). 


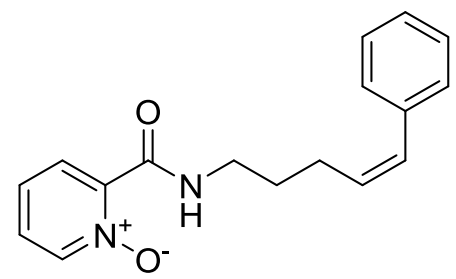

The title compound was prepared according to general procedure 3 from $(Z)$-5-phenylpent-4-en-1amine S6 (100 mg, $0.62 \mathrm{mmol}, 1.00$ equiv) and 2-carboxypyridine 1-oxide (95 mg, $0.68 \mathrm{mmol}$, 1.10 equiv). The crude product was purified via flash column chromatography (3:1 Hex/EtOAc) to afford colorless oil (120 mg, $0.43 \mathrm{mmol}, 69 \%)$.

${ }^{1} \mathbf{H}$ NMR $\left(400 \mathrm{MHz}, \mathrm{CD}_{3} \mathrm{CN}, 25^{\circ} \mathrm{C}\right) \delta 11.32(\mathrm{~s}, 1 \mathrm{H}), 8.30-8.24(\mathrm{~m}, 1 \mathrm{H}), 8.24-8.20(\mathrm{~m}, 1 \mathrm{H}), 7.52$ $7.40(\mathrm{~m}, 2 \mathrm{H}), 7.33-7.24(\mathrm{~m}, 4 \mathrm{H}), 7.24-7.13(\mathrm{~m}, 1 \mathrm{H}), 6.45(\mathrm{dt}, J=11.7,1.8 \mathrm{~Hz}, 1 \mathrm{H}), 5.72(\mathrm{dt}, J=$ 11.7, 7.4 Hz, 1H), $3.43(\mathrm{td}, J=7.0,5.8 \mathrm{~Hz}, 2 \mathrm{H}), 2.40(\mathrm{qd}, J=7.4,1.8 \mathrm{~Hz}, 2 \mathrm{H}), 1.81-1.71(\mathrm{~m}, 2 \mathrm{H})$; ${ }^{13} \mathrm{C}$ NMR $\left(100 \mathrm{MHz}, \mathrm{CD}_{3} \mathrm{CN}, 25{ }^{\circ} \mathrm{C}\right) \delta 160.6,141.7,141.3,138.4,132.9,130.2,129.6,129.22$, 129.16, 128.7, 128.1, 127.6, 39.6, 30.2, 26.8; FT-IR (neat) 3390, 3110, 3054, 3011, 2865, 1667, 1602, 1541, 1475, 1431, 1304, 1182, 786, 763, 701, 673, $682 \mathrm{~cm}^{-1}$; HRMS (ESI) $\mathrm{m} / \mathrm{z}$ calculated for $\mathrm{C}_{17} \mathrm{H}_{19} \mathrm{~N}_{2} \mathrm{O}_{2}[\mathrm{M}+\mathrm{H}]^{+} 283.1441$, found 283.1437; TLC: $R_{f}=0.30$ (EtOAc).

(Z)- $N$-(5-phenylpent-4-en-1-yl)pyrazine-2-carboxamide (S11)

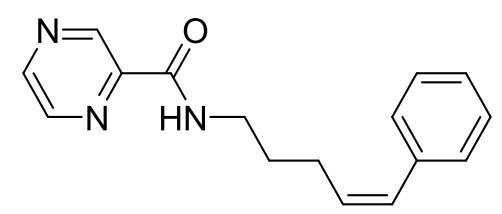

The title compound was prepared according to general procedure 3 from (Z)-5-phenylpent-4-en-1amine S6 (150 mg, $0.93 \mathrm{mmol}, 1.00$ equiv) and pyrazine-2-carboxylic acid (115 mg, $0.93 \mathrm{mmol}$, 1.00 equiv). The crude product was purified via flash column chromatography (2:1 Hex/EtOAc) to afford an amorphous white solid (226 mg, $0.85 \mathrm{mmol}, 91 \%)$.

${ }^{1} \mathbf{H}$ NMR $\left(400 \mathrm{MHz}, \mathrm{CDCl}_{3}, 25{ }^{\circ} \mathrm{C}\right) \delta 9.39(\mathrm{~d}, J=1.5 \mathrm{~Hz}, 1 \mathrm{H}), 8.73(\mathrm{~d}, J=2.5 \mathrm{~Hz}, 1 \mathrm{H}), 8.48(\mathrm{dd}, J=$ 2.5, $1.5 \mathrm{~Hz}, 1 \mathrm{H}), 7.78(\mathrm{~s}, 1 \mathrm{H}), 7.39-7.10(\mathrm{~m}, 5 \mathrm{H}), 6.48(\mathrm{dt}, J=11.6,1.9 \mathrm{~Hz}, 1 \mathrm{H}), 5.68(\mathrm{dt}, J=11.6$, $7.3 \mathrm{~Hz}, 1 \mathrm{H}), 3.50(\mathrm{td}, J=7.1,6.2 \mathrm{~Hz}, 2 \mathrm{H}), 2.44(\mathrm{qd}, J=7.4,1.8 \mathrm{~Hz}, 2 \mathrm{H}), 1.84-1.75(\mathrm{~m}, 2 \mathrm{H}) ;{ }^{13} \mathbf{C}$ NMR $\left(100 \mathrm{MHz}, \mathrm{CDCl}_{3}, 25^{\circ} \mathrm{C}\right) \delta 163.0,147.3,144.6,144.5,142.6,137.5,131.4,130.1,128.8$, 128.3, 126.8, 39.1, 29.7, 25.9; FT-IR (neat) 3334, 3055, 3009, 2931, 2864, 1667, 1529, 1494, 1466, 1400, 1299, 1155, 1020, 773, $699 \mathrm{~cm}^{-1}$; HRMS (ESI) $\mathrm{m} / z$ calculated for $\mathrm{C}_{16} \mathrm{H}_{18} \mathrm{~N}_{3} \mathrm{O}[\mathrm{M}+\mathrm{H}]^{+}$ 268.1444, found 268.1448; Melting point 59.0-60.8 ${ }^{\circ} \mathrm{C}$; TLC: $R_{f}=0.16(3: 2 \mathrm{Hex} / \mathrm{EtOAc})$. 


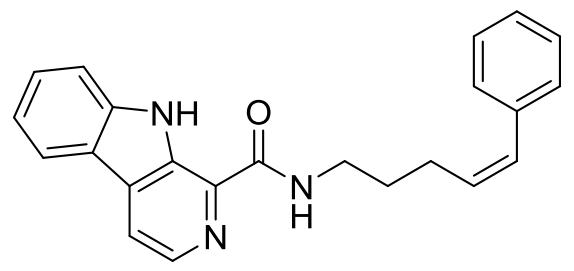

Based on a literature protocol, ${ }^{13}$ lithium $9 \mathrm{H}$-pyrido[3,4-b]indole-1-carboxylate ${ }^{14}$ (135 mg, $0.62 \mathrm{mmol}$, 1.00 equiv) was suspended in DMF (10.0 mL), HATU (259 mg, $0.68 \mathrm{mmol}, 1.10$ equiv) was added and the reaction was stirred for $30 \mathrm{~min}$. A solution of (Z)-5-phenylpent-4-en-1-amine S6 (100 mg, $0.62 \mathrm{mmol}, 1.00$ equiv) and $N, N$-diisopropylethylamine $(0.12 \mathrm{~mL}, 0.68 \mathrm{mmol}, 1.10$ equiv) in DMF $(3 \mathrm{~mL})$ was added dropwise, then the reaction was stirred for $2 \mathrm{~h}$. The reaction was diluted with sat aq $\mathrm{NaHCO}_{3}(50 \mathrm{~mL})$, extracted with EtOAc $(2 \times 50 \mathrm{~mL})$, the combined organic phases were washed with brine $(1 \times 50 \mathrm{~mL})$, dried over $\mathrm{Na}_{2} \mathrm{SO}_{4}$, and concentrated. The crude product was purified via flash column chromatography (6:1 Hex/EtOAc) to afford an off-white solid (210 mg, $0.59 \mathrm{mmol}, 95 \%)$.

${ }^{1} \mathbf{H}$ NMR $\left(400 \mathrm{MHz}, \mathrm{CDCl}_{3}, 25{ }^{\circ} \mathrm{C}\right) \delta 8.36(\mathrm{~d}, J=5.0 \mathrm{~Hz}, 1 \mathrm{H}), 8.28-8.11(\mathrm{~m}, 2 \mathrm{H}), 8.08(\mathrm{dd}, J=5.1$, $0.7 \mathrm{~Hz}, 1 \mathrm{H}), 7.65-7.52$ (m, 2H), $7.34-7.25(\mathrm{~m}, 5 \mathrm{H}), 7.22-7.16(\mathrm{~m}, 1 \mathrm{H}), 6.49$ (dt, $J=11.6,1.8 \mathrm{~Hz}$, $1 \mathrm{H}), 5.72(\mathrm{dt}, J=11.6,7.3 \mathrm{~Hz}, 1 \mathrm{H}), 3.57(\mathrm{td}, J=7.2,6.2 \mathrm{~Hz}, 2 \mathrm{H}), 2.50(\mathrm{qd}, J=7.4,1.8 \mathrm{~Hz}, 2 \mathrm{H}), 1.95$ $-1.81(\mathrm{~m}, 2 \mathrm{H}) ;{ }^{13} \mathrm{C}$ NMR $\left(100 \mathrm{MHz}, \mathrm{CDCl}_{3}, 25{ }^{\circ} \mathrm{C}\right) \delta 166.5,141.1,137.4,137.2,135.5,132.1,131.5$, 131.3, 129.9, 129.2, 128.7, 128.2, 126.6, 121.8, 120.6, 120.3, 117.7, 111.9, 38.7, 30.0, 26.0; FT-IR (neat) 3364, 3057, 3009, 2931, 1655, 1626, 1527, 1493, 1321, 1250, 766, 728, $634 \mathrm{~cm}^{-1}$; HRMS (ESI) $m / z$ calculated for $\mathrm{C}_{23} \mathrm{H}_{22} \mathrm{~N}_{3} \mathrm{O}[\mathrm{M}+\mathrm{H}]^{+} 356.1757$, found 356.1761 ; Melting point $91.8-93{ }^{\circ} \mathrm{C}$; TLC: $R_{f}=0.55$ (3:1 Hex/EtOAc).

\section{(Z)-5-phenyl- $N$-(2-(pyridin-2-yl)propan-2-yl)pent-4-enamide (S13)}<smiles>CC(C)(NC(=O)CC/C=C\c1ccccn1)c1ccccn1</smiles>

The title compound was prepared according to general procedure 3 from 2-(pyridin-2-yl)propan-2amine (100 mg, $0.74 \mathrm{mmol}, 1.00$ equiv) and (Z)-5-phenylpent-4-enoic acid $^{3}$ (142 mg, $0.81 \mathrm{mmol}$, 1.10 equiv). The crude product was purified via flash column chromatography (3:2 Hex/EtOAc) to afford an amorphous white solid (205 mg, $0.70 \mathrm{mmol}, 95 \%)$.

${ }^{1} \mathbf{H}$ NMR $\left(400 \mathrm{MHz}, \mathrm{CDCl}_{3}, 25{ }^{\circ} \mathrm{C}\right) \delta 8.48(\mathrm{ddd}, J=4.9,1.8,1.0 \mathrm{~Hz}, 1 \mathrm{H}), 7.77-7.67(\mathrm{~m}, 2 \mathrm{H}), 7.39$ $(\mathrm{dt}, J=8.1,1.0 \mathrm{~Hz}, 1 \mathrm{H}), 7.35-7.27(\mathrm{~m}, 4 \mathrm{H}), 7.24-7.17(\mathrm{~m}, 2 \mathrm{H}), 6.46(\mathrm{dt}, J=11.6,1.9 \mathrm{~Hz}, 1 \mathrm{H})$, $5.68(\mathrm{dt}, J=11.6,7.2 \mathrm{~Hz}, 1 \mathrm{H}), 2.72(\mathrm{qd}, J=7.6,1.9 \mathrm{~Hz}, 2 \mathrm{H}), 2.40(\mathrm{dd}, J=8.4,6.8 \mathrm{~Hz}, 2 \mathrm{H}), 1.74(\mathrm{~s}$, 
$6 \mathrm{H}) ;{ }^{13} \mathrm{C}$ NMR $\left(100 \mathrm{MHz}, \mathrm{CDCl}_{3}, 25{ }^{\circ} \mathrm{C}\right) \delta 171.5,164.6,147.6,137.4,137.4,131.2,129.9,128.9$, 128.3, 126.8, 122.0, 119.7, 56.5, 37.7, 27.7, 24.9; FT-IR (neat) 3313, 3056, 3008, 2978, 2933, 1648, 1591, 1569, 1539, 1508, 1495, 1447, 1430, 1380, 1204, 1127, 994, 787,748, 700, $624 \mathrm{~cm}^{-1}$; HRMS (ESI) $m / z$ calculated for $\mathrm{C}_{19} \mathrm{H}_{22} \mathrm{~N}_{2} \mathrm{NaO}[\mathrm{M}+\mathrm{Na}]^{+} 317.1624$, found 317.1631 ; Melting point $46.8-$ $48.3{ }^{\circ} \mathrm{C}$; TLC: $R_{f}=0.43(1: 1 \mathrm{Hex} / \mathrm{EtOAc})$. 


\section{Synthesis of alkenyl iodides}

\section{(E)- $N$-(4-iodo-5-phenylpent-4-en-1-yl)picolinamide (2a)}<smiles>O=C(NCCC/C(I)=C\c1ccccc1)c1ccccn1</smiles>

The title compound was prepared according to general procedure 4 from ( $Z$ )- $N$-(5-phenylpent-4-en-1yl)picolinamide 1a $(80 \mathrm{mg}, 0.30 \mathrm{mmol})$. The crude product was purified via flash column chromatography (97:3 DCM/EtOAc) to afford a faint yellow oil (75 mg, $0.19 \mathrm{mmol}, 64 \%)$.

${ }^{1} \mathbf{H}$ NMR $\left(400 \mathrm{MHz}, \mathrm{CDCl}_{3}, 25{ }^{\circ} \mathrm{C}\right) \delta 8.51(\mathrm{dt}, J=4.8,1.3 \mathrm{~Hz}, 1 \mathrm{H}), 8.16(\mathrm{dt}, J=7.8,1.2 \mathrm{~Hz}, 1 \mathrm{H})$, $8.02(\mathrm{~s}, 1 \mathrm{H}), 7.83(\mathrm{td}, J=7.7,1.7 \mathrm{~Hz}, 1 \mathrm{H}), 7.41$ (ddd, $J=7.6,4.8,1.3 \mathrm{~Hz}, 1 \mathrm{H}), 7.33(\mathrm{~s}, 1 \mathrm{H}), 7.27-$ $7.11(\mathrm{~m}, 5 \mathrm{H}), 3.47(\mathrm{q}, J=6.8 \mathrm{~Hz}, 2 \mathrm{H}), 2.71-2.66(\mathrm{~m}, 2 \mathrm{H}), 1.95(\mathrm{dq}, J=9.6,7.1 \mathrm{~Hz}, 2 \mathrm{H}) ;{ }^{13} \mathbf{C} \mathbf{~ N M R}$ $\left(100 \mathrm{MHz}, \mathrm{CDCl}_{3}, 25{ }^{\circ} \mathrm{C}\right) \delta 164.4,149.9,148.1,141.6,137.5,137.4,128.6,128.0,127.4,126.2$, 122.3, 107.4, 38.2, 36.8, 30.1; FT-IR (neat) 3377, 3055, 3022, 2928, 2866, 1667, 1590, 1569, 1521, 1464, 1433, 1287, 1095, 997, 856, 819, 747, 698, 620; HRMS (ESI) $m / z$ calculated for $\mathrm{C}_{17} \mathrm{H}_{18} \mathrm{IN}_{2} \mathrm{O}$ $[\mathrm{M}+\mathrm{H}]^{+} 393.0458$, found 393.0457; TLC: $R_{f}=0.44$ (3:2 Hex/EtOAc).

(E)- $N$-(4-iodo-5-(naphthalen-2-yl)pent-4-en-1-yl)picolinamide (2b)<smiles>O=C(NCCC/C(I)=C\c1ccc2ccccc2c1)c1ccccn1</smiles>

The title compound was prepared according to general procedure 4 from (Z)- $N$-(5-(naphthalen-2yl)pent-4-en-1-yl)picolinamide $\mathbf{1 b}(80 \mathrm{mg}, 0.30 \mathrm{mmol})$. The crude product was purified via flash column chromatography (97:3 DCM/EtOAc) to afford a colorless oil (83 mg, $0.19 \mathrm{mmol}, 63 \%)$.

${ }^{1} \mathbf{H}$ NMR $\left(400 \mathrm{MHz}, \mathrm{CDCl}_{3}, 25{ }^{\circ} \mathrm{C}\right) \delta 8.27(\mathrm{~d}, J=4.0 \mathrm{~Hz}, 1 \mathrm{H}), 8.11(\mathrm{~d}, J=7.8 \mathrm{~Hz}, 1 \mathrm{H}), 7.96(\mathrm{~s}, 2 \mathrm{H})$, $7.85-7.69(\mathrm{~m}, 4 \mathrm{H}), 7.64(\mathrm{~s}, 1 \mathrm{H}), 7.49(\mathrm{~s}, 1 \mathrm{H}), 7.48-7.39(\mathrm{~m}, 2 \mathrm{H}), 7.36-7.27$ (m, 2H), 3.49 (q, $J=$ $6.7 \mathrm{~Hz}, 2 \mathrm{H}), 2.82-2.75(\mathrm{~m}, 2 \mathrm{H}), 2.00(\mathrm{dq}, J=9.1,6.9 \mathrm{~Hz}, 2 \mathrm{H}) ;{ }^{13} \mathbf{C ~ N M R}\left(100 \mathrm{MHz}, \mathrm{CDCl}_{3}, 25{ }^{\circ} \mathrm{C}\right)$ $\delta 164.4,149.8,148.0,141.8,137.3,135.0,133.4,132.5,128.3,128.2,127.8,127.1,126.5,126.3$, 126.1, 122.2, 107.8, 38.1, 36.8, 30.0; FT-IR (neat) 3379, 3054, 2928, 1672, 1523, 1434, 1288, 1040, 
997, 818, 748, $621 \mathrm{~cm}^{-1}$; HRMS (ESI) $\mathrm{m} / z$ calculated for $\mathrm{C}_{21} \mathrm{H}_{19} \mathrm{IN}_{2} \mathrm{NaO}[\mathrm{M}+\mathrm{Na}]^{+} 465.0434$, found 465.0436; TLC: $R_{f}=0.46$ (97:3 DCM/EtOAc).

(E)-N-(4-iodo-5-(4-methoxyphenyl)pent-4-en-1-yl)picolinamide (2c)<smiles>COc1ccc(/C=C(/I)CCCNC(=O)c2ccccn2)cc1</smiles>

The title compound was prepared according to general procedure 5 from (Z)- $N-(5-(4-$ methoxyphenyl)pent-4-en-1-yl)picolinamide 1 c $(89 \mathrm{mg}, 0.30 \mathrm{mmol})$. The crude product was purified via flash column chromatography (97:3 DCM/EtOAc) to afford a pale yellow oil (76 mg, $0.18 \mathrm{mmol}$, $60 \%)$.

${ }^{1} \mathbf{H}$ NMR $\left(400 \mathrm{MHz}, \mathrm{CDCl}_{3}, 25{ }^{\circ} \mathrm{C}\right) \delta 8.51(\mathrm{ddd}, J=4.8,1.7,0.9 \mathrm{~Hz}, 1 \mathrm{H}), 8.17(\mathrm{dt}, J=7.8,1.1 \mathrm{~Hz}$, $1 \mathrm{H}), 8.00(\mathrm{~s}, 1 \mathrm{H}), 7.83(\mathrm{td}, J=7.7,1.7 \mathrm{~Hz}, 1 \mathrm{H}), 7.41(\mathrm{ddd}, J=7.6,4.8,1.2 \mathrm{~Hz}, 1 \mathrm{H}), 7.27(\mathrm{~s}, 1 \mathrm{H}), 7.12$ $-7.06(\mathrm{~m}, 2 \mathrm{H}), 6.80-6.74(\mathrm{~m}, 2 \mathrm{H}), 3.75(\mathrm{~s}, 3 \mathrm{H}), 3.48(\mathrm{td}, J=7.0,6.1 \mathrm{~Hz}, 2 \mathrm{H}), 2.76-2.64(\mathrm{~m}, 2 \mathrm{H})$, $2.01-1.89(\mathrm{~m}, 2 \mathrm{H}) ;{ }^{13} \mathrm{C}$ NMR $\left(100 \mathrm{MHz}, \mathrm{CDCl}_{3}, 25^{\circ} \mathrm{C}\right) \delta 164.4,158.9,150.0,148.1,141.2,137.4$, 130.2, 129.4, 126.2, 122.3, 114.0, 105.9, 55.3, 38.2, 36.7, 30.0; FT-IR (neat) 3377, 3056, 3031, 2932 , 2835, 1668, 1604, 1569, 1463, 1433, 1287, 1247, 1176, 1033, 819, 749, $621 \mathrm{~cm}^{-1}$; HRMS (ESI) $\mathrm{m} / \mathrm{z}$ calculated for $\mathrm{C}_{18} \mathrm{H}_{19} \mathrm{IN}_{2} \mathrm{NaO}_{2}[\mathrm{M}+\mathrm{Na}]^{+}$445.0383, found 445.0383 .

(E)- $N$-(5-(4-formylphenyl)-4-iodopent-4-en-1-yl)picolinamide (2d)<smiles>O=Cc1ccc(/C=C(/I)CCCNC(=O)c2ccccn2)cc1</smiles>

The title compound was prepared according to general procedure 4 from (Z)- $N$-(5-(4formylphenyl)pent-4-en-1-yl)picolinamide $1 \mathbf{d}(88 \mathrm{mg}, 0.30 \mathrm{mmol})$. The crude product was purified via flash column chromatography (95:5 to 90:10 DCM/EtOAc) to afford an orange oil ( $84 \mathrm{mg}, 0.20 \mathrm{mmol}$, $66 \%)$. 
${ }^{1} \mathbf{H}$ NMR $\left(400 \mathrm{MHz}, \mathrm{CDCl}_{3}, 25{ }^{\circ} \mathrm{C}\right) \delta 9.89(\mathrm{~s}, 1 \mathrm{H}), 8.48(\mathrm{ddd}, J=4.8,1.7,0.9 \mathrm{~Hz}, 1 \mathrm{H}), 8.14(\mathrm{dt}, J=$ 7.8, 1.1 Hz, 1H), $7.97(\mathrm{~s}, 1 \mathrm{H}), 7.84(\mathrm{td}, J=7.7,1.7 \mathrm{~Hz}, 1 \mathrm{H}), 7.74-7.68(\mathrm{~m}, 2 \mathrm{H}), 7.42$ (ddd, $J=7.6$, 4.8, $1.3 \mathrm{~Hz}, 1 \mathrm{H}), 7.36(\mathrm{~s}, 1 \mathrm{H}), 7.34-7.29(\mathrm{~m}, 2 \mathrm{H}), 3.48(\mathrm{q}, J=6.6 \mathrm{~Hz}, 2 \mathrm{H}), 2.71(\mathrm{ddd}, J=8.7,6.2$, $1.0 \mathrm{~Hz}, 2 \mathrm{H}), 2.03-1.92(\mathrm{~m}, 2 \mathrm{H}) ;{ }^{13} \mathbf{C}$ NMR $\left(100 \mathrm{MHz}, \mathrm{CDCl}_{3}, 25{ }^{\circ} \mathrm{C}\right) \delta 191.6,164.4,149.7,148.1$, 143.4, 140.6, 137.5, 135.1, 130.1, 128.7, 126.4, 122.3, 110.0, 38.0, 36.9, 30.0; FT-IR (neat) 3381, 3056, 2928, 2850, 2736, 1696, 1670, 1600, 1567, 1525, 1464, 1434, 1213, 1168, 997, 750, 724, $621 \mathrm{~cm}^{-1}$; HRMS (ESI) $\mathrm{m} / z$ calculated for $\mathrm{C}_{18} \mathrm{H}_{18} \mathrm{IN}_{2} \mathrm{O}_{2}[\mathrm{M}+\mathrm{H}]^{+} 421.0407$, found 421.0408; TLC: $R_{f}=0.42(97: 3 \mathrm{DCM} / \mathrm{EtOAc})$.

(E)- $N$-(4-iodo-5-(4-(trifluoromethyl)phenyl)pent-4-en-1-yl)picolinamide (2e)<smiles>O=C(NCCC/C(I)=C\c1ccc(C(F)(F)F)cc1)c1ccccn1</smiles>

The title compound was prepared according to general procedure 4 from (Z)- $N$-(5-(4(trifluoromethyl)phenyl)pent-4-en-1-yl)picolinamide 1e $(100 \mathrm{mg}, 0.30 \mathrm{mmol})$. The crude product was purified via flash column chromatography (97:3 DCM/EtOAc) to afford an amorphous white solid (87 mg, $0.19 \mathrm{mmol}, 63 \%)$.

${ }^{1} \mathbf{H}$ NMR $\left(500 \mathrm{MHz}, \mathrm{CDCl}_{3}, 25{ }^{\circ} \mathrm{C}\right) \delta 8.49(\mathrm{ddd}, J=4.8,1.7,0.9 \mathrm{~Hz}, 1 \mathrm{H}), 8.15(\mathrm{dt}, J=7.8,1.1 \mathrm{~Hz}$, $1 \mathrm{H}), 8.05-7.93(\mathrm{~m}, 1 \mathrm{H}), 7.84(\mathrm{td}, J=7.7,1.7 \mathrm{~Hz}, 1 \mathrm{H}), 7.46-7.41(\mathrm{~m}, 3 \mathrm{H}), 7.33(\mathrm{~d}, J=1.4 \mathrm{~Hz}, 1 \mathrm{H})$, $7.27-7.21$ (m, 2H), 3.48 (q, $J=6.6 \mathrm{~Hz}, 2 \mathrm{H}), 2.67(\mathrm{ddd}, J=8.8,6.1,1.0 \mathrm{~Hz}, 2 \mathrm{H}), 2.01-1.90(\mathrm{~m}, 2 \mathrm{H})$; ${ }^{13} \mathbf{C}$ NMR $\left(126 \mathrm{MHz}, \mathrm{CDCl}_{3}, 25{ }^{\circ} \mathrm{C}\right) \delta 164.5,149.8,148.1,140.9(\mathrm{~d}, J=1.4 \mathrm{~Hz}), 140.2,137.5,129.4$ (q, $J=32.6 \mathrm{~Hz}), 128.4,126.4,125.6(\mathrm{q}, J=3.8 \mathrm{~Hz}), 124.1$ (q, $J=272.0 \mathrm{~Hz}), 122.3,38.0,36.7,30.1$; ${ }^{19} \mathbf{F}$ NMR (471 MHz, $\left.\mathrm{CDCl}_{3}, 25{ }^{\circ} \mathrm{C}\right) \delta-62.47$; FT-IR (neat) 3385, 3058, 2932, 1669, 1615, 1524, 1322, 1164, 1122, 1067, 873, 821, $621 \mathrm{~cm}^{-1}$; HRMS (ESI) $\mathrm{m} / z$ calculated for $\mathrm{C}_{18} \mathrm{H}_{17} \mathrm{~F}_{3} \mathrm{IN}_{2} \mathrm{O}[\mathrm{M}+\mathrm{H}]^{+}$ 461.0332, found 461.0332; Melting point $83.7-84.8^{\circ} \mathrm{C}$; TLC: $R_{f}=0.27$ (97:3 DCM/EtOAc). 
<smiles>O=C(NCCC/C(I)=C\c1cc(C(F)(F)F)cc(C(F)(F)F)c1)c1ccccn1</smiles>

The title compound was prepared according to general procedure 4 from (Z)- $N$-(5-(3,5bis(trifluoromethyl)phenyl)pent-4-en-1-yl)picolinamide $\mathbf{1 f}(121 \mathrm{mg}, 0.30 \mathrm{mmol})$. The crude product was purified via flash column chromatography (98:2 DCM/EtOAc) to afford a pale yellow oil (91 mg, $0.17 \mathrm{mmol}, 57 \%)$.

${ }^{1} \mathbf{H}$ NMR $\left(500 \mathrm{MHz}, \mathrm{CDCl}_{3}, 25{ }^{\circ} \mathrm{C}\right) \delta 8.48-8.44(\mathrm{~m}, 1 \mathrm{H}), 8.12(\mathrm{dt}, J=7.8,1.1 \mathrm{~Hz}, 1 \mathrm{H}), 7.96(\mathrm{~s}, 1 \mathrm{H})$, $7.82(\mathrm{td}, J=7.7,1.7 \mathrm{~Hz}, 1 \mathrm{H}), 7.69(\mathrm{~s}, 1 \mathrm{H}), 7.60(\mathrm{~s}, 2 \mathrm{H}), 7.40(\mathrm{ddd}, J=7.6,4.8,1.2 \mathrm{~Hz}, 1 \mathrm{H}), 7.36(\mathrm{~s}$, $1 \mathrm{H}), 3.47(\mathrm{q}, J=6.7 \mathrm{~Hz}, 2 \mathrm{H}), 2.70-2.62(\mathrm{~m}, 2 \mathrm{H}), 1.97(\mathrm{dq}, J=9.1,6.9 \mathrm{~Hz}, 2 \mathrm{H}) ;{ }^{13} \mathbf{C} \mathbf{N M R}(126$ $\left.\mathrm{MHz}, \mathrm{CDCl}_{3}, 25^{\circ} \mathrm{C}\right) \delta 164.4,149.7,148.0,139.2,138.6,137.5,132.0(\mathrm{q}, J=33.5 \mathrm{~Hz}), 128.4-127.7$ $(\mathrm{m}), 126.3,123.2(\mathrm{q}, J=272.9 \mathrm{~Hz}), 122.3,121.5-120.7(\mathrm{~m}), 111.0,37.9,36.5,30.2 ;{ }^{19} \mathbf{F}$ NMR $(471$ $\left.\mathrm{MHz}, \mathrm{CDCl}_{3}, 25{ }^{\circ} \mathrm{C}\right) \delta-62.83$; FT-IR (neat) 3390, 3060, 2932, 1676, 1524, 1377, 1276, 1171, 1130, 899, $682 \mathrm{~cm}^{-1}$; HRMS (ESI) $\mathrm{m} / z$ calculated for $\mathrm{C}_{19} \mathrm{H}_{16} \mathrm{~F}_{6} \mathrm{IN}_{2} \mathrm{O}[\mathrm{M}+\mathrm{H}]^{+}$529.0206, found 529.0204; TLC: $R_{f}=0.44(97: 3 \mathrm{DCM} / \mathrm{EtOAc})$.

\section{(E)- $N$-(5-(4-fluorophenyl)-4-iodopent-4-en-1-yl)picolinamide (2g)}<smiles>O=C(NCCC/C(I)=C\c1ccc(F)cc1)c1ccccn1</smiles>

The title compound was prepared according to general procedure 4 from (Z)- $N$-(5-(4fluorophenyl)pent-4-en-1-yl)picolinamide $1 \mathbf{g}(85 \mathrm{mg}, 0.30 \mathrm{mmol})$. The crude product was purified via flash column chromatography (97:3 DCM/EtOAc) to afford a colorless oil (87 mg, $0.21 \mathrm{mmol}, 71 \%)$.

${ }^{1} \mathbf{H}$ NMR $\left(400 \mathrm{MHz}, \mathrm{CDCl}_{3}, 25{ }^{\circ} \mathrm{C}\right) \delta 8.53-8.47(\mathrm{~m}, 1 \mathrm{H}), 8.17-8.12(\mathrm{~m}, 1 \mathrm{H}), 7.96(\mathrm{~s}, 1 \mathrm{H}), 7.84(\mathrm{td}$, $J=7.7,1.7 \mathrm{~Hz}, 1 \mathrm{H}), 7.42(\mathrm{ddd}, J=7.6,4.8,1.2 \mathrm{~Hz}, 1 \mathrm{H}), 7.28(\mathrm{~s}, 1 \mathrm{H}), 7.16-7.08(\mathrm{~m}, 2 \mathrm{H}), 6.91(\mathrm{t}, J=$ $8.7 \mathrm{~Hz}, 2 \mathrm{H}), 3.46(\mathrm{td}, J=6.8,6.1 \mathrm{~Hz}, 2 \mathrm{H}), 2.72-2.58(\mathrm{~m}, 2 \mathrm{H}), 2.02-1.86(\mathrm{~m}, 2 \mathrm{H}) ;{ }^{13} \mathbf{C}$ NMR $(100$ $\left.\mathrm{MHz}, \mathrm{CDCl}_{3}, 25{ }^{\circ} \mathrm{C}\right) \delta 13 \mathrm{C} \mathrm{NMR}(101 \mathrm{MHz}$, Chloroform-d) $\delta 164.4,161.9(\mathrm{~d}, J=247.3 \mathrm{~Hz}), 149.8$, 148.1, 140.5, 137.5, 133.6 (d, $J=3.5 \mathrm{~Hz}), 129.8$ (d, $J=8.0 \mathrm{~Hz}), 126.3,122.3,115.6$ (d, $J=21.6 \mathrm{~Hz})$, $107.4(\mathrm{~d}, J=1.6 \mathrm{~Hz}), 38.0,36.4,29.9 ;{ }^{19} \mathbf{F}$ NMR $\left(376 \mathrm{MHz}, \mathrm{CDCl}_{3}, 25{ }^{\circ} \mathrm{C}\right) \delta-113.86$; FT-IR (neat) 
3382, 3057, 2928, 1669, 1590, 1523, 1506, 1564, 1434, 1223, 1158, 997, 821, 749, $621 \mathrm{~cm}^{-1}$; HRMS (ESI) $\mathrm{m} / \mathrm{z}$ calculated for $\mathrm{C}_{17} \mathrm{H}_{17} \mathrm{FIN}_{2} \mathrm{O}[\mathrm{M}+\mathrm{H}]^{+} 411.0364$, found 411.0366; TLC: $R_{f}=0.50(3: 2$ $\mathrm{Hex} / \mathrm{EtOAc})$.

(E)- $N$-(5-(4-bromophenyl)-4-iodopent-4-en-1-yl)picolinamide (2h)<smiles>O=C(NCCC/C(I)=C\c1ccc(Br)cc1)c1ccccn1</smiles>

The title compound was prepared according to general procedure 4 from (Z)- $N$-(5-(4bromophenyl)pent-4-en-1-yl)picolinamide $\mathbf{1 h}(104 \mathrm{mg}, 0.30 \mathrm{mmol})$. The crude product was purified via flash column chromatography (97:3 DCM/EtOAc) to afford a colorless oil (101 mg, $0.21 \mathrm{mmol}$, $72 \%)$.

${ }^{1} \mathbf{H}$ NMR $\left(400 \mathrm{MHz}, \mathrm{CDCl}_{3}, 25{ }^{\circ} \mathrm{C}\right) \delta 8.53(\mathrm{ddd}, J=4.7,1.7,0.9 \mathrm{~Hz}, 1 \mathrm{H}), 8.16(\mathrm{dt}, J=7.8,1.1 \mathrm{~Hz}$, 1H), 7.97 (s, 1H), $7.85(\mathrm{td}, J=7.7,1.7 \mathrm{~Hz}, 1 \mathrm{H}), 7.44(\mathrm{ddd}, J=7.6,4.8,1.3 \mathrm{~Hz}, 1 \mathrm{H}), 7.36-7.30(\mathrm{~m}$, 2H), $7.25(\mathrm{~s}, 1 \mathrm{H}), 7.06-6.99(\mathrm{~m}, 2 \mathrm{H}), 3.46(\mathrm{q}, J=6.6 \mathrm{~Hz}, 2 \mathrm{H}), 2.66(\mathrm{ddd}, J=8.7,6.2,1.0 \mathrm{~Hz}, 2 \mathrm{H})$, 1.99 - $1.90(\mathrm{~m}, 2 \mathrm{H}) ;{ }^{13} \mathbf{C}$ NMR $\left(100 \mathrm{MHz}, \mathrm{CDCl}_{3}, 25^{\circ} \mathrm{C}\right) \delta 164.4,149.8,148.2,140.4,137.5,136.4$, 131.8, 129.7, 126.3, 122.3, 121.5, 108.0, 38.0, 36.6, 29.9; FT-IR (neat) 3380, 3056, 2929, 2866, 1669, 1523, 1485, 1433, 1288, 1187, 1072, 1009, 867, $748 \mathrm{~cm}^{-1}$; HRMS (ESI) $\mathrm{m} / z$ calculated for $\mathrm{C}_{17} \mathrm{H}_{17} \mathrm{BrIN}_{2} \mathrm{O}[\mathrm{M}+\mathrm{H}]^{+}$470.9563, found 470.9559; TLC: $R_{f}=0.43$ (97:3 DCM/EtOAC).

(E)- $N$-(5-(2-chlorophenyl)-4-iodopent-4-en-1-yl)picolinamide (2i)<smiles>O=C(NCCC/C(I)=C\c1ccccc1Cl)c1ccccn1</smiles>

The title compound was prepared according to general procedure 4 from (Z)- $N$-(5-(2chlorophenyl)pent-4-en-1-yl)picolinamide $\mathbf{1 i}(90 \mathrm{mg}, 0.30 \mathrm{mmol})$. The crude product was purified via flash column chromatography (97:3 DCM/EtOAc) to afford a faint yellow oil (96 mg, $0.23 \mathrm{mmol}$, $75 \%)$.

${ }^{1} \mathbf{H}$ NMR $\left(500 \mathrm{MHz}, \mathrm{CDCl}_{3}, 25{ }^{\circ} \mathrm{C}\right) \delta 8.50(\mathrm{ddd}, J=4.8,1.7,0.9 \mathrm{~Hz}, 1 \mathrm{H}), 8.15(\mathrm{dt}, J=7.8,1.1 \mathrm{~Hz}$, $1 \mathrm{H}), 8.01-7.94(\mathrm{~m}, 1 \mathrm{H}), 7.83(\mathrm{td}, J=7.7,1.7 \mathrm{~Hz}, 1 \mathrm{H}), 7.41(\mathrm{ddd}, J=7.5,4.7,1.2 \mathrm{~Hz}, 1 \mathrm{H}), 7.35-$ 
$7.33(\mathrm{~m}, 1 \mathrm{H}), 7.30-7.29(\mathrm{~m}, 1 \mathrm{H}), 7.21-7.09(\mathrm{~m}, 3 \mathrm{H}), 3.42(\mathrm{td}, J=7.0,6.2 \mathrm{~Hz}, 2 \mathrm{H}), 2.55$ (ddd, $J=$ 8.6, 6.3, $1.0 \mathrm{~Hz}, 2 \mathrm{H}), 1.95-1.88(\mathrm{~m}, 2 \mathrm{H}) ;{ }^{13} \mathbf{C} \mathbf{~ N M R}\left(126 \mathrm{MHz}, \mathrm{CDCl}_{3}, 25{ }^{\circ} \mathrm{C}\right) \delta 164.4,150.0,148.1$, 139.0, 137.4, 135.9, 133.1, 130.0, 129.7, 129.0, 126.8, 126.2, 122.2, 108.5, 38.1, 36.6, 29.9; FT-IR (neat) 3382, 3056, 2928, 2867, 1670, 1522, 1465, 1434, 1288, 1053, 749, $621 \mathrm{~cm}^{-1}$; HRMS (ESI) $\mathrm{m} / \mathrm{z}$ calculated for $\mathrm{C}_{17} \mathrm{H}_{16} \mathrm{ClIN}_{2} \mathrm{NaO}_{2}[\mathrm{M}+\mathrm{Na}]^{+} 448.9888$, found 448.9886; TLC: $R_{f}=0.34$ (3:2 Hex/EtOAc).

\section{$1 \mathrm{mmol}$ scale reaction}<smiles>O=C(NCCC/C(I)=C\c1ccccc1Cl)c1ccccn1</smiles>

To a $15 \mathrm{~mL}$ microwave vial under ambient atmosphere was $(Z)-N$-(5-(2-chlorophenyl)pent-4-en-1yl)picolinamide $1 \mathbf{i}$ (301 mg, $1.00 \mathrm{mmol}, 1.00$ equiv), pivalic acid (102 mg, $1.00 \mathrm{mmol}, 1.00$ equiv), tetrabutylammonium iodide (480 mg, $1.30 \mathrm{mmol}, 1.30$ equiv), bis(tert-butylcarbonyloxy)iodobenzene (528 mg, $1.30 \mathrm{mmol}, 1.30$ equiv), $\mathrm{Pd}(\mathrm{OAc})_{2}(11.2 \mathrm{mg}, 0.0050 \mathrm{mmol}, 0.05$ equiv), water $(2.0 \mathrm{~mL})$, and acetonitrile $(8.0 \mathrm{~mL})$. The vial was sealed, placed in a heating block pre-heated to $90{ }^{\circ} \mathrm{C}$, and stirred at this temperature for $8 \mathrm{~h}$. The reaction was allowed to cool to room temperature, quenched by addition of aq. $\mathrm{Na}_{2} \mathrm{~S}_{2} \mathrm{O}_{3}(5 \mathrm{wt} \%, 50 \mathrm{~mL})$ and sat. aq. $\mathrm{NaHCO}_{3}(50 \mathrm{~mL})$, brine $(50 \mathrm{~mL})$, extracted with EtOAc, dried over $\mathrm{Na}_{2} \mathrm{SO}_{4}$ and concentrated. The crude product was obtained by flash column chromatography (97:3 DCM/EtOAc) to afford a faint yellow oil (322 mg, $0.75 \mathrm{mmol}, 75 \%)$. The characterization data was identical to those reported above.

\section{(E)-N-(5-(2,6-dimethylphenyl)-4-iodopent-4-en-1-yl)picolinamide (2j)}<smiles>Cc1cccc(C)c1/C=C(/I)CCCNC(=O)c1ccccn1</smiles>

The title compound was prepared according to general procedure 4 from (Z)- $N-(5-(2,6-$ dimethylphenyl)pent-4-en-1-yl)picolinamide $\mathbf{1 j}$ ( $88 \mathrm{mg}, 0.30 \mathrm{mmol})$. The crude product was purified via flash column chromatography (97:3 DCM/EtOAc) to afford a yellow oil (105 mg, $0.25 \mathrm{mmol}$, $83 \%)$. 
${ }^{1} \mathbf{H}$ NMR $\left(400 \mathrm{MHz}, \mathrm{CDCl}_{3}, 25{ }^{\circ} \mathrm{C}\right) \delta 8.49(\mathrm{ddd}, J=4.8,1.7,0.9 \mathrm{~Hz}, 1 \mathrm{H}), 8.14(\mathrm{dt}, J=7.8,1.1 \mathrm{~Hz}$, $1 \mathrm{H}), 7.91(\mathrm{~s}, 1 \mathrm{H}), 7.83(\mathrm{td}, J=7.7,1.7 \mathrm{~Hz}, 1 \mathrm{H}), 7.41(\mathrm{ddd}, J=7.6,4.8,1.3 \mathrm{~Hz}, 1 \mathrm{H}), 7.13(\mathrm{~s}, 1 \mathrm{H}), 7.05$ (dd, $J=8.4,6.5 \mathrm{~Hz}, 1 \mathrm{H}), 6.97(\mathrm{~d}, J=7.5 \mathrm{~Hz}, 2 \mathrm{H}), 3.33(\mathrm{td}, J=7.3,6.1 \mathrm{~Hz}, 2 \mathrm{H}), 2.27-2.17(\mathrm{~m}, 8 \mathrm{H})$, $1.85-1.73(\mathrm{~m}, 2 \mathrm{H}) ;{ }^{13} \mathbf{C}$ NMR $\left(100 \mathrm{MHz}, \mathrm{CDCl}_{3}, 25^{\circ} \mathrm{C}\right) \delta 164.3,149.9,148.0,140.6,137.5,137.0$, 135.7, 127.6, 127.5, 126.2, 122.3, 106.0, 38.2, 36.2, 29.6, 20.5; FT-IR (neat) 3383, 3058, 3014, 2925 , 2865, 1674, 1523, 1464, 1434, 1288, 1245, 1095, 997, 771, 749, 693, $621 \mathrm{~cm}^{-1}$; HRMS (ESI) $\mathrm{m} / \mathrm{z}$ calculated for $\mathrm{C}_{19} \mathrm{H}_{21} \mathrm{~N}_{2} \mathrm{NaO}[\mathrm{M}+\mathrm{Na}]^{+} 443.0591$, found 443.0589; TLC: $R_{f}=0.75(1: 1 \mathrm{Hex} / \mathrm{EtOAc})$.

\section{(E)- $N$-(5-(2,6-dichlorophenyl)-4-iodopent-4-en-1-yl)picolinamide (2k)}<smiles>O=C(NCCC/C(I)=C\c1c(Cl)cccc1Cl)c1ccccn1</smiles>

The title compound was prepared according to general procedure 4 from (Z)- $N$-(5-(2,6dichlorophenyl)pent-4-en-1-yl)picolinamide $\mathbf{1 k}(101 \mathrm{mg}, 0.30 \mathrm{mmol})$. The crude product was purified via flash column chromatography (97:3 DCM/EtOAc) to afford a colorless oil (117 mg, $0.25 \mathrm{mmol}$, $85 \%)$.

${ }^{1} \mathbf{H}$ NMR $\left(500 \mathrm{MHz}, \mathrm{CDCl}_{3}, 25{ }^{\circ} \mathrm{C}\right) \delta 8.49(\mathrm{ddd}, J=4.8,1.7,0.9 \mathrm{~Hz}, 1 \mathrm{H}), 8.14(\mathrm{dt}, J=7.8,1.2 \mathrm{~Hz}$, $1 \mathrm{H}), 7.99-7.91(\mathrm{~m}, 1 \mathrm{H}), 7.82(\mathrm{td}, J=7.7,1.7 \mathrm{~Hz}, 1 \mathrm{H}), 7.40(\mathrm{ddd}, J=7.6,4.7,1.2 \mathrm{~Hz}, 1 \mathrm{H}), 7.28-$ $7.27(\mathrm{~m}, 1 \mathrm{H}), 7.26-7.25(\mathrm{~m}, 1 \mathrm{H}), 7.13(\mathrm{ddd}, J=8.5,7.6,0.8 \mathrm{~Hz}, 1 \mathrm{H}), 7.01-6.97(\mathrm{~m}, 1 \mathrm{H}), 3.40-$ $3.32(\mathrm{~m}, 2 \mathrm{H}), 2.30(\mathrm{ddd}, J=7.5,6.6,1.0 \mathrm{~Hz}, 2 \mathrm{H}), 1.92-1.81(\mathrm{~m}, 2 \mathrm{H}) ;{ }^{13} \mathbf{C} \mathbf{N M R}\left(126 \mathrm{MHz}, \mathrm{CDCl}_{3}\right.$, $\left.25^{\circ} \mathrm{C}\right) \delta 164.3,150.0,148.0,137.4,135.6,135.2,134.4,129.3,128.2,126.2,122.3,110.0,38.1,37.1$, 29.3; FT-IR (neat) 3386, 3056, 2931, 2867, 1673, 1523, 1429, 1288, 1194, 997, 830, 774, 749, $621 \mathrm{~cm}^{-1}$; HRMS (ESI) $\mathrm{m} / z$ calculated for $\mathrm{C}_{17} \mathrm{H}_{16} \mathrm{Cl}_{2} \mathrm{IN}_{2} \mathrm{O}[\mathrm{M}+\mathrm{H}]^{+} 460.9679$, found 460.9685; TLC: $R_{f}=0.34(3: 2 \mathrm{Hex} / \mathrm{EtOAc})$.

(E)- $N$-(5-(2-bromo-6-chlorophenyl)-4-iodopent-4-en-1-yl)picolinamide (2l)<smiles>O=C(NCCC/C(I)=C\c1c(Cl)cccc1Br)c1ccccn1</smiles> 
The title compound was prepared according to general procedure 4 from (Z)- $N$-(5-(2-bromo-6chlorophenyl)pent-4-en-1-yl)picolinamide 11 (114 mg, $0.30 \mathrm{mmol})$. The crude product was purified via flash column chromatography (97:3 DCM/EtOAc) to afford a colorless oil (126 mg, $0.25 \mathrm{mmol}$, $84 \%)$.

${ }^{1} \mathbf{H}$ NMR $\left(500 \mathrm{MHz}, \mathrm{CDCl}_{3}, 25{ }^{\circ} \mathrm{C}\right) \delta 8.48(\mathrm{ddd}, J=4.8,1.7,0.9 \mathrm{~Hz}, 1 \mathrm{H}), 8.13(\mathrm{dt}, J=7.8,1.1 \mathrm{~Hz}$, $1 \mathrm{H}), 7.94(\mathrm{~d}, J=6.7 \mathrm{~Hz}, 1 \mathrm{H}), 7.81(\mathrm{td}, J=7.7,1.7 \mathrm{~Hz}, 1 \mathrm{H}), 7.45(\mathrm{dd}, J=8.1,1.1 \mathrm{~Hz}, 1 \mathrm{H}), 7.40$ (ddd, $J=7.5,4.8,1.3 \mathrm{~Hz}, 1 \mathrm{H}), 7.31(\mathrm{dd}, J=8.1,1.1 \mathrm{~Hz}, 1 \mathrm{H}), 7.05(\mathrm{td}, J=8.1,0.8 \mathrm{~Hz}, 1 \mathrm{H}), 6.97(\mathrm{~d}, J=1.1$ $\mathrm{Hz}, 1 \mathrm{H}), 3.39-3.34(\mathrm{~m}, 2 \mathrm{H}), 2.33-2.23(\mathrm{~m}, 2 \mathrm{H}), 1.87$ (p, $J=7.2 \mathrm{~Hz}, 2 \mathrm{H}) ;{ }^{13} \mathbf{C}$ NMR $(126 \mathrm{MHz}$, $\left.\mathrm{CDCl}_{3}, 25{ }^{\circ} \mathrm{C}\right) \delta 164.3,150.0,148.0,137.7,137.4,137.0,134.2,131.3,129.7,128.8,126.1,124.2$, 122.2, 109.8, 38.1, 37.0, 29.3; FT-IR (neat) 3384, 3055, 2929, 2866, 1670, 1520, 1424, 820, 774, 747, $621 \mathrm{~cm}^{-1}$; HRMS (ESI) $\mathrm{m} / z$ calculated for $\mathrm{C}_{17} \mathrm{H}_{15} \mathrm{BrClIN}_{2} \mathrm{NaO}[\mathrm{M}+\mathrm{H}]^{+}$526.8993, found 526.8993; TLC: $R_{f}=0.30$ (97:3 DCM/EtOAc).

\section{(E)- $N$-(4-iodo-2-methyl-5-phenylpent-4-en-1-yl)picolinamide (2m)}<smiles>CC(CNC(=O)c1ccccn1)C/C(I)=C\c1ccccc1</smiles>

The title compound was prepared according to general procedure 4 from (Z)- $N$-(2-methyl-5phenylpent-4-en-1-yl)picolinamide $1 \mathbf{m}(84 \mathrm{mg}, 0.30 \mathrm{mmol})$. The crude product was purified via flash column chromatography (97:3 DCM/EtOAc) to afford a pale yellow oil (55 mg, $0.14 \mathrm{mmol}, 45 \%)$.

${ }^{1} \mathbf{H}$ NMR $\left(400 \mathrm{MHz}, \mathrm{CDCl}_{3}, 25{ }^{\circ} \mathrm{C}\right) \delta 8.52(\mathrm{ddd}, J=4.8,1.7,0.9 \mathrm{~Hz}, 1 \mathrm{H}), 8.17(\mathrm{dt}, J=7.8,1.1 \mathrm{~Hz}$, $1 \mathrm{H}), 8.05(\mathrm{~s}, 1 \mathrm{H}), 7.83(\mathrm{td}, J=7.7,1.7 \mathrm{~Hz}, 1 \mathrm{H}), 7.45-7.39(\mathrm{~m}, 2 \mathrm{H}), 7.34-7.16(\mathrm{~m}, 5 \mathrm{H}), 3.41(\mathrm{dt}, J=$ 13.4, $6.1 \mathrm{~Hz}, 1 \mathrm{H}), 3.30$ (dt, $J=13.6,6.8 \mathrm{~Hz}, 1 \mathrm{H}), 2.63$ (ddd, $J=14.3,6.3,1.3 \mathrm{~Hz}, 1 \mathrm{H}), 2.53$ (ddd, $J=$ 14.4, 7.9, $1.0 \mathrm{~Hz}, 1 \mathrm{H}), 2.33-2.21(\mathrm{~m}, 1 \mathrm{H}), 0.94(\mathrm{~d}, J=6.7 \mathrm{~Hz}, 3 \mathrm{H}) ;{ }^{13} \mathbf{C} \mathbf{N M R}\left(100 \mathrm{MHz}, \mathrm{CDCl}_{3}, 25\right.$ $\left.{ }^{\circ} \mathrm{C}\right) \delta 164.5,150.0,148.1,142.7,137.7,137.4,128.6,128.3,127.4,126.2,122.4,107.4,44.2,43.0$, 35.0, 17.0; FT-IR (neat) 3384, 3056, 3023, 2959, 2926, 2871, 1674, 1524, 1464, 1434, 1288, 1142, 998, 750, 700, $621 \mathrm{~cm}^{-1}$; HRMS (ESI) $\mathrm{m} / z$ calculated for $\mathrm{C}_{18} \mathrm{H}_{20} \mathrm{IN}_{2} \mathrm{O}[\mathrm{M}+\mathrm{H}]^{+} 407.0615$, found 407.0612; TLC: $R_{f}=0.36$ (3:2 Hex/EtOAc). 
<smiles>CC(C)/C=C(/I)CCCNC(=O)c1ccccn1</smiles>

The title compound was prepared according to general procedure 5 from (Z)- $N$-(6-methylhept-4-en-1yl)picolinamide $1 \mathrm{n}(70 \mathrm{mg}, 0.30 \mathrm{mmol})$. The crude product was purified via flash column chromatography (97:3 DCM/EtOAc) to afford a pale yellow oil (63 mg, $0.18 \mathrm{mmol}, 58 \%$ ).

${ }^{1} \mathbf{H}$ NMR $\left(400 \mathrm{MHz}, \mathrm{CDCl}_{3}, 25{ }^{\circ} \mathrm{C}\right) \delta 8.54(\mathrm{ddd}, J=4.7,1.7,0.9 \mathrm{~Hz}, 1 \mathrm{H}), 8.19(\mathrm{dt}, J=7.8,1.1 \mathrm{~Hz}$, $1 \mathrm{H}), 8.11(\mathrm{~s}, 1 \mathrm{H}), 7.85(\mathrm{td}, J=7.7,1.7 \mathrm{~Hz}, 1 \mathrm{H}), 7.48-7.38(\mathrm{~m}, 1 \mathrm{H}), 6.06(\mathrm{~d}, J=9.8 \mathrm{~Hz}, 1 \mathrm{H}), 3.56-$ $3.43(\mathrm{~m}, 2 \mathrm{H}), 2.67-2.53(\mathrm{~m}, 1 \mathrm{H}), 2.49(\mathrm{ddd}, J=7.6,6.7,0.9 \mathrm{~Hz}, 2 \mathrm{H}), 1.86(\mathrm{td}, J=7.1,0.7 \mathrm{~Hz}, 2 \mathrm{H})$, $0.95(\mathrm{~d}, J=6.6 \mathrm{~Hz}, 6 \mathrm{H}) ;{ }^{13} \mathbf{C}$ NMR $\left(100 \mathrm{MHz}, \mathrm{CDCl}_{3}, 25{ }^{\circ} \mathrm{C}\right) \delta 164.4,150.0,149.4,148.1,137.5$, 126.3, 122.3, 100.2, 38.3, 36.1, 30.9, 29.5, 22.7; FT-IR (neat) 3386, 3057, 2960, 2927, 2867, 1671, 1569, 1525, 1464, 1434, 1288, 1146, 998, 749, $634 \mathrm{~cm}^{-1}$; HRMS (ESI) $\mathrm{m} / z$ calculated for $\mathrm{C}_{14} \mathrm{H}_{20} \mathrm{IN}_{2} \mathrm{O}$ $[\mathrm{M}+\mathrm{H}]^{+} 359.0615$, found 359.0617; TLC: $R_{f}=0.49$ (97:3 DCM/EtOAc).

(E)-N-(4-iodo-6,6-dimethylhept-4-en-1-yl)picolinamide (2o)<smiles>CC(C)(C)/C=C(/I)CCCNC(=O)c1ccccn1</smiles>

The title compound was prepared according to general procedure 5 from (Z)- $N$-(6,6-dimethylhept-4en-1-yl)picolinamide 1o $(74 \mathrm{mg}, 0.30 \mathrm{mmol})$. The crude product was purified via flash column chromatography (97:3 DCM/EtOAc) to afford a colorless oil (61 mg, $0.16 \mathrm{mmol}, 55 \%$ ).

${ }^{1} \mathbf{H}$ NMR $\left(400 \mathrm{MHz}, \mathrm{CDCl}_{3}, 25{ }^{\circ} \mathrm{C}\right) \delta 8.54(\mathrm{ddd}, J=4.8,1.7,0.9 \mathrm{~Hz}, 1 \mathrm{H}), 8.19(\mathrm{dt}, J=7.8,1.1 \mathrm{~Hz}$, $1 \mathrm{H}), 8.12(\mathrm{~s}, 1 \mathrm{H}), 7.85(\mathrm{td}, J=7.7,1.7 \mathrm{~Hz}, 1 \mathrm{H}), 7.42(\mathrm{ddd}, J=7.6,4.8,1.2 \mathrm{~Hz}, 1 \mathrm{H}), 6.27(\mathrm{~s}, 1 \mathrm{H}), 3.52$ $(\mathrm{td}, J=7.2,6.1 \mathrm{~Hz}, 2 \mathrm{H}), 2.62-2.54(\mathrm{~m}, 2 \mathrm{H}), 1.96-1.86(\mathrm{~m}, 2 \mathrm{H}), 1.11(\mathrm{~s}, 9 \mathrm{H}) ;{ }^{13} \mathbf{C}$ NMR $(100 \mathrm{MHz}$, $\left.\mathrm{CDCl}_{3}, 25{ }^{\circ} \mathrm{C}\right) \delta 164.4,151.9,150.0,148.1,137.5,126.3,122.3,104.1,38.5,38.0,37.1,31.0,30.6$; FT-IR (neat) 3383, 3056, 2957, 2867, 1667, 1522, 1464, 1433, 1364, 1287, 1242, 1092, 997, 748, $620 \mathrm{~cm}^{-1}$; HRMS (ESI) $\mathrm{m} / z$ calculated for $\mathrm{C}_{15} \mathrm{H}_{22} \mathrm{IN}_{2} \mathrm{O}[\mathrm{M}+\mathrm{H}]^{+} 373.0771$, found 373.0779; TLC: $R_{f}=$ $0.40(3: 2 \mathrm{Hex} / \mathrm{EtOAc})$. 


\section{(E)- $N$-(5-cyclohexyl-4-iodopent-4-en-1-yl)picolinamide (2p)}

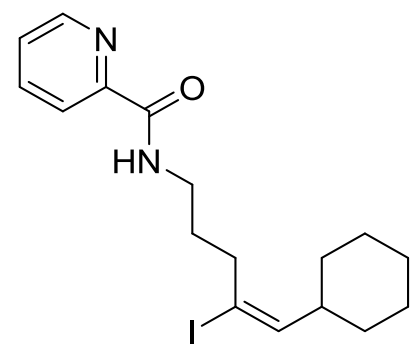

The title compound was prepared according to general procedure 5 from ( $Z$ )- $N$-(5-cyclohexylpent-4en-1-yl)picolinamide 1p $(82 \mathrm{mg}, 0.30 \mathrm{mmol})$. The crude product was purified via flash column chromatography (97:3 DCM/EtOAc) to afford a pale yellow oil ( $80 \mathrm{mg}, 0.20 \mathrm{mmol}, 67 \%)$.

${ }^{1} \mathbf{H}$ NMR $\left(400 \mathrm{MHz}, \mathrm{CDCl}_{3}, 25{ }^{\circ} \mathrm{C}\right) \delta 8.55(\mathrm{ddd}, J=4.8,1.7,0.9 \mathrm{~Hz}, 1 \mathrm{H}), 8.22-8.18(\mathrm{~m}, 1 \mathrm{H}), 8.11$ (s, 1H), $7.85(\mathrm{td}, J=7.7,1.7 \mathrm{~Hz}, 1 \mathrm{H}), 7.43(\mathrm{ddd}, J=7.6,4.8,1.3 \mathrm{~Hz}, 1 \mathrm{H}), 6.07(\mathrm{~d}, J=9.7 \mathrm{~Hz}, 1 \mathrm{H})$, $3.48(\mathrm{td}, J=7.1,6.2 \mathrm{~Hz}, 2 \mathrm{H}), 2.49(\mathrm{ddd}, J=7.6,6.7,0.9 \mathrm{~Hz}, 2 \mathrm{H}), 2.30-2.18(\mathrm{~m}, 1 \mathrm{H}), 1.86(\mathrm{td}, J=$ 7.1, $1.0 \mathrm{~Hz}, 2 \mathrm{H}), 1.73-1.54(\mathrm{~m}, 5 \mathrm{H}), 1.26-1.00(\mathrm{~m}, 5 \mathrm{H}) ;{ }^{13} \mathbf{C ~ N M R}\left(100 \mathrm{MHz}, \mathrm{CDCl}_{3}, 25{ }^{\circ} \mathrm{C}\right) \delta$ $164.5,150.0,148.2,148.2,137.5,126.3,122.3,100.9,40.6,38.1,36.0,32.9,29.5$, 25.9, 25.8 ; FT-IR (neat) 3387, 3056, 2922, 2849, 1668, 1591, 1521, 1464, 1447, 1286, 1143, 1040, 997, 896, 748, 691, $621 \mathrm{~cm}^{-1}$; HRMS (ESI) $\mathrm{m} / z$ calculated for $\mathrm{C}_{17} \mathrm{H}_{24} \mathrm{IN}_{2} \mathrm{O}[\mathrm{M}+\mathrm{H}]^{+} 399.0928$, found 399.0926; TLC: $R_{f}=$ $0.40(2: 1 \mathrm{Hex} / \mathrm{EtOAc})$.

\section{(E)-tert-butyl 4-(2-iodo-5-(picolinamido)pent-1-en-1-yl)piperidine-1-carboxylate (2q)}<smiles>CC(C)(C)OC(=O)N1CCC(/C=C(/I)CCCNC(=O)c2ccccn2)CC1</smiles>

To a $7 \mathrm{~mL}$ microwave vial was added (Z)-tert-butyl 4-(5-(picolinamido)pent-1-en-1-yl)piperidine-1carboxylate 1q (112 mg, $0.30 \mathrm{mmol}, 1.00$ equiv), pivalic acid ( $31 \mathrm{mg}, 0.30 \mathrm{mmol}, 1.00$ equiv), tetrabutylammonium iodide (144 $\mathrm{mg}, \quad 0.39 \mathrm{mmol}, \quad 1.30$ equiv $) \quad$ Oxone $\left(\mathrm{KHSO}_{5} \cdot 0.5 \mathrm{KHSO}_{4} \cdot 0.5 \mathrm{~K}_{2} \mathrm{SO}_{4}, 120 \mathrm{mg}, 0.39 \mathrm{mmol}, 1.30\right.$ equiv), $\mathrm{Pd}(\mathrm{OAc})_{2}(10.1 \mathrm{mg}, 0.045 \mathrm{mmol}$, 0.15 equiv), and anhydrous acetonitrile $(3 \mathrm{~mL})$. The vial was sealed, placed in a heating block preheated to $70{ }^{\circ} \mathrm{C}$, and stirred at this temperature for $8 \mathrm{~h}$. The reaction was allowed to cool to room temperature, filtered through silica and concentrated. The yield of the reaction was determined to be 
$55 \%$ by ${ }^{1} \mathrm{H}-\mathrm{NMR}$ analysis of the crude reaction mixture using hexamethyldisilane as internal standard. A sample with sufficient purity for characterization was obtained by preparative TLC of an aliquote of the crude reaction mixture (4:1 DCM/EtOAc followed by 1:1 Hex/EtOAc).

${ }^{1} \mathbf{H}$ NMR $\left(500 \mathrm{MHz}, \mathrm{CDCl}_{3}, 25{ }^{\circ} \mathrm{C}\right) \delta 8.55(\mathrm{ddd}, J=4.8,1.7,0.9 \mathrm{~Hz}, 1 \mathrm{H}), 8.20(\mathrm{dt}, J=7.8,1.1 \mathrm{~Hz}$, $1 \mathrm{H}), 8.10(\mathrm{~s}, 1 \mathrm{H}), 7.86(\mathrm{td}, J=7.8,1.7 \mathrm{~Hz}, 1 \mathrm{H}), 7.44(\mathrm{ddd}, J=7.6,4.7,1.2 \mathrm{~Hz}, 1 \mathrm{H}), 6.06(\mathrm{~d}, J=9.6$ $\mathrm{Hz}, 1 \mathrm{H}), 4.03(\mathrm{~s}, 2 \mathrm{H}), 3.49(\mathrm{td}, J=7.0,6.1 \mathrm{~Hz}, 2 \mathrm{H}), 2.71-2.57(\mathrm{~m}, 2 \mathrm{H}), 2.55-2.49(\mathrm{~m}, 2 \mathrm{H}), 2.45-$ $2.35(\mathrm{~m}, 1 \mathrm{H}), 1.89(\mathrm{p}, J=7.1 \mathrm{~Hz}, 2 \mathrm{H}), 1.61-1.53(\mathrm{~m}, 2 \mathrm{H}), 1.45(\mathrm{~s}, 9 \mathrm{H}), 1.35-1.21(\mathrm{~m}, 2 \mathrm{H}) ;{ }^{13} \mathbf{C}$ NMR $\left(126 \mathrm{MHz}, \mathrm{CDCl}_{3}, 2{ }^{\circ} \mathrm{C}\right) \delta 164.5,154.9,150.0,148.2,145.9,137.6,126.4,122.4,102.2,79.6$, 43.6 (broad), 38.6, 38.1, 36.1, 31.7, 29.6, 28.6; FT-IR (neat) 3384, 3057, 2974, 2930, 2852, 1678, 1523, 1424, 1365, 1284, 1227, 1168, 1139, 998, 750, $621 \mathrm{~cm}^{-1}$; HRMS (ESI) $\mathrm{m} / z$ calculated for $\mathrm{C}_{21} \mathrm{H}_{31} \mathrm{IN}_{3} \mathrm{O}_{3}[\mathrm{M}+\mathrm{H}]^{+}$500.1405, found 500.1401.

\section{$N$-(2-(2-iodocyclohex-2-en-1-yl)ethyl)picolinamide (2r)}<smiles>O=C(NCCC1CCCC=C1I)c1ccccn1</smiles>

The title compound was prepared according to general procedure 5 from $N$-(2-(cyclohex-1-en-1yl)ethyl)picolinamide 1r $(69 \mathrm{mg}, 0.30 \mathrm{mmol})$. The crude product was purified via flash column chromatography (97:3 DCM/EtOAc) to afford a brown oil (52 mg, $0.15 \mathrm{mmol}, 49 \%)$.

${ }^{1} \mathbf{H}$ NMR $\left(400 \mathrm{MHz}, \mathrm{CDCl}_{3}, 25{ }^{\circ} \mathrm{C}\right) \delta 8.55(\mathrm{ddd}, J=4.8,1.7,0.9 \mathrm{~Hz}, 1 \mathrm{H}), 8.19(\mathrm{dt}, J=7.9,1.1 \mathrm{~Hz}$, $1 \mathrm{H}), 8.08(\mathrm{~s}, 1 \mathrm{H}), 7.84(\mathrm{td}, J=7.7,1.7 \mathrm{~Hz}, 1 \mathrm{H}), 7.42(\mathrm{ddd}, J=7.6,4.8,1.2 \mathrm{~Hz}, 1 \mathrm{H}), 6.40(\mathrm{td}, J=4.1$, $1.5 \mathrm{~Hz}, 1 \mathrm{H}), 3.60-3.45(\mathrm{~m}, 2 \mathrm{H}), 2.50-2.38(\mathrm{~m}, 1 \mathrm{H}), 2.19-2.01(\mathrm{~m}, 3 \mathrm{H}), 1.91(\mathrm{~m}, 1 \mathrm{H}), 1.83-1.57$ $(\mathrm{m}, 4 \mathrm{H}) ;{ }^{13} \mathbf{C}$ NMR $\left(100 \mathrm{MHz}, \mathrm{CDCl}_{3}, 25{ }^{\circ} \mathrm{C}\right) \delta 164.4,150.1,148.2,139.3,137.5,126.2,122.3,105.4$, 43.0, 37.2, 34.8, 29.6, 28.3, 18.5; FT-IR (neat) 3380, 3055, 2931, 2859, 2832, 1667, 1590, 1569, 1521, 1464, 1432, 1286, 1244, 1165, 1041, 997, 819, 748, $621 \mathrm{~cm}^{-1}$; HRMS (ESI) $\mathrm{m} / z$ calculated for $\mathrm{C}_{14} \mathrm{H}_{18} \mathrm{IN}_{2} \mathrm{O}[\mathrm{M}+\mathrm{H}]^{+} 357.0458$, found 357.0462; TLC: $R_{f}=0.39$ (2:1 Hex/EtOAc). 


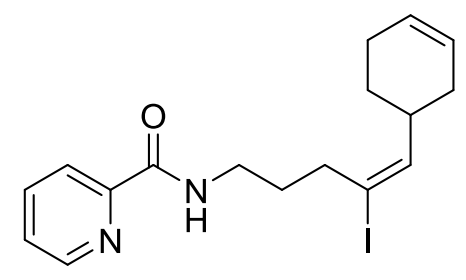

The title compound was prepared according to general procedure 5 from $(Z)-N$-(5-(cyclohex-3-en-1yl)pent-4-en-1-yl)picolinamide $1 \mathrm{~s}(81 \mathrm{mg}, 0.30 \mathrm{mmol})$. The crude product was purified via flash column chromatography (97:3 DCM/EtOAc) to afford a brown oil (67 mg, $0.17 \mathrm{mmol}, 56 \%)$.

${ }^{1} \mathbf{H}$ NMR $\left(400 \mathrm{MHz}, \mathrm{CDCl}_{3}, 25{ }^{\circ} \mathrm{C}\right) \delta 8.54(\mathrm{ddd}, J=4.8,1.7,0.9 \mathrm{~Hz}, 1 \mathrm{H}), 8.19(\mathrm{dt}, J=7.8,1.1 \mathrm{~Hz}$, $1 \mathrm{H}), 8.11(\mathrm{~s}, 1 \mathrm{H}), 7.84(\mathrm{td}, J=7.7,1.7 \mathrm{~Hz}, 1 \mathrm{H}), 7.42(\mathrm{ddd}, J=7.6,4.8,1.3 \mathrm{~Hz}, 1 \mathrm{H}), 6.16(\mathrm{dt}, J=9.7$, $1.0 \mathrm{~Hz}, 1 \mathrm{H}), 5.68-5.55(\mathrm{~m}, 2 \mathrm{H}), 3.52-3.45(\mathrm{~m}, 2 \mathrm{H}), 2.61-2.47$ (m, 3H), $2.09-1.97(\mathrm{~m}, 3 \mathrm{H}), 1.92$ - $1.75(\mathrm{~m}, 3 \mathrm{H}), 1.71-1.60(\mathrm{~m}, 1 \mathrm{H}), 1.40(\mathrm{dddd}, J=12.9,11.0,9.0,6.9 \mathrm{~Hz}, 1 \mathrm{H}) ;{ }^{13} \mathbf{C}$ NMR $(100$ $\left.\mathrm{MHz}, \mathrm{CDCl}_{3}, 25^{\circ} \mathrm{C}\right) \delta 164.5,150.0,148.2,147.2,137.5,127.0,126.3,125.6,122.3,101.5,38.2,36.3$, 36.1, 31.2, 29.6, 28.6, 24.5; FT-IR (neat) 3385, 3056, 3021, 2918, 2834, 1674, 1591, 1569, 1524, $1434,1288,998,749,621 \mathrm{~cm}^{-1}$; HRMS (ESI) $\mathrm{m} / z$ calculated for $\mathrm{C}_{17} \mathrm{H}_{22} \mathrm{IN}_{2} \mathrm{O}[\mathrm{M}+\mathrm{H}]^{+} 397.0771$, found 397.0775 .

$N$-((4E,6E)-4-iodo-7-phenylhepta-4,6-dien-1-yl)picolinamide (2t)<smiles>O=C(NCCC/C(I)=C\C=C\c1ccccc1)c1ccccn1</smiles>

The title compound was prepared according to general procedure 5 from $N-((4 Z, 6 E)-7$-phenylhepta4,6-dien-1-yl)picolinamide 1t $(88 \mathrm{mg}, 0.30 \mathrm{mmol})$. The crude product was purified via flash column chromatography (97:3 DCM/EtOAc) to afford a yellow oil (62 mg, $0.15 \mathrm{mmol}, 49 \%)$.

${ }^{1} \mathbf{H}$ NMR $\left(400 \mathrm{MHz}, \mathrm{CD}_{2} \mathrm{Cl}_{2}, 25{ }^{\circ} \mathrm{C}\right) \delta 8.48(\mathrm{ddd}, J=4.8,1.7,0.9 \mathrm{~Hz}, 1 \mathrm{H}), 8.20-8.04(\mathrm{~m}, 2 \mathrm{H}), 7.83$ $(\mathrm{td}, J=7.7,1.7 \mathrm{~Hz}, 1 \mathrm{H}), 7.42(\mathrm{ddd}, J=7.6,4.8,1.2 \mathrm{~Hz}, 1 \mathrm{H}), 7.38-7.32(\mathrm{~m}, 2 \mathrm{H}), 7.30-7.23(\mathrm{~m}, 3 \mathrm{H})$, $7.02(\mathrm{dd}, J=10.9,0.8 \mathrm{~Hz}, 1 \mathrm{H}), 6.90(\mathrm{dd}, J=15.2,11.0 \mathrm{~Hz}, 1 \mathrm{H}), 6.53(\mathrm{~d}, J=15.2 \mathrm{~Hz}, 1 \mathrm{H}), 3.49$ (q, $J$ $=6.7 \mathrm{~Hz}, 2 \mathrm{H}), 2.84-2.73(\mathrm{~m}, 2 \mathrm{H}), 1.93(\mathrm{p}, J=7.0 \mathrm{~Hz}, 2 \mathrm{H}) ;{ }^{13} \mathbf{C ~ N M R}\left(100 \mathrm{MHz}, \mathrm{CD}_{2} \mathrm{Cl}_{2}, 25{ }^{\circ} \mathrm{C}\right) \delta$ 164.7, 150.6, 148.7, 142.1, 137.9, 137.2, 134.2, 129.2, 128.5, 127.1, 126.6, 124.0, 122.5, 106.3, 38.3, 37.1, 29.9; FT-IR (neat) 3382, 3056, 3030, 2928, 1673, 1590, 1569, 1525, 1465, 1434, 1287, 1144, 
963, 748, 691, $621 \mathrm{~cm}^{-1}$; HRMS (ESI) $\mathrm{m} / z$ calculated for $\mathrm{C}_{19} \mathrm{H}_{20} \mathrm{IN}_{2} \mathrm{O}[\mathrm{M}+\mathrm{H}]^{+} 419.0615$, found 419.0616; TLC: $R_{f}=0.24$ (3:2 Hex/EtOAc).

(S,E)-N-(4-iodo-5-(4-(prop-1-en-2-yl)cyclohex-1-en-1-yl)pent-4-en-1-yl)picolinamide (2u)<smiles>C=C(C)C1CC=C(/C=C(/I)CCCNC(=O)c2ccccn2)CC1</smiles>

The title compound was prepared according to general procedure 5 from $(S, Z)-N$-(5-(4-(prop-1-en-2yl)cyclohex-1-en-1-yl)pent-4-en-1-yl)picolinamide $1 \mathbf{l u}(93 \mathrm{mg}, 0.30 \mathrm{mmol})$. The crude product was purified via flash column chromatography (6:1 Hex/EtOAc) to afford a yellow oil (74 mg, $0.17 \mathrm{mmol}$, $56 \%)$.

${ }^{1} \mathbf{H}$ NMR $\left(400 \mathrm{MHz}, \mathrm{CDCl}_{3}, 25{ }^{\circ} \mathrm{C}\right) \delta 8.53(\mathrm{ddd}, J=4.8,1.8,0.9 \mathrm{~Hz}, 1 \mathrm{H}), 8.20(\mathrm{dt}, J=7.8,1.1 \mathrm{~Hz}$, $1 \mathrm{H}), 8.10(\mathrm{~s}, 1 \mathrm{H}), 7.85(\mathrm{td}, J=7.7,1.7 \mathrm{~Hz}, 1 \mathrm{H}), 7.42(\mathrm{ddd}, J=7.6,4.8,1.3 \mathrm{~Hz}, 1 \mathrm{H}), 6.64(\mathrm{~s}, 1 \mathrm{H}), 5.61$ $(\mathrm{s}, 1 \mathrm{H}), 4.74-4.68(\mathrm{~m}, 1 \mathrm{H}), 4.66-4.62(\mathrm{~m}, 1 \mathrm{H}), 3.50(\mathrm{q}, J=6.7 \mathrm{~Hz}, 2 \mathrm{H}), 2.70-2.63(\mathrm{~m}, 2 \mathrm{H}), 2.19-$ $1.99(\mathrm{~m}, 4 \mathrm{H}), 1.96-1.83(\mathrm{~m}, 3 \mathrm{H}), 1.78-1.71(\mathrm{~m}, 1 \mathrm{H}), 1.68(\mathrm{~s}, 3 \mathrm{H}), 1.46-1.34(\mathrm{~m}, 1 \mathrm{H}) ;{ }^{13} \mathbf{C} \mathbf{N M R}$ $\left(100 \mathrm{MHz}, \mathrm{CDCl}_{3}, 25{ }^{\circ} \mathrm{C}\right) \delta 164.4,150.0,149.5,148.2,144.1,137.5,135.6,127.7,126.3,122.4$, 108.9, 104.8, 40.4, 38.2, 37.1, 30.9, 30.3, 29.1, 27.6, 20.9; FT-IR (neat) 3385, 3058, 2924, 2834, 1672, 1523, 1434, 1288, 997, 889, 749, $621 \mathrm{~cm}^{-1}$; HRMS (ESI) $\mathrm{m} / z$ calculated for $\mathrm{C}_{20} \mathrm{H}_{25} \mathrm{IN}_{2} \mathrm{NaO}$ $[\mathrm{M}+\mathrm{Na}]^{+} 459.0904$, found 459.0905; O.R. $[\alpha]_{\mathrm{D}}^{24}=-59.4\left(c 1.0, \mathrm{CHCl}_{3}\right)$; TLC: $R_{f}=0.55(2: 1 \mathrm{Hex} /$ EtOAc).

\section{$N$-((E)-5-((1R,5S)-6,6-dimethylbicyclo[3.1.1] hept-2-en-2-yl)-4-iodopent-4-en-1-yl)picolinamide} $(2 \mathbf{v})$

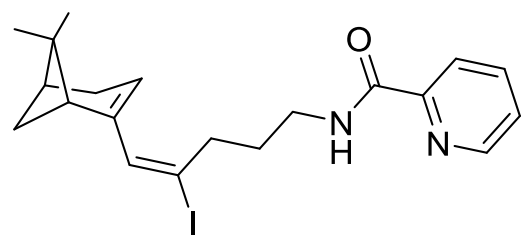

The title compound was prepared according to general procedure 5 (reaction stopped after $6 \mathrm{~h}$ ) from $N$-((Z)-5-((1R,5S)-6,6-dimethylbicyclo[3.1.1]hept-2-en-2-yl)pent-4-en-1-yl)picolinamide $1 \mathbf{v}$ (93 mg, $0.30 \mathrm{mmol})$. The crude product was purified via flash column chromatography (97:3 DCM/EtOAc) to afford an orange oil (65 mg, $0.15 \mathrm{mmol}, 50 \%)$. 
${ }^{1} \mathbf{H}$ NMR $\left(500 \mathrm{MHz}, \mathrm{CDCl}_{3}, 25{ }^{\circ} \mathrm{C}\right) \delta 8.54(\mathrm{ddd}, J=4.8,1.7,0.9 \mathrm{~Hz}, 1 \mathrm{H}), 8.19(\mathrm{dt}, J=7.8,1.1 \mathrm{~Hz}$, $1 \mathrm{H}), 8.11(\mathrm{~s}, 1 \mathrm{H}), 7.84(\mathrm{td}, J=7.7,1.7 \mathrm{~Hz}, 1 \mathrm{H}), 7.43-7.40(\mathrm{~m}, 1 \mathrm{H}), 6.58(\mathrm{tq}, J=1.9,0.9 \mathrm{~Hz}, 1 \mathrm{H})$, $5.47(\mathrm{tq}, J=3.2,1.5 \mathrm{~Hz}, 1 \mathrm{H}), 3.50(\mathrm{td}, J=7.0,6.1 \mathrm{~Hz}, 2 \mathrm{H}), 2.69-2.62(\mathrm{~m}, 2 \mathrm{H}), 2.36-2.32(\mathrm{~m}, 1 \mathrm{H})$, $2.27-2.12(\mathrm{~m}, 3 \mathrm{H}), 2.07-2.00(\mathrm{~m}, 1 \mathrm{H}), 1.93-1.85(\mathrm{~m}, 2 \mathrm{H}), 1.23(\mathrm{~s}, 3 \mathrm{H}), 1.14(\mathrm{~d}, J=8.8 \mathrm{~Hz}, 1 \mathrm{H})$, $0.82(\mathrm{~s}, 3 \mathrm{H}) ;{ }^{13} \mathrm{C}$ NMR $\left(126 \mathrm{MHz}, \mathrm{CDCl}_{3}, 25{ }^{\circ} \mathrm{C}\right) \delta 164.4,150.0,148.1,145.6,142.4,137.5,126.2$, 123.3, 122.3, 104.7, 46.1 , 40.4, 38.5, 38.0, 37.5, 31.9, 31.5, 30.5, 26.3, 21.2; FT-IR (neat) 3386, 3055, 2981, 2918, 2880, 2828, 172, 1522, 1465, 1433, 1288, 1191, 997, 748, $621 \mathrm{~cm}^{-1}$; HRMS (ESI) $\mathrm{m} / \mathrm{z}$ calculated for $\mathrm{C}_{20} \mathrm{H}_{26} \mathrm{IN}_{2} \mathrm{O}[\mathrm{M}+\mathrm{H}]^{+} 437.1084$, found 437.1081; O.R. $[\alpha]_{\mathrm{D}}^{24}=-12.5\left(c 1.0, \mathrm{CHCl}_{3}\right)$; TLC: $R_{f}=0.60(3: 2 \mathrm{Hex} / \mathrm{EtOAc})$.

(E)- $N$-(4-iodo-7-phenylhept-4-en-6-yn-1-yl)picolinamide (2w)<smiles>O=C(NCCC/C(I)=C\C#Cc1ccccc1)c1ccccn1</smiles>

The title compound was prepared according to general procedure 5 from (Z)- $N$-(7-phenylhept-4-en-6yn-1-yl)picolinamide $1 \mathbf{w}(29 \mathrm{mg}, 0.10 \mathrm{mmol})$. Instead of an aqueous workup the reaction was filtered through celite, concentrated, and purified via flash column chromatography (97:3 DCM/EtOAc) to afford a yellow oil (17 mg, $0.041 \mathrm{mmol}, 41 \%)$.

${ }^{1} \mathbf{H}$ NMR $\left(400 \mathrm{MHz}, \mathrm{CDCl}_{3}, 25{ }^{\circ} \mathrm{C}\right) \delta 8.30(\mathrm{ddd}, J=4.8,1.8,0.9 \mathrm{~Hz}, 1 \mathrm{H}), 8.23-8.12(\mathrm{~m}, 2 \mathrm{H}), 7.79$ (td, $J=7.7,1.7 \mathrm{~Hz}, 1 \mathrm{H}), 7.38-7.20(\mathrm{~m}, 6 \mathrm{H}), 6.49(\mathrm{~s}, 1 \mathrm{H}), 3.55$ (q, $J=6.6 \mathrm{~Hz}, 2 \mathrm{H}), 2.96-2.85(\mathrm{~m}$, 2H), $2.04-1.85(\mathrm{~m}, 2 \mathrm{H}) ;{ }^{13} \mathbf{C} \mathbf{~ N M R}\left(100 \mathrm{MHz}, \mathrm{CDCl}_{3}, 25{ }^{\circ} \mathrm{C}\right) \delta 164.5,150.0,148.1,137.4,131.6$, 128.6, 128.4, 126.1, 122.9, 122.3, 121.7, 117.3, 94.4, 86.4, 38.7, 38.0, 29.1; FT-IR (neat) 3384, 3056, 3030, 2928, 2196, 1673, 1523, 1433, 998, 755, 690, $621 \mathrm{~cm}^{-1}$; HRMS (ESI) $\mathrm{m} / \mathrm{z}$ calculated for $\mathrm{C}_{19} \mathrm{H}_{18} \mathrm{IN}_{2} \mathrm{O}[\mathrm{M}+\mathrm{H}]^{+} 417.0458$, found 417.0457; TLC: $R_{f}=0.25$ (97:3 DCM/EtOAc).

$N$-(2-(2-iodocyclohex-1-en-1-yl)ethyl)picolinamide (2x)<smiles>O=C(NCCC1=C(I)CCCC1)c1ccccn1</smiles>

The title compound was prepared according to general procedure 4 from $N$-(2-(cyclohex-1-en-1yl)ethyl)picolinamide $\mathbf{1} \mathbf{x}^{15}$ (88 $\mathrm{mg}, 0.30 \mathrm{mmol}$ ) using anhydrous acetonitrile as solvent, $20 \mathrm{~mol} \%$ 
$\mathrm{Pd}(\mathrm{OAc})_{2}$ as catalyst, and conducting the reaction at $70{ }^{\circ} \mathrm{C}$. The crude product was purified via flash column chromatography (97:3 DCM/EtOAc) to afford a yellow oil (51 mg, $0.14 \mathrm{mmol}, 48 \%)$.

${ }^{1} \mathbf{H}$ NMR $\left(400 \mathrm{MHz}, \mathrm{CDCl}_{3}, 25{ }^{\circ} \mathrm{C}\right) \delta 8.57-8.55(\mathrm{~m}, 1 \mathrm{H}), 8.23-8.18(\mathrm{~m}, 1 \mathrm{H}), 8.14(\mathrm{~s}, 1 \mathrm{H}), 7.84(\mathrm{td}$, $J=7.7,1.7 \mathrm{~Hz}, 1 \mathrm{H}), 7.42(\mathrm{ddd}, J=7.6,4.8,1.3 \mathrm{~Hz}, 1 \mathrm{H}), 3.62-3.52(\mathrm{~m}, 2 \mathrm{H}), 2.69-2.60(\mathrm{~m}, 2 \mathrm{H})$, $2.52(\mathrm{t}, J=7.8 \mathrm{~Hz}, 2 \mathrm{H}), 2.30-2.22(\mathrm{~m}, 2 \mathrm{H}), 1.79-1.67(\mathrm{~m}, 2 \mathrm{H}), 1.67-1.56(\mathrm{~m}, 2 \mathrm{H}) ;{ }^{13} \mathbf{C}$ NMR $(100$ $\left.\mathrm{MHz}, \mathrm{CDCl}_{3}, 25^{\circ} \mathrm{C}\right) \delta 164.4,150.1,148.2,138.8,137.5,126.2,122.3,100.1,42.7,41.9,37.3,31.2$, 25.8, 22.9; FT-IR (neat) 3382, 3056, 2929, 1857,1669, 1520, 1464, 1433, 1287, 1165, 997, 819, 749, $621 \mathrm{~cm}^{-1}$; HRMS (ESI) $\mathrm{m} / z$ calculated for $\mathrm{C}_{14} \mathrm{H}_{18} \mathrm{IN}_{2} \mathrm{O}[\mathrm{M}+\mathrm{H}]^{+} 357.0458$, found 357.0461; TLC: $R_{f}=$ 0.45 (3:2 Hex/EtOAc).

\section{$N$-(2-((1R,5R)-3-iodo-6,6-dimethylbicyclo[3.1.1]hept-2-en-2-yl)ethyl)picolinamide (2y)}<smiles>CC1CCCC(I)=C1CCNC(=O)c1ccccn1</smiles>

The title compound was prepared according to general procedure 4 from $N$-(2-((1R,5S)-6,6dimethylbicyclo[3.1.1]hept-2-en-2-yl)ethyl)picolinamide $1 \mathbf{y}(81 \mathrm{mg}, 0.30 \mathrm{mmol})$ using anhydrous acetonitrile as solvent, and conducting the reaction at $70^{\circ} \mathrm{C}$ for $2 \mathrm{~h}$. The crude product was purified via flash column chromatography $(8: 1 \mathrm{Hex} / \mathrm{EtOAc})$ to afford a pale yellow oil $(79 \mathrm{mg}, 0.20 \mathrm{mmol}$, $66 \%)$.

${ }^{1} \mathbf{H}$ NMR $\left(500 \mathrm{MHz}, \mathrm{CDCl}_{3}, 25{ }^{\circ} \mathrm{C}\right) \delta 8.55(\mathrm{ddd}, J=4.8,1.7,0.9 \mathrm{~Hz}, 1 \mathrm{H}), 8.18(\mathrm{dt}, J=7.8,1.1 \mathrm{~Hz}$, $1 \mathrm{H}), 8.11(\mathrm{~s}, 1 \mathrm{H}), 7.84(\mathrm{td}, J=7.7,1.7 \mathrm{~Hz}, 1 \mathrm{H}), 7.41(\mathrm{ddd}, J=7.6,4.7,1.2 \mathrm{~Hz}, 1 \mathrm{H}), 3.56-3.41(\mathrm{~m}$, 2H), $2.80(\mathrm{ddt}, J=17.3,2.9,1.3 \mathrm{~Hz}, 1 \mathrm{H}), 2.70(\mathrm{ddt}, J=17.2,2.6,1.2 \mathrm{~Hz}, 1 \mathrm{H}), 2.52-2.39(\mathrm{~m}, 4 \mathrm{H})$, $1.96-1.91(\mathrm{~m}, 1 \mathrm{H}), 1.35(\mathrm{~d}, J=8.7 \mathrm{~Hz}, 1 \mathrm{H}), 1.27(\mathrm{~s}, 3 \mathrm{H}), 0.87(\mathrm{~s}, 3 \mathrm{H}) ;{ }^{13} \mathbf{C} \mathbf{N M R}\left(126 \mathrm{MHz}, \mathrm{CDCl}_{3}\right.$, $\left.25^{\circ} \mathrm{C}\right) \delta 164.4,150.1,149.5,148.2,137.4,126.2,122.2,93.5,48.5,46.3,43.9,40.8,39.9,36.9,32.0$, 25.8, 21.8; FT-IR (neat) 3387, 3056, 2976, 2918, 2835, 1677, 1522, 1465, 1433, 1287, 1042, 749, $621 \mathrm{~cm}^{-1}$; HRMS (ESI) $\mathrm{m} / z$ calculated for $\mathrm{C}_{17} \mathrm{H}_{22} \mathrm{IN}_{2} \mathrm{O}[\mathrm{M}+\mathrm{H}]^{+}$387.0771, found 397.0769; O.R. $[\alpha]_{\mathrm{D}}^{24}=-21.1\left(c 1.0, \mathrm{CHCl}_{3}\right)$. 


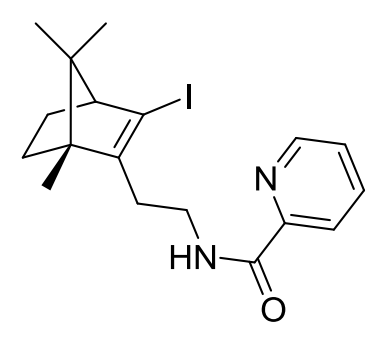

The title compound was prepared according to general procedure 4 from $N$-(2- $((1 R, 4 R)-1,7,7-$ trimethylbicyclo[2.2.1]hept-2-en-2-yl)ethyl)picolinamide $\mathbf{1 z}(28 \mathrm{mg}, 0.10 \mathrm{mmol})$ using anhydrous acetonitrile as solvent, and conducting the reaction at $70{ }^{\circ} \mathrm{C}$ for $90 \mathrm{~min}$. The crude product was purified via flash column chromatography (97:3 DCM/EtOAc) to afford colorless oil (34 mg, $0.083 \mathrm{mmol}, 83 \%)$.

${ }^{1} \mathbf{H}$ NMR $\left(500 \mathrm{MHz}, \mathrm{CDCl}_{3}, 25{ }^{\circ} \mathrm{C}\right) \delta 8.55(\mathrm{ddd}, J=4.8,1.7,0.9 \mathrm{~Hz}, 1 \mathrm{H}), 8.18(\mathrm{dt}, J=7.8,1.1 \mathrm{~Hz}$, $1 \mathrm{H}), 8.12(\mathrm{~s}, 1 \mathrm{H}), 7.83(\mathrm{td}, J=7.7,1.7 \mathrm{~Hz}, 1 \mathrm{H}), 7.41(\mathrm{ddd}, J=7.6,4.7,1.2 \mathrm{~Hz}, 1 \mathrm{H}), 3.59-3.42(\mathrm{~m}$, $2 \mathrm{H}), 2.48-2.33(\mathrm{~m}, 3 \mathrm{H}), 1.76-1.68(\mathrm{~m}, 1 \mathrm{H}), 1.56-1.48(\mathrm{~m}, 1 \mathrm{H}), 1.14(\mathrm{~s}, 3 \mathrm{H}), 1.11-1.03(\mathrm{~m}, 2 \mathrm{H})$, 0.87 (s, 3H), 0.78 (s, 3H); ${ }^{13} \mathbf{C}$ NMR $\left(126 \mathrm{MHz}, \mathrm{CDCl}_{3}, 25{ }^{\circ} \mathrm{C}\right) \delta 164.4,150.1,150.1,148.2,137.4$, 126.2, 122.2, 98.3, 63.1, 57.2, 56.3, 37.7, 32.7, 30.3, 24.9, 19.6, 19.5, 12.0; FT-IR (neat) 3385, 3056, 2953, 2871, 1677, 1521, 1464, 1433, 1286, 997, 749, 701, $621 \mathrm{~cm}^{-1}$; HRMS (ESI) $\mathrm{m} / z$ calculated for $\mathrm{C}_{18} \mathrm{H}_{23} \mathrm{IN}_{2} \mathrm{NaO}[\mathrm{M}+\mathrm{Na}]^{+} 433.0747$, found 433.0744. O.R. $[\alpha]_{\mathrm{D}}^{24}=+5.2$ (c 1.0, $\left.\mathrm{CHCl}_{3}\right)$; TLC: $R_{f}=$ 0.33 (97:3 DCM/EtOAc).

(E)- $N$-(4-iodo-5-phenylpent-4-en-1-yl)pyrazine-2-carboxamide (S14)

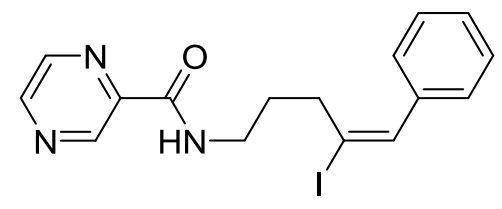

The title compound was prepared according to general procedure 4 from ( $Z$ )- $N$-(5-phenylpent-4-en-1yl)pyrazine-2-carboxamide $\mathbf{S 1 1}(27 \mathrm{mg}, 0.10 \mathrm{mmol}$ ). The crude product was purified via flash column chromatography (95:5 DCM/EtOAc to pure EtOAc) to afford a pale yellow oil (22 mg, $0.056 \mathrm{mmol}$, $56 \%$ ).

${ }^{1} \mathbf{H}$ NMR $\left(400 \mathrm{MHz}, \mathrm{CDCl}_{3}, 25{ }^{\circ} \mathrm{C}\right) \delta 9.36(\mathrm{~d}, J=1.5 \mathrm{~Hz}, 1 \mathrm{H}), 8.73(\mathrm{~d}, J=2.5 \mathrm{~Hz}, 1 \mathrm{H}), 8.47(\mathrm{dd}, J=$ 2.5, $1.5 \mathrm{~Hz}, 1 \mathrm{H}), 7.70(\mathrm{~s}, 1 \mathrm{H}), 7.35(\mathrm{~s}, 1 \mathrm{H}), 7.28-7.23(\mathrm{~m}, 2 \mathrm{H}), 7.22-7.13(\mathrm{~m}, 3 \mathrm{H}), 3.48(\mathrm{td}, J=7.0$, $6.1 \mathrm{~Hz}, 2 \mathrm{H}), 2.73-2.65(\mathrm{~m}, 2 \mathrm{H}), 1.95(\mathrm{dq}, J=9.0,7.1 \mathrm{~Hz}, 2 \mathrm{H}) ;{ }^{13} \mathbf{C} \mathbf{~ N M R}\left(100 \mathrm{MHz}, \mathrm{CDCl}_{3}, 25{ }^{\circ} \mathrm{C}\right)$ $\delta 163.1,147.3,144.5,142.5,141.9,137.5,128.6,128.1,127.5,107.1,38.1,36.5,29.9$; FT-IR (neat) 
3384, 3346, 3055, 2928, 1671, 1530, 1400, 1165, 1020, 863, $700 \mathrm{~cm}^{-1}$; HRMS (ESI) $\mathrm{m} / \mathrm{z}$ calculated for $\mathrm{C}_{16} \mathrm{H}_{16} \mathrm{IN}_{3} \mathrm{NaO}[\mathrm{M}+\mathrm{H}]^{+}$416.0230, found 416.0230; TLC: $R_{f}=0.17$ (3:2 Hex/EtOAc).

(E)- $N$-(4-iodo-5-phenylpent-4-en-1-yl)-9H-pyrido[3,4-b]indole-1-carboxamide (S15)<smiles>O=C(NCCC/C(I)=C\c1ccccc1)c1nccc2c1[nH]c1ccccc12</smiles>

The title compound was prepared according to general procedure 4 from $(Z)-N$-(5-phenylpent-4-en-1yl)-9H-pyrido[3,4-b]indole-1-carboxamide $\mathbf{S 1 2}(35 \mathrm{mg}, 0.10 \mathrm{mmol})$. The crude product was purified via flash column chromatography (20:1 toluene/EtOAc) to afford a pale yellow oil (29 mg, $0.060 \mathrm{mmol}, 60 \%)$.

${ }^{1} \mathbf{H}$ NMR $\left(500 \mathrm{MHz}, \mathrm{CDCl}_{3}, 25{ }^{\circ} \mathrm{C}\right) \delta 10.29(\mathrm{~s}, 1 \mathrm{H}), 8.35(\mathrm{~d}, J=5.1 \mathrm{~Hz}, 1 \mathrm{H}), 8.19-8.07(\mathrm{~m}, 3 \mathrm{H})$, $7.63-7.54(\mathrm{~m}, 2 \mathrm{H}), 7.35(\mathrm{~s}, 1 \mathrm{H}), 7.32(\mathrm{ddd}, J=8.0,7.0,1.2 \mathrm{~Hz}, 1 \mathrm{H}), 7.22-7.16(\mathrm{~m}, 4 \mathrm{H}), 7.15-7.11$ (m, 1H), $3.55(\mathrm{td}, J=7.0,6.2 \mathrm{~Hz}, 2 \mathrm{H}), 2.75(\mathrm{ddd}, J=8.6,6.2,1.0 \mathrm{~Hz}, 2 \mathrm{H}), 2.06-1.99(\mathrm{~m}, 2 \mathrm{H}) ;{ }^{13} \mathbf{C}$ NMR $\left(126 \mathrm{MHz}, \mathrm{CDCl}_{3}, 25{ }^{\circ} \mathrm{C}\right) \delta 166.5$ (weak, confirmed by HMBC), 141.8, 141.2, 137.6, 137.2, 135.6, 132.0 (weak, confirmed by HMBC), 131.5 (weak, confirmed by HMBC), 129.4, 128.6, 128.1, 127.4, 122.0, 120.7, 120.5, 117.8, 112.1, 107.3, 37.9, 36.7, 30.2; FT-IR (neat) 3420, 3364, 3058, 2927, 1655, 1627, 1527, 1492, 1451, 1321, 1251, 1219, $749 \mathrm{~cm}^{-1}$; HRMS (ESI) $\mathrm{m} / z$ calculated for $\mathrm{C}_{23} \mathrm{H}_{21} \mathrm{IN}_{3} \mathrm{O}[\mathrm{M}+\mathrm{H}]^{+} 482.0724$, found 482.0725; TLC: $R_{f}=0.59$ (3:1 Hex/EtOAc).

(E)-4-iodo-5-phenyl- $N$-(2-(pyridin-2-yl)propan-2-yl)pent-4-enamide (S16)<smiles>CC(C)(NC(=O)CC/C(I)=C\c1ccccc1)c1ccccn1</smiles>

The title compound was prepared according to general procedure 4 from (Z)-5-phenyl- $N$-(2-(pyridin-2yl)propan-2-yl)pent-4-enamide $\mathbf{S 1 3}$ (29 $\mathrm{mg}, 0.10 \mathrm{mmol}$ ). The crude product was purified via flash column chromatography (4:1 to 2:1 Hex/EtAOc) to afford a yellow oil (11 mg, $0.026 \mathrm{mmol}, 26 \%)$.

${ }^{1} \mathbf{H}$ NMR $\left(400 \mathrm{MHz}, \mathrm{CDCl}_{3}, 25{ }^{\circ} \mathrm{C}\right) \delta 8.51(\mathrm{ddd}, J=4.9,1.8,1.0 \mathrm{~Hz}, 1 \mathrm{H}), 7.84-7.79(\mathrm{~m}, 1 \mathrm{H}), 7.71$ (ddd, $J=8.1,7.4,1.8 \mathrm{~Hz}, 1 \mathrm{H}), 7.39(\mathrm{dt}, J=8.1,1.0 \mathrm{~Hz}, 1 \mathrm{H}), 7.35-7.30(\mathrm{~m}, 3 \mathrm{H}), 7.27-7.22(\mathrm{~m}, 3 \mathrm{H})$, 
$7.19(\mathrm{ddd}, J=7.4,4.9,1.1 \mathrm{~Hz}, 1 \mathrm{H}), 2.97-2.92(\mathrm{~m}, 2 \mathrm{H}), 2.60-2.54(\mathrm{~m}, 2 \mathrm{H}), 1.73(\mathrm{~s}, 6 \mathrm{H}) ;{ }^{13} \mathbf{C} \mathbf{~ N M R}$ $\left(100 \mathrm{MHz}, \mathrm{CDCl}_{3}, 25{ }^{\circ} \mathrm{C}\right) \delta 170.1,164.4,147.6,142.0,137.4,128.7,128.2,127.6,122.1,119.7$, 107.0, 56.6, 38.0, 35.4, 27.6; FT-IR (neat) 3315, 3056, 2976, 2931, 1651, 1613, 1591, 1570, 1536, 1508, 1473, 1447, 1431, 1127, 1036, 786, 748, $699 \mathrm{~cm}^{-1}$; HRMS (ESI) $\mathrm{m} / z$ calculated for $\mathrm{C}_{19} \mathrm{H}_{22} \mathrm{IN}_{2} \mathrm{O}$ $[\mathrm{M}+\mathrm{H}]^{+}$421.0771, found 421.0768; TLC: $R_{f}=0.48(1: 1 \mathrm{Hex} / \mathrm{EtOAc})$. 


\section{Derivatization of $2 \mathbf{a}$}

\section{(E)-tert-butyl (4-iodo-5-phenylpent-4-en-1-yl)carbamate (4)}<smiles>CC(C)(C)OC(=O)NCCC/C(I)=C\c1ccccc1</smiles>

To a round bottom flask under ambient atmosphere ( $1 \mathrm{~atm}$, balloon) was added (E)- $N$-(4-iodo-5phenylpent-4-en-1-yl)picolinamide $\mathbf{2 a}$ (40 $\mathrm{mg}, \quad 0.10 \mathrm{mmol}, 1.0$ equiv), 4-dimethylaminopyridine (15 mg, $0.12 \mathrm{mmol}, 1.2$ equiv), di-tert-butyl dicarbonate (66.8 mg, $0.31 \mathrm{mmol}, 3.0$ equiv), and acetonitrile $(0.5 \mathrm{~mL})$. The reaction was stirred at room temperature for $14 \mathrm{~h}$, an additional 2 equiv of di-tert-butyl dicarbonate were added and the reaction was heated to $50{ }^{\circ} \mathrm{C}$. Additional di-tert-butyl dicarbonate ( 3 equiv) was added in portions until complete consumption of starting material was observed by TLC and LCMS (ca $12 \mathrm{~h}$ at $50{ }^{\circ} \mathrm{C}$ ). The reaction was allowed to cool to room temperature, a solution of lithium hydroxide monohydrate $(214 \mathrm{mg}, 5.10 \mathrm{mmol}, 50$ equiv) in water $(1 \mathrm{~mL})$ was added, the reaction was re-heated to $50{ }^{\circ} \mathrm{C}$ and stirred at this temperature for $3 \mathrm{~h}$. The reaction was allowed to cool to room temperature, diluted with $1 \mathrm{M}$ aq $\mathrm{NaOH}(30 \mathrm{~mL})$, extracted with DCM $(2 \times 30 \mathrm{~mL})$, dried over $\mathrm{Na}_{2} \mathrm{SO}_{4}$ and concentrated. Purification by flash column chromatography (5:1 Hex/EtOAc) afforded a colorless oil (35 mg, $0.089 \mathrm{mmol}, 89 \%)$.

${ }^{1} \mathbf{H}$ NMR $\left(400 \mathrm{MHz}, \mathrm{CDCl}_{3}, 25{ }^{\circ} \mathrm{C}\right) \delta 7.37-7.31(\mathrm{~m}, 3 \mathrm{H}), 7.30-7.25(\mathrm{~m}, 1 \mathrm{H}), 7.20-7.14(\mathrm{~m}, 2 \mathrm{H})$, $4.38(\mathrm{~s}, 1 \mathrm{H}), 3.10(\mathrm{q}, J=6.6 \mathrm{~Hz}, 2 \mathrm{H}), 2.60(\mathrm{ddd}, J=8.7,6.0,1.0 \mathrm{~Hz}, 2 \mathrm{H}), 1.82-1.74(\mathrm{~m}, 2 \mathrm{H}), 1.41$ (s, 9H); ${ }^{13} \mathrm{C}$ NMR $\left(100 \mathrm{MHz}, \mathrm{CDCl}_{3}, 25{ }^{\circ} \mathrm{C}\right) \delta 156.0,141.5,137.6,128.7,128.1,127.6,107.7,79.3$, 39.3, 36.6, 30.4, 28.5; FT-IR (neat) 3354, 2975, 2931, 1693, 1512, 1446, 1391, 1366, 1252, 1170, 750, $699 \mathrm{~cm}^{-1}$; HRMS (ESI) $\mathrm{m} / z$ calculated for $\mathrm{C}_{16} \mathrm{H}_{22} \mathrm{INNaO}_{2}[\mathrm{M}+\mathrm{Na}]^{+} 410.0587$, found 410.0588; TLC: $R_{f}=0.51$ (3:2 Hex/EtOAc); TLC: $R_{f}=0.73$ (3:2 Hex/EtOAc).

\section{(E)-(2-benzylidenepyrrolidin-1-yl)(pyridin-2-yl)methanone (5)}

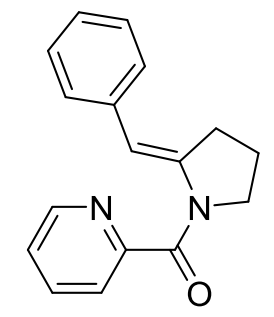

Based on a literature protocol, ${ }^{16}$ a screw-cap glass vial was charged with $(E)-N$-(4-iodo-5-phenylpent4-en-1-yl)picolinamide $2 \mathbf{a}$ ( $30 \mathrm{mg}, 0.076 \mathrm{mmol}, 1.00$ equiv), cesium carbonate ( $37 \mathrm{mg}, 0.115 \mathrm{mmol}$, 
1.50 equiv), $\mathrm{N}, \mathrm{N}^{\prime}$-dimethylethane-1,2-diamine ( $2.7 \mathrm{mg}, 0.031 \mathrm{mmol}, 0.40$ equiv), and anhydrous THF $(0.4 \mathrm{~mL})$. The reaction was sparged with argon for $30 \mathrm{~s}$, CuI $(2.9 \mathrm{mg}, 0.015 \mathrm{mmol}, 0.20$ equiv) was added, the vial was sealed and placed in a pre-heated heating block at $50{ }^{\circ} \mathrm{C}$ and stirred for $90 \mathrm{~min}$. The reaction allowed to cool to room temperature, filtered through a silica plug (washed with EtOAc) and concentrated. Purification by flash column chromatography (2:1 Hex/EtOAc) afforded a pale yellow oil (19 mg, $0.072 \mathrm{mmol}, 93 \%)$.

${ }^{1} \mathbf{H}$ NMR $\left(400 \mathrm{MHz}, \mathrm{DMSO}-d_{6}, 25^{\circ} \mathrm{C}\right) \delta 8.61(\mathrm{ddd}, J=4.8,1.8,1.0 \mathrm{~Hz}, 1 \mathrm{H}), 7.97(\mathrm{td}, J=7.7,1.8 \mathrm{~Hz}$, $1 \mathrm{H}), 7.66(\mathrm{dt}, J=7.8,1.2 \mathrm{~Hz}, 1 \mathrm{H}), 7.51(\mathrm{ddd}, J=7.6,4.9,1.2 \mathrm{~Hz}, 1 \mathrm{H}), 7.38-7.23(\mathrm{~m}, 4 \mathrm{H}), 7.21-$ $7.12(\mathrm{~m}, 1 \mathrm{H}), 3.71(\mathrm{t}, J=7.0 \mathrm{~Hz}, 2 \mathrm{H}), 2.84(\mathrm{td}, J=7.4,2.1 \mathrm{~Hz}, 2 \mathrm{H}), 1.91-1.74(\mathrm{~m}, 2 \mathrm{H})$. Note that the vinylic proton is not visible due to line broadening at room temperature. ${ }^{13} \mathbf{C}$ NMR (100 MHz, DMSO$\left.d_{6}, 25{ }^{\circ} \mathrm{C}\right) \delta 166.8,155.0,148.3,142.0,137.8,137.5,128.3,128.1,125.6,124.9,122.7,111.5,50.9$ (broad), 30.0, 22.7; ${ }^{1} \mathbf{H}$ NMR (400 MHz, DMSO- $\left.d_{6}, 80{ }^{\circ} \mathrm{C}\right) \delta 8.61(\mathrm{ddd}, J=4.8,1.7,0.9 \mathrm{~Hz}, 1 \mathrm{H}), 7.94$ $(\mathrm{td}, J=7.7,1.8 \mathrm{~Hz}, 1 \mathrm{H}), 7.65(\mathrm{dt}, J=7.8,1.1 \mathrm{~Hz}, 1 \mathrm{H}), 7.48(\mathrm{ddd}, J=7.6,4.8,1.2 \mathrm{~Hz}, 1 \mathrm{H}), 7.41(\mathrm{~s}$, $1 \mathrm{H}), 7.34-7.31(\mathrm{~m}, 2 \mathrm{H}), 7.28-7.24(\mathrm{~m}, 2 \mathrm{H}), 7.19-7.13(\mathrm{~m}, 1 \mathrm{H}), 3.76-3.72(\mathrm{~m}, 2 \mathrm{H}), 2.84(\mathrm{td}, J=$ 7.4, $2.2 \mathrm{~Hz}, 2 \mathrm{H}), 1.91-1.82(\mathrm{~m}, 2 \mathrm{H}) ;{ }^{13} \mathbf{C}$ NMR $\left(100 \mathrm{MHz}, \mathrm{DMSO}-d_{6}, 80{ }^{\circ} \mathrm{C}\right) \delta 166.3,154.8,147.9$, 141.4, 137.5, 136.9, 127.8, 127.6, 125.2, 124.3, 122.2, 111.4, 50.1, 29.4, 22.0; FT-IR (neat) 3053, 3020, 2976, 2893, 1651, 1625, 1596, 1585, 1566, 1469, 1435, 1388, 1353, 1339, 1226, 1159, 994, 895, 804, 771, 746, $696 \mathrm{~cm}^{-1}$; HRMS (ESI) $\mathrm{m} / z$ calculated for $\mathrm{C}_{17} \mathrm{H}_{16} \mathrm{~N}_{2} \mathrm{NaO}[\mathrm{M}+\mathrm{Na}]^{+} 287.1155$, found 287.1158; TLC: $R_{f}=0.15$ (2:1 Hex/EtOAc).

\section{(E)- $N$-(4-benzylidene-6-(1,3-dioxan-2-yl)hexyl)picolinamide (6)}

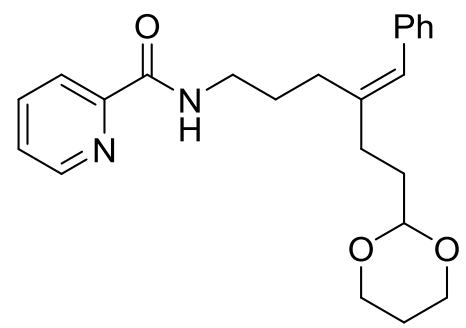

A screw-cap glass vial was charged with (E)-N-(4-iodo-5-phenylpent-4-en-1-yl)picolinamide 2a (25 mg, $0.064 \mathrm{mmol}, 1.0$ equiv) and anhydrous THF $(0.6 \mathrm{~mL})$. The reaction was sparged with argon for $30 \mathrm{~s}, \mathrm{Pd}\left(\mathrm{PPh}_{3}\right)_{4}(7.4 \mathrm{mg}, 0.0064 \mathrm{mmol}, 0.10$ equiv) was added, the reaction was sparged with argon for an additional $30 \mathrm{~s}$, (2-(1,3-dioxan-2-yl)ethyl)zinc(II) bromide (0.5M in THF, $0.38 \mathrm{~mL}, 0.19 \mathrm{mmol}$, 3.0 equiv) was added and the vial was sealed. The vial was placed in a heating block pre-heated to $50{ }^{\circ} \mathrm{C}$ and stirred for $4 \mathrm{~h}$ at this temperature. The reaction was allowed to cool to room temperature, ethylenediaminetetraacetic acid disodium salt dihydrate $(119 \mathrm{mg}, 0.32 \mathrm{mmol}, 5.0$ equiv) was added, the reaction was stirred for $15 \mathrm{~min}$ and then poured into $30 \mathrm{~mL}$ aqueous EDTA solution $(500 \mathrm{mg}$ 
ethylenediaminetetraacetic acid disodium salt dihydrate dissolved in $30 \mathrm{~mL}$ water). The aqueous phase was extracted with EtOAc $(1 \times 50 \mathrm{~mL})$, the organic phase was washed with a basic EDTA solution (500 mg ethylenediaminetetraacetic acid disodium salt dihydrate dissolved in $30 \mathrm{~mL}$ sat. aq. $\mathrm{NaHCO}_{3}$ ), dried over $\mathrm{Na}_{2} \mathrm{SO}_{4}$ and concentrated. Purification by flash column chromatography (2:1 to 1:1 Hex/EtOAc) afforded a pale yellow oil (21 $\mathrm{mg}, 0.055 \mathrm{mmol}, 87 \%)$.

${ }^{1} \mathbf{H}$ NMR $\left(500 \mathrm{MHz}, \mathrm{CDCl}_{3}, 25{ }^{\circ} \mathrm{C}\right) \delta 8.52(\mathrm{ddd}, J=4.8,1.7,0.9 \mathrm{~Hz}, 1 \mathrm{H}), 8.17(\mathrm{dt}, J=7.8,1.1 \mathrm{~Hz}$, 1H), $8.02(\mathrm{~s}, 1 \mathrm{H}), 7.84(\mathrm{td}, J=7.7,1.7 \mathrm{~Hz}, 1 \mathrm{H}), 7.43-7.39$ (m, 1H), $7.26-7.10(\mathrm{~m}, 5 \mathrm{H}), 6.33$ (s, $1 \mathrm{H}), 4.57(\mathrm{t}, J=5.1 \mathrm{~Hz}, 1 \mathrm{H}), 4.12(\mathrm{ddt}, J=10.4,5.0,1.4 \mathrm{~Hz}, 2 \mathrm{H}), 3.77$ (dddd, $J=12.6,10.5,2.6,1.6$ $\mathrm{Hz}, 2 \mathrm{H}), 3.43(\mathrm{td}, J=7.1,6.1 \mathrm{~Hz}, 2 \mathrm{H}), 2.39-2.26(\mathrm{~m}, 5 \mathrm{H}), 2.09$ (dtt, $J=13.4,12.4,5.0 \mathrm{~Hz}, 1 \mathrm{H}), 1.88$ $-1.77(\mathrm{~m}, 4 \mathrm{H}), 1.35(\mathrm{dtt}, J=13.5,2.7,1.4 \mathrm{~Hz}, 1 \mathrm{H}) ;{ }^{13} \mathbf{C} \mathbf{N M R}\left(126 \mathrm{MHz}, \mathrm{CDCl}_{3}, 25{ }^{\circ} \mathrm{C}\right) \delta 164.3$, $150.1,148.1,141.5,138.4,137.4,128.7,128.3,126.2,126.2,126.1,122.3,102.0,67.1,39.4,33.9$, 31.2, 28.3, 28.3, 26.0; FT-IR (neat) 3384, 3054, 2958, 2928, 2852, 1675, 1525, 1465, 1434, 1134, 998, 750, 700, $621 \mathrm{~cm}^{-1}$; HRMS (ESI) $\mathrm{m} / z$ calculated for $\mathrm{C}_{23} \mathrm{H}_{29} \mathrm{~N}_{2} \mathrm{O}_{3}[\mathrm{M}+\mathrm{H}]^{+} 381.2173$, found 381.2170; TLC: $R_{f}=0.14$ (2:1 Hex/EtOAc).

\section{(E)-N-(4-benzylidene-6-(trimethylsilyl)hex-5-yn-1-yl)picolinamide (7)}

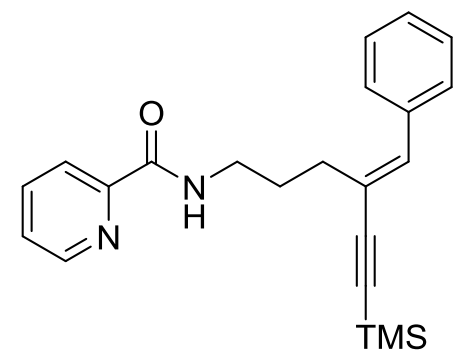

A flame dried flask under $\mathrm{N}_{2}$ was charged with anhydrous THF $(0.40 \mathrm{~mL})$, diisopropylamine $\left(0.030 \mathrm{~mL}, 0.21 \mathrm{mmol}, 3.3\right.$ equiv) and cooled to $0{ }^{\circ} \mathrm{C} . n \mathrm{BuLi}(1.6 \mathrm{M}$ in hexanes, $0.12 \mathrm{~mL}, 0.19 \mathrm{mmol}$, 3.0 equiv) was added dropwise, the reaction was stirred for $10 \mathrm{~min}$ at $0{ }^{\circ} \mathrm{C}$ and subsequently cooled to $-78{ }^{\circ} \mathrm{C}$. Ethynyltrimethylsilane $(0.027 \mathrm{~mL}, 0.19 \mathrm{mmol}, 3.0$ equiv $)$ was added, the reaction was stirred at $-78{ }^{\circ} \mathrm{C}$ for $20 \mathrm{~min}$, and a solution of anhydrous $\mathrm{ZnBr}_{2}(47 \mathrm{mg}, 0.21 \mathrm{mmol}, 3.3$ equiv) in THF $(0.47 \mathrm{~mL})$ was added. After $10 \mathrm{~min}$ the reaction was warmed to $0{ }^{\circ} \mathrm{C}$, stirred for an additional $10 \mathrm{~min}$, (E)- $N$-(4-iodo-5-phenylpent-4-en-1-yl)picolinamide 2a (25 mg, $0.064 \mathrm{mmol}, 1.0$ equiv) and $\mathrm{Pd}\left(\mathrm{PPh}_{3}\right)_{4}$ (7.4 mg, $0.0064 \mathrm{mmol}, 0.10$ equiv) were added, and the reaction was heated to $50{ }^{\circ} \mathrm{C}$ for $2 \mathrm{~h}$. The reaction was allowed to cool to room temperature, ethylenediaminetetraacetic acid disodium salt dihydrate (119 mg, $0.32 \mathrm{mmol}, 5.0$ equiv) was added, the reaction was stirred for $15 \mathrm{~min}$ and then poured into $30 \mathrm{~mL}$ aqueous EDTA solution $(500 \mathrm{mg}$ ethylenediaminetetraacetic acid disodium salt dihydrate dissolved in $30 \mathrm{~mL}$ water). The aqueous phase was extracted with EtOAc $(1 \times 50 \mathrm{~mL})$, the organic phase was washed with a basic EDTA solution (500 mg ethylenediaminetetraacetic acid 
disodium salt dihydrate dissolved in $30 \mathrm{~mL}$ sat. aq. $\mathrm{NaHCO}_{3}$ ), dried over $\mathrm{Na}_{2} \mathrm{SO}_{4}$ and concentrated. Purification by flash column chromatography (4:1 Hex/EtOAc) afforded a pale yellow oil $(21 \mathrm{mg}$, $0.058 \mathrm{mmol}, 91 \%)$.

${ }^{1} \mathbf{H}$ NMR $\left(400 \mathrm{MHz}, \mathrm{CDCl}_{3}, 25{ }^{\circ} \mathrm{C}\right) \delta 8.52(\mathrm{ddd}, J=4.8,1.8,0.9 \mathrm{~Hz}, 1 \mathrm{H}), 8.18(\mathrm{dt}, J=7.8,1.1 \mathrm{~Hz}$, $1 \mathrm{H}), 8.06(\mathrm{~s}, 1 \mathrm{H}), 7.83(\mathrm{td}, J=7.7,1.7 \mathrm{~Hz}, 1 \mathrm{H}), 7.41$ (ddd, $J=7.6,4.7,1.3 \mathrm{~Hz}, 1 \mathrm{H}), 7.31-7.17$ (m, $5 \mathrm{H}), 6.94(\mathrm{~s}, 1 \mathrm{H}), 3.52(\mathrm{q}, J=6.7 \mathrm{~Hz}, 2 \mathrm{H}), 2.49(\mathrm{ddd}, J=9.1,6.2,1.1 \mathrm{~Hz}, 2 \mathrm{H}), 1.97(\mathrm{dq}, J=9.1,7.0$ $\mathrm{Hz}, 2 \mathrm{H}), 0.23$ (s, 9H); ${ }^{13} \mathbf{C} \mathbf{~ N M R}\left(100 \mathrm{MHz}, \mathrm{CDCl}_{3}, 25{ }^{\circ} \mathrm{C}\right) \delta 164.4,150.2,148.1,137.6,137.4,136.5$, 128.9, 128.5, 127.5, 126.1, 124.3, 122.3, 107.5, 94.5, 38.9, 28.7, 28.6, 0.2; FT-IR (neat) 3388, 3057, 3022, 2957, 2139, 1676, 1522, 1465, 1434, 886, 840, 749, 698, $621 \mathrm{~cm}^{-1}$; HRMS (ESI) $\mathrm{m} / \mathrm{z}$ calculated for $\mathrm{C}_{22} \mathrm{H}_{27} \mathrm{~N}_{2} \mathrm{OSi}[\mathrm{M}+\mathrm{H}]^{+} 363.1887$, found 363.1887; TLC: $R_{f}=0.40(2: 1 \mathrm{Hex} / \mathrm{EtOAc})$. 


\section{Kinetic Isotope Effects}

\section{Intermolecular competition}<smiles>[2H]/C(=C/c1c(Cl)cccc1Cl)CC([2H])([2H])CNC(=O)O</smiles>

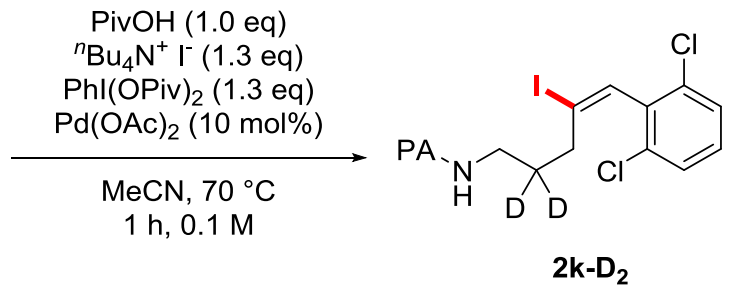

An approximately equal amount of $\mathbf{1 k}$ and $\mathbf{1 k}-\mathbf{D}_{3}$ were dissolved in $\mathrm{CD}_{3} \mathrm{CN}$. The deuterium content of the mixture was determined to be $47 \%$ by ${ }^{1} \mathrm{H}$ NMR spectroscopy. The mixture was concentrated and the resulting solid (26 mg, $0.077 \mathrm{mmol}, 1.0$ equiv) was placed in a $1.6 \mathrm{~mL}$ screw-cap vial. To the vial was added pivalic acid $(7.9 \mathrm{mg}, 0.077 \mathrm{mmol}, 1.0$ equiv), tetrabutylammonium iodide $(37 \mathrm{mg}$, $0.10 \mathrm{mmol}, 1.3$ equiv), bis(tert-butylcarbonyloxy)iodobenzene (41 mg, $0.10 \mathrm{mmol}, 1.3$ equiv), $\mathrm{Pd}(\mathrm{OAc})_{2}\left(1.7 \mathrm{mg}, 0.0077 \mathrm{mmol}, 0.10\right.$ equiv), and $\mathrm{CH}_{3} \mathrm{CN}(0.77 \mathrm{~mL})$. The vial was sealed, placed in a pre-heated heating block at $70{ }^{\circ} \mathrm{C}$ and stirred for $1 \mathrm{~h}$. The reaction was cooled in an ice-bath and subsequently concentrated under reduced pressure. The yield of $2 \mathbf{k}-\mathbf{D}_{\mathbf{2}}$ was determined to be $10 \%$ by ${ }^{1} \mathrm{H}-\mathrm{NMR}$ analysis of the unpurified reaction mixture using ethylene carbonate as internal standard. The crude reaction mixture was purified by preparative TLC (95:5 DCM/EtOAc) to afford $2 \mathbf{k}-\mathbf{D}_{\mathbf{2}}$, for which a deuterium content of $21 \%$ was determined by ${ }^{1} \mathrm{H}$ NMR spectroscopy. $k_{H} / k_{D}$ was calculated according to the literature. ${ }^{17}$

$\mathrm{SM}_{\mathrm{D}}$ (Deuterium content of starting material): $47 \%$

$\mathrm{SM}_{\mathrm{H}}$ (Proton content of starting material): $53 \%$

$\mathrm{P}_{\mathrm{D}}$ (Deuterium content of product): $21 \%$

$\mathrm{P}_{\mathrm{H}}$ (Proton content of product): $79 \%$

$$
\frac{k_{H}}{k_{D}}=\frac{S M_{D} * P_{H}}{S M_{H} * P_{D}}=\frac{0.47 * 0.79}{0.53 * 0.21}=3.34
$$


${ }^{\mathbf{1}} \mathbf{H}$ NMR $\left(500 \mathrm{MHz}, \mathrm{CD}_{3} \mathrm{CN}, 25^{\circ} \mathrm{C}\right)$ of the mixture of $\mathbf{2 k}$ and $\mathbf{2 k - \mathbf { D } _ { \mathbf { 3 } }}$

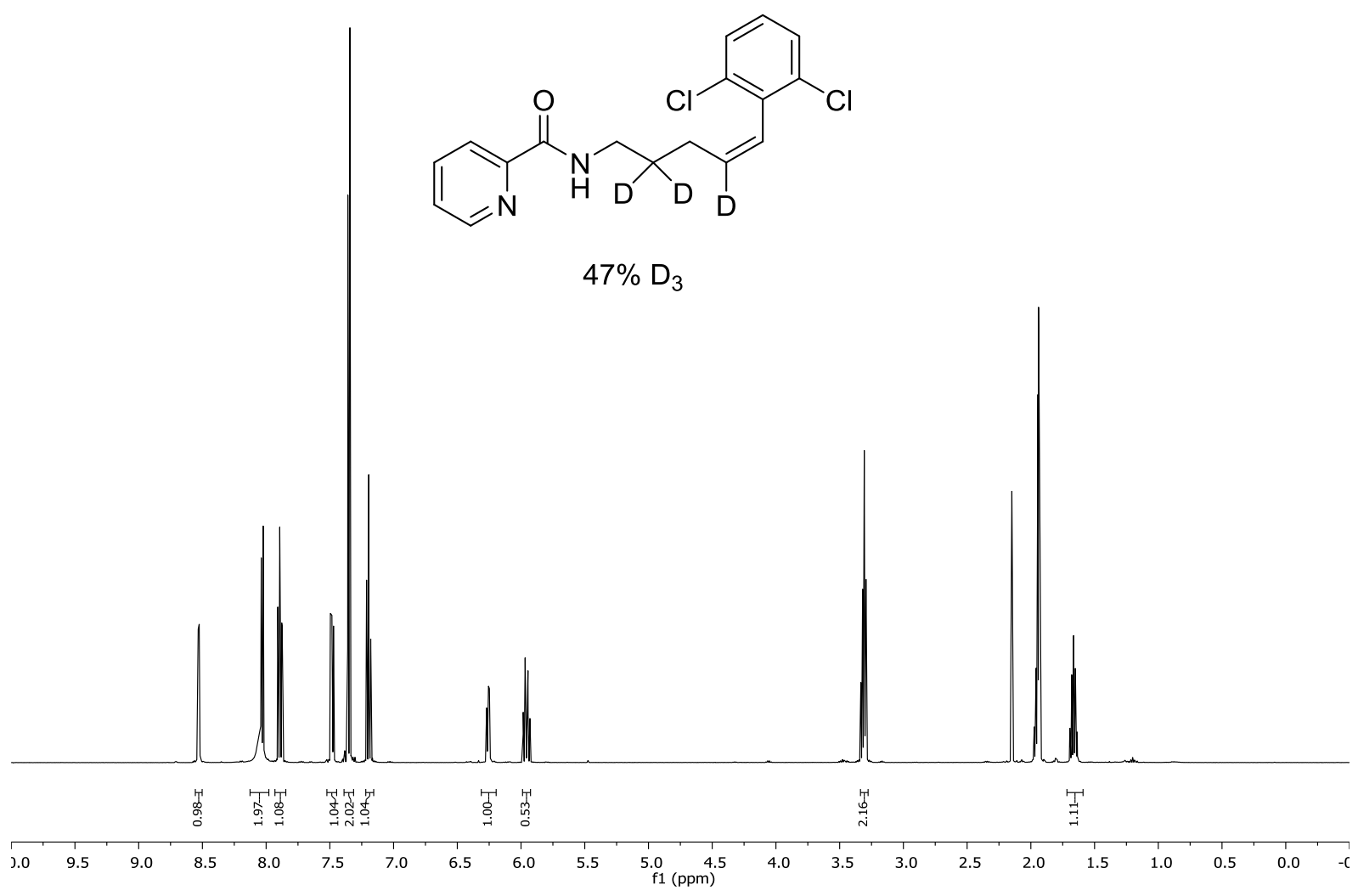

${ }^{1} \mathbf{H}$ NMR $\left(500 \mathrm{MHz}, \mathrm{CD}_{3} \mathrm{CN}, 25{ }^{\circ} \mathrm{C}\right)$ of $2 \mathbf{k}-\mathbf{D}_{\mathbf{2}}$

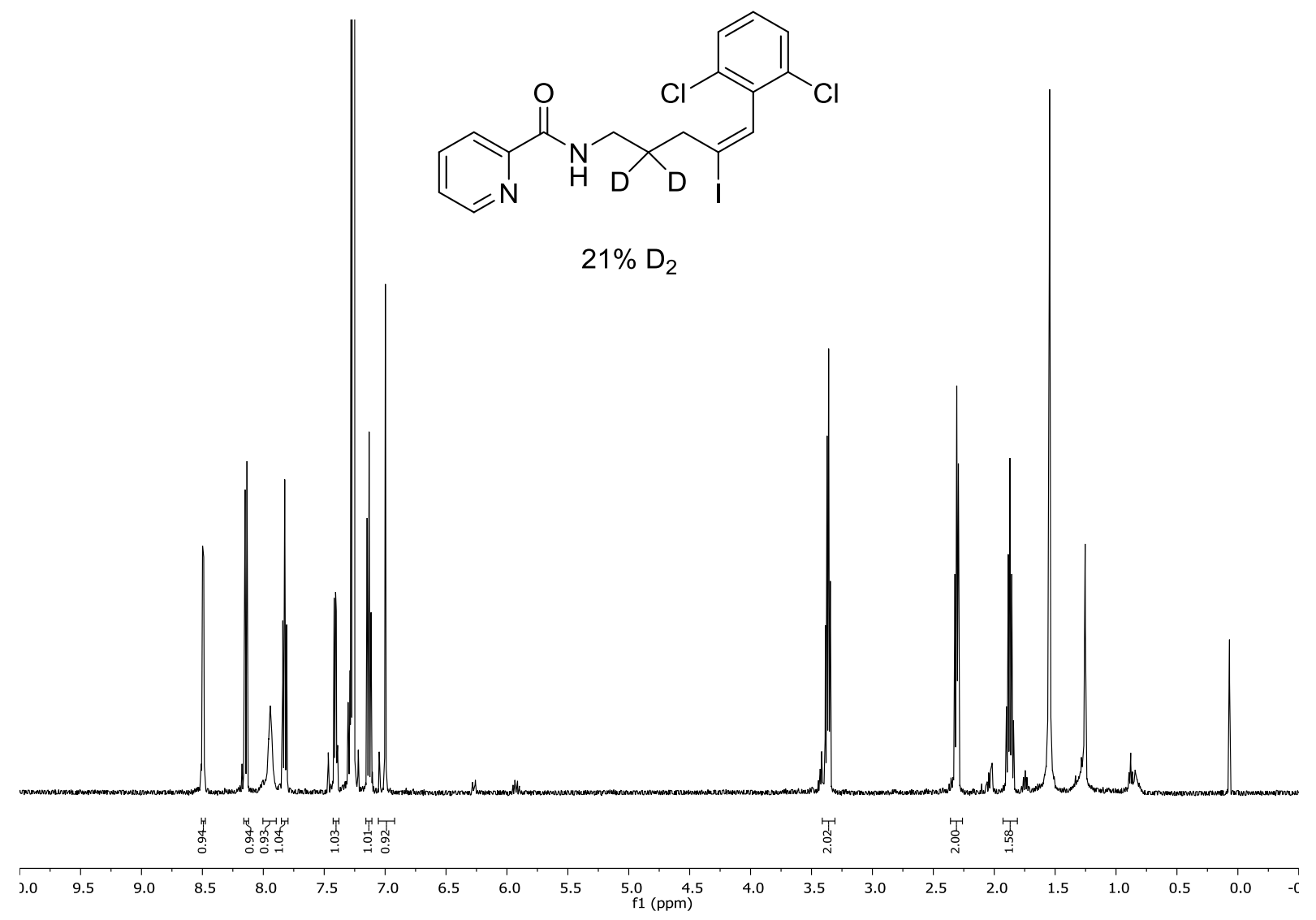

S62 

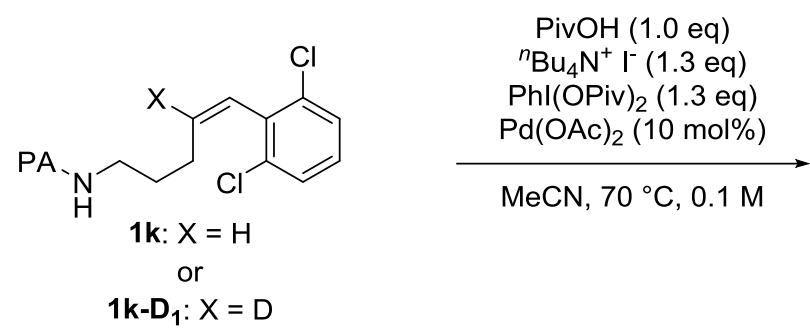<smiles>O=C(NCCC/C(I)=C\c1c(Cl)cccc1Cl)c1ccccc1</smiles>

A $1.6 \mathrm{~mL}$ screw-cap vial was charged with (Z)-N-(5-(2,6-dichlorophenyl)pent-4-en-1-yl)picolinamide $1 \mathbf{k}$ or 1k-D $(23 \mathrm{mg}, 0.07 \mathrm{mmol}, 1.0$ equiv), pivalic acid (7.15 mg, $0.070 \mathrm{mmol}, 1.0$ equiv), tetrabutylammonium iodide (34 mg, $0.091 \mathrm{mmol}, 1.3 \mathrm{equiv})$, bis(tert-butylcarbonyloxy)iodobenzene (37 mg, $0.091 \mathrm{mmol}, 1.3$ equiv), $\mathrm{Pd}(\mathrm{OAc})_{2}(1.6 \mathrm{mg}, 0.0070 \mathrm{mmol}, 0.10$ equiv), and ethylene carbonate (6.2 mg, $0.070 \mathrm{mmol}, 1.0$ equiv). $\mathrm{CD}_{3} \mathrm{CN}(0.70 \mathrm{~mL})$ was added, the resulting solution was transferred to an NMR tube and immediately placed in an NMR Spectrometer, which was pre-heated to $70^{\circ} \mathrm{C}$. The reaction progress was monitored by ${ }^{1} \mathrm{H}$ NMR spectroscopy ( 8 scans every 5 minutes) and the concentration of $\mathbf{2 k}$ was determined by integration of the vinylic proton (7.00 ppm) against ethylene carbonate as internal standard. NMR spectra were analyzed using Mestrenova. The resulting data was analyzed using Microsoft Excel. A linear regression model $\mathrm{y}=\mathrm{m}^{*} \mathrm{x}$ was used to determine the initial rates of the reactions.

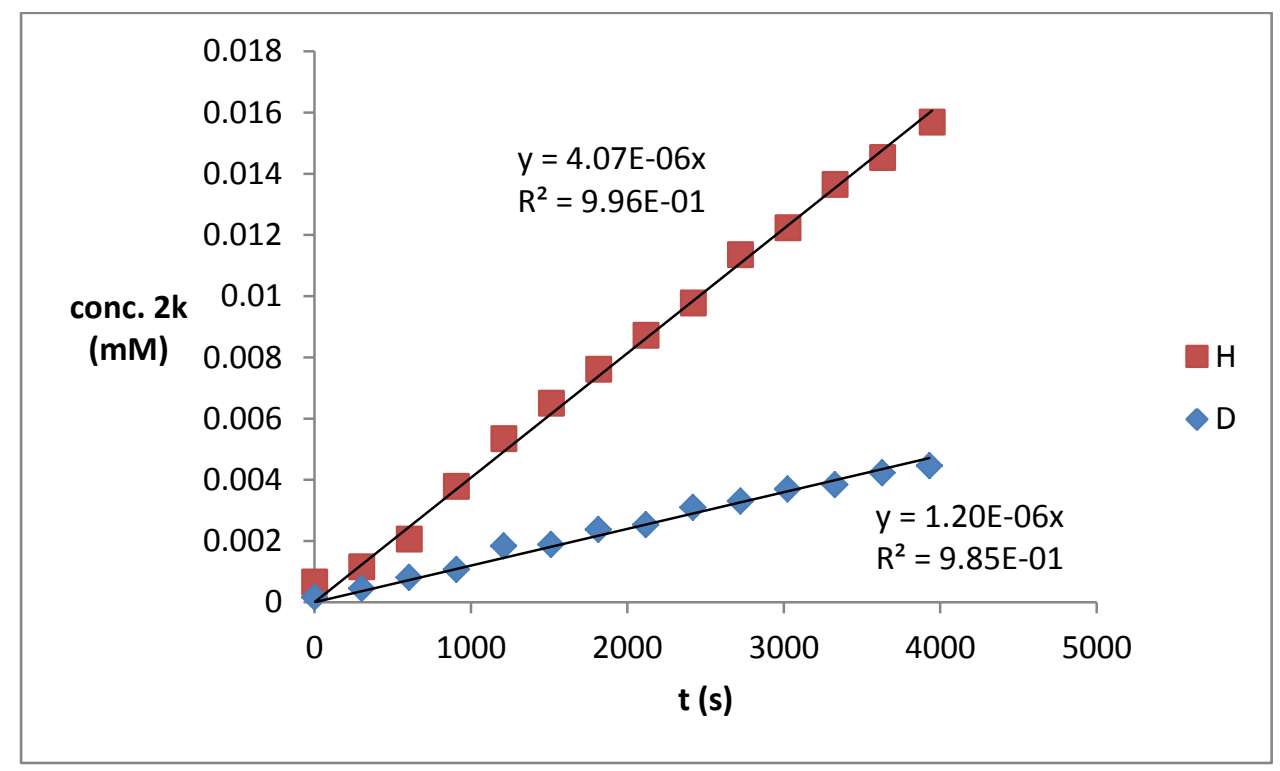

$$
\frac{k_{H}}{k_{D}}=\frac{4.07}{1.20}=3.39
$$




\section{References}

(1) Rosenau, C. P.; Jelier, B. J.; Gossert, A. D.; Togni, A. Exposing the Origins of Irreproducibility in Fluorine NMR Spectroscopy. Angewandte Chemie International Edition 2018, 57 (30), 9528-9533.

(2) Bowman, W. R.; Clark, D. N.; Marmon, R. J. Generation of Aminyl Radicals Using Sulfenamides as Synthetic Precursors. Tetrahedron 1994, 50, 1275-1294.

(3) Zheng, T.; Narayan, R. S.; Schomaker, J. M.; Borhan, B. One-Pot Regio- and Stereoselective Cyclization of 1,2,n-Triols. J. Am. Chem. Soc. 2005, 127 (19), 6946-6947.

(4) Liu, M.; Yang, P.; Karunananda, M. K.; Wang, Y.; Liu, P.; Engle, K. M. C(Alkenyl)-H Activation via Six-Membered Palladacycles: Catalytic 1,3-Diene Synthesis. J. Am. Chem. Soc. 2018, 140 (17), 5805-5813.

(5) Denmark, S. E.; Burk, M. T. Enantioselective Bromocycloetherification by Lewis Base/Chiral Brønsted Acid Cooperative Catalysis. Org. Lett. 2012, 14 (1), 256-259.

(6) Reitz, A. B.; Maryanoff, B. E. Deuteration of Non-Stabilized Phosphorus Ylides With DMSO$\mathrm{d}_{6}$ : Convenient Synthesis of Mono-Deuterated Alkenes. Synthetic Communications 1983, 13 (10), 845-852.

(7) Wu, X.; Riedel, J.; Dong, V. M. Transforming Olefins into $\gamma, \delta$-Unsaturated Nitriles through Copper Catalysis. Angewandte Chemie International Edition 2017, 56 (38), 11589-11593.

(8) Lovick, H. M.; Michael, F. E. Metal-Free Highly Regioselective Aminotrifluoroacetoxylation of Alkenes. J. Am. Chem. Soc. 2010, 132 (4), 1249-1251.

(9) Hörnfeldt, K.; Langström, B. Synthesis of $\left[{ }^{11} \mathrm{C}\right]$ Cyanoalkylphosphoranes and their Use in the Preparation of ${ }^{11}$ C-Olefins, Acta Chem. Scand. 1994, 48, 665.

(10) Akgun, B.; Hall, D. G. Fast and Tight Boronate Formation for Click Bioorthogonal Conjugation. Angewandte Chemie International Edition 2016, 55 (12), 3909-3913.

(11) Meuzelaar, G. J.; Blom, A.; Maat, L.; Sheldon, R. A. Unprecedented Formation of a Benzo[d]Azepine by Acid-Catalyzed Cyclization of a Camphor-Derived $N$-Formylenamine. European Journal of Organic Chemistry 1999, 1999 (2), 519-522.

(12) Gerpe, A.; Bollini, M.; González, M.; Cerecetto, H. Convenient Route to Primary (Z)-Allyl Amines and Homologs. Synthetic Communications 2008, 39 (1), 29-47. 
(13) Goodreid, J. D.; Duspara, P. A.; Bosch, C.; Batey, R. A. Amidation Reactions from the Direct Coupling of Metal Carboxylate Salts with Amines. J. Org. Chem. 2014, 79 (3), 943-954.

(14) Baiget, J.; Llona-Minguez, S.; Lang, S.; MacKay, S. P.; Suckling, C. J.; Sutcliffe, O. B. Manganese Dioxide Mediated One-Pot Synthesis of Methyl 9H-Pyrido[3,4-b]Indole-1Carboxylate: Concise Synthesis of Alangiobussinine. Beilstein Journal of Organic Chemistry 2011, 7 (1), 1407-1411.

(15) Zang, Z.-L.; Zhao, S.; Karnakanti, S.; Liu, C.-L.; Shao, P.-L.; He, Y. Catalytic MultisiteSelective Acetoxylation Reactions at $\mathrm{sp}^{2} \mathrm{vs} \mathrm{sp}^{3} \mathrm{C}-\mathrm{H}$ Bonds in Cyclic Olefins. Org. Lett. 2016, $18(19), 5014-5017$.

(16) Jiang, L.; Job, G. E.; Klapars, A.; Buchwald, S. L. Copper-Catalyzed Coupling of Amides and Carbamates with Vinyl Halides. Org. Lett. 2003, 5 (20), 3667-3669.

(17) Wolfsberg, M.; Van Hook, W. A.; Paneth, P. Instrumentation and Experimental Techniques. In Isotope Effects: in the Chemical, Geological, and Bio Sciences; Wolfsberg, M., Hook, W. A., Paneth, P., Rebelo, L. P. N., Eds.; Springer Netherlands: Dordrecht, 2009; pp 203-244. 
${ }^{1} \mathbf{H}$ NMR $\left(400 \mathrm{MHz}, \mathrm{DMSO}-d_{6}, 25^{\circ} \mathrm{C}\right)$ of $\mathbf{S 1}$

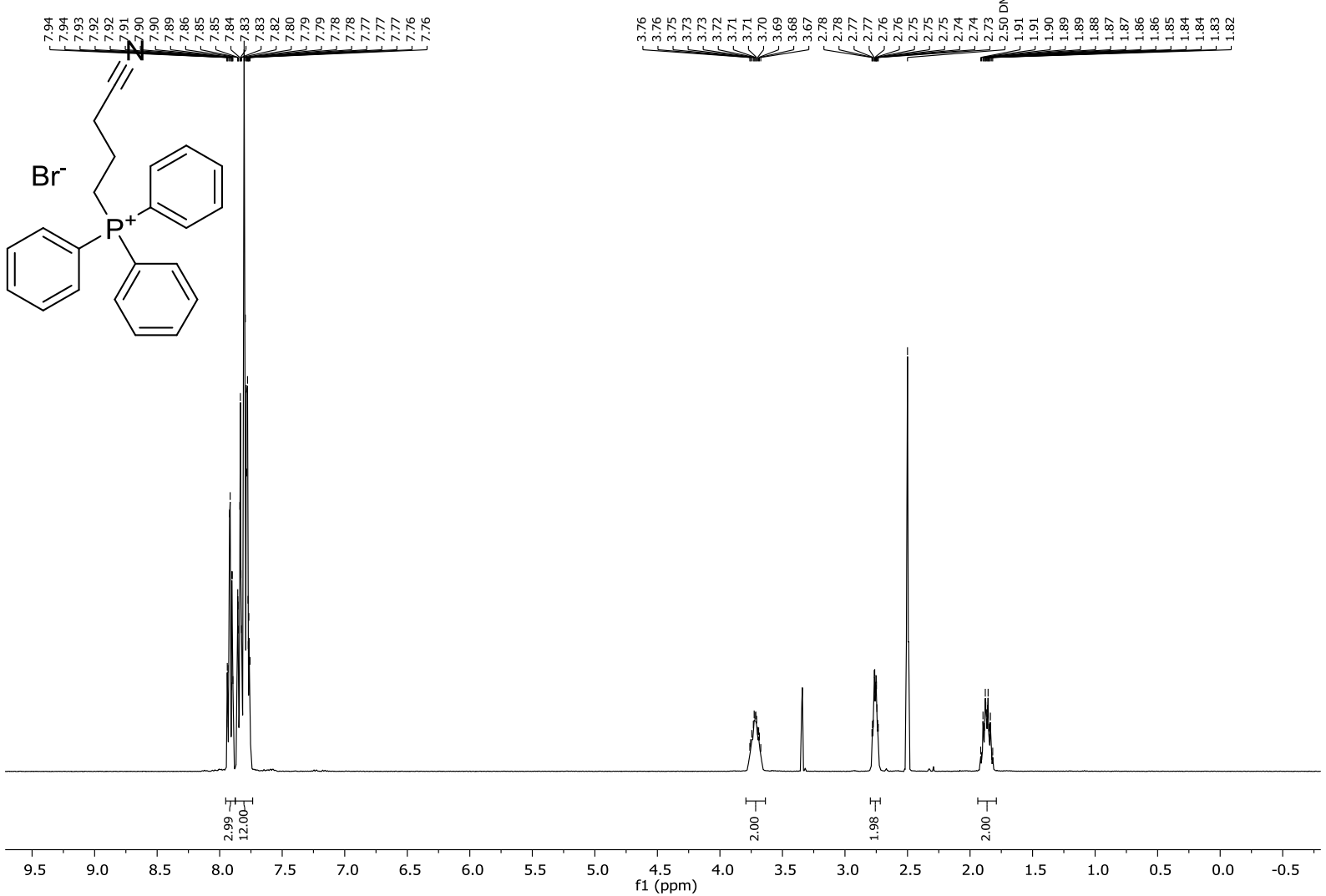

${ }^{13} \mathbf{C}$ NMR $\left(100 \mathrm{MHz}, \mathrm{DMSO}-d_{6}, 25^{\circ} \mathrm{C}\right)$ of $\mathbf{S 1}$

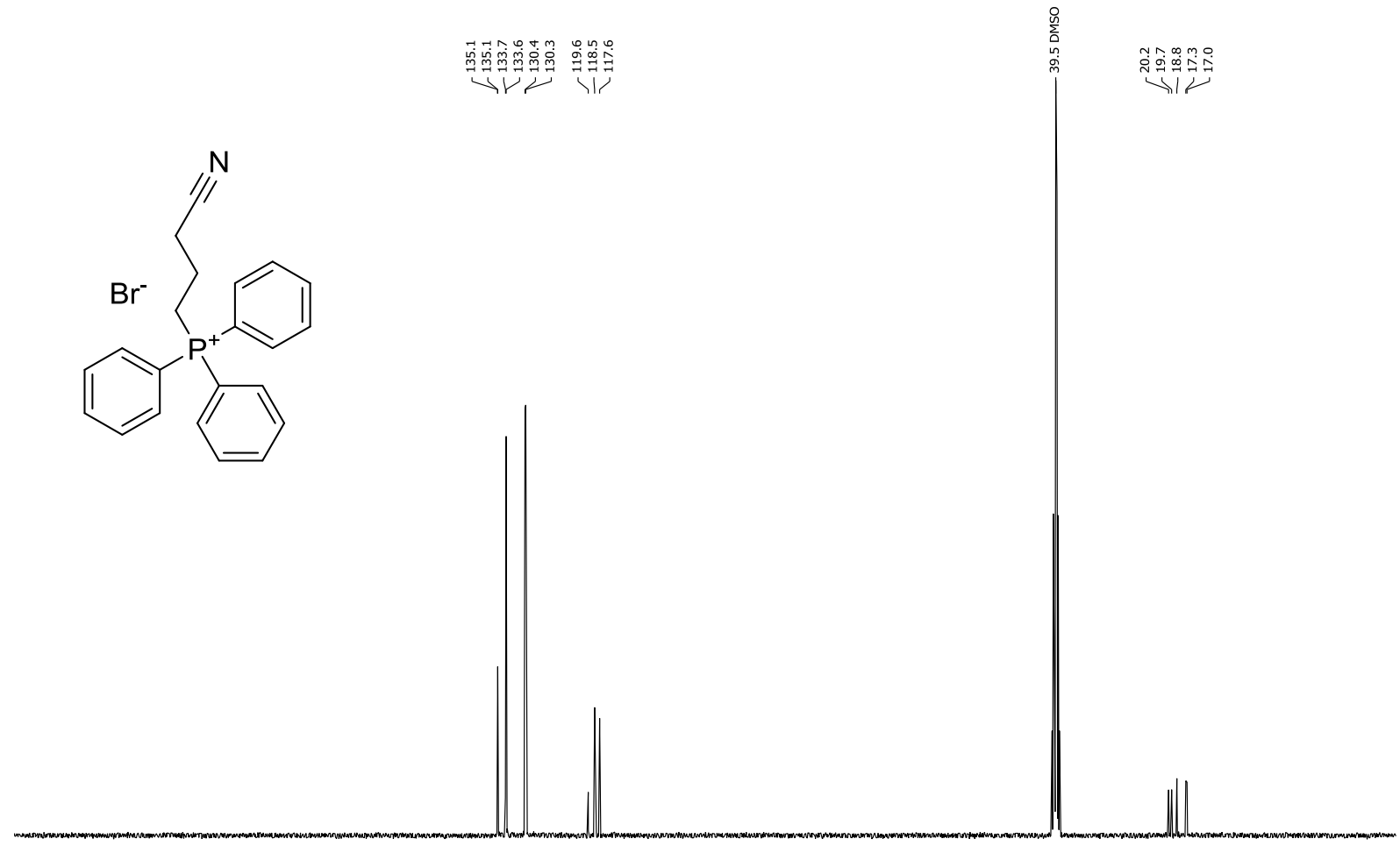

$\begin{array}{llllllllllllllllllllllll}1 & 1 & 200 & 190 & 180 & 170 & 160 & 150 & 140 & 130 & 120 & 110 & 100 & 100 & 80 & 70 & 60 & 50 & 40 & 30 & 20 & 10 & 0 & -10\end{array}$ 
${ }^{31} \mathbf{P}$ NMR $\left(162 \mathrm{MHz}, \mathrm{DMSO}-d_{6}, 25^{\circ} \mathrm{C}\right)$ of $\mathbf{S 1}$
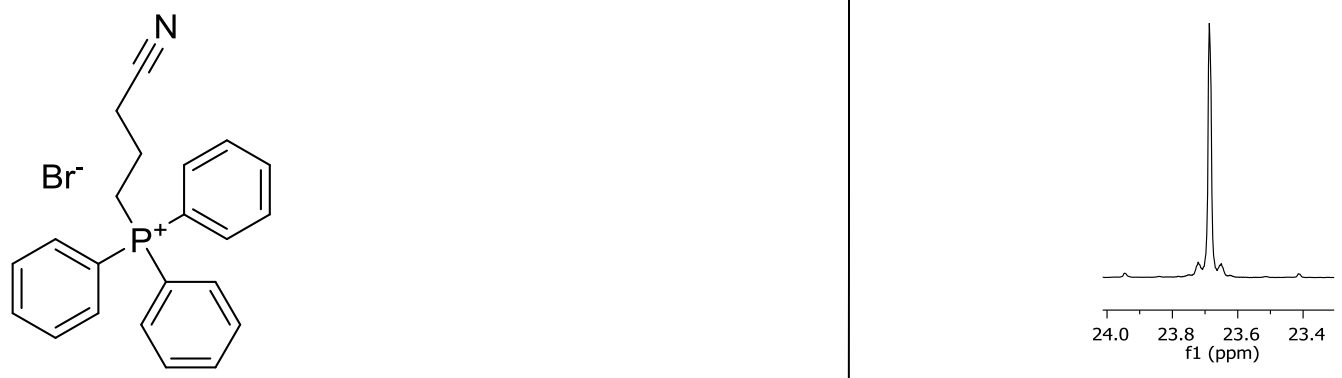

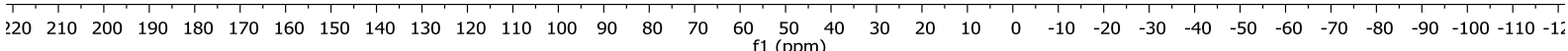


${ }^{\mathbf{1}} \mathbf{H}$ NMR $\left(400 \mathrm{MHz}, \mathrm{CDCl}_{3}, 25^{\circ} \mathrm{C}\right)$ of $\mathbf{S 2 a}$

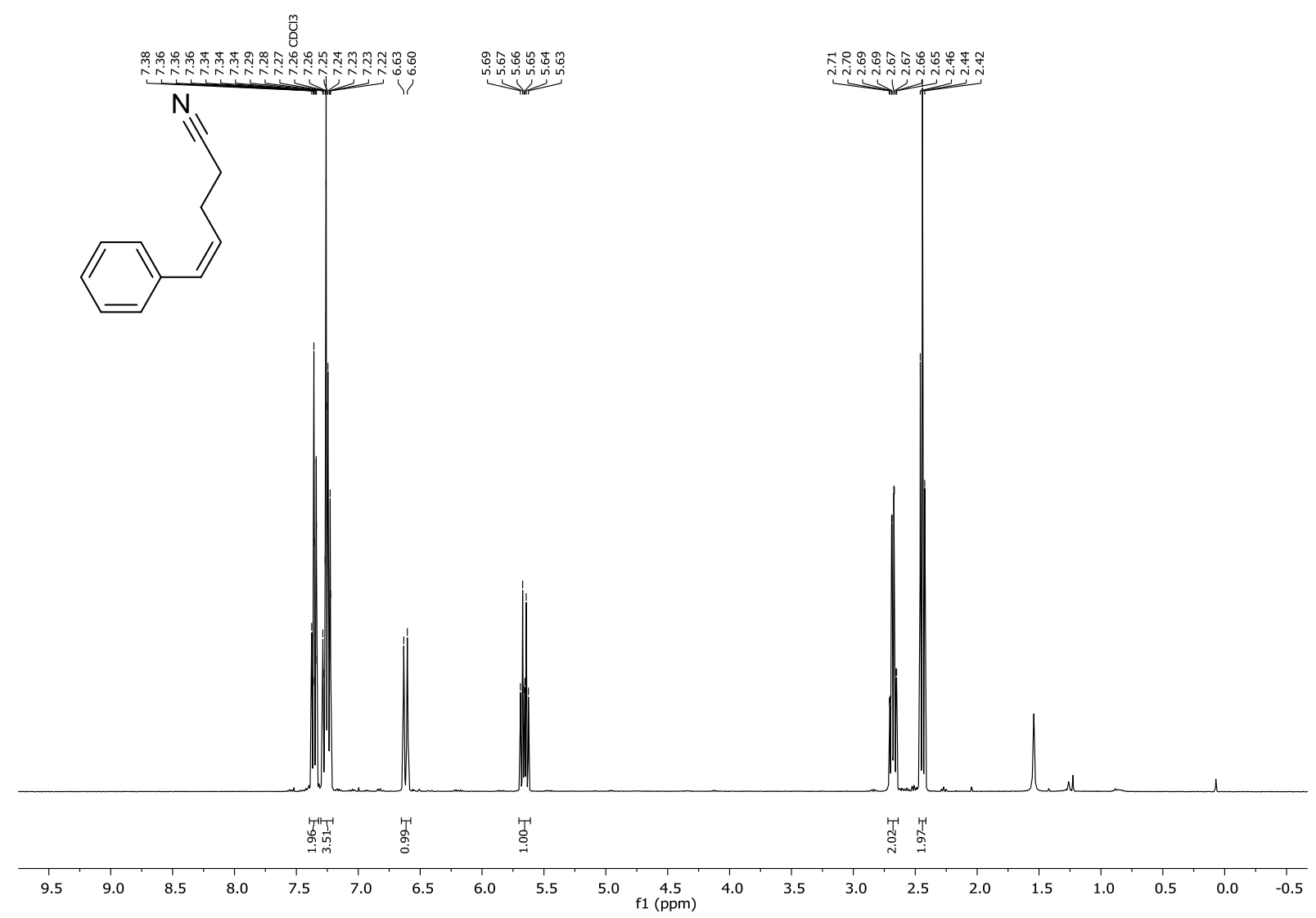

${ }^{13} \mathbf{C} \mathbf{N M R}\left(100 \mathrm{MHz}, \mathrm{CDCl}_{3}, 25^{\circ} \mathrm{C}\right)$ of $\mathbf{S 2 a}$

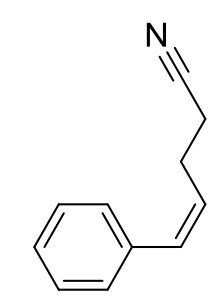

نَّ
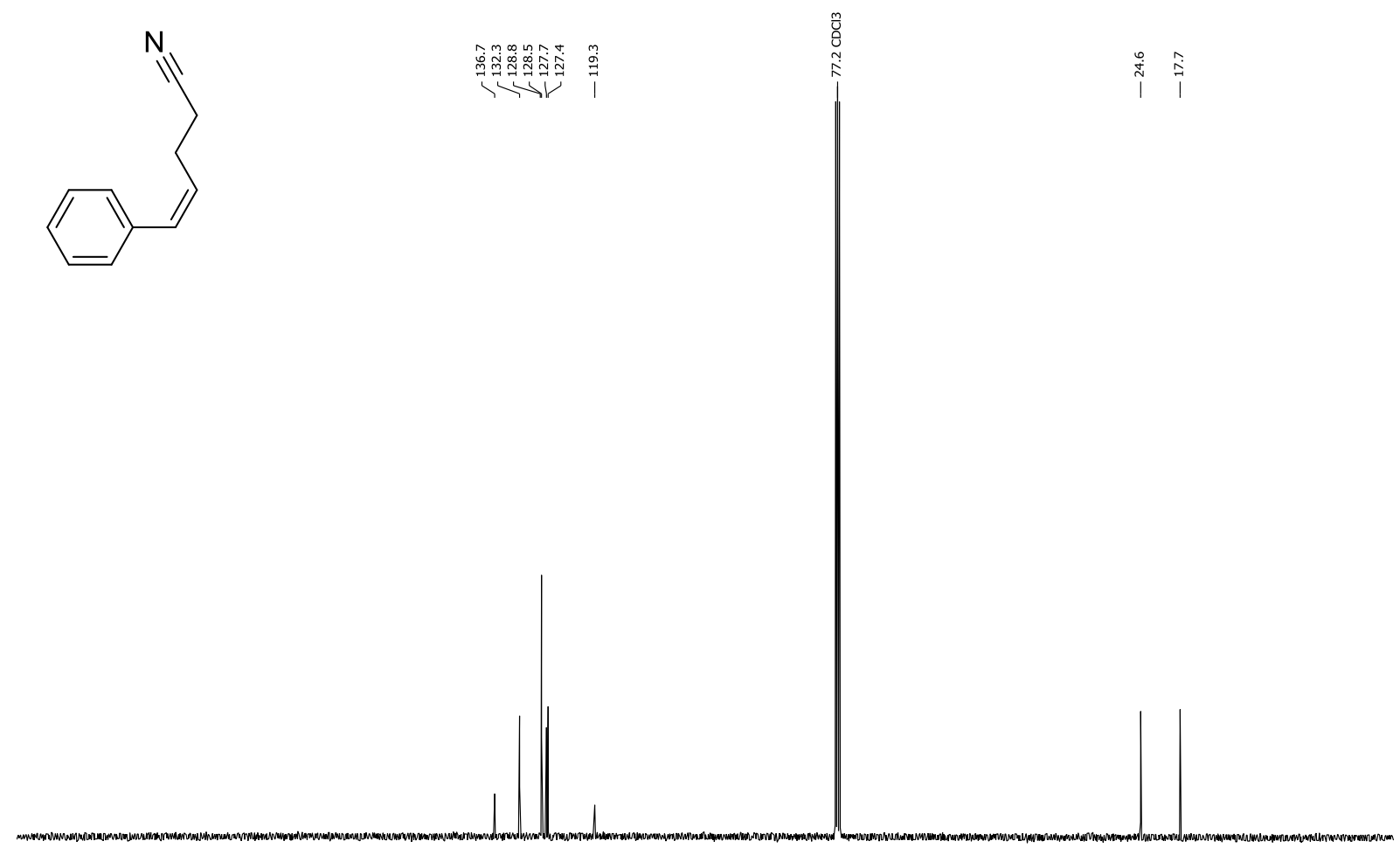

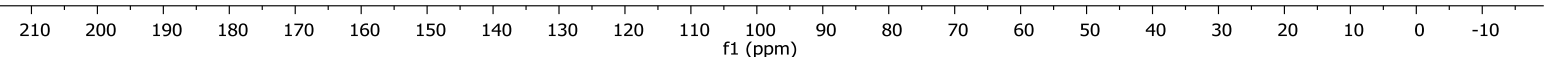


${ }^{\mathbf{1}} \mathbf{H}$ NMR $\left(400 \mathrm{MHz}, \mathrm{CDCl}_{3}, 25^{\circ} \mathrm{C}\right)$ of $\mathbf{1 a}$

范

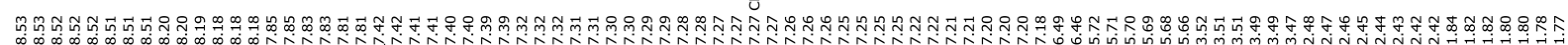

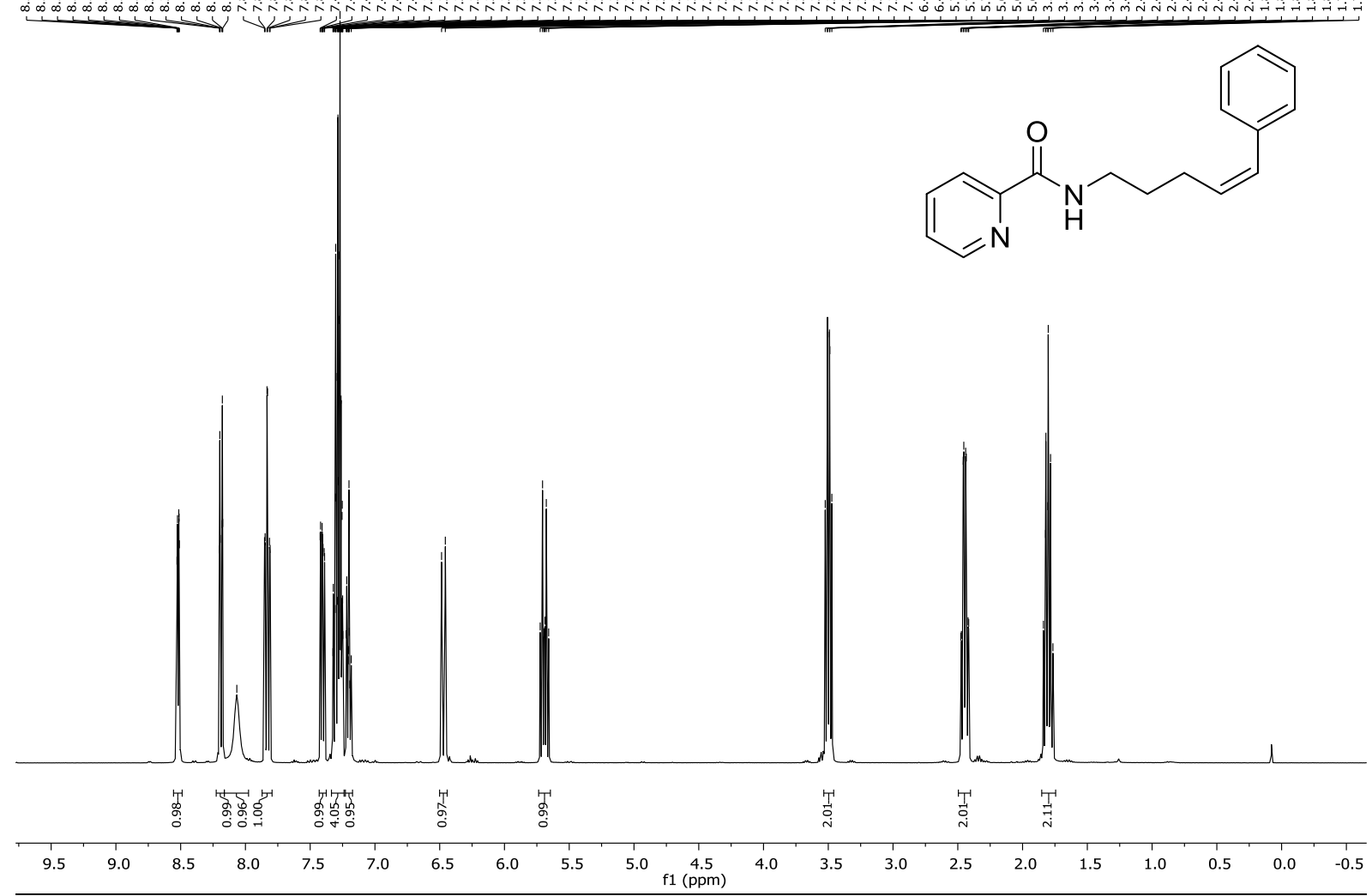

${ }^{13} \mathbf{C}$ NMR $\left(100 \mathrm{MHz}, \mathrm{CDCl}_{3}, 25^{\circ} \mathrm{C}\right)$ of $\mathbf{1 a}$

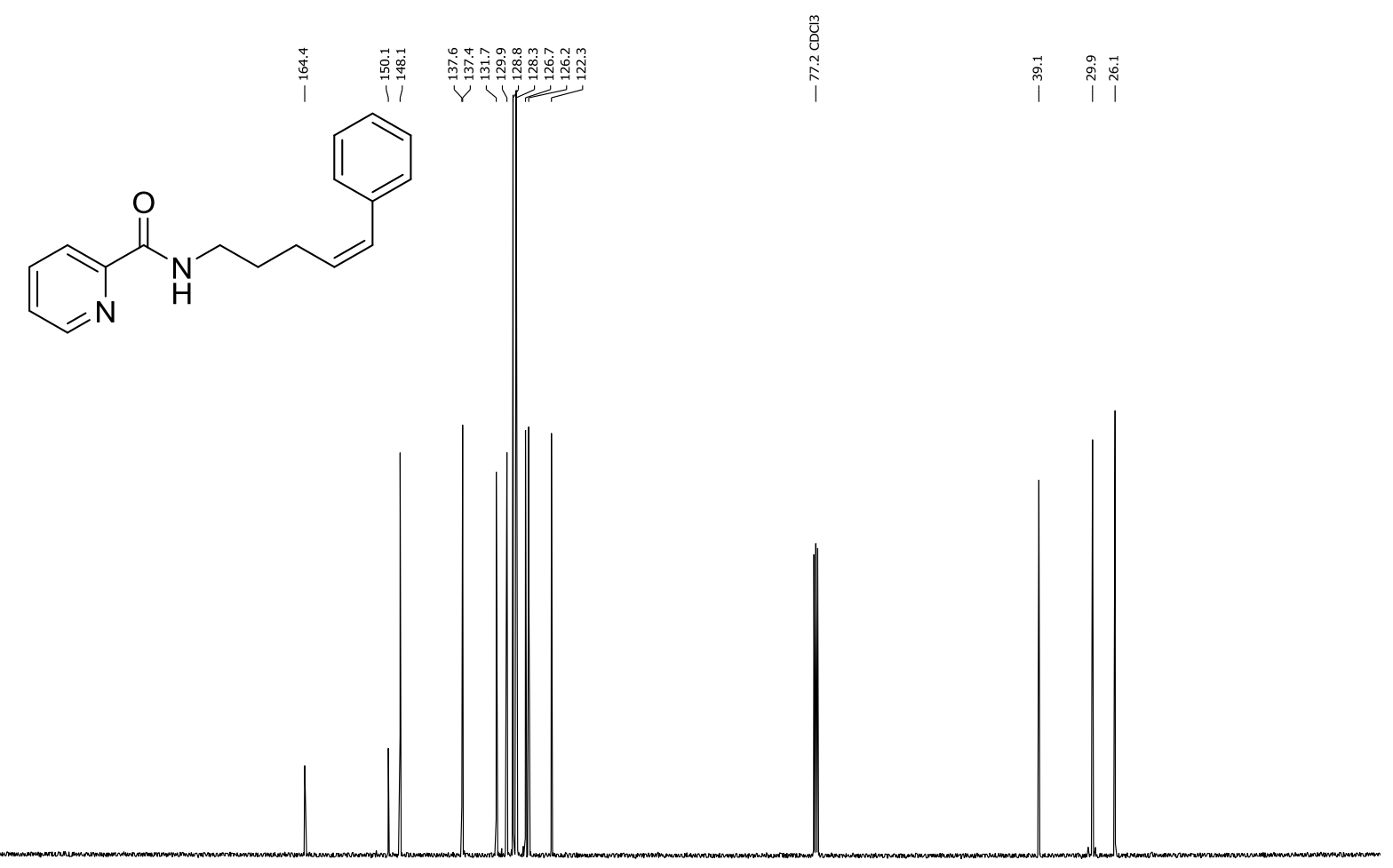

$\begin{array}{llllllllllllllllllllllllllllll} & 210 & 200 & 190 & 180 & 170 & 160 & 150 & 140 & 130 & 120 & 110 & 100 & 90 & 80 & 70 & 60 & 50 & 40 & 30 & 20 & 10 & 0 & -10\end{array}$ 
${ }^{\mathbf{1}} \mathbf{H}$ NMR $\left(400 \mathrm{MHz}, \mathrm{CDCl}_{3}, 25^{\circ} \mathrm{C}\right)$ of $\mathbf{1 b}$

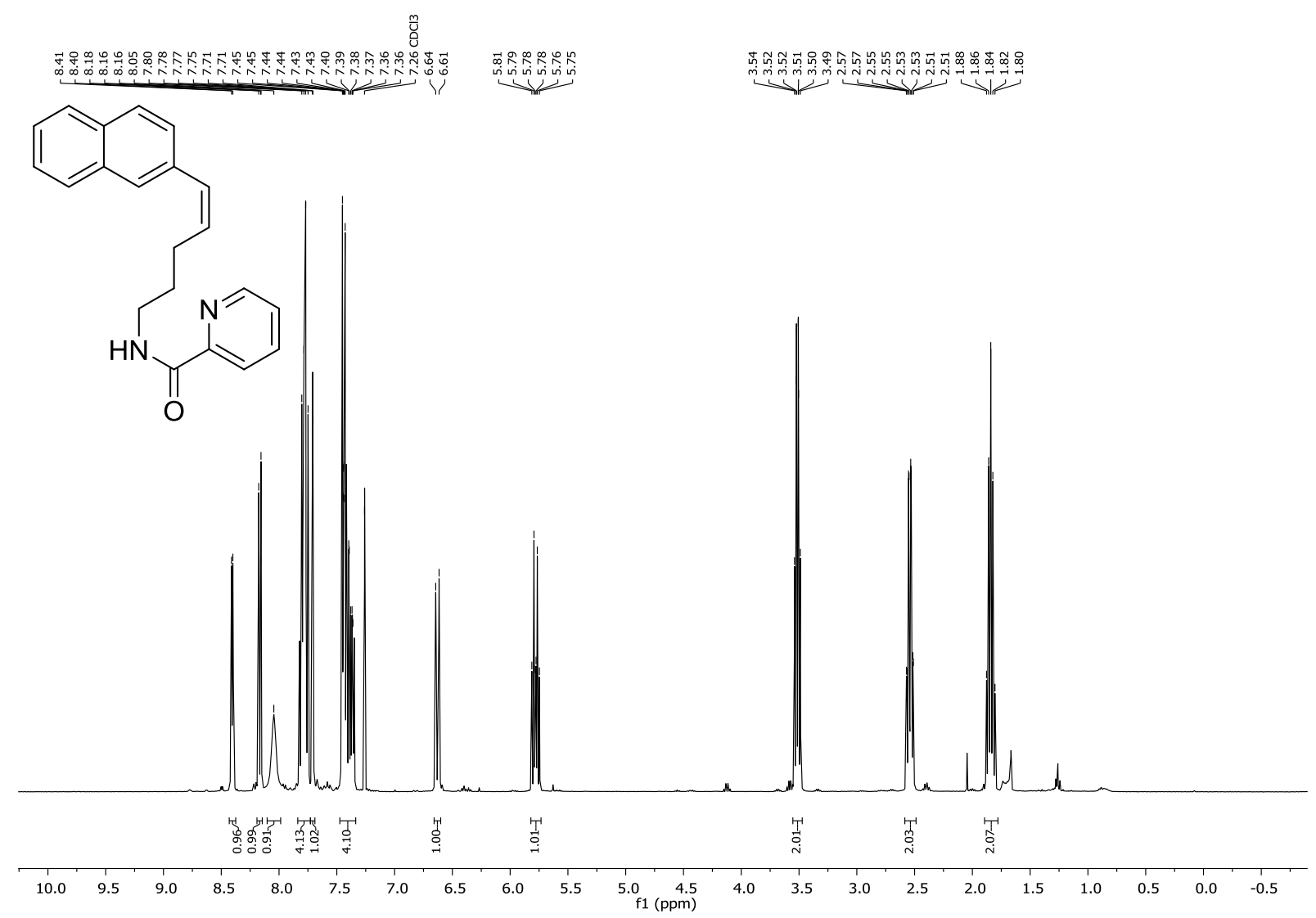

${ }^{13} \mathbf{C}$ NMR $\left(100 \mathrm{MHz}, \mathrm{CDCl}_{3}, 25^{\circ} \mathrm{C}\right)$ of $\mathbf{1 b}$

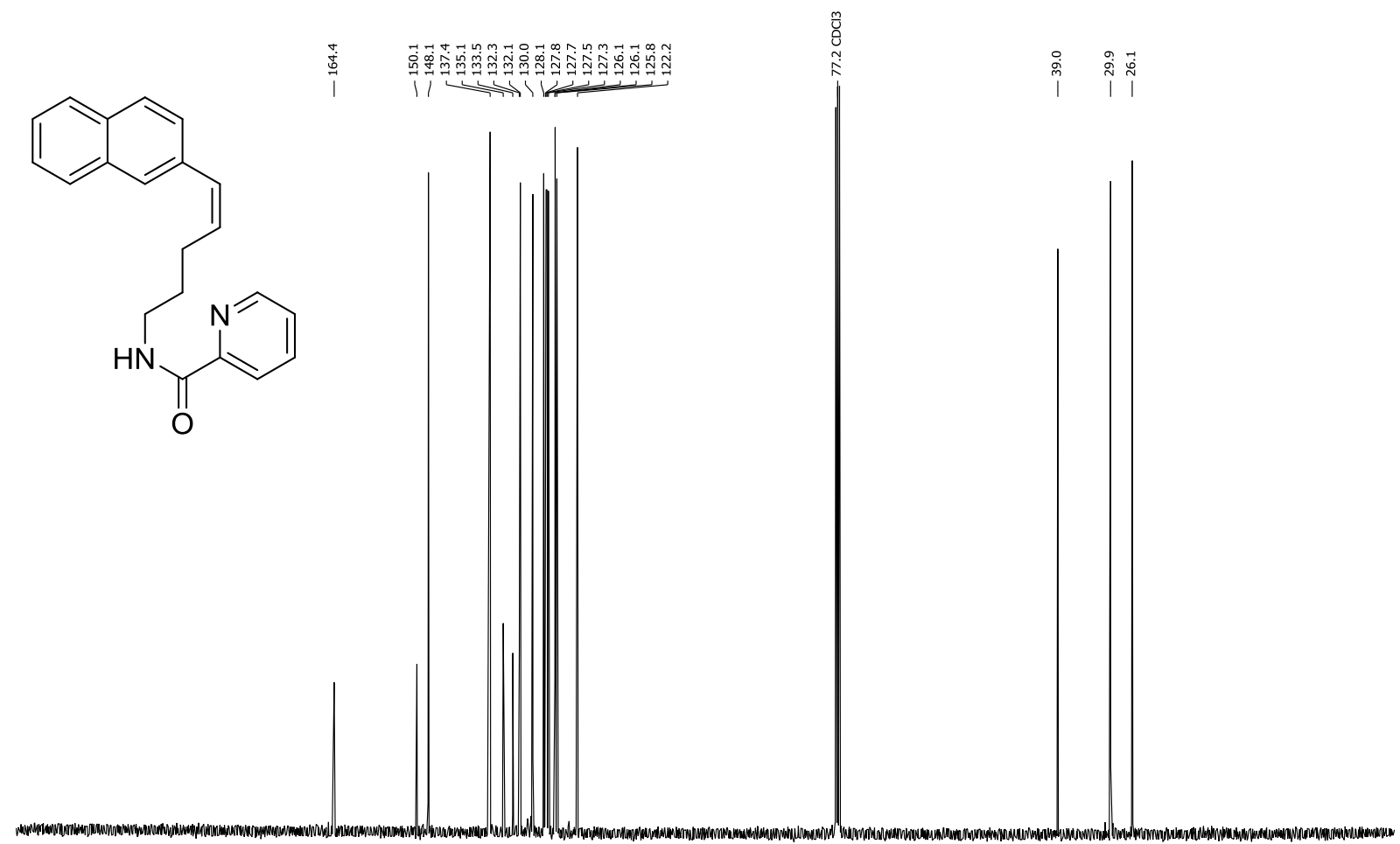

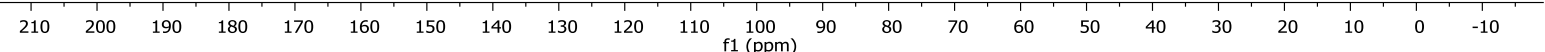


${ }^{1} \mathbf{H}$ NMR $\left(400 \mathrm{MHz}, \mathrm{CDCl}_{3}, 25^{\circ} \mathrm{C}\right)$ of $\mathbf{S 2 c}$

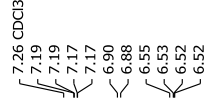

을<smiles>COc1ccc(/C=C\CCC#N)cc1</smiles>

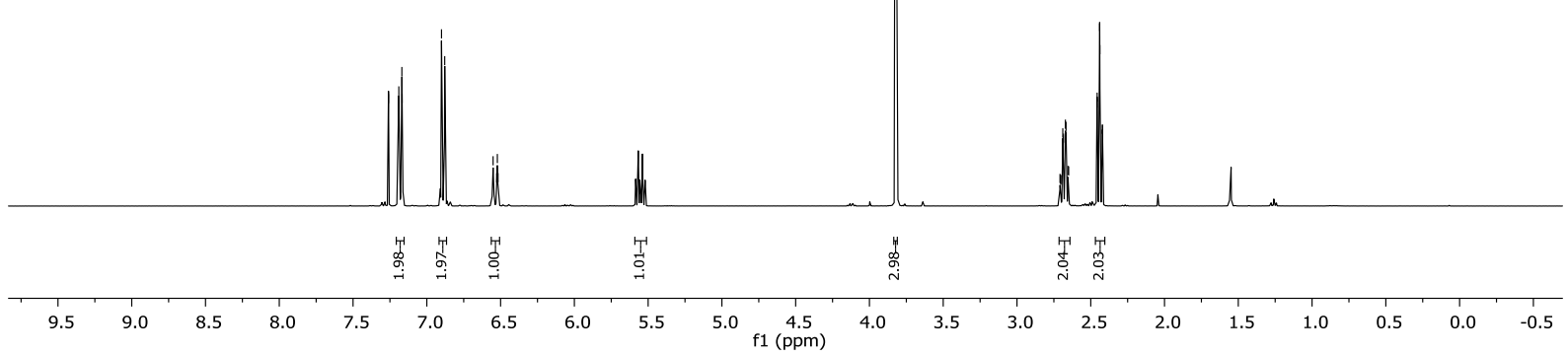

${ }^{13} \mathbf{C ~ N M R}\left(100 \mathrm{MHz}, \mathrm{CDCl}_{3}, 25^{\circ} \mathrm{C}\right)$ of $\mathbf{S 2 c}$

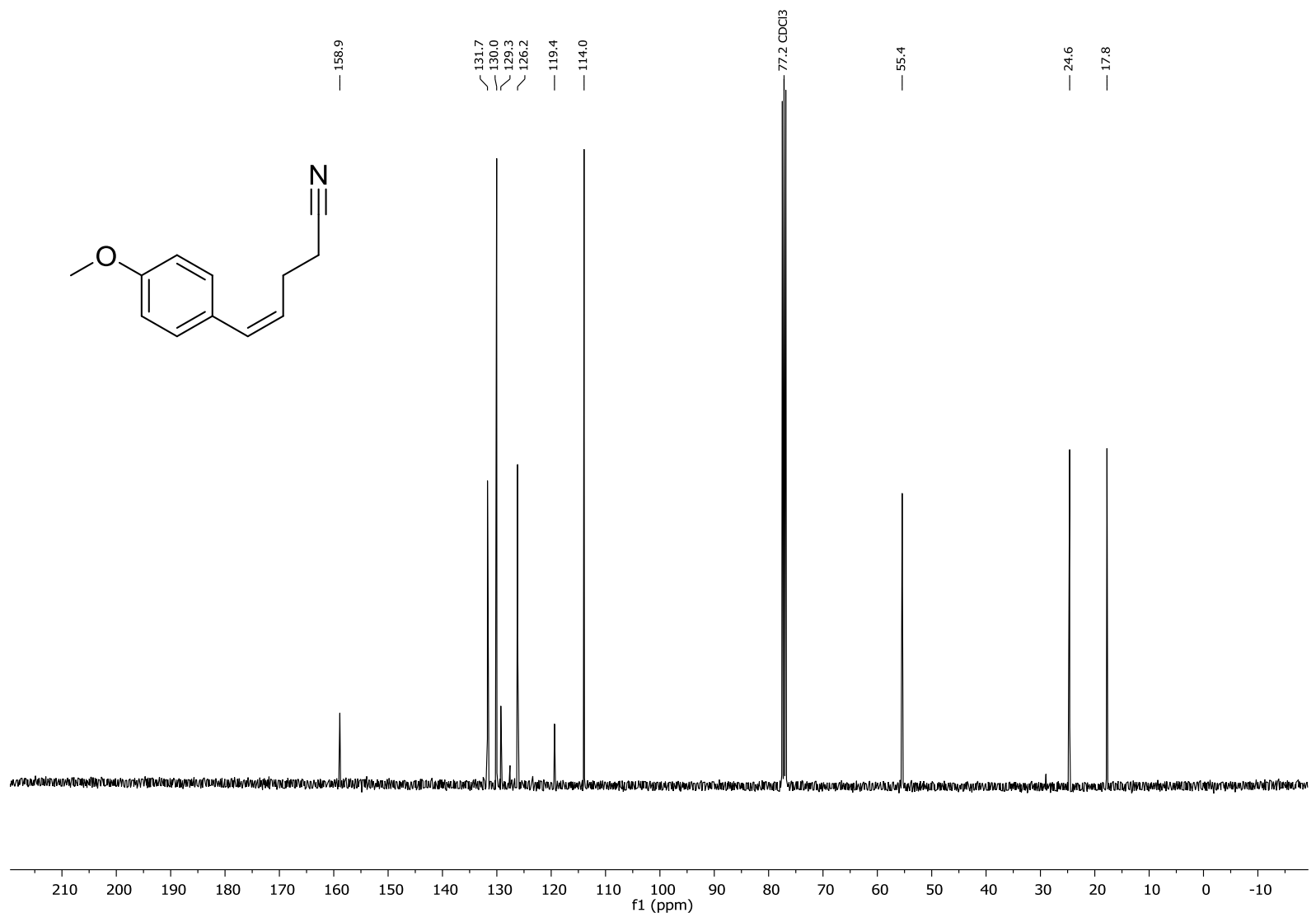

S71 
${ }^{\mathbf{1}} \mathbf{H}$ NMR $\left(400 \mathrm{MHz}, \mathrm{CDCl}_{3}, 25^{\circ} \mathrm{C}\right)$ of $\mathbf{1 c}$

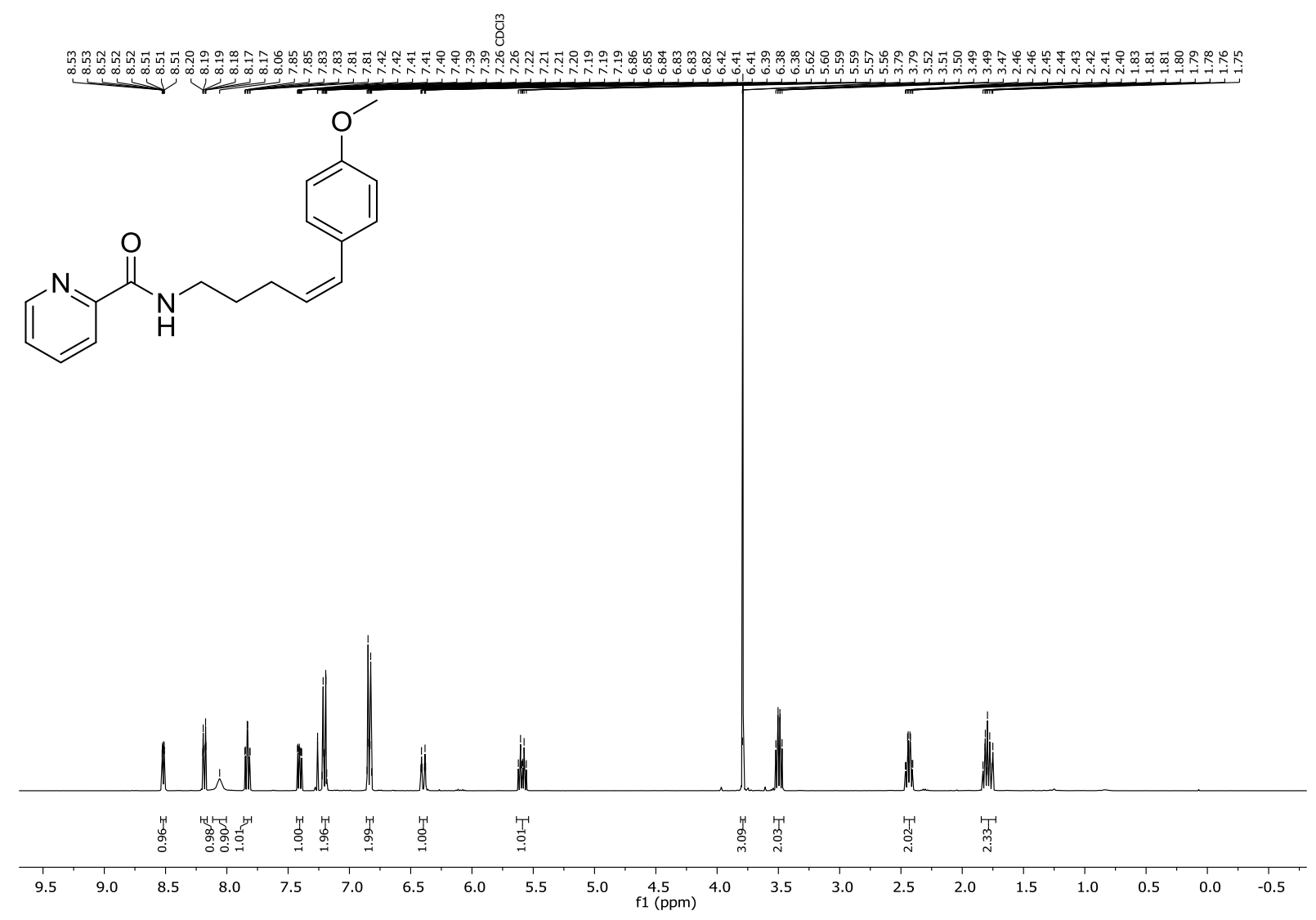

${ }^{13} \mathbf{C}$ NMR $\left(100 \mathrm{MHz}, \mathrm{CDCl}_{3}, 25{ }^{\circ} \mathrm{C}\right)$ of $\mathbf{1 c}$

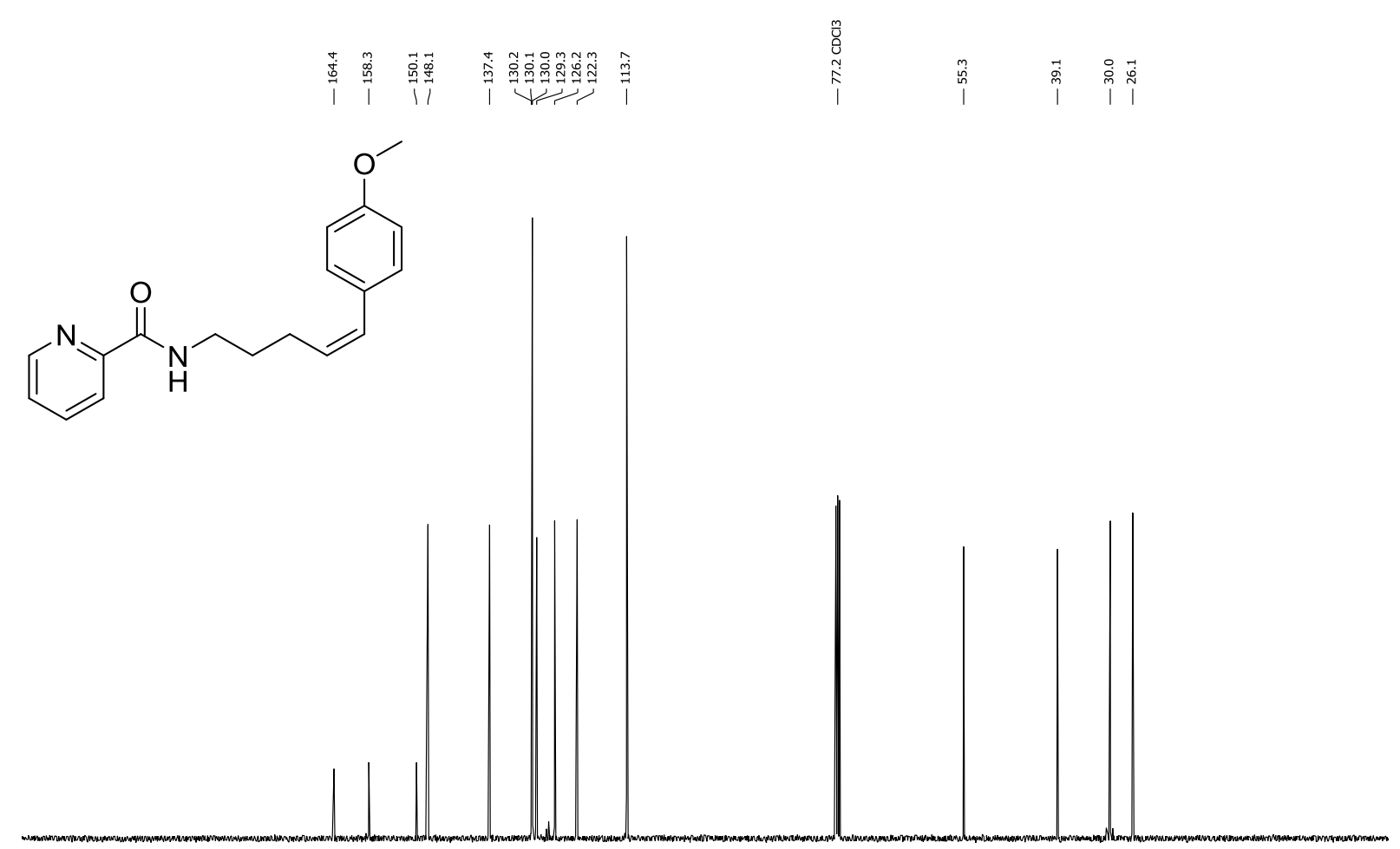

$\begin{array}{lllllllllllllllllllllllllllllllll}210 & 200 & 190 & 180 & 170 & 160 & 150 & 140 & 130 & 120 & 110 & 100 & 90 & 80 & 70 & 60 & 50 & 40 & 30 & 20 & 10 & 0 & -10\end{array}$ 
${ }^{1} \mathbf{H}$ NMR $\left(400 \mathrm{MHz}, \mathrm{CDCl}_{3}, 25^{\circ} \mathrm{C}\right)$ of $\mathbf{1 d}$

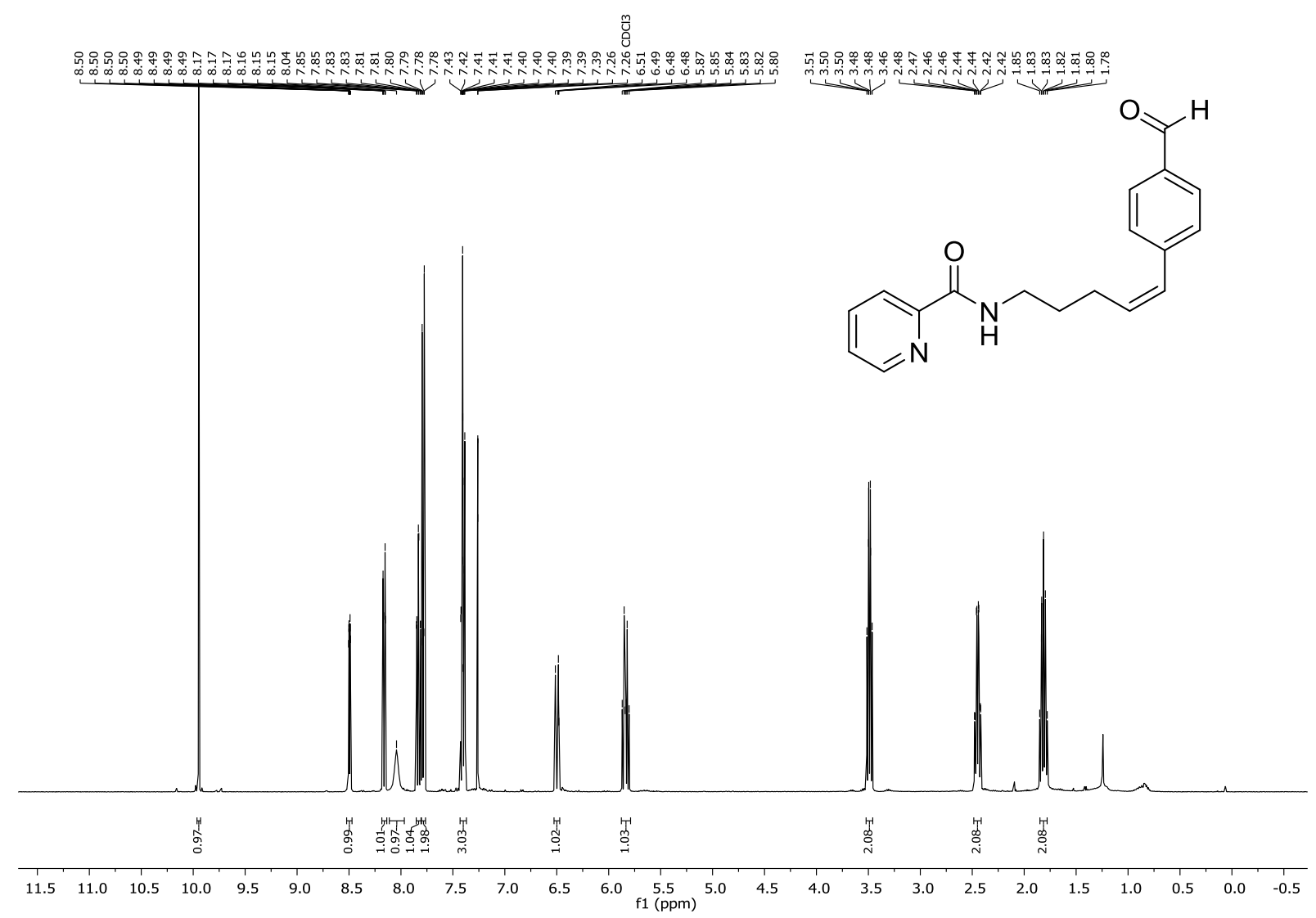

${ }^{13} \mathbf{C} \mathbf{N M R}\left(100 \mathrm{MHz}, \mathrm{CDCl}_{3}, 25{ }^{\circ} \mathrm{C}\right)$ of $\mathbf{1 d}$

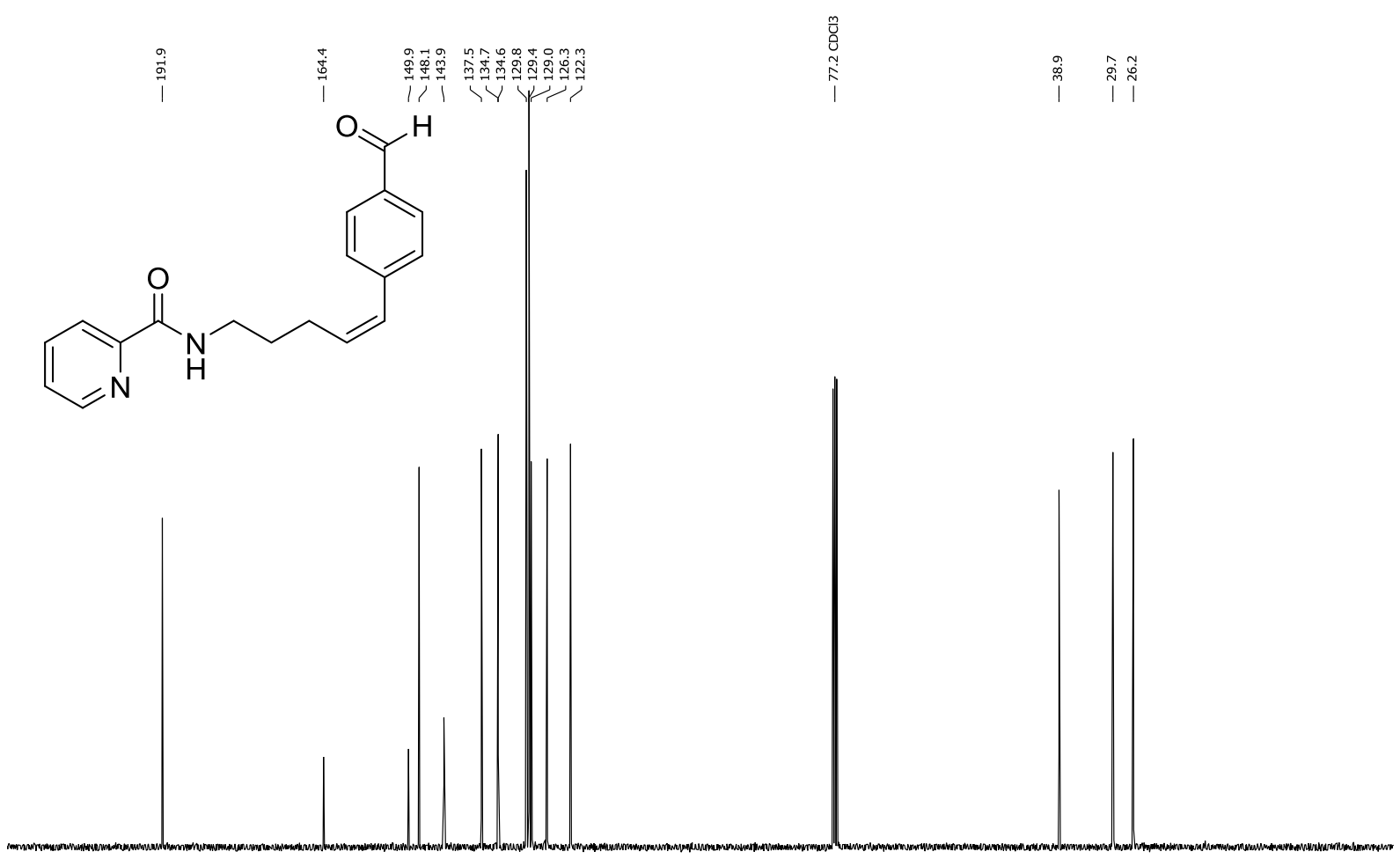

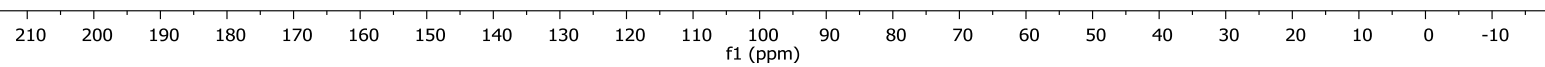


${ }^{1} \mathbf{H}$ NMR $\left(400 \mathrm{MHz}, \mathrm{CDCl}_{3}, 25^{\circ} \mathrm{C}\right)$ of $\mathbf{S 2 e}$
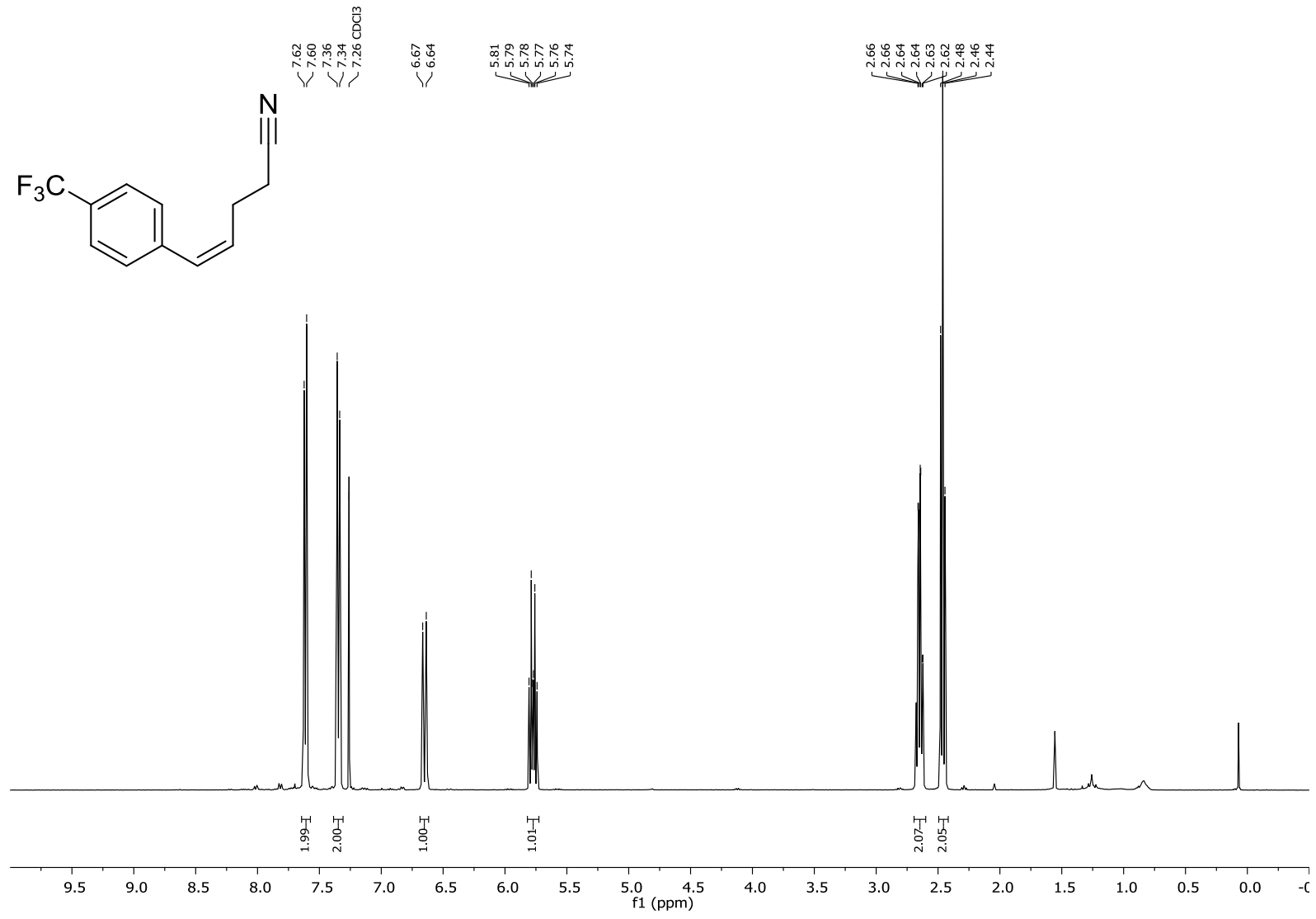

${ }^{13} \mathbf{C}$ NMR $\left(100 \mathrm{MHz}, \mathrm{CDCl}_{3}, 25^{\circ} \mathrm{C}\right)$ of $\mathbf{S 2 e}$
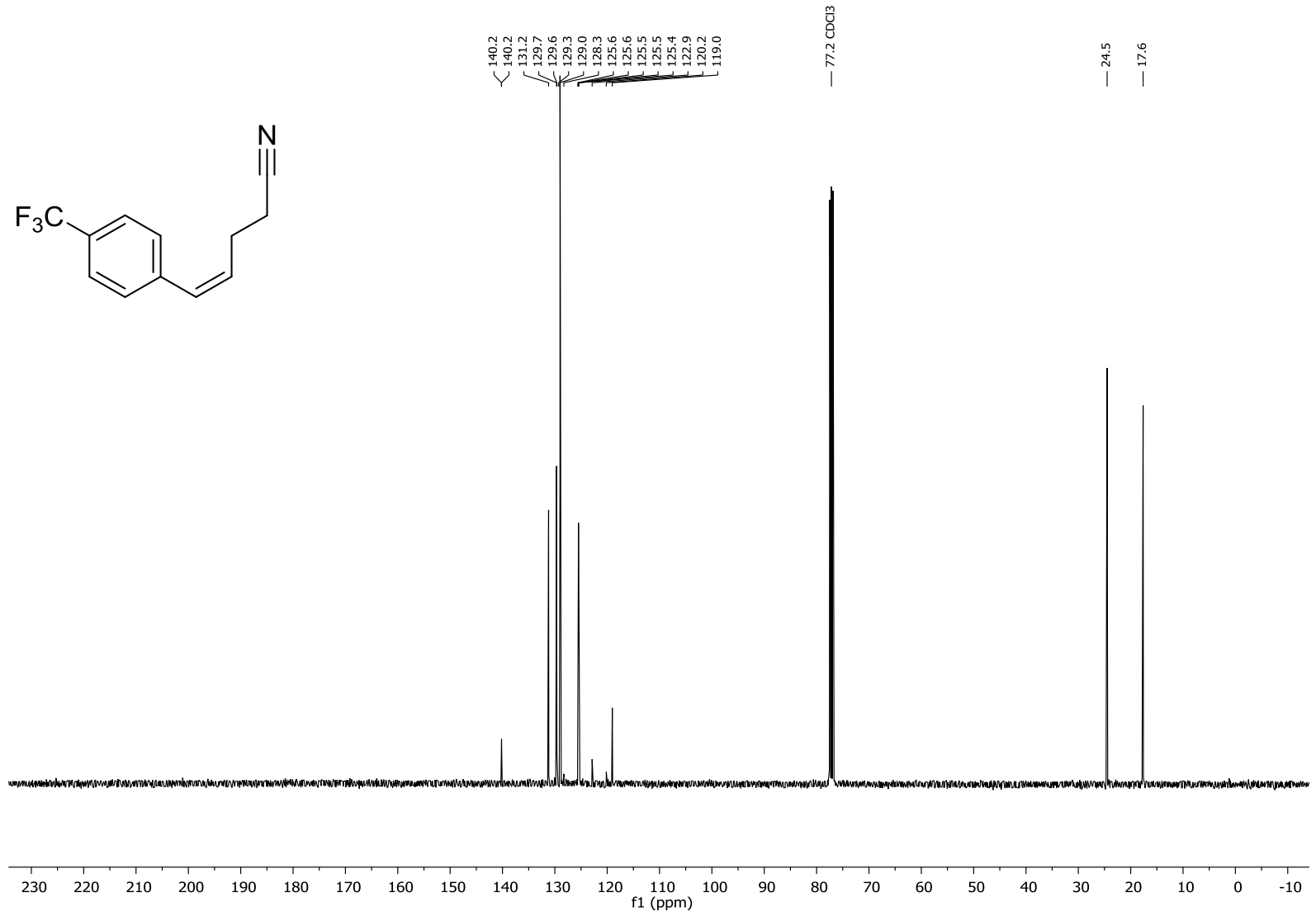

S74 
${ }^{19}$ F NMR (376 MHz, $\left.\mathrm{CDCl}_{3}, 25^{\circ} \mathrm{C}\right)$ of $\mathbf{S 2 e}$

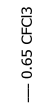
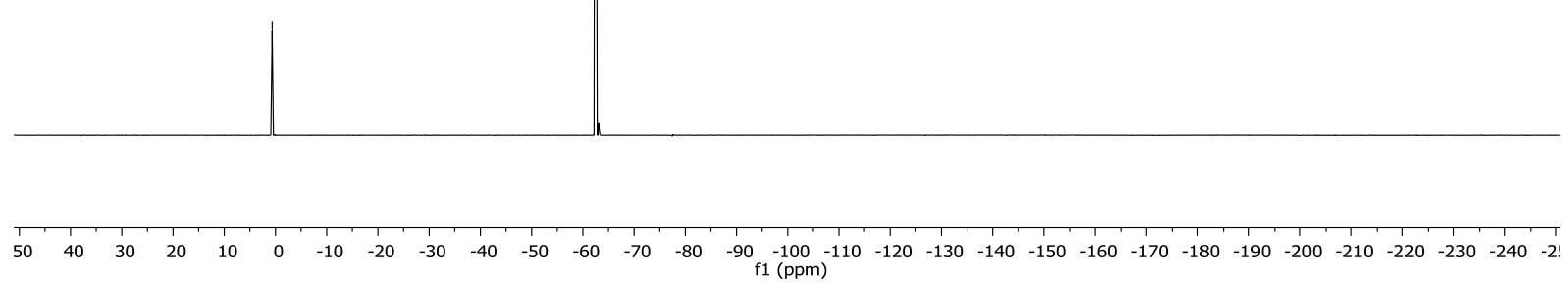

S75 
${ }^{\mathbf{1}} \mathbf{H}$ NMR $\left(400 \mathrm{MHz}, \mathrm{CDCl}_{3}, 25^{\circ} \mathrm{C}\right)$ of $\mathbf{1 e}$

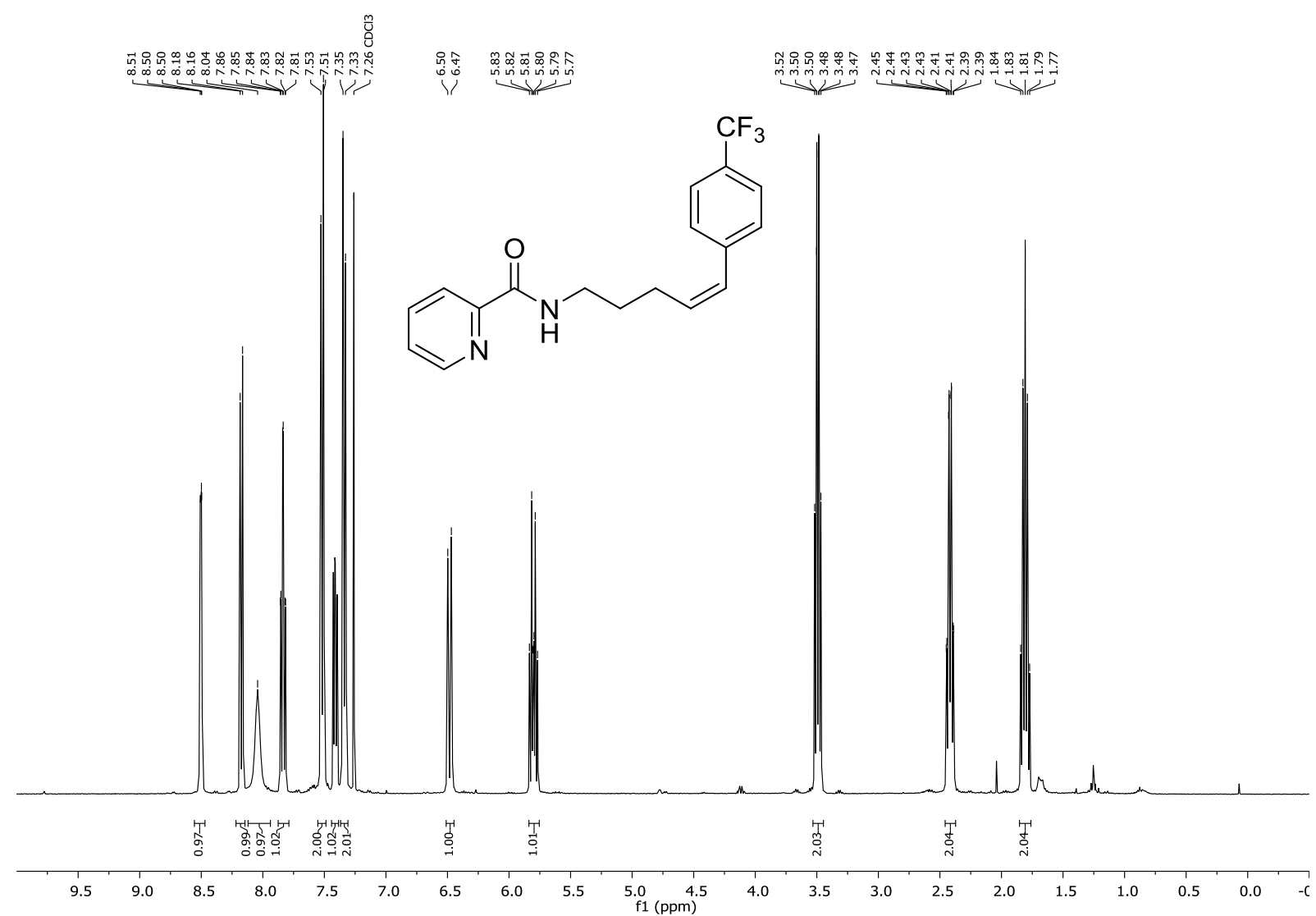

${ }^{13} \mathbf{C}$ NMR $\left(100 \mathrm{MHz}, \mathrm{CDCl}_{3}, 25{ }^{\circ} \mathrm{C}\right)$ of $\mathbf{1 e}$

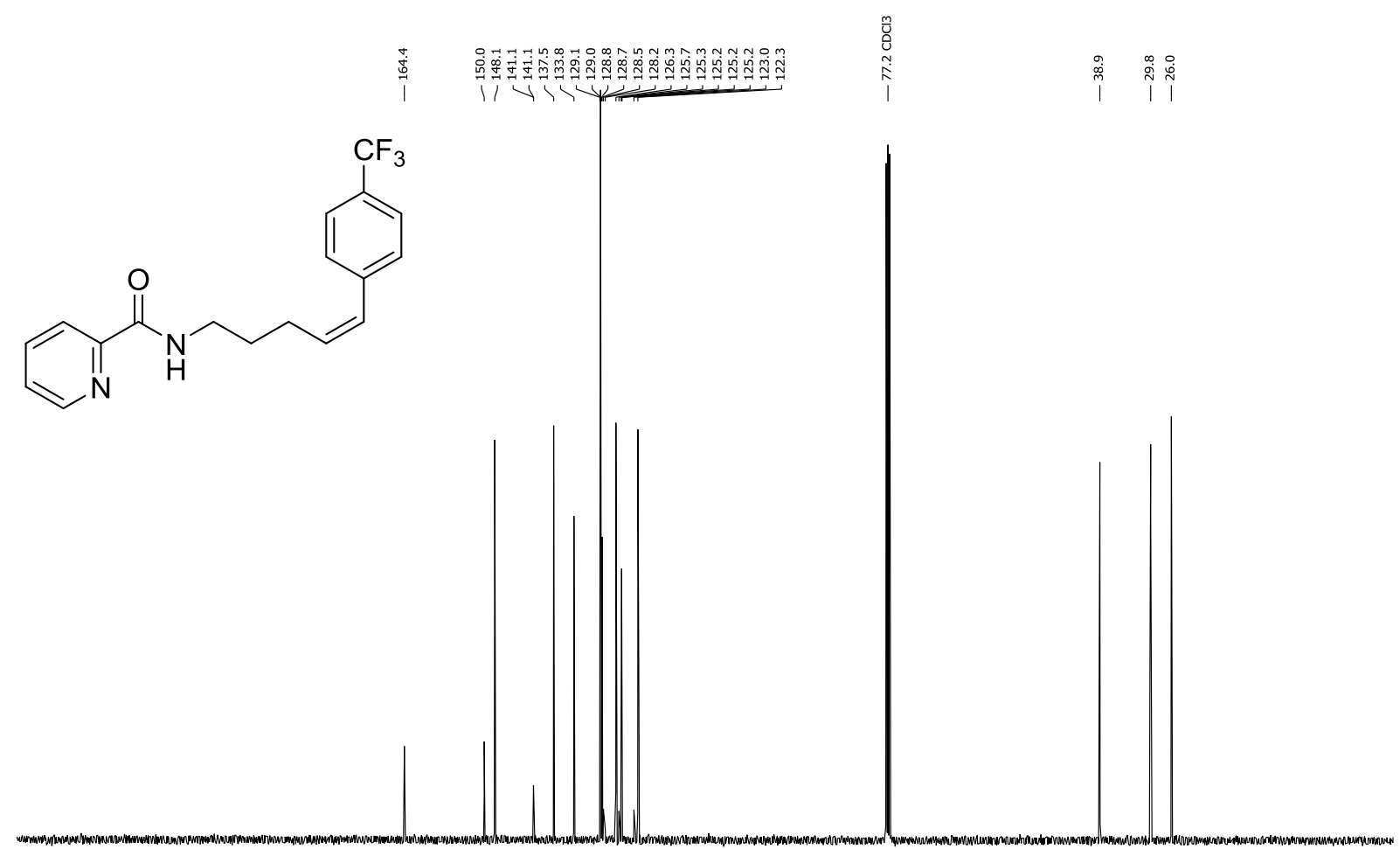

$\begin{array}{rlllllllllllllllllllllllllll}130 & 220 & 210 & 200 & 190 & 180 & 170 & 160 & 150 & 140 & 130 & 120 & 110 & 100 & 90 & 80 & 70 & 60 & 50 & 40 & 30 & 20 & 10 & 0 & -10\end{array}$ 
${ }^{19}$ F NMR $\left(376 \mathrm{MHz}, \mathrm{CDCl}_{3}, 25{ }^{\circ} \mathrm{C}\right)$ of $\mathbf{1 e}$

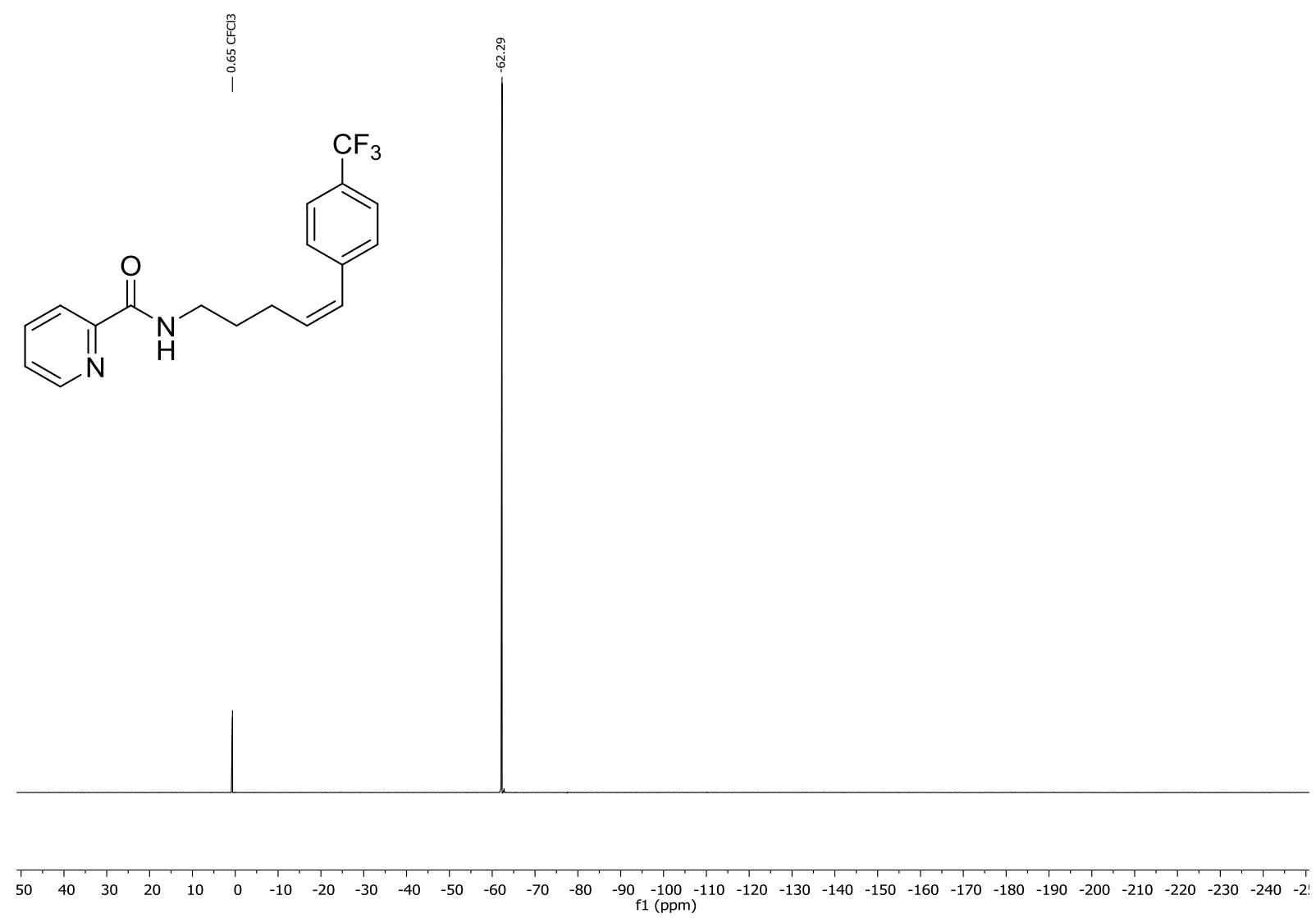


${ }^{1} \mathbf{H}$ NMR $\left(500 \mathrm{MHz}, \mathrm{CDCl}_{3}, 25^{\circ} \mathrm{C}\right)$ of $\mathbf{S 2 f}$

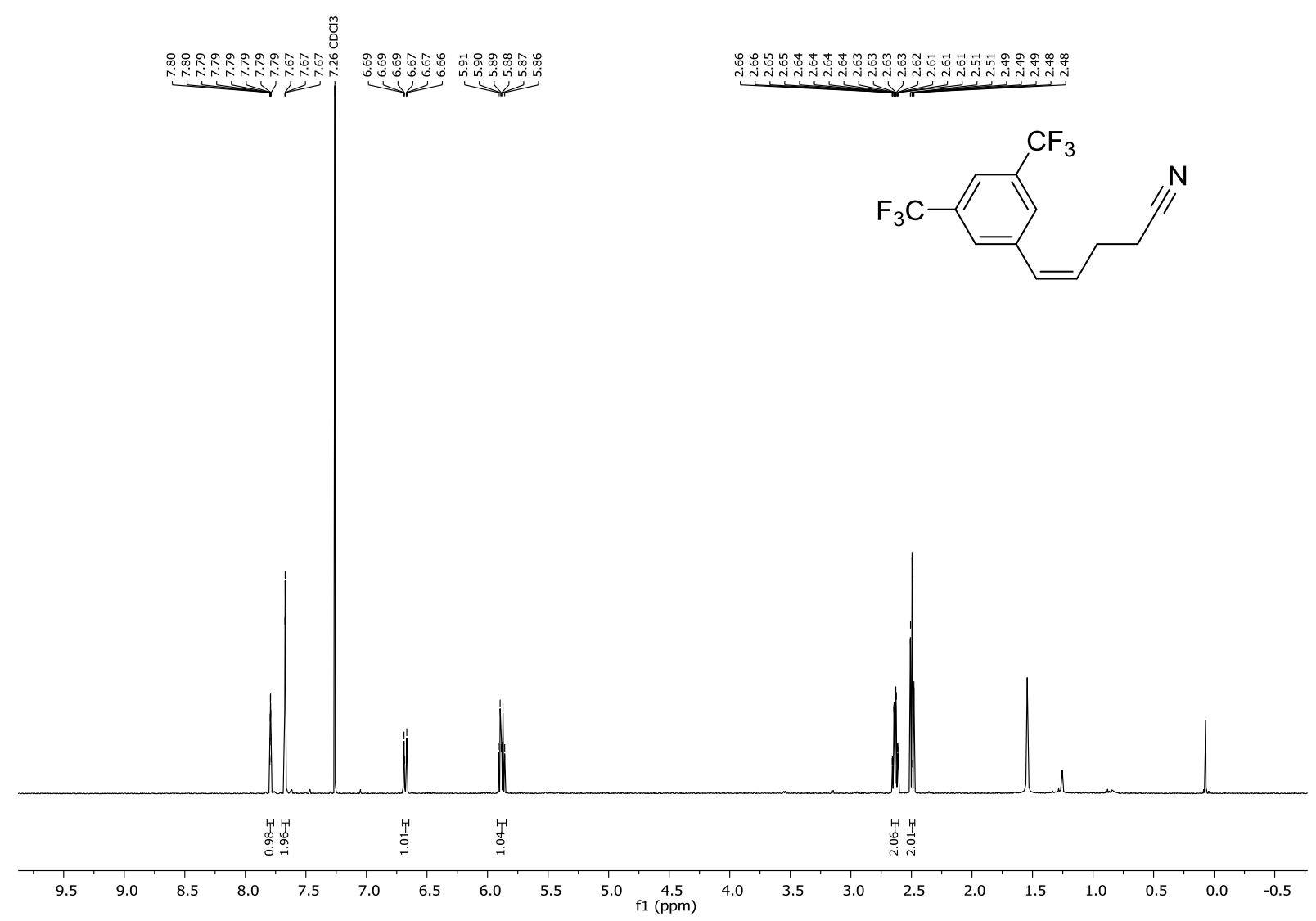

${ }^{13} \mathbf{C}$ NMR $\left(126 \mathrm{MHz}, \mathrm{CDCl}_{3}, 25^{\circ} \mathrm{C}\right)$ of $\mathbf{S 2 f}$<smiles>N#CCC/C=C\c1cc(C(F)(F)F)cc(C(F)(F)F)c1</smiles>
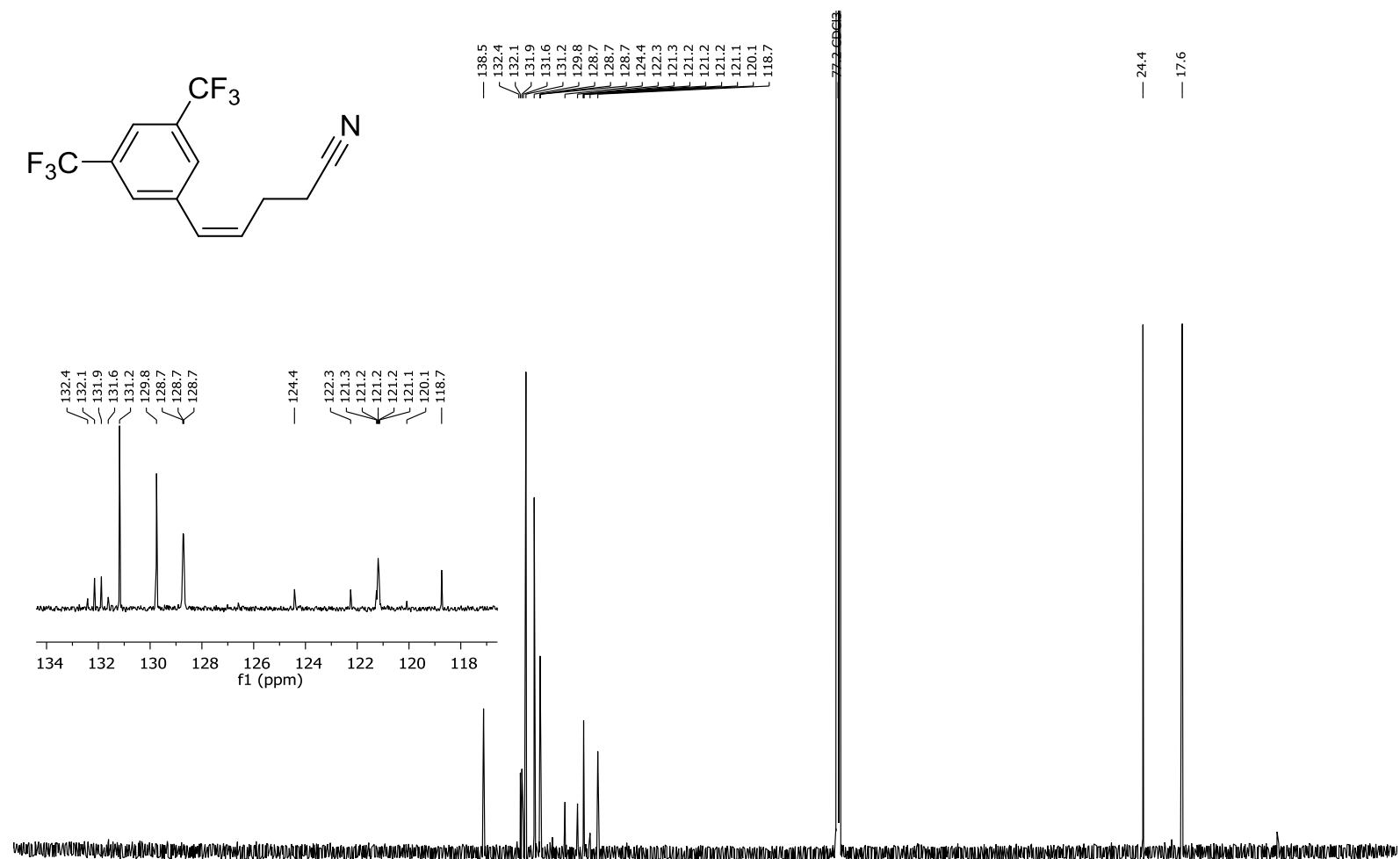

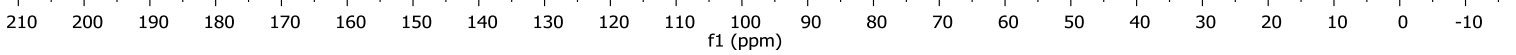


${ }^{19} \mathbf{F}$ NMR $\left(471 \mathrm{MHz}, \mathrm{CDCl}_{3}, 25^{\circ} \mathrm{C}\right)$ of $\mathbf{S 2 f}$

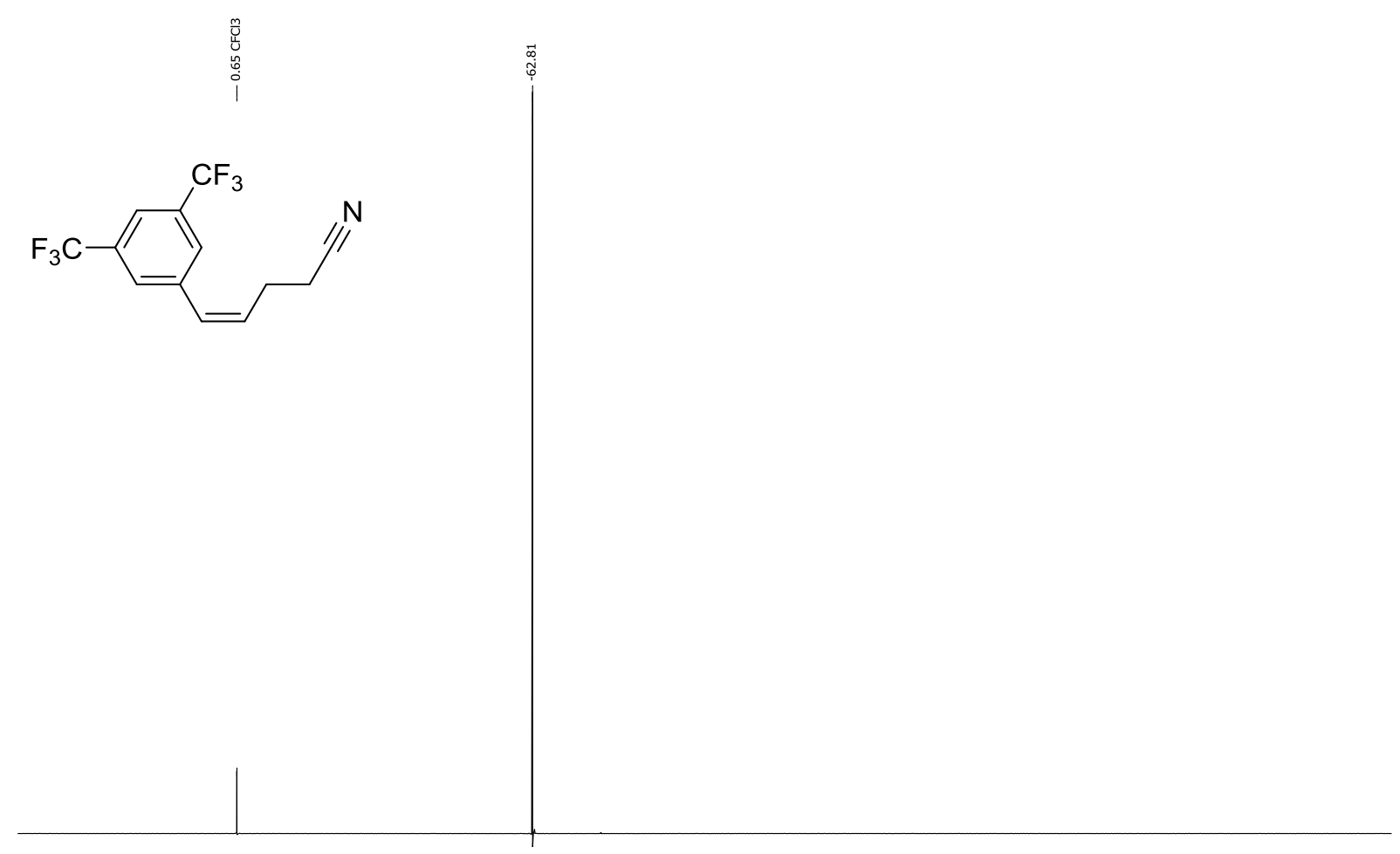

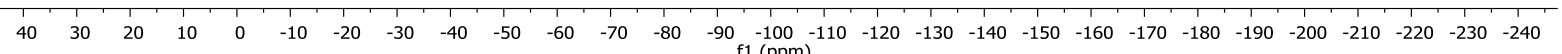


${ }^{1} \mathbf{H}$ NMR $\left(500 \mathrm{MHz}, \mathrm{CDCl}_{3}, 25^{\circ} \mathrm{C}\right)$ of $\mathbf{1 f}$

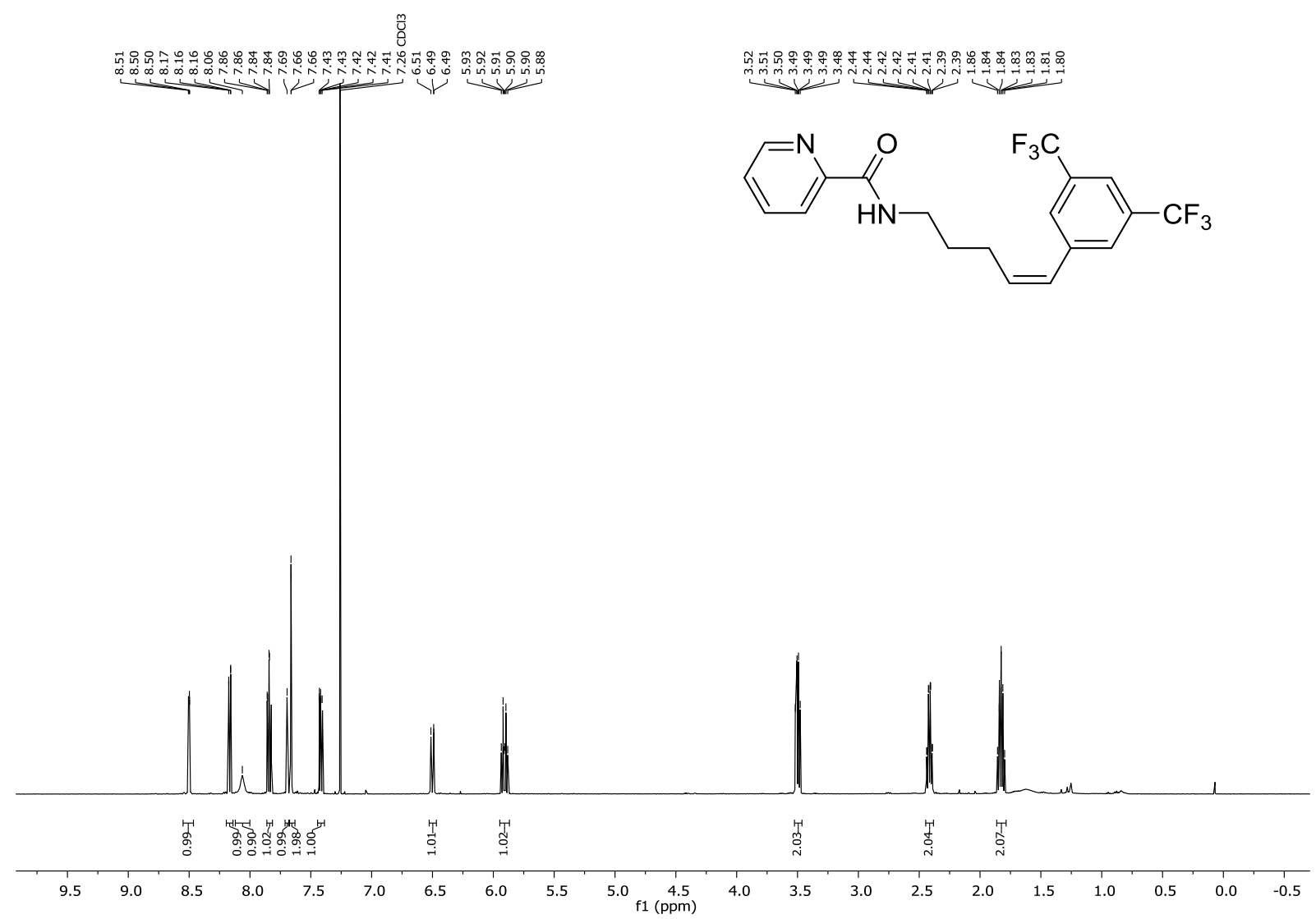

${ }^{13} \mathbf{C}$ NMR $\left(126 \mathrm{MHz}, \mathrm{CDCl}_{3}, 25^{\circ} \mathrm{C}\right)$ of $\mathbf{1 f}$

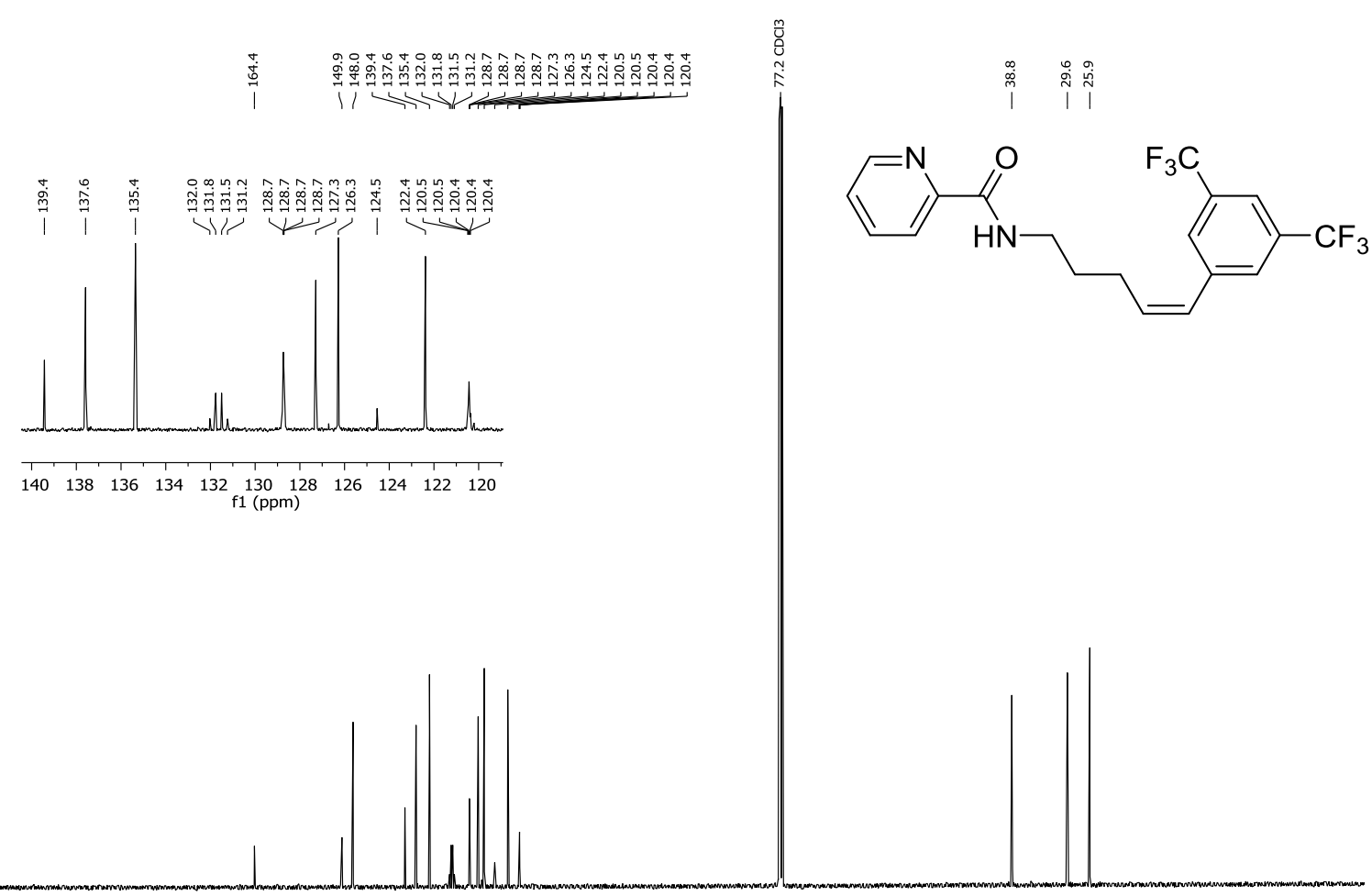

$\begin{array}{lllllllllllllllllllllll}210 & 200 & 190 & 180 & 170 & 160 & 150 & 140 & 130 & 120 & 110 & 100 & 100 & 10 & 70 & 60 & 50 & 40 & 30 & 20 & 10 & 0 & -10\end{array}$ 
${ }^{19}$ F NMR $\left(471 \mathrm{MHz}, \mathrm{CDCl}_{3}, 25^{\circ} \mathrm{C}\right)$ of $\mathbf{1 f}$

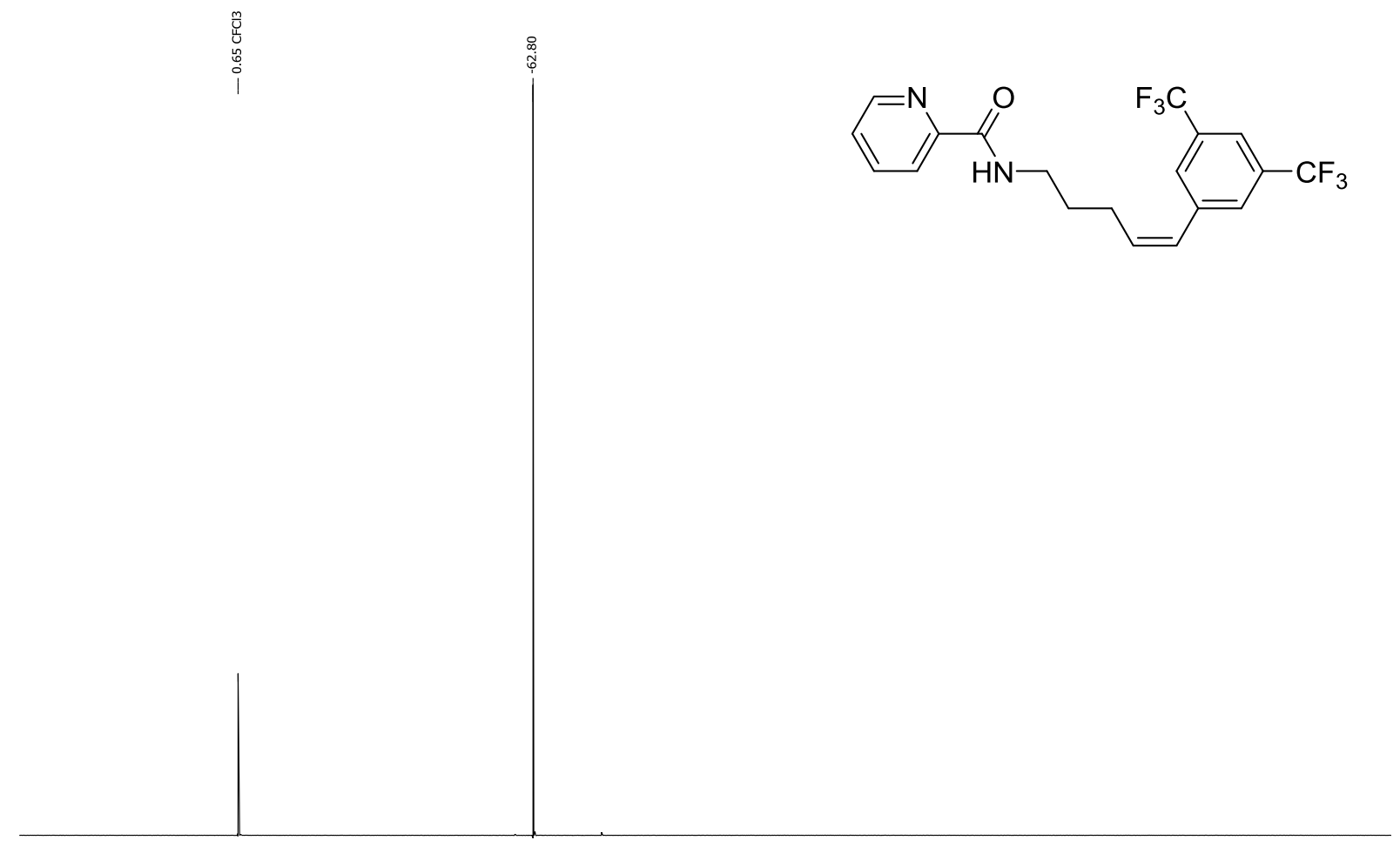

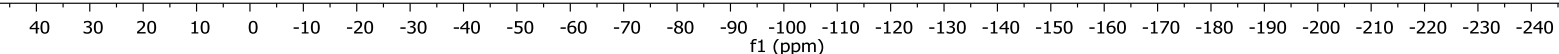


${ }^{\mathbf{1}} \mathbf{H}$ NMR $\left(400 \mathrm{MHz}, \mathrm{CDCl}_{3}, 25^{\circ} \mathrm{C}\right)$ of $\mathbf{S 2 g}$

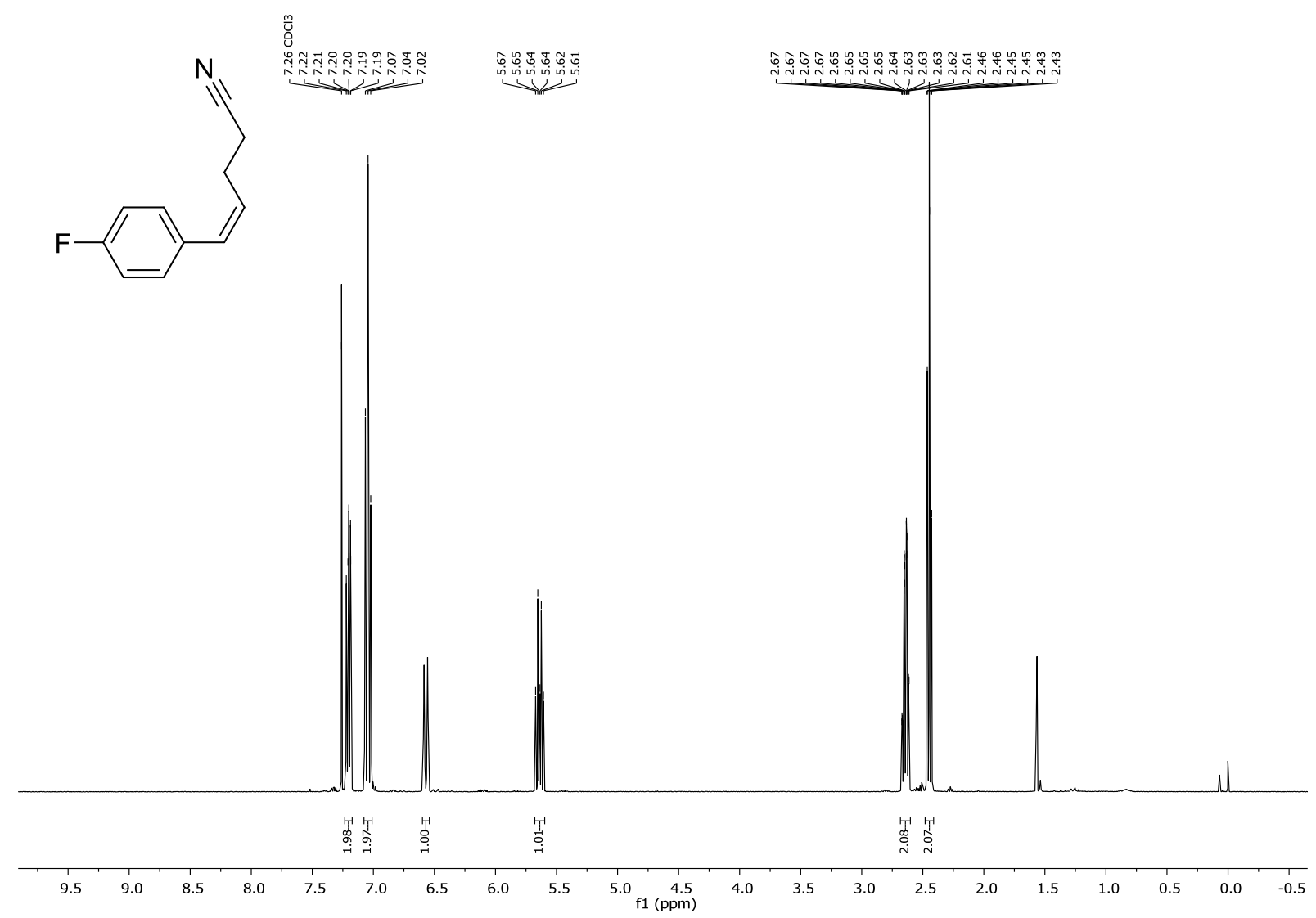

${ }^{13} \mathbf{C}$ NMR $\left(100 \mathrm{MHz}, \mathrm{CDCl}_{3}, 25^{\circ} \mathrm{C}\right)$ of $\mathbf{S 2 g}$

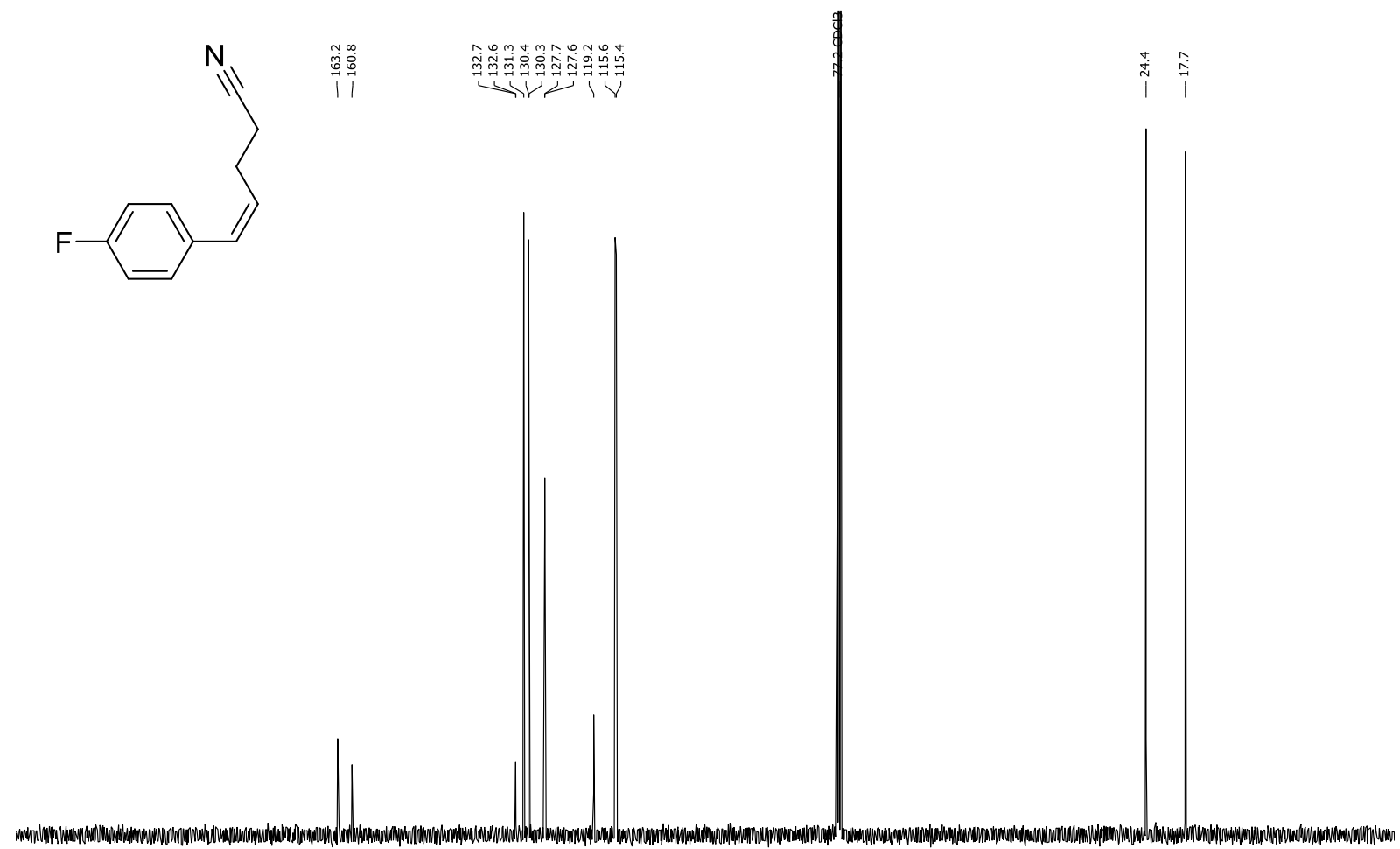

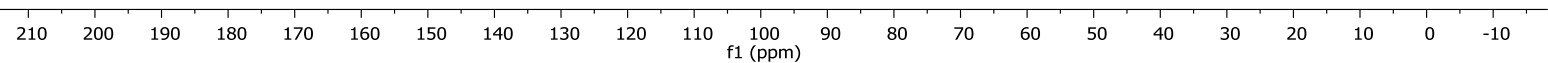


${ }^{19}$ F NMR $\left(377 \mathrm{MHz}, \mathrm{CDCl}_{3}, 25{ }^{\circ} \mathrm{C}\right)$ of $\mathbf{S 2 g}$<smiles>N#CCC/C=C\c1ccc(F)cc1</smiles>

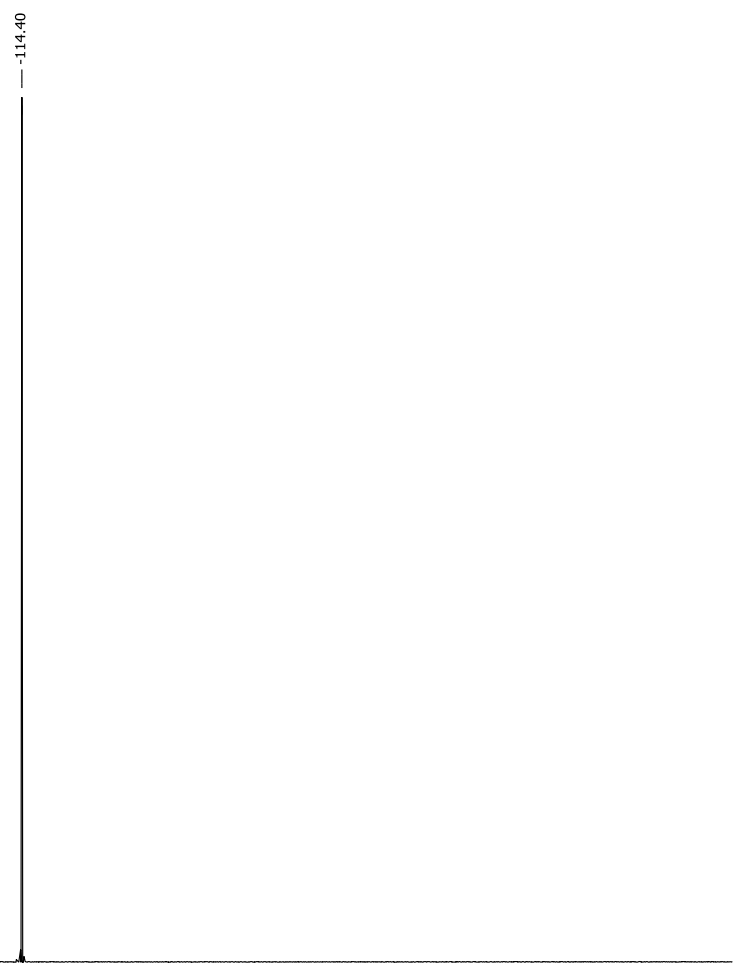

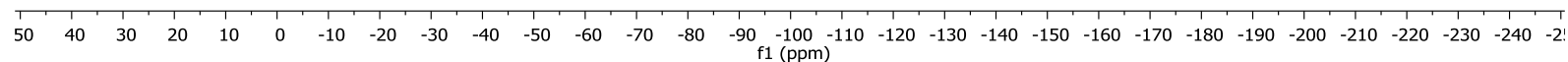


${ }^{1} \mathbf{H}$ NMR $\left(400 \mathrm{MHz}, \mathrm{CDCl}_{3}, 25^{\circ} \mathrm{C}\right)$ of $\mathbf{1 g}$

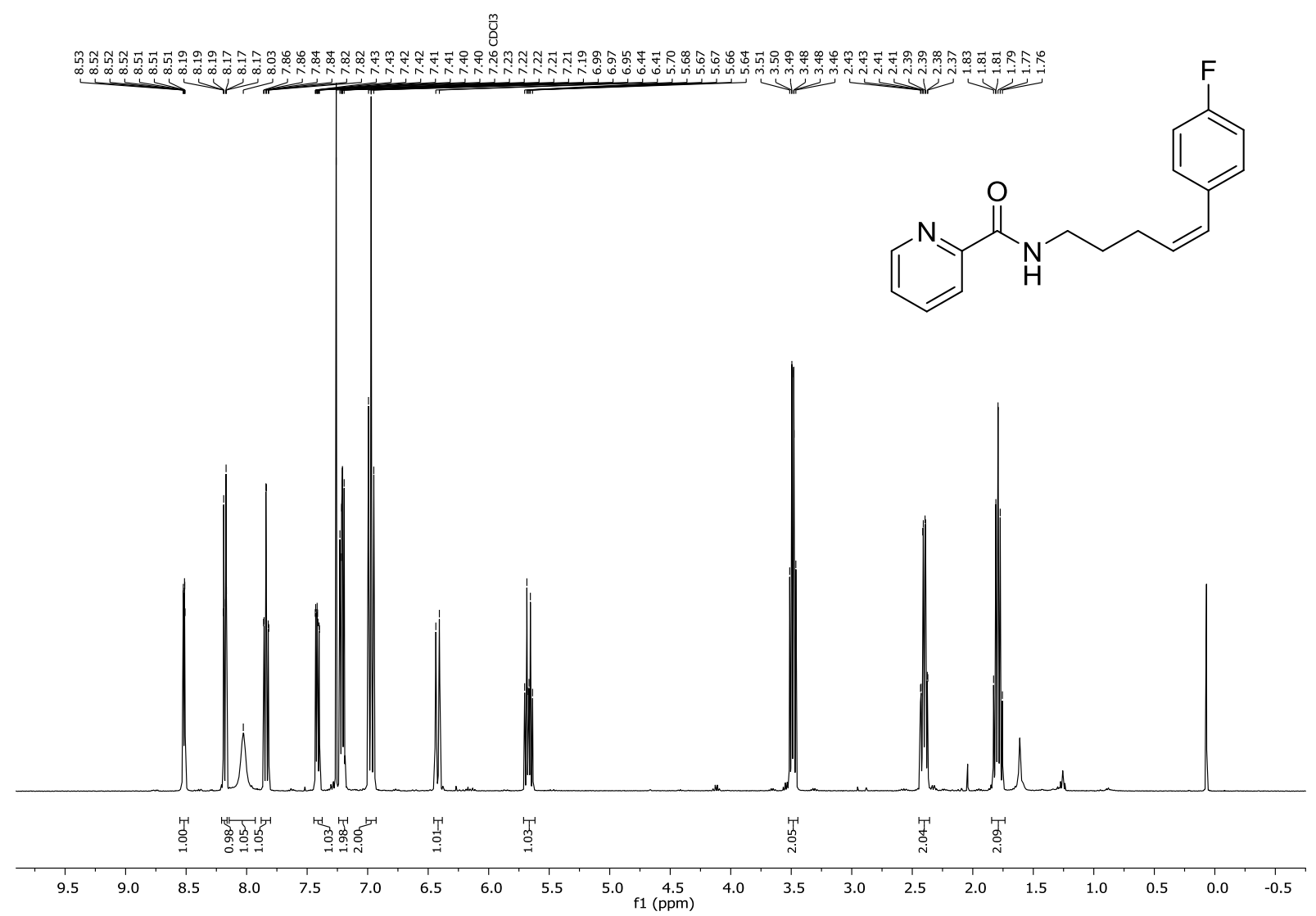

${ }^{13} \mathbf{C}$ NMR $\left(100 \mathrm{MHz}, \mathrm{CDCl}_{3}, 25^{\circ} \mathrm{C}\right)$ of $\mathbf{1 g}$

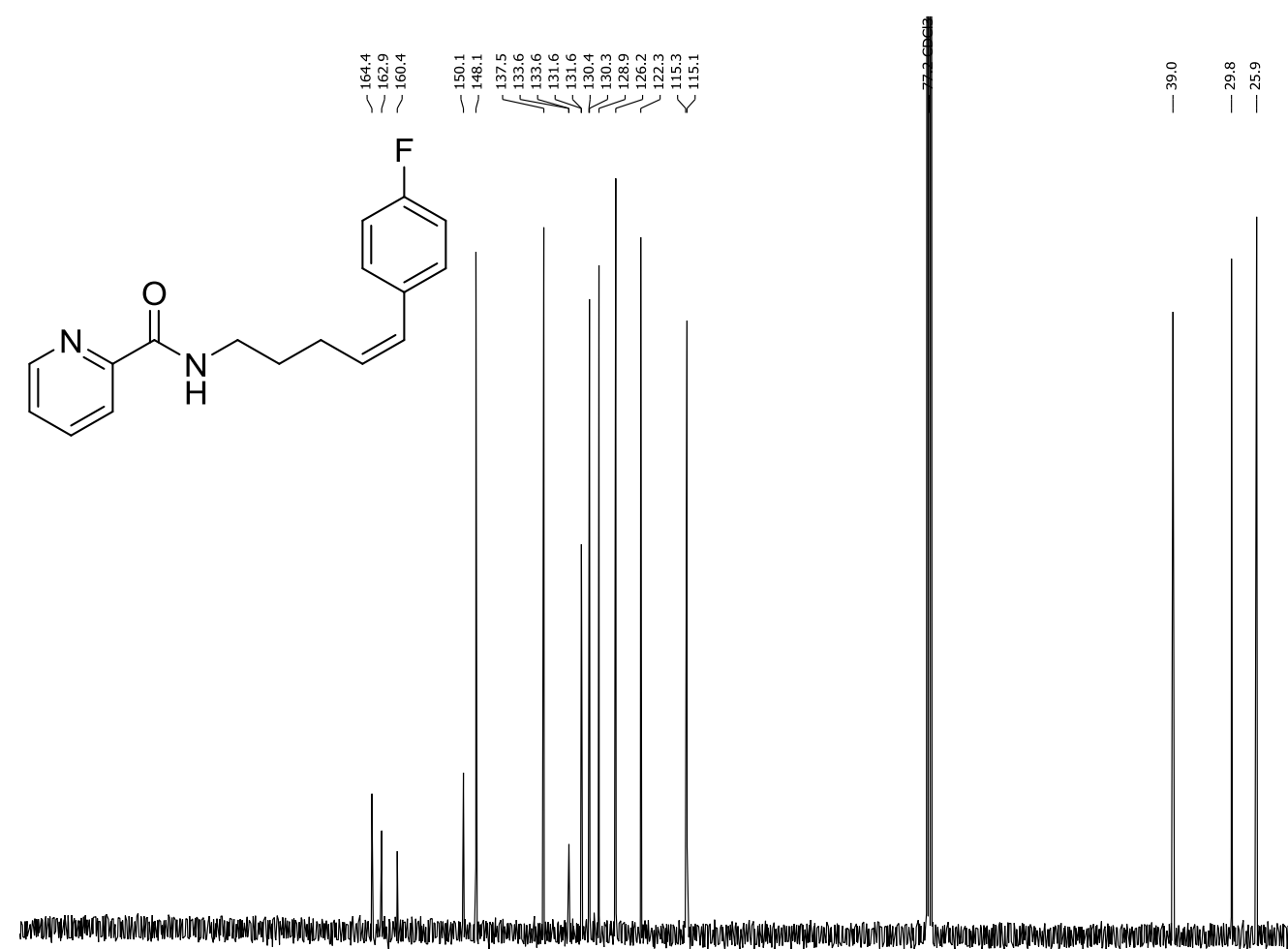

$\begin{array}{llllllllllllllllllllllll}210 & 200 & 190 & 180 & 170 & 160 & 150 & 140 & 130 & 120 & 110 & 100 & 90 & 80 & 70 & 60 & 50 & 40 & 30 & 20 & 10 & 0 & -10 & \end{array}$ 
${ }^{19}$ F NMR $\left(377 \mathrm{MHz}, \mathrm{CDCl}_{3}, 25^{\circ} \mathrm{C}\right)$ of $\mathbf{1 g}$

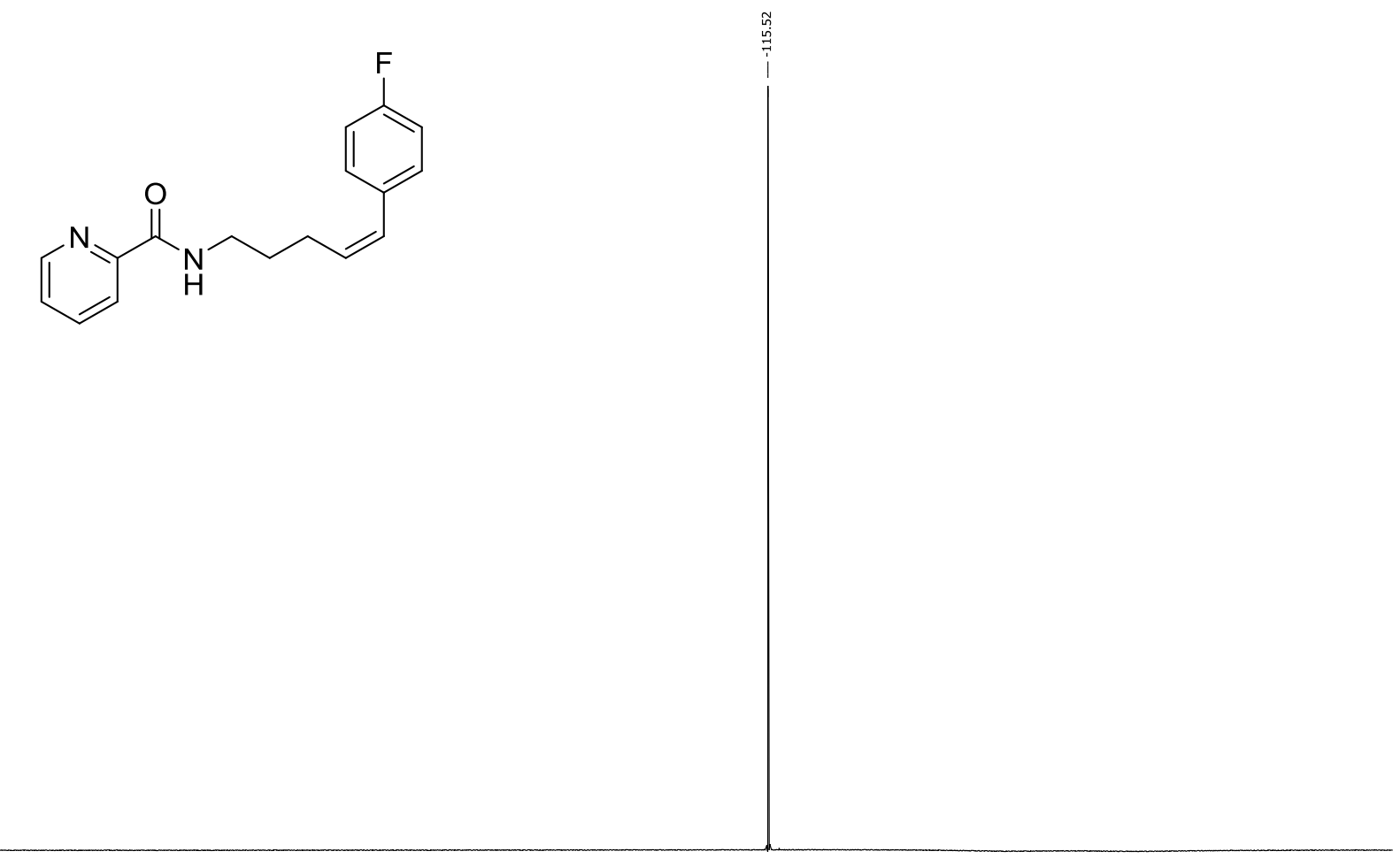

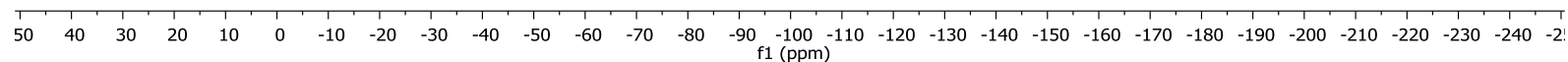


${ }^{\mathbf{1}} \mathbf{H} \mathbf{N M R}\left(400 \mathrm{MHz}, \mathrm{CDCl}_{3}, 25^{\circ} \mathrm{C}\right)$ of $\mathbf{S 2 h}$

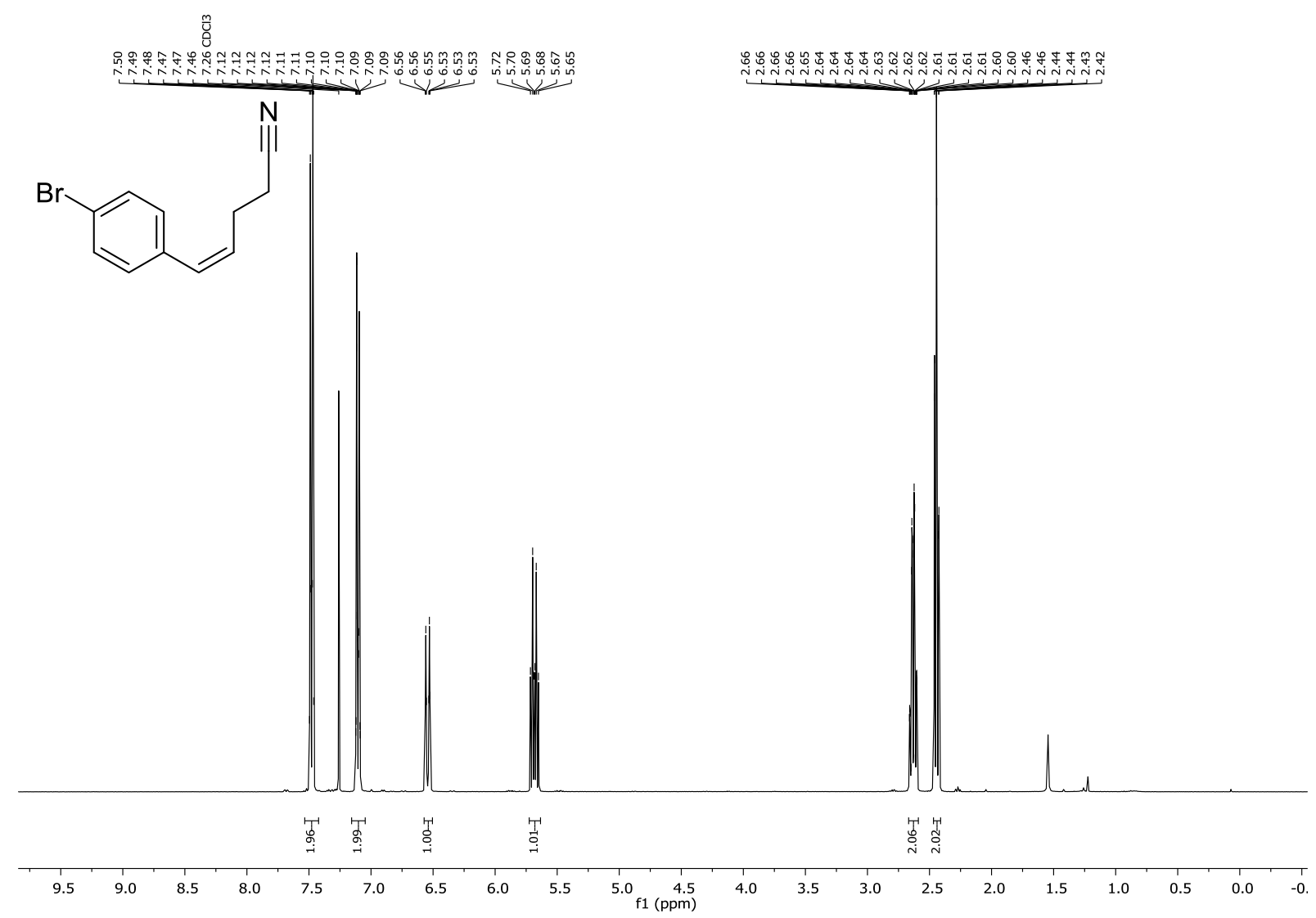

${ }^{13} \mathbf{C}$ NMR $\left(100 \mathrm{MHz}, \mathrm{CDCl}_{3}, 25^{\circ} \mathrm{C}\right)$ of $\mathbf{S 2 h}$

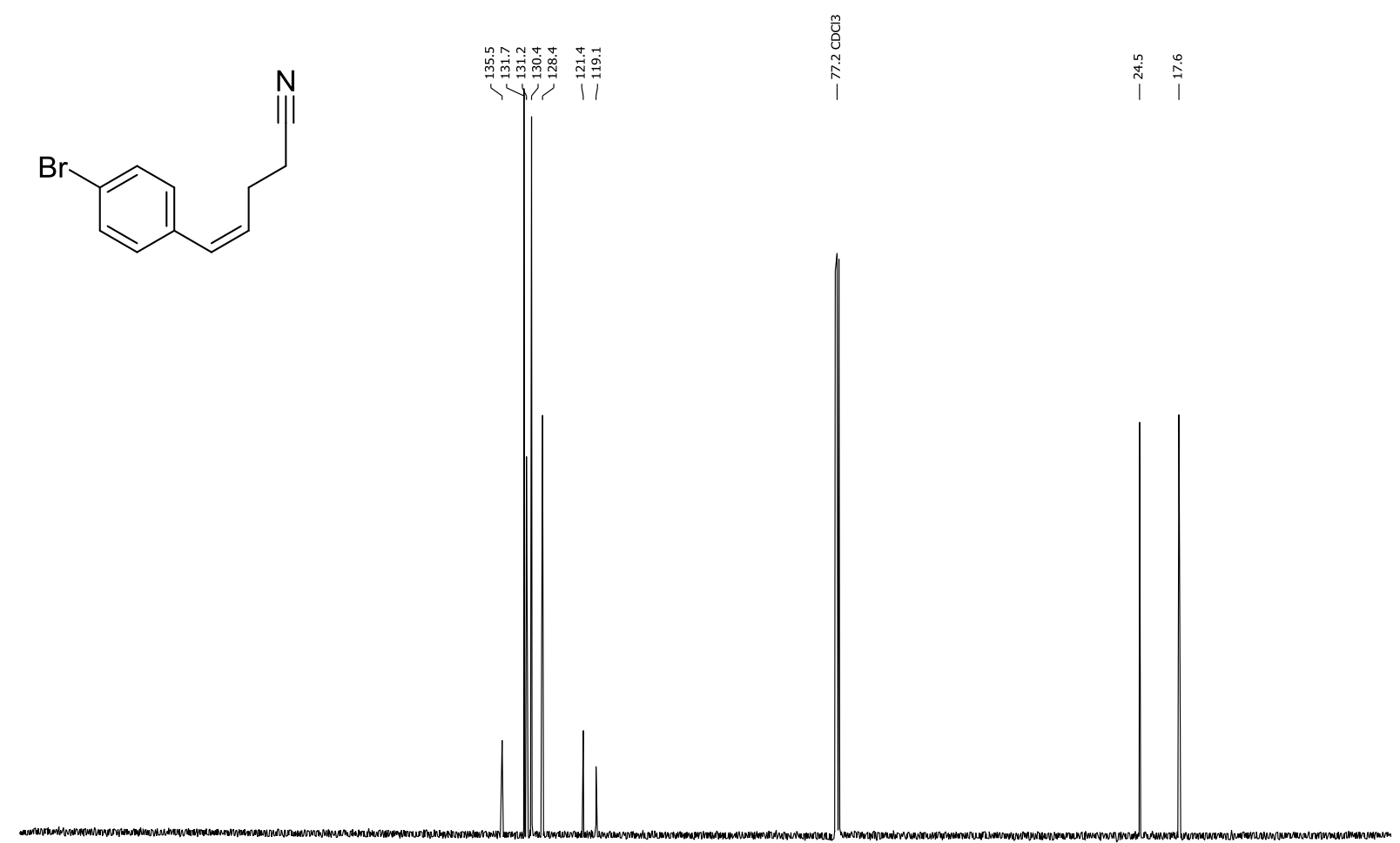

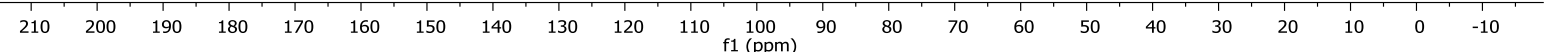


${ }^{\mathbf{1}} \mathbf{H}$ NMR $\left(400 \mathrm{MHz}, \mathrm{CDCl}_{3}, 25^{\circ} \mathrm{C}\right)$ of $\mathbf{1 h}$

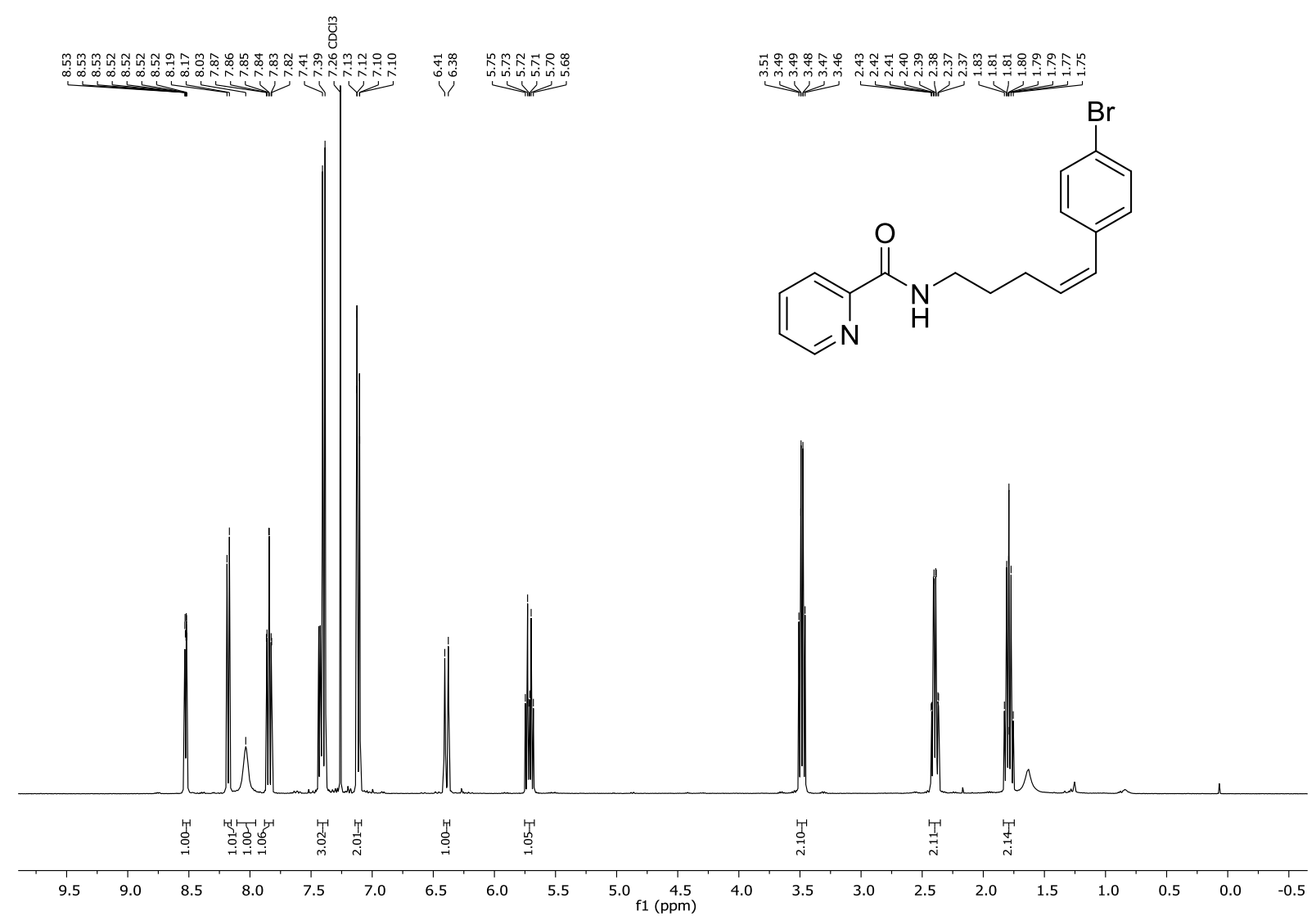

${ }^{13} \mathbf{C ~ N M R}\left(100 \mathrm{MHz}, \mathrm{CDCl}_{3}, 25^{\circ} \mathrm{C}\right)$ of $\mathbf{1 h}$

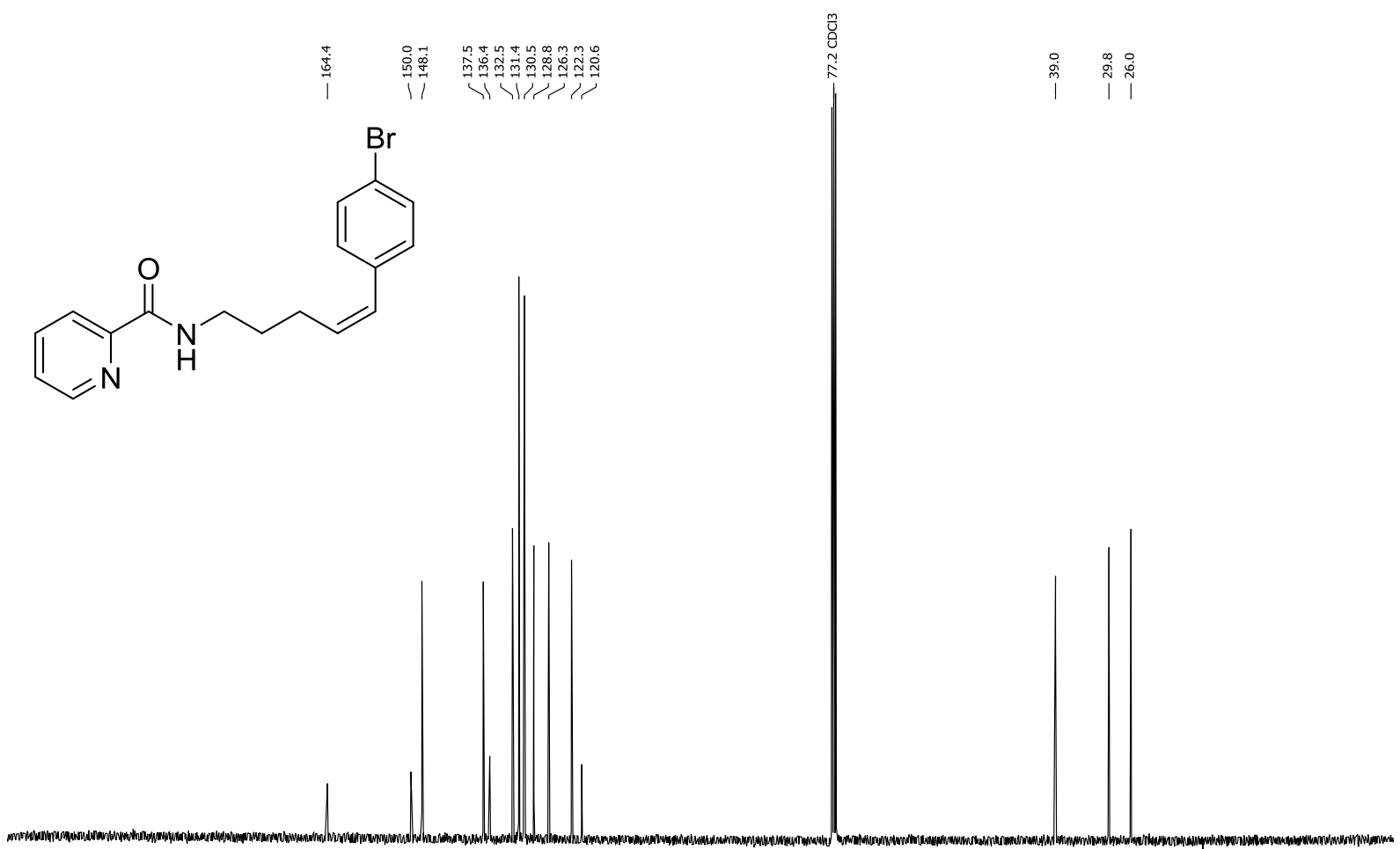

$\begin{array}{llllllllllllllllllllllllll}210 & 200 & 190 & 180 & 170 & 160 & 150 & 140 & 130 & 120 & 110 & \begin{array}{c}100 \\ \mathrm{f} 1\end{array}(\mathrm{ppm}) & 90 & 80 & 70 & 60 & 50 & 40 & 30 & 20 & 10 & 0 & -10 & \end{array}$ 
${ }^{\mathbf{1}} \mathbf{H}$ NMR $\left(400 \mathrm{MHz}, \mathrm{CDCl}_{3}, 25^{\circ} \mathrm{C}\right)$ of $\mathbf{S 2 i}$

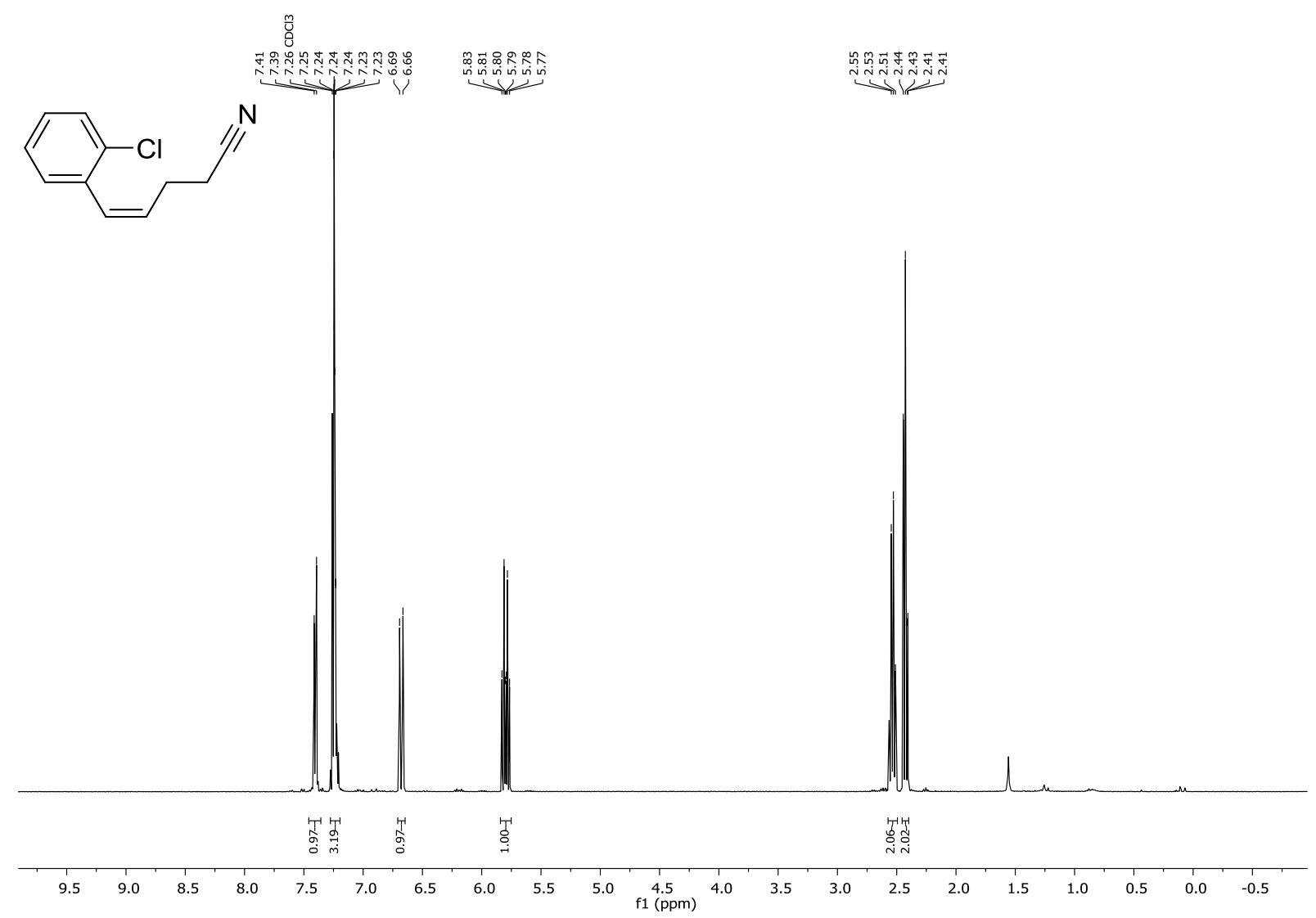

${ }^{13} \mathbf{C}$ NMR $\left(100 \mathrm{MHz}, \mathrm{CDCl}_{3}, 25^{\circ} \mathrm{C}\right)$ of $\mathbf{S 2 i}$

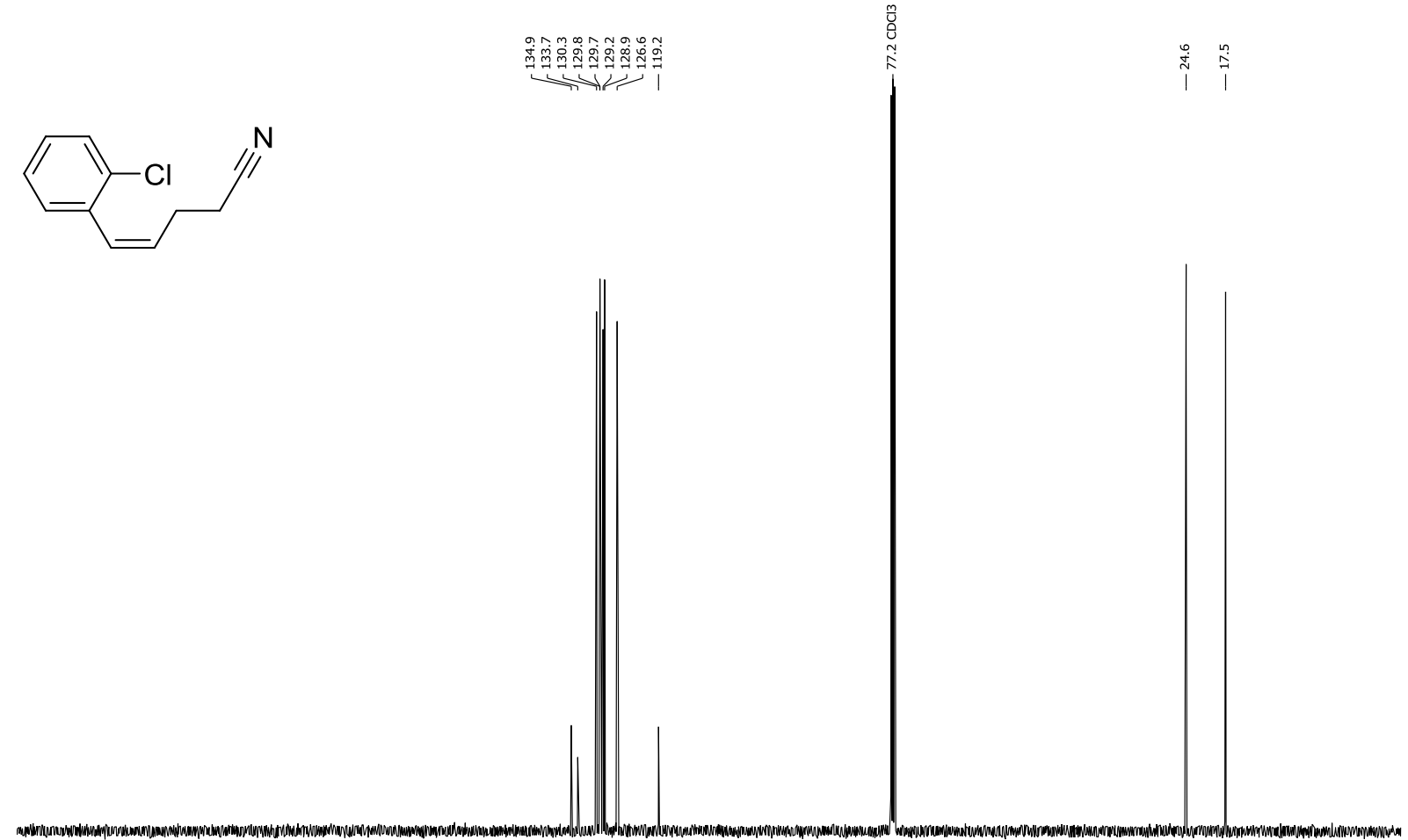

$\begin{array}{llllllllllllllllllllllllllllllllll}230 & 220 & 210 & 200 & 190 & 180 & 170 & 160 & 150 & 140 & 130 & 120 & 110 & 100 & 90 & 80 & 70 & 60 & 50 & 40 & 30 & 20 & 10 & 0 & -10\end{array}$ 
${ }^{\mathbf{1}} \mathbf{H}$ NMR $\left(500 \mathrm{MHz}, \mathrm{CDCl}_{3}, 25^{\circ} \mathrm{C}\right)$ of $\mathbf{1 i}$

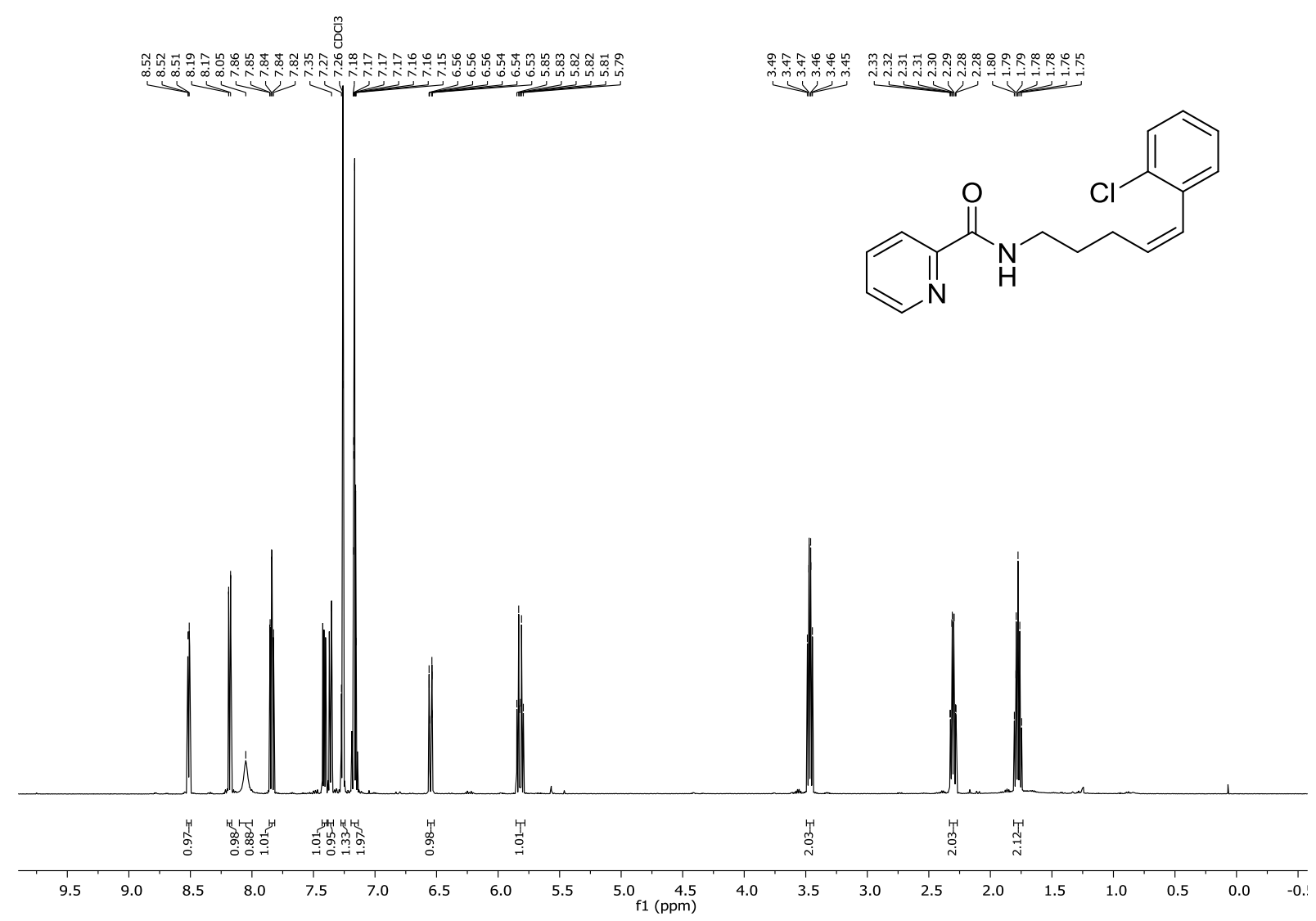

${ }^{13} \mathbf{C ~ N M R ~}\left(126 \mathrm{MHz}, \mathrm{CDCl}_{3}, 25^{\circ} \mathrm{C}\right)$ of $\mathbf{1 i}$

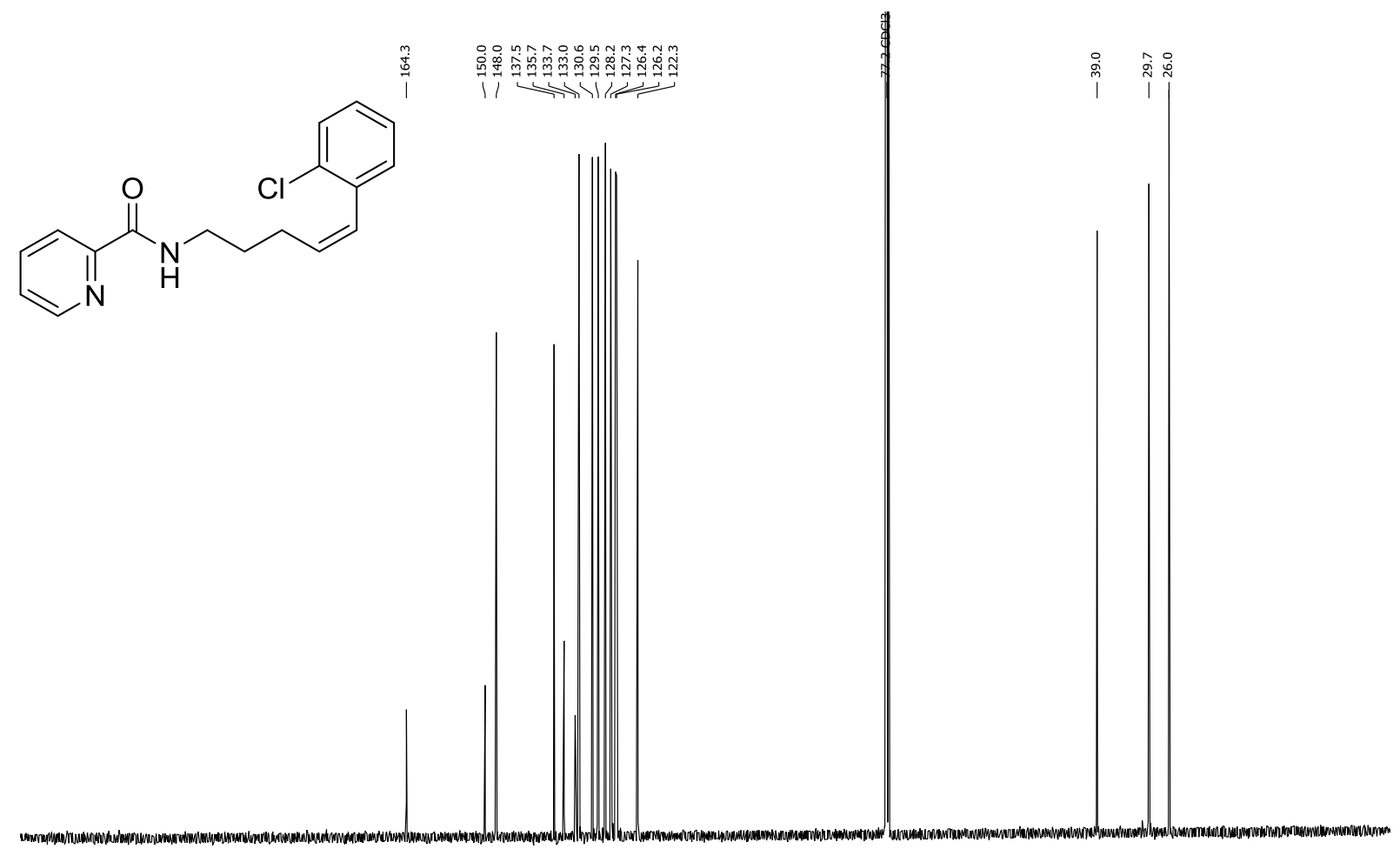

$\begin{array}{lllllllllllllllllllllllllll}230 & 220 & 210 & 200 & 190 & 180 & 170 & 160 & 150 & 140 & 130 & 120 & 110 & 100 & 90 & 80 & 70 & 60 & 50 & 40 & 30 & 20 & 10 & 0 & -10\end{array}$ 
${ }^{1} \mathbf{H}$ NMR $\left(400 \mathrm{MHz}, \mathrm{CDCl}_{3}, 25^{\circ} \mathrm{C}\right)$ of $\mathbf{1 j}$

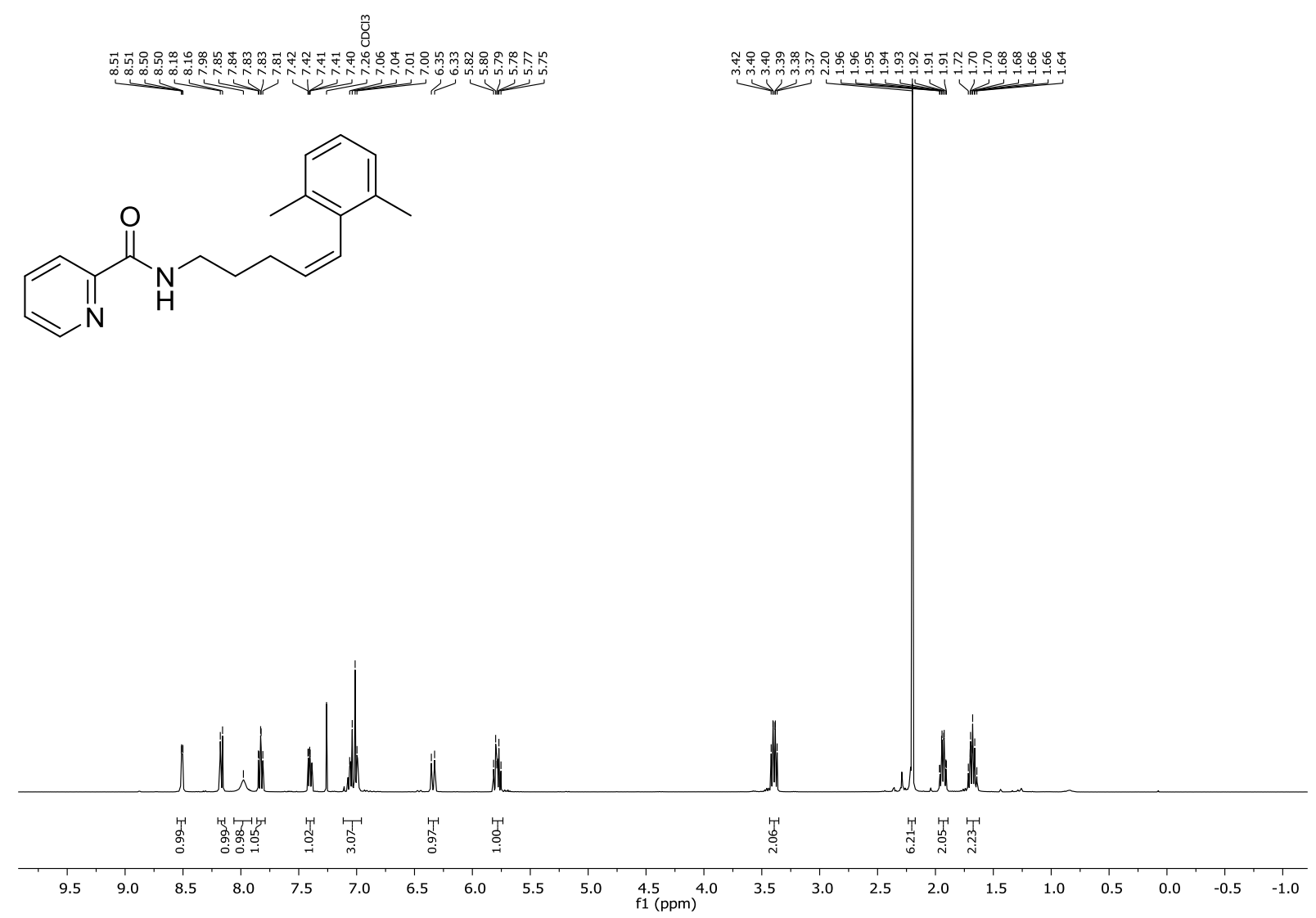

${ }^{13} \mathbf{C}$ NMR $\left(100 \mathrm{MHz}, \mathrm{CDCl}_{3}, 25{ }^{\circ} \mathrm{C}\right)$ of $\mathbf{1 j}$

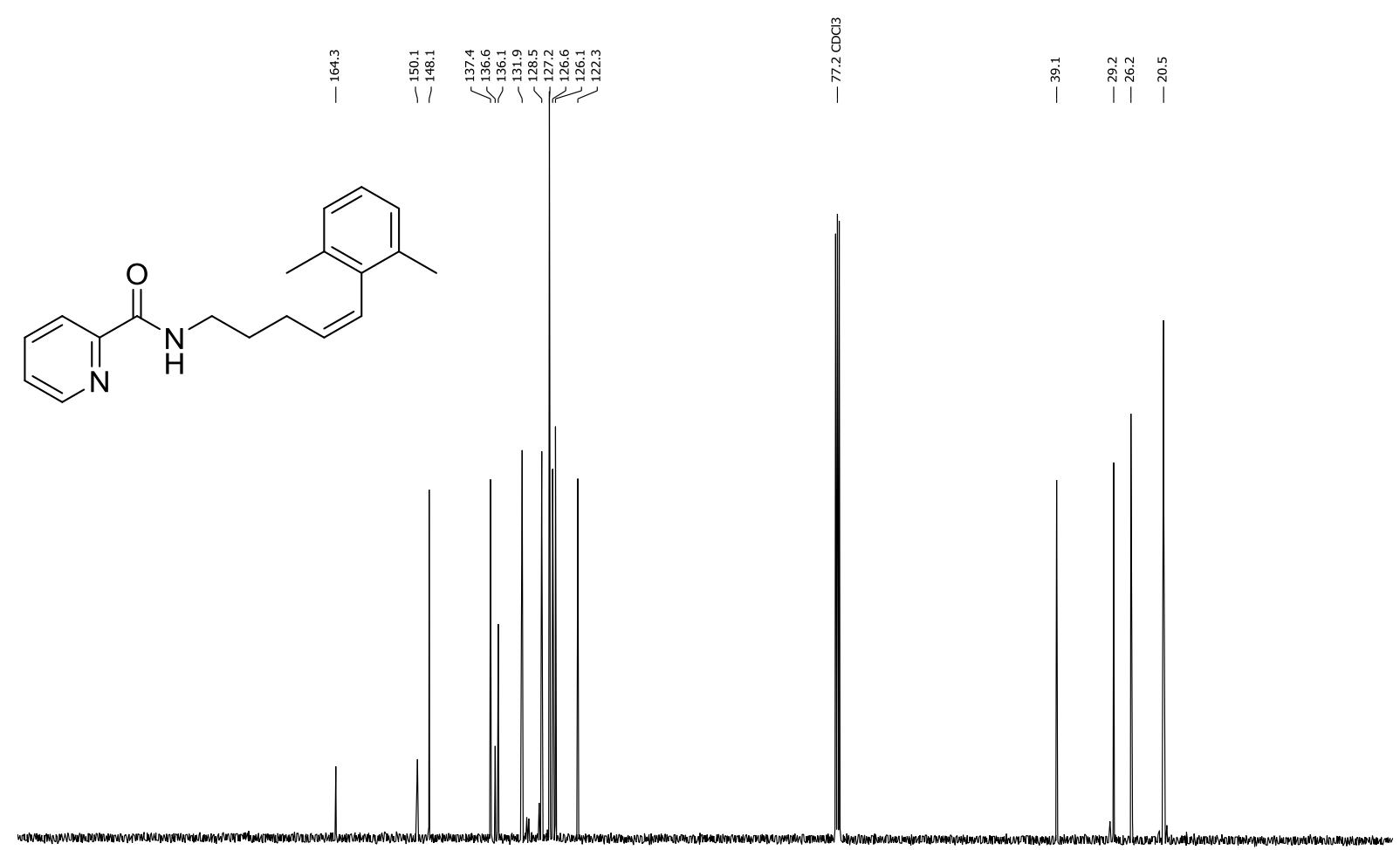

$\begin{array}{llllllllllllllllllllllllll}210 & 200 & 190 & 180 & 170 & 160 & 150 & 140 & 130 & 120 & 110 & \begin{array}{c}100 \\ \mathrm{f} 1\end{array}(\mathrm{ppm}) & 90 & 80 & 70 & 60 & 50 & 40 & 30 & 20 & 10 & 0 & -10 & \end{array}$ 
${ }^{1} \mathbf{H}$ NMR $\left(400 \mathrm{MHz}, \mathrm{CDCl}_{3}, 25^{\circ} \mathrm{C}\right)$ of $\mathbf{S 2 k}$

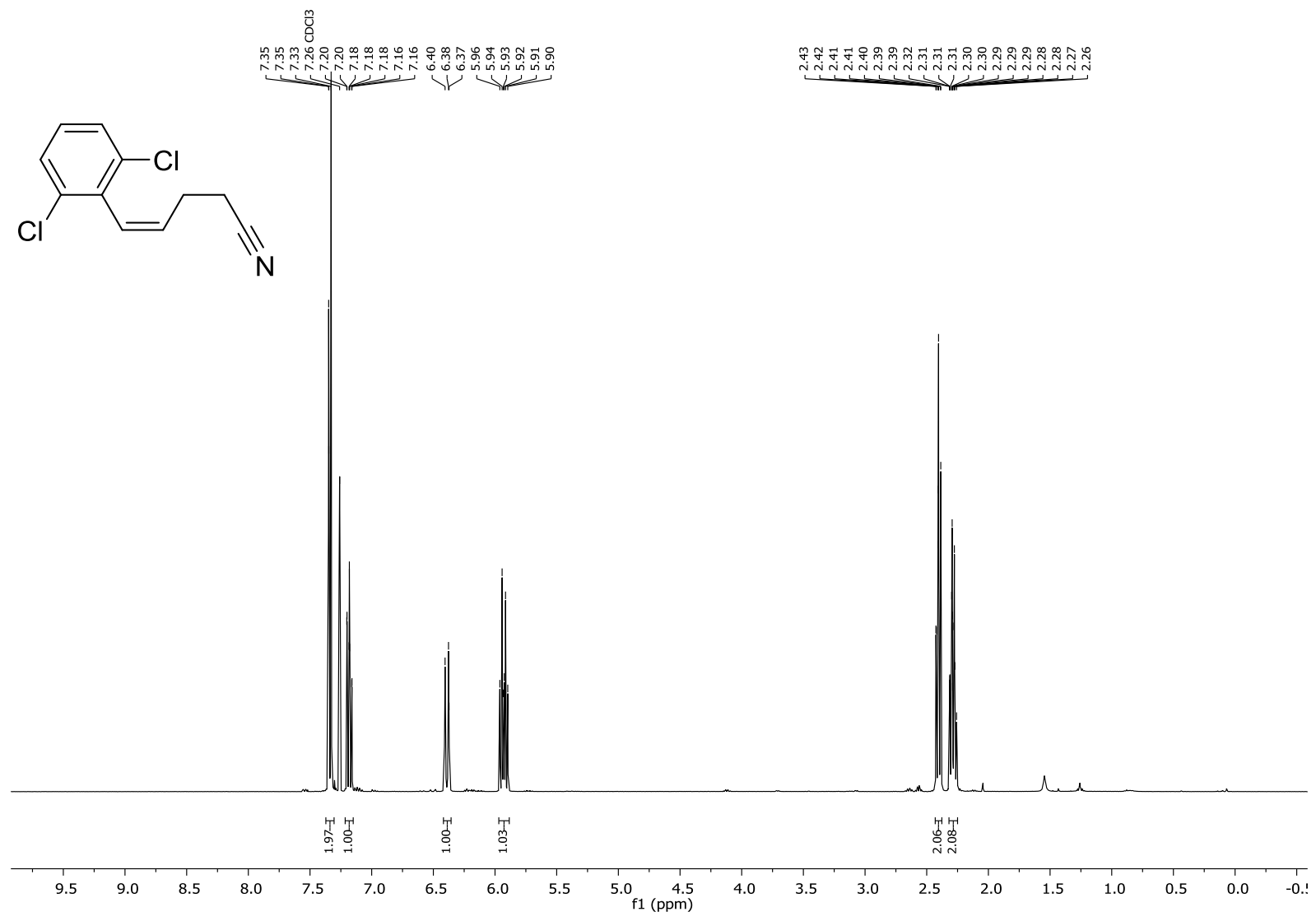

${ }^{13} \mathbf{C}$ NMR $\left(100 \mathrm{MHz}, \mathrm{CDCl}_{3}, 25{ }^{\circ} \mathrm{C}\right)$ of $\mathbf{S 2 k}$
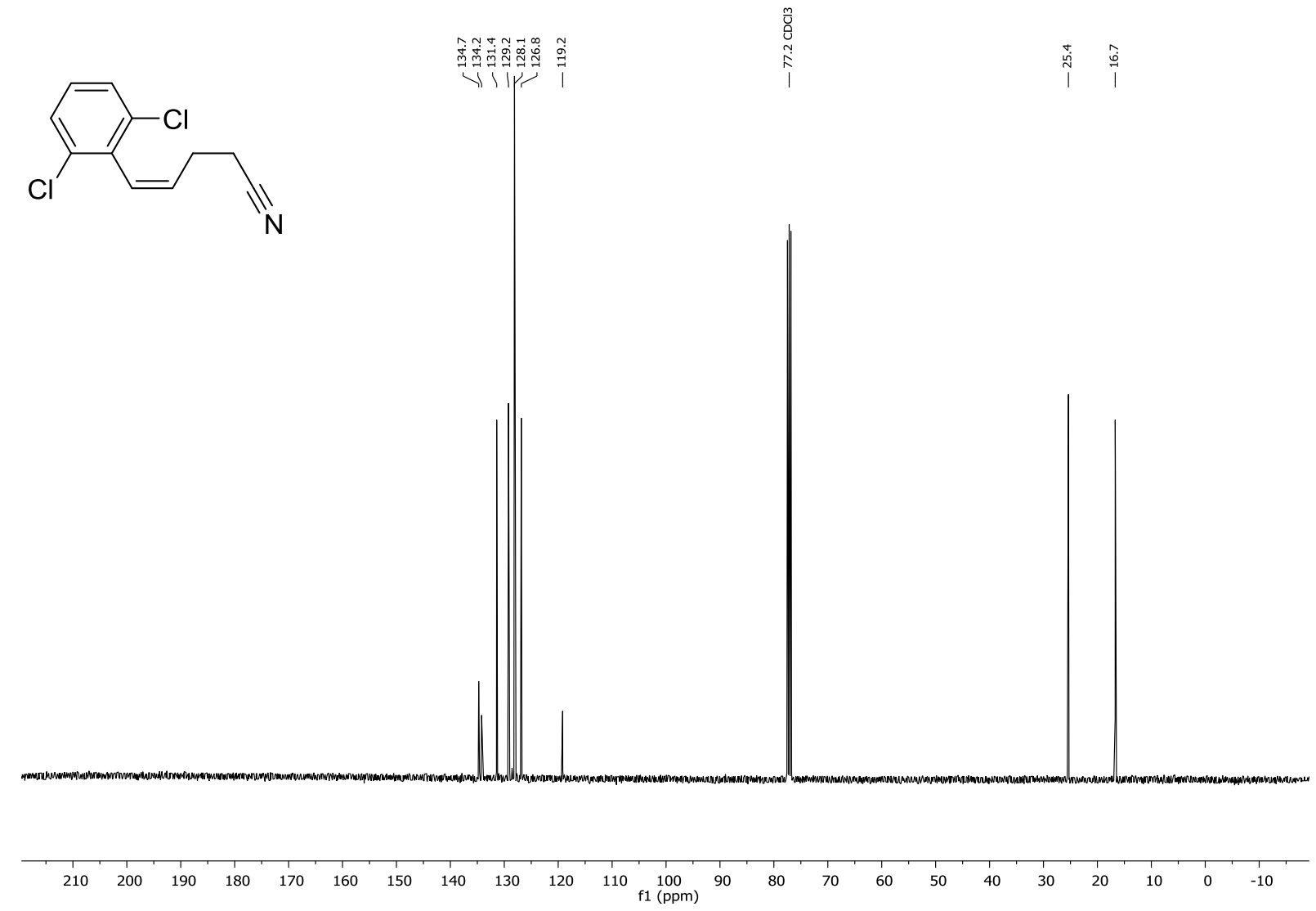

S91 
${ }^{\mathbf{1}} \mathbf{H}$ NMR $\left(400 \mathrm{MHz}, \mathrm{CDCl}_{3}, 25^{\circ} \mathrm{C}\right)$ of $\mathbf{1} \mathbf{k}$

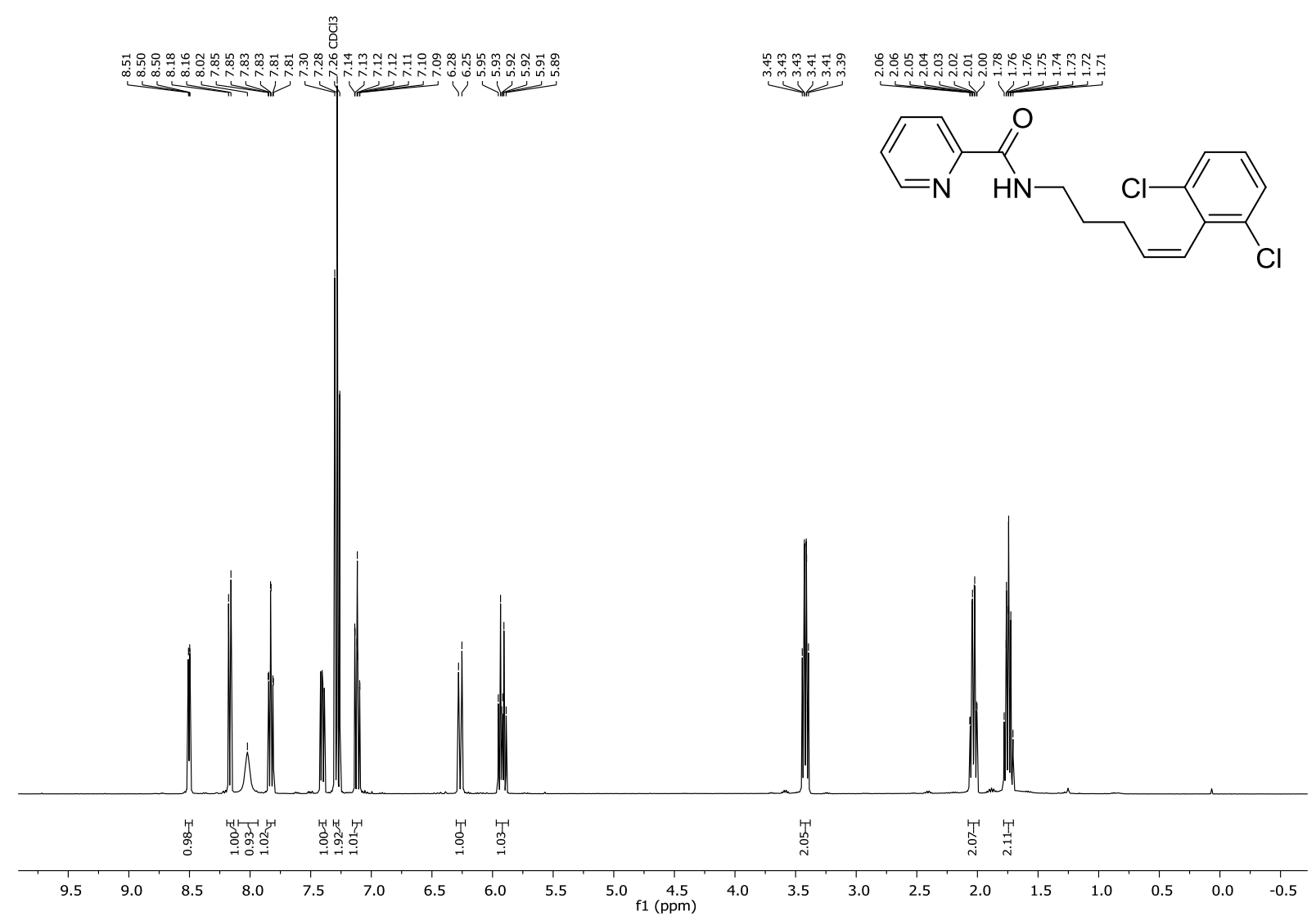

${ }^{13} \mathbf{C}$ NMR $\left(100 \mathrm{MHz}, \mathrm{CDCl}_{3}, 25^{\circ} \mathrm{C}\right)$ of $\mathbf{1 k}$

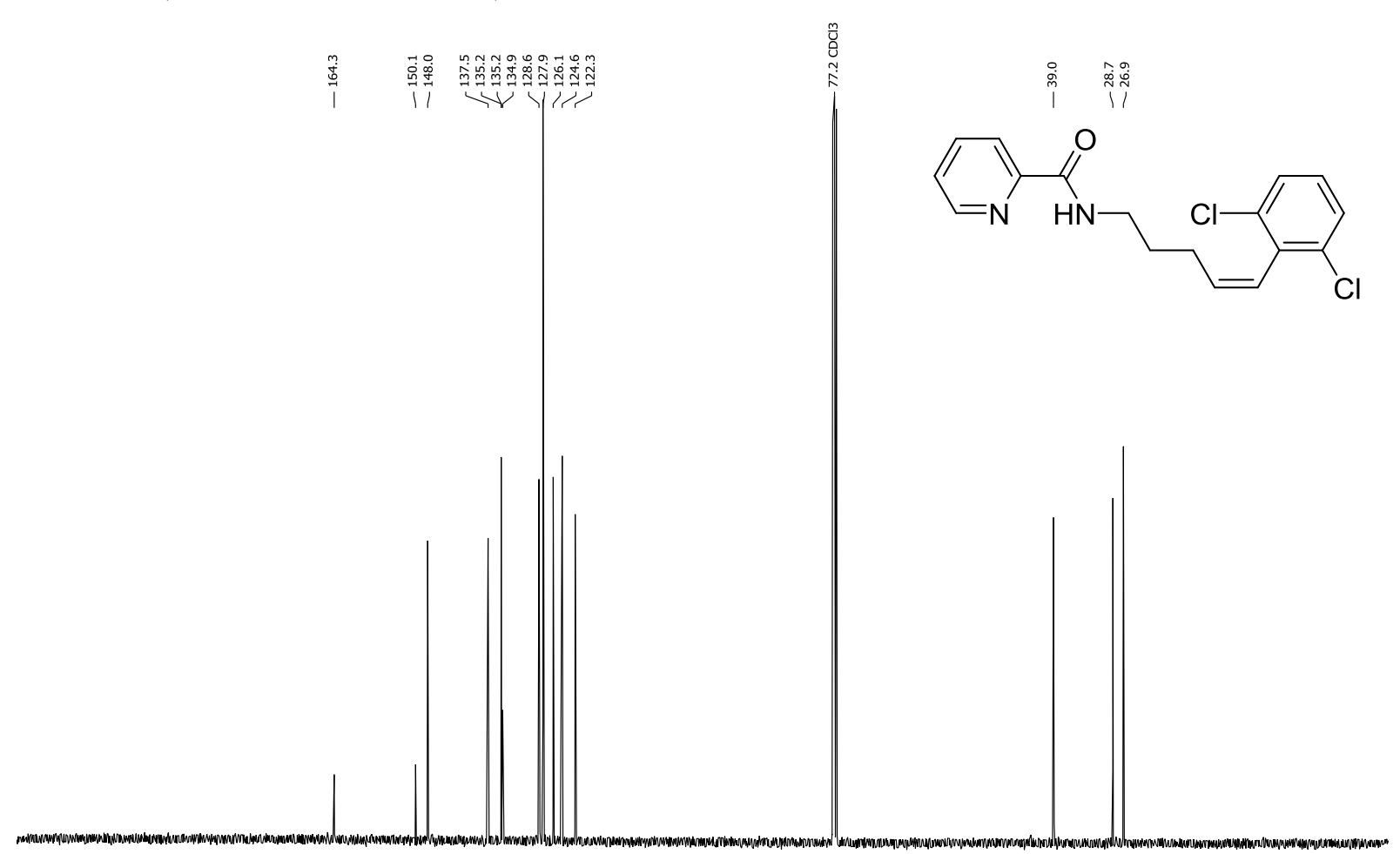

$\begin{array}{llllllllllllllllllllllllll}210 & 200 & 190 & 180 & 170 & 160 & 150 & 140 & 130 & 120 & 110 & 100 & 10 & 80 & 70 & 60 & 50 & 40 & 30 & 20 & 10 & 0 & -10\end{array}$ 
${ }^{\mathbf{1}} \mathbf{H}$ NMR $\left(500 \mathrm{MHz}, \mathrm{CDCl}_{3}, 25^{\circ} \mathrm{C}\right)$ of $\mathbf{1} \mathbf{k}-\mathbf{D}_{\mathbf{1}}$

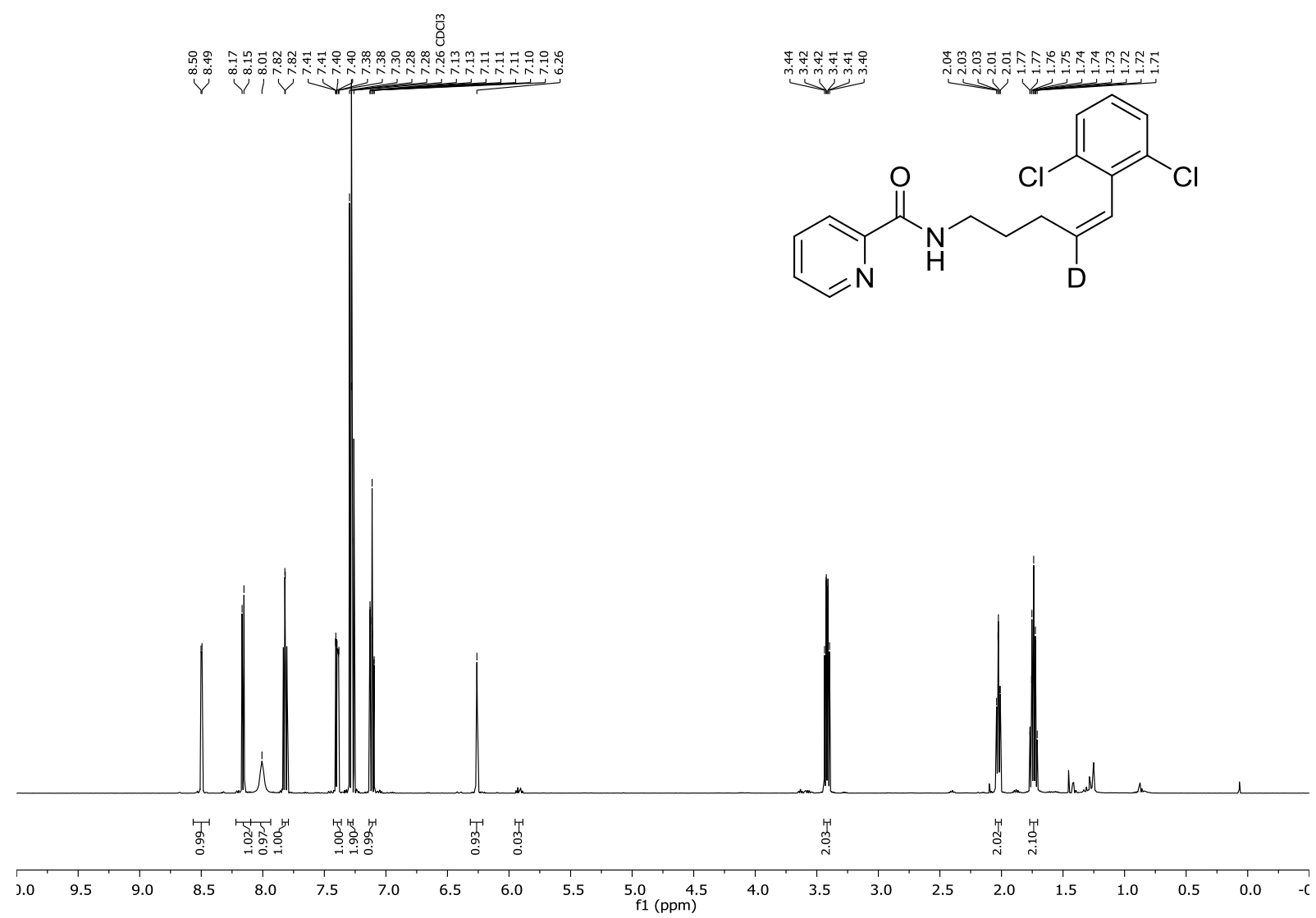

${ }^{13} \mathbf{C}$ NMR $\left(126 \mathrm{MHz}, \mathrm{CDCl}_{3}, 25^{\circ} \mathrm{C}\right)$ of $\mathbf{1 k}-\mathbf{D}_{\mathbf{1}}$

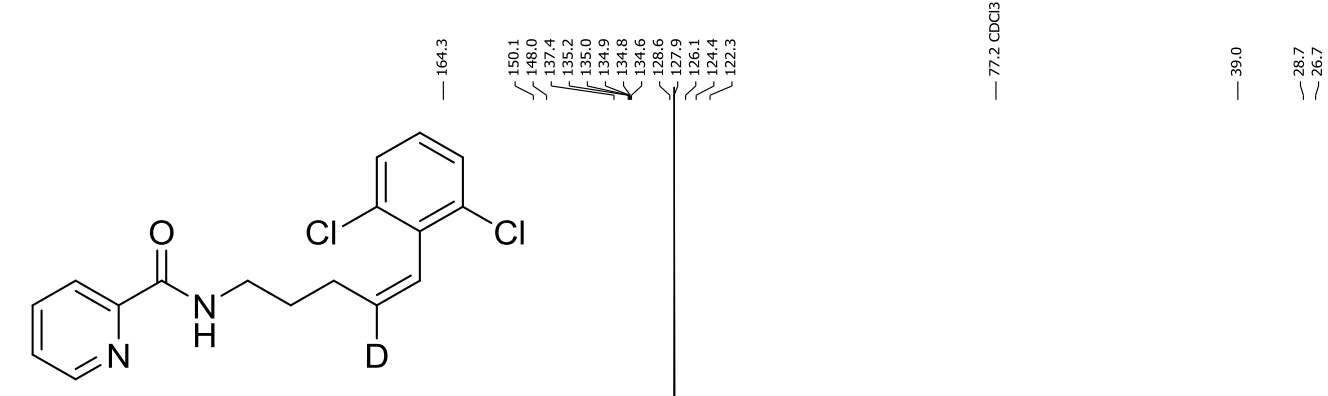

$\begin{array}{llllllllllllllllllllllllllllllllll}230 & 220 & 210 & 200 & 190 & 180 & 170 & 160 & 150 & 140 & 130 & 120 & 110 & 100 & 90 & 80 & 70 & 60 & 50 & 40 & 30 & 20 & 10 & 0 & -10\end{array}$ 
${ }^{\mathbf{1}} \mathbf{H}$ NMR $\left(500 \mathrm{MHz}, \mathrm{CD}_{3} \mathrm{CN}, 25^{\circ} \mathrm{C}\right)$ of $\mathbf{1 k}-\mathbf{D}_{\mathbf{3}}$

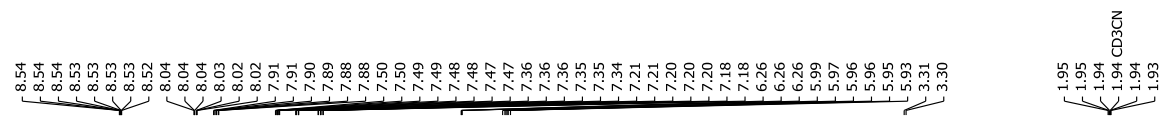

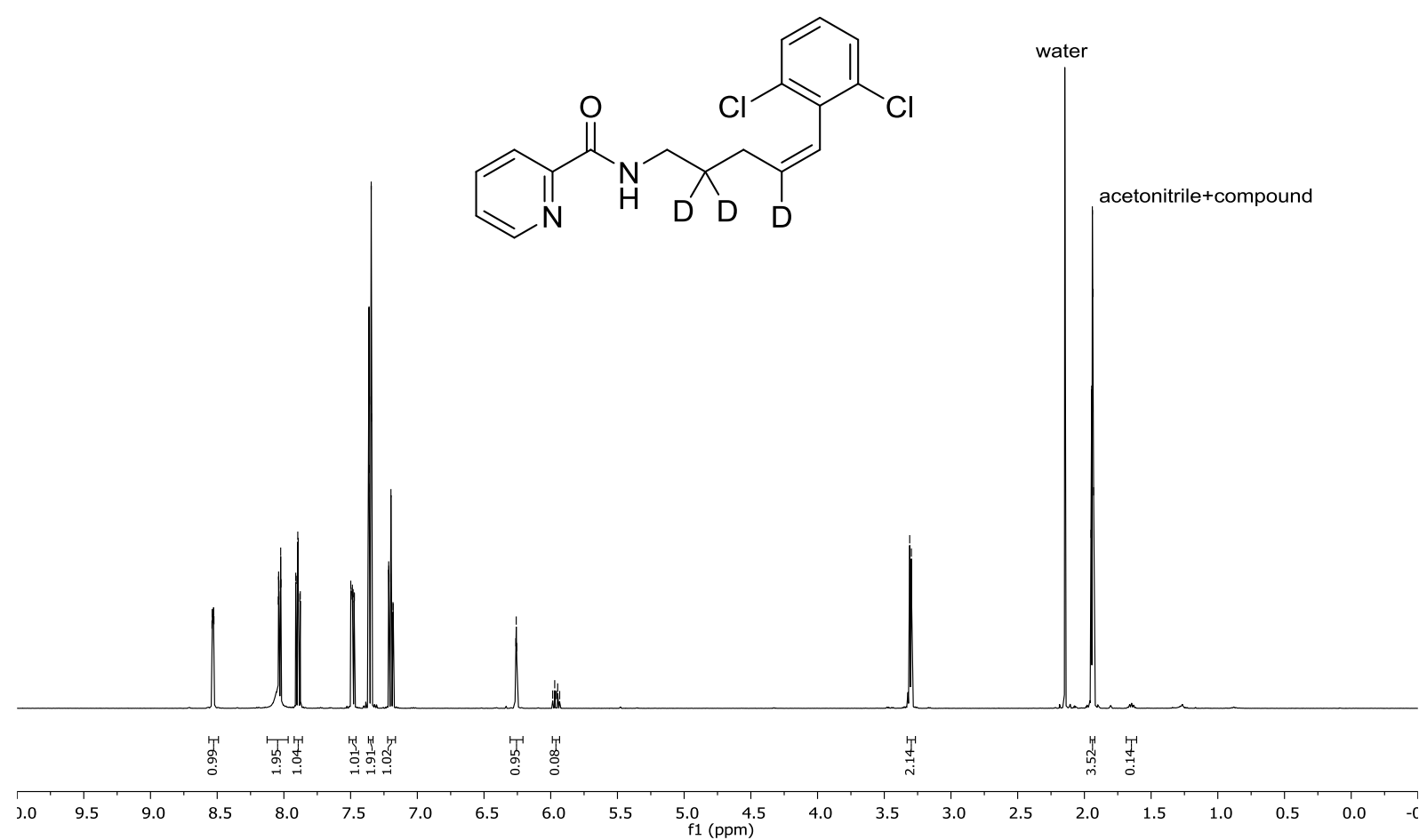

${ }^{13} \mathbf{C}$ NMR $\left(126 \mathrm{MHz}, \mathrm{CD}_{3} \mathrm{CN}, 25{ }^{\circ} \mathrm{C}\right)$ of $\mathbf{1 k}-\mathbf{D}_{\mathbf{3}}$

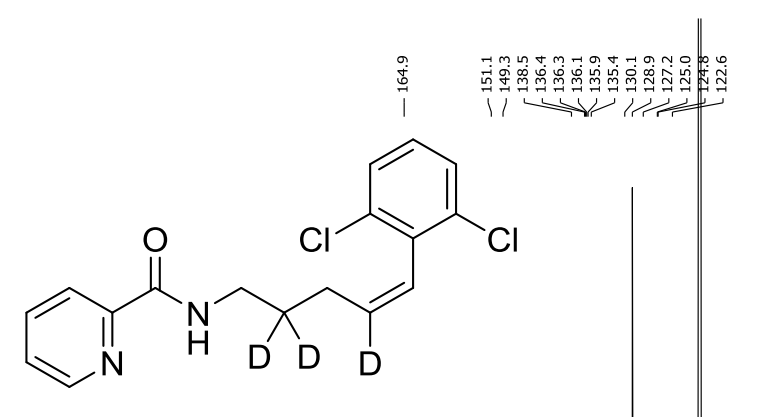

$\begin{array}{llllllllllllllllllllllllll}230 & 220 & 210 & 200 & 190 & 180 & 170 & 160 & 150 & 140 & 130 & 120 & 110 & 100 & 90 & 80 & 70 & 60 & 50 & 40 & 30 & 20 & 10 & 0 & -10\end{array}$ 
${ }^{1} \mathbf{H}$ NMR $\left(400 \mathrm{MHz}, \mathrm{CDCl}_{3}, 25^{\circ} \mathrm{C}\right)$ of $\mathbf{S 2 l}$

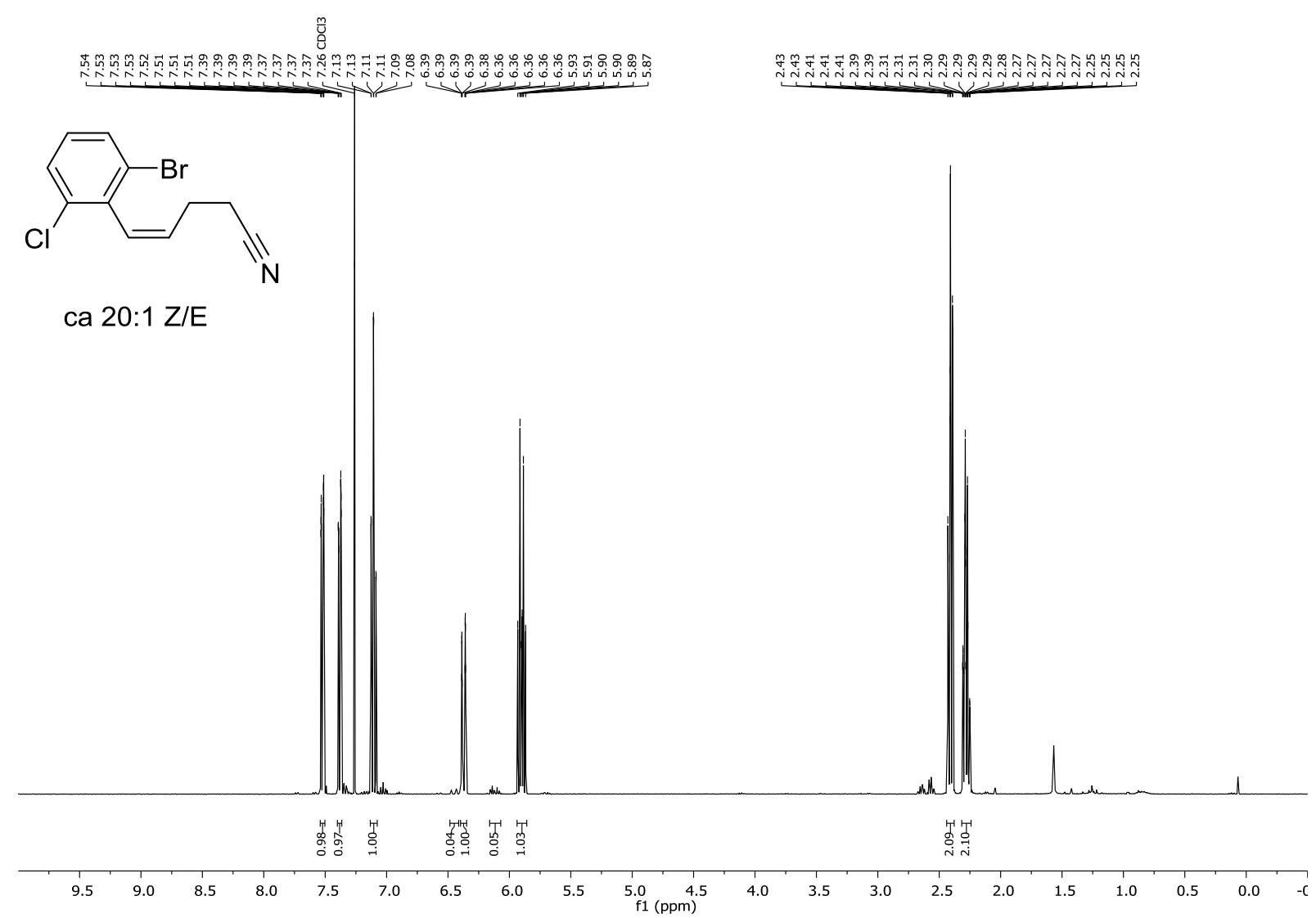

${ }^{13} \mathbf{C}$ NMR $\left(126 \mathrm{MHz}, \mathrm{CDCl}_{3}, 25^{\circ} \mathrm{C}\right)$ of $\mathbf{S 2 1}$
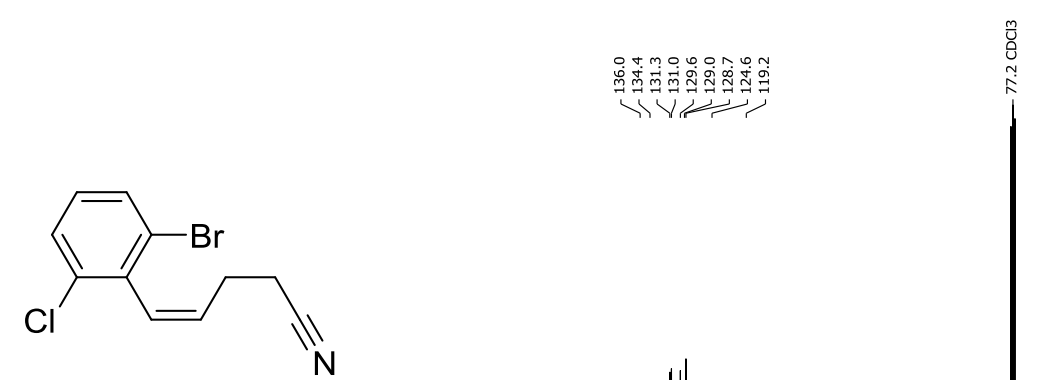

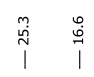

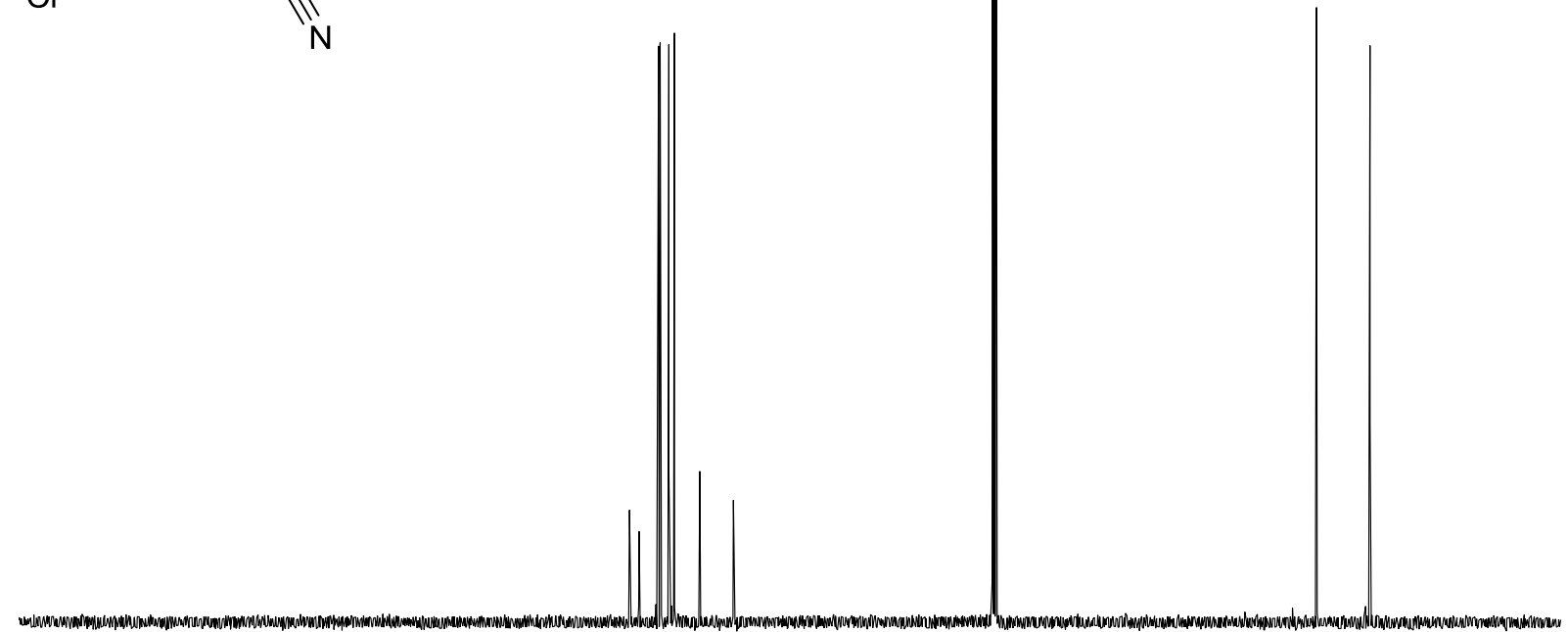

\begin{tabular}{lllllllllllllllllllllllll}
\hline 230 & 220 & 210 & 200 & 190 & 180 & 170 & 160 & 150 & 140 & 130 & 120 & 110 & 100 & 90 & 80 & 70 & 60 & 50 & 40 & 30 & 20 & 10 & 0 & -10
\end{tabular} 
${ }^{\mathbf{1}} \mathbf{H}$ NMR $\left(400 \mathrm{MHz}, \mathrm{CDCl}_{3}, 25^{\circ} \mathrm{C}\right)$ of $\mathbf{1 l}$

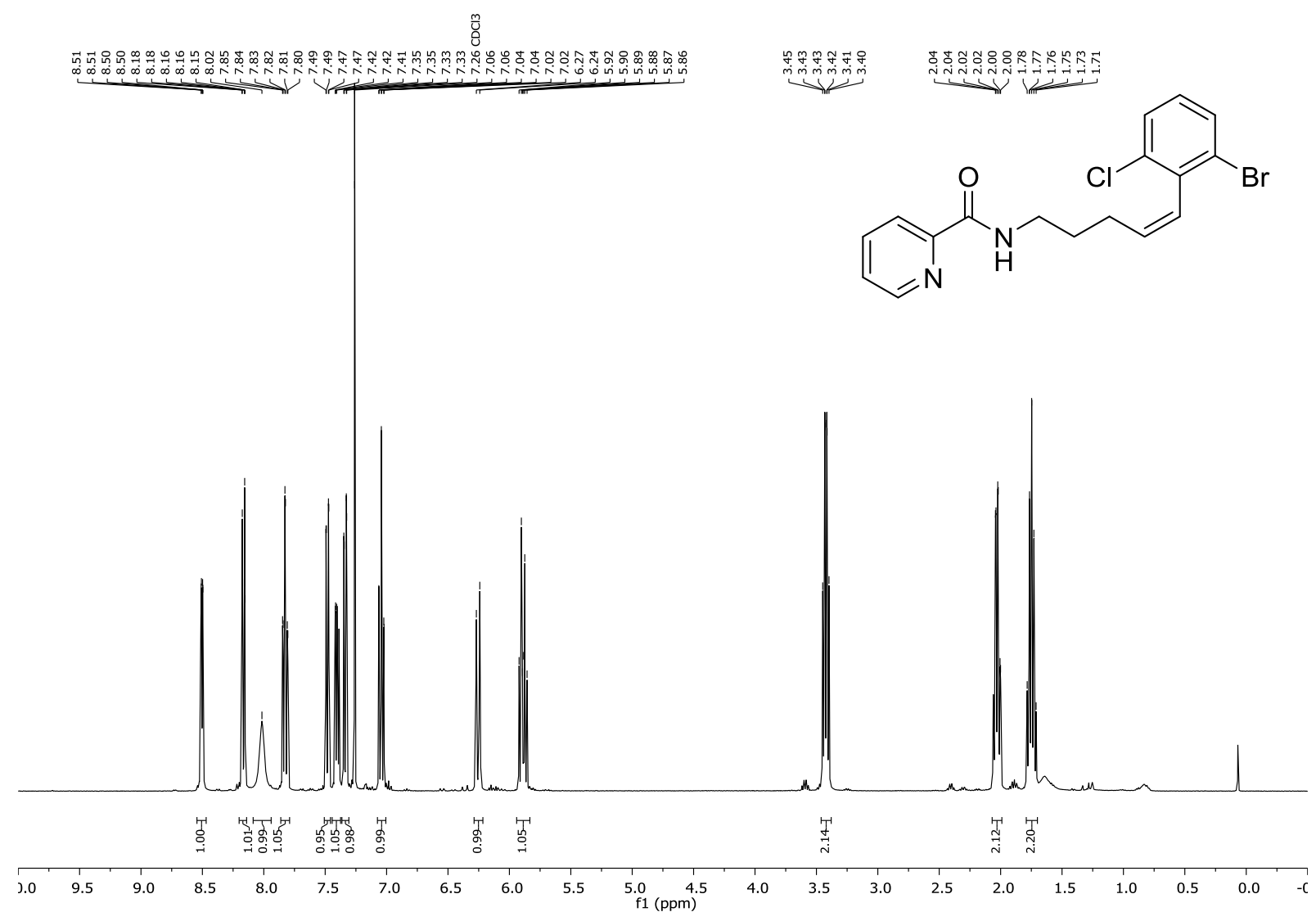

${ }^{13} \mathbf{C}$ NMR $\left(100 \mathrm{MHz}, \mathrm{CDCl}_{3}, 25^{\circ} \mathrm{C}\right)$ of $\mathbf{1 l}$

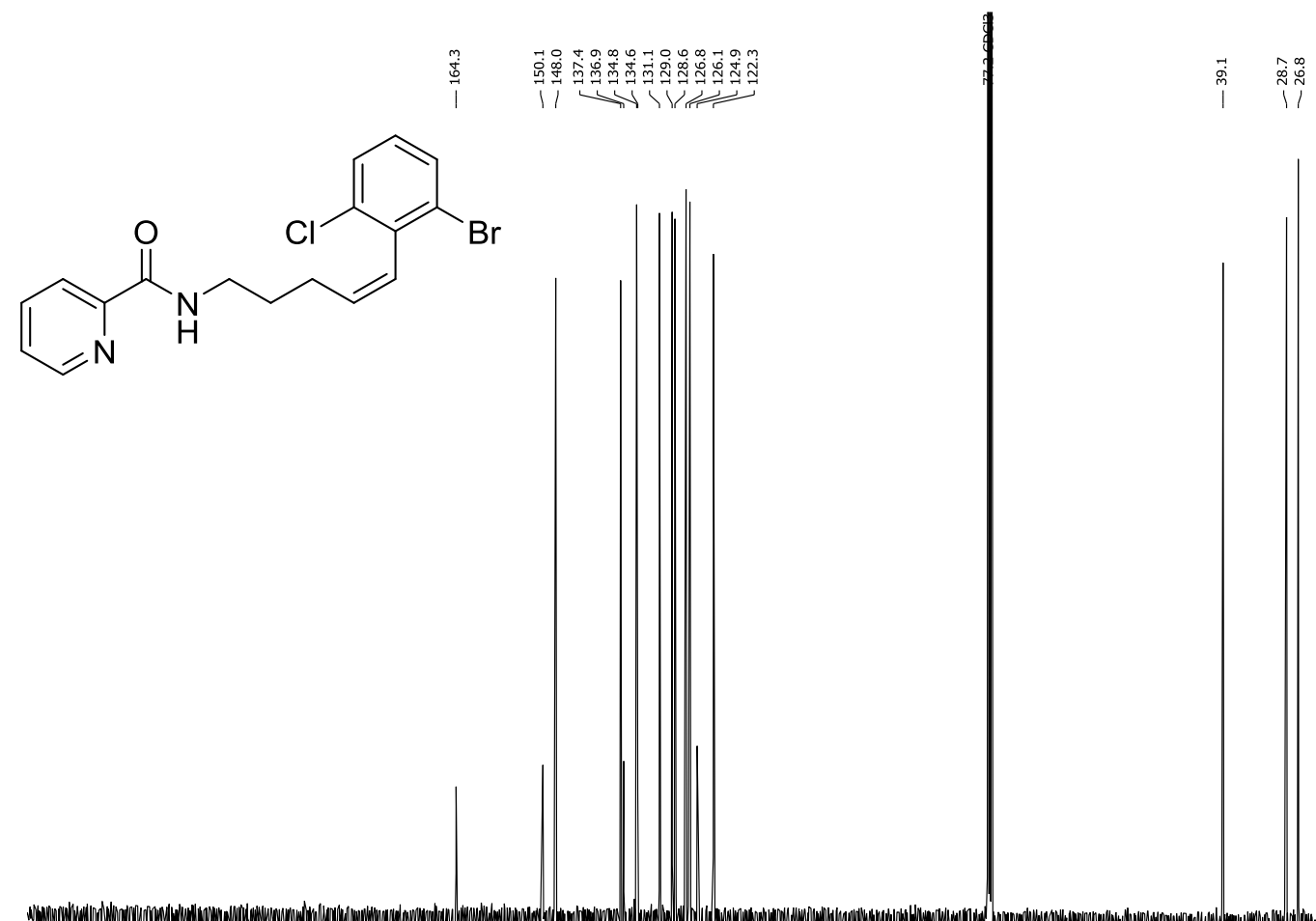

$\begin{array}{lllllllllllllllllllllllllll}230 & 220 & 210 & 200 & 190 & 180 & 170 & 160 & 150 & 140 & 130 & 120 & 110 & 100 & 90 & 80 & 70 & 60 & 50 & 40 & 30 & 20 & 10 & 0 & -10\end{array}$ 
${ }^{1} \mathbf{H}$ NMR $\left(400 \mathrm{MHz}, \mathrm{CDCl}_{3}, 25^{\circ} \mathrm{C}\right)$ of $\mathbf{S 2 m}$

雚

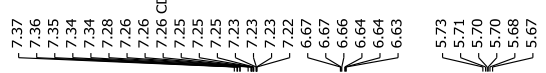

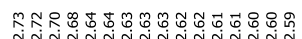
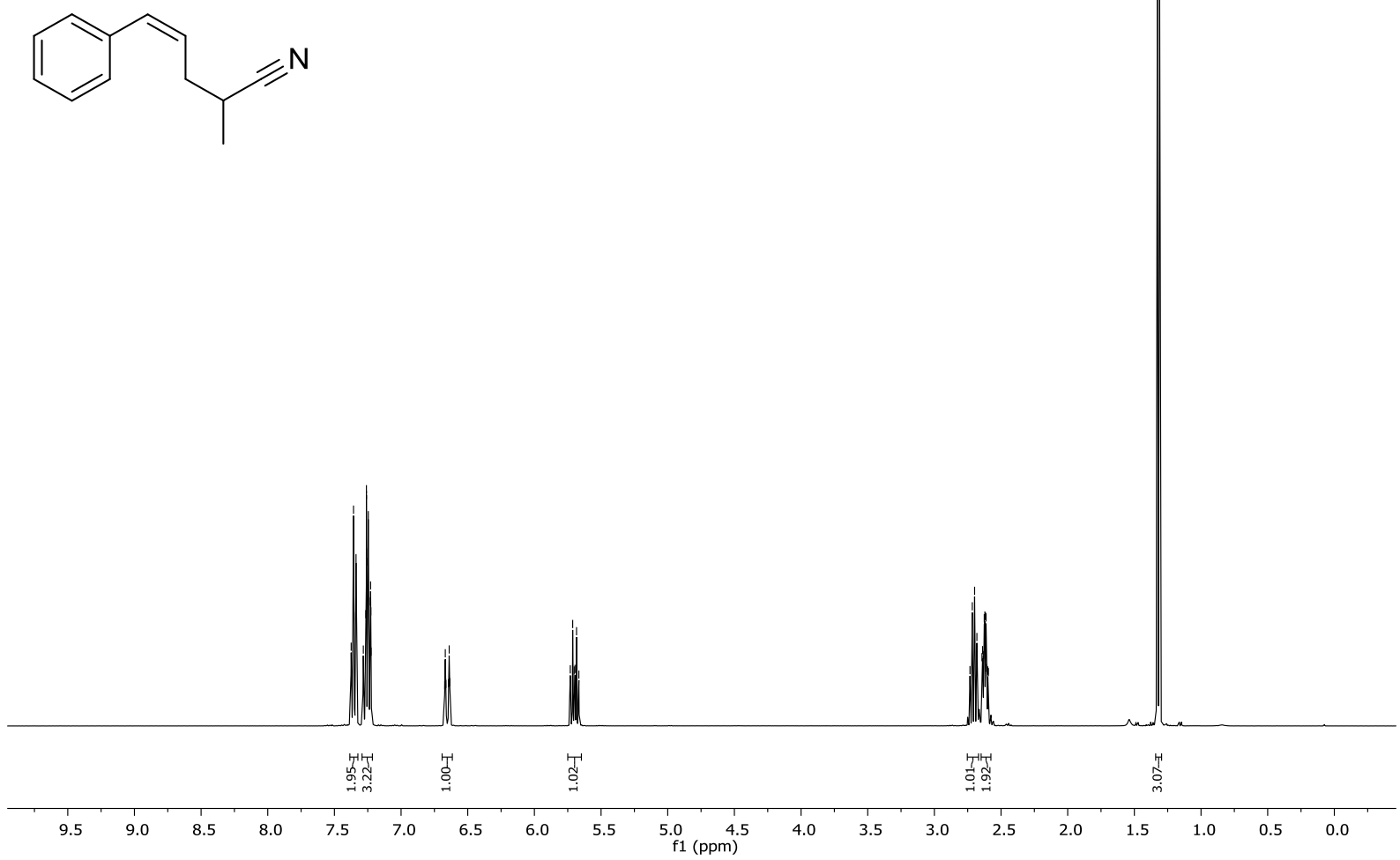

${ }^{13} \mathbf{C}$ NMR $\left(100 \mathrm{MHz}, \mathrm{CDCl}_{3}, 25{ }^{\circ} \mathrm{C}\right)$ of $\mathbf{S 2 m}$

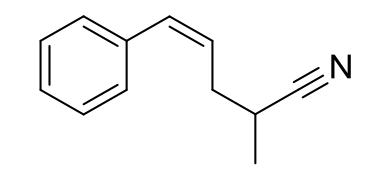

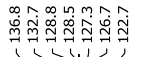
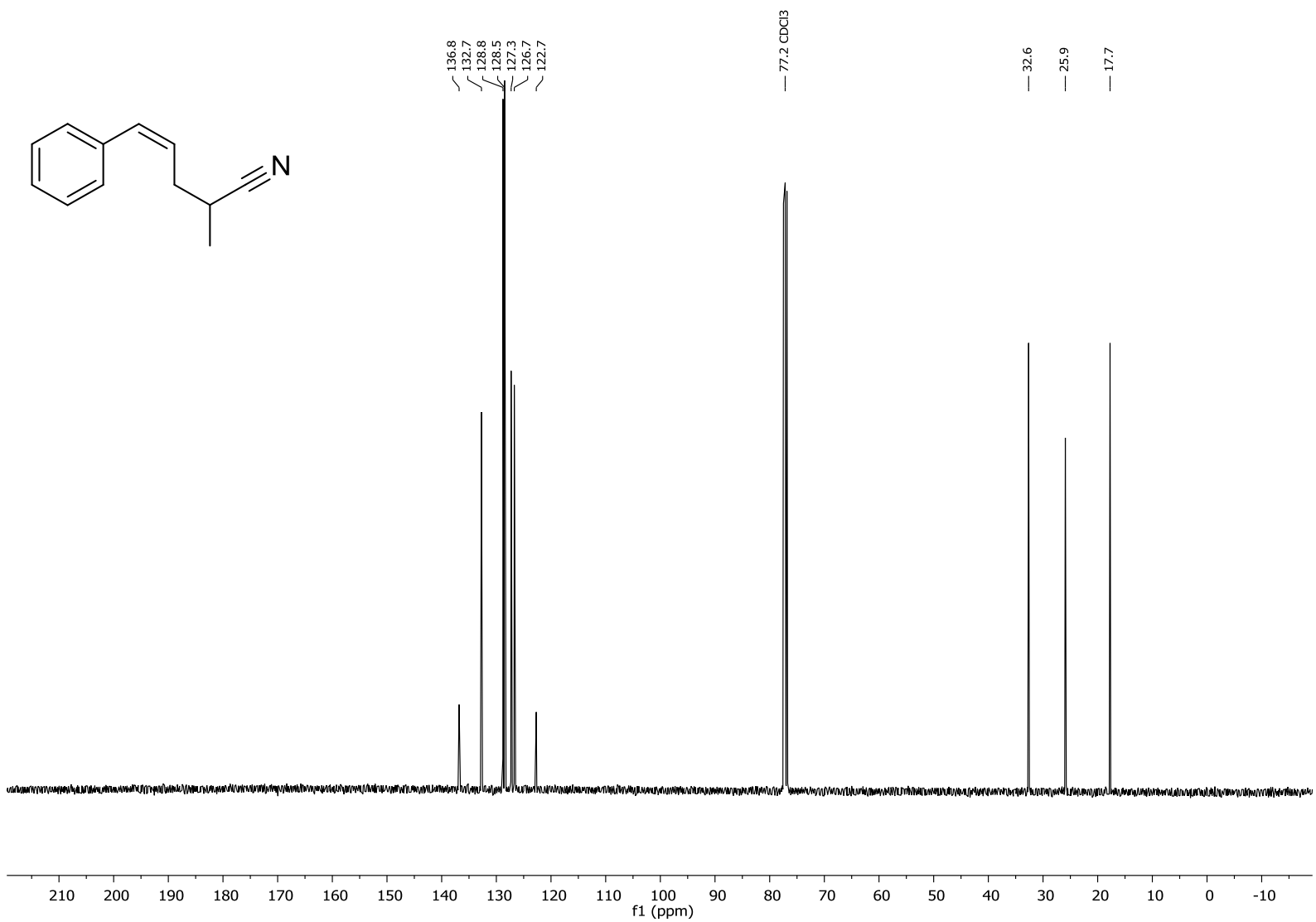

S97 
${ }^{\mathbf{1}} \mathbf{H}$ NMR $\left(400 \mathrm{MHz}, \mathrm{CDCl}_{3}, 25^{\circ} \mathrm{C}\right)$ of $\mathbf{1 m}$

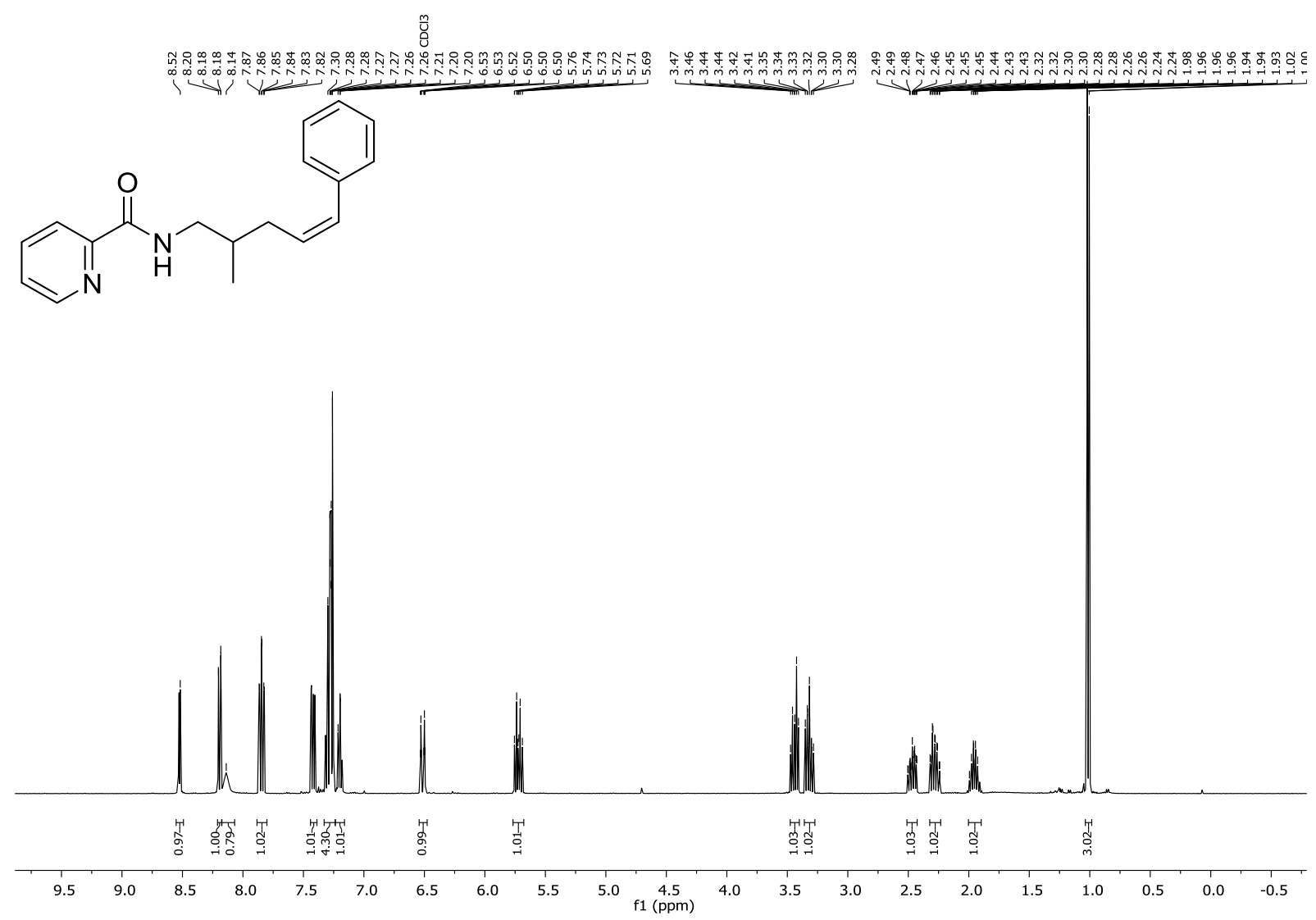

${ }^{13} \mathbf{C ~ N M R}\left(100 \mathrm{MHz}, \mathrm{CDCl}_{3}, 25{ }^{\circ} \mathrm{C}\right)$ of $\mathbf{1 m}$

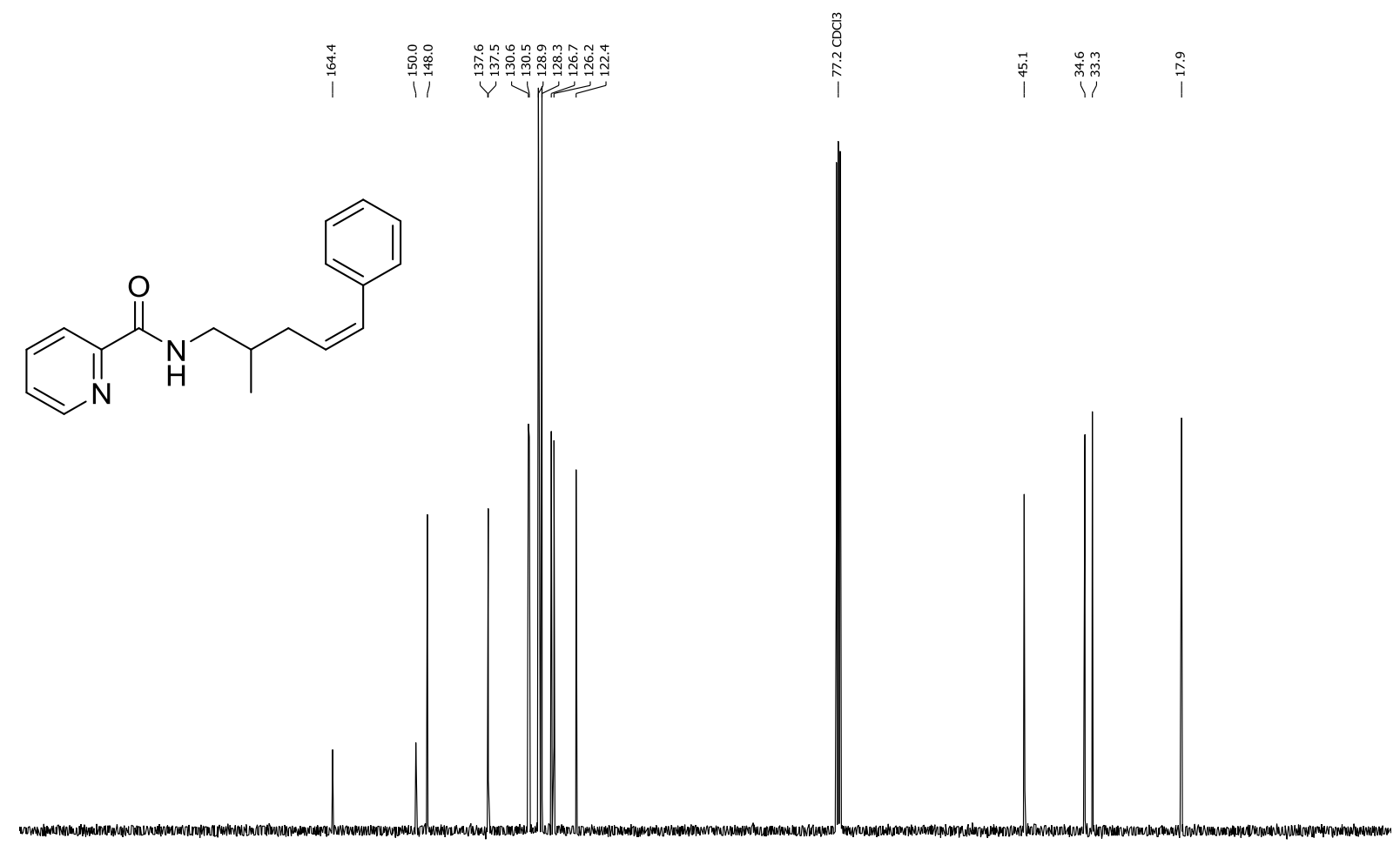

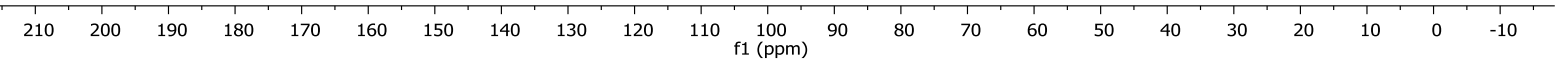


${ }^{1} \mathbf{H}$ NMR $\left(400 \mathrm{MHz}, \mathrm{CDCl}_{3}, 25^{\circ} \mathrm{C}\right)$ of $\mathbf{S 2 n}$

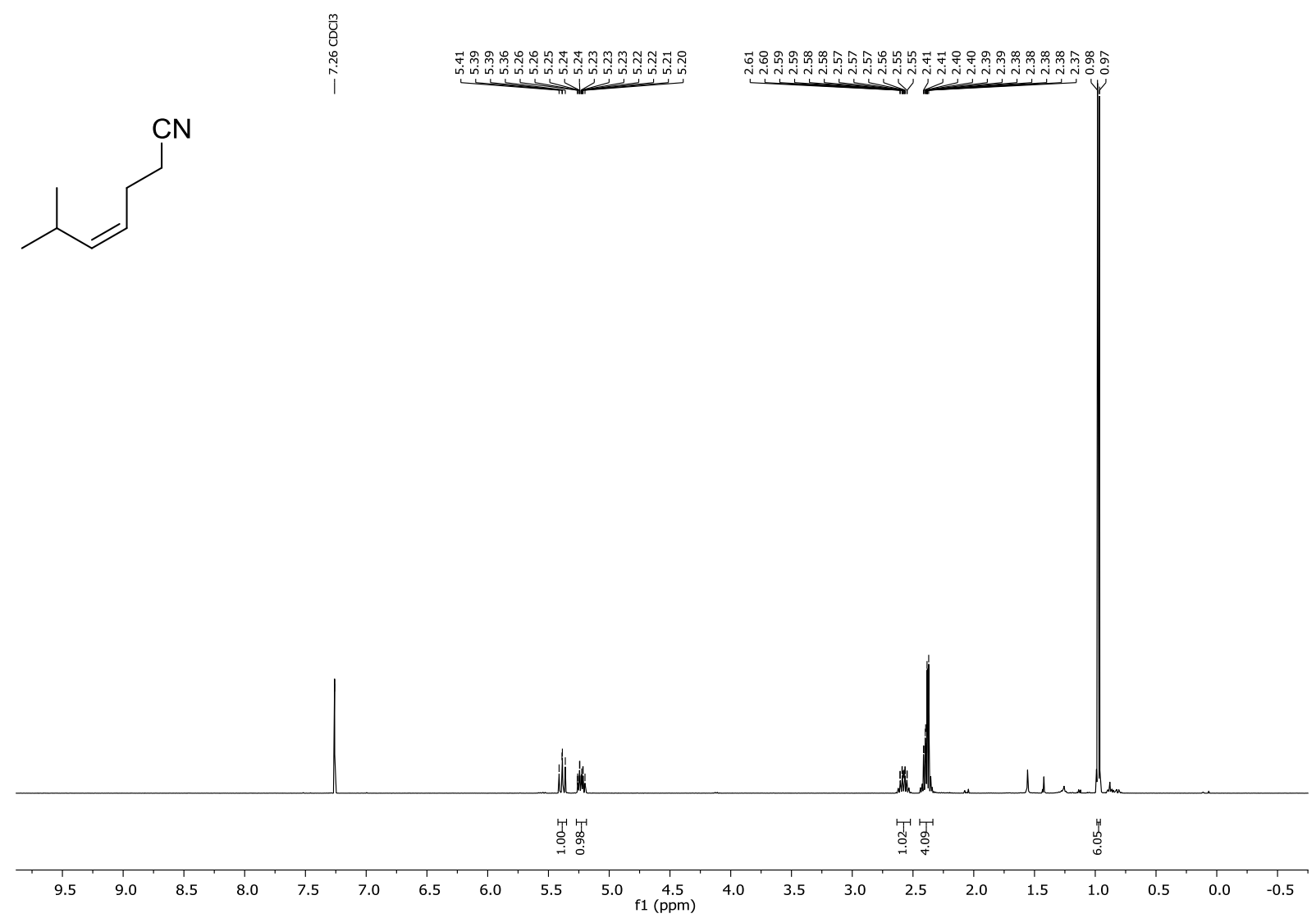

${ }^{13} \mathbf{C}$ NMR $\left(100 \mathrm{MHz}, \mathrm{CDCl}_{3}, 25^{\circ} \mathrm{C}\right)$ of $\mathbf{S 2 n}$
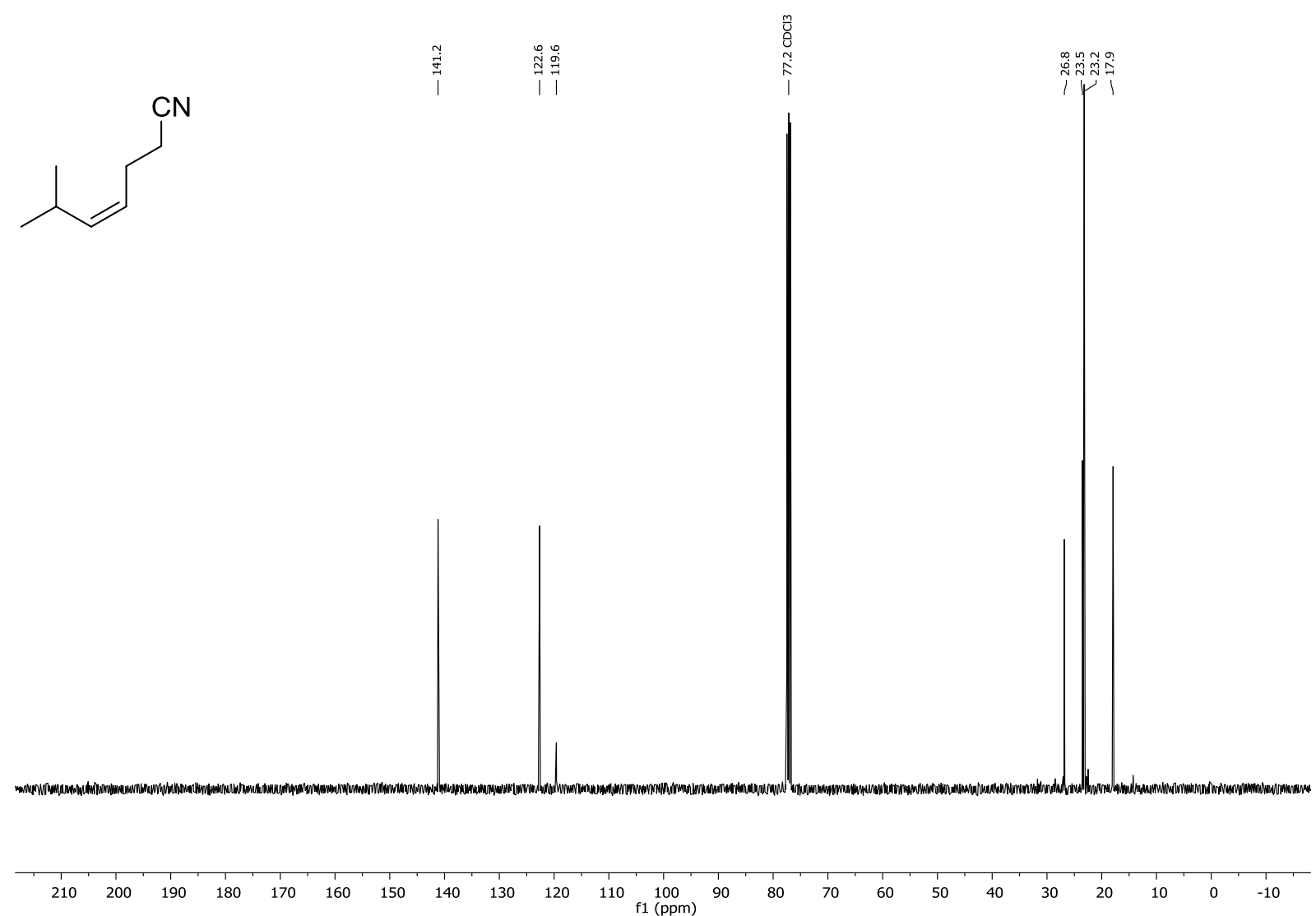
${ }^{\mathbf{1}} \mathbf{H} \mathbf{N M R}\left(400 \mathrm{MHz}, \mathrm{CDCl}_{3}, 25^{\circ} \mathrm{C}\right)$ of $\mathbf{1 n}$
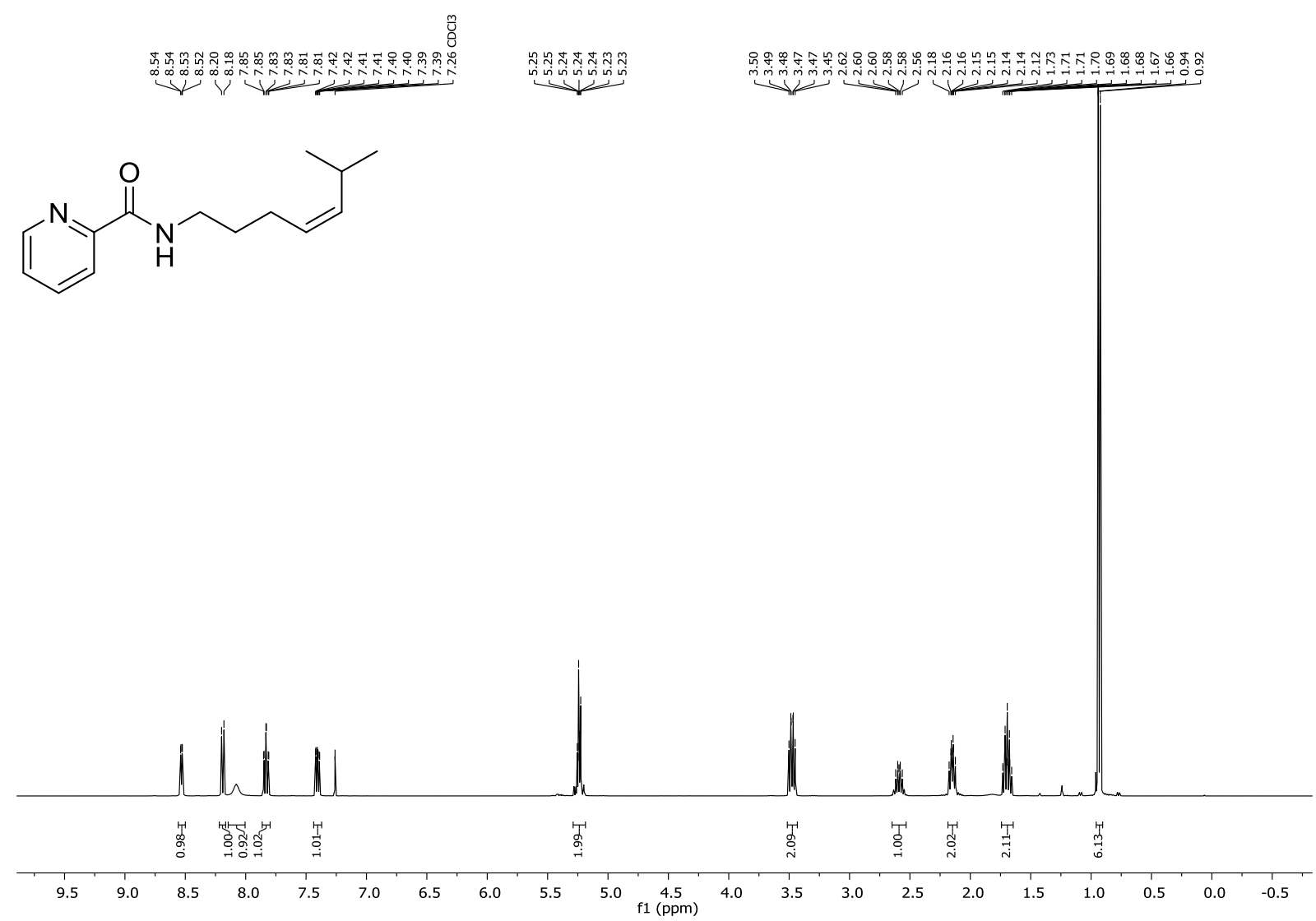

${ }^{13} \mathbf{C}$ NMR $\left(100 \mathrm{MHz}, \mathrm{CDCl}_{3}, 25{ }^{\circ} \mathrm{C}\right)$ of $\mathbf{1 n}$

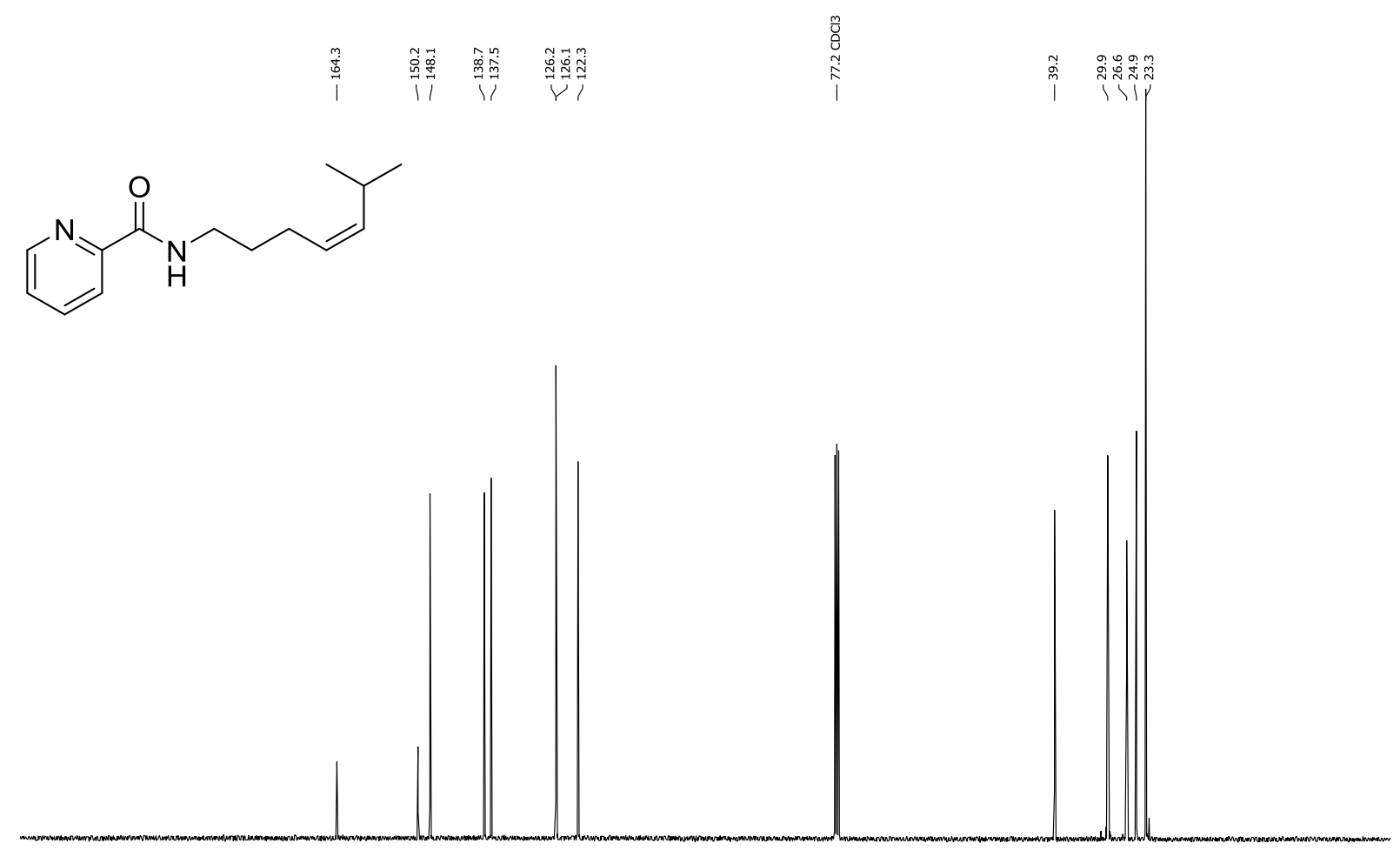

$\begin{array}{lllllllllllllllllllllllllllll}210 & 200 & 190 & 180 & 170 & 160 & 150 & 140 & 130 & 120 & 110 & 100 & 90 & 80 & 70 & 60 & 50 & 40 & 30 & 20 & 10 & 0 & -10\end{array}$ 
${ }^{1} \mathbf{H}$ NMR $\left(400 \mathrm{MHz}, \mathrm{CDCl}_{3}, 25^{\circ} \mathrm{C}\right)$ of $\mathbf{S 2 o}$

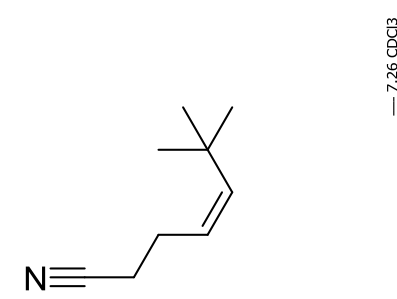

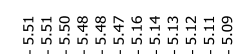

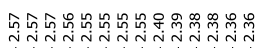
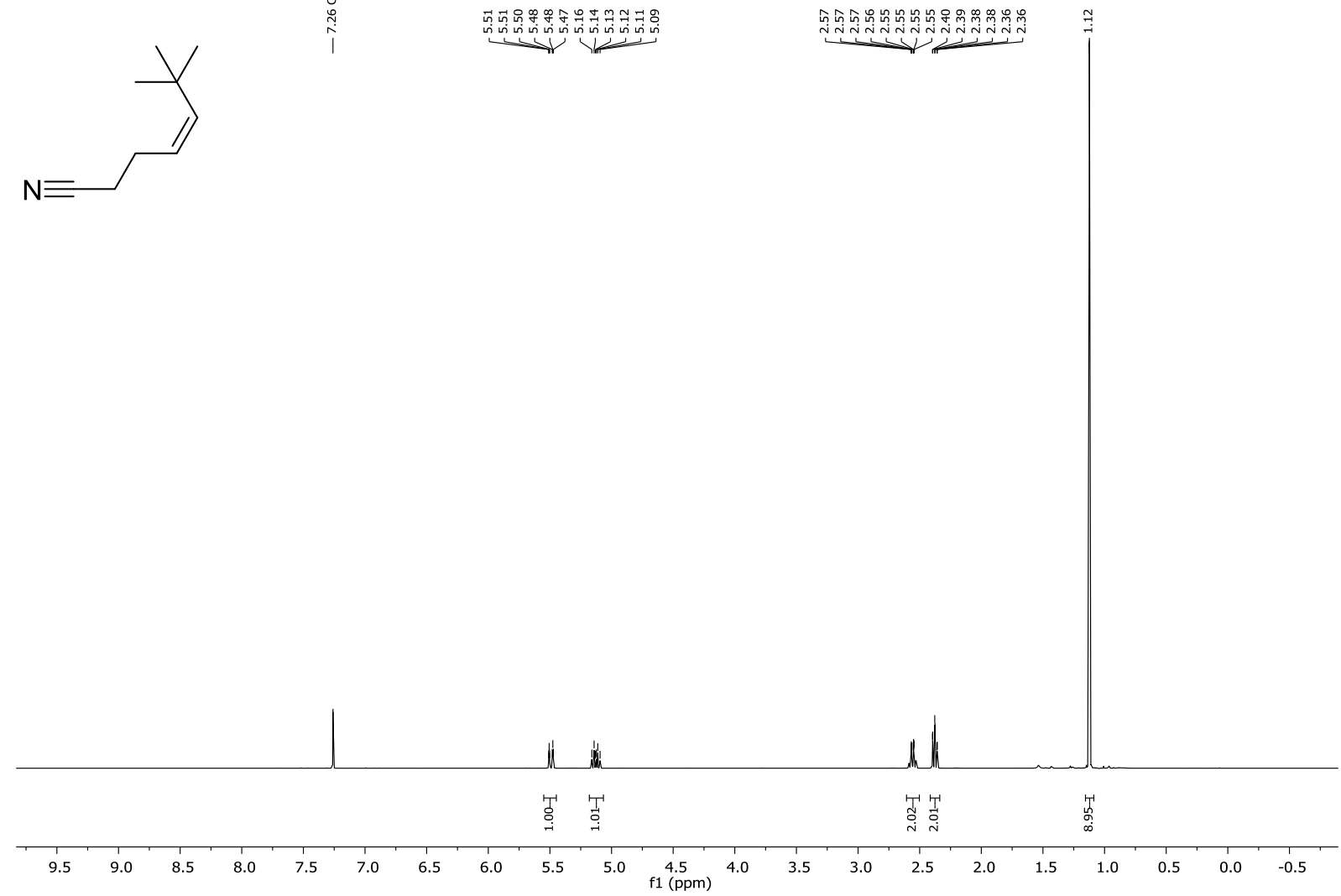

${ }^{13} \mathbf{C}$ NMR (100 MHz, $\left.\mathrm{CDCl}_{3}, 25^{\circ} \mathrm{C}\right)$ of $\mathbf{S 2 0}$

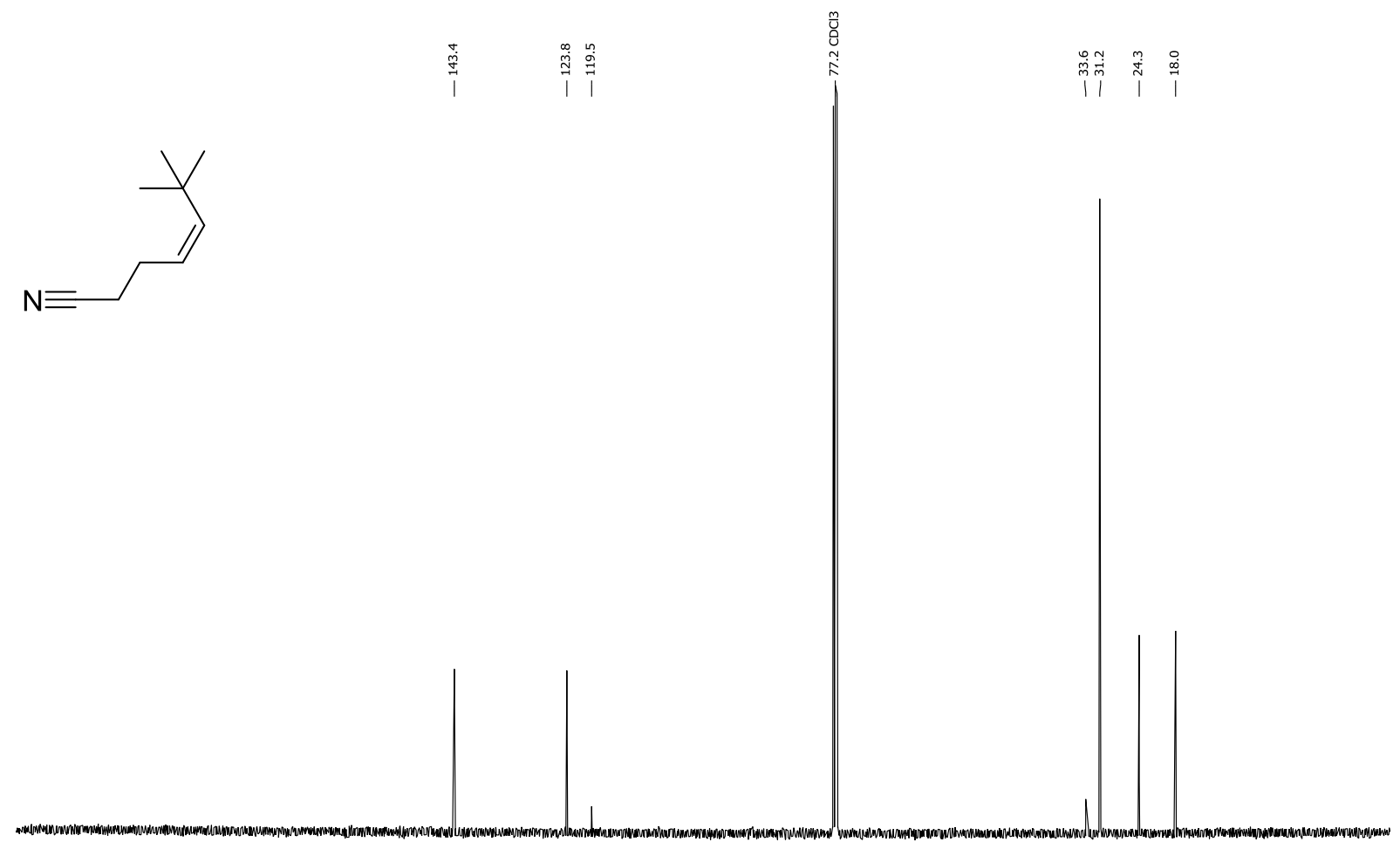

$\begin{array}{lllllllllllllllllllllllll}210 & 200 & 190 & 180 & 170 & 160 & 150 & 140 & 130 & 120 & 110 & 100 & 90 & 80 & 70 & 60 & 50 & 40 & 30 & 20 & 10 & 0 & -10\end{array}$ 
${ }^{\mathbf{1}} \mathbf{H}$ NMR $\left(400 \mathrm{MHz}, \mathrm{CDCl}_{3}, 25^{\circ} \mathrm{C}\right)$ of $\mathbf{1 o}$

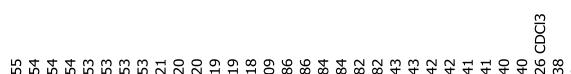

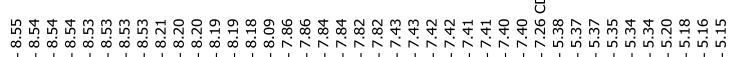

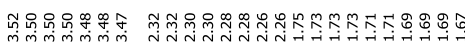

$\underbrace{(}_{\mathrm{N}}$

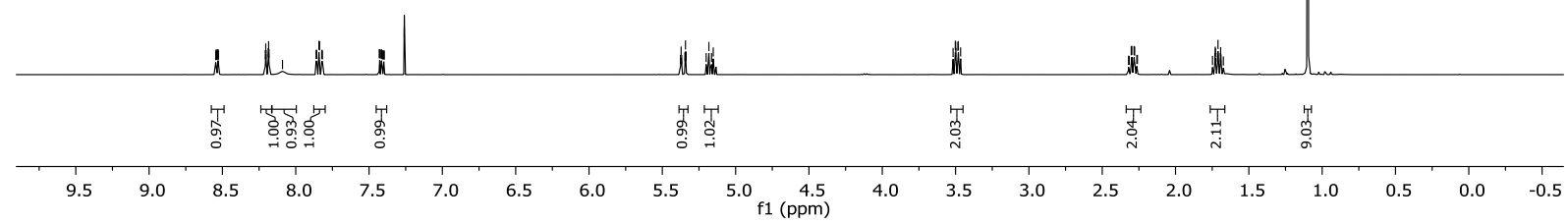

${ }^{13} \mathrm{C}$ NMR $\left(100 \mathrm{MHz}, \mathrm{CDCl}_{3}, 25{ }^{\circ} \mathrm{C}\right)$ of $\mathbf{1 o}$
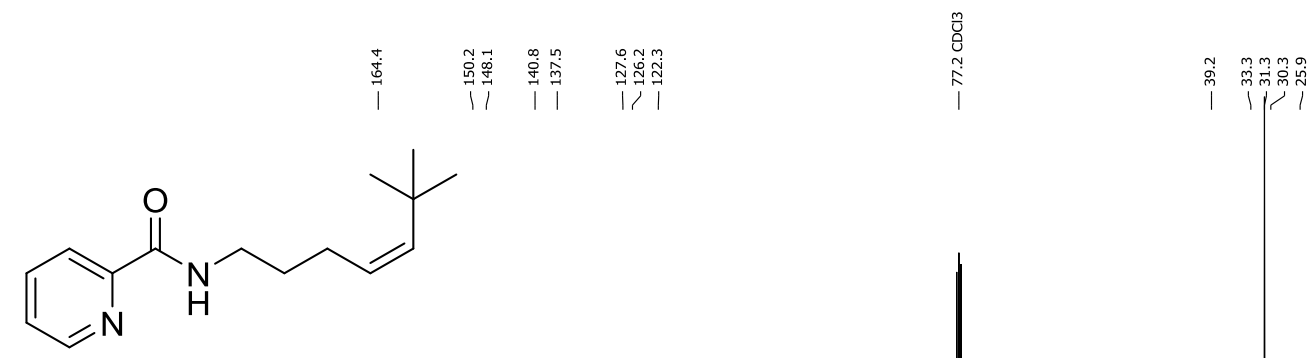

$\begin{array}{lllllllllllllllllllllll}210 & 200 & 190 & 180 & 170 & 160 & 150 & 140 & 130 & 120 & 110 & 100 & 90 & 80 & 70 & 60 & 50 & 40 & 30 & 20 & 10 & 0 & -10\end{array}$ 
${ }^{1} \mathbf{H}$ NMR $\left(400 \mathrm{MHz}, \mathrm{CDCl}_{3}, 25^{\circ} \mathrm{C}\right)$ of $\mathbf{S 2 p}$

葛

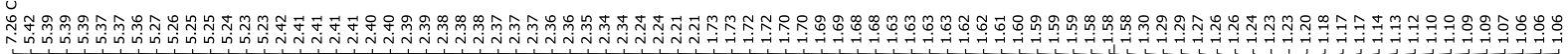<smiles>N#CCC/C=C\C1CCCCC1</smiles>

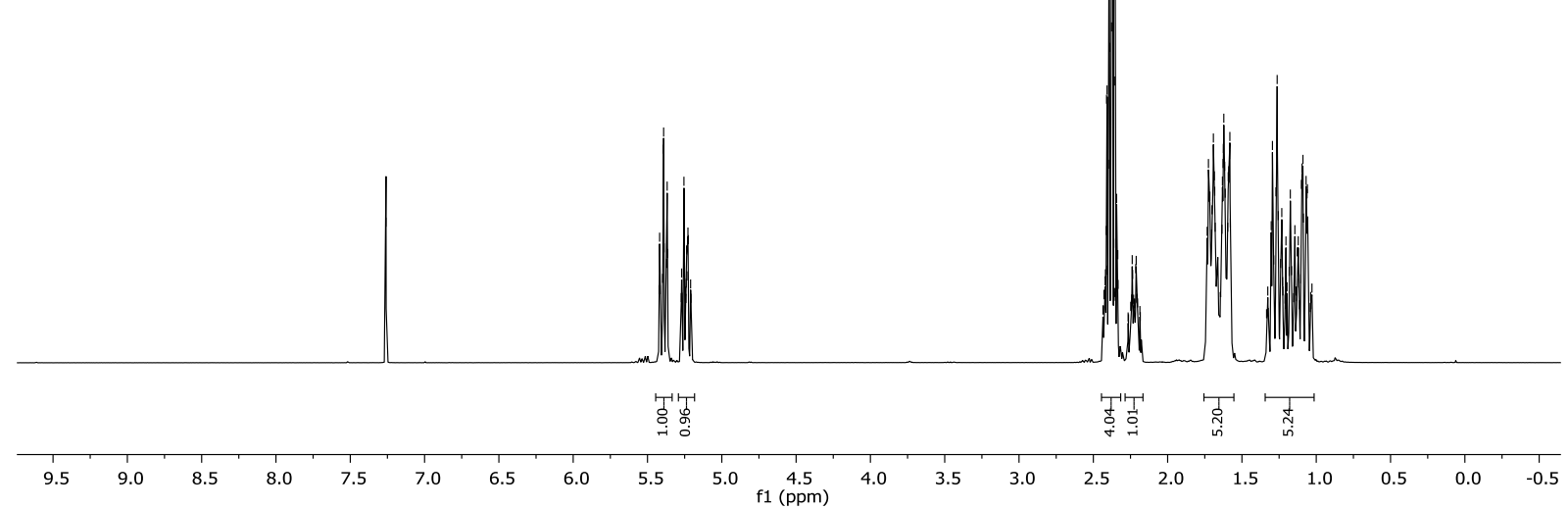

${ }^{13} \mathbf{C ~ N M R}\left(100 \mathrm{MHz}, \mathrm{CDCl}_{3}, 25^{\circ} \mathrm{C}\right)$ of $\mathbf{S 2 p}$
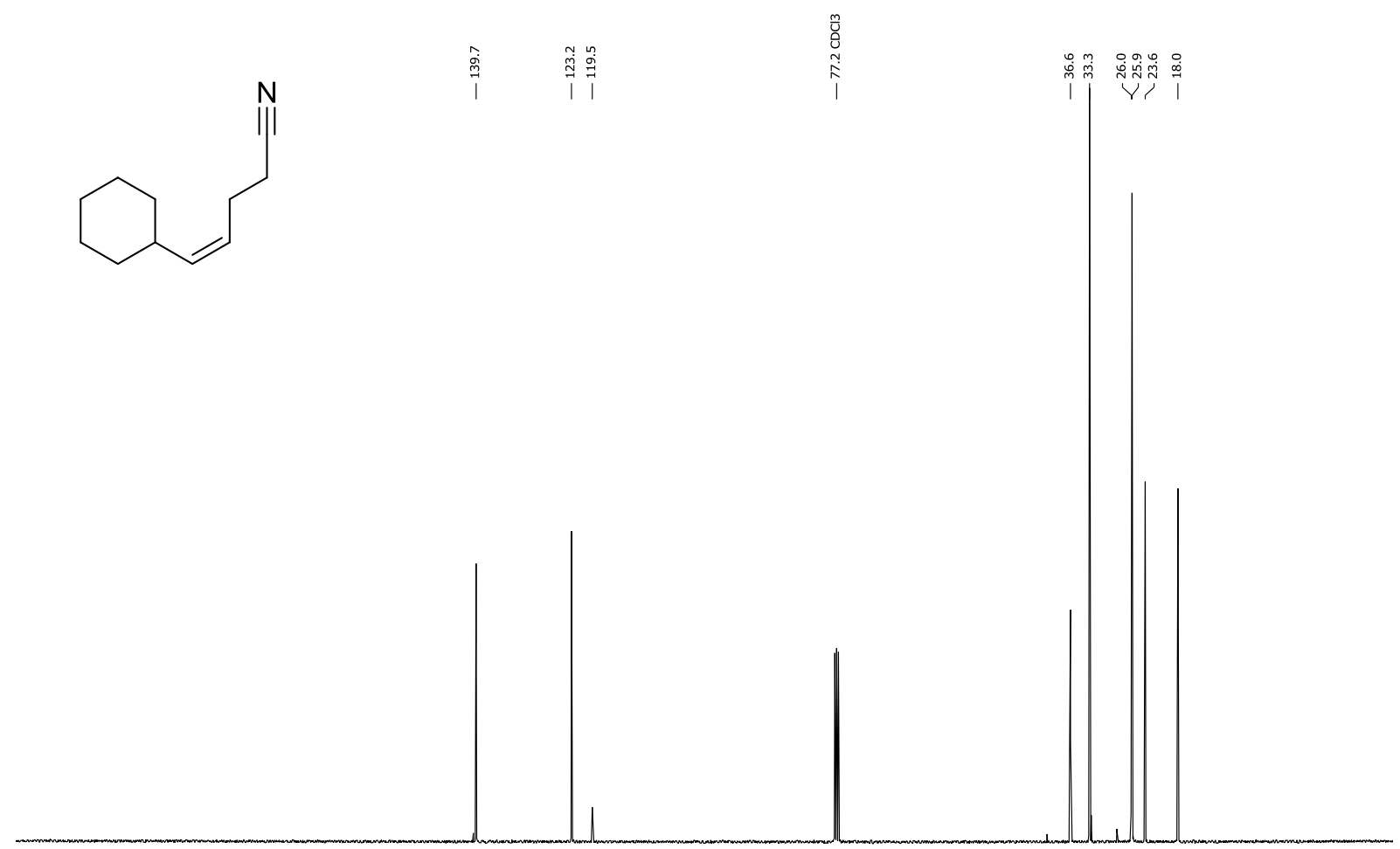

$\begin{array}{llllllllllllllllllllllllllllllll}210 & 200 & 190 & 180 & 170 & 160 & 150 & 140 & 130 & 120 & 110 & 100 & 90 & 80 & 70 & 60 & 50 & 40 & 30 & 20 & 10 & 0 & -10 & 10\end{array}$ 
${ }^{1} \mathbf{H}$ NMR $\left(400 \mathrm{MHz}, \mathrm{CDCl}_{3}, 25^{\circ} \mathrm{C}\right)$ of $\mathbf{1 p}$

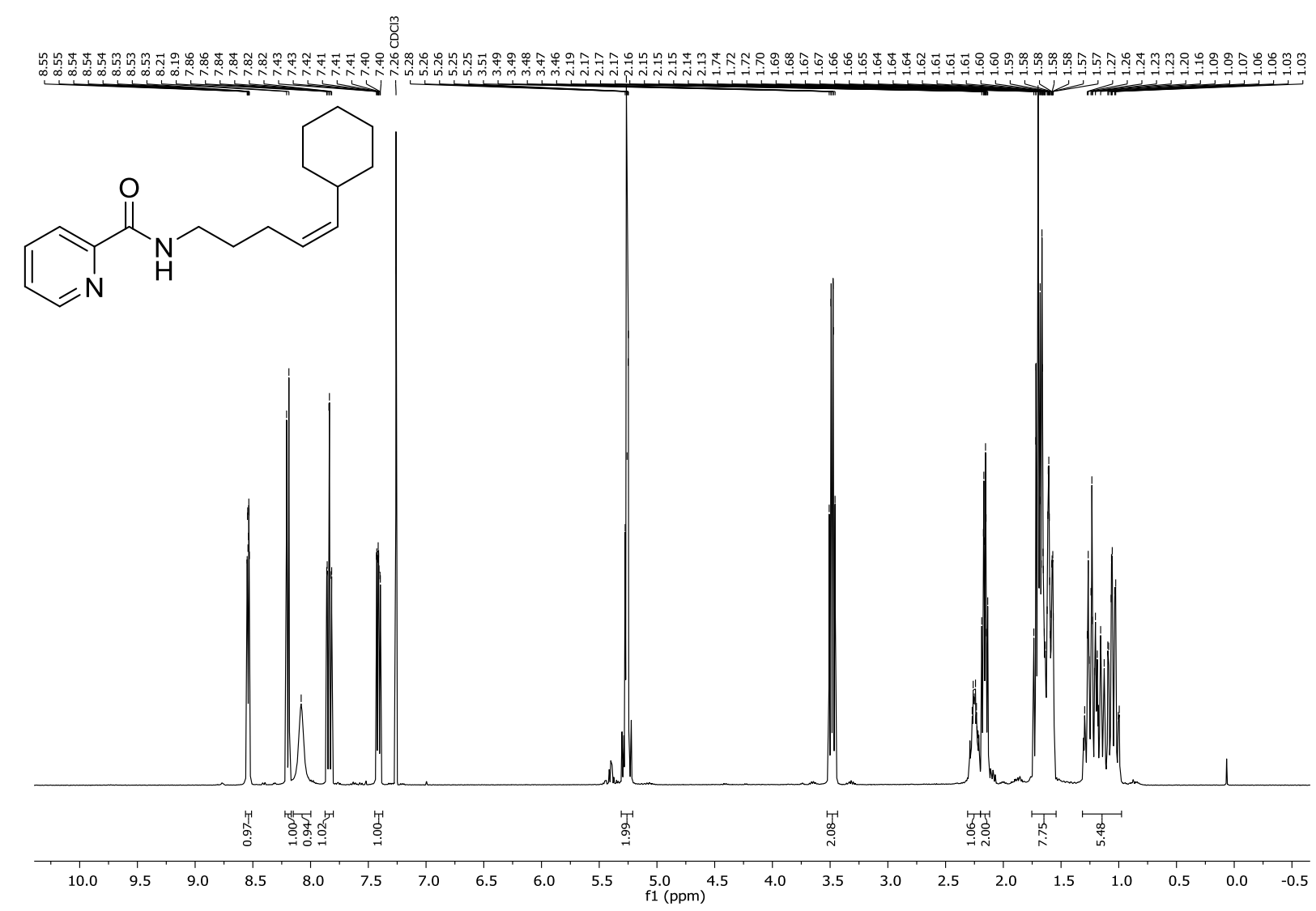

${ }^{13} \mathbf{C}$ NMR $\left(100 \mathrm{MHz}, \mathrm{CDCl}_{3}, 25^{\circ} \mathrm{C}\right)$ of $\mathbf{1 p}$

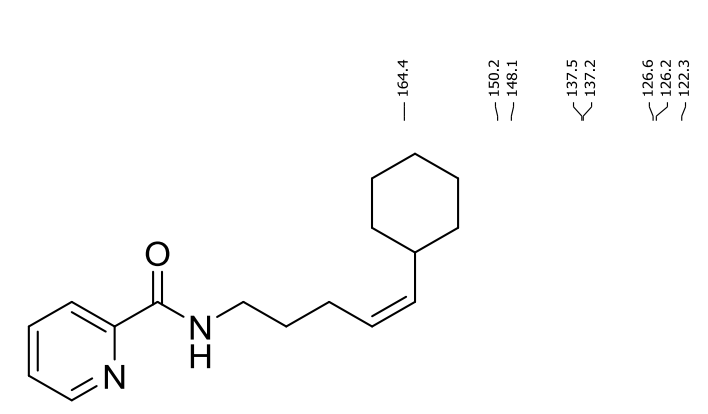

$\begin{array}{llllllllllllllllllllllllll}210 & 200 & 190 & 180 & 170 & 160 & 150 & 140 & 130 & 120 & 110 & 100 & 90 & 80 & 70 & 60 & 50 & 40 & 30 & 20 & 10 & 0 & -10 & \end{array}$ 
${ }^{\mathbf{1}} \mathbf{H}$ NMR (400 MHz, $\left.\mathrm{CDCl}_{3}, 25^{\circ} \mathrm{C}\right)$ of $\mathbf{S 2 q}$<smiles>CC(C)(C)OC(=O)N1CCC(/C=C\CCC#N)CC1</smiles>

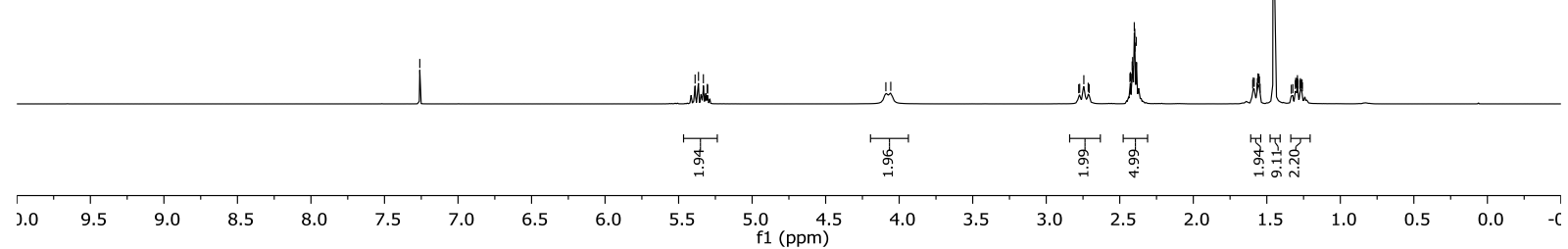

${ }^{13} \mathbf{C}$ NMR $\left(100 \mathrm{MHz}, \mathrm{CDCl}_{3}, 2{ }^{\circ} \mathrm{C}\right)$ of $\mathbf{S 2 q}$

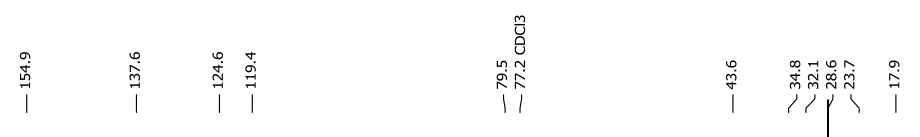<smiles>CC(C)(C)OC(=O)N1CCC(/C=C\CCC#N)CC1</smiles>

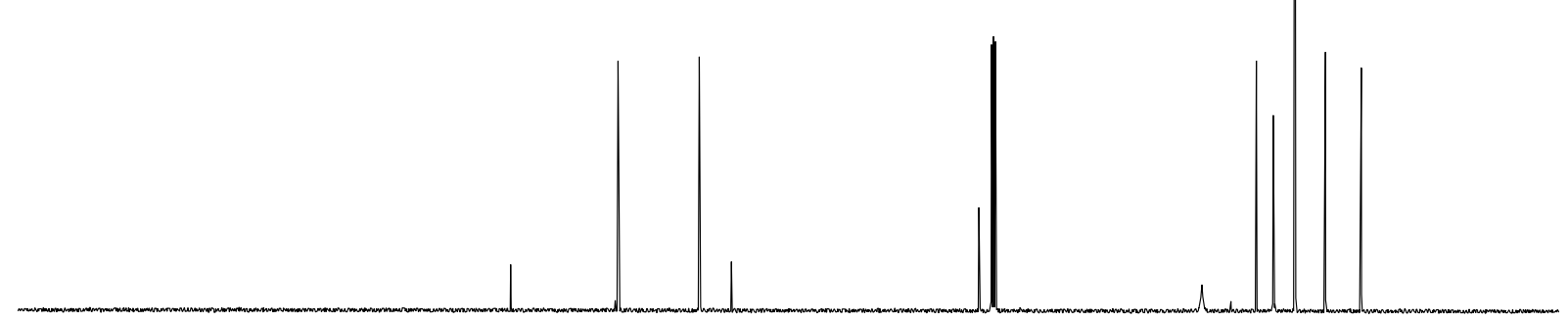

$\begin{array}{lllllllllllllllllllllllllllllllllll}230 & 220 & 210 & 200 & 190 & 180 & 170 & 160 & 150 & 140 & 130 & 120 & 110 & 100 & 90 & 80 & 70 & 60 & 50 & 40 & 30 & 20 & 10 & 0 & -10\end{array}$ 
${ }^{\mathbf{1}} \mathbf{H}$ NMR $\left(400 \mathrm{MHz}, \mathrm{CDCl}_{3}, 25^{\circ} \mathrm{C}\right)$ of $\mathbf{1 q}$

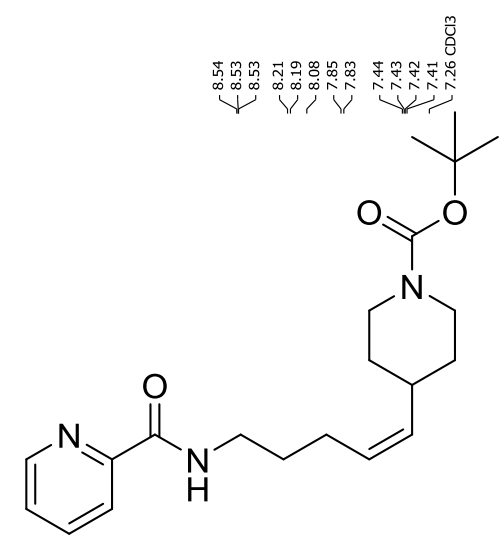

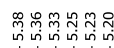

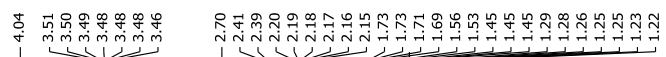

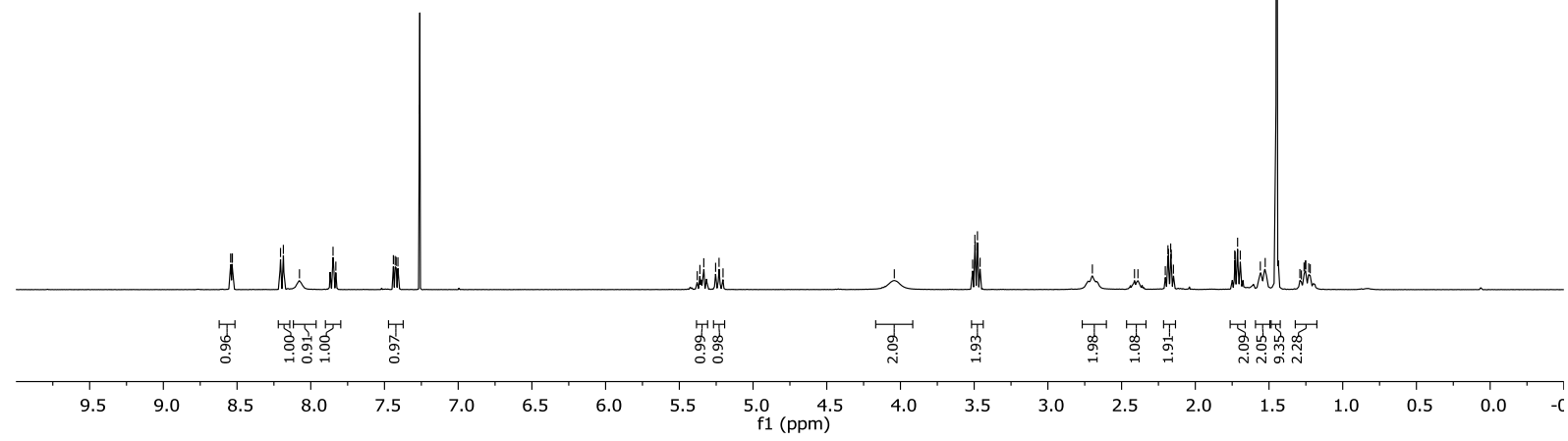

${ }^{13} \mathbf{C ~ N M R}\left(100 \mathrm{MHz}, \mathrm{CDCl}_{3}, 25^{\circ} \mathrm{C}\right)$ of $\mathbf{1 q}$
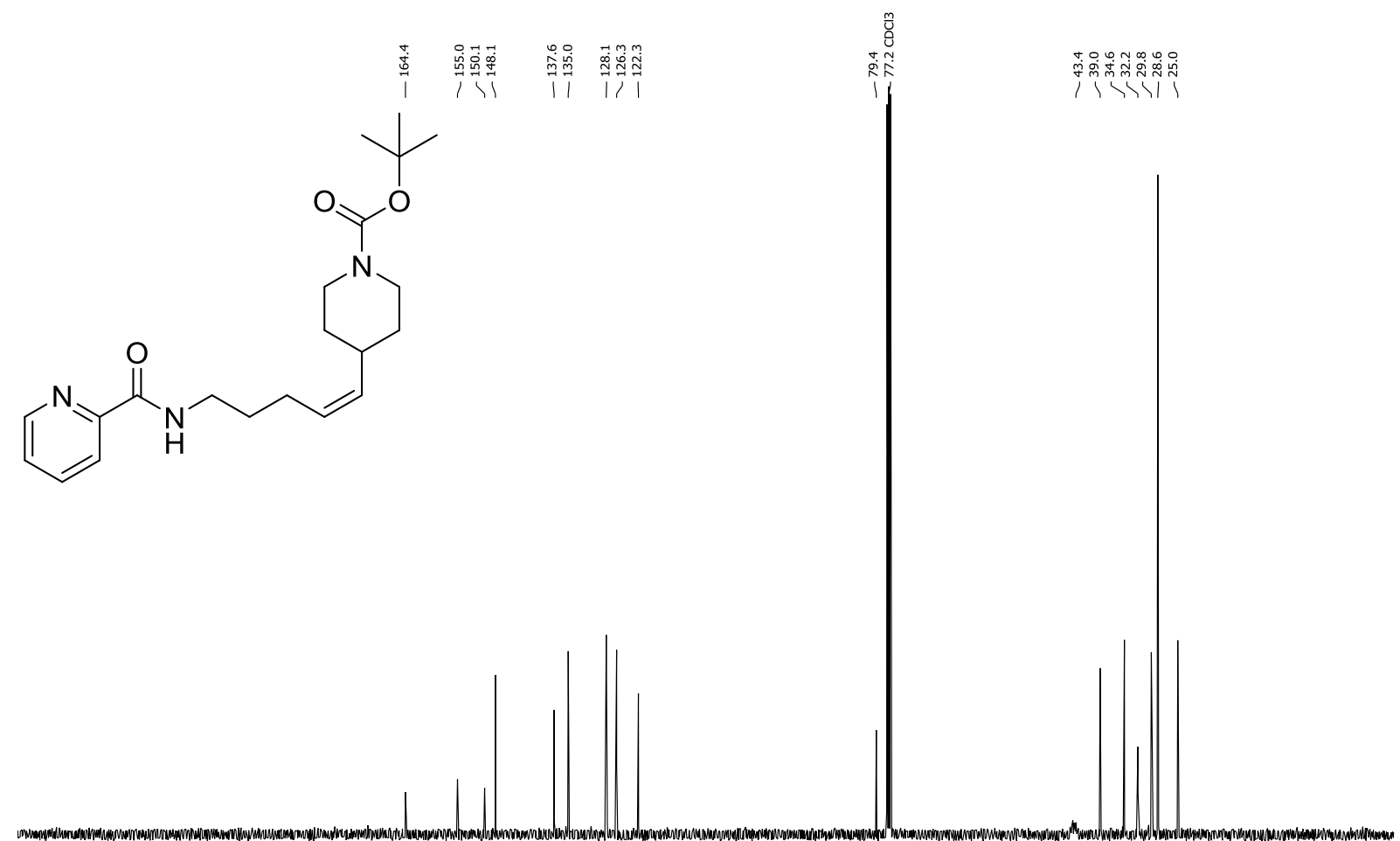

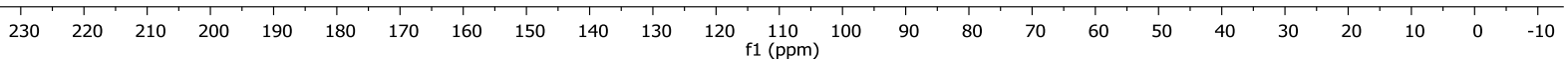


${ }^{1} \mathbf{H}$ NMR $\left(400 \mathrm{MHz}, \mathrm{CDCl}_{3}, 25^{\circ} \mathrm{C}\right)$ of $\mathbf{1 r}$

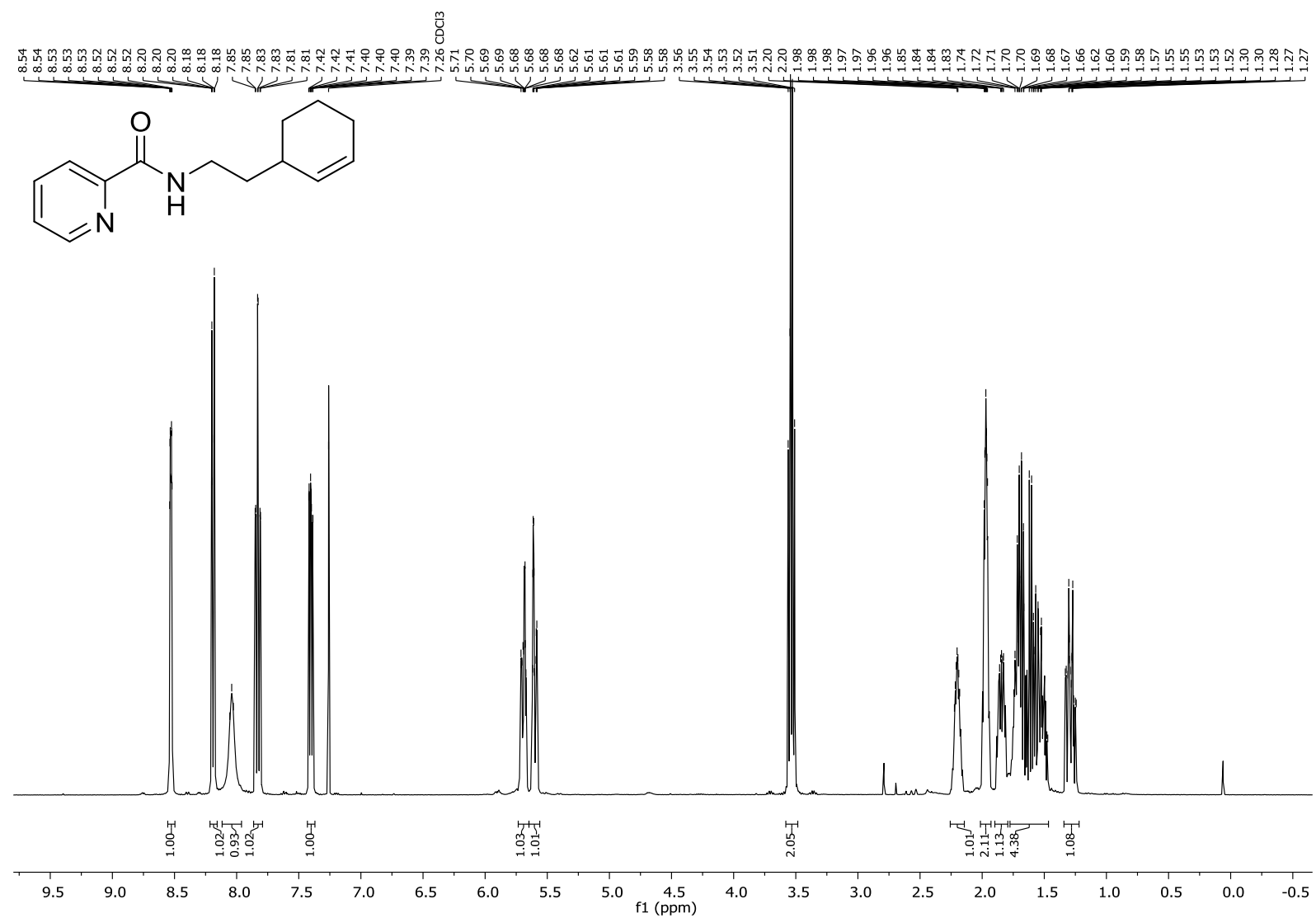

$\left.{ }^{13} \mathbf{C ~ N M R ~ ( 1 0 0 ~ M H z , ~} \mathrm{CDCl}_{3}, 25{ }^{\circ} \mathrm{C}\right)$ of $\mathbf{1 r}$

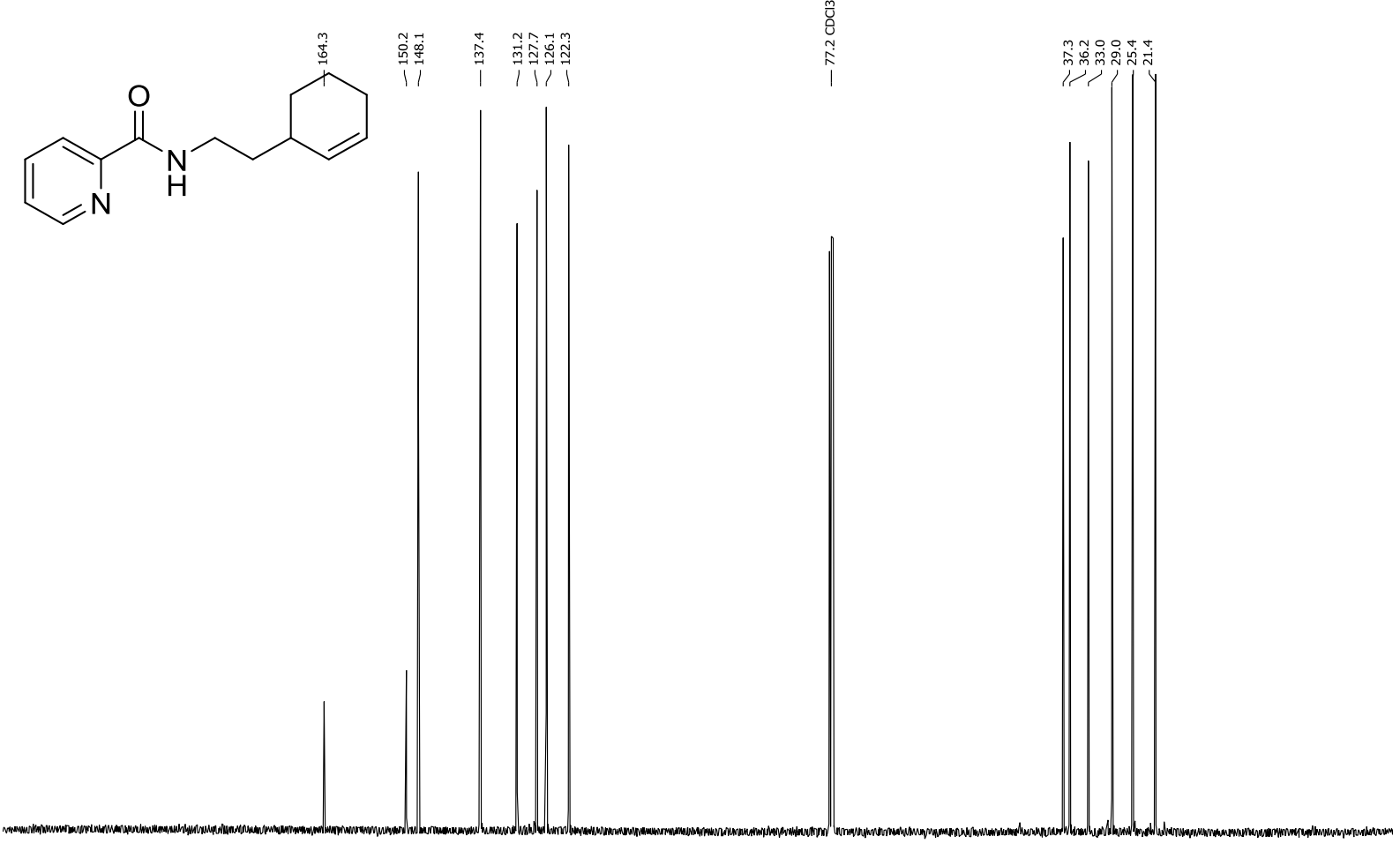

$\begin{array}{llllllllllllllllllllllllll}210 & 200 & 190 & 180 & 170 & 160 & 150 & 140 & 130 & 120 & 110 & 100 & 90 & 80 & 70 & 60 & 50 & 40 & 30 & 20 & 10 & 0 & -10\end{array}$ 
${ }^{1} \mathbf{H}$ NMR $\left(400 \mathrm{MHz}, \mathrm{CDCl}_{3}, 25^{\circ} \mathrm{C}\right)$ of $\mathbf{S 2 s}$

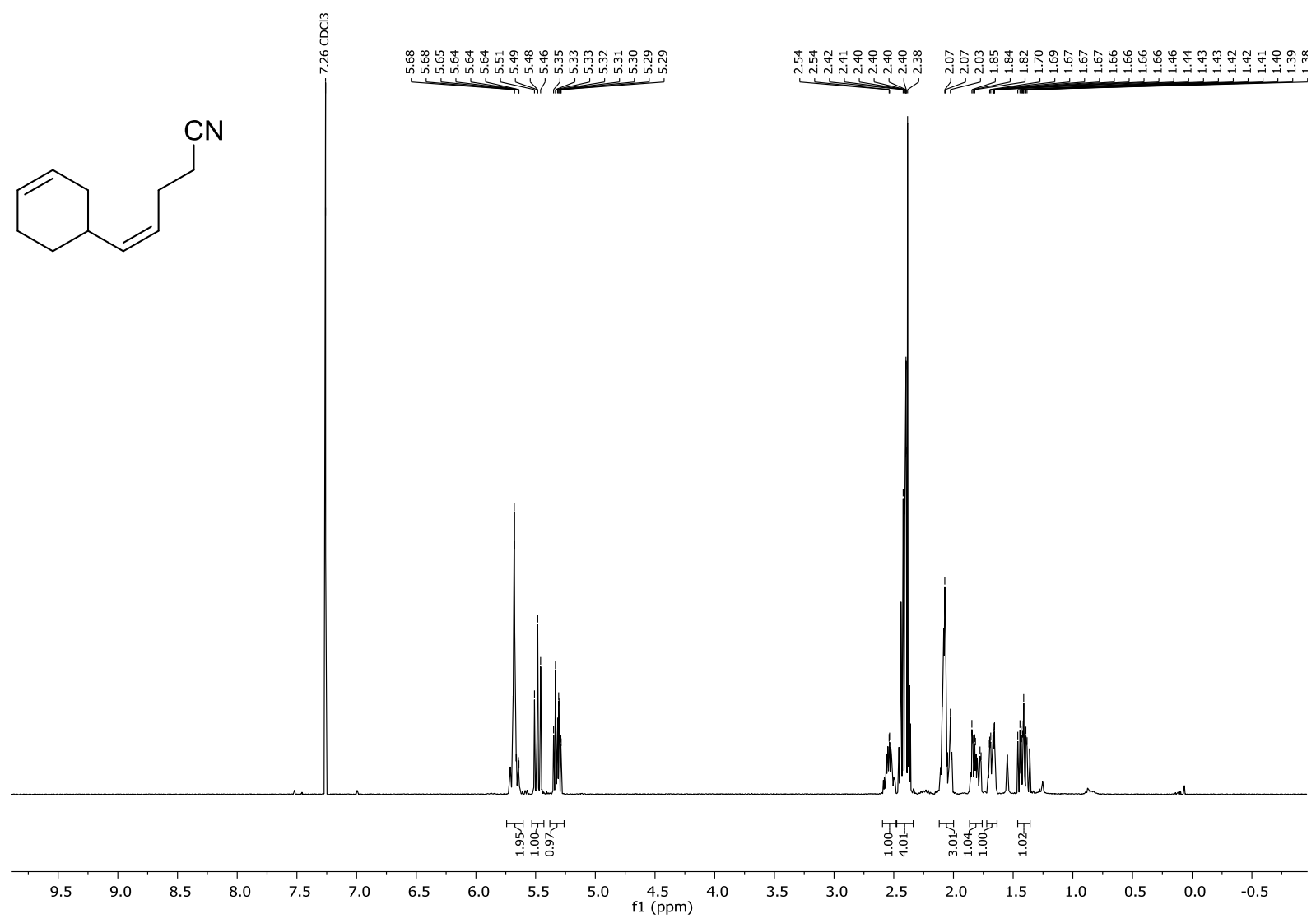

${ }^{13} \mathbf{C}$ NMR (100 MHz, $\left.\mathrm{CDCl}_{3}, 25{ }^{\circ} \mathrm{C}\right)$ of $\mathbf{S 2} \mathbf{s}$<smiles>N#CCC/C=C\C1CC=CCC1</smiles>

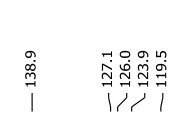

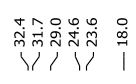
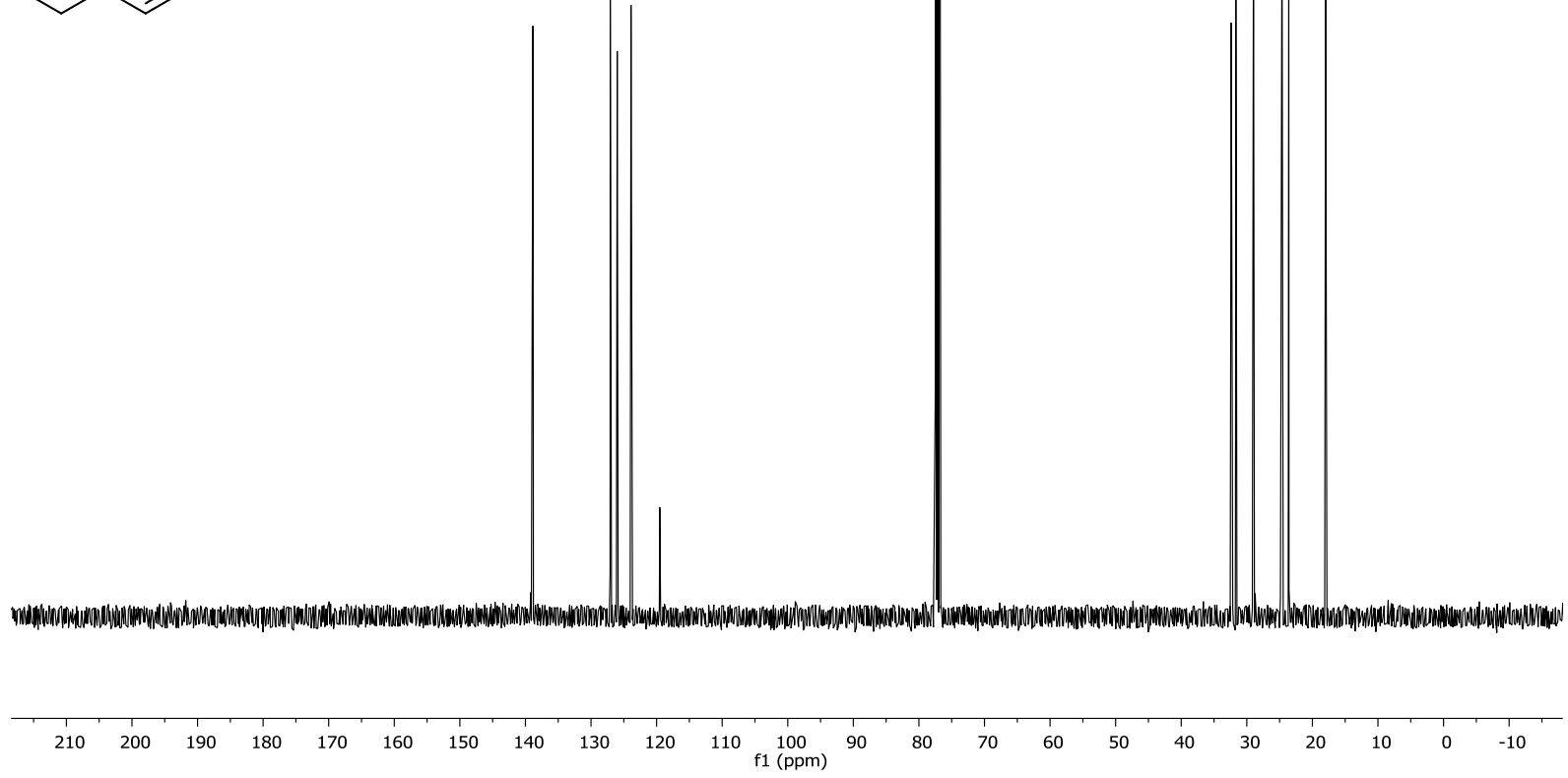

S108 
${ }^{\mathbf{1}} \mathbf{H}$ NMR $\left(500 \mathrm{MHz}, \mathrm{CDCl}_{3}, 25^{\circ} \mathrm{C}\right)$ of $\mathbf{1 s}$

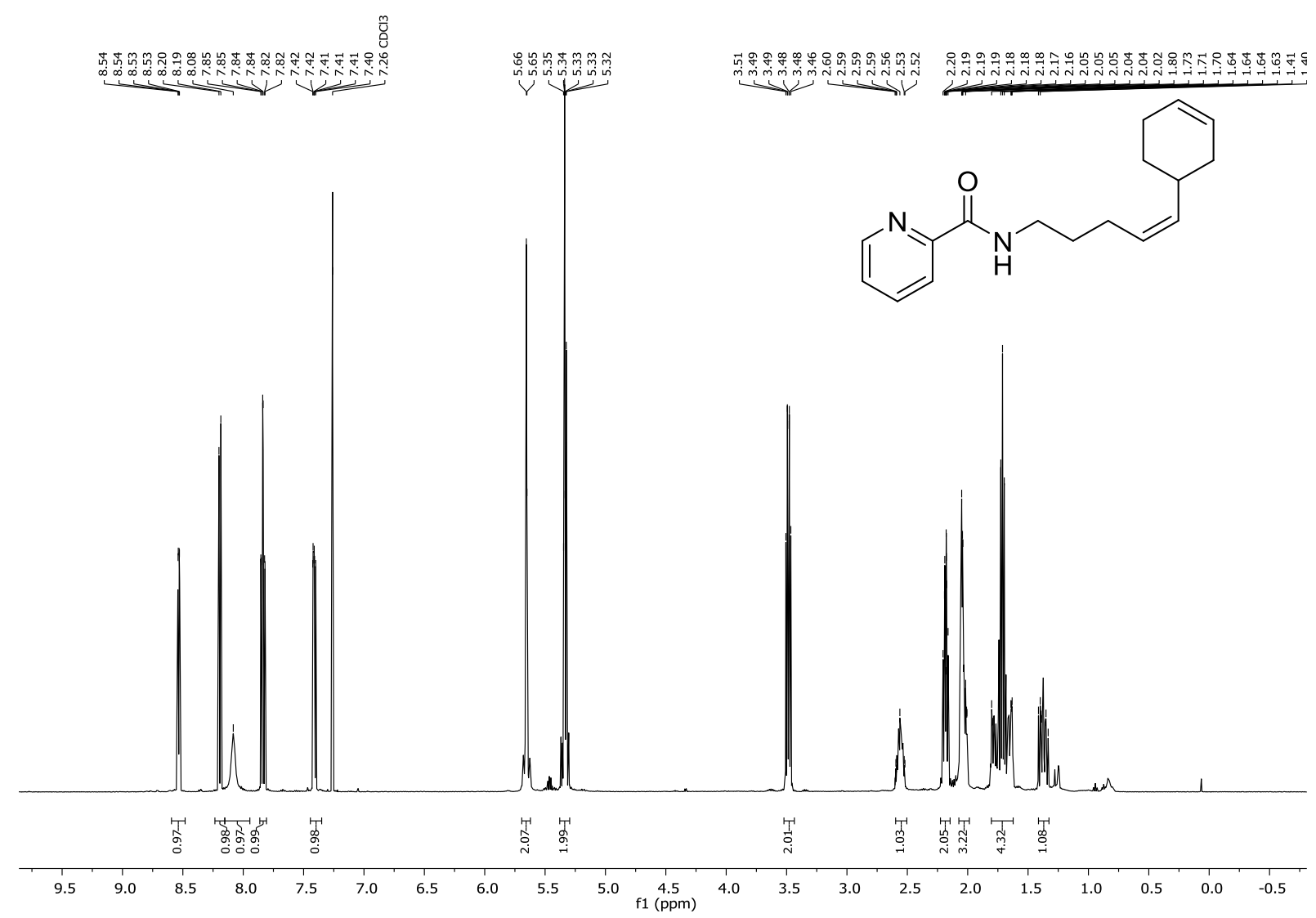

${ }^{13} \mathrm{C}$ NMR $\left(126 \mathrm{MHz}, \mathrm{CDCl}_{3}, 25^{\circ} \mathrm{C}\right)$ of $1 \mathrm{~s}$

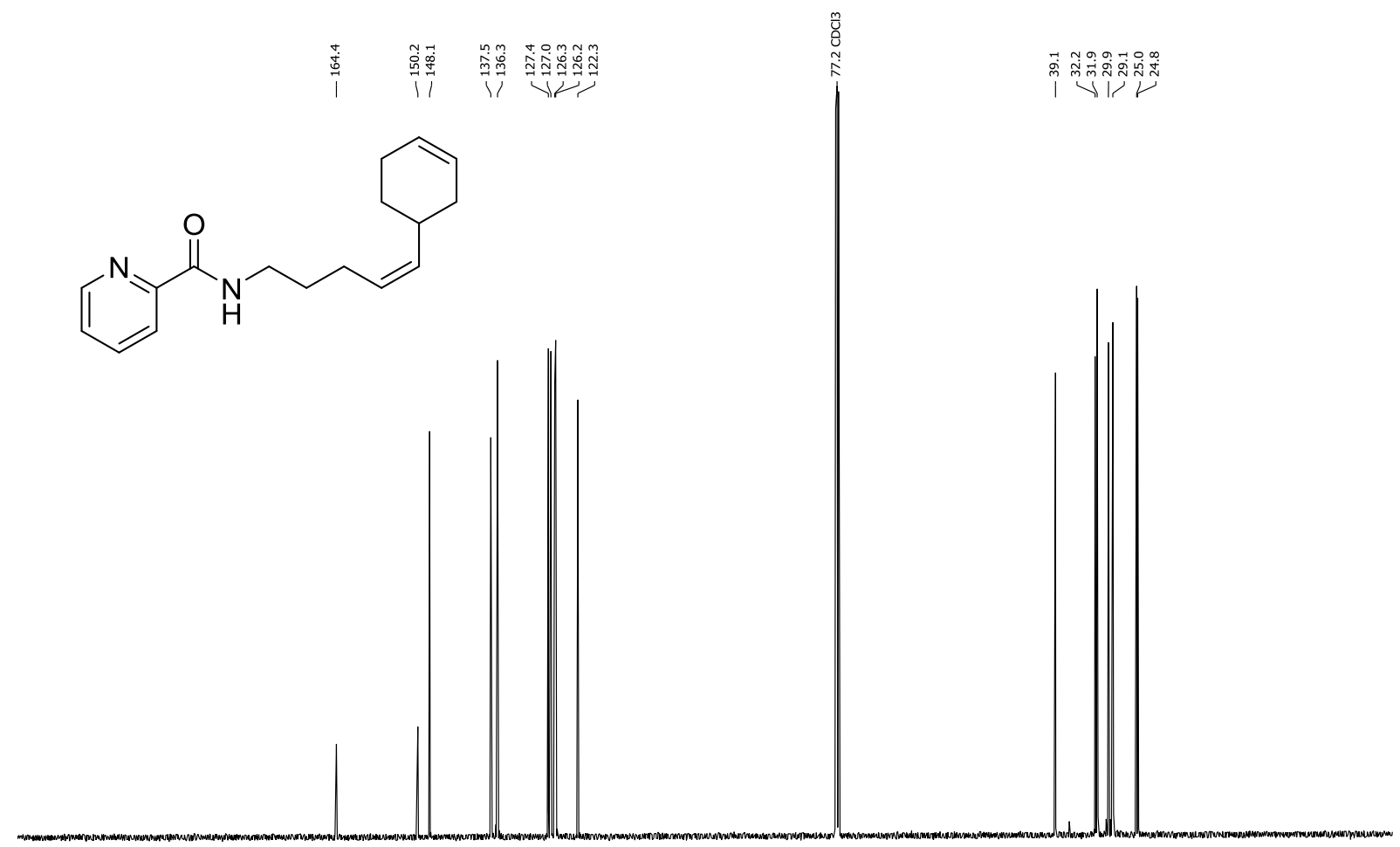

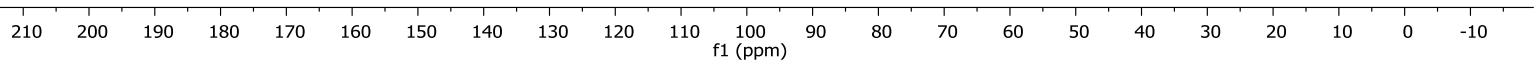


${ }^{1} \mathbf{H}$ NMR $\left(400 \mathrm{MHz}, \mathrm{CD}_{2} \mathrm{Cl}_{2}, 25^{\circ} \mathrm{C}\right)$ of $\mathbf{S 2 t}$

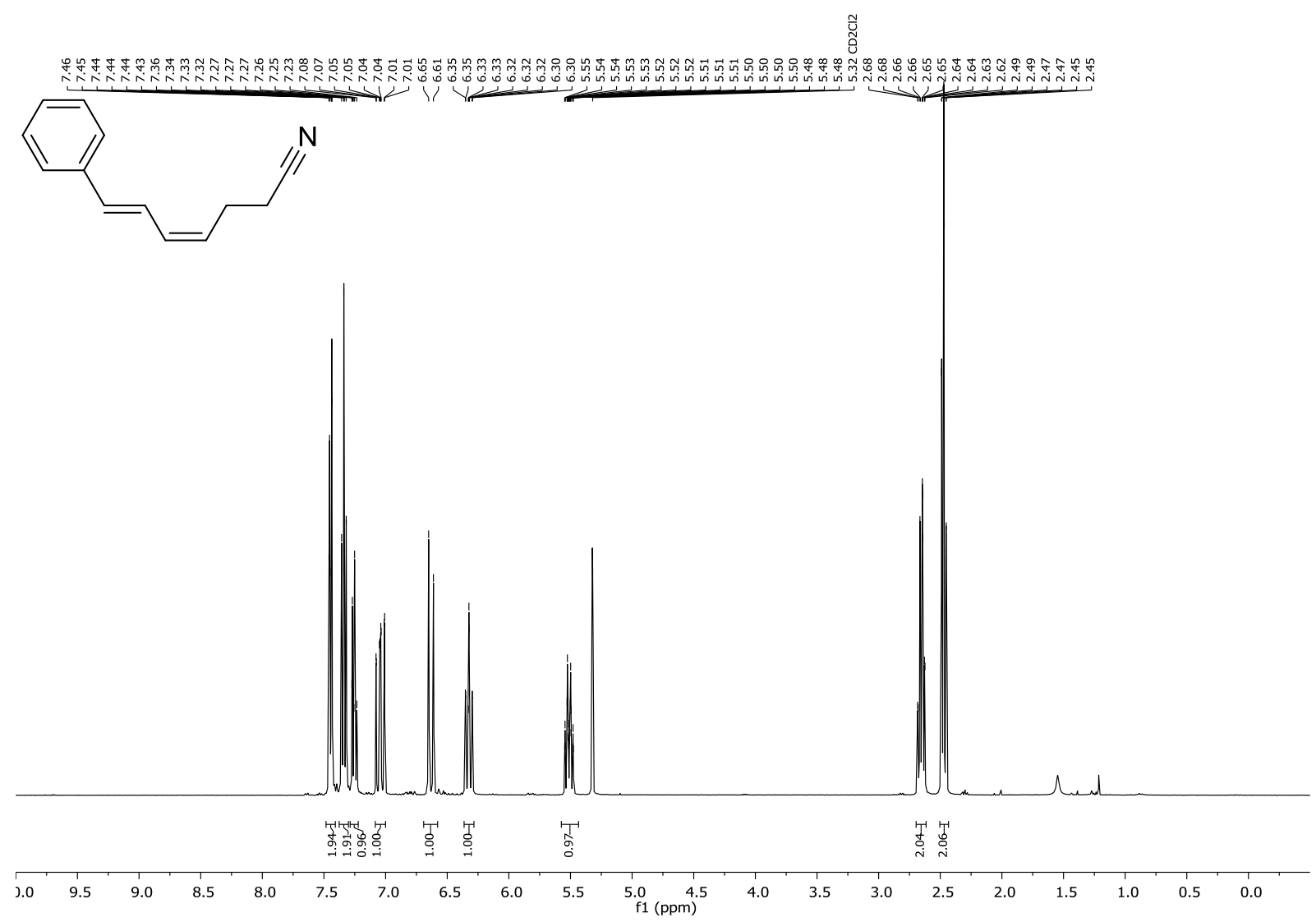

${ }^{13} \mathbf{C} \mathbf{N M R}\left(100 \mathrm{MHz}, \mathrm{CD}_{2} \mathrm{Cl}_{2}, 25{ }^{\circ} \mathrm{C}\right)$ of $\mathbf{S 2 t}$

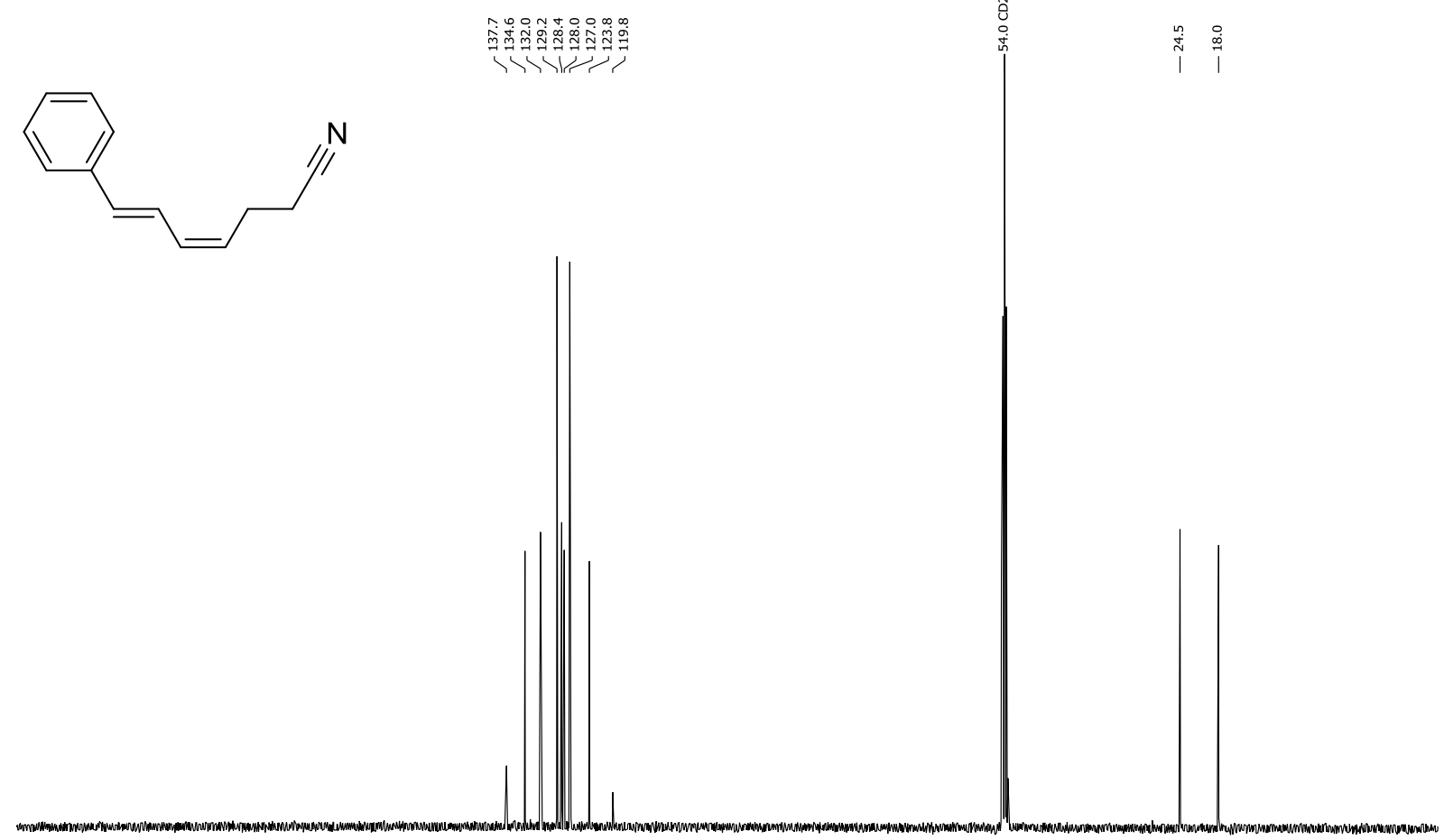

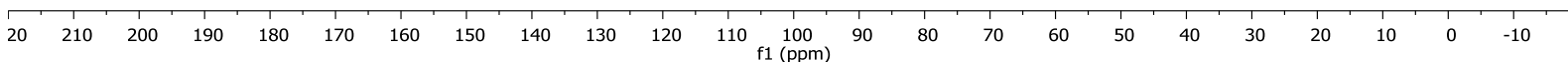


${ }^{\mathbf{1}} \mathbf{H}$ NMR $\left(400 \mathrm{MHz}, \mathrm{CD}_{2} \mathrm{Cl}_{2}, 25^{\circ} \mathrm{C}\right)$ of $\mathbf{1 t}$

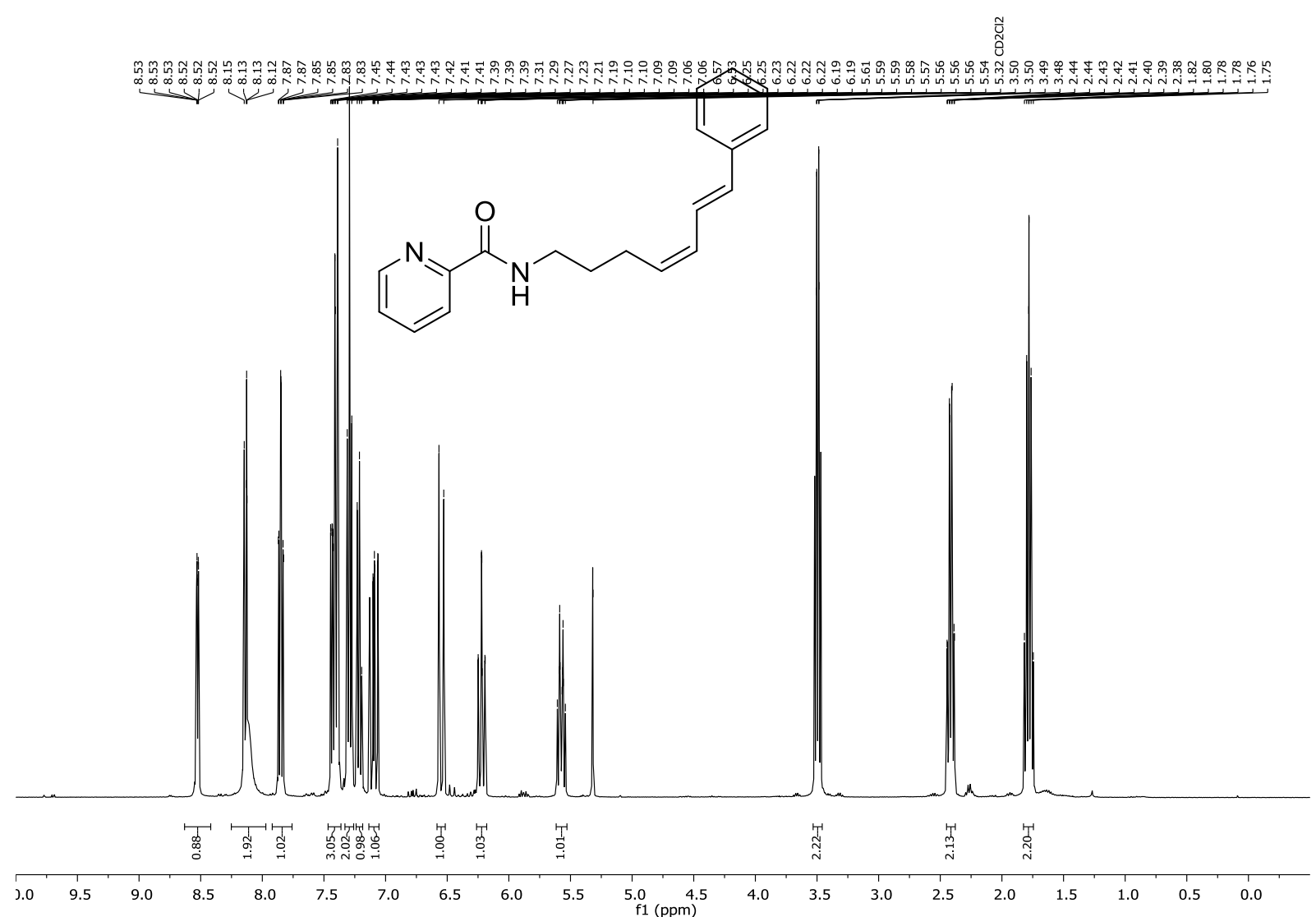

${ }^{13} \mathbf{C}$ NMR $\left(100 \mathrm{MHz}, \mathrm{CD}_{2} \mathrm{Cl}_{2}, 25{ }^{\circ} \mathrm{C}\right)$ of $\mathbf{1 t}$

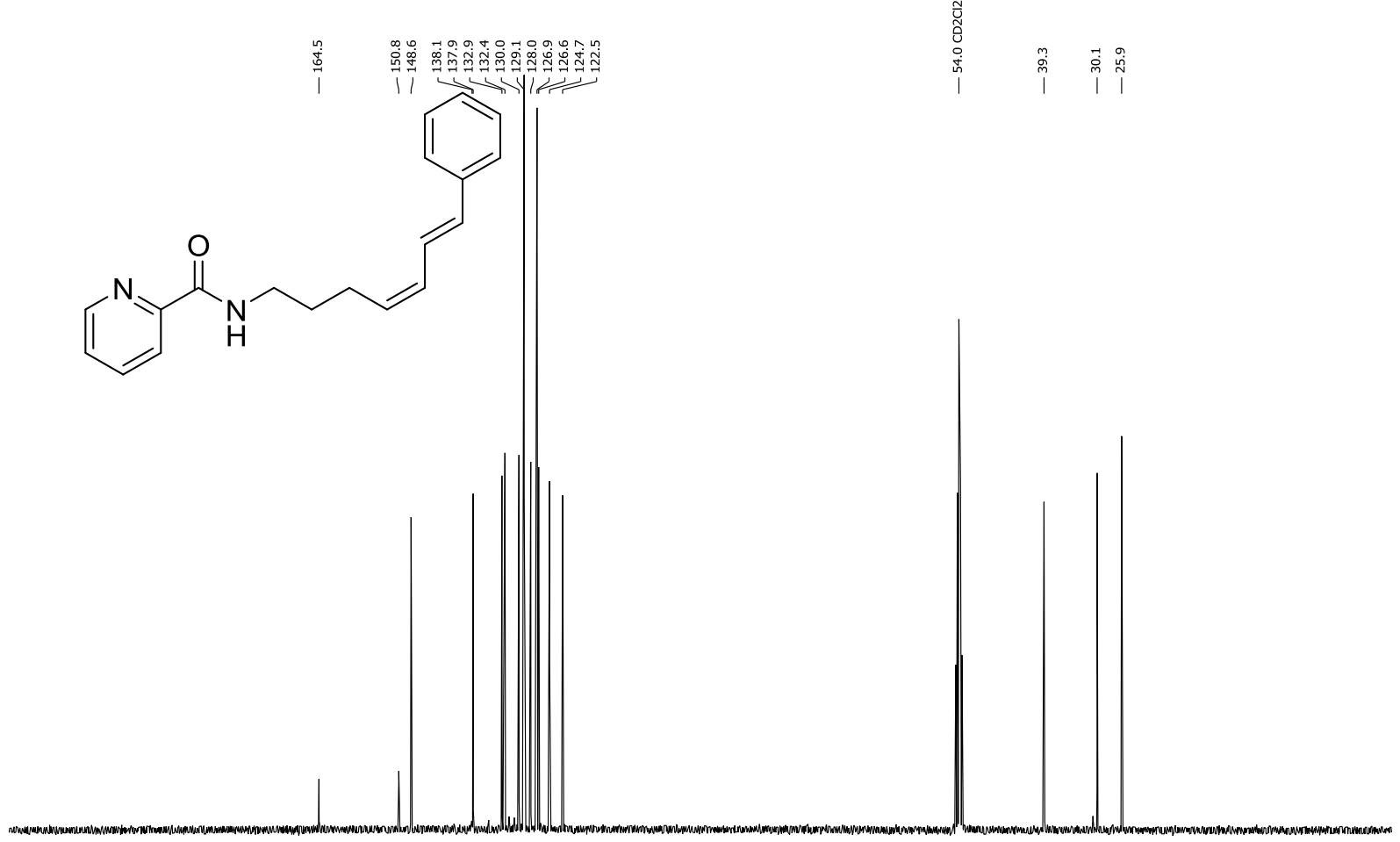

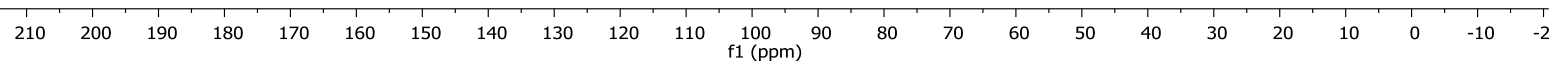


${ }^{\mathbf{1}} \mathbf{H}$ NMR $\left(400 \mathrm{MHz}, \mathrm{CDCl}_{3}, 25^{\circ} \mathrm{C}\right)$ of $\mathbf{1 u}$

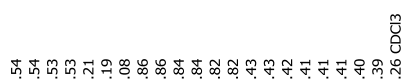

年

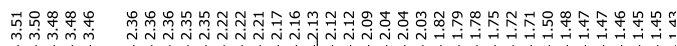<smiles>C=C(C)[C@H]1CC=C(/C=C\CCCNC(=O)c2ccccn2)CC1</smiles>

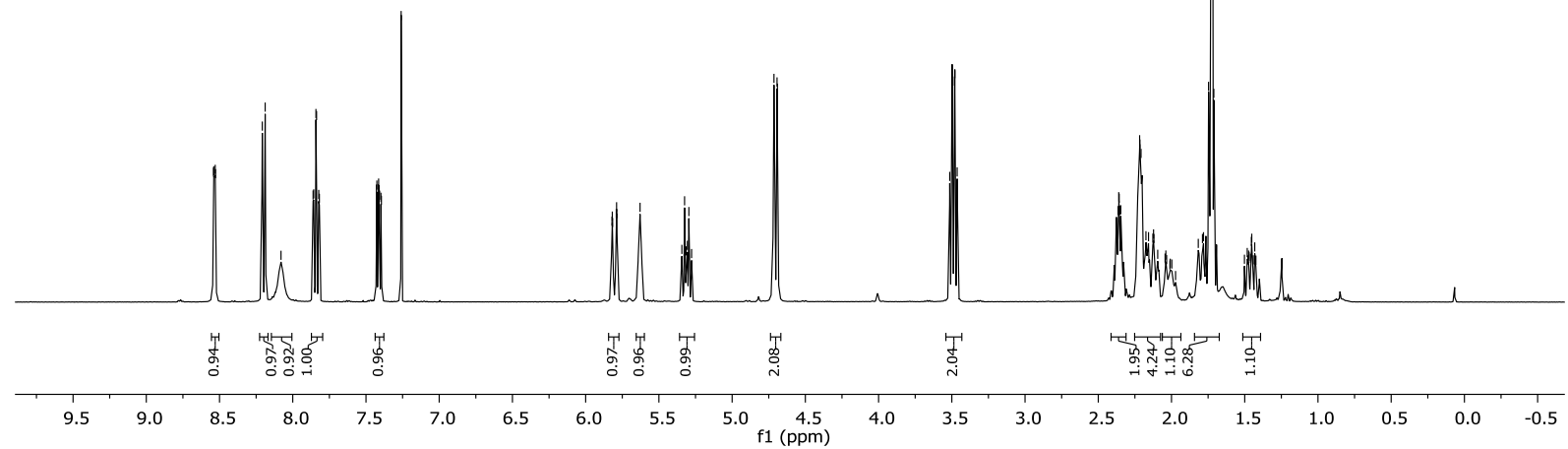

${ }^{13} \mathbf{C ~ N M R ~}\left(100 \mathrm{MHz}, \mathrm{CDCl}_{3}, 25{ }^{\circ} \mathrm{C}\right)$ of $\mathbf{1 u}$

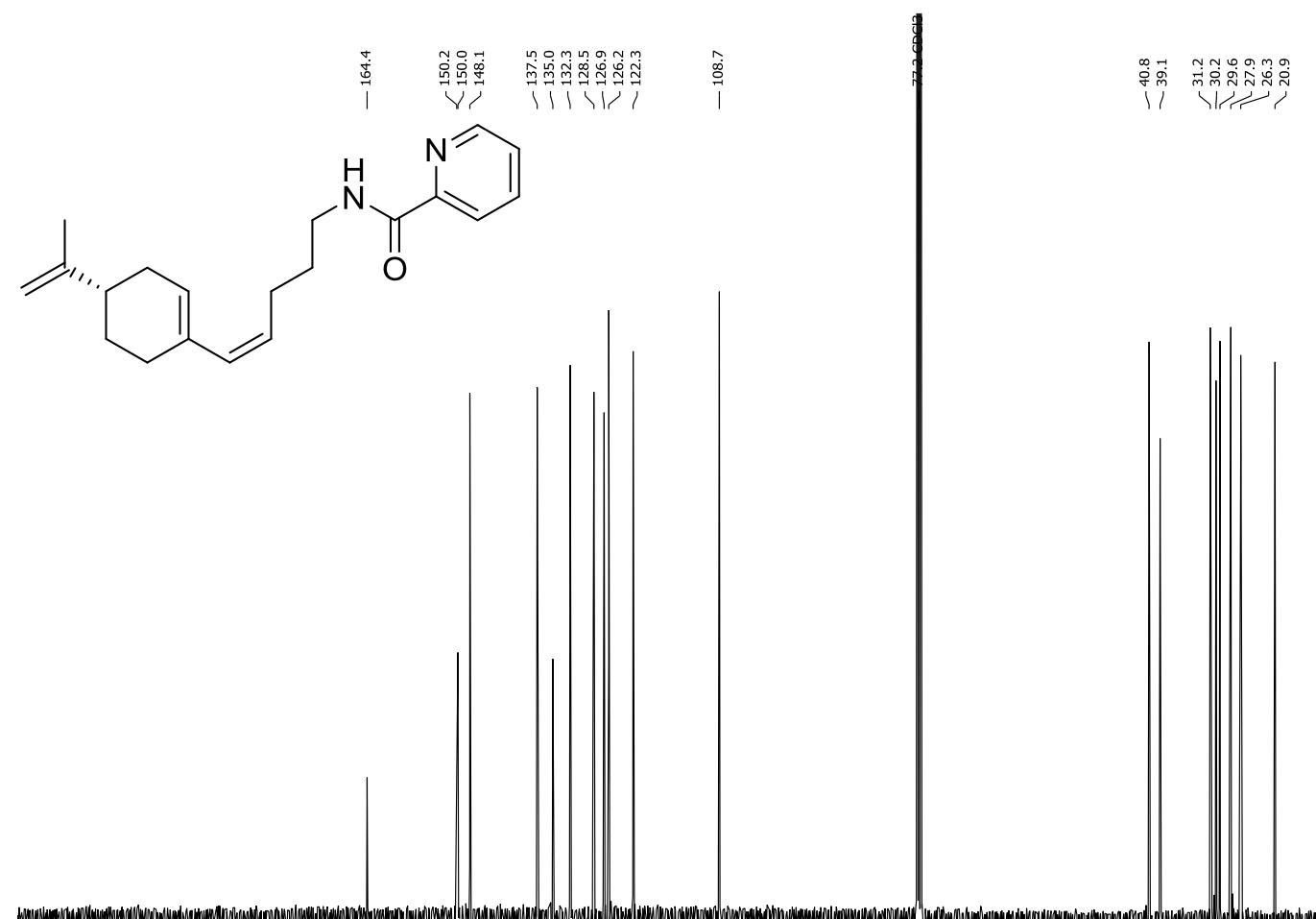

$\begin{array}{lllllllllllllllllllllllll}210 & 200 & 190 & 180 & 170 & 160 & 150 & 140 & 130 & 120 & 110 & 100 & 100 & 80 & 70 & 60 & 50 & 40 & 30 & 20 & 10 & 0 & -10\end{array}$ 
${ }^{\mathbf{1}} \mathbf{H}$ NMR $\left(400 \mathrm{MHz}, \mathrm{CDCl}_{3}, 25^{\circ} \mathrm{C}\right)$ of $\mathbf{1 v}$
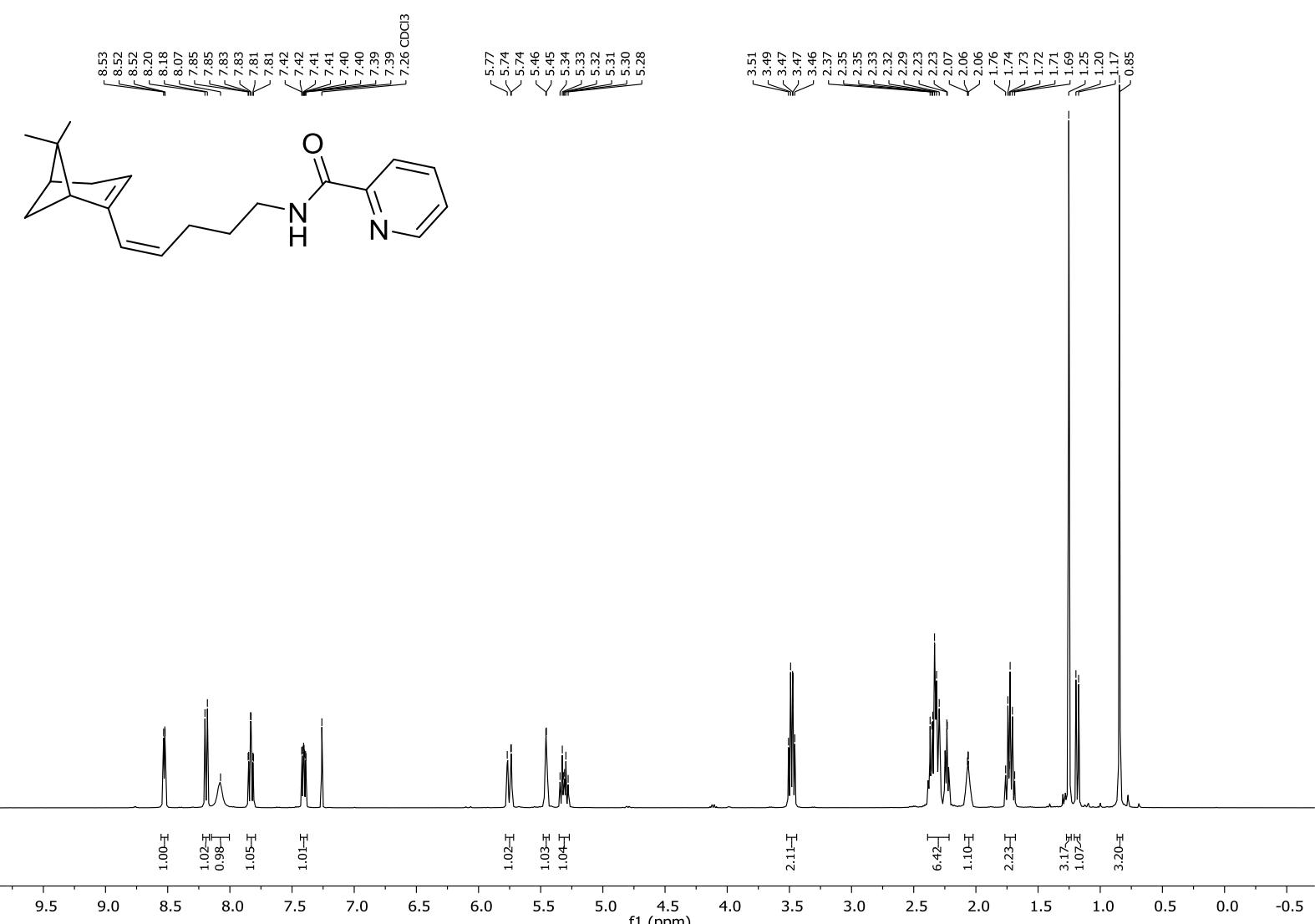

${ }^{13} \mathbf{C}$ NMR $\left(100 \mathrm{MHz}, \mathrm{CDCl}_{3}, 25{ }^{\circ} \mathrm{C}\right)$ of $\mathbf{1 v}$

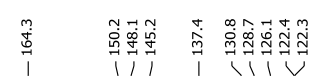<smiles>CC1(C)CCC2CC1CC2/C=C\CCCNC(=O)c1ccccn1</smiles>

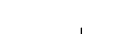


${ }^{\mathbf{1}} \mathbf{H} \mathbf{N M R}\left(400 \mathrm{MHz}, \mathrm{CDCl}_{3}, 25^{\circ} \mathrm{C}\right)$ of $\mathbf{S 2} \mathbf{w}$

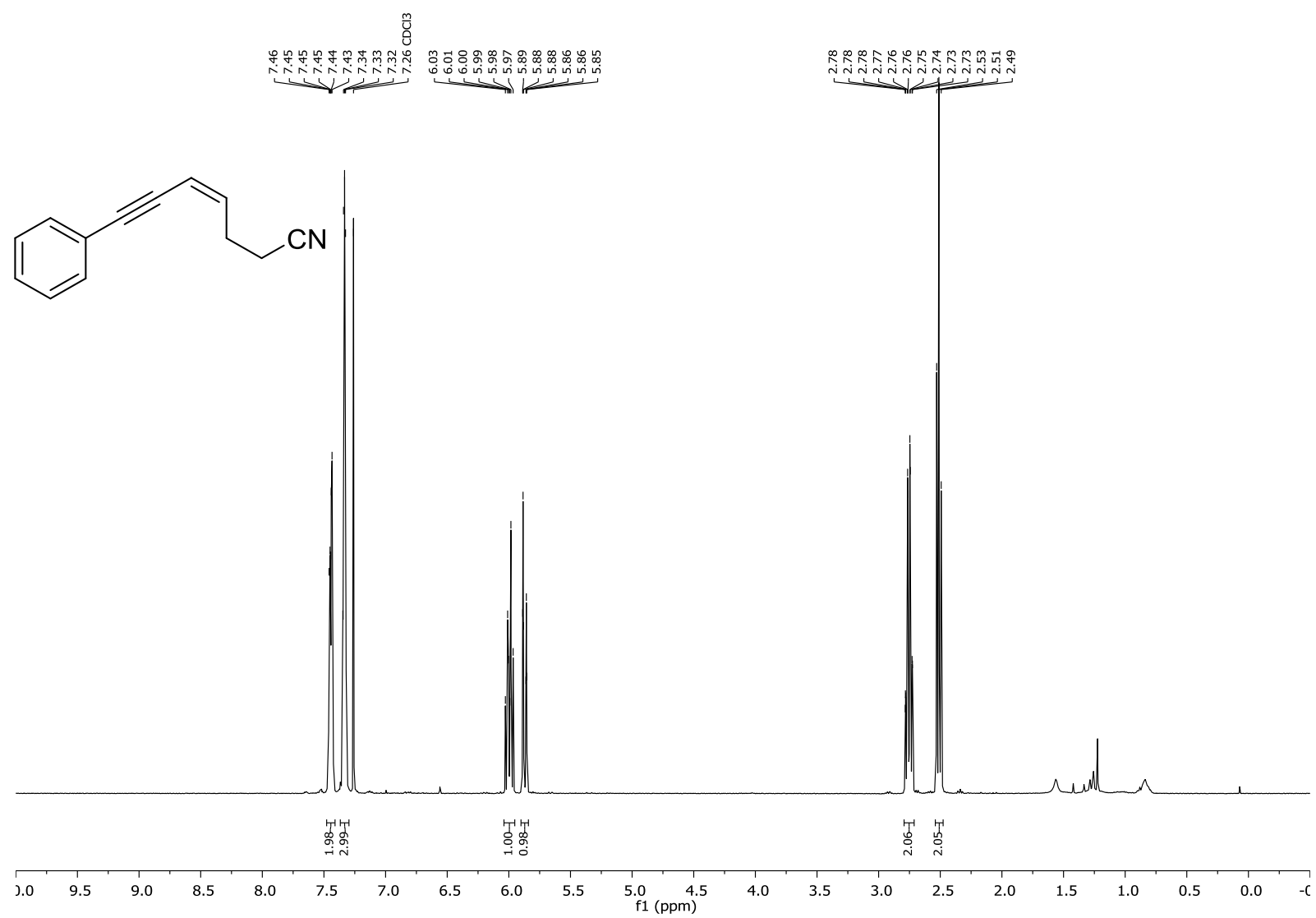

${ }^{13} \mathbf{C}$ NMR $\left(100 \mathrm{MHz}, \mathrm{CDCl}_{3}, 25^{\circ} \mathrm{C}\right)$ of $\mathbf{S 2 w}$<smiles>N#CCC/C=C\C#Cc1ccccc1</smiles>

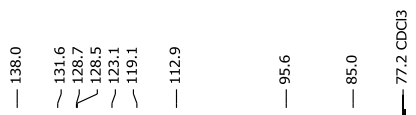

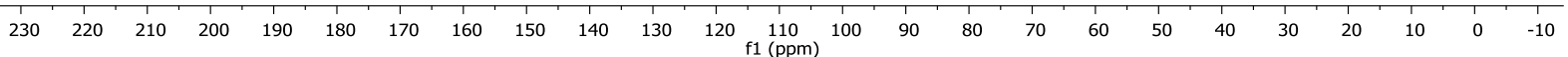


${ }^{\mathbf{1}} \mathbf{H}$ NMR $\left(400 \mathrm{MHz}, \mathrm{CDCl}_{3}, 25^{\circ} \mathrm{C}\right)$ of $\mathbf{1 w}$

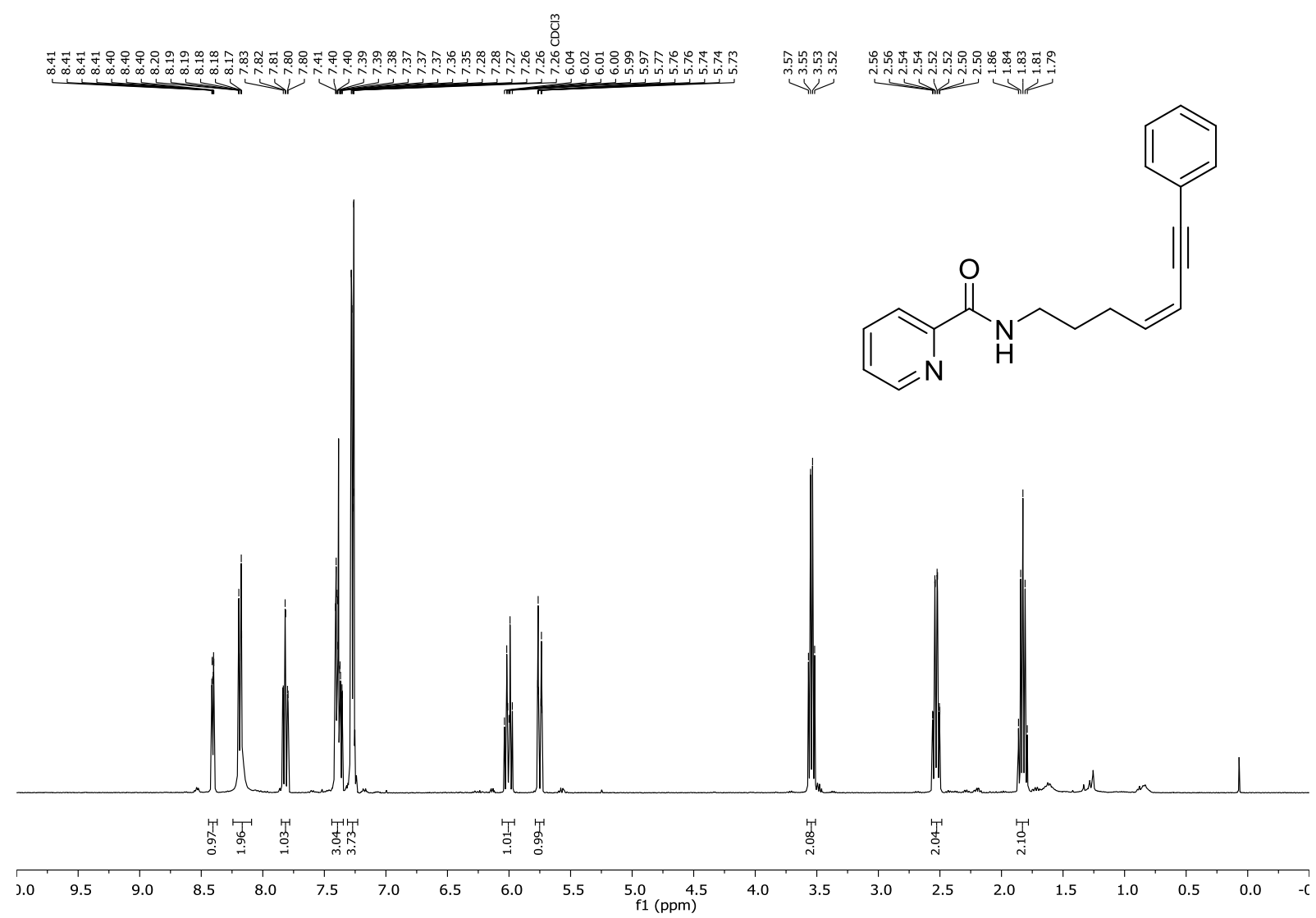

${ }^{13} \mathbf{C}$ NMR $\left(100 \mathrm{MHz}, \mathrm{CDCl}_{3}, 25^{\circ} \mathrm{C}\right)$ of $\mathbf{1 w}$

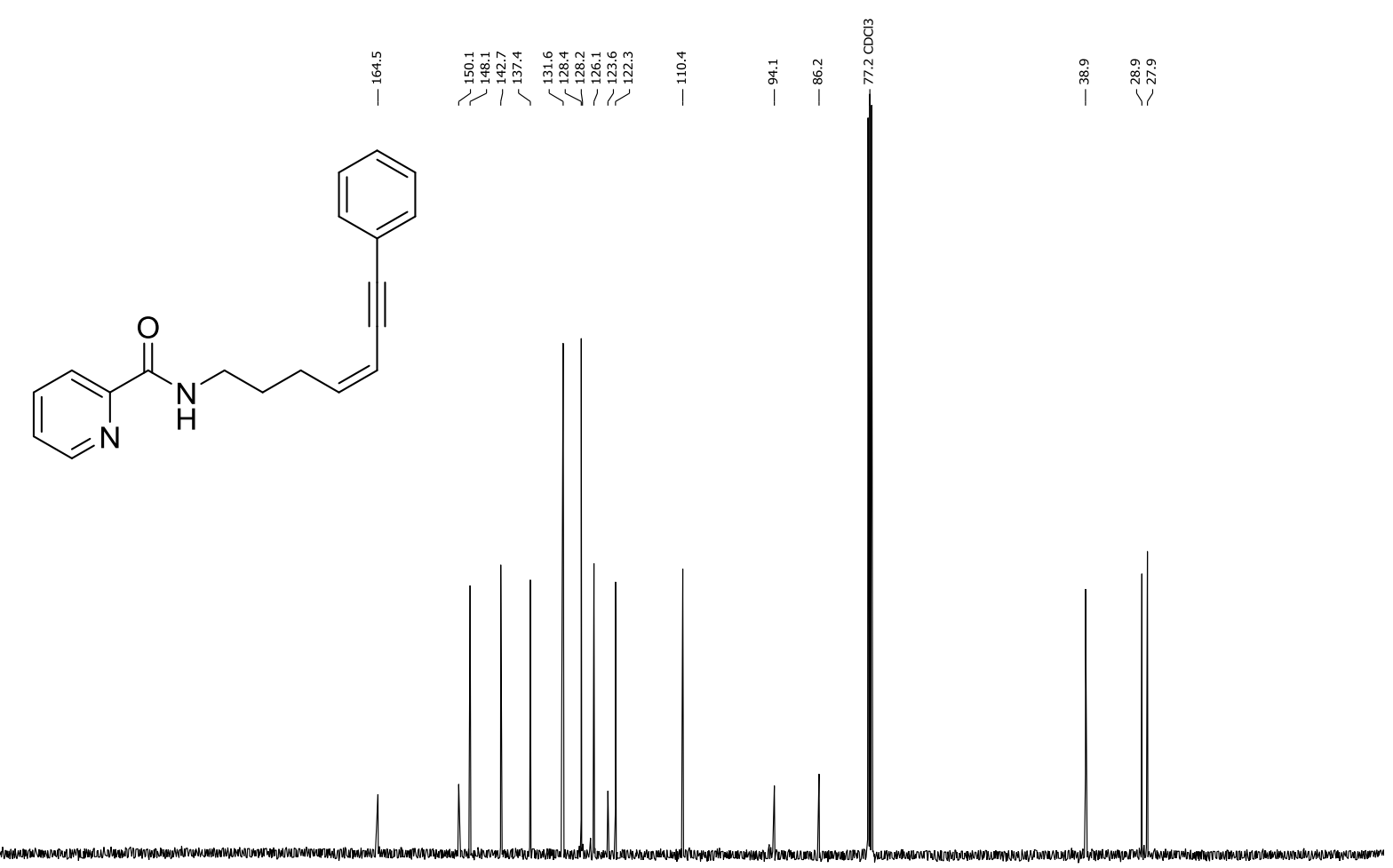

$\begin{array}{llllllllllllllllllllllllllllll}230 & 220 & 210 & 200 & 190 & 180 & 170 & 160 & 150 & 140 & 130 & 120 & 110 & 100 & 90 & 80 & 70 & 60 & 50 & 40 & 30 & 20 & 10 & 0 & -10\end{array}$ 
${ }^{\mathbf{1}} \mathbf{H}$ NMR $\left(400 \mathrm{MHz}, \mathrm{CDCl}_{3}, 25^{\circ} \mathrm{C}\right)$ of $\mathbf{1 y}$<smiles>CC1(C)CCC=C(CCNC(=O)c2ccccn2)C1</smiles>

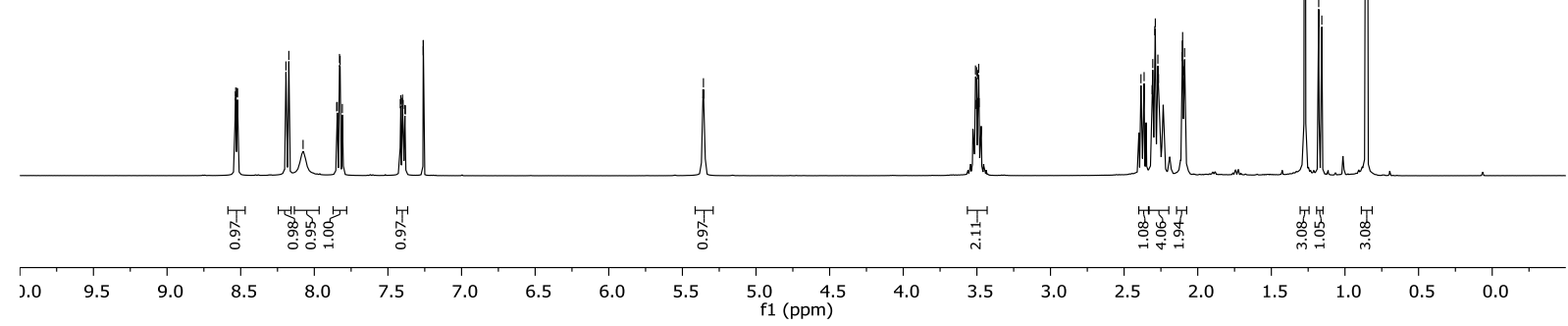

${ }^{13} \mathbf{C ~ N M R}\left(100 \mathrm{MHz}, \mathrm{CDCl}_{3}, 25^{\circ} \mathrm{C}\right)$ of $\mathbf{1 y}$

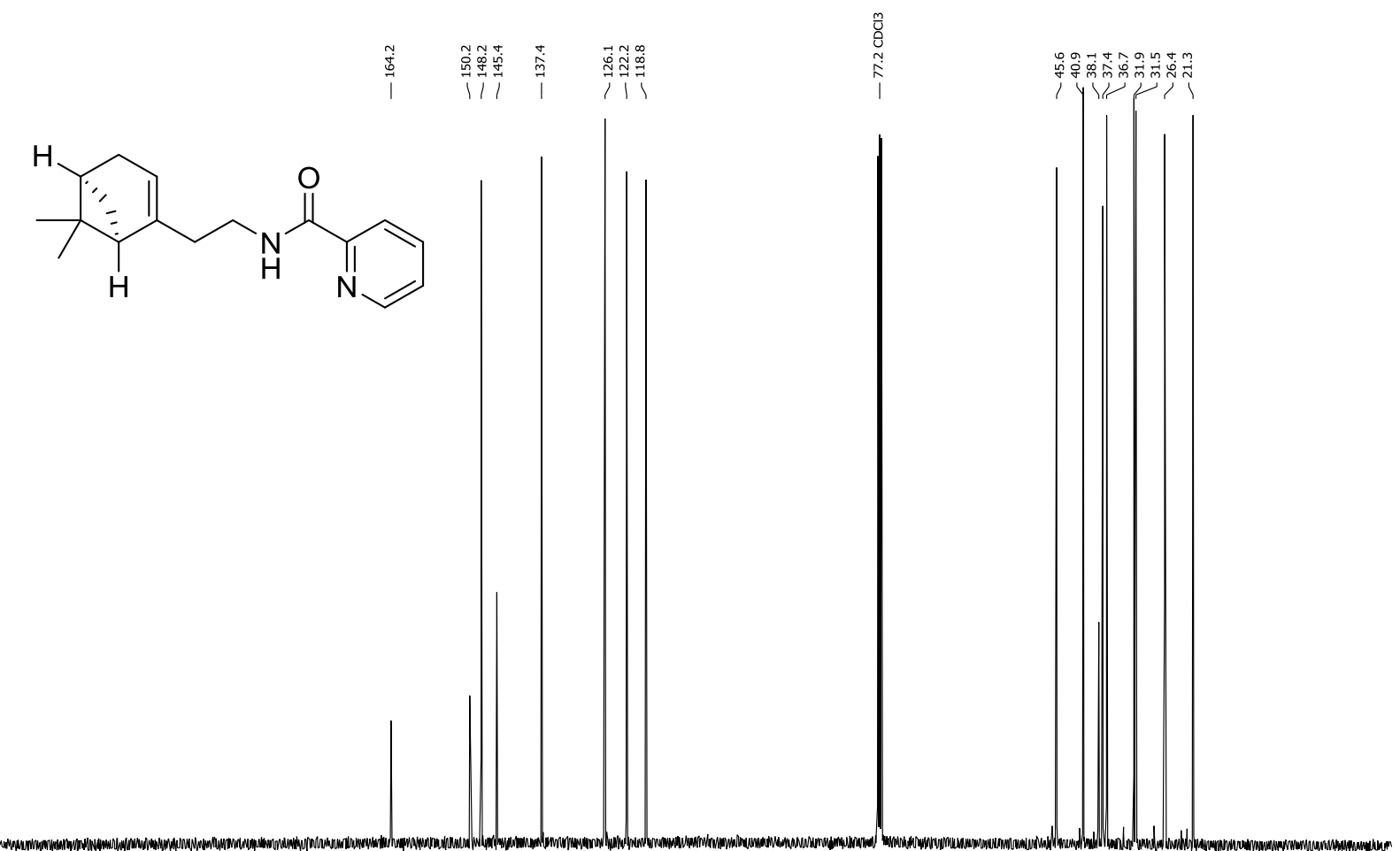

$\begin{array}{lllllllllllllllllllllllllllll}230 & 220 & 210 & 200 & 190 & 180 & 170 & 160 & 150 & 140 & 130 & 120 & 110 & 100 & 90 & 80 & 70 & 60 & 50 & 40 & 30 & 20 & 10 & 0 & -10\end{array}$ 
${ }^{\mathbf{1}} \mathbf{H} \mathbf{N M R}\left(500 \mathrm{MHz}, \mathrm{CDCl}_{3}, 25^{\circ} \mathrm{C}\right)$ of $\mathbf{S 5}$

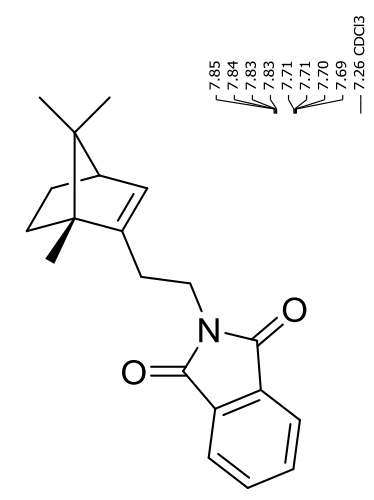

$\underbrace{\circ 00}$

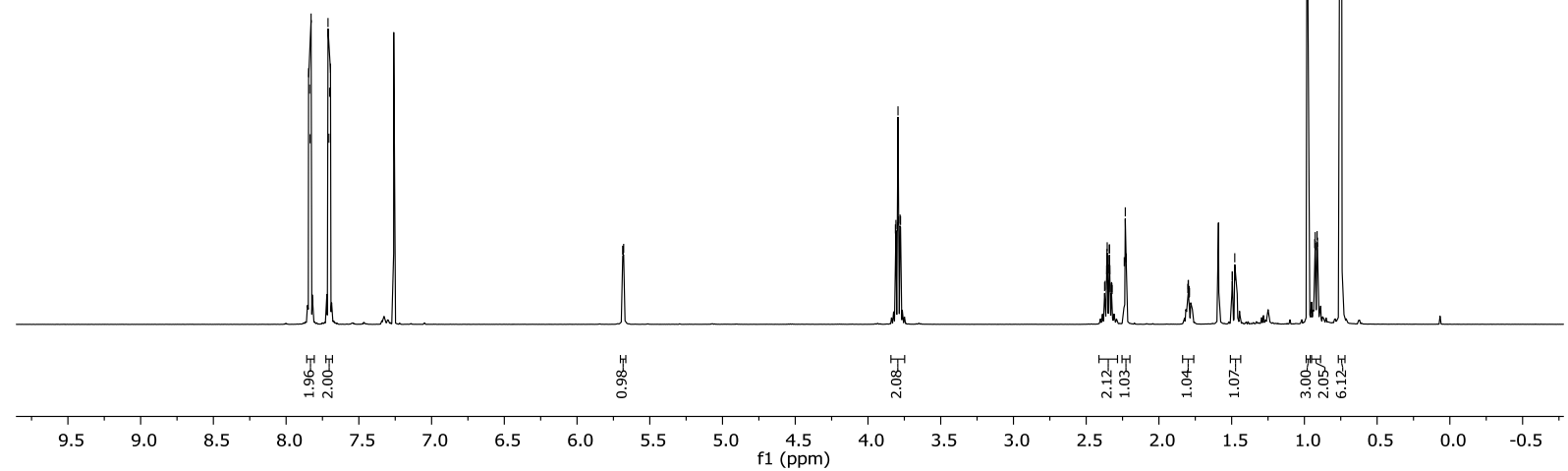

${ }^{13} \mathbf{C ~ N M R}\left(126 \mathrm{MHz}, \mathrm{CDCl}_{3}, 25^{\circ} \mathrm{C}\right)$ of $\mathbf{S 5}$

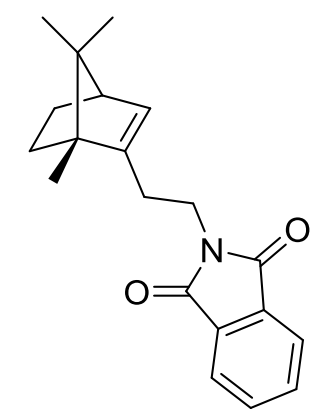


${ }^{\mathbf{1}} \mathbf{H}$ NMR $\left(500 \mathrm{MHz}, \mathrm{CDCl}_{3}, 25^{\circ} \mathrm{C}\right)$ of $\mathbf{1 z}$

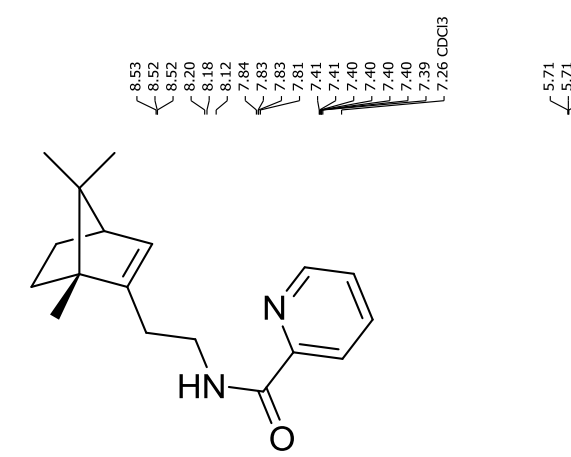

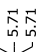

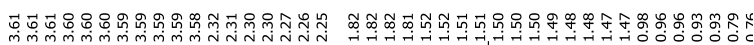

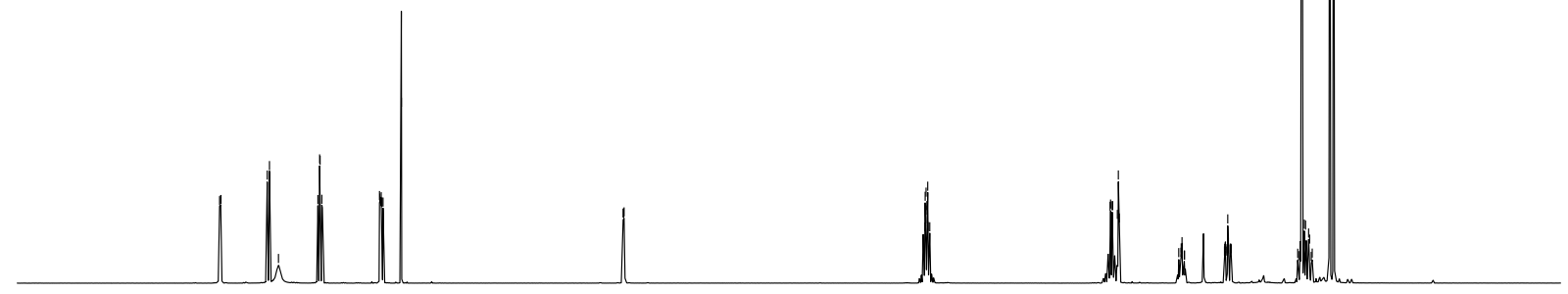

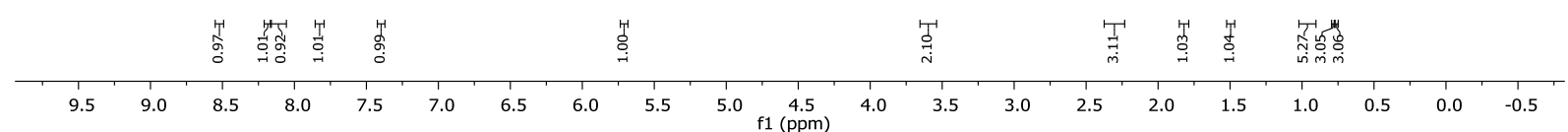

${ }^{13} \mathbf{C ~ N M R}\left(126 \mathrm{MHz}, \mathrm{CDCl}_{3}, 25^{\circ} \mathrm{C}\right)$ of $\mathbf{1 z}$

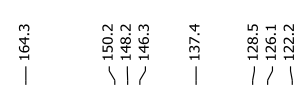

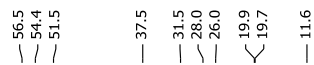

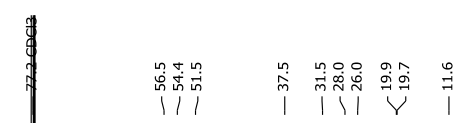

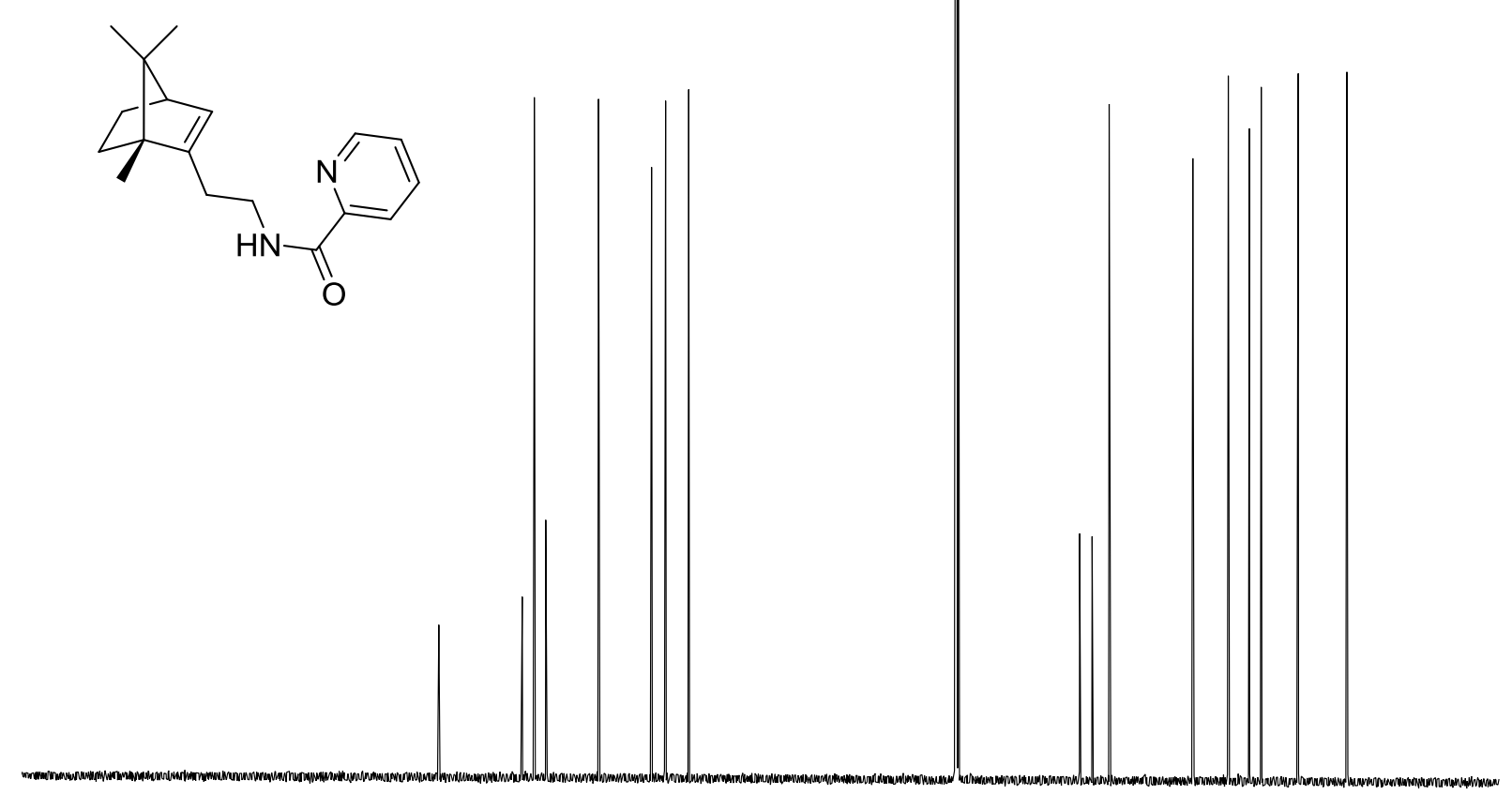

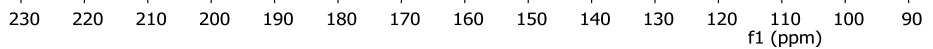


${ }^{1} \mathbf{H}$ NMR $\left(400 \mathrm{MHz}, \mathrm{CDCl}_{3}, 25^{\circ} \mathrm{C}\right)$ of $\mathbf{S 6}$

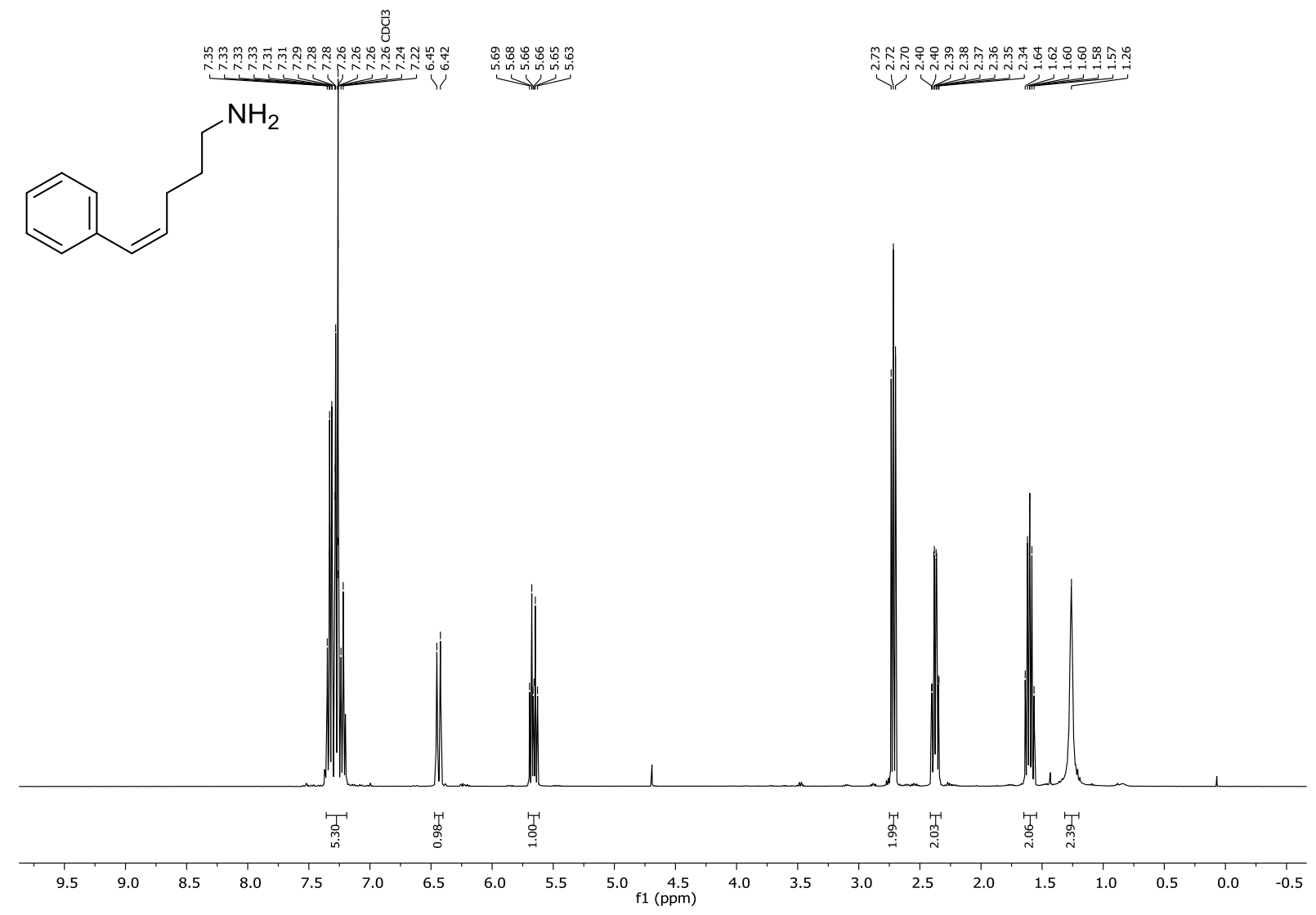

${ }^{13} \mathbf{C}$ NMR $\left(100 \mathrm{MHz}, \mathrm{CDCl}_{3}, 25{ }^{\circ} \mathrm{C}\right)$ of $\mathbf{S 6}$<smiles>NCCC/C=C\c1ccccc1</smiles>
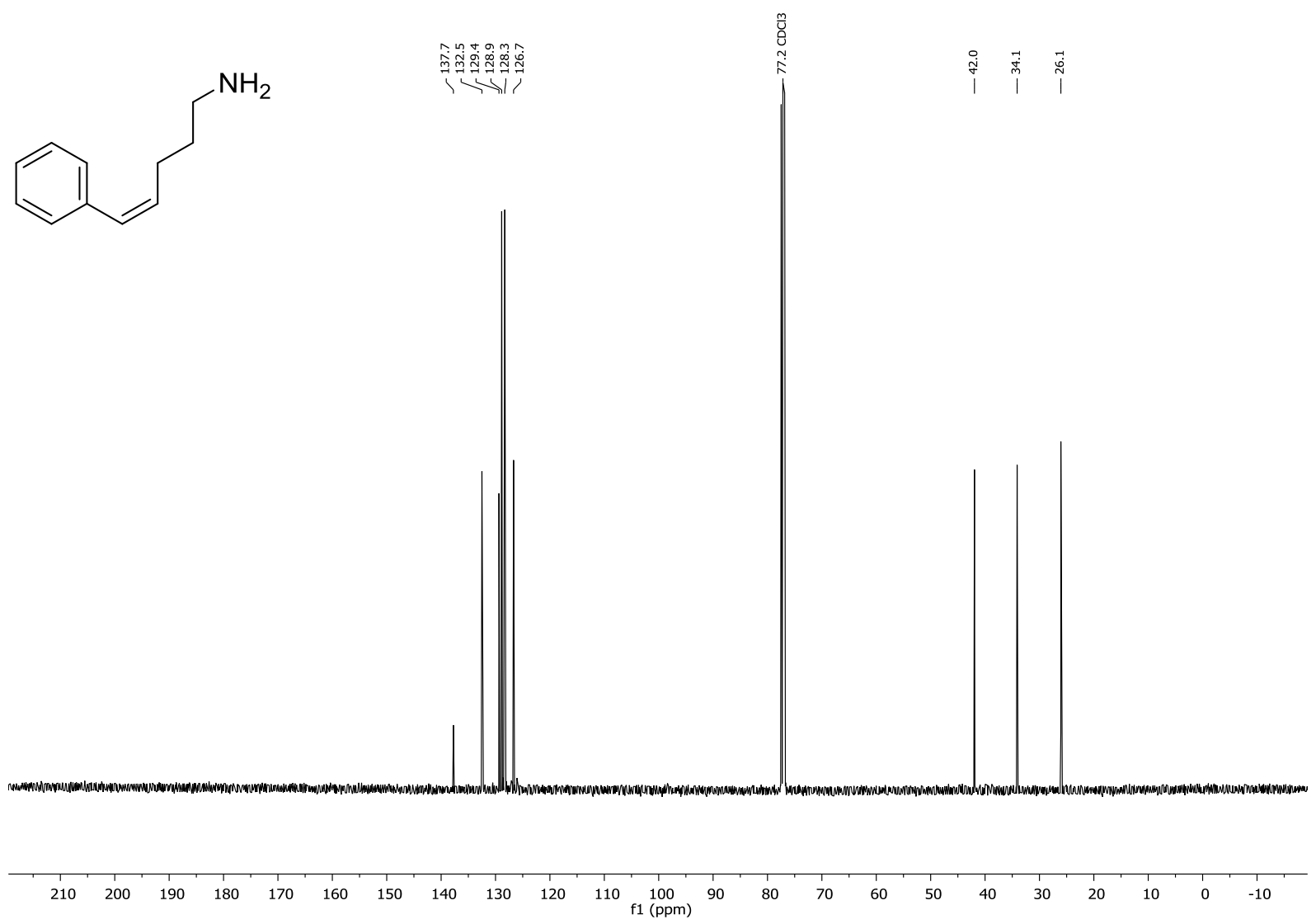

S119 
${ }^{1} \mathbf{H}$ NMR $\left(400 \mathrm{MHz}, \mathrm{CDCl}_{3}, 25^{\circ} \mathrm{C}\right)$ of $\mathbf{S} 7$

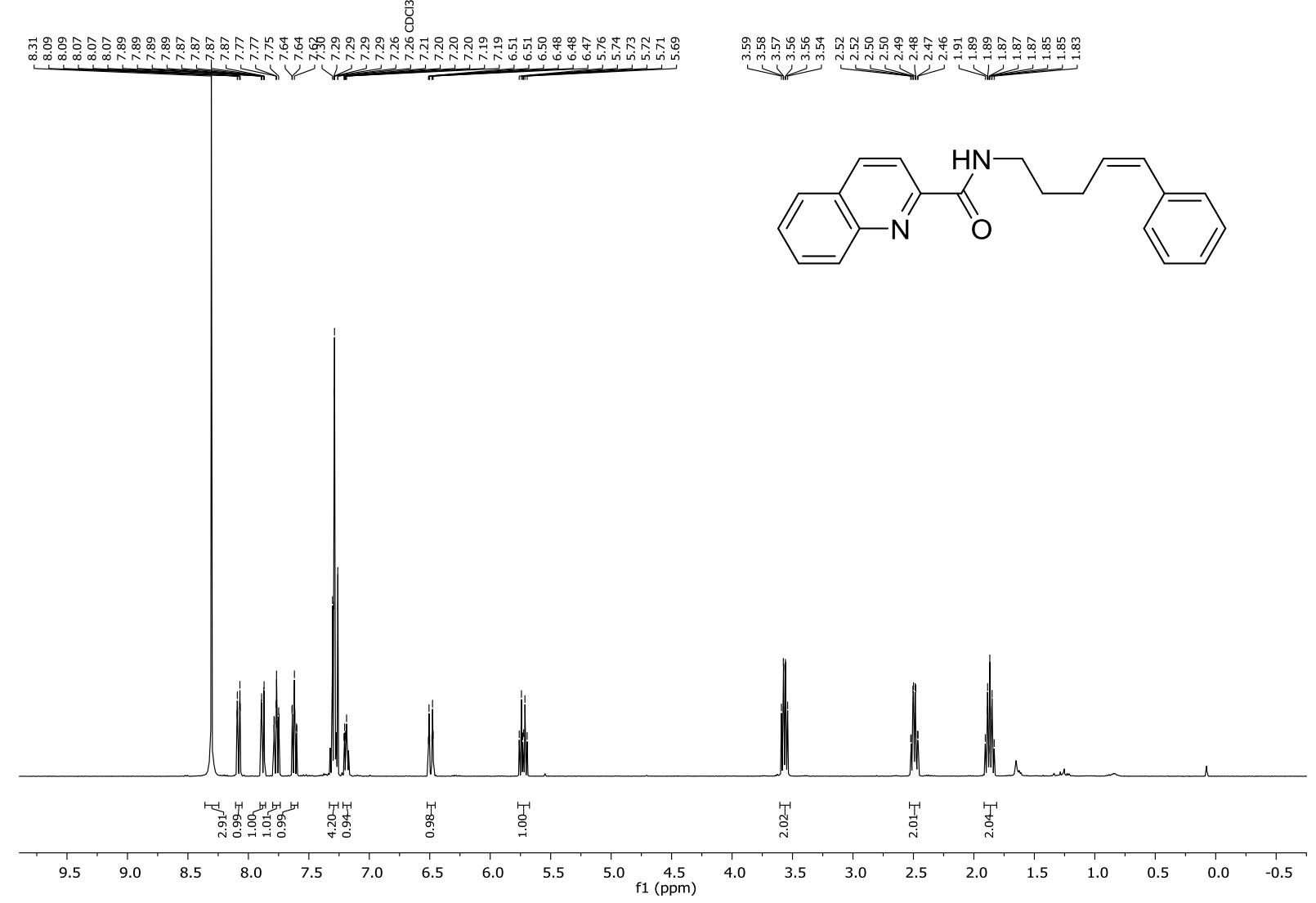

${ }^{13} \mathbf{C}$ NMR $\left(100 \mathrm{MHz}, \mathrm{CDCl}_{3}, 25{ }^{\circ} \mathrm{C}\right)$ of $\mathbf{S 7}$

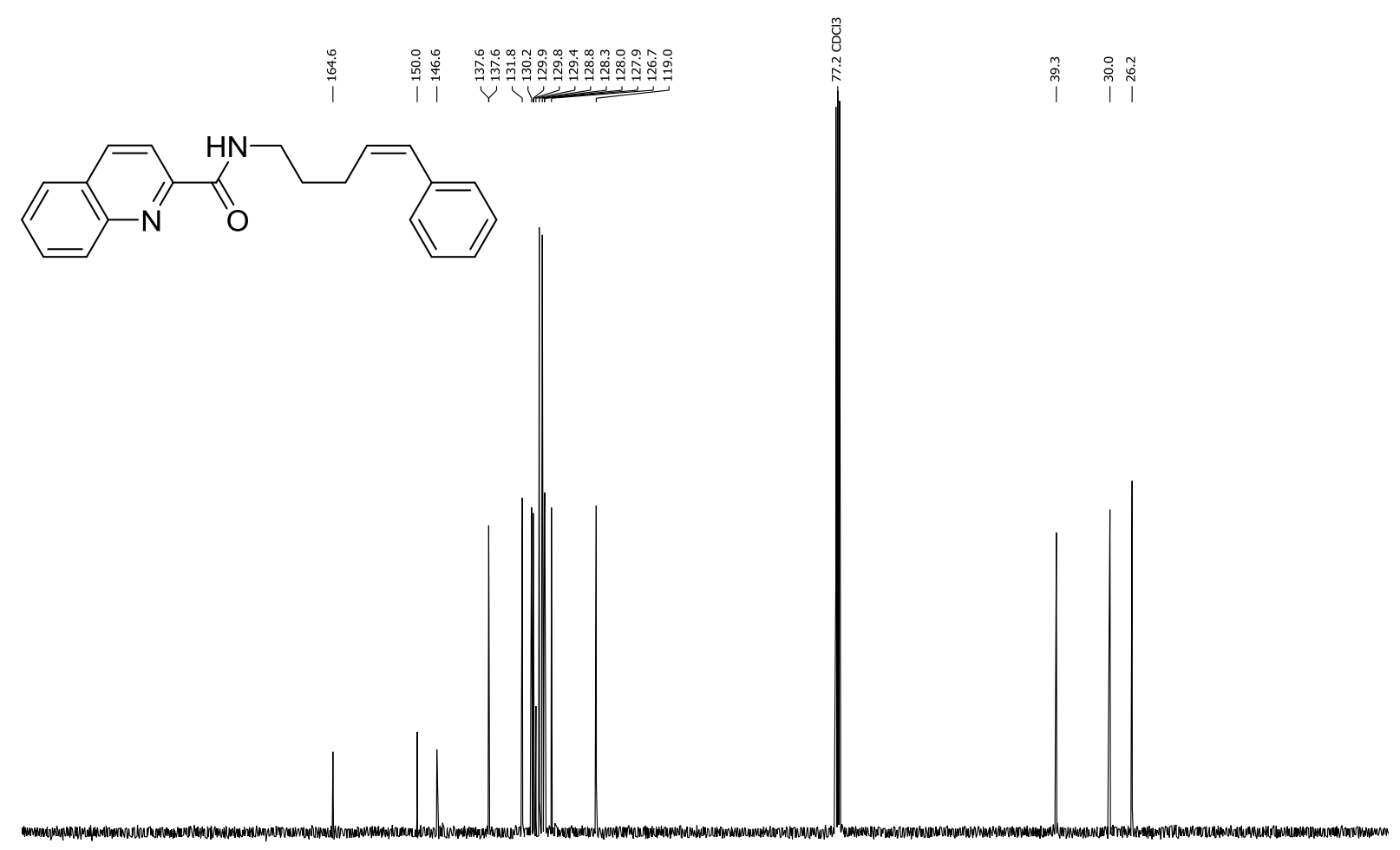

$\begin{array}{lllllllllllllllllllllllllllll} & 210 & 200 & 190 & 180 & 170 & 160 & 150 & 140 & 130 & 120 & 110 & 100 & 90 & 80 & 70 & 60 & 50 & 40 & 30 & 20 & 10 & 0 & -10\end{array}$ 
${ }^{1} \mathbf{H} \mathbf{N M R}\left(400 \mathrm{MHz}, \mathrm{CDCl}_{3}, 25^{\circ} \mathrm{C}\right)$ of $\mathbf{S 8}$

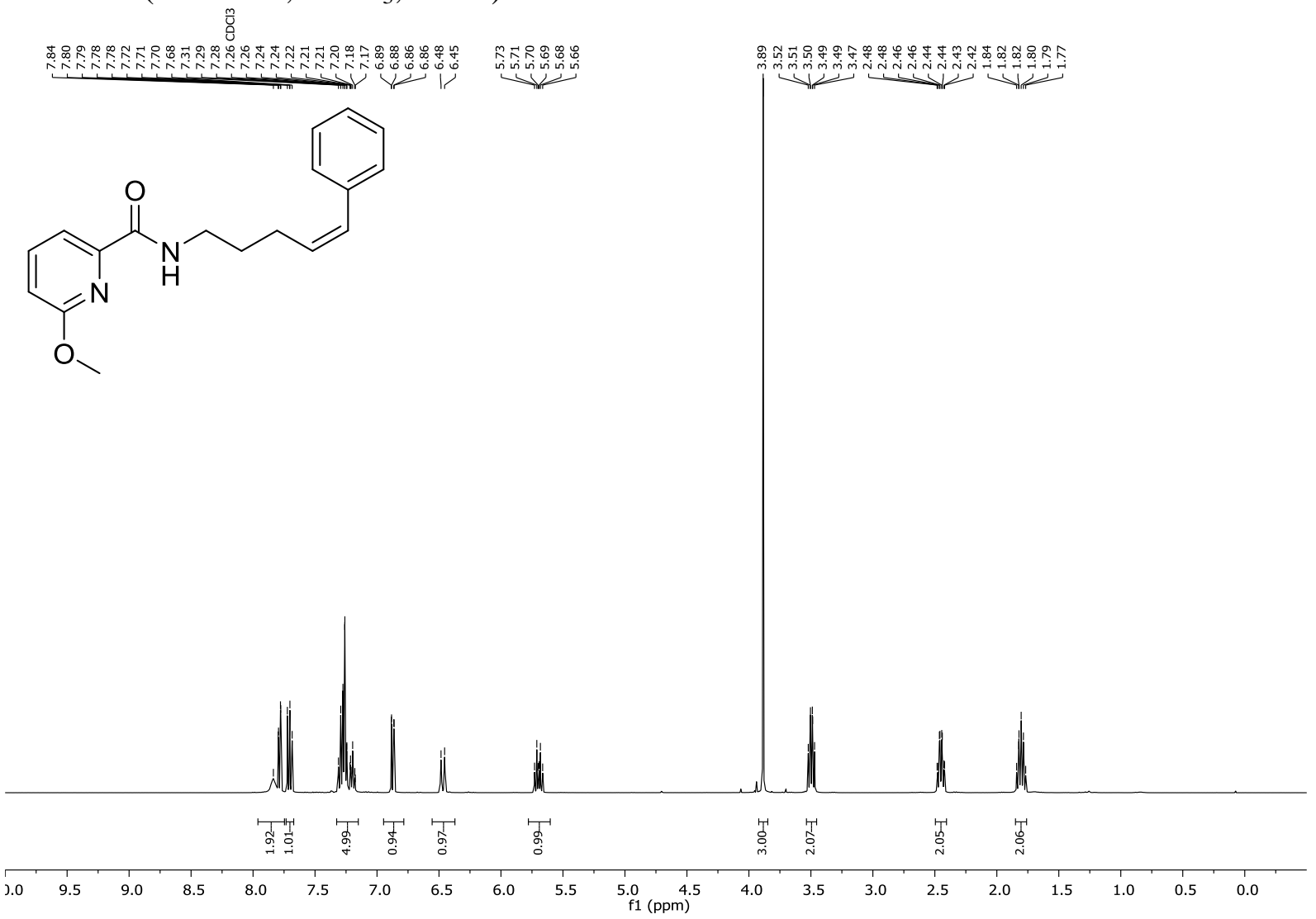

${ }^{13} \mathbf{C}$ NMR $\left(126 \mathrm{MHz}, \mathrm{CDCl}_{3}, 25{ }^{\circ} \mathrm{C}\right)$ of $\mathbf{S 8}$

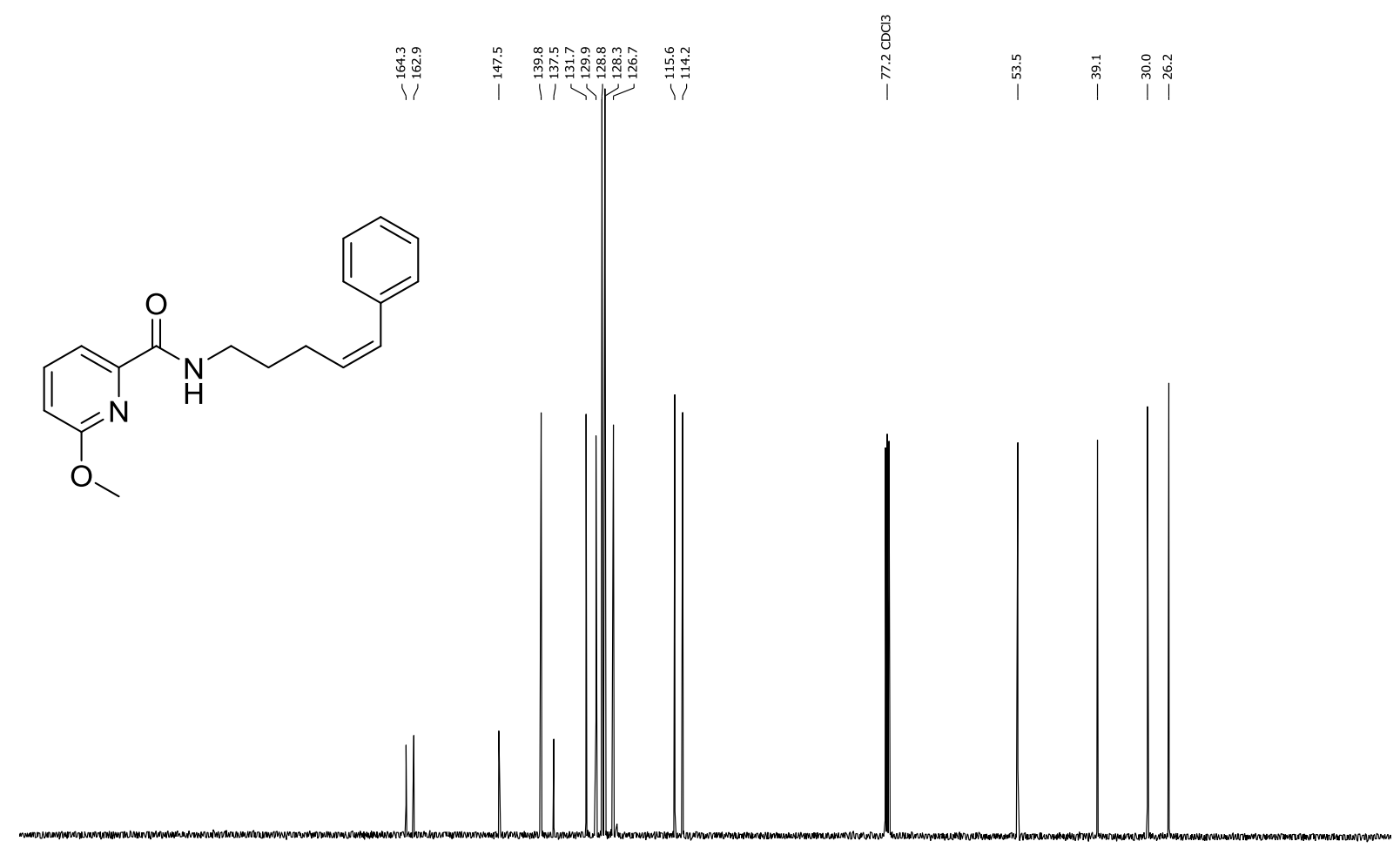

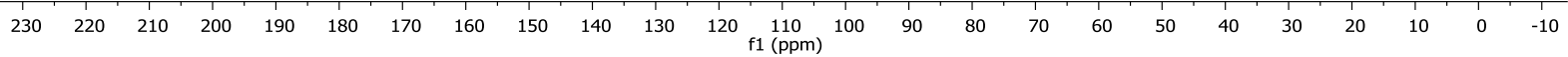


${ }^{1} \mathbf{H}$ NMR $\left(500 \mathrm{MHz}, \mathrm{CDCl}_{3}, 25^{\circ} \mathrm{C}\right)$ of $\mathbf{S 9}$

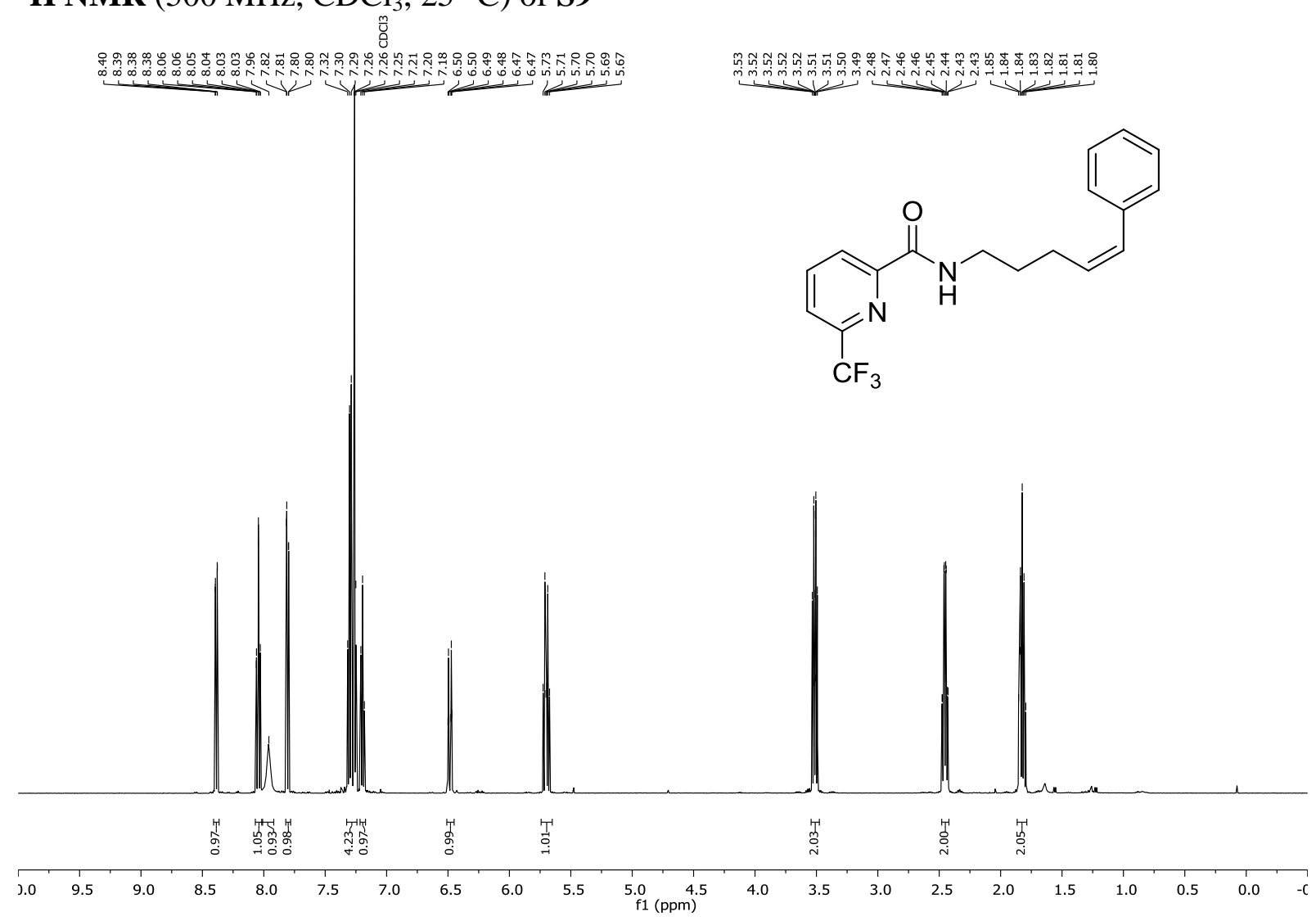

${ }^{13} \mathbf{C}$ NMR $\left(126 \mathrm{MHz}, \mathrm{CDCl}_{3}, 25^{\circ} \mathrm{C}\right)$ of $\mathbf{S 9}$

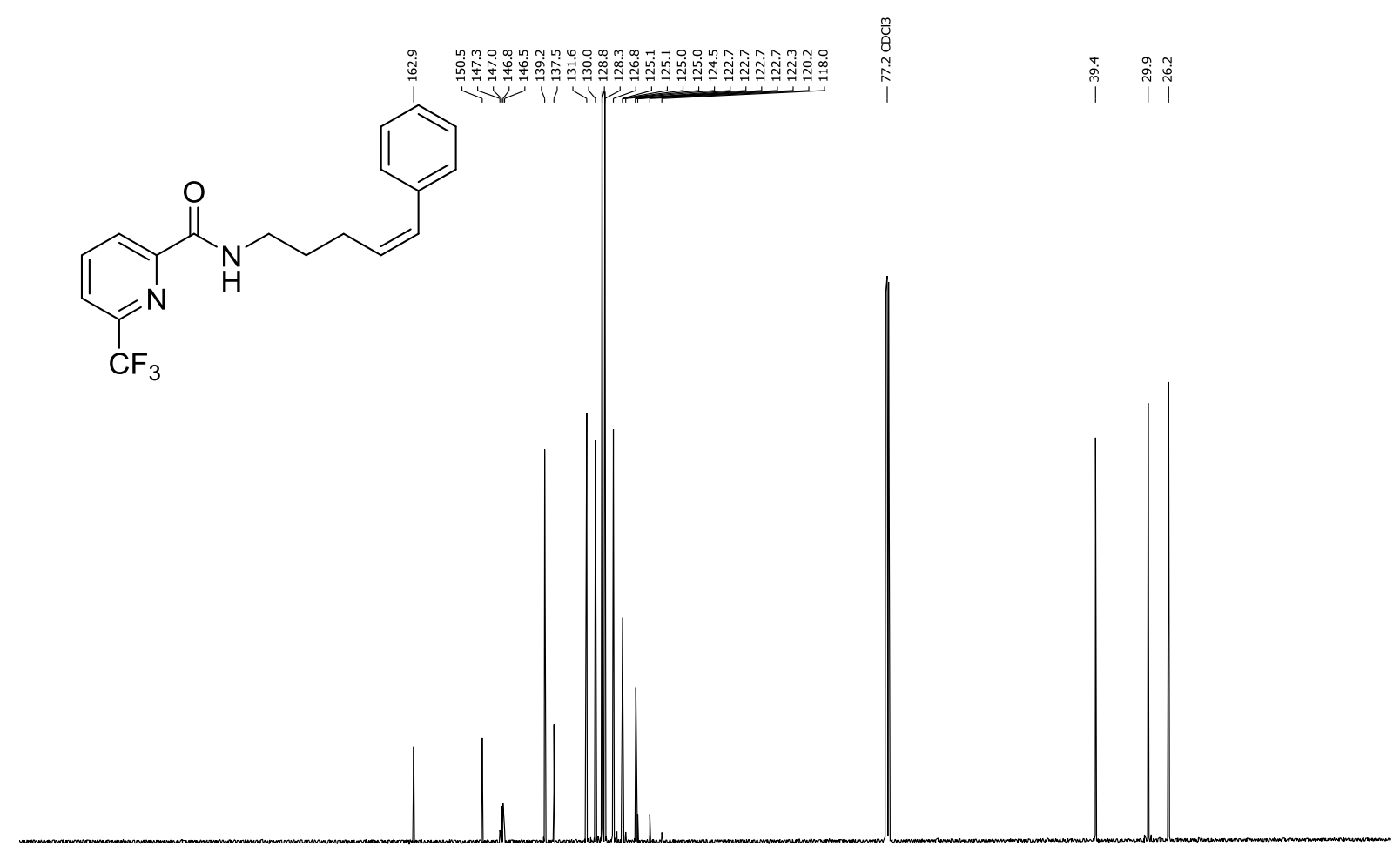

$\begin{array}{lllllllllllllllllllllllllllll}230 & 220 & 210 & 200 & 190 & 180 & 170 & 160 & 150 & 140 & 130 & 120 & 110 & 100 & 90 & 80 & 70 & 60 & 50 & 40 & 30 & 20 & 10 & 0 & -10\end{array}$ 
${ }^{19} \mathbf{F}$ NMR $\left(471 \mathrm{MHz}, \mathrm{CDCl}_{3}, 25^{\circ} \mathrm{C}\right)$ of $\mathbf{S 9}$
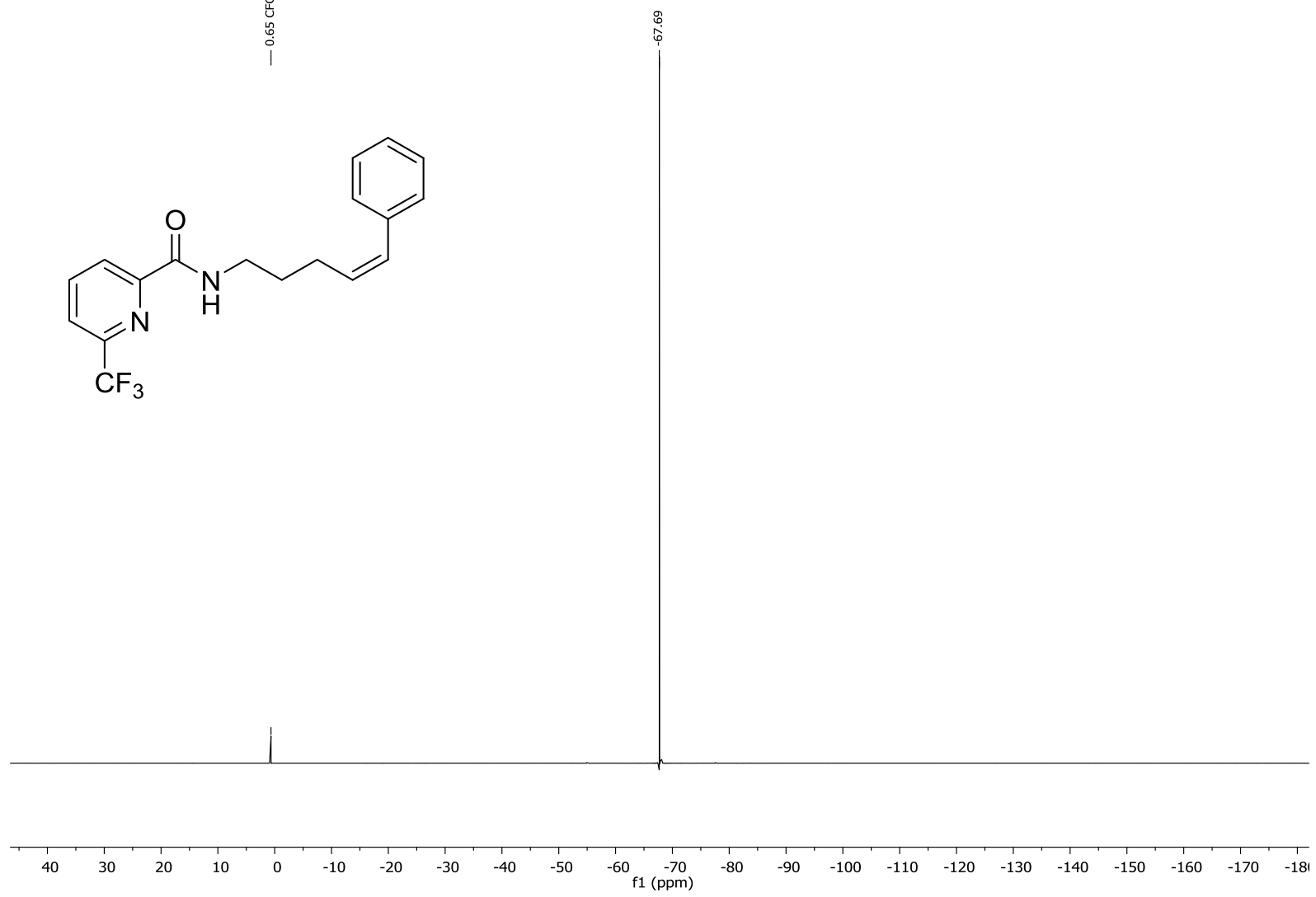

S123 
${ }^{1} \mathbf{H}$ NMR $\left(400 \mathrm{MHz}, \mathrm{CD}_{3} \mathrm{CN}, 25^{\circ} \mathrm{C}\right.$ ) of $\mathbf{S 1 0}$

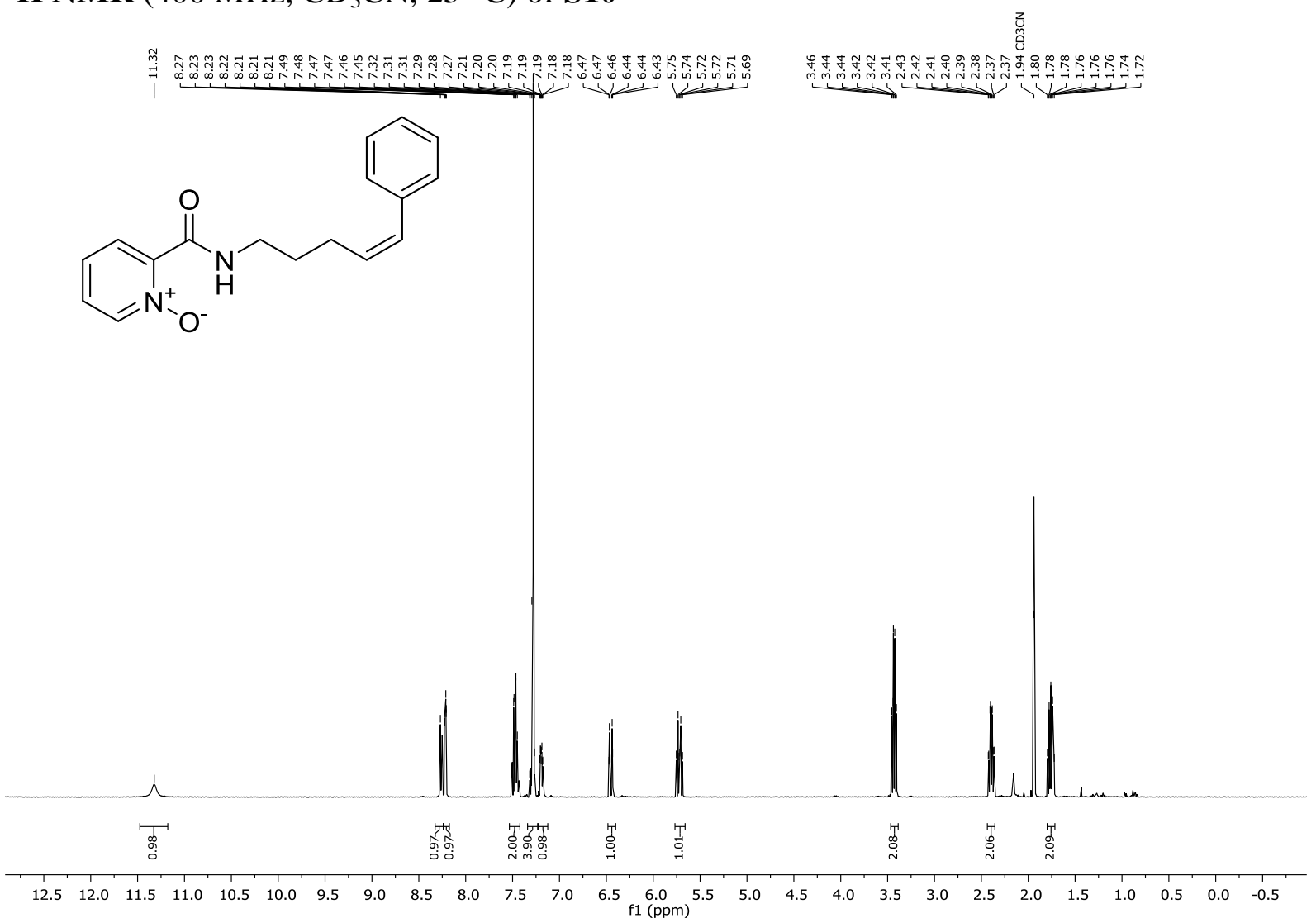

${ }^{13} \mathbf{C}$ NMR $\left(100 \mathrm{MHz}, \mathrm{CD}_{3} \mathrm{CN}, 25{ }^{\circ} \mathrm{C}\right)$ of $\mathbf{S 1 0}$

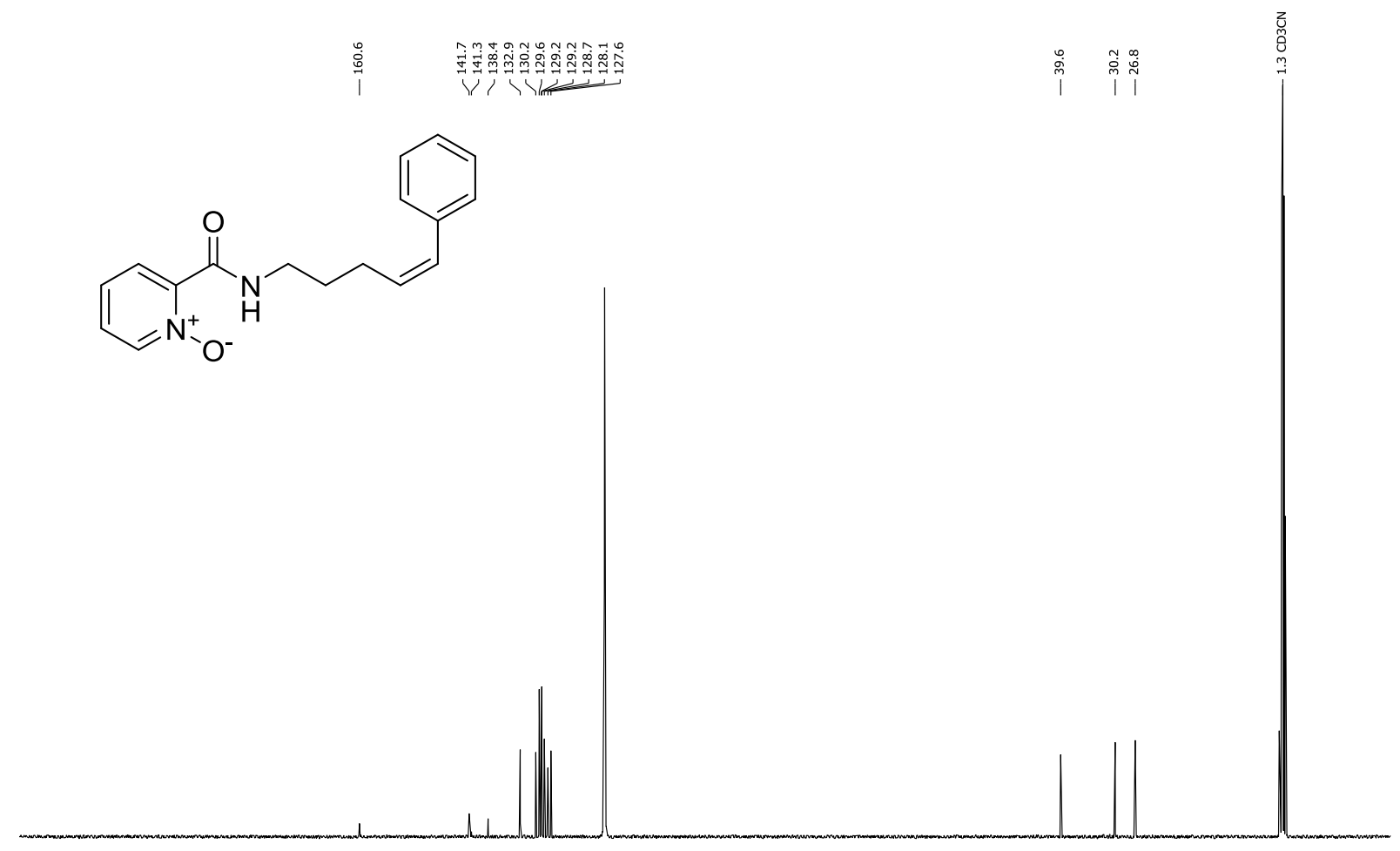

$\begin{array}{lllllllllllllllllllllllllll}210 & 200 & 190 & 180 & 170 & 160 & 150 & 140 & 130 & 120 & 110 & 100 & 90 & 80 & 70 & 60 & 50 & 40 & 30 & 20 & 10 & 0 & -10\end{array}$ 
${ }^{1} \mathbf{H} \mathbf{N M R}\left(400 \mathrm{MHz}, \mathrm{CDCl}_{3}, 25^{\circ} \mathrm{C}\right)$ of $\mathbf{S 1 1}$

要

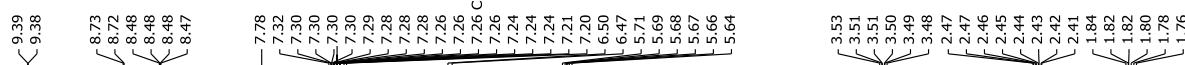

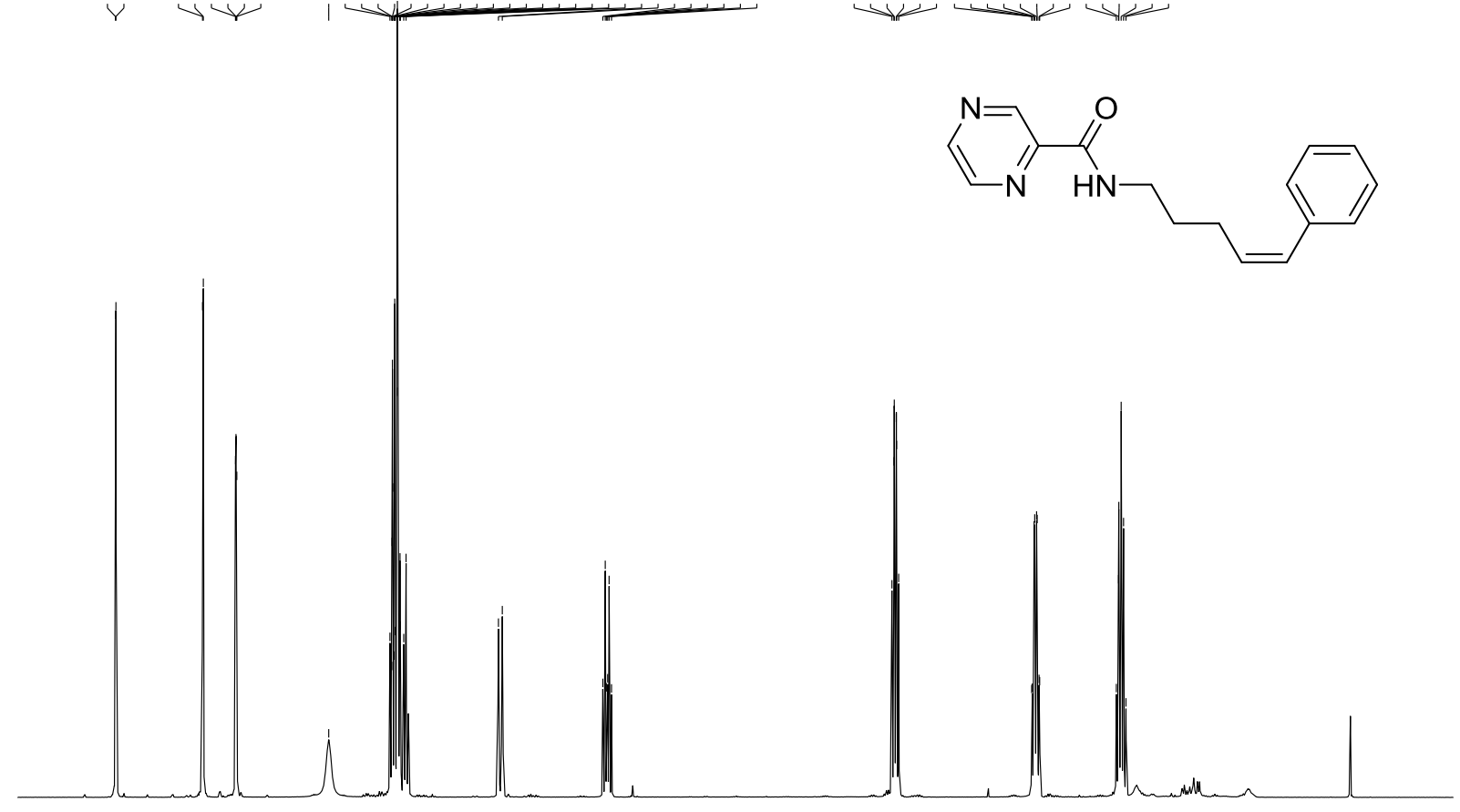

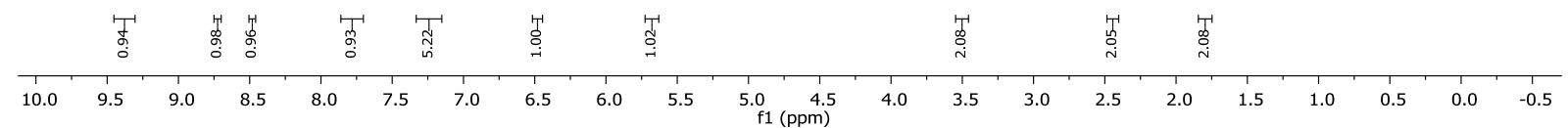

${ }^{13} \mathbf{C}$ NMR $\left(100 \mathrm{MHz}, \mathrm{CDCl}_{3}, 25^{\circ} \mathrm{C}\right)$ of $\mathbf{S 1 1}$

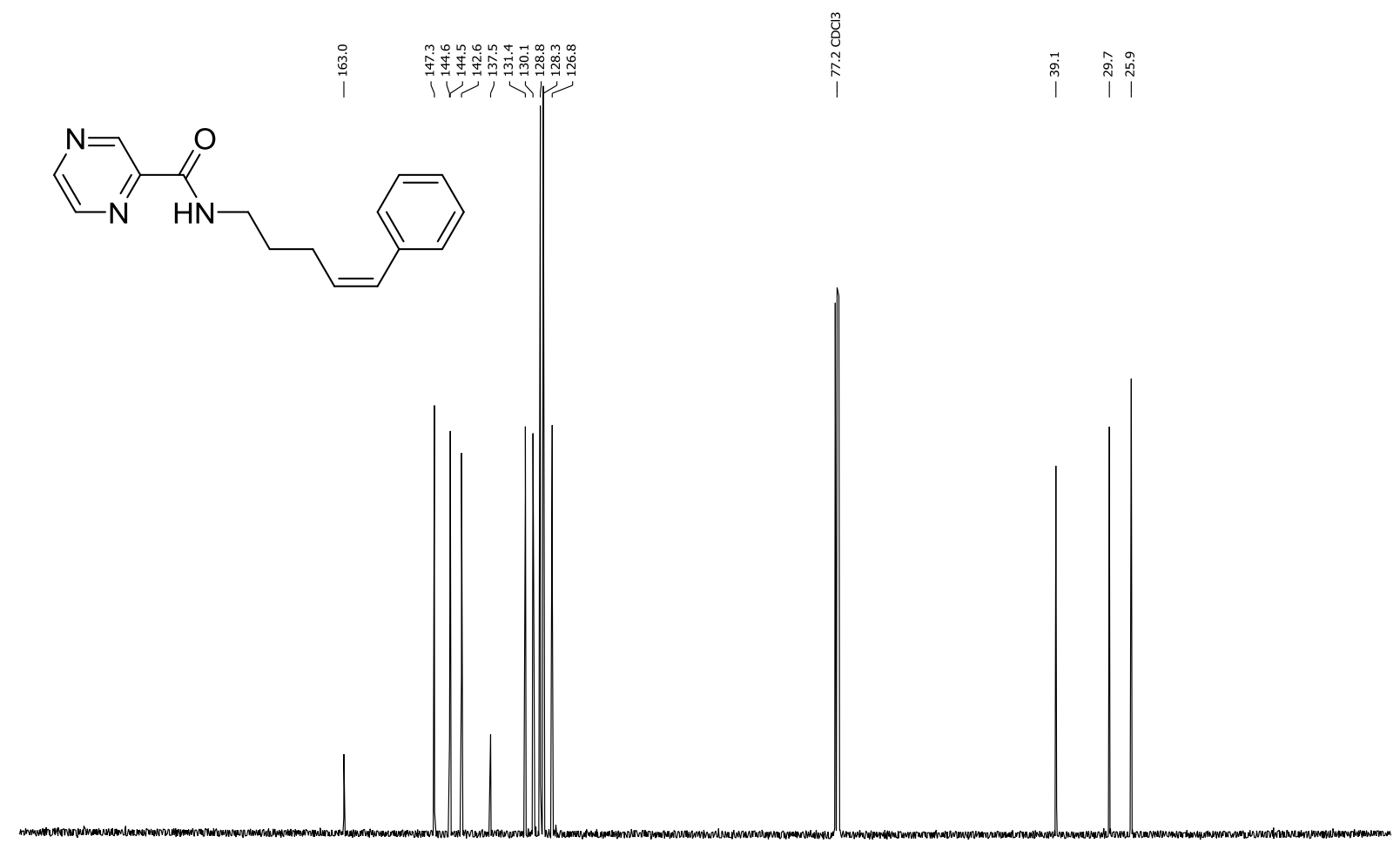

$\begin{array}{lllllllllllllllllllllllllllllllllll}210 & 200 & 190 & 180 & 170 & 160 & 150 & 140 & 130 & 120 & 110 & 100 & 90 & 80 & 70 & 60 & 50 & 40 & 30 & 20 & 10 & 0 & -10\end{array}$ 
${ }^{1} \mathbf{H}$ NMR $\left(400 \mathrm{MHz}, \mathrm{CDCl}_{3}, 25^{\circ} \mathrm{C}\right)$ of $\mathbf{S 1 2}$

莟<smiles>O=C(NCCC/C=C\c1ccccc1)c1nccc2c1[nH]c1ccccc12</smiles>

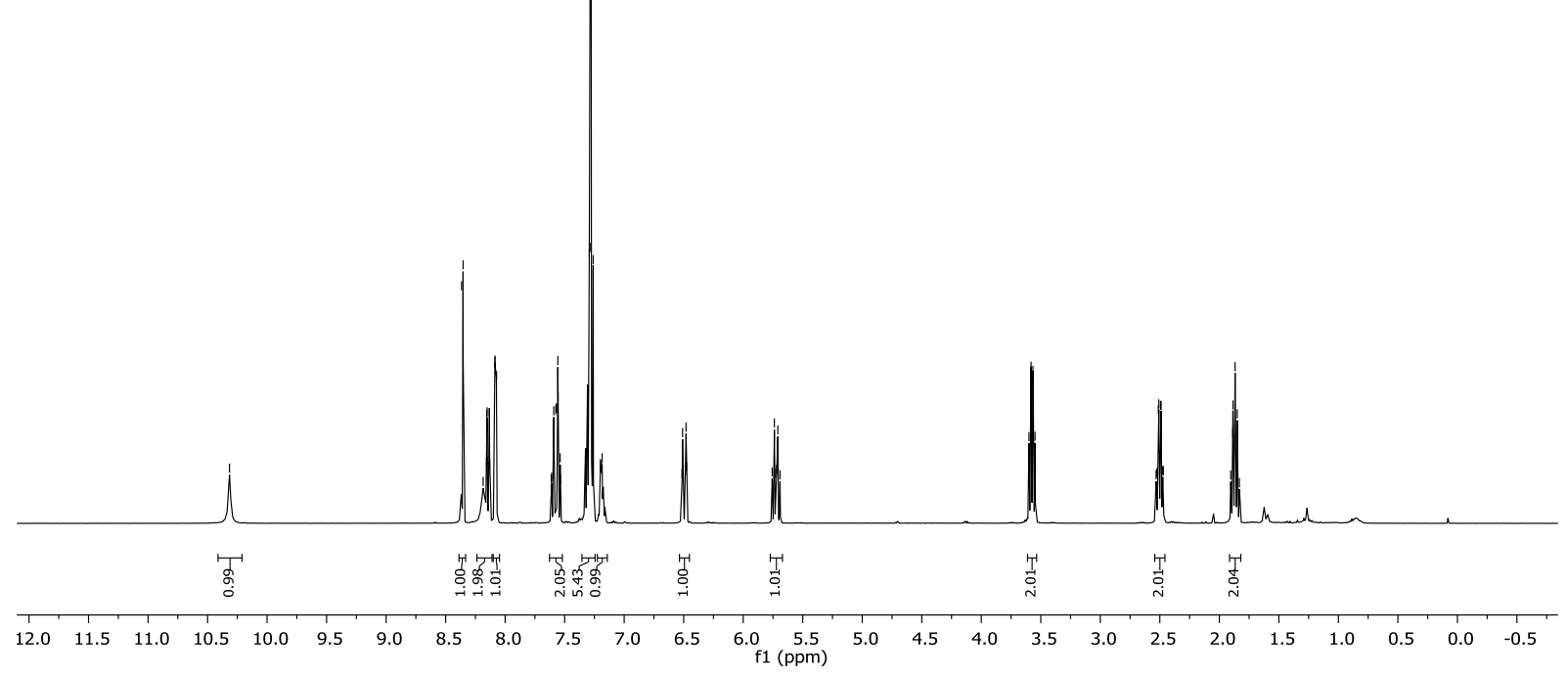

${ }^{13} \mathbf{C}$ NMR $\left(100 \mathrm{MHz}, \mathrm{CDCl}_{3}, 25^{\circ} \mathrm{C}\right)$ of $\mathbf{S 1 2}$

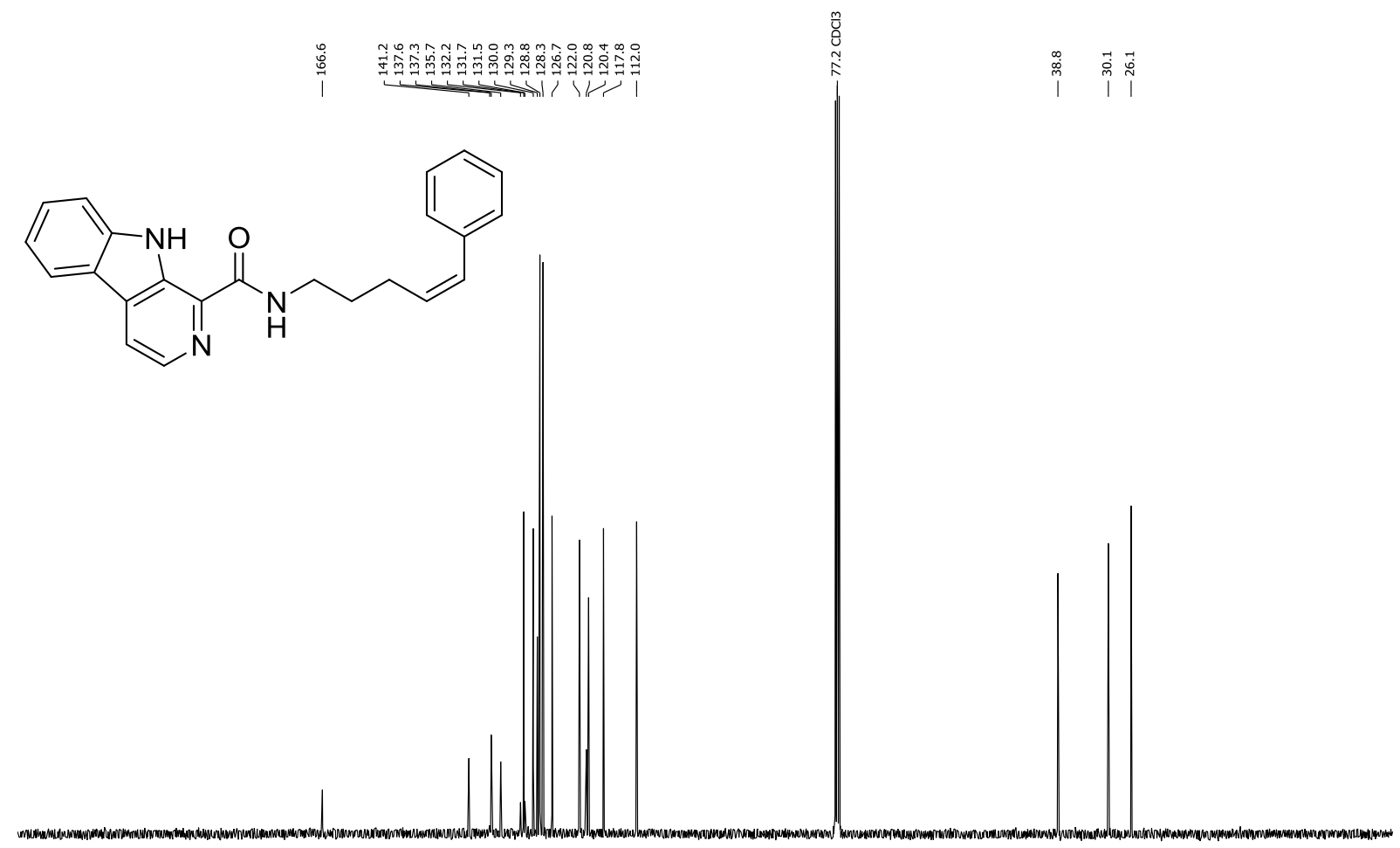

$\begin{array}{llllllllllllllllllllllllll}210 & 200 & 190 & 180 & 170 & 160 & 150 & 140 & 130 & 120 & 110 & 100 & 90 & 80 & 70 & 60 & 50 & 40 & 30 & 20 & 10 & 0 & -10\end{array}$ 
${ }^{1} \mathbf{H}$ NMR $\left(400 \mathrm{MHz}, \mathrm{CDCl}_{3}, 25^{\circ} \mathrm{C}\right)$ of $\mathbf{S 1 3}$

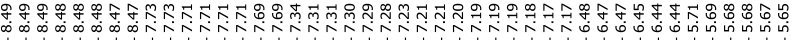<smiles>CC(C)(NC(=O)CC/C=C/c1ccccc1)c1ccccn1</smiles>

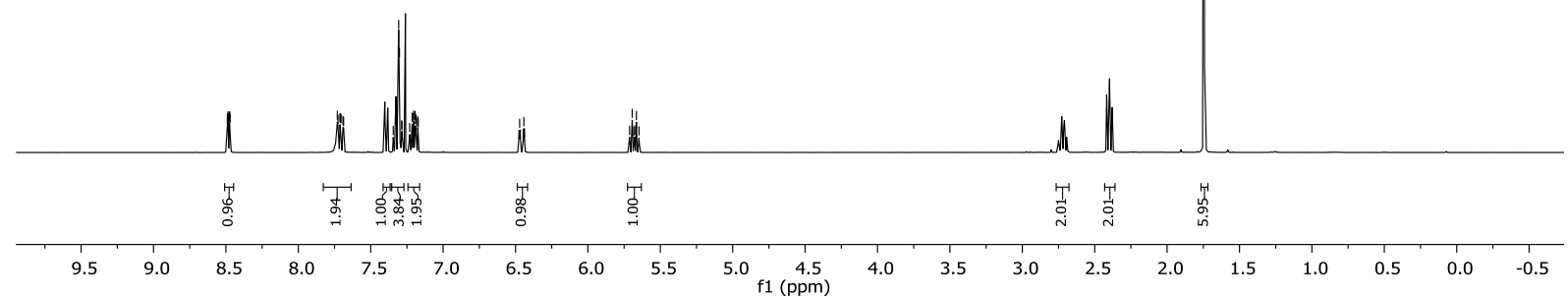

${ }^{13} \mathbf{C}$ NMR $\left(100 \mathrm{MHz}, \mathrm{CDCl}_{3}, 25^{\circ} \mathrm{C}\right)$ of $\mathbf{S 1 3}$

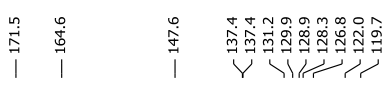<smiles>CC(C)(NC(=O)CC/C=C\c1ccccc1)c1ccccn1</smiles>

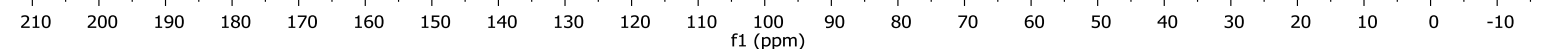


${ }^{\mathbf{1}} \mathbf{H}$ NMR $\left(400 \mathrm{MHz}, \mathrm{CDCl}_{3}, 25^{\circ} \mathrm{C}\right)$ of $\mathbf{2 a}$

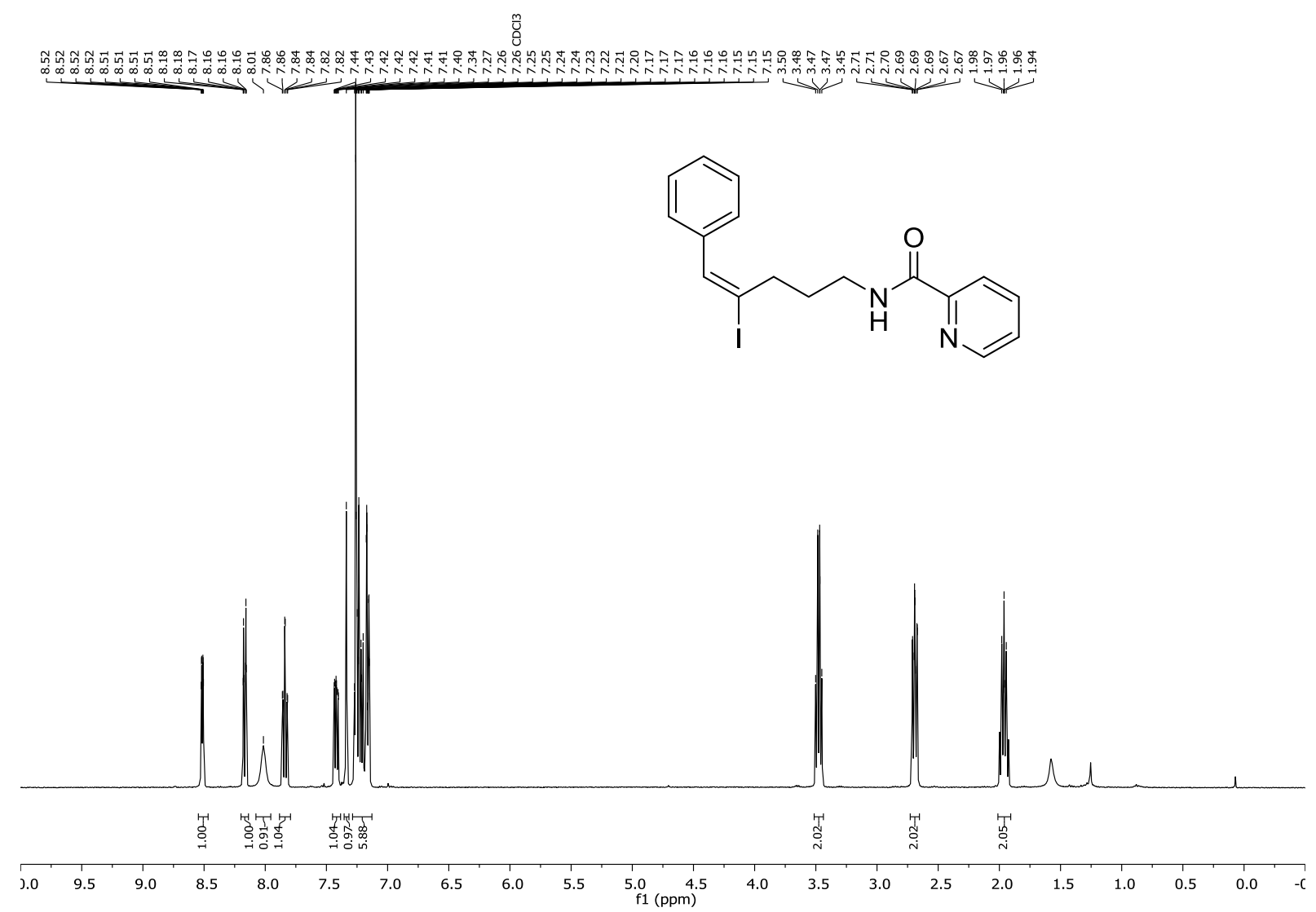

${ }^{13} \mathbf{C ~ N M R}\left(100 \mathrm{MHz}, \mathrm{CDCl}_{3}, 25^{\circ} \mathrm{C}\right)$ of $\mathbf{2 a}$

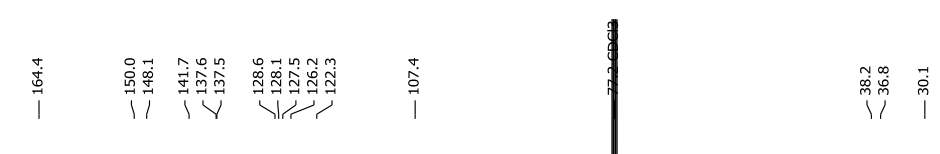<smiles>O=C(NCCC/C(I)=C\c1ccccc1)c1ccccn1</smiles>

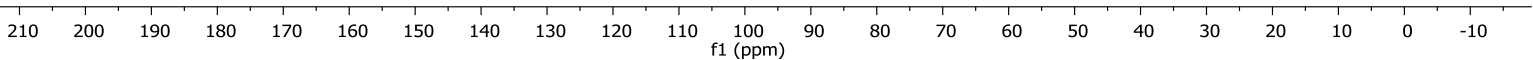


$\operatorname{NOESY}\left(500 \mathrm{MHz}, \mathrm{CDCl}_{3} .25{ }^{\circ} \mathrm{C}, \mathrm{t}_{\mathrm{m}}=500 \mathrm{~ms}\right)$ of $\mathbf{2 a}$

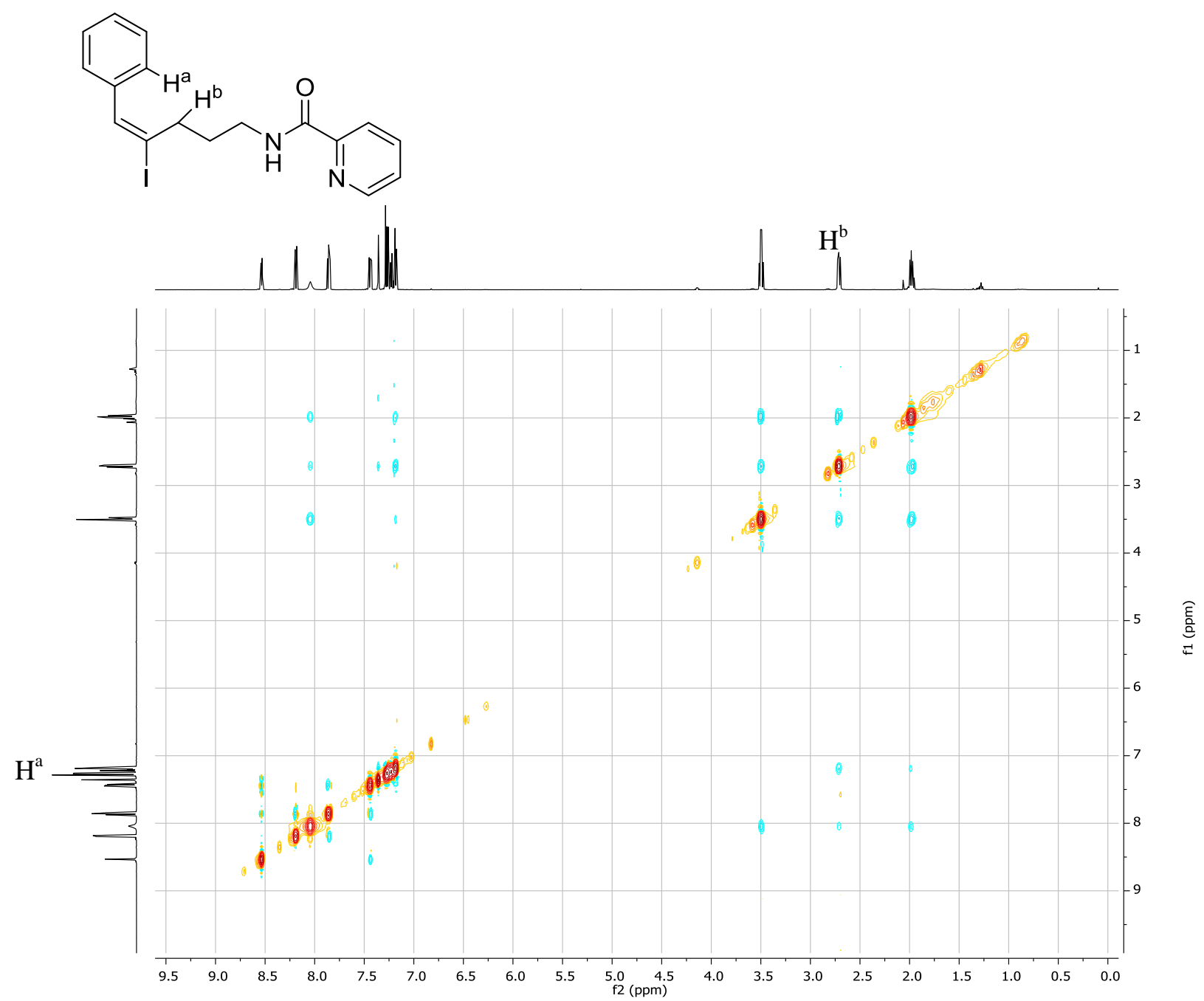


${ }^{\mathbf{1}} \mathbf{H}$ NMR $\left(400 \mathrm{MHz}, \mathrm{CDCl}_{3}, 25^{\circ} \mathrm{C}\right)$ of $\mathbf{2 b}$

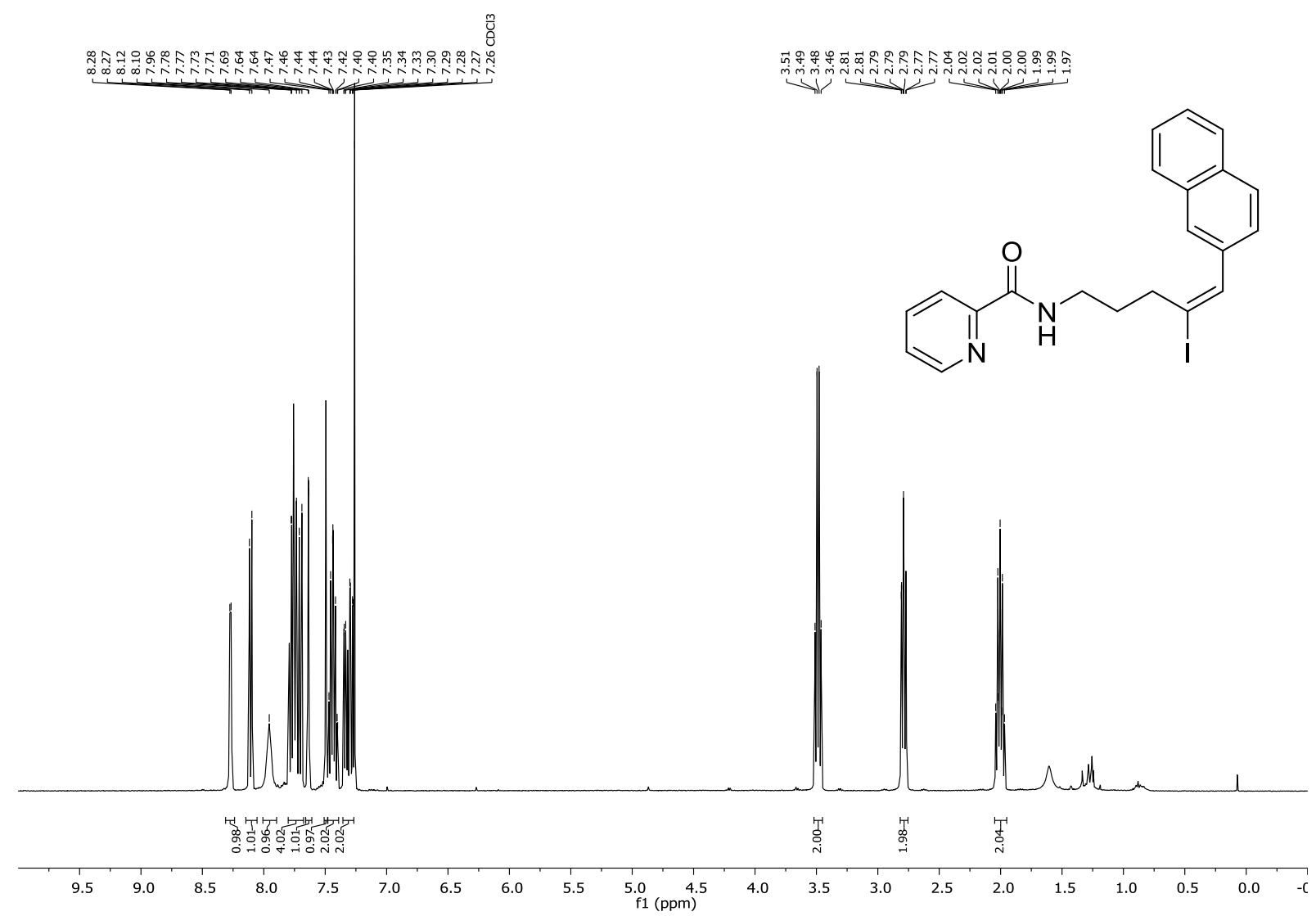

${ }^{13} \mathbf{C}$ NMR $\left(100 \mathrm{MHz}, \mathrm{CDCl}_{3}, 25^{\circ} \mathrm{C}\right)$ of $\mathbf{2 b}$

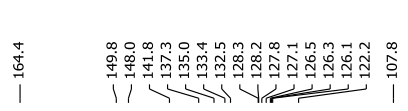

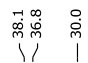

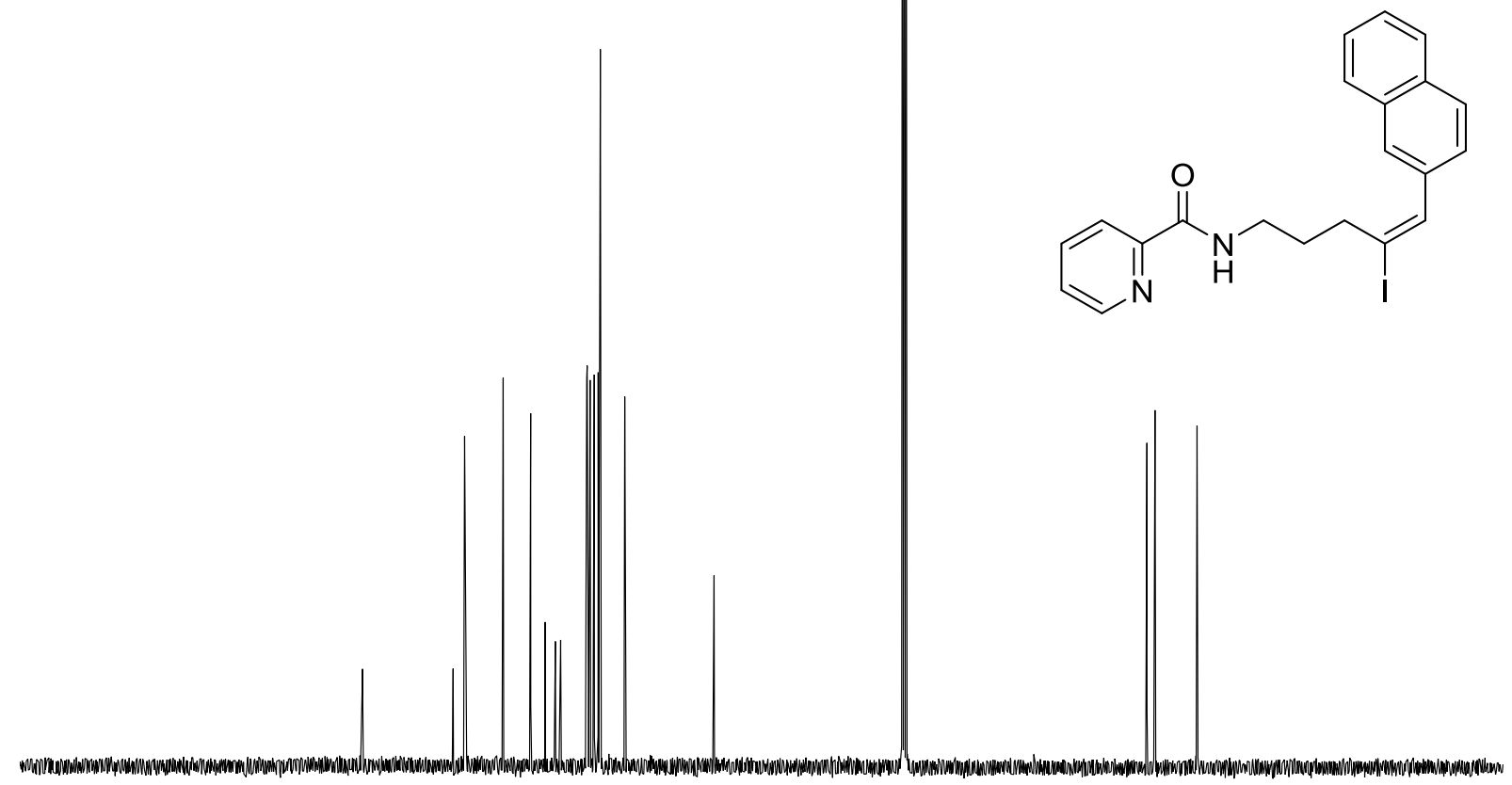

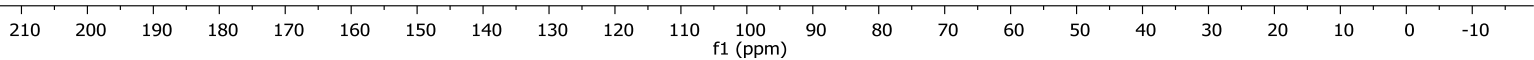


${ }^{\mathbf{1}} \mathbf{H}$ NMR $\left(400 \mathrm{MHz}, \mathrm{CDCl}_{3}, 25^{\circ} \mathrm{C}\right)$ of $\mathbf{2 c}$

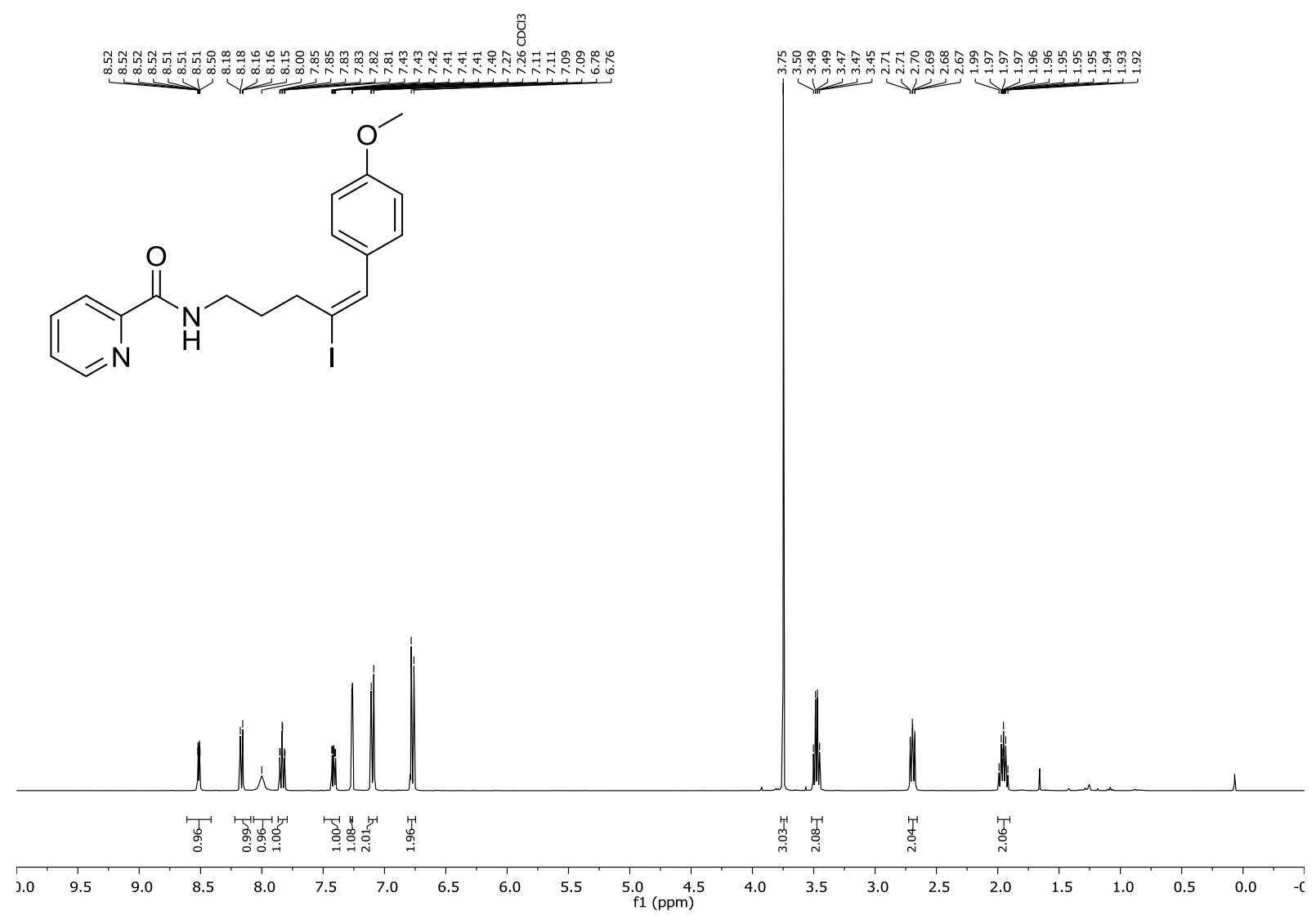

${ }^{13} \mathbf{C ~ N M R}\left(100 \mathrm{MHz}, \mathrm{CDCl}_{3}, 25^{\circ} \mathrm{C}\right)$ of $\mathbf{2 c}$

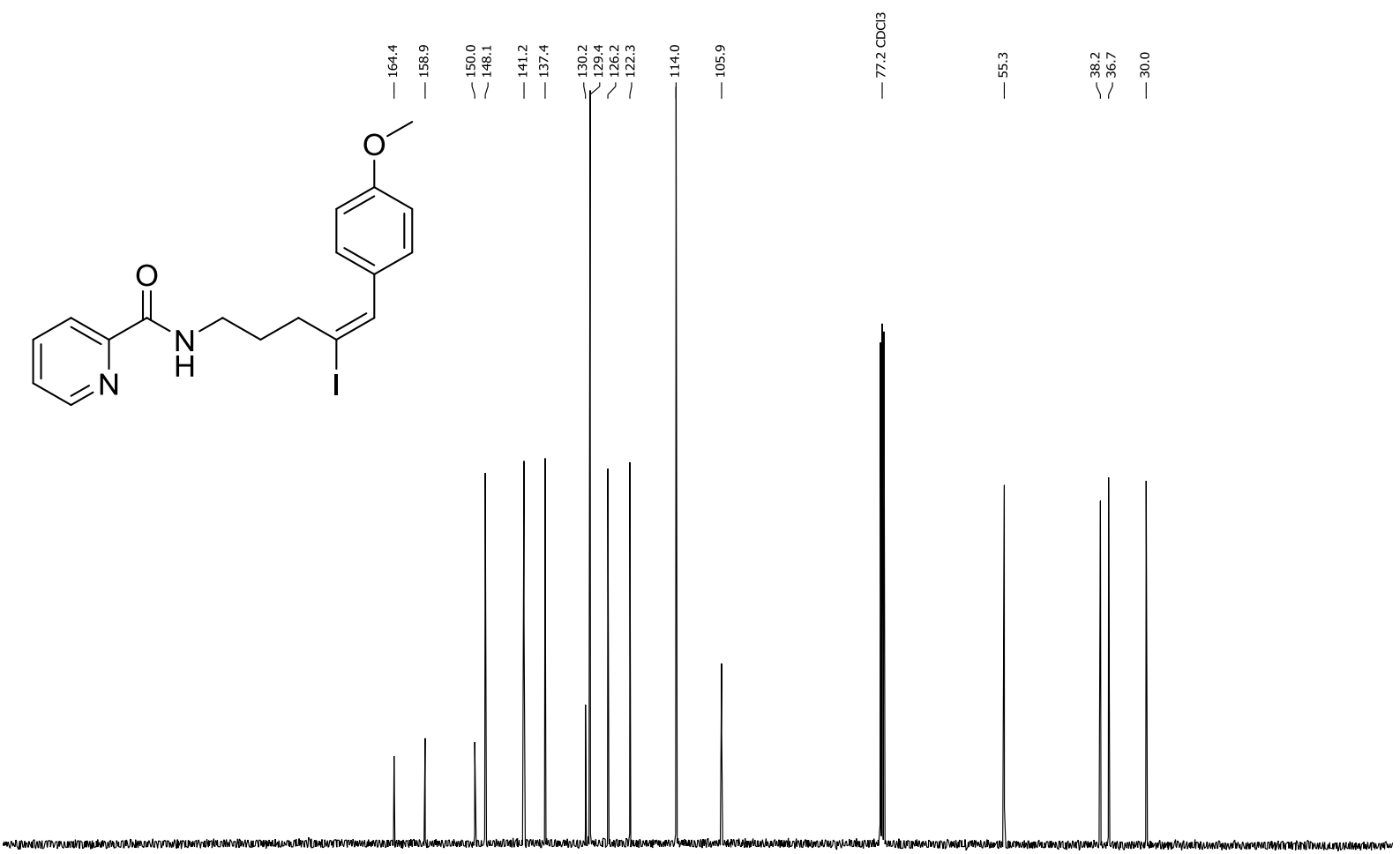

$\begin{array}{llllllllllllllllllllllllllll}230 & 220 & 210 & 200 & 190 & 180 & 170 & 160 & 150 & 140 & 130 & 120 & 110 & 100 & 90 & 80 & 70 & 60 & 50 & 40 & 30 & 20 & 10 & 0 & -10\end{array}$ 
NOESY $\left(500 \mathrm{MHz}, \mathrm{CDCl}_{3} .25^{\circ} \mathrm{C}, \mathrm{t}_{\mathrm{m}}=500 \mathrm{~ms}\right)$ of $\mathbf{2 c}$

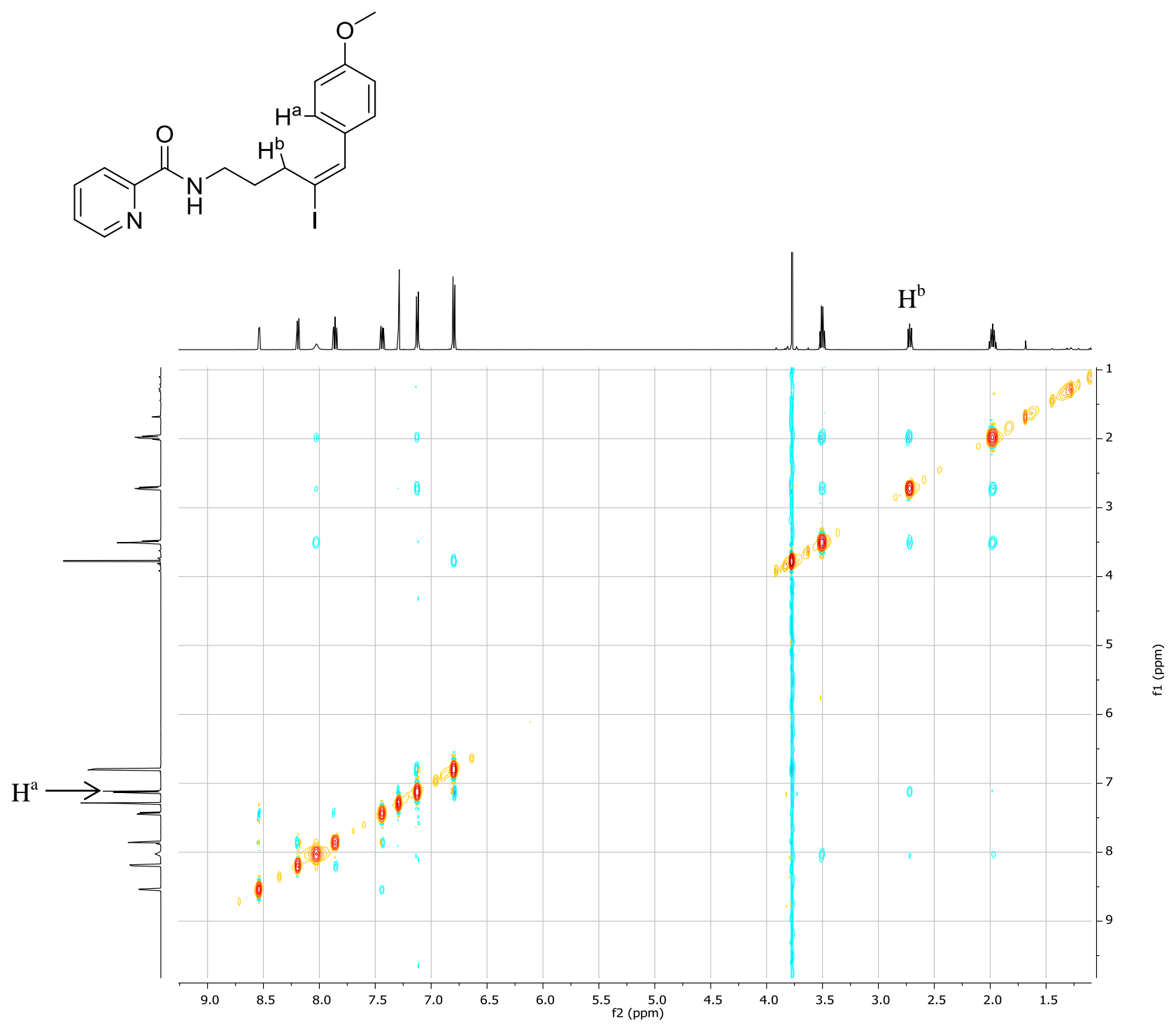


${ }^{\mathbf{1}} \mathbf{H}$ NMR $\left(400 \mathrm{MHz}, \mathrm{CDCl}_{3}, 25^{\circ} \mathrm{C}\right)$ of $\mathbf{2 d}$

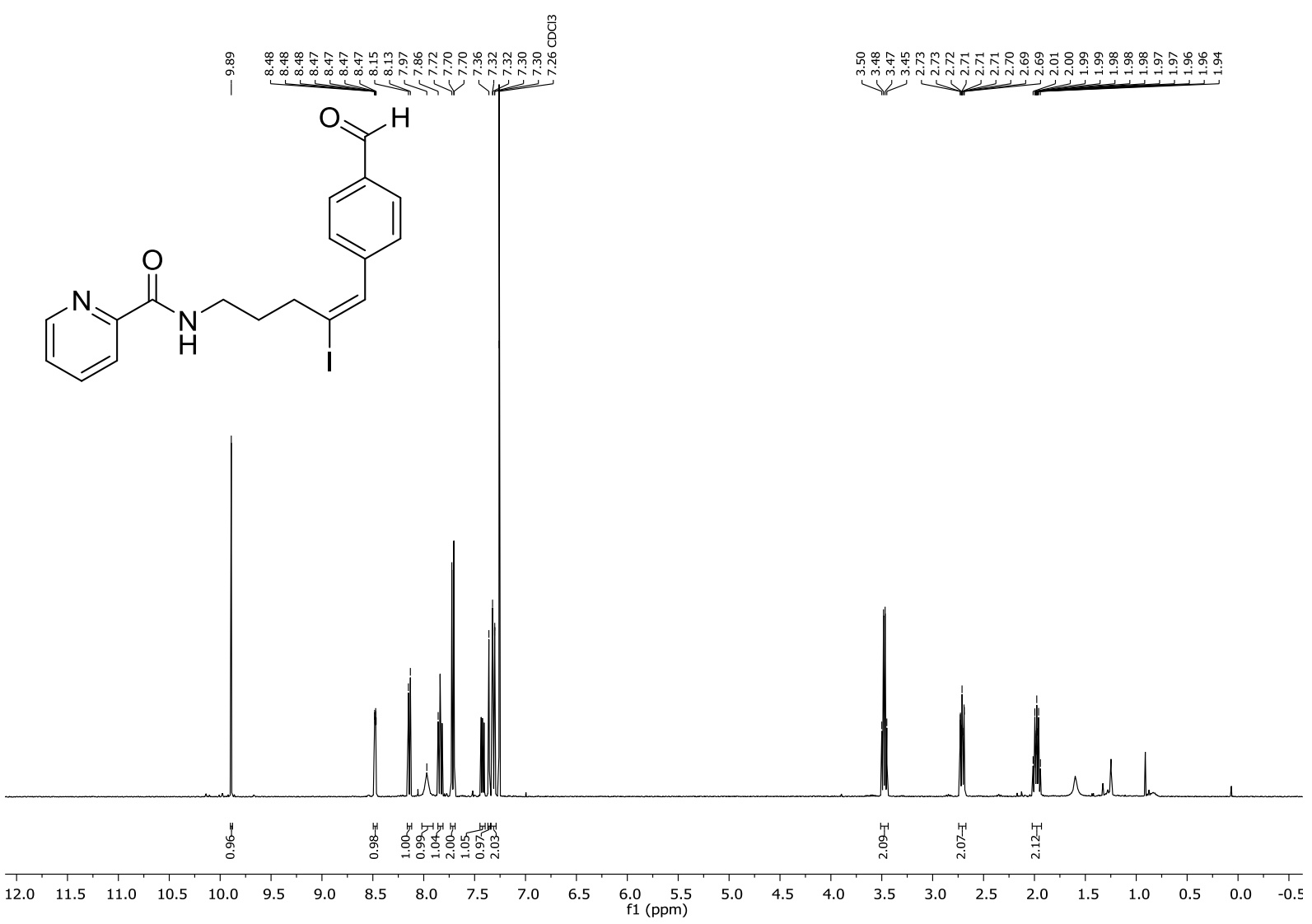

${ }^{13} \mathbf{C}$ NMR $\left(100 \mathrm{MHz}, \mathrm{CDCl}_{3}, 25^{\circ} \mathrm{C}\right)$ of $\mathbf{2 d}$

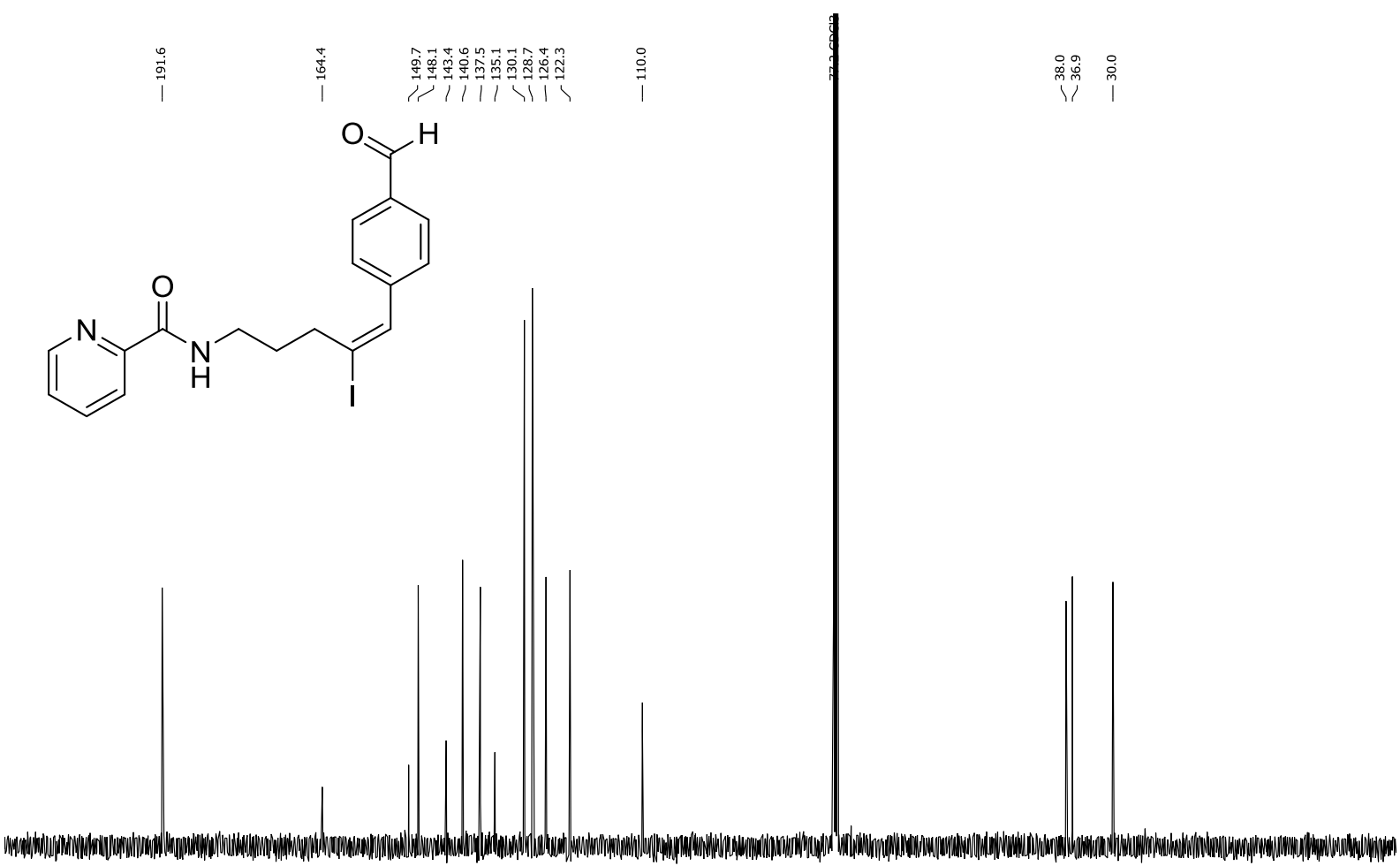

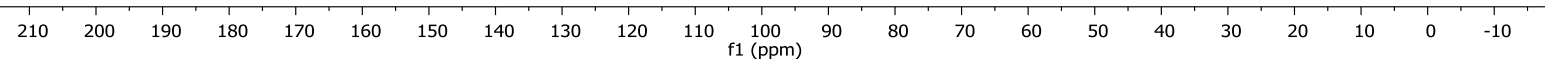


${ }^{\mathbf{1}} \mathbf{H}$ NMR $\left(500 \mathrm{MHz}, \mathrm{CDCl}_{3}, 25^{\circ} \mathrm{C}\right)$ of $\mathbf{2 e}$

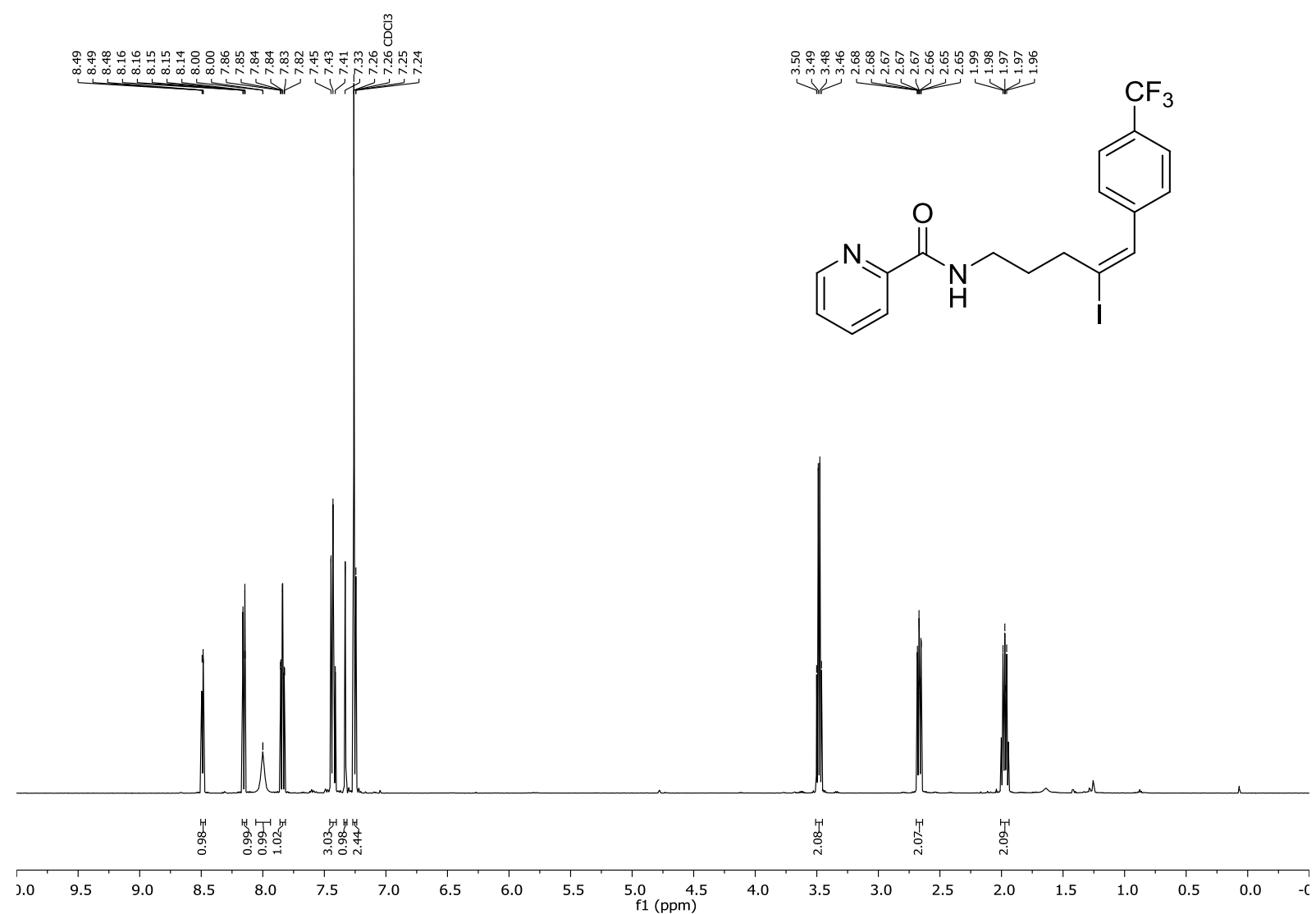

${ }^{13} \mathbf{C}$ NMR $\left(126 \mathrm{MHz}, \mathrm{CDCl}_{3}, 25{ }^{\circ} \mathrm{C}\right)$ of $\mathbf{2 e}$

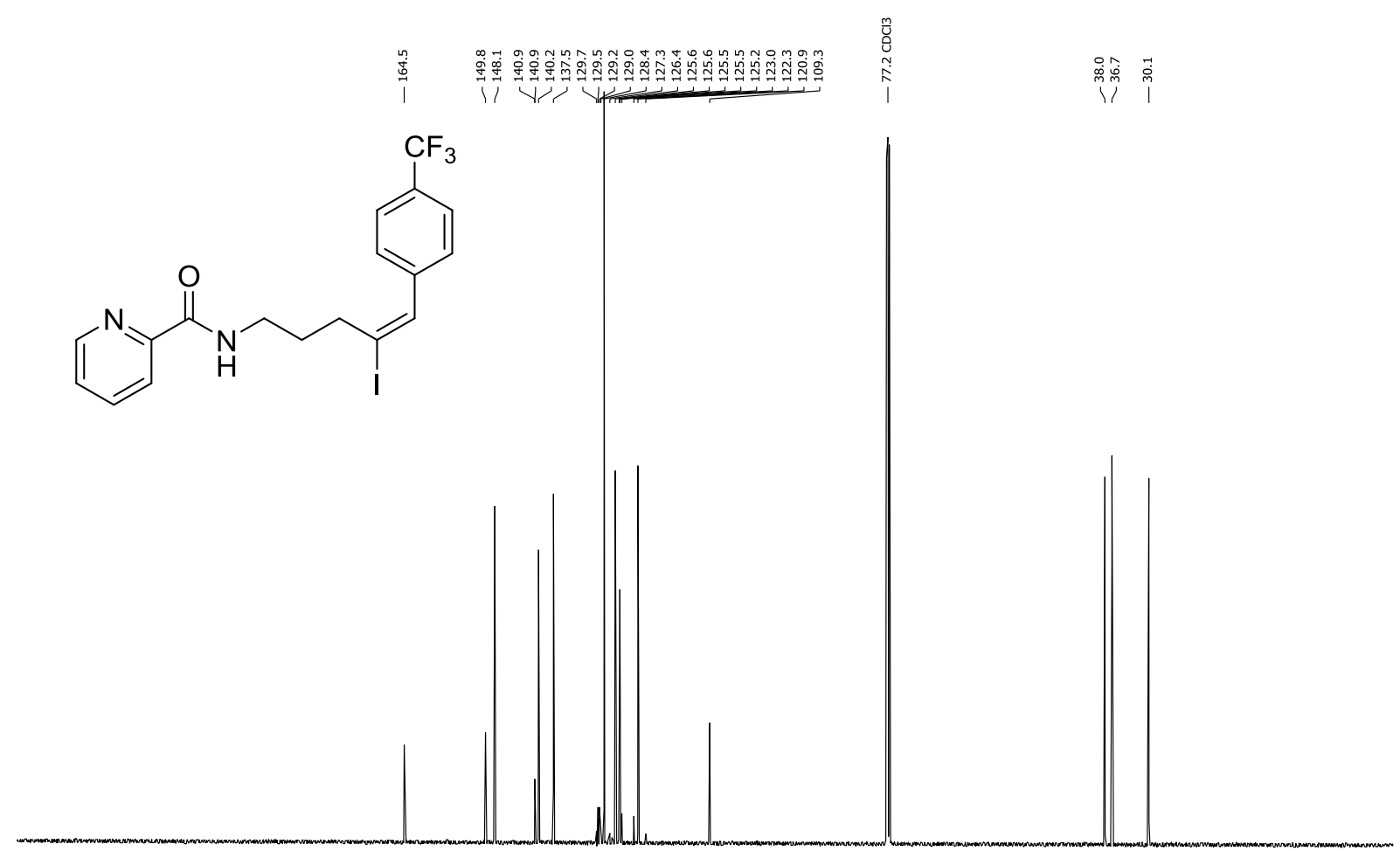

$\begin{array}{llllllllllllllllllllllllllll}130 & 220 & 210 & 200 & 190 & 180 & 170 & 160 & 150 & 140 & 130 & 120 & 110 & 100 & 90 & 80 & 70 & 60 & 50 & 40 & 30 & 20 & 10 & 0 & -10\end{array}$ 
${ }^{19}$ F NMR $\left(471 \mathrm{MHz}, \mathrm{CDCl}_{3}, 25{ }^{\circ} \mathrm{C}\right)$ of $\mathbf{2 e}$

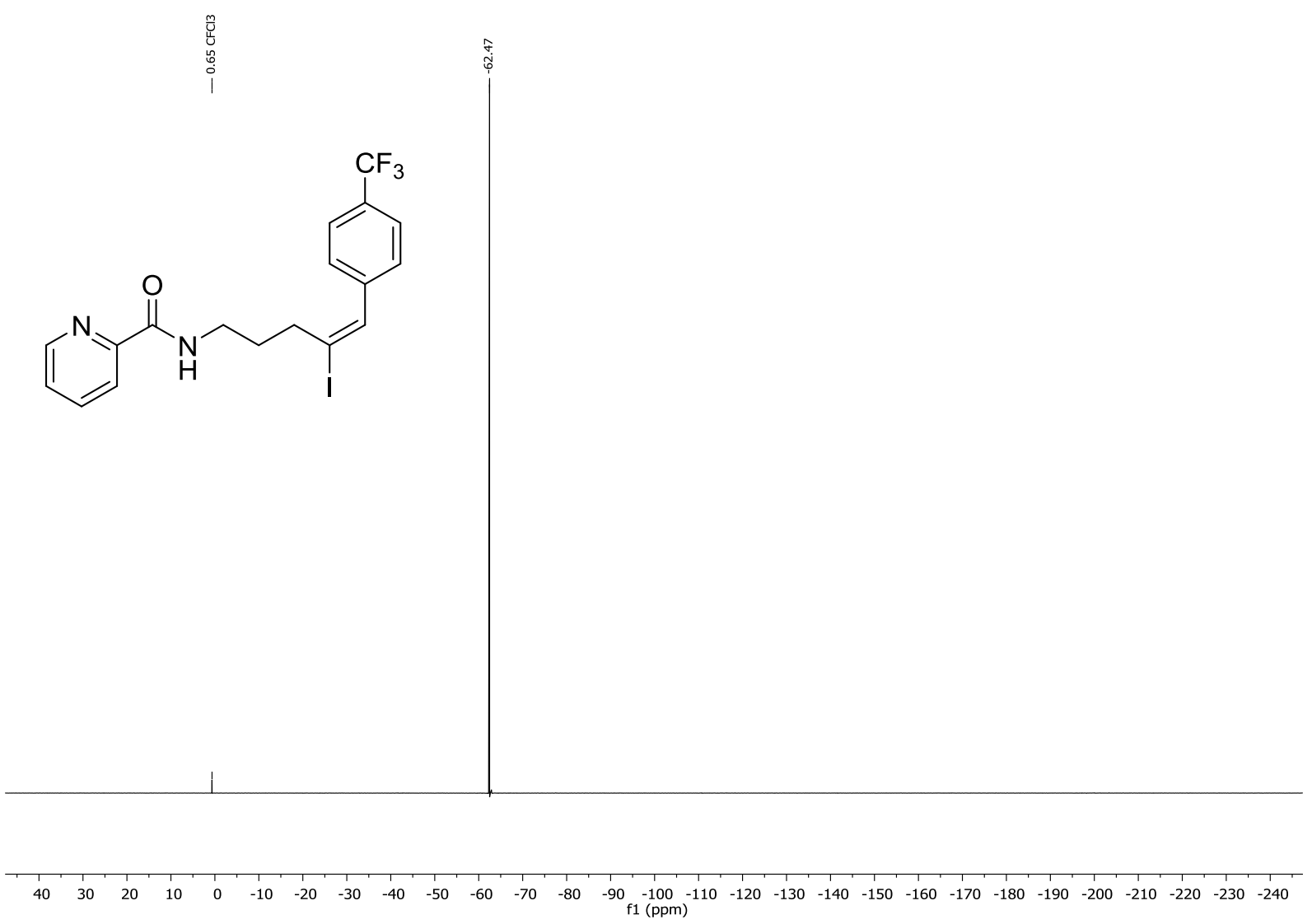


${ }^{\mathbf{1}} \mathbf{H}$ NMR $\left(500 \mathrm{MHz}, \mathrm{CDCl}_{3}, 25^{\circ} \mathrm{C}\right)$ of $\mathbf{2 f}$

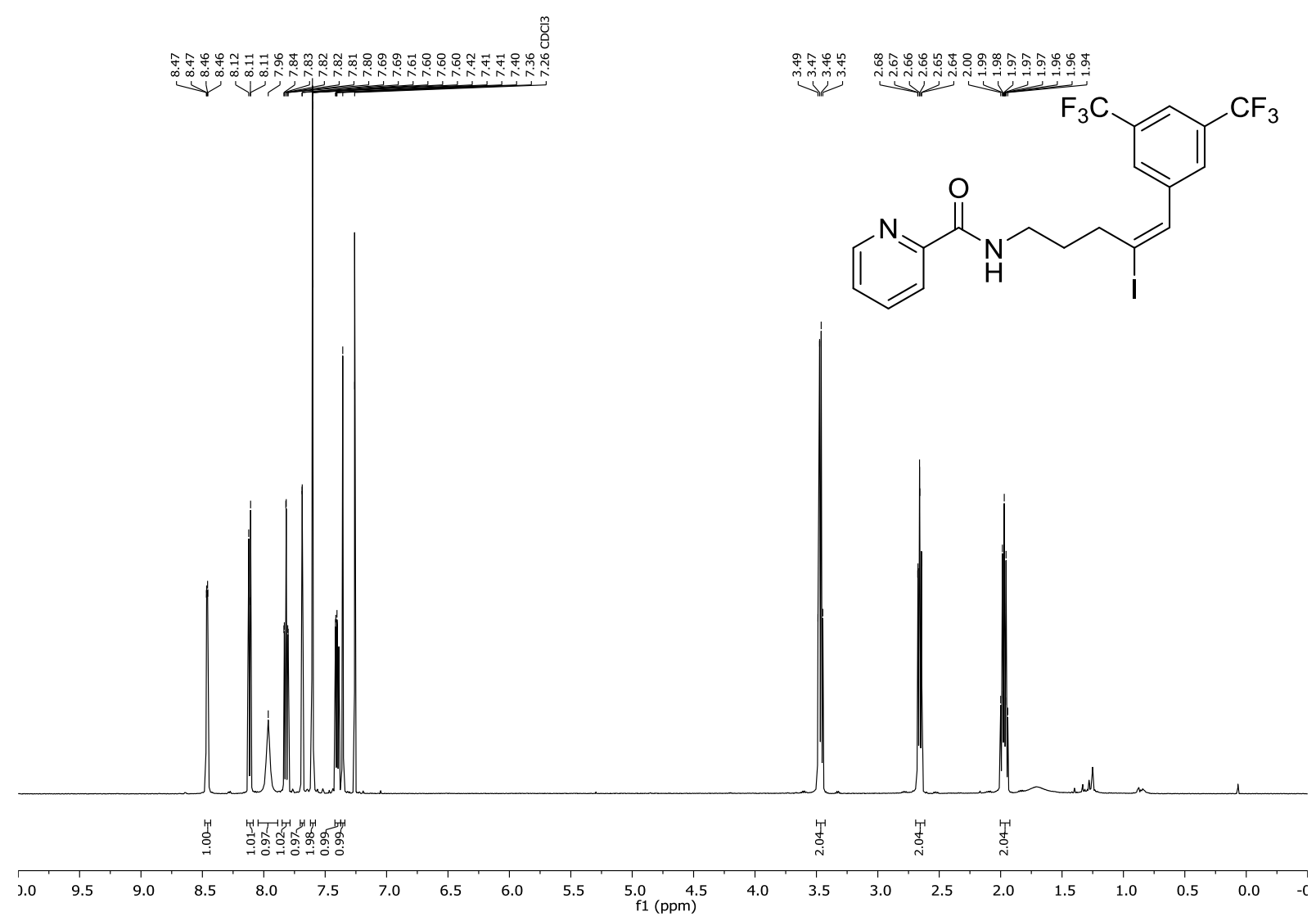

${ }^{13} \mathbf{C}$ NMR $\left(126 \mathrm{MHz}, \mathrm{CDCl}_{3}, 25^{\circ} \mathrm{C}\right)$ of $\mathbf{2 f}$

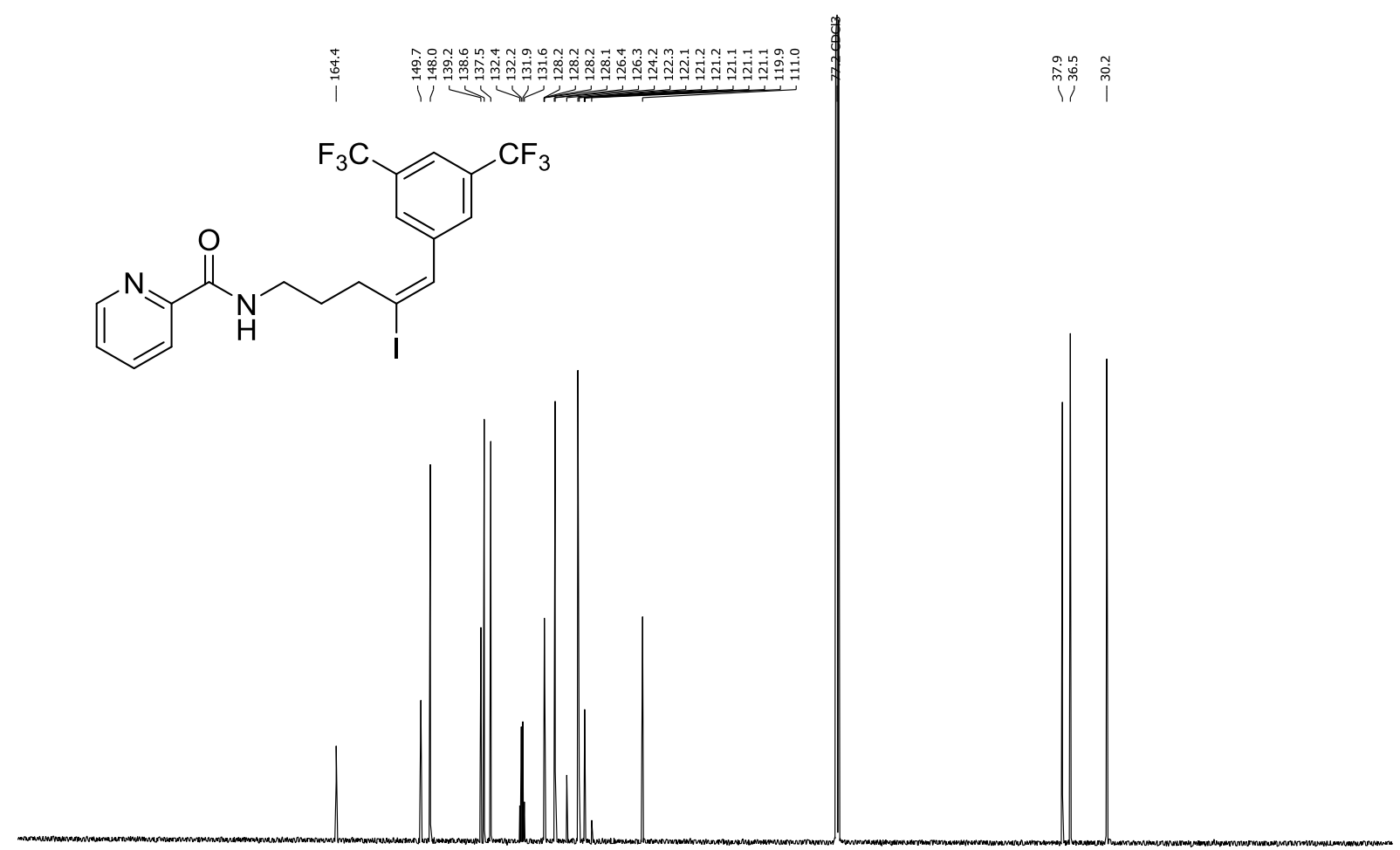

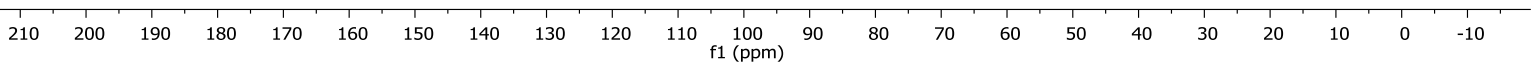


${ }^{19}$ F NMR $\left(471 \mathrm{MHz}, \mathrm{CDCl}_{3}, 25^{\circ} \mathrm{C}\right)$ of $\mathbf{2 f}$

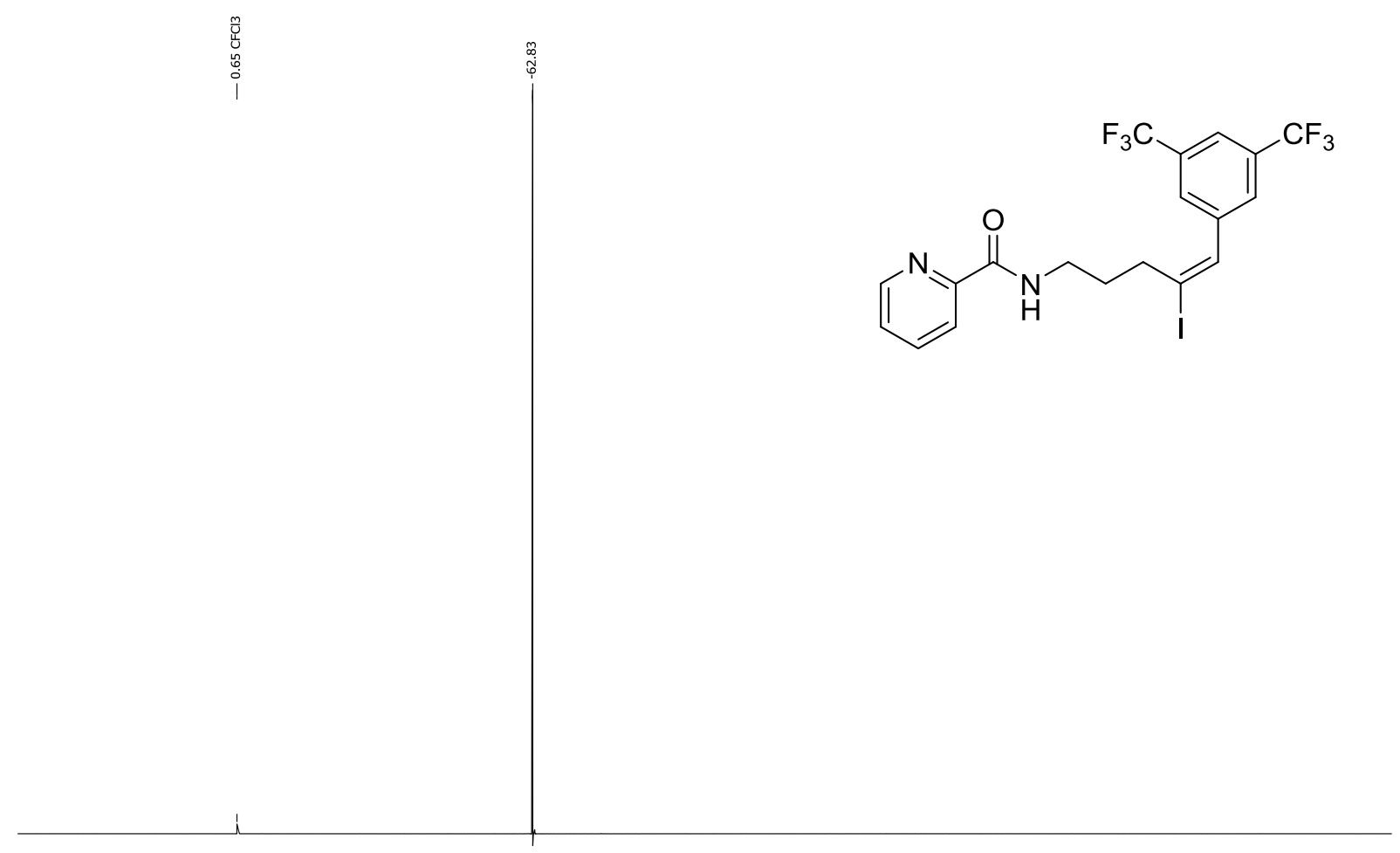

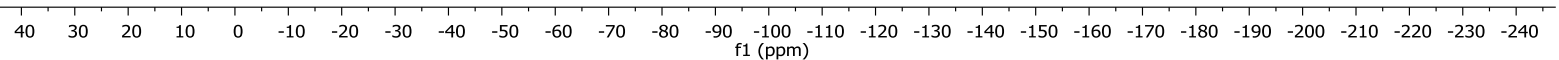


${ }^{\mathbf{1}} \mathbf{H}$ NMR $\left(400 \mathrm{MHz}, \mathrm{CDCl}_{3}, 25^{\circ} \mathrm{C}\right)$ of $\mathbf{2 g}$

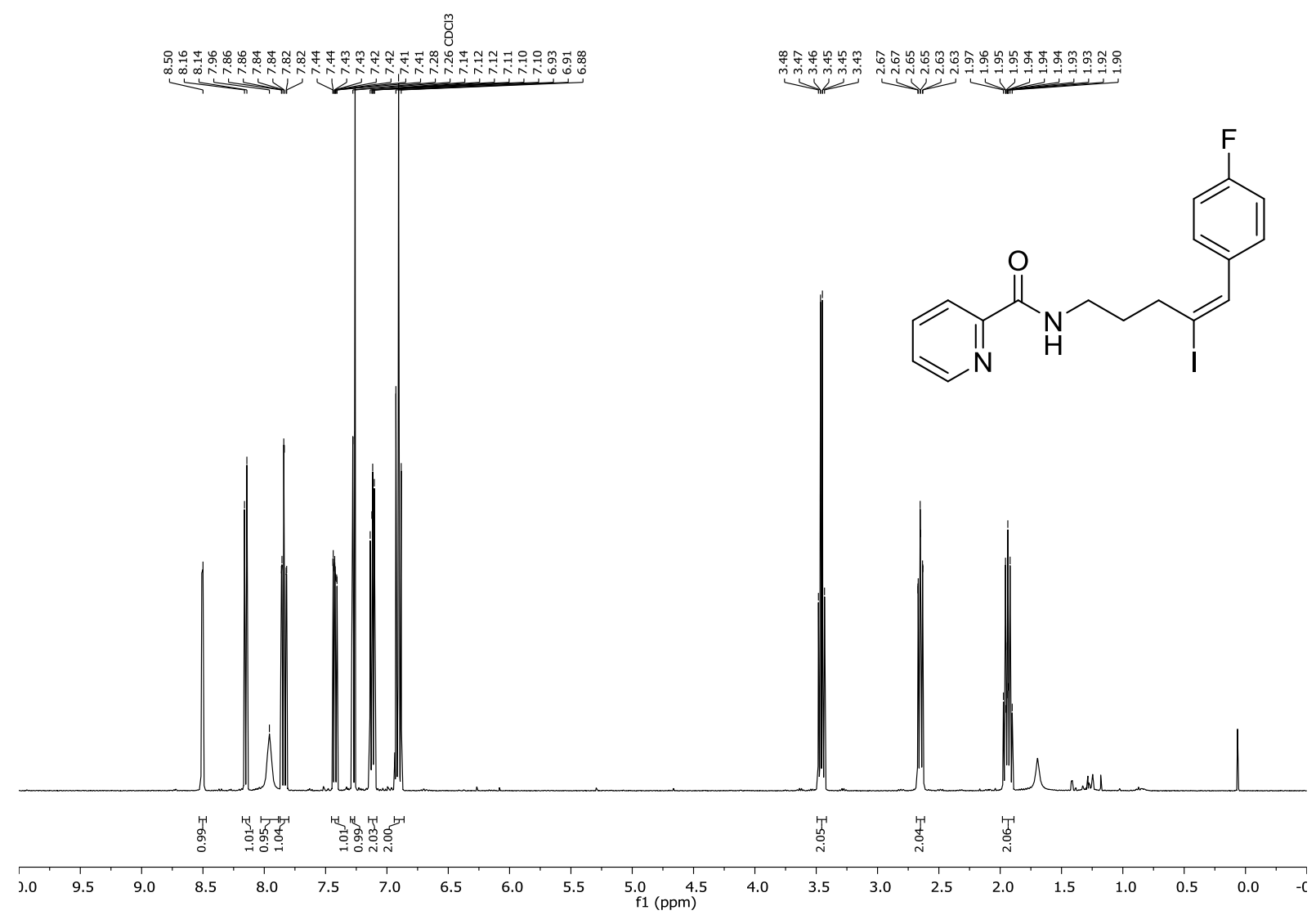

${ }^{13} \mathbf{C}$ NMR $\left(100 \mathrm{MHz}, \mathrm{CDCl}_{3}, 25^{\circ} \mathrm{C}\right)$ of $\mathbf{2 g}$

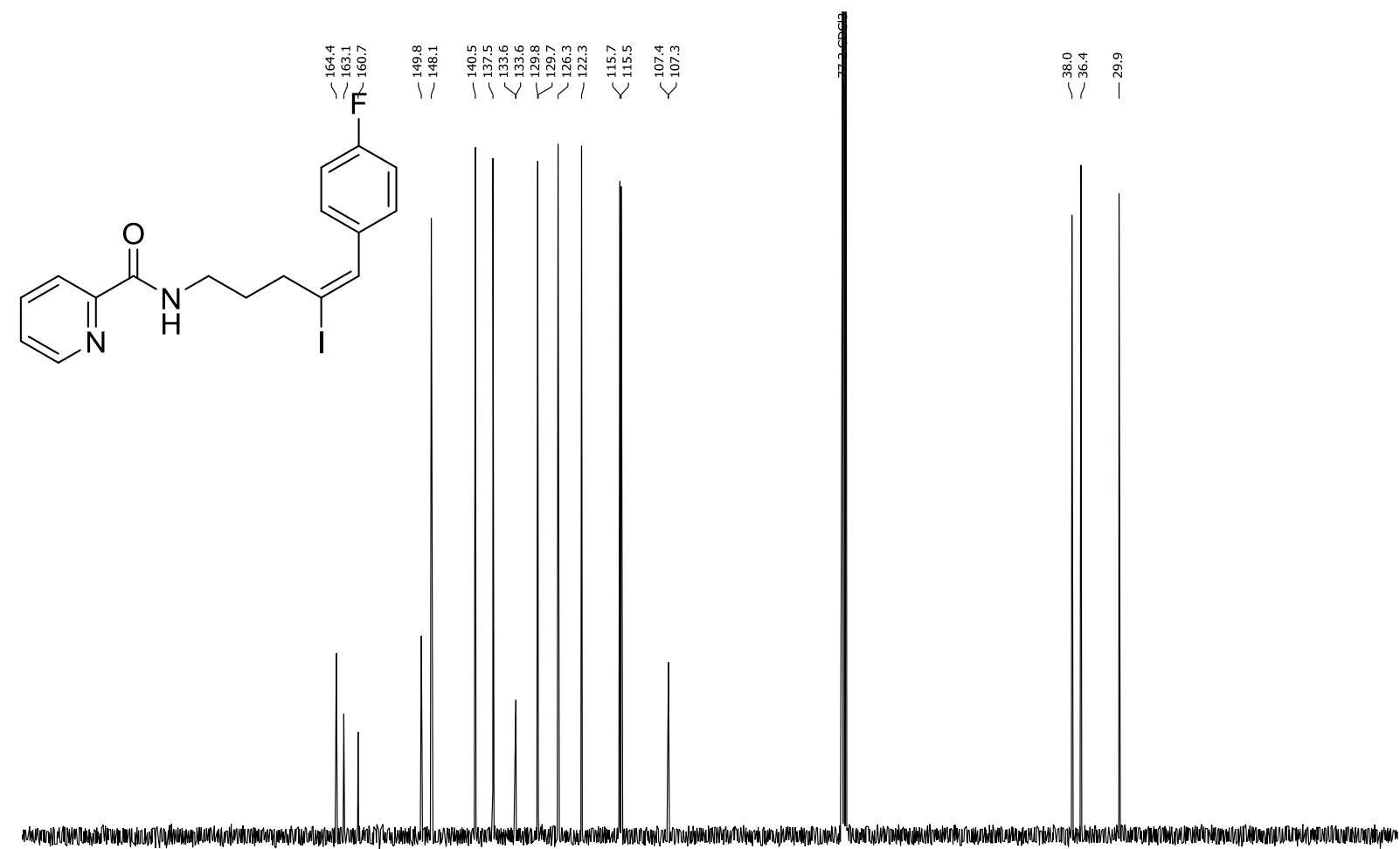

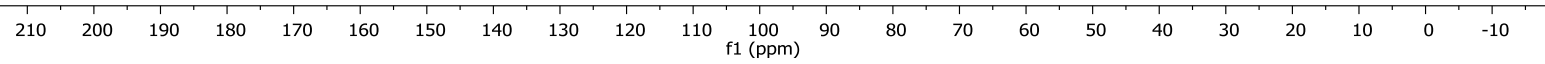


${ }^{19}$ F NMR $\left(376 \mathrm{MHz}, \mathrm{CDCl}_{3}, 25^{\circ} \mathrm{C}\right)$ of $\mathbf{2 g}$<smiles>O=C(NCCC/C(I)=C\c1ccc(F)cc1)c1ccccn1</smiles>

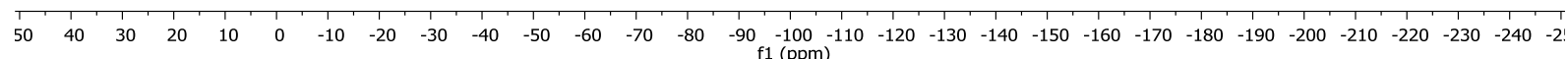


${ }^{\mathbf{1}} \mathbf{H}$ NMR $\left(400 \mathrm{MHz}, \mathrm{CDCl}_{3}, 25^{\circ} \mathrm{C}\right)$ of $\mathbf{2 h}$

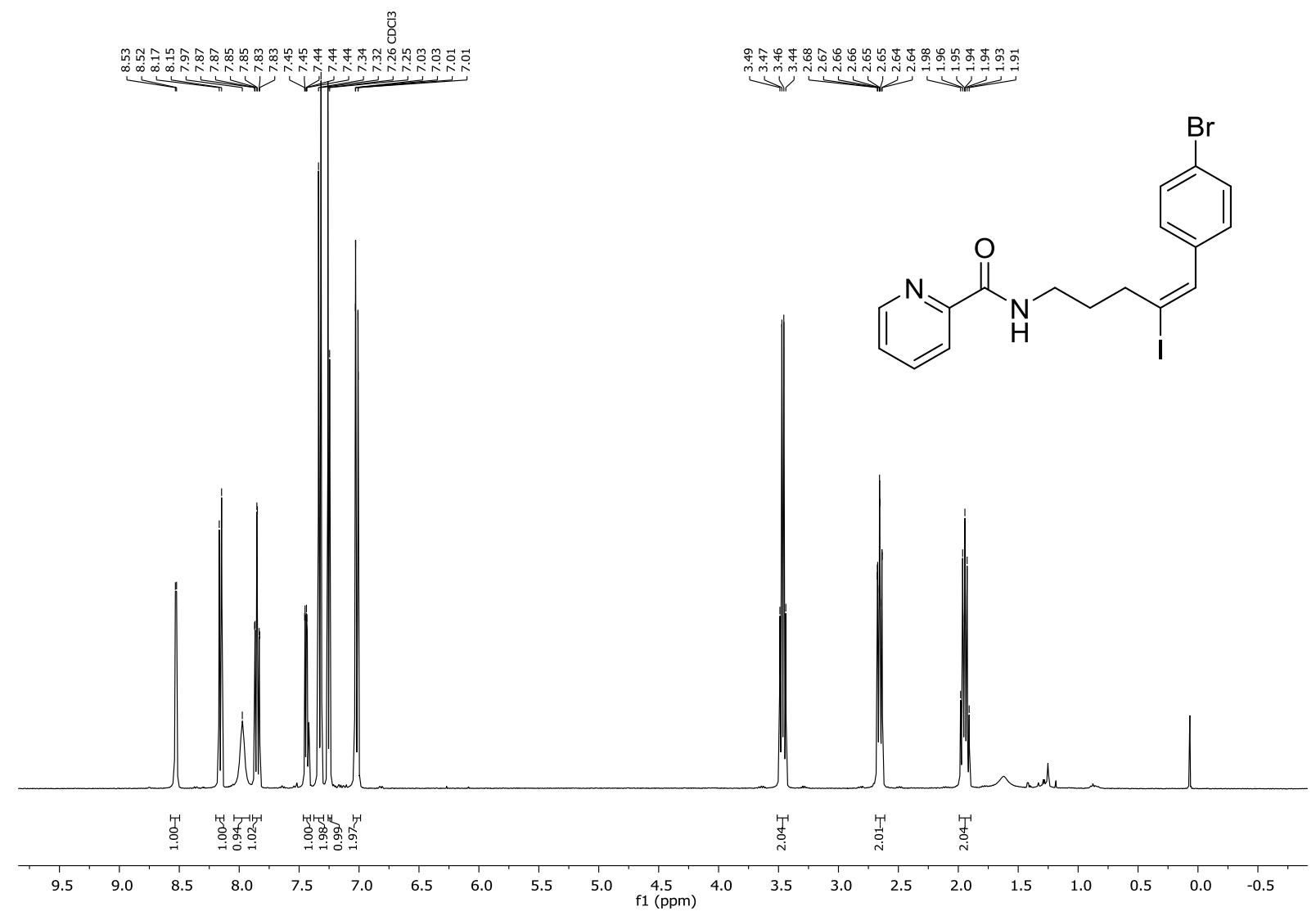

${ }^{13} \mathbf{C ~ N M R}\left(100 \mathrm{MHz}, \mathrm{CDCl}_{3}, 25^{\circ} \mathrm{C}\right)$ of $\mathbf{2 h}$

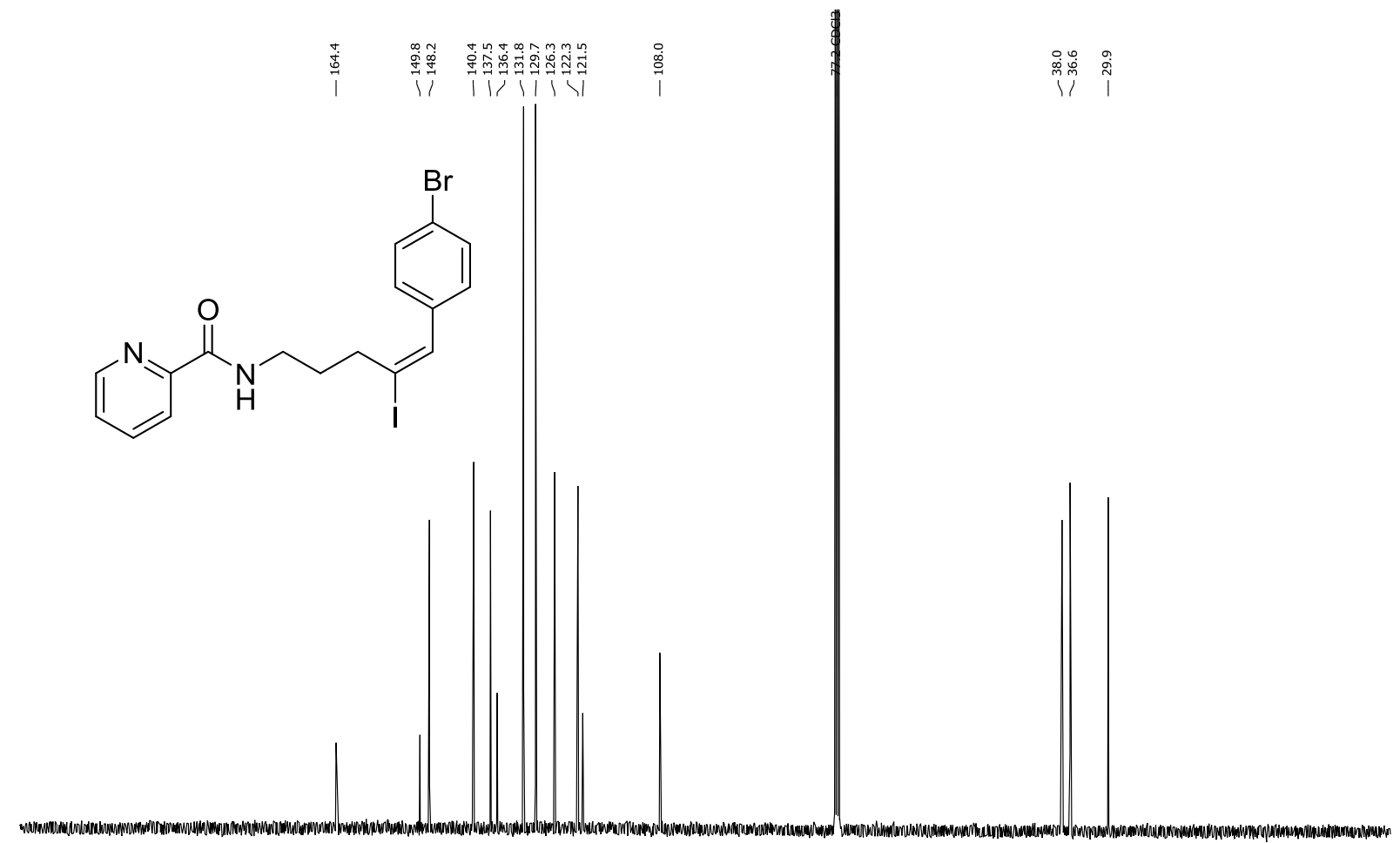

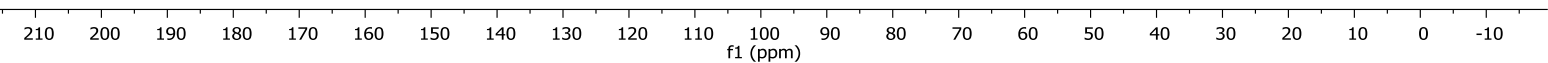


${ }^{1} \mathbf{H}$ NMR $\left(500 \mathrm{MHz}, \mathrm{CDCl}_{3}, 25^{\circ} \mathrm{C}\right)$ of $\mathbf{2 i}$

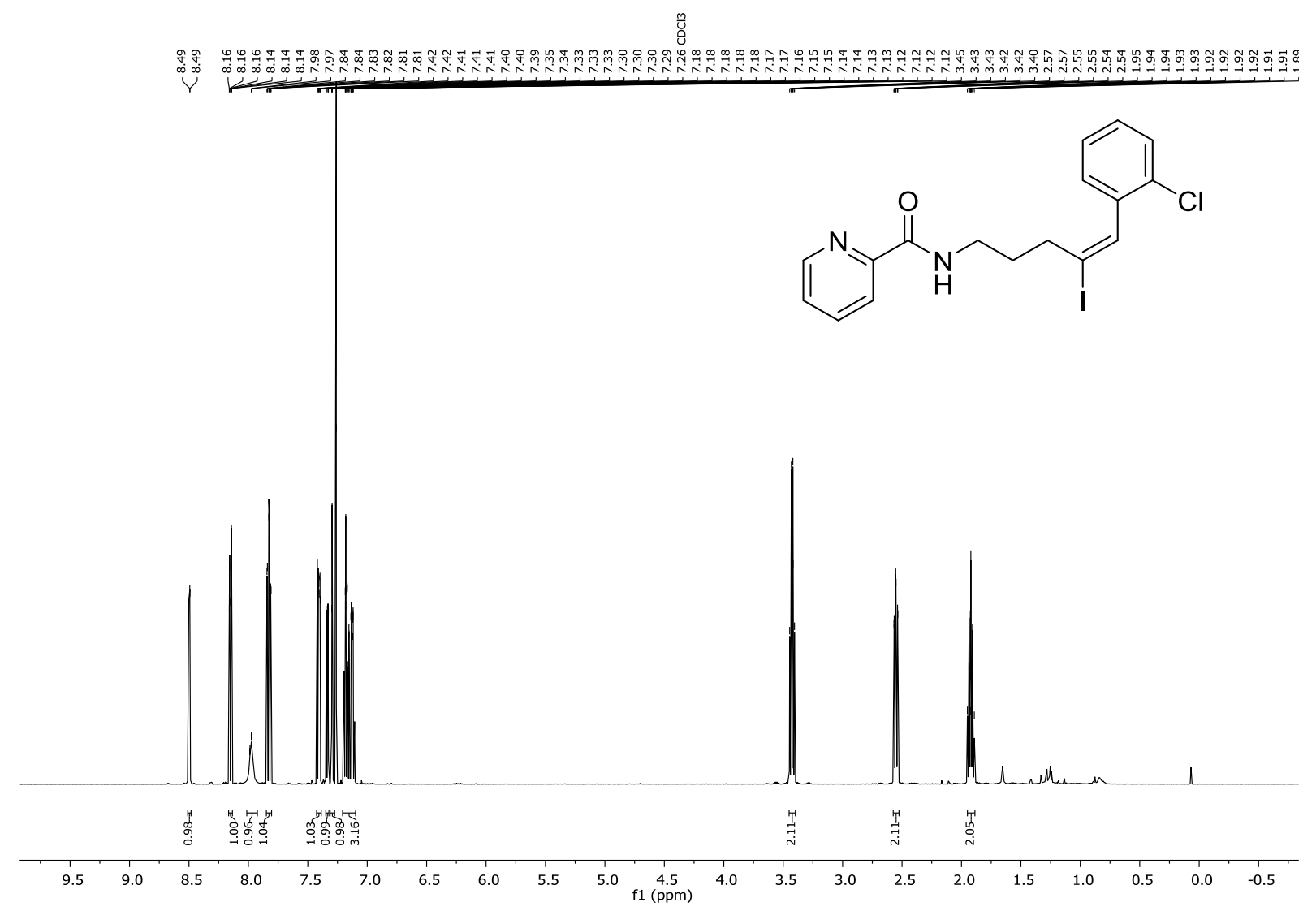

${ }^{13} \mathbf{C}$ NMR $\left(126 \mathrm{MHz}, \mathrm{CDCl}_{3}, 25^{\circ} \mathrm{C}\right)$ of $\mathbf{2 i}$
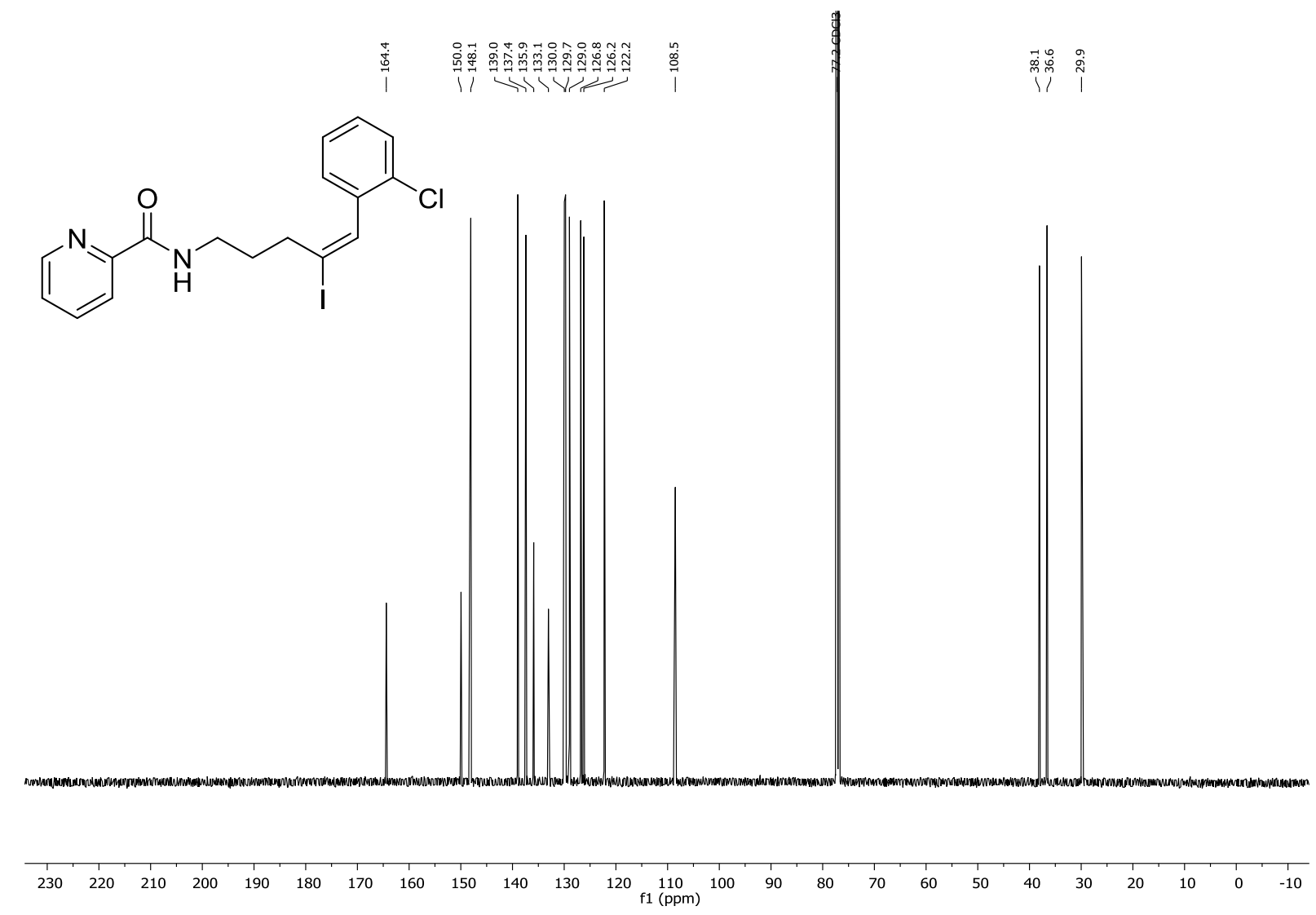

S141 
${ }^{\mathbf{1}} \mathbf{H}$ NMR $\left(400 \mathrm{MHz}, \mathrm{CDCl}_{3}, 25^{\circ} \mathrm{C}\right)$ of $\mathbf{2} \mathbf{j}$

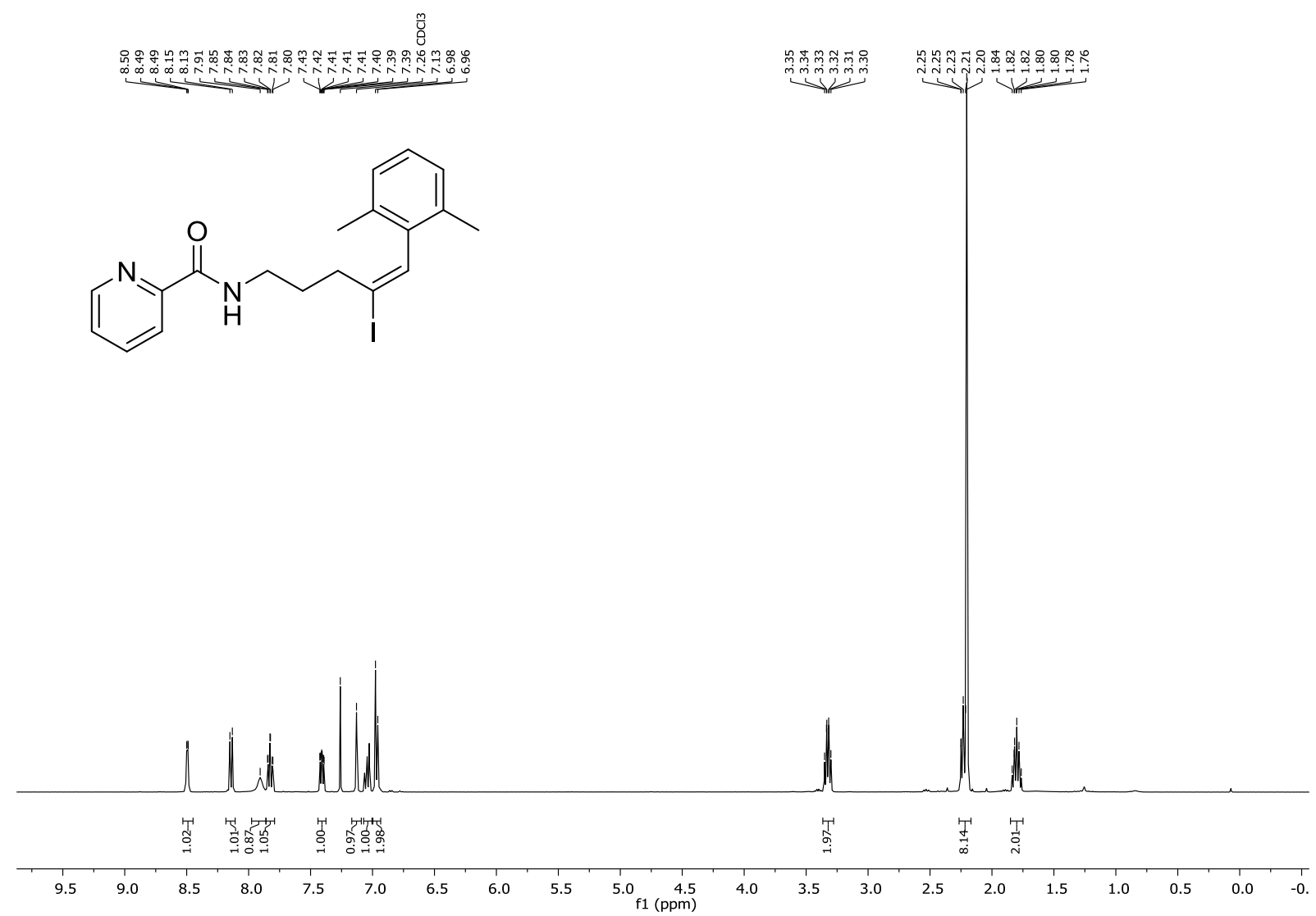

${ }^{13} \mathbf{C}$ NMR $\left(100 \mathrm{MHz}, \mathrm{CDCl}_{3}, 25{ }^{\circ} \mathrm{C}\right)$ of $\mathbf{2 j}$

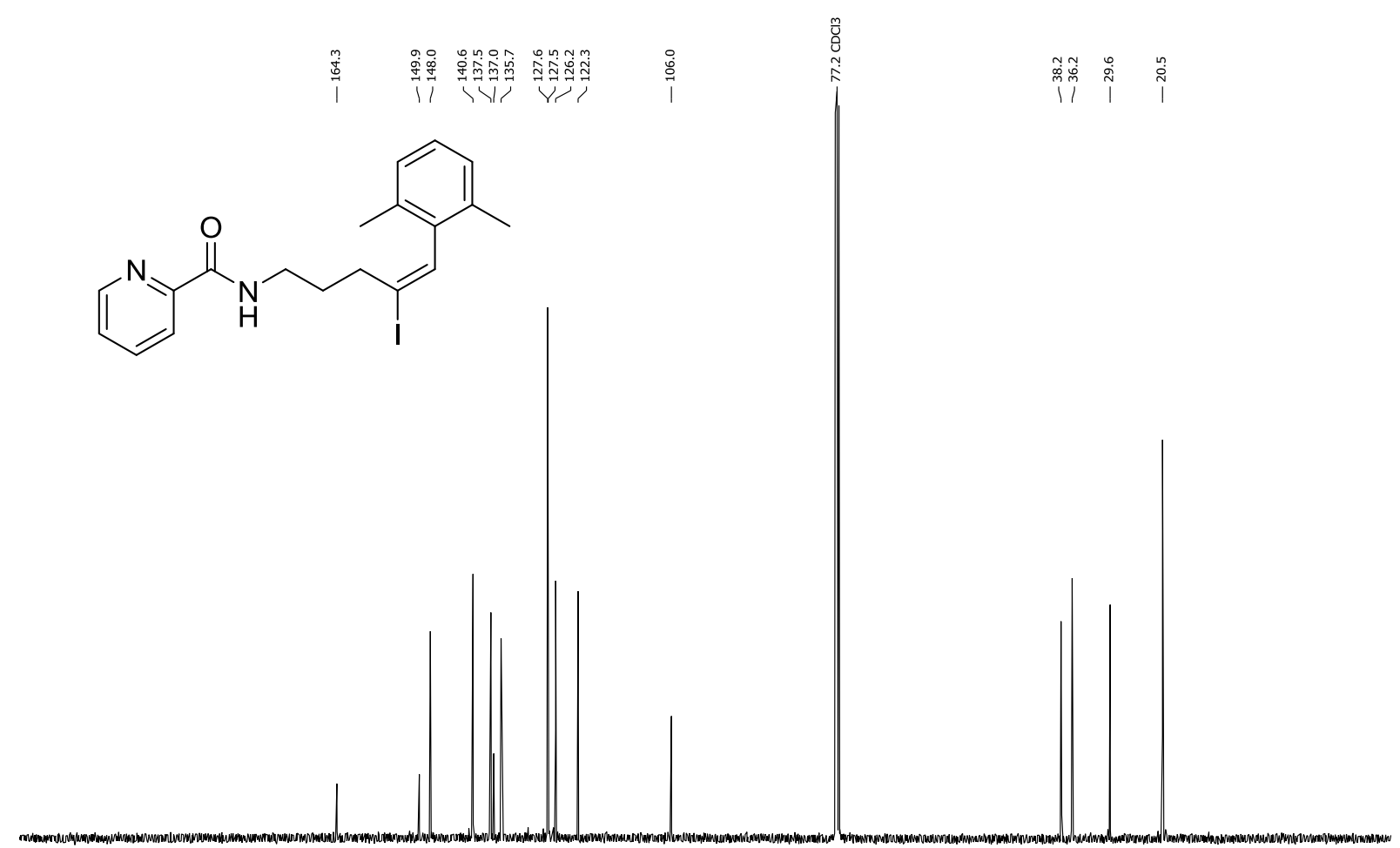

$\begin{array}{llllllllllllllllllllllllll}1 & 210 & 200 & 190 & 180 & 170 & 160 & 150 & 140 & 130 & 120 & 110 & \begin{array}{c}100 \\ \mathrm{f} 1(\mathrm{ppm})\end{array} & 90 & 80 & 70 & 60 & 50 & 40 & 30 & 20 & 10 & 0 & -10 & \end{array}$ 
${ }^{\mathbf{1}} \mathbf{H}$ NMR $\left(500 \mathrm{MHz}, \mathrm{CDCl}_{3}, 25^{\circ} \mathrm{C}\right)$ of $\mathbf{2 k}$

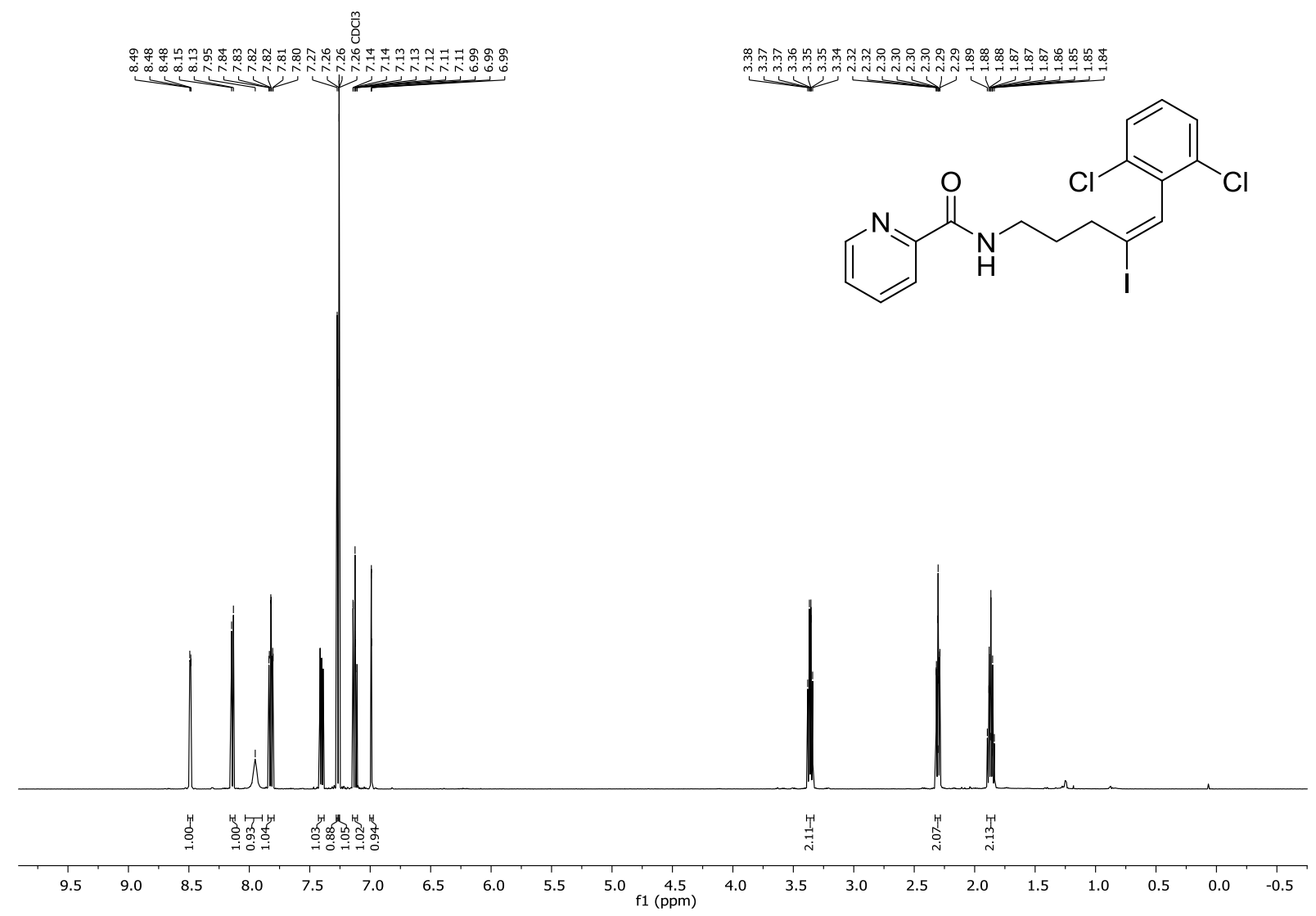

${ }^{13} \mathbf{C ~ N M R}\left(126 \mathrm{MHz}, \mathrm{CDCl}_{3}, 25^{\circ} \mathrm{C}\right)$ of $\mathbf{2 k}$

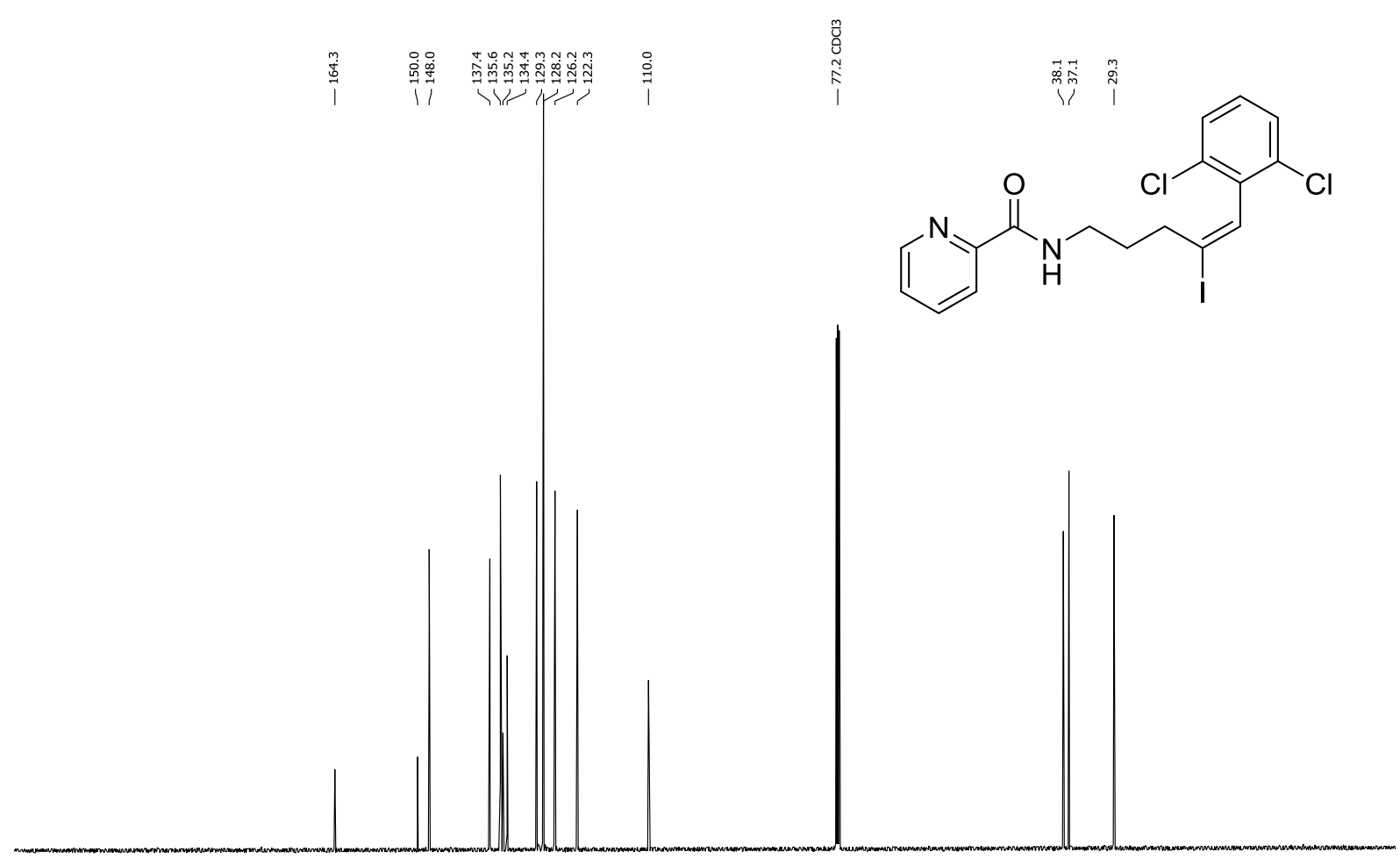

$\begin{array}{lllllllllllllllllllllll}210 & 200 & 190 & 180 & 170 & 160 & 150 & 140 & 130 & 120 & 110 & \begin{array}{c}100 \\ \mathrm{f} 1(\mathrm{ppm})\end{array} & 90 & 80 & 70 & 60 & 50 & 40 & 30 & 20 & 10 & 0 & -10\end{array}$ 
${ }^{\mathbf{1}} \mathbf{H}$ NMR $\left(500 \mathrm{MHz}, \mathrm{CDCl}_{3}, 25^{\circ} \mathrm{C}\right)$ of $\mathbf{2 l}$

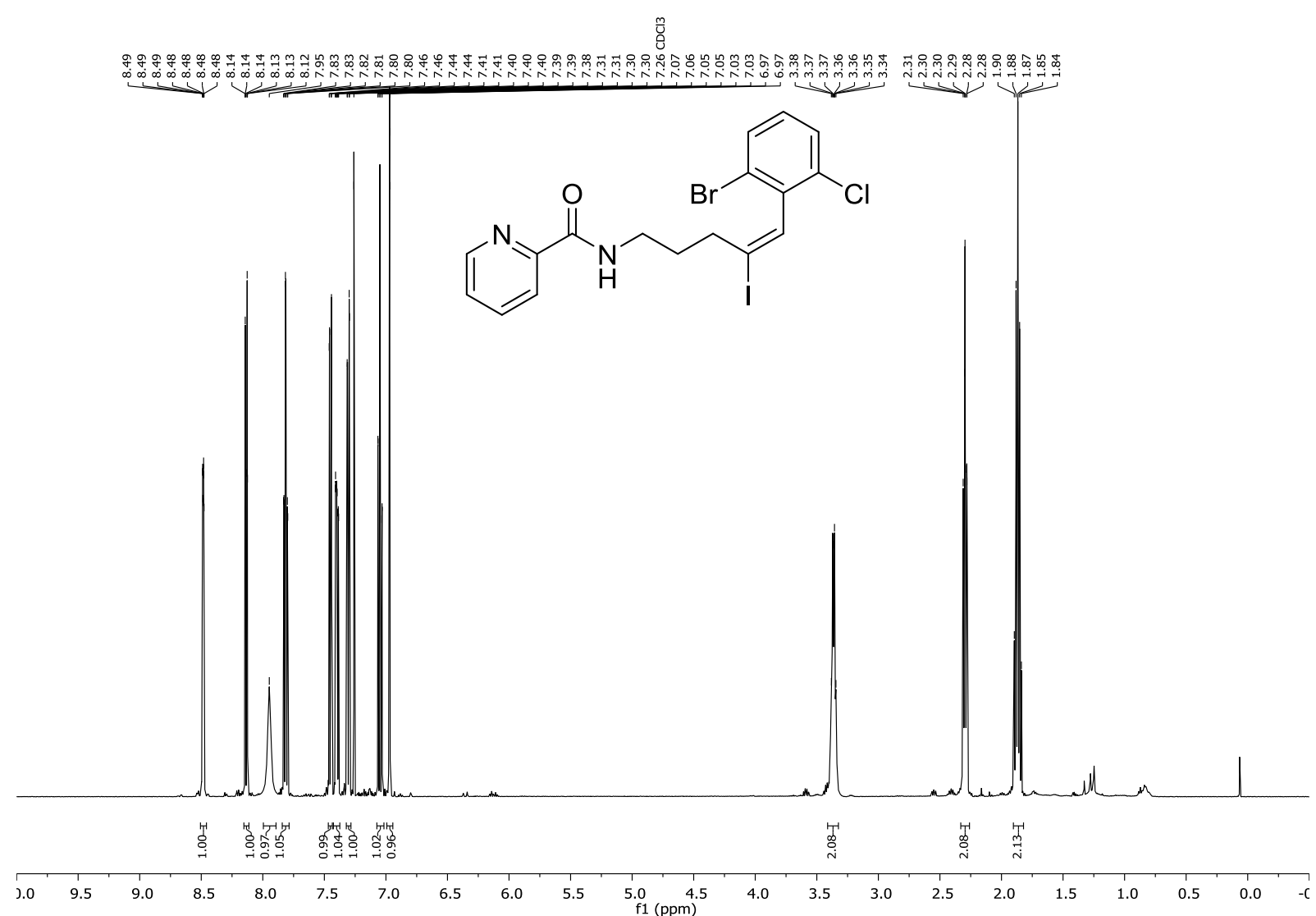

${ }^{13} \mathbf{C}$ NMR $\left(126 \mathrm{MHz}, \mathrm{CDCl}_{3}, 25^{\circ} \mathrm{C}\right)$ of $\mathbf{2 l}$

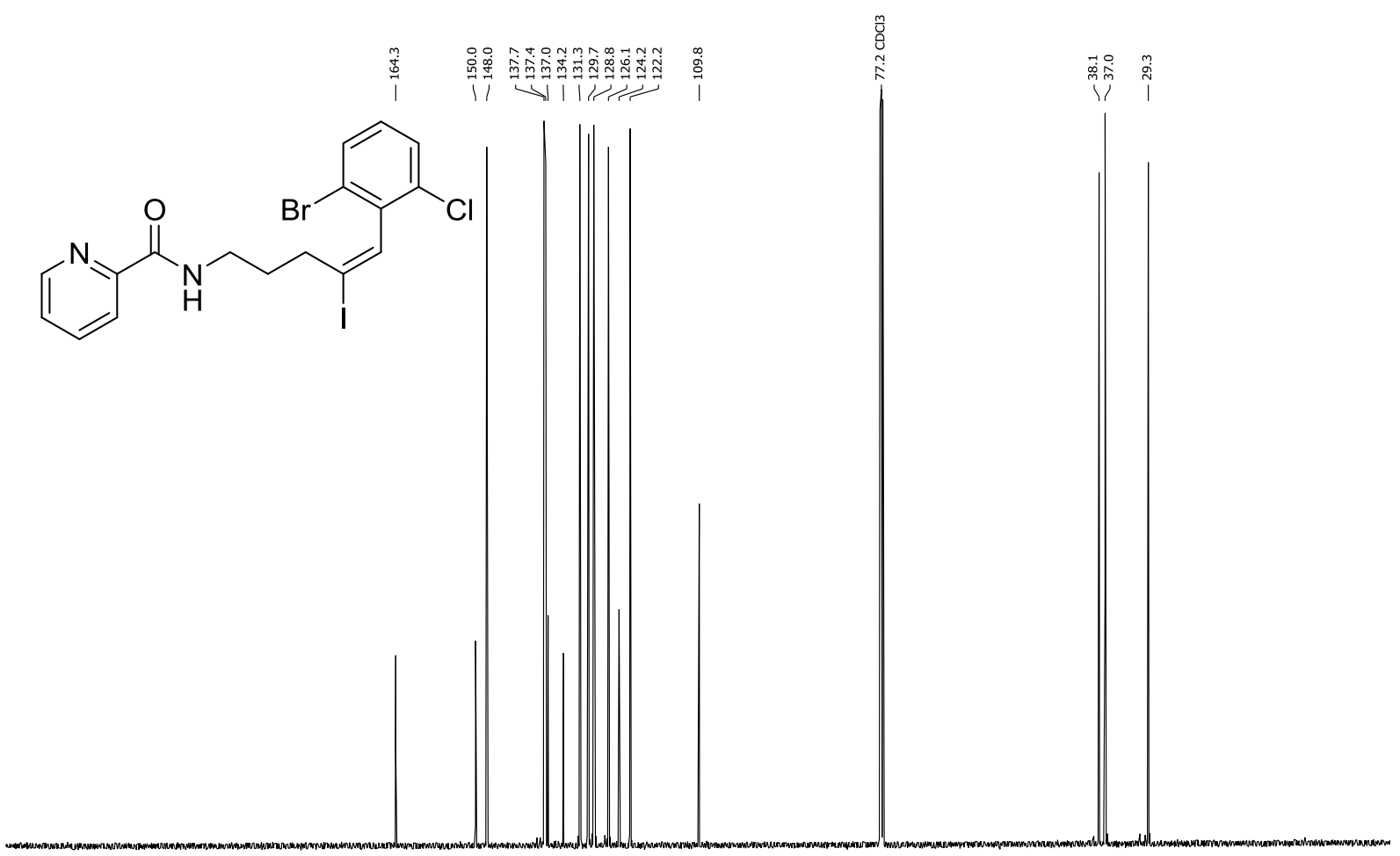

$\begin{array}{llllllllllllllllllllllllllll}230 & 220 & 210 & 200 & 190 & 180 & 170 & 160 & 150 & 140 & 130 & 120 & 110 & 100 & 90 & 80 & 70 & 60 & 50 & 40 & 30 & 20 & 10 & 0 & -10\end{array}$ 
${ }^{1} \mathbf{H}$ NMR $\left(400 \mathrm{MHz}, \mathrm{CDCl}_{3}, 25^{\circ} \mathrm{C}\right)$ of $\mathbf{2} \mathbf{m}$
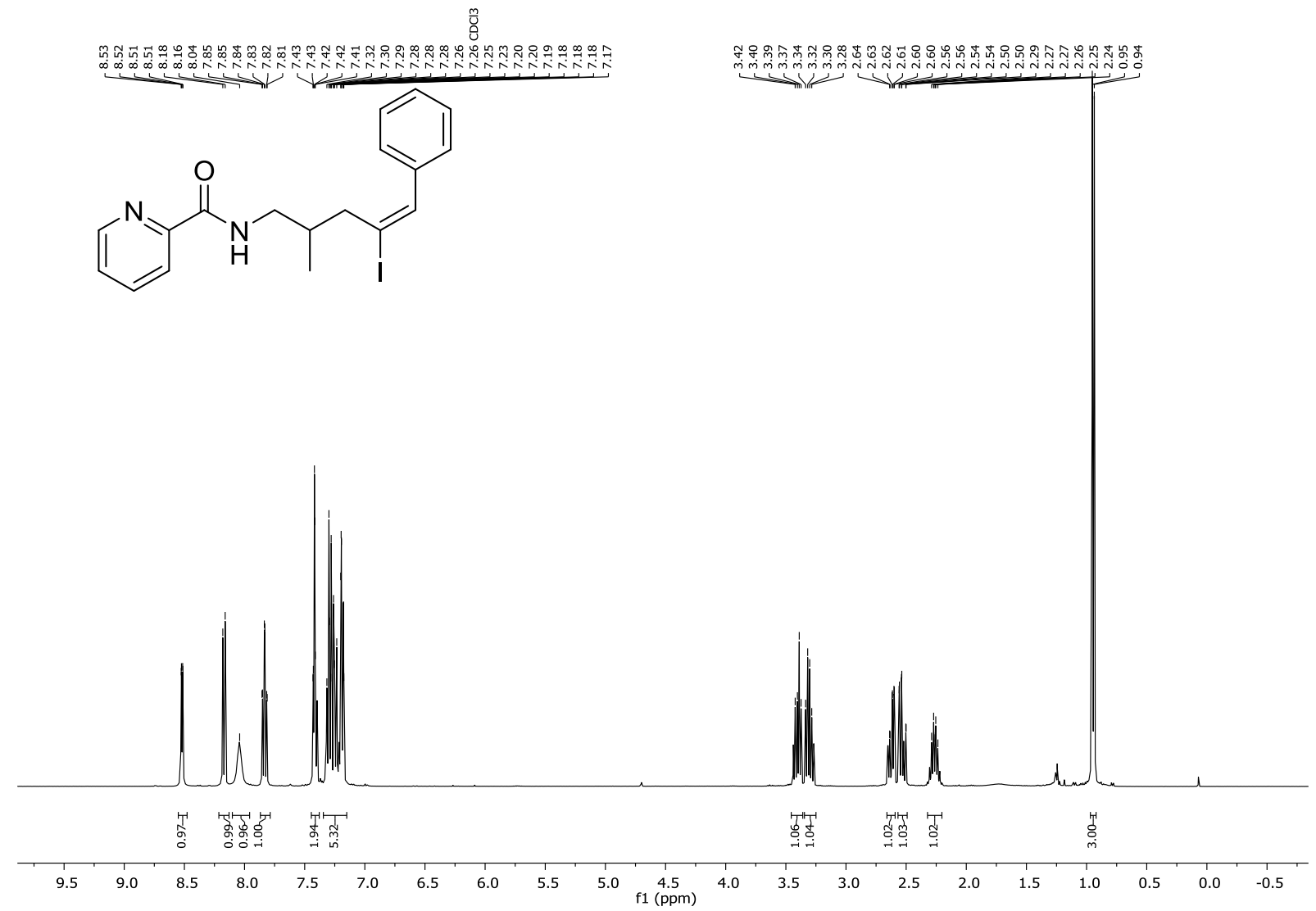

${ }^{13} \mathbf{C}$ NMR $\left(100 \mathrm{MHz}, \mathrm{CDCl}_{3}, 25{ }^{\circ} \mathrm{C}\right)$ of $\mathbf{2 m}$

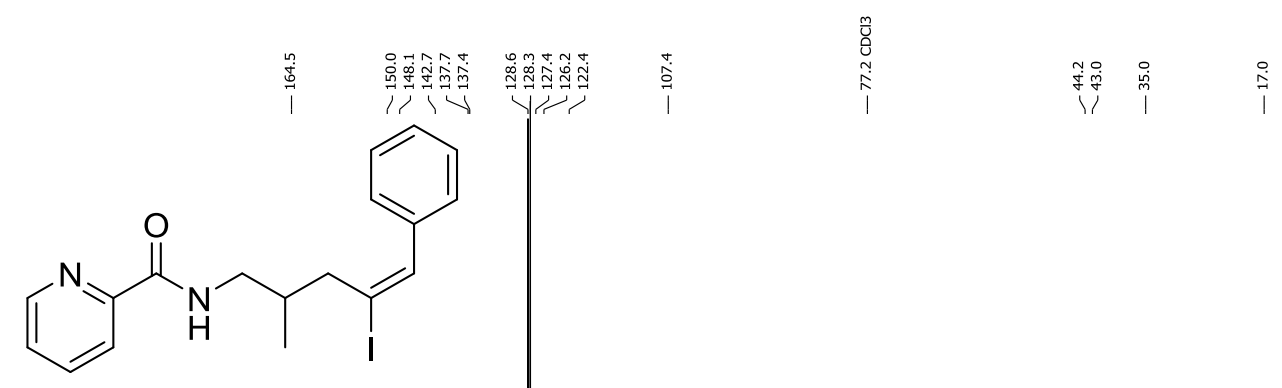

$\begin{array}{llllllllllllllllllllllllll}210 & 200 & 190 & 180 & 170 & 160 & 150 & 140 & 130 & 120 & 110 & 100 & 90 & 80 & 70 & 60 & 50 & 40 & 30 & 20 & 10 & 0 & -10\end{array}$ 
${ }^{1} \mathbf{H}$ NMR $\left(400 \mathrm{MHz}, \mathrm{CDCl}_{3}, 25^{\circ} \mathrm{C}\right)$ of $\mathbf{2 n}$

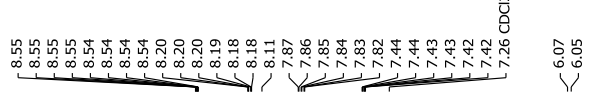

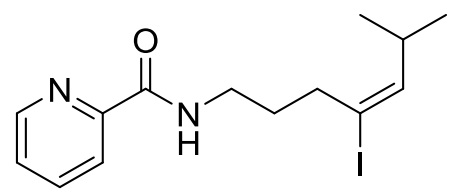

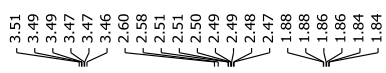

ֻू.

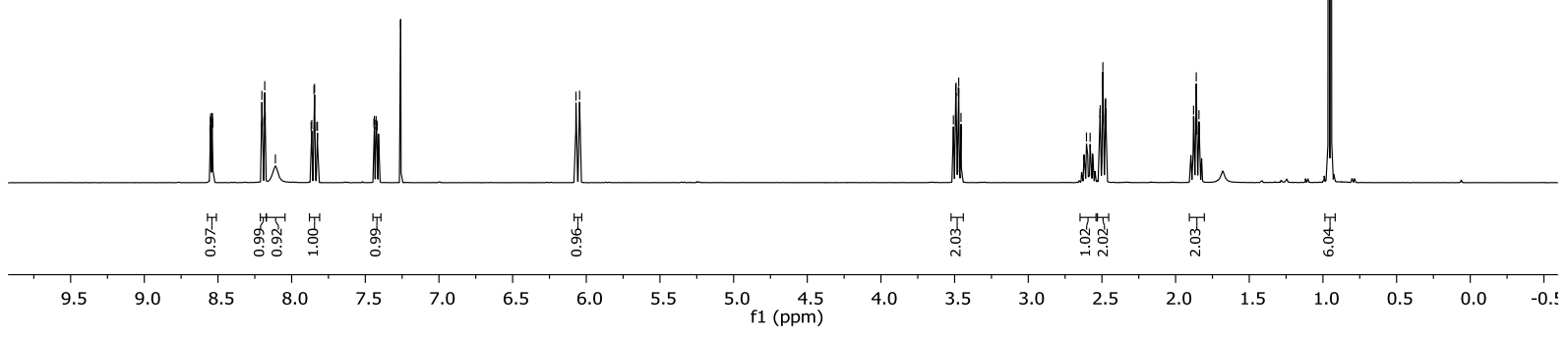

${ }^{13} \mathbf{C}$ NMR $\left(100 \mathrm{MHz}, \mathrm{CDCl}_{3}, 25^{\circ} \mathrm{C}\right)$ of $\mathbf{2 n}$

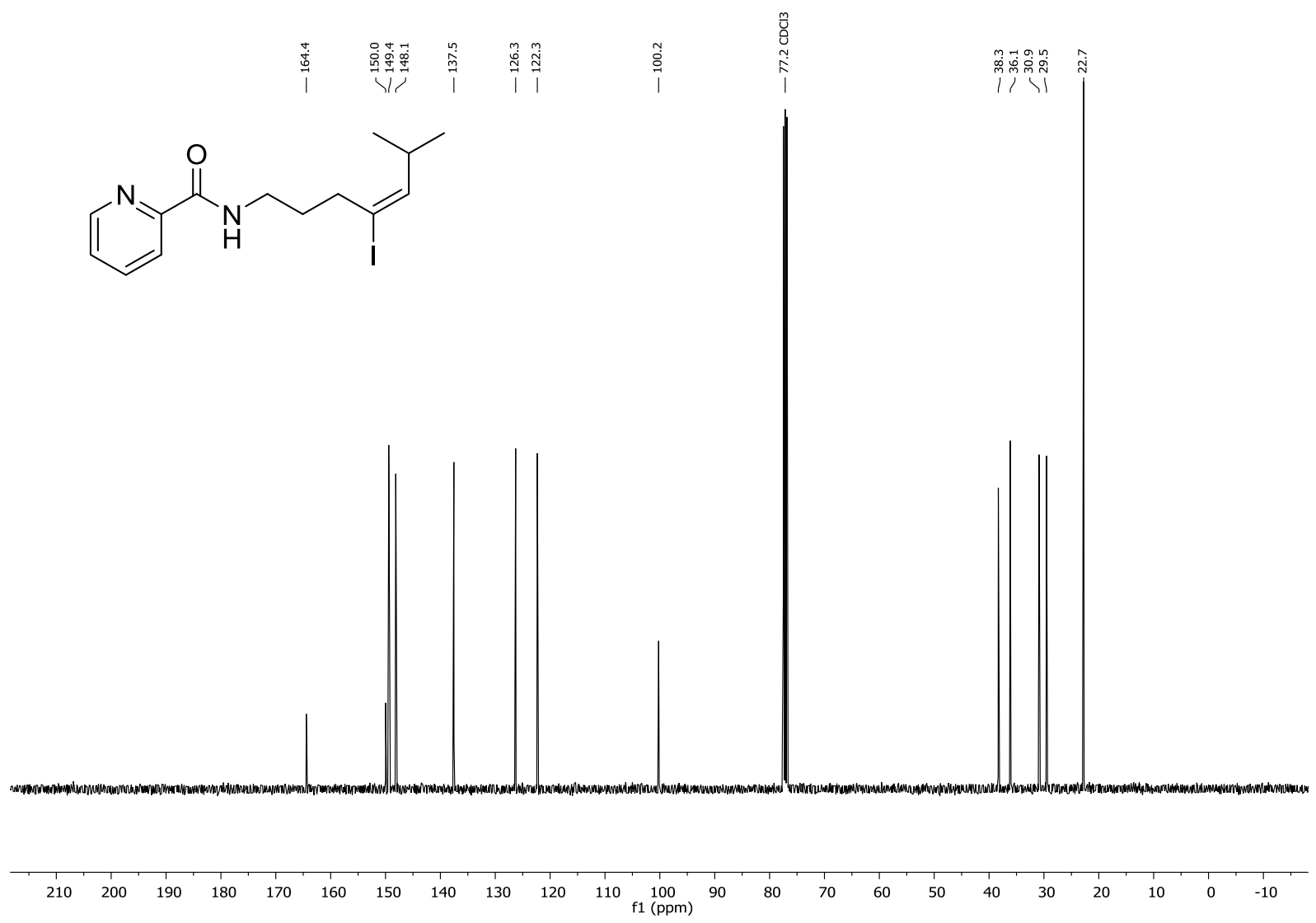

S146 
${ }^{1} \mathbf{H}$ NMR $\left(400 \mathrm{MHz}, \mathrm{CDCl}_{3}, 25^{\circ} \mathrm{C}\right)$ of $\mathbf{2 0}$<smiles>CC(C)(C)/C=C(/I)CCCNC(=O)c1ccccn1</smiles>

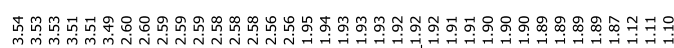

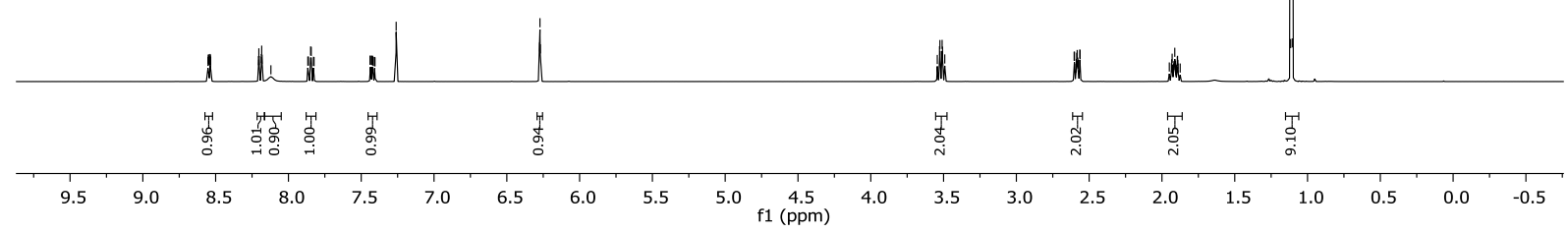

${ }^{13} \mathbf{C}$ NMR $\left(100 \mathrm{MHz}, \mathrm{CDCl}_{3}, 25^{\circ} \mathrm{C}\right)$ of $\mathbf{2 0}$

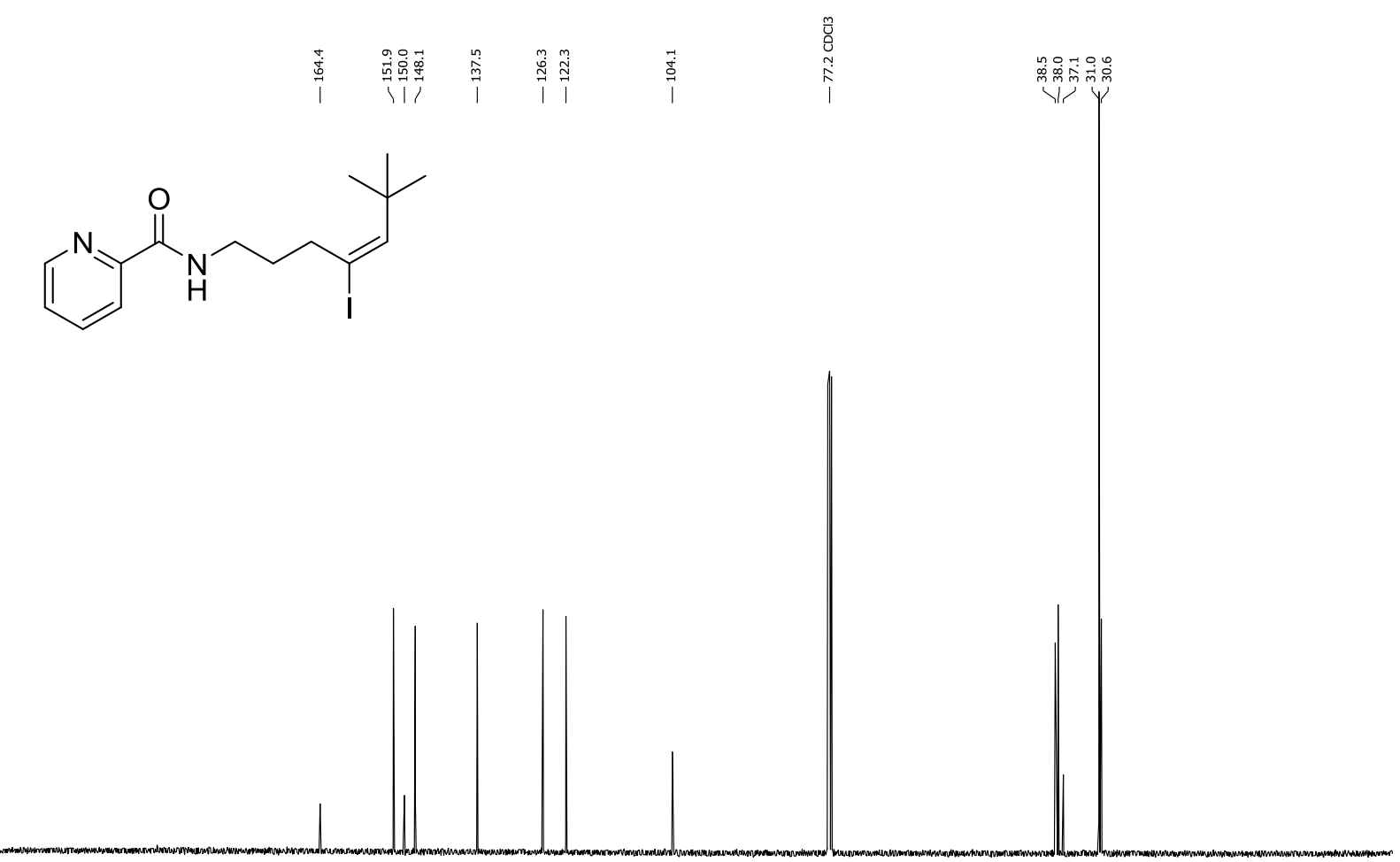

$\begin{array}{lllllllllllllllllllllllll} & 210 & 200 & 190 & 180 & 170 & 160 & 150 & 140 & 130 & 120 & 110 & \begin{array}{l}100 \\ \mathrm{f} 1(\mathrm{ppm})\end{array} & 90 & 80 & 70 & 60 & 50 & 40 & 30 & 20 & 10 & 0 & -10\end{array}$ 
${ }^{\mathbf{1}} \mathbf{H}$ NMR $\left(400 \mathrm{MHz}, \mathrm{CDCl}_{3}, 25^{\circ} \mathrm{C}\right)$ of $\mathbf{2 p}$

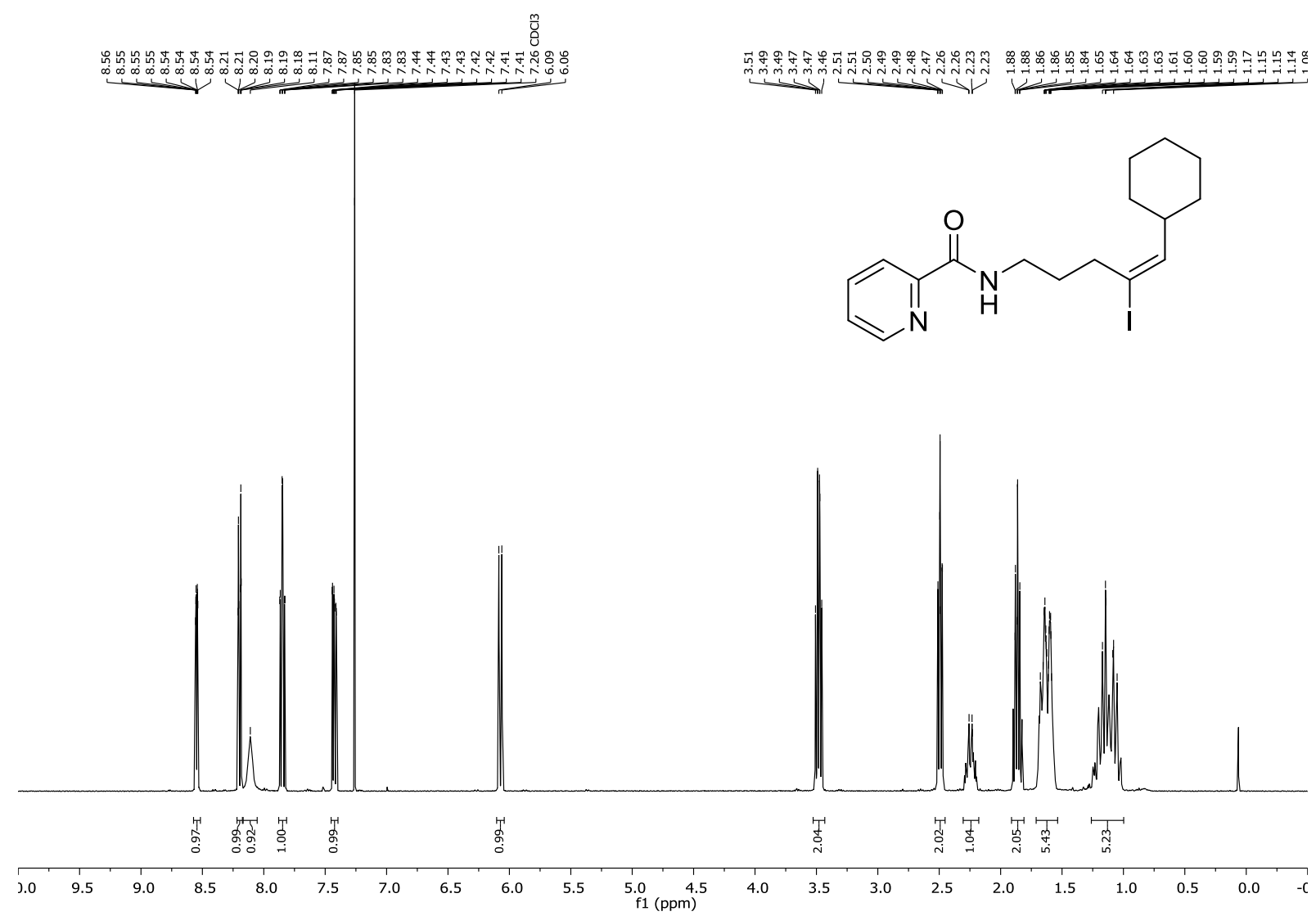

${ }^{13} \mathbf{C}$ NMR $\left(100 \mathrm{MHz}, \mathrm{CDCl}_{3}, 25^{\circ} \mathrm{C}\right)$ of $\mathbf{2 p}$

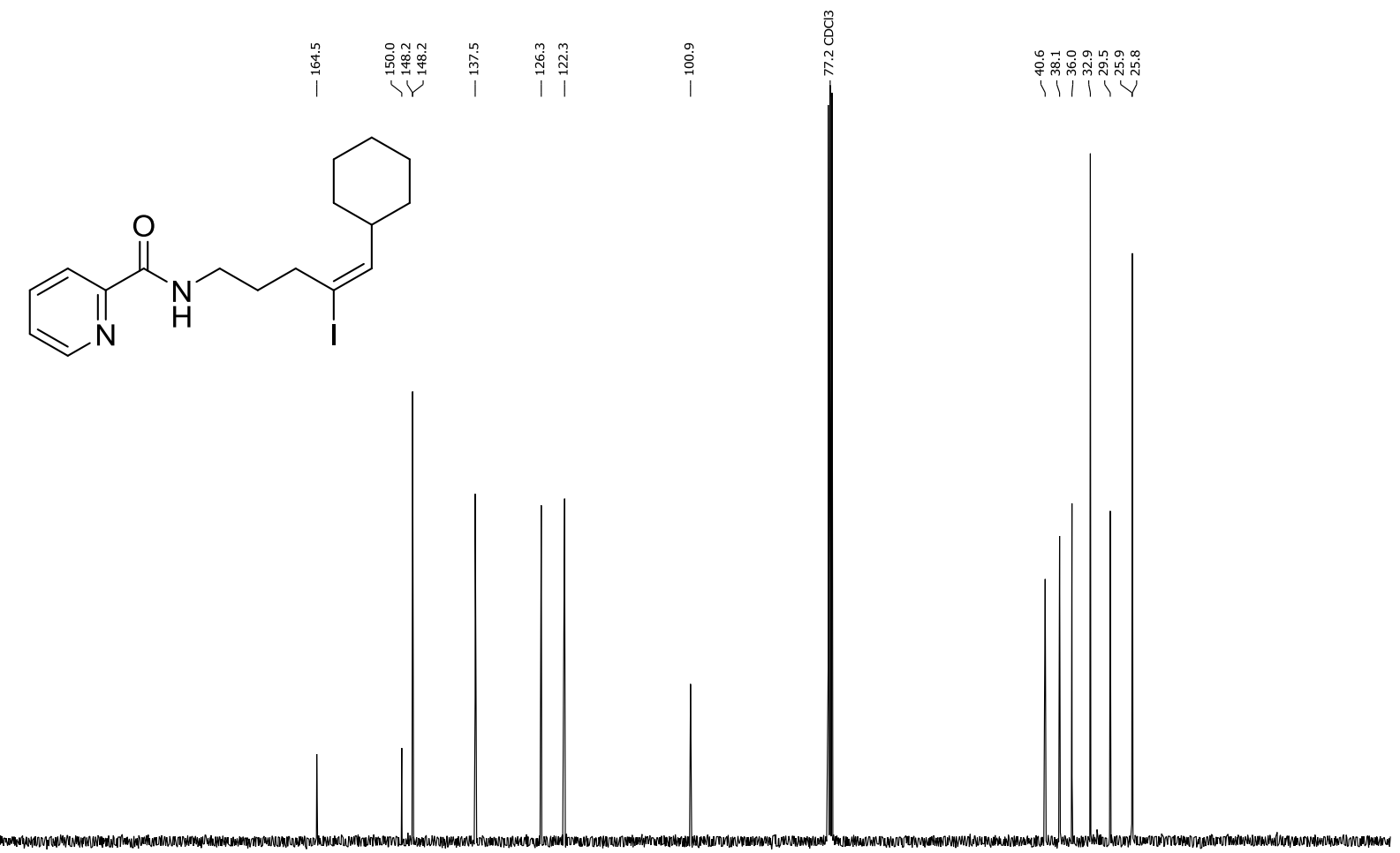

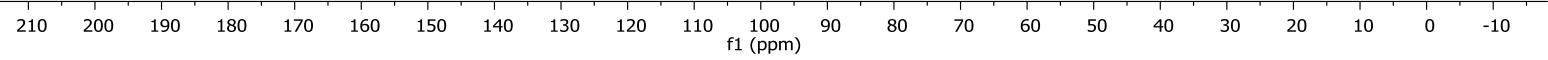


NOESY $\left(500 \mathrm{MHz}, \mathrm{CDCl}_{3} .25^{\circ} \mathrm{C}, \mathrm{t}_{\mathrm{m}}=500 \mathrm{~ms}\right)$ of $\mathbf{2 p}$
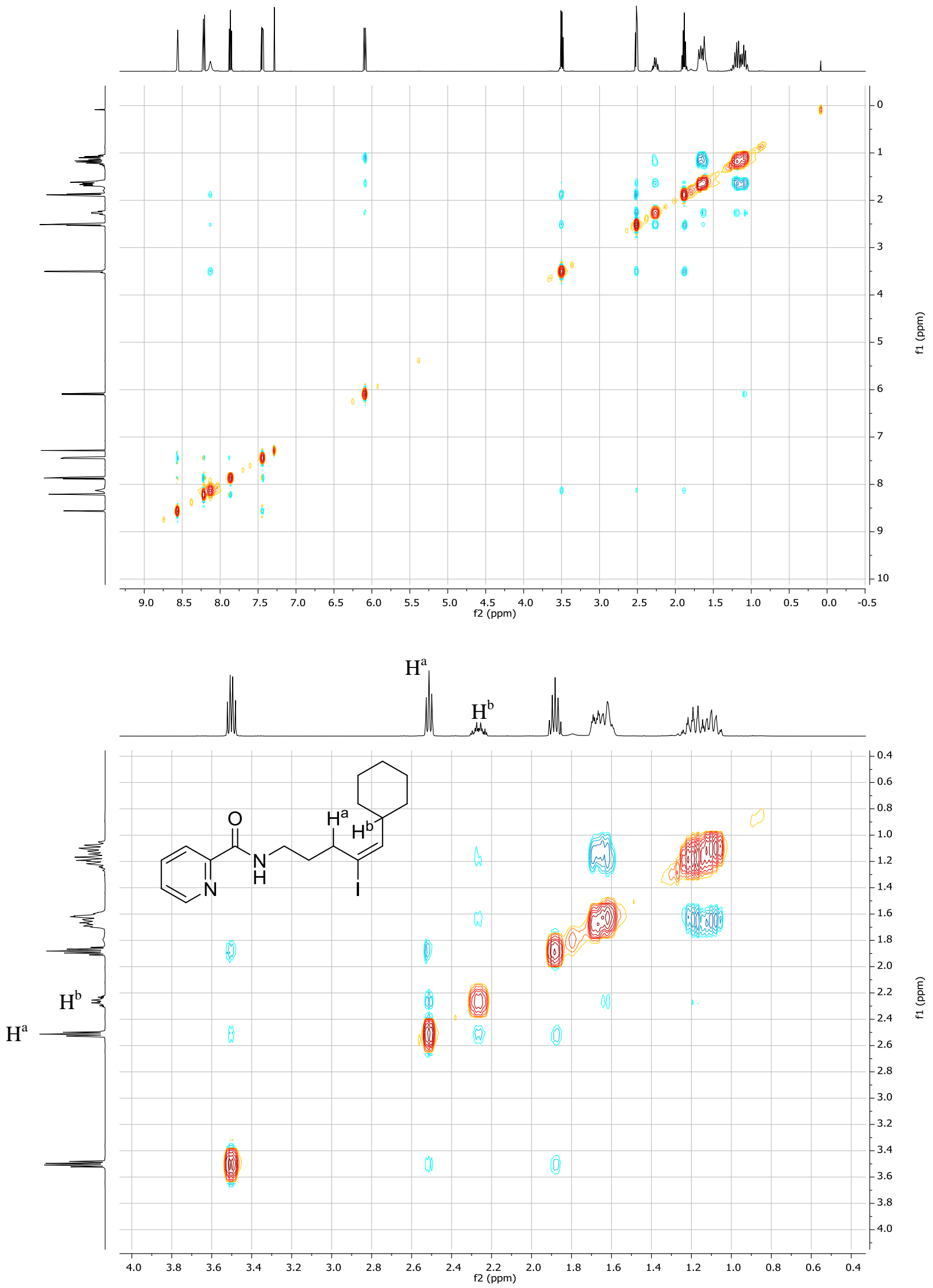
${ }^{\mathbf{1}} \mathbf{H} \mathbf{N M R}\left(500 \mathrm{MHz}, \mathrm{CDCl}_{3}, 25^{\circ} \mathrm{C}\right)$ of $\mathbf{2 q}$<smiles>CC(C)(C)OC(=O)N1CCC(/C=C(/I)CCCNC(=O)c2ccccn2)CC1</smiles>

$\underbrace{\text { 象 }}$

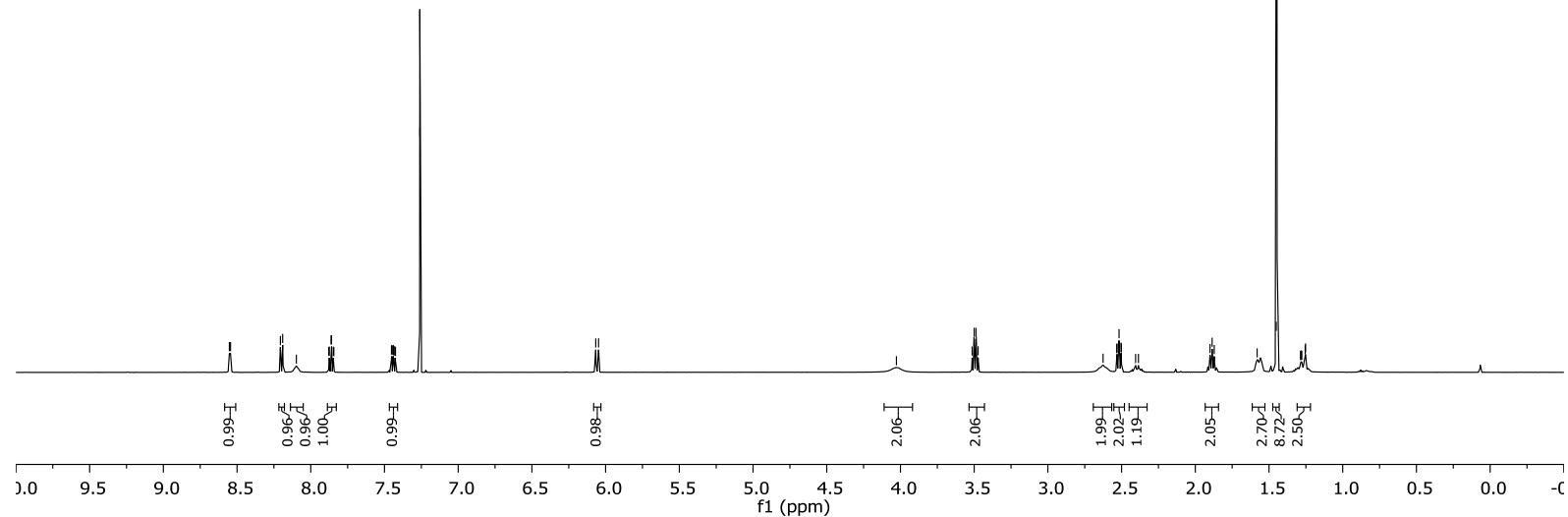

${ }^{13} \mathbf{C}$ NMR $\left(126 \mathrm{MHz}, \mathrm{CDCl}_{3}, 25{ }^{\circ} \mathrm{C}\right)$ of $\mathbf{2 q}$

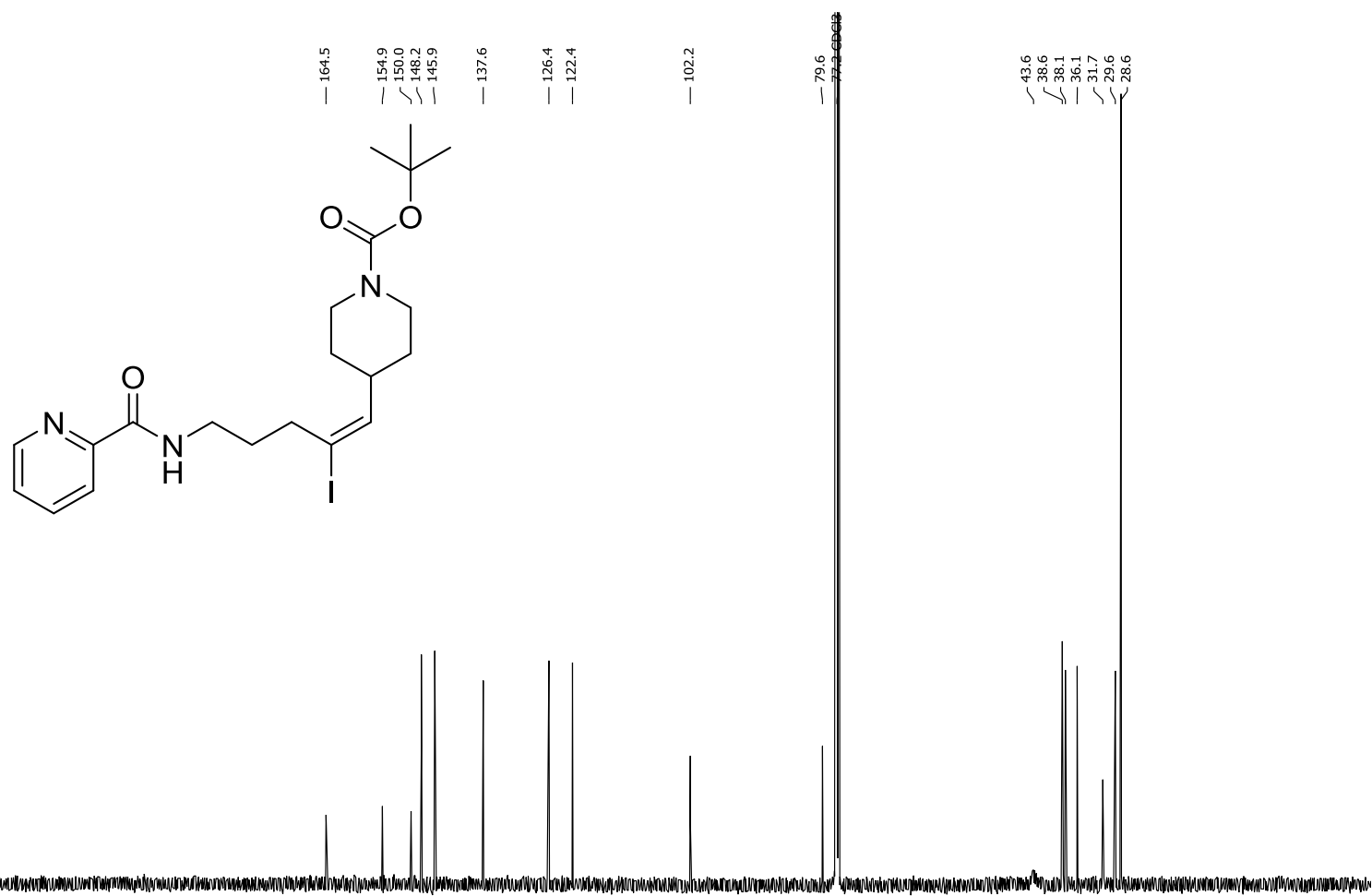

$\begin{array}{llllllllllllllllllllllllllll}230 & 220 & 210 & 200 & 190 & 180 & 170 & 160 & 150 & 140 & 130 & 120 & 110 & 100 & 90 & 80 & 70 & 60 & 50 & 40 & 30 & 20 & 10 & 0 & -10\end{array}$ 
${ }^{\mathbf{1}} \mathbf{H}$ NMR $\left(400 \mathrm{MHz}, \mathrm{CDCl}_{3}, 25^{\circ} \mathrm{C}\right)$ of $\mathbf{2 r}$

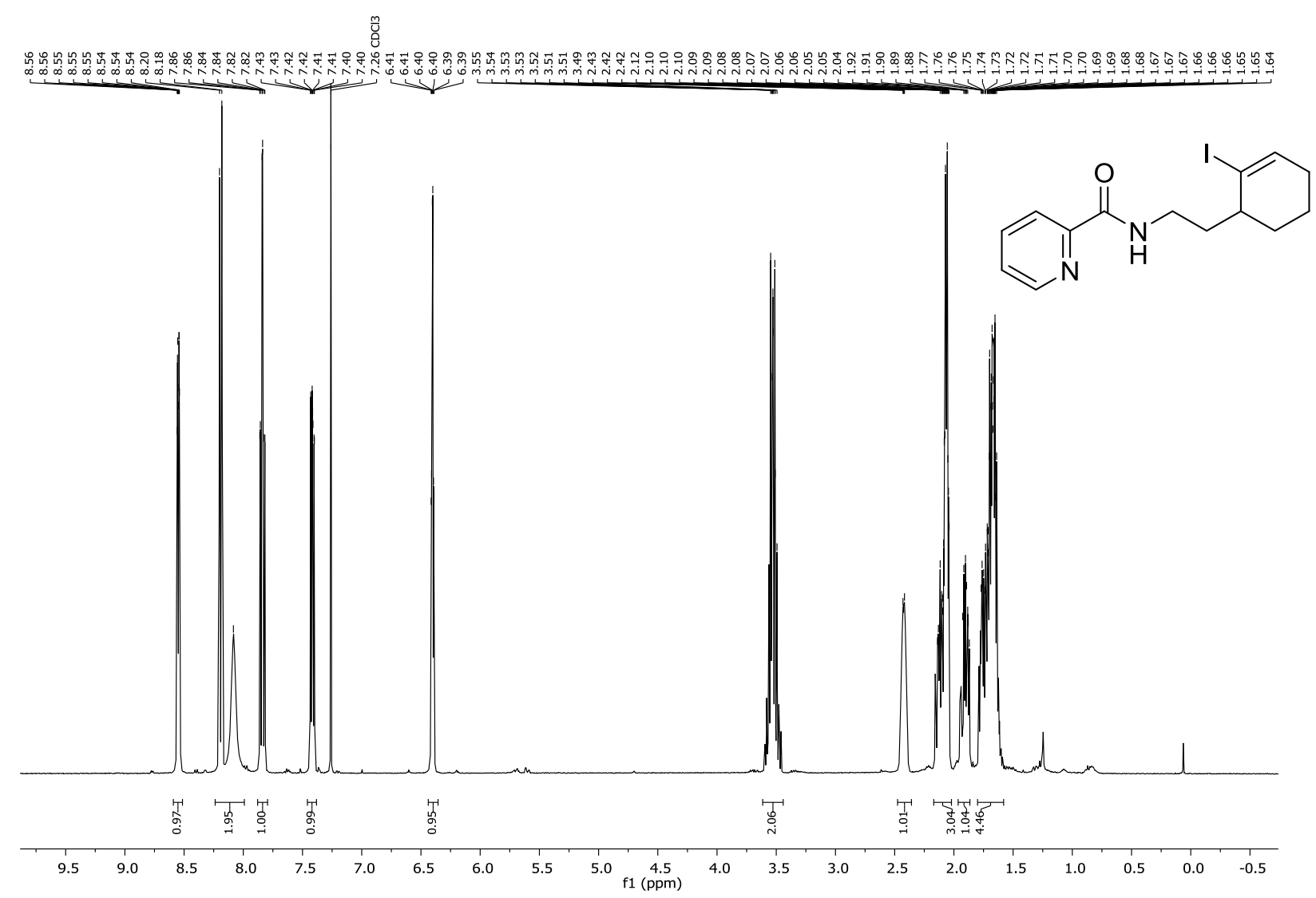

${ }^{13} \mathbf{C ~ N M R}\left(100 \mathrm{MHz}, \mathrm{CDCl}_{3}, 25^{\circ} \mathrm{C}\right)$ of $\mathbf{2 r}$

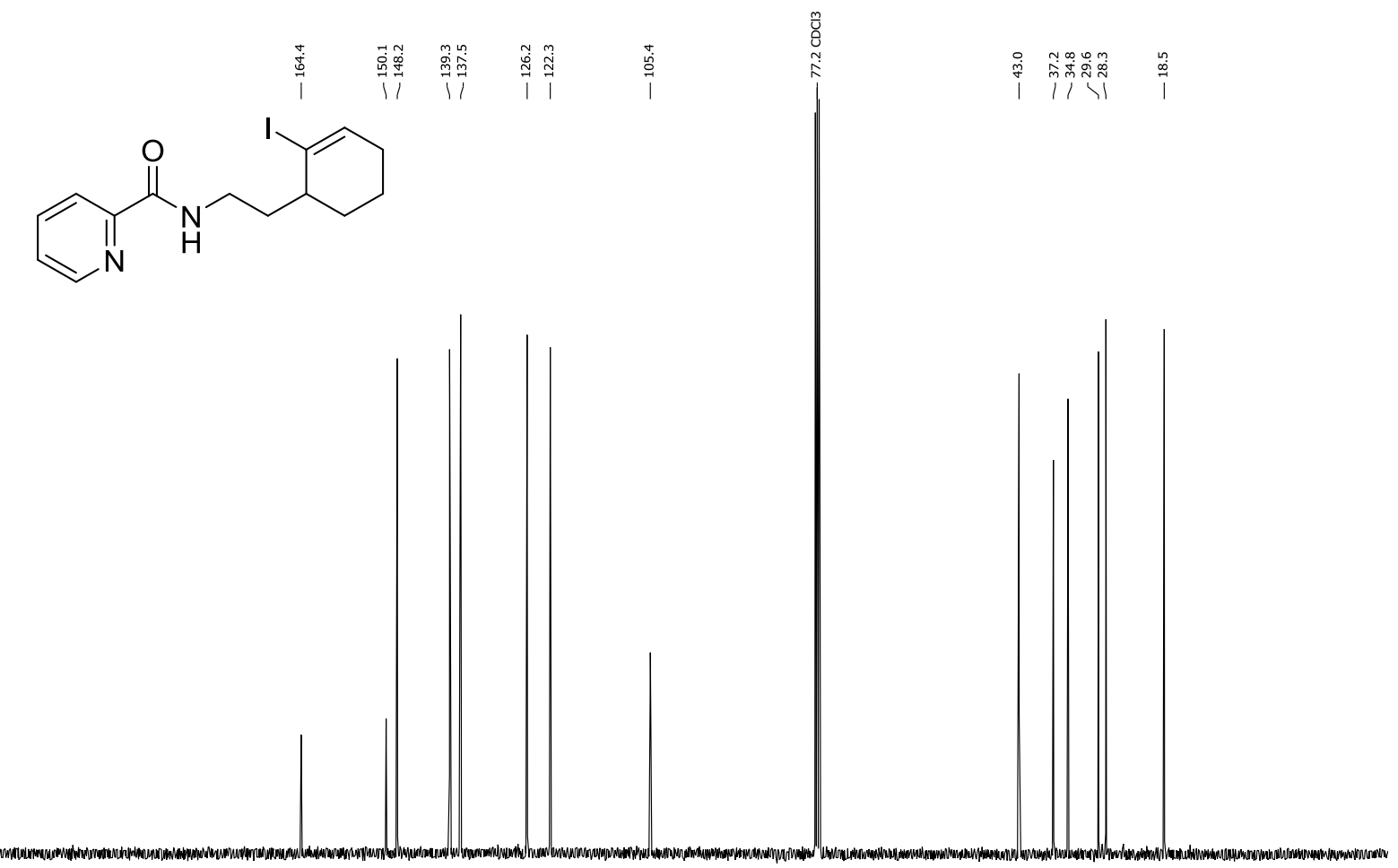

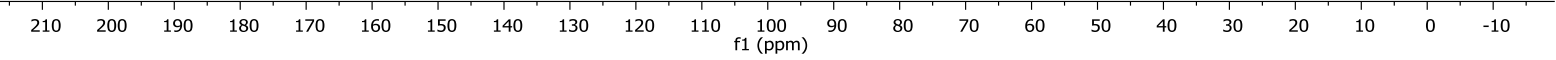


${ }^{1} \mathbf{H}$ NMR $\left(400 \mathrm{MHz}, \mathrm{CDCl}_{3}, 25^{\circ} \mathrm{C}\right)$ of $\mathbf{2 s}$

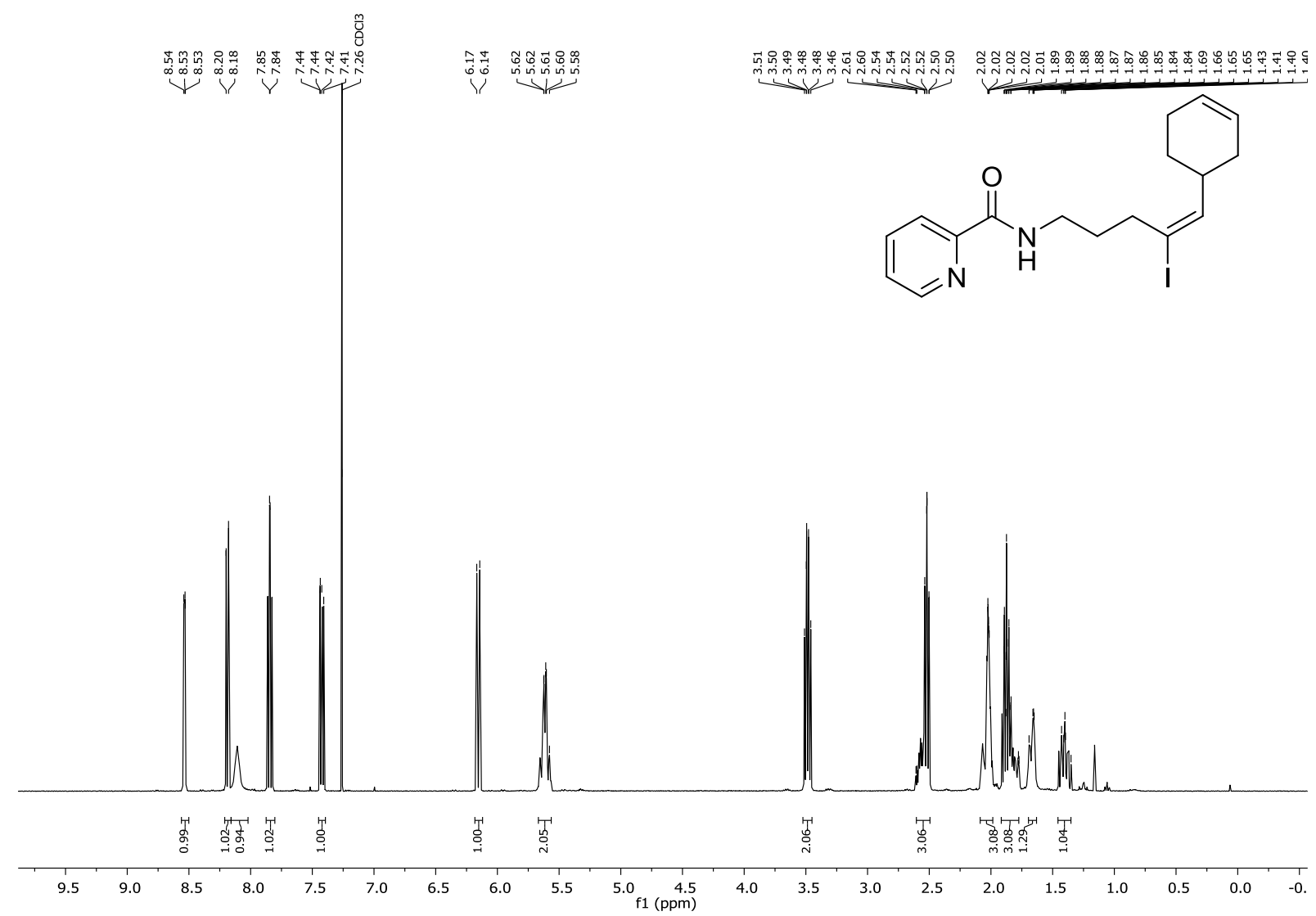

${ }^{13} \mathbf{C}$ NMR $\left(100 \mathrm{MHz}, \mathrm{CDCl}_{3}, 25^{\circ} \mathrm{C}\right)$ of $2 \mathrm{~s}$

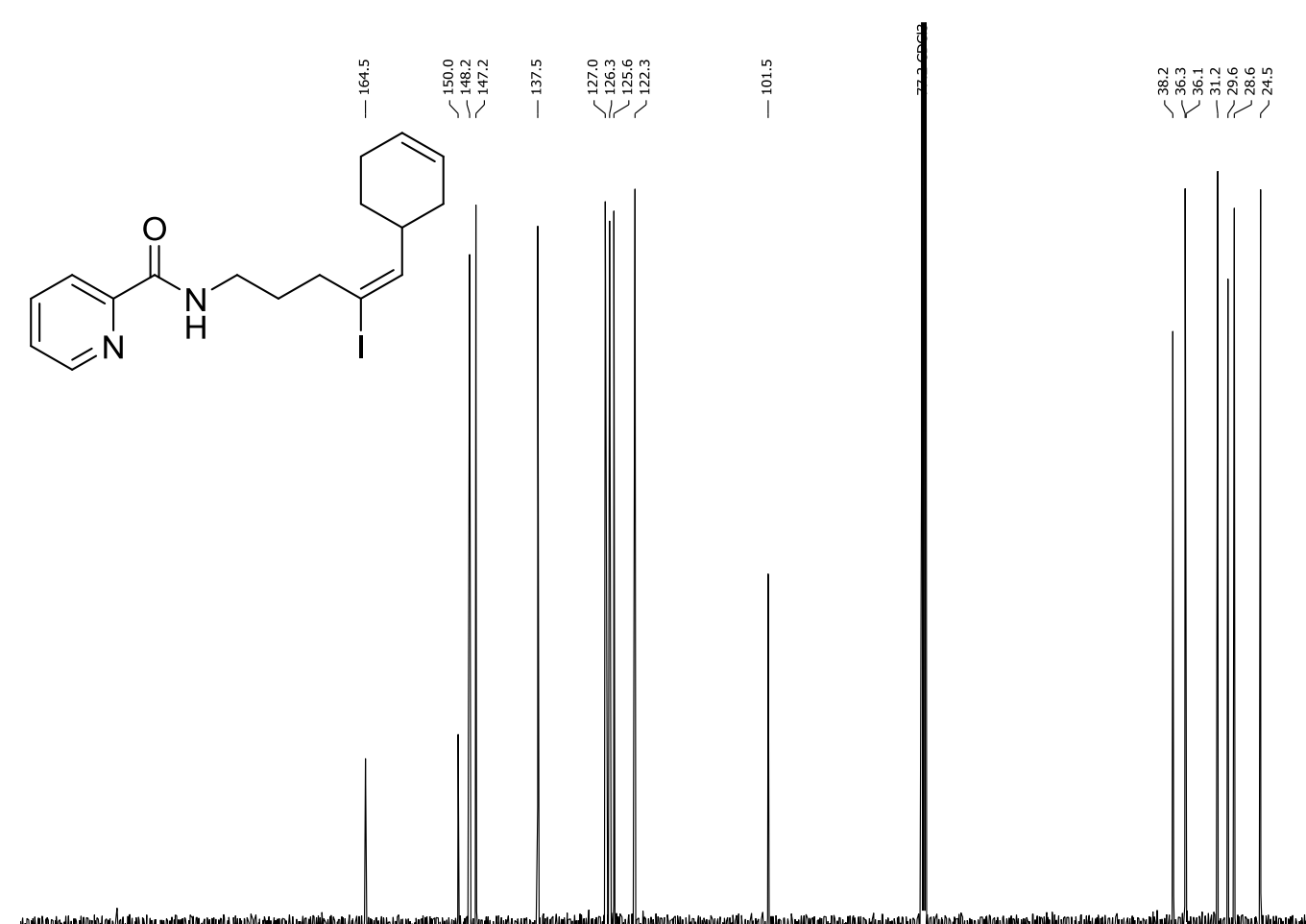

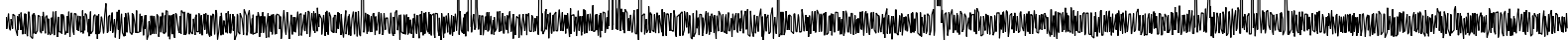

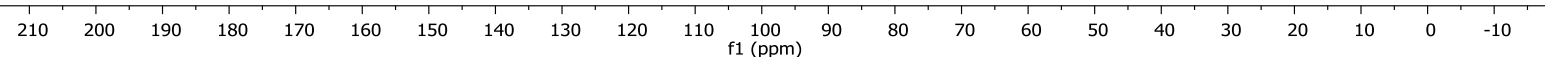


${ }^{1} \mathbf{H}$ NMR $\left(400 \mathrm{MHz}, \mathrm{CD}_{2} \mathrm{Cl}_{2}, 25^{\circ} \mathrm{C}\right)$ of $\mathbf{2 t}$

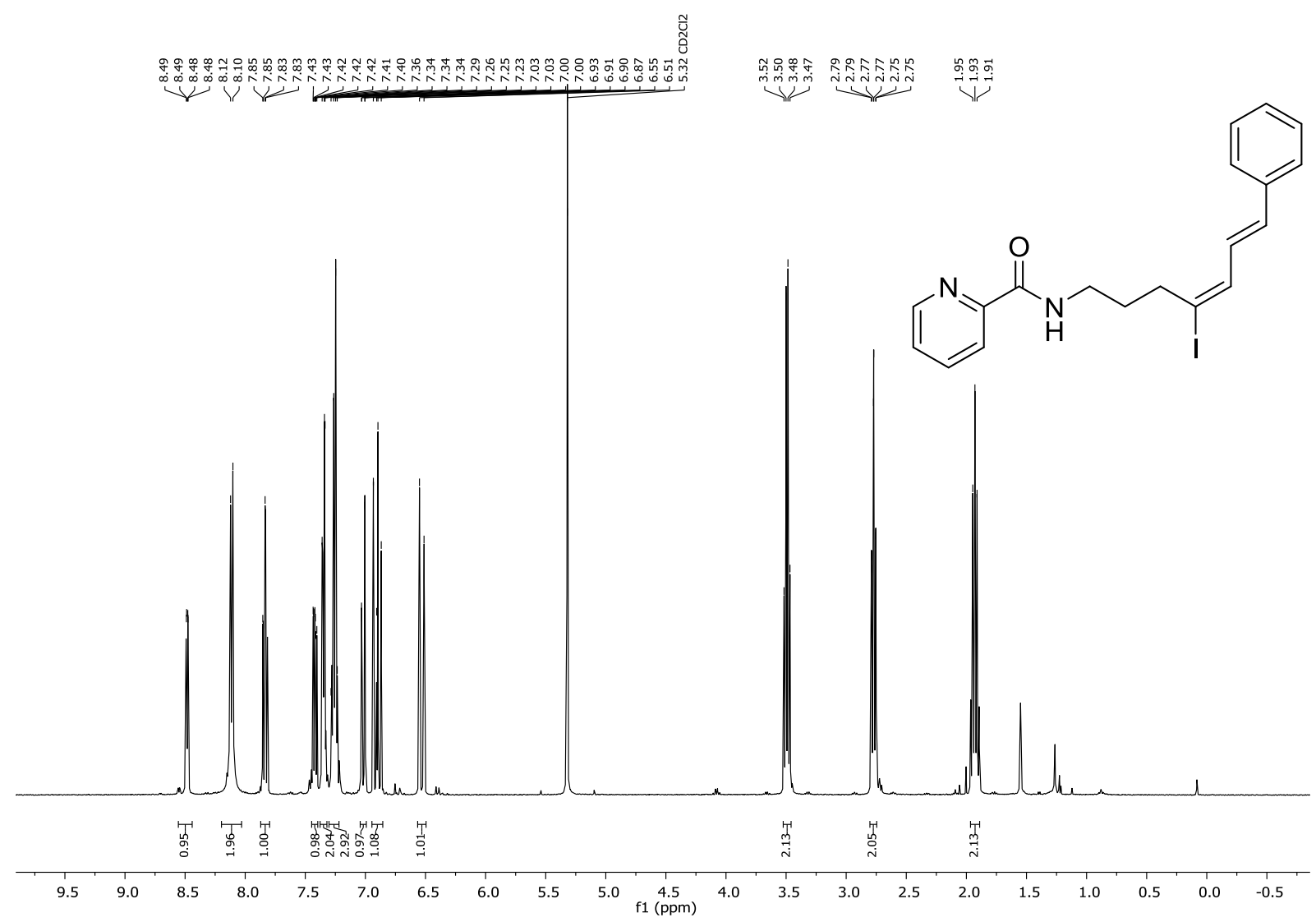

${ }^{13} \mathbf{C ~ N M R}\left(100 \mathrm{MHz}, \mathrm{CD}_{2} \mathrm{Cl}_{2}, 25{ }^{\circ} \mathrm{C}\right)$ of $\mathbf{2 t}$

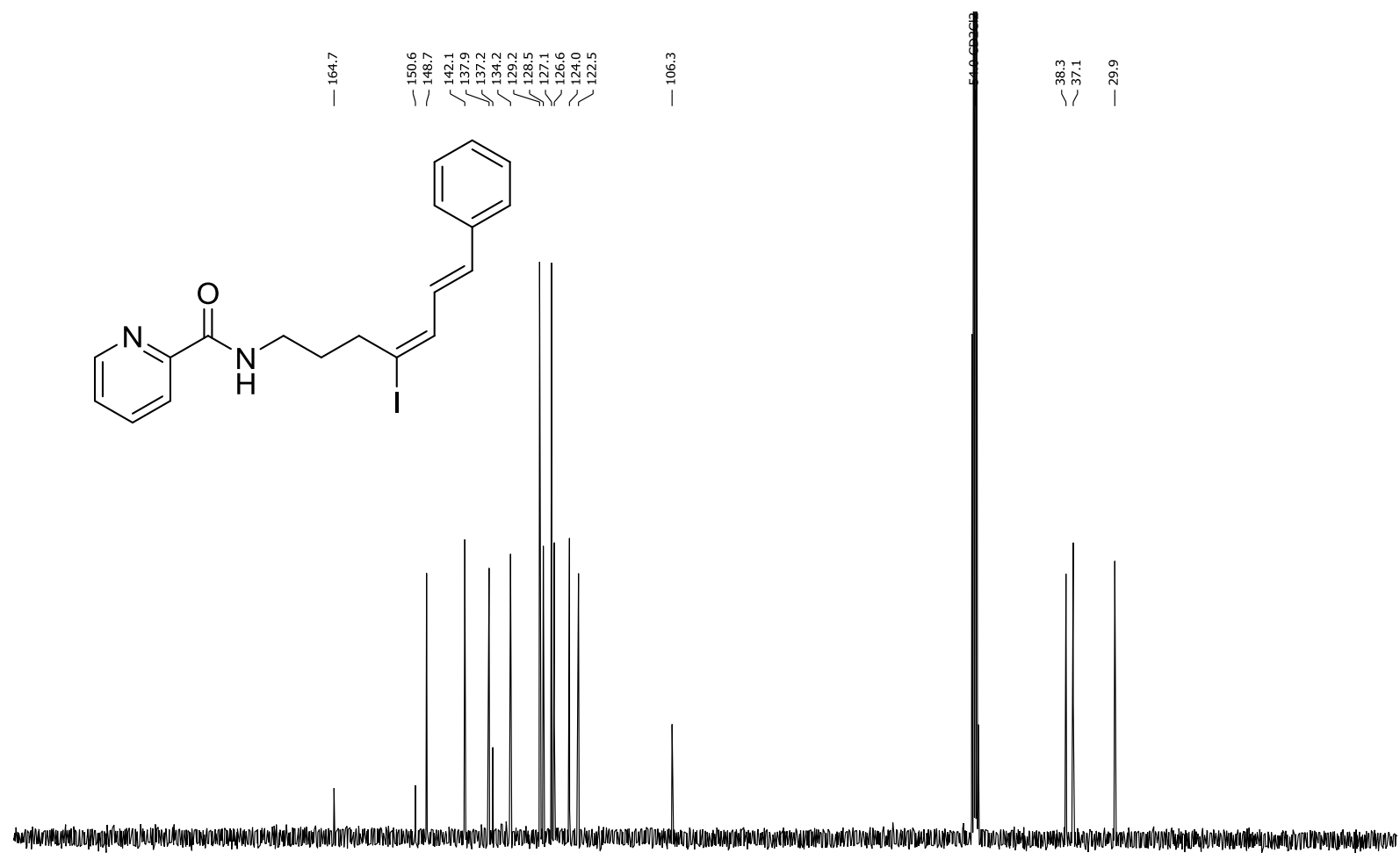

\begin{tabular}{llllllllllllllllllllllllll}
\hline & 210 & 200 & 190 & 180 & 170 & 160 & 150 & 140 & 130 & 120 & 110 & $\begin{array}{l}100 \\
\mathrm{f} 1\end{array}(\mathrm{ppm})$ & 90 & 80 & 70 & 60 & 50 & 40 & 30 & 20 & 10 & 0 & -10
\end{tabular} 
${ }^{\mathbf{1}} \mathbf{H}$ NMR $\left(400 \mathrm{MHz}, \mathrm{CDCl}_{3}, 25^{\circ} \mathrm{C}\right)$ of $\mathbf{2 u}$

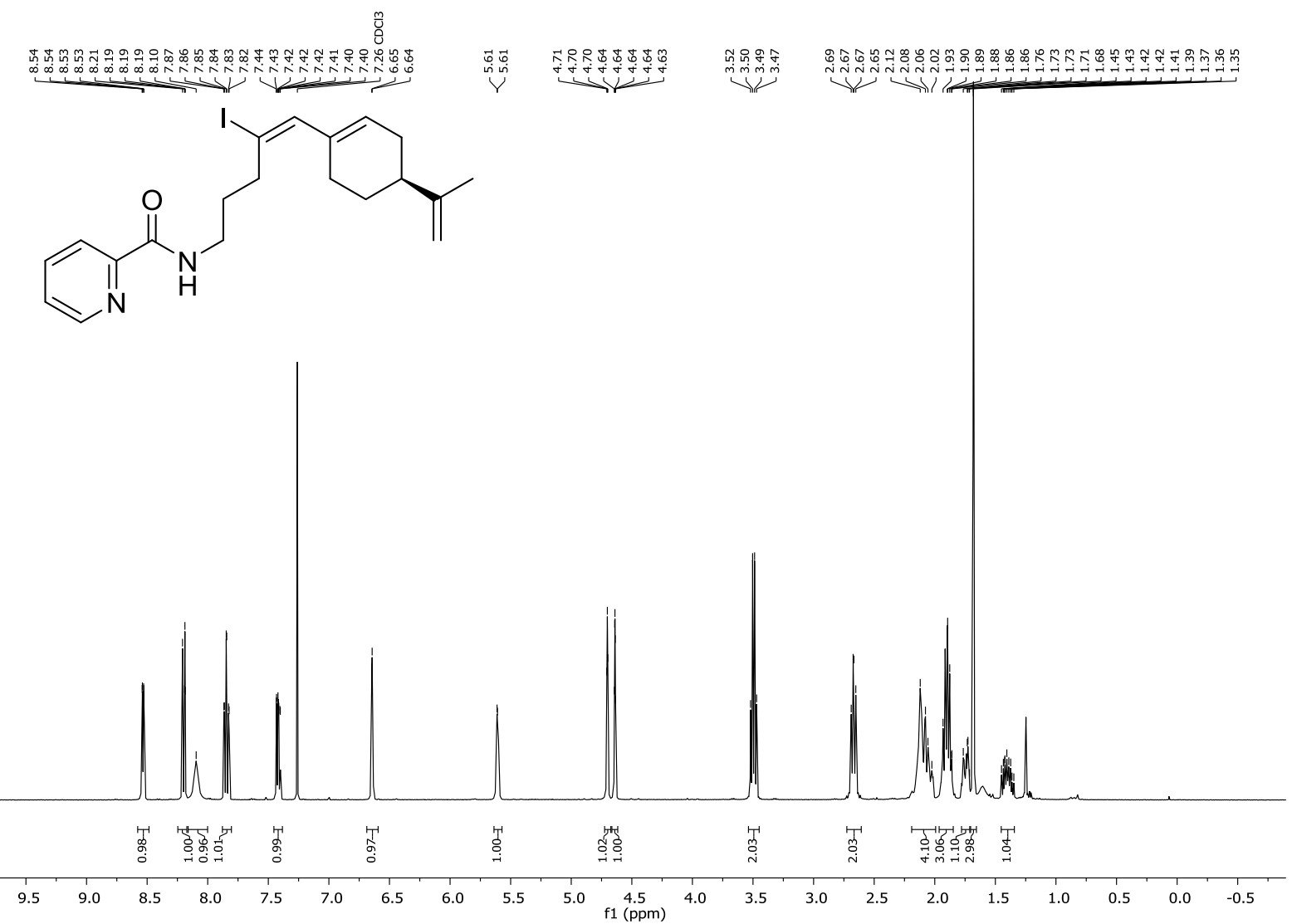

${ }^{13} \mathbf{C}$ NMR $\left(100 \mathrm{MHz}, \mathrm{CDCl}_{3}, 25^{\circ} \mathrm{C}\right)$ of $\mathbf{2 u}$

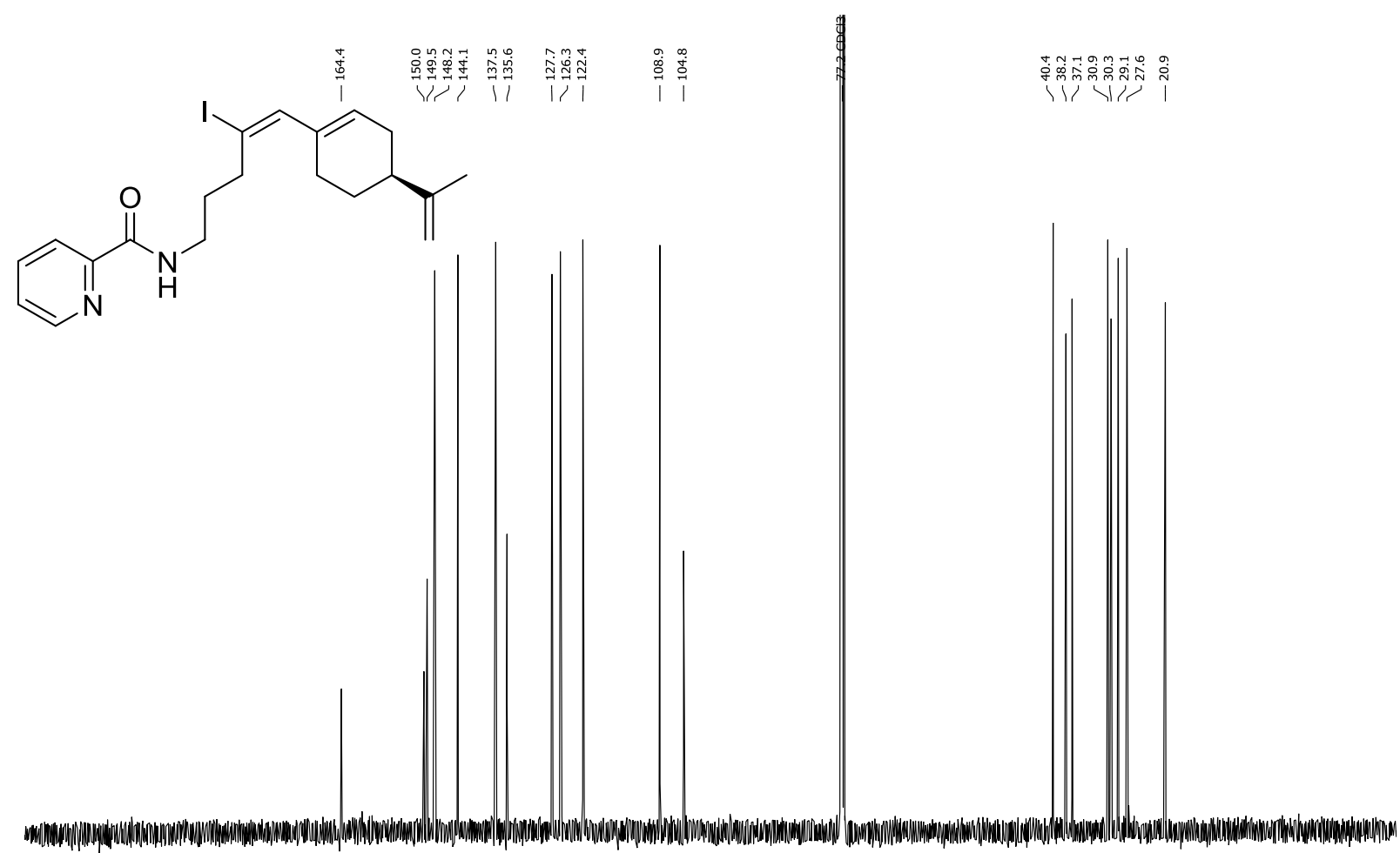

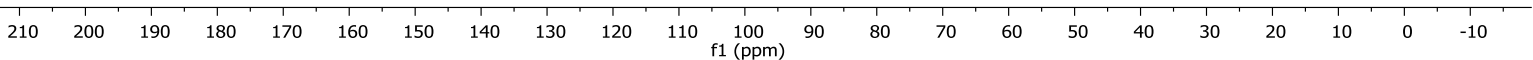


${ }^{1} \mathbf{H}$ NMR $\left(500 \mathrm{MHz}, \mathrm{CDCl}_{3}, 25^{\circ} \mathrm{C}\right)$ of $\mathbf{2 v}$

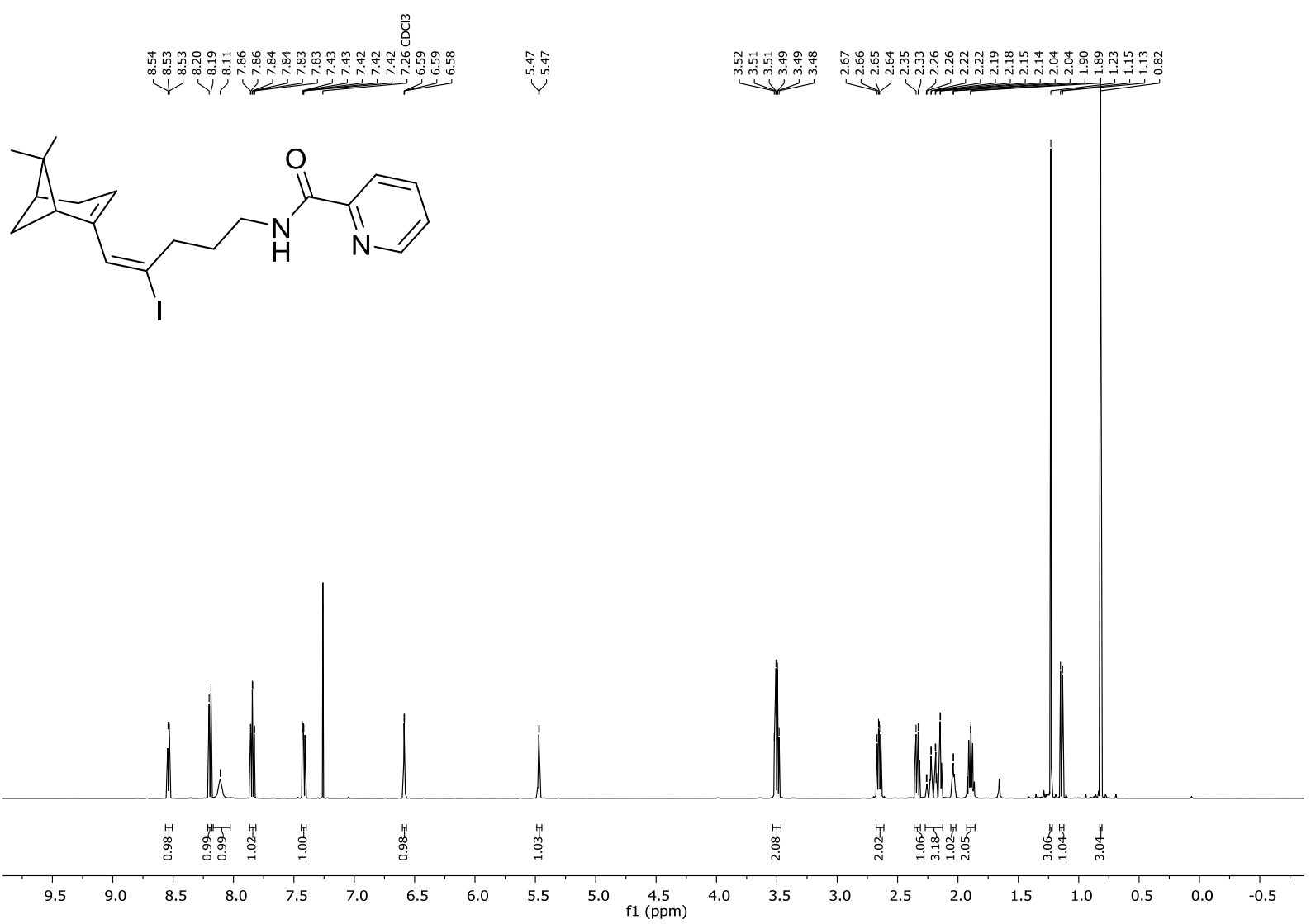

${ }^{13} \mathbf{C}$ NMR $\left(126 \mathrm{MHz}, \mathrm{CDCl}_{3}, 25^{\circ} \mathrm{C}\right)$ of $\mathbf{2 v}$

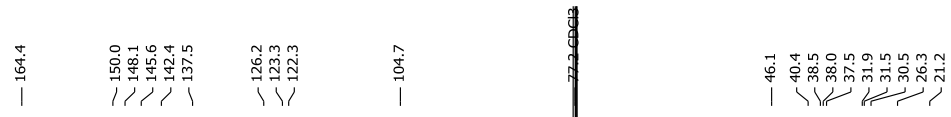

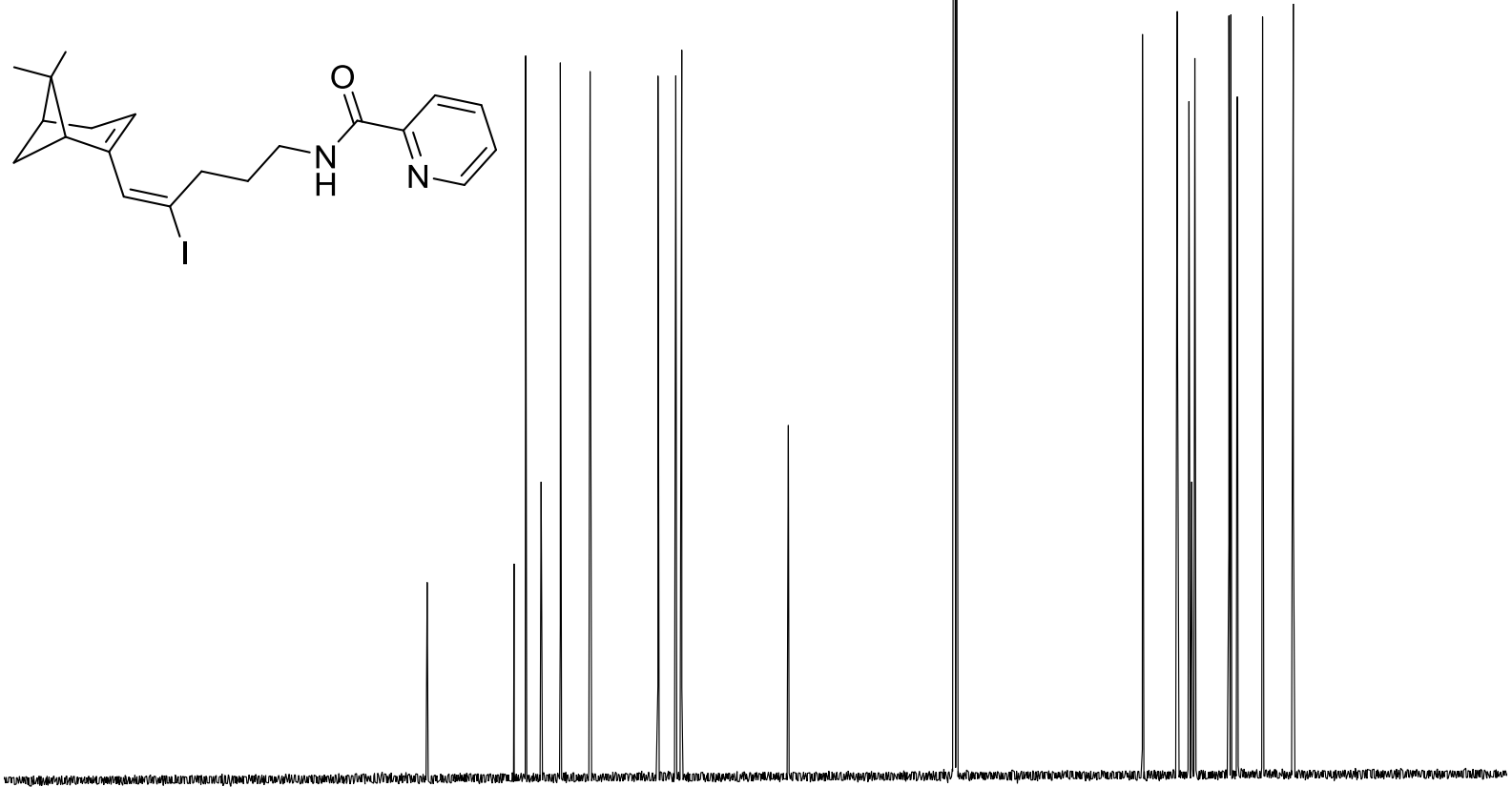

$\begin{array}{lllllllllllllllllllllllllllllllllll}230 & 220 & 210 & 200 & 190 & 180 & 170 & 160 & 150 & 140 & 130 & 120 & 110 & 100 & 90 & 80 & 70 & 60 & 50 & 40 & 30 & 20 & 10 & 0 & -10\end{array}$ 
${ }^{1} \mathbf{H}$ NMR $\left(400 \mathrm{MHz}, \mathrm{CDCl}_{3}, 25^{\circ} \mathrm{C}\right)$ of $\mathbf{2 w}$

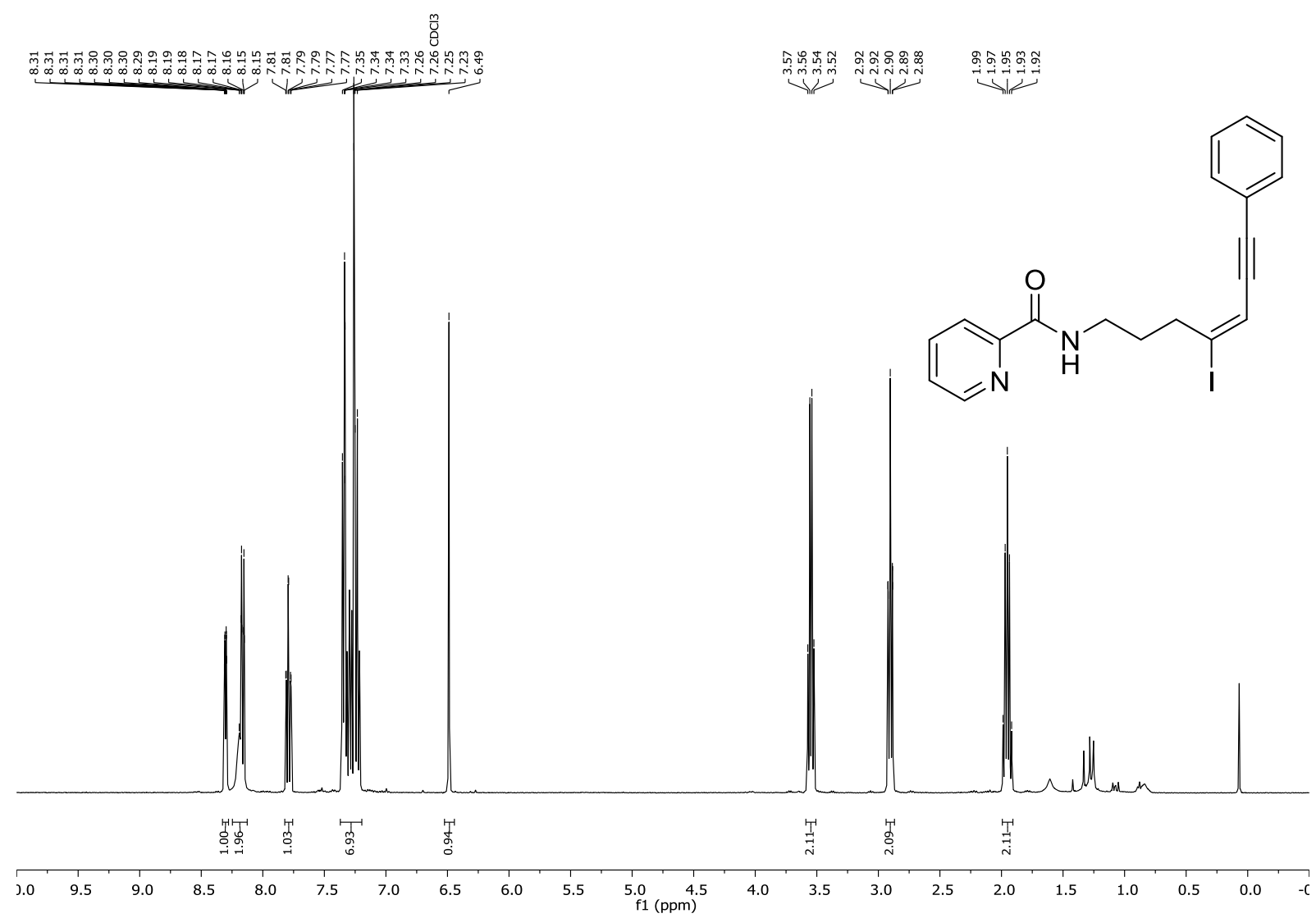

${ }^{13} \mathbf{C}$ NMR $\left(100 \mathrm{MHz}, \mathrm{CDCl}_{3}, 25^{\circ} \mathrm{C}\right)$ of $\mathbf{2 w}$

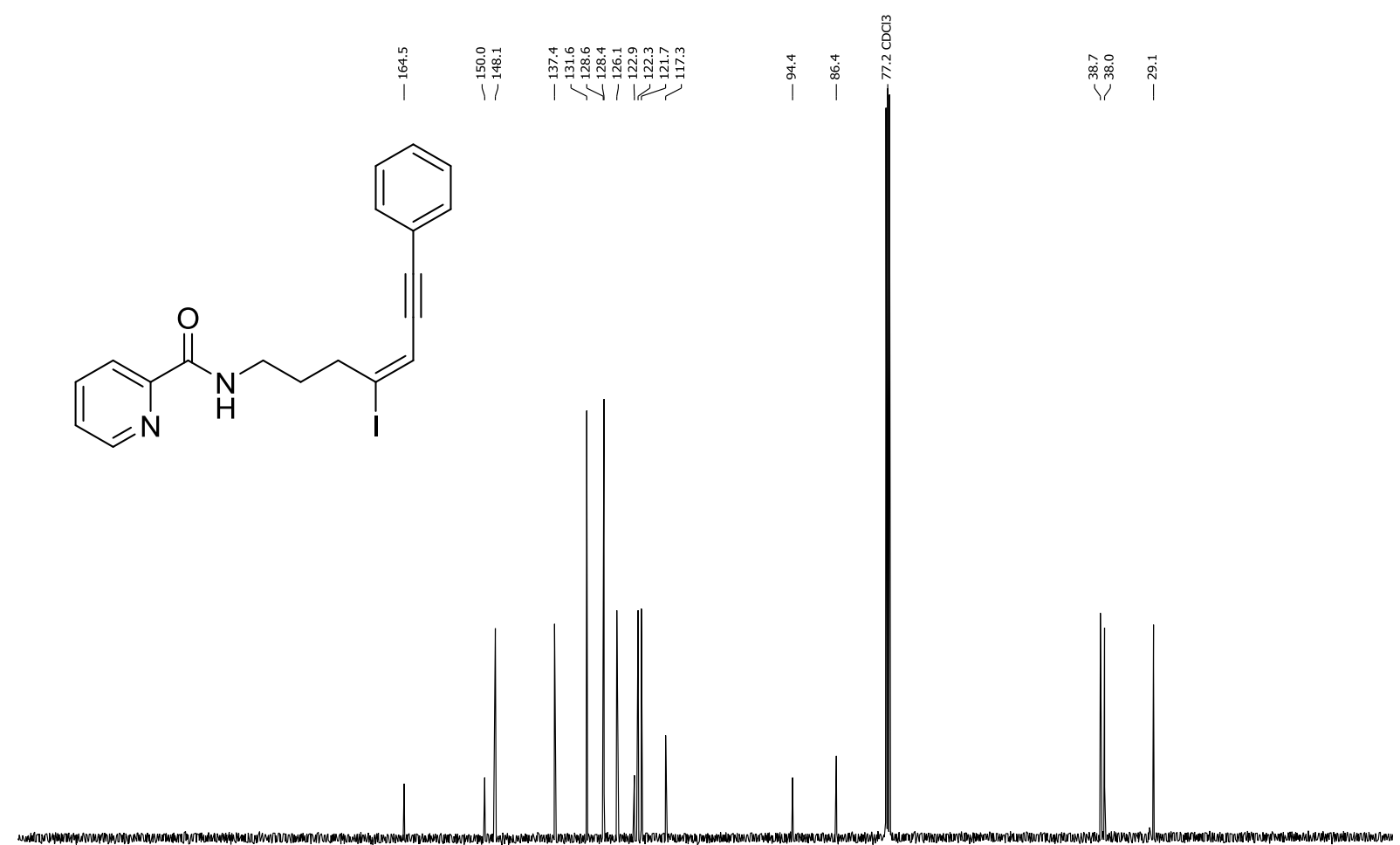

$\begin{array}{lllllllllllllllllllllllllll}230 & 220 & 210 & 200 & 190 & 180 & 170 & 160 & 150 & 140 & 130 & 120 & 110 & 100 & 90 & 80 & 70 & 60 & 50 & 40 & 30 & 20 & 10 & 0 & -10\end{array}$ 
${ }^{\mathbf{1}} \mathbf{H}$ NMR $\left(400 \mathrm{MHz}, \mathrm{CDCl}_{3}, 25^{\circ} \mathrm{C}\right)$ of $\mathbf{2 x}$

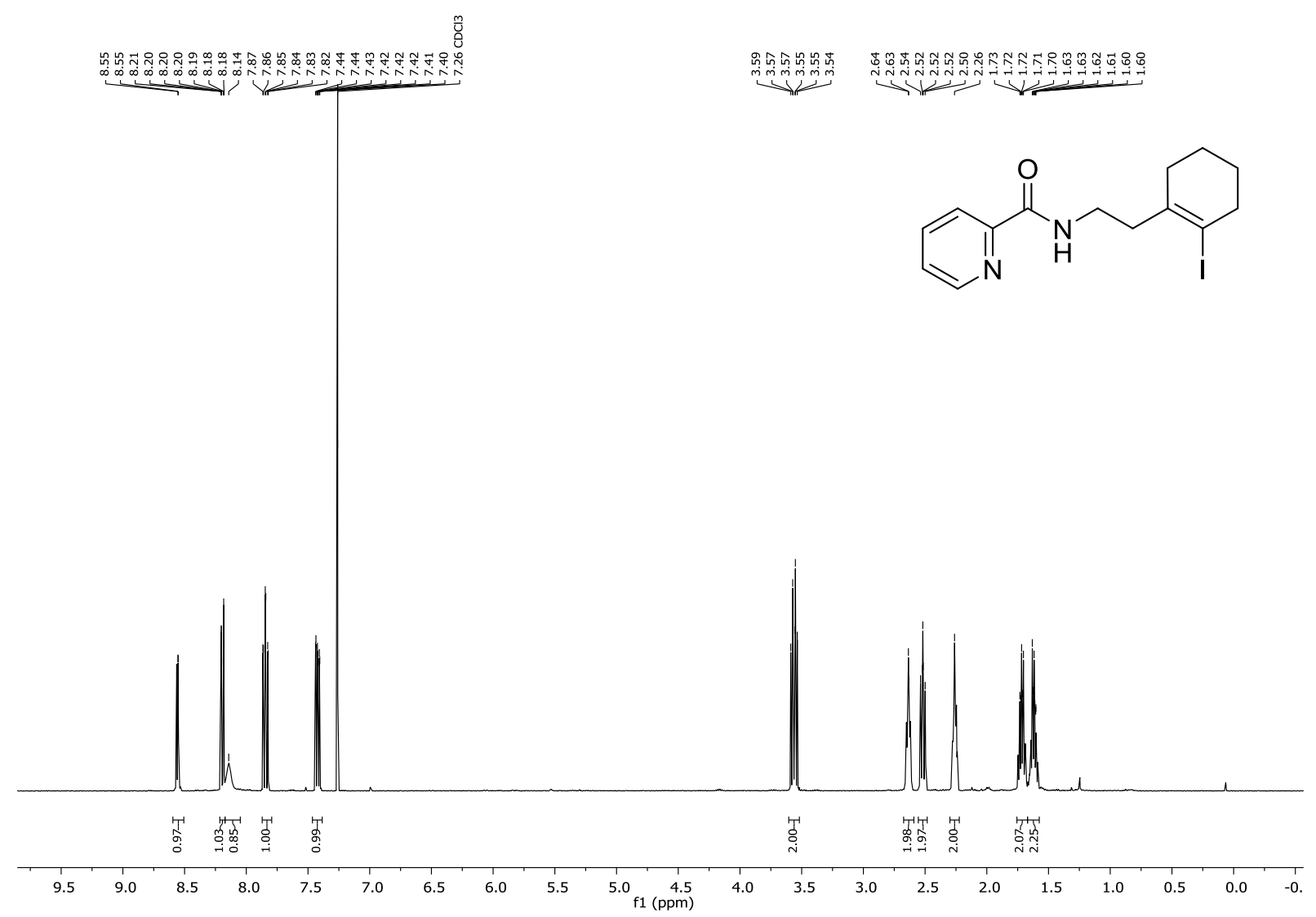

${ }^{13} \mathbf{C ~ N M R}\left(100 \mathrm{MHz}, \mathrm{CDCl}_{3}, 25^{\circ} \mathrm{C}\right)$ of $\mathbf{2 x}$

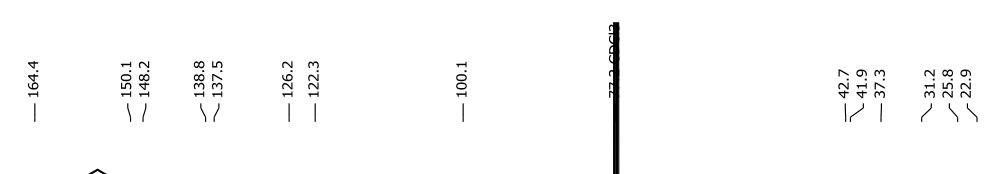<smiles>O=C(NCCC1=C(I)CCCC1)c1ccccn1</smiles>

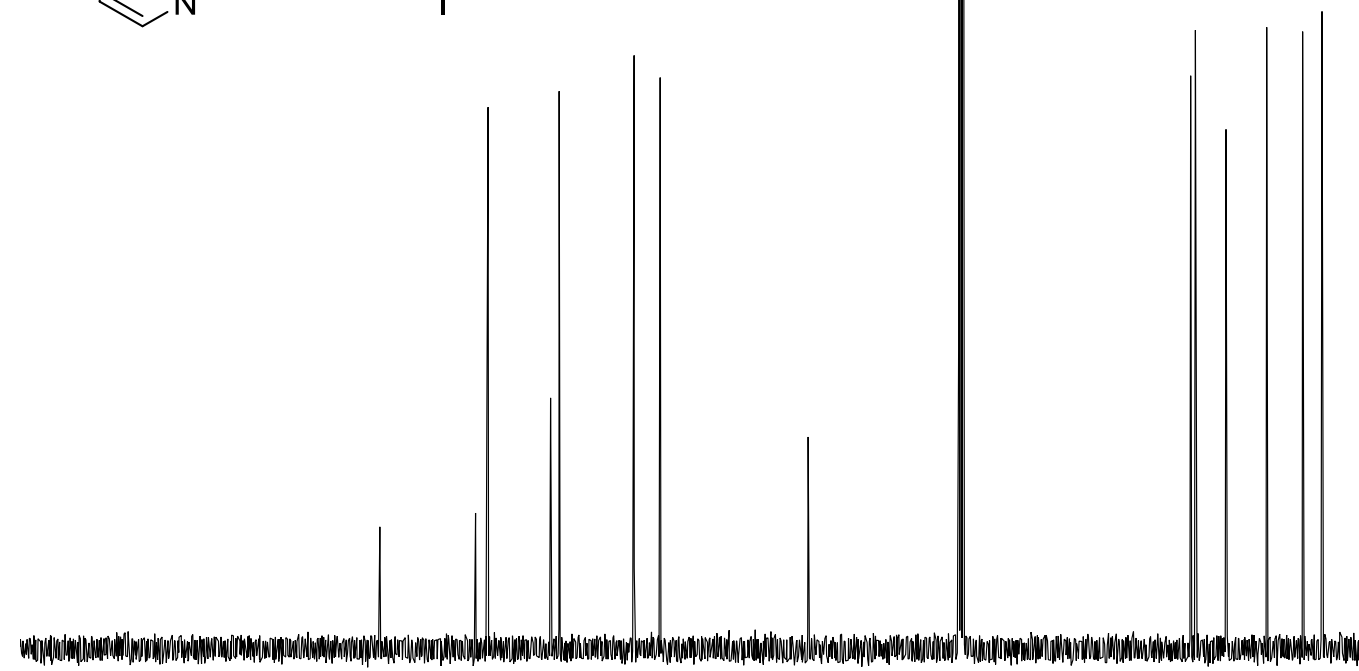

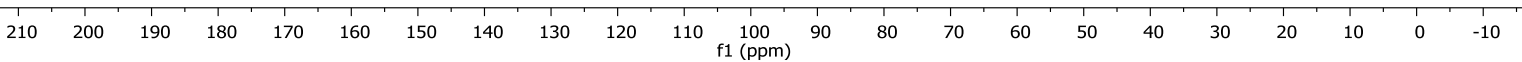


${ }^{\mathbf{1}} \mathbf{H} \mathbf{N M R}\left(500 \mathrm{MHz}, \mathrm{CDCl}_{3}, 25^{\circ} \mathrm{C}\right)$ of $\mathbf{2 y}$

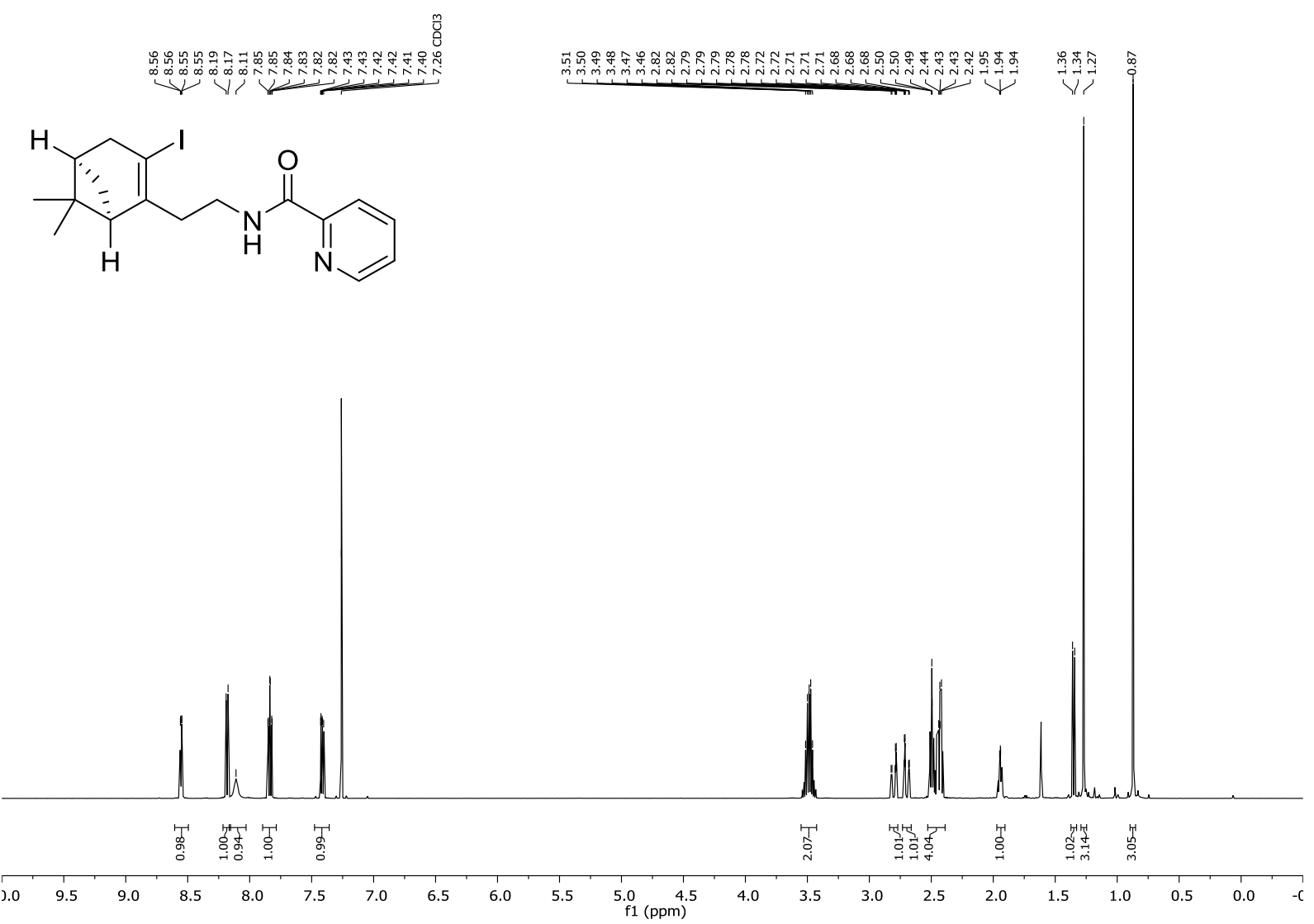

${ }^{13} \mathbf{C ~ N M R}\left(126 \mathrm{MHz}, \mathrm{CDCl}_{3}, 25^{\circ} \mathrm{C}\right)$ of $\mathbf{2 y}$

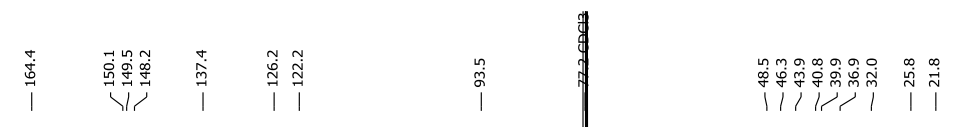<smiles>CC1(C)CCC(I)=C(CCNC(=O)c2ccccn2)C1</smiles>

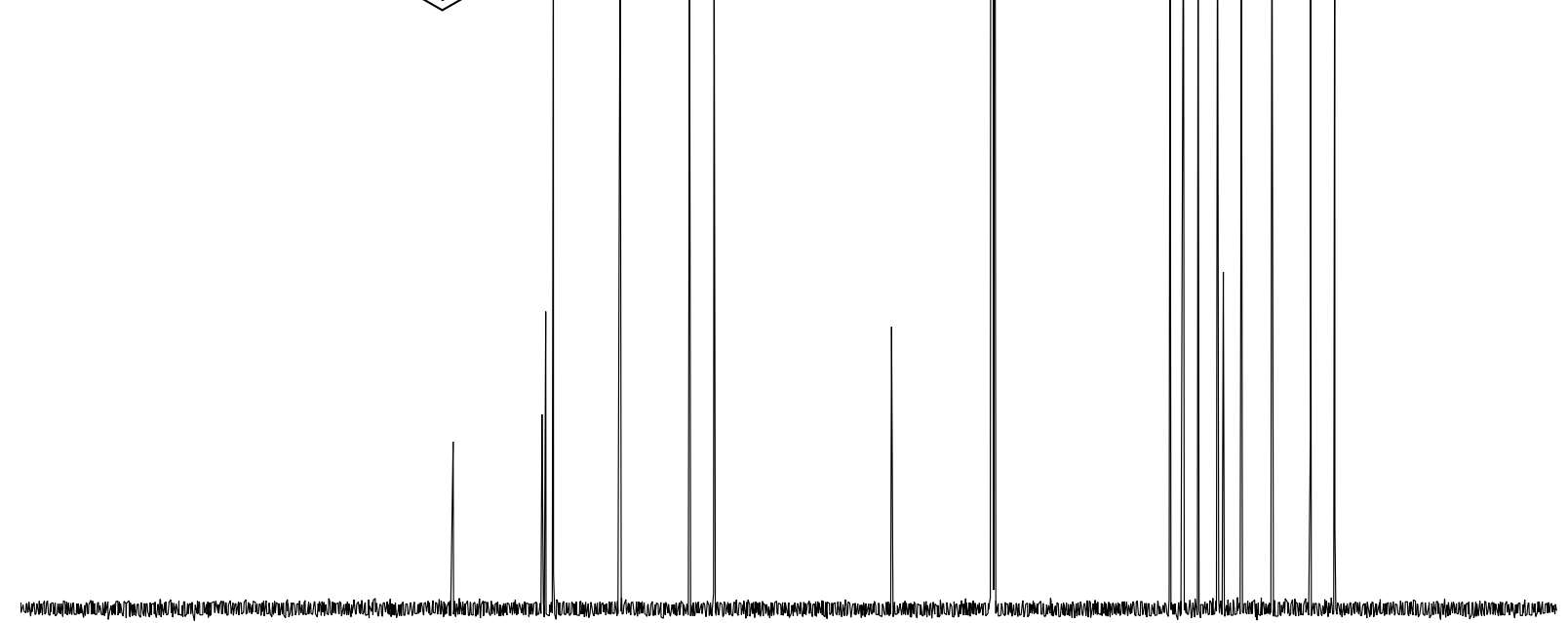

$\begin{array}{llllllllllllllllllllllllllllllllll}230 & 220 & 210 & 200 & 190 & 180 & 170 & 160 & 150 & 140 & 130 & 120 & 110 & 100 & 90 & 80 & 70 & 60 & 50 & 40 & 30 & 20 & 10 & 0 & -10\end{array}$ 
${ }^{\mathbf{1}} \mathbf{H}$ NMR $\left(500 \mathrm{MHz}, \mathrm{CDCl}_{3}, 25^{\circ} \mathrm{C}\right)$ of $\mathbf{2 z}$

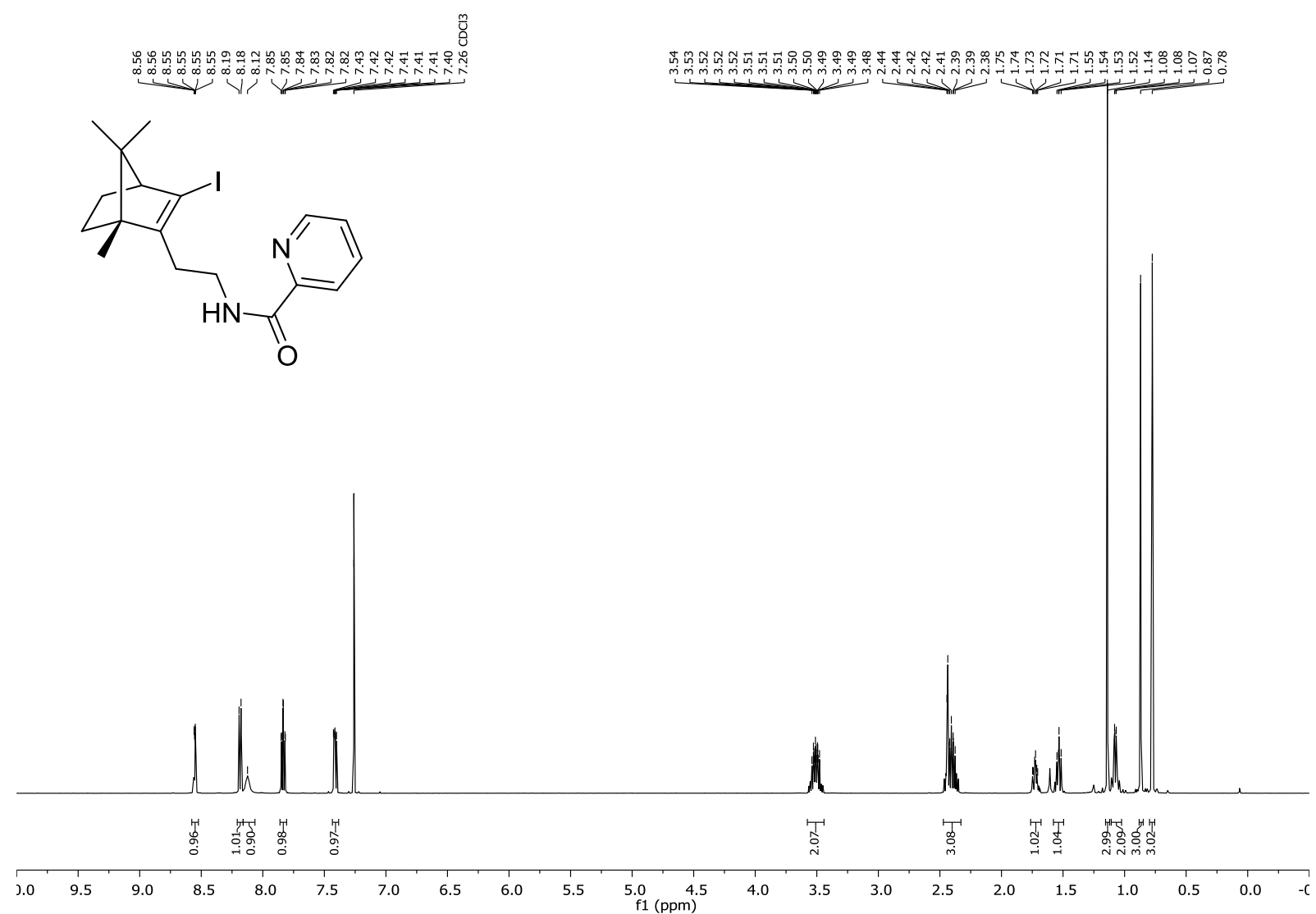

${ }^{13} \mathrm{C}$ NMR $\left(126 \mathrm{MHz}, \mathrm{CDCl}_{3}, 25^{\circ} \mathrm{C}\right)$ of $\mathbf{2 z}$

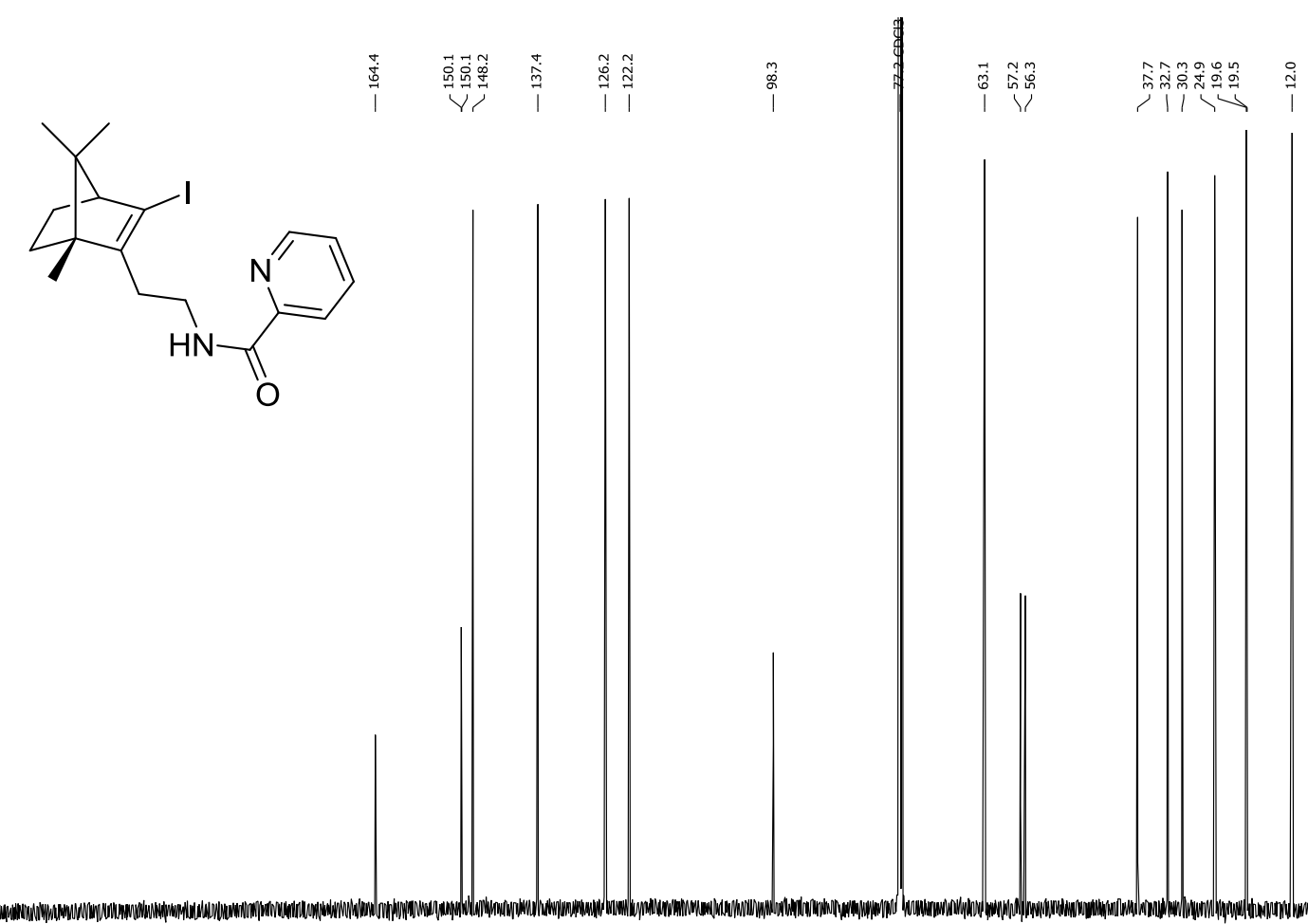

$\begin{array}{lllllllllllllllllllllllllllll}230 & 220 & 210 & 200 & 190 & 180 & 170 & 160 & 150 & 140 & 130 & 120 & 110 & 100 & 90 & 80 & 70 & 60 & 50 & 40 & 30 & 20 & 10 & 0 & -10\end{array}$ 
${ }^{\mathbf{1}} \mathbf{H}$ NMR $\left(400 \mathrm{MHz}, \mathrm{CDCl}_{3}, 25^{\circ} \mathrm{C}\right)$ of $\mathbf{S 1 4}$

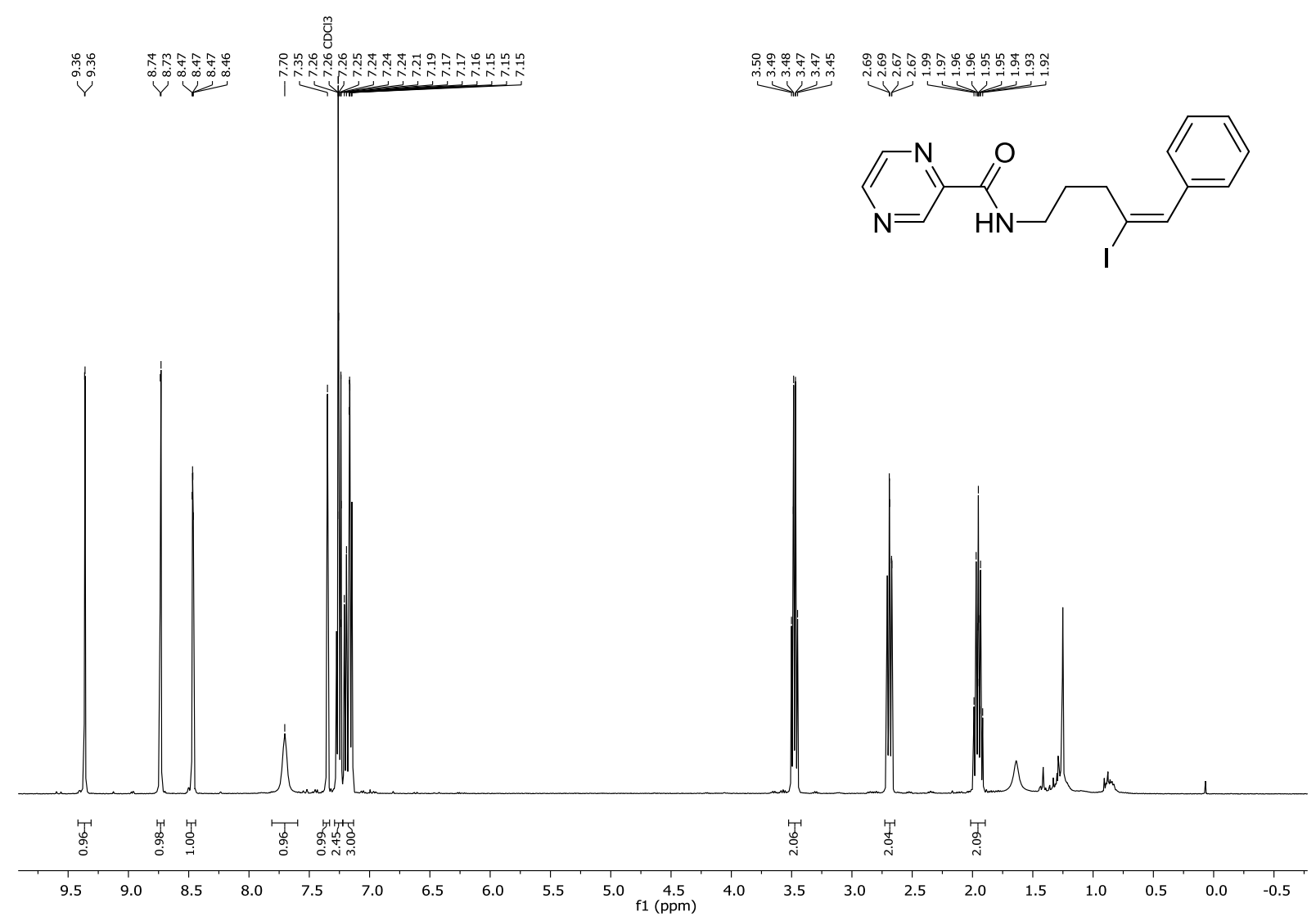

${ }^{13} \mathbf{C ~ N M R}\left(100 \mathrm{MHz}, \mathrm{CDCl}_{3}, 25^{\circ} \mathrm{C}\right)$ of $\mathbf{S 1 4}$

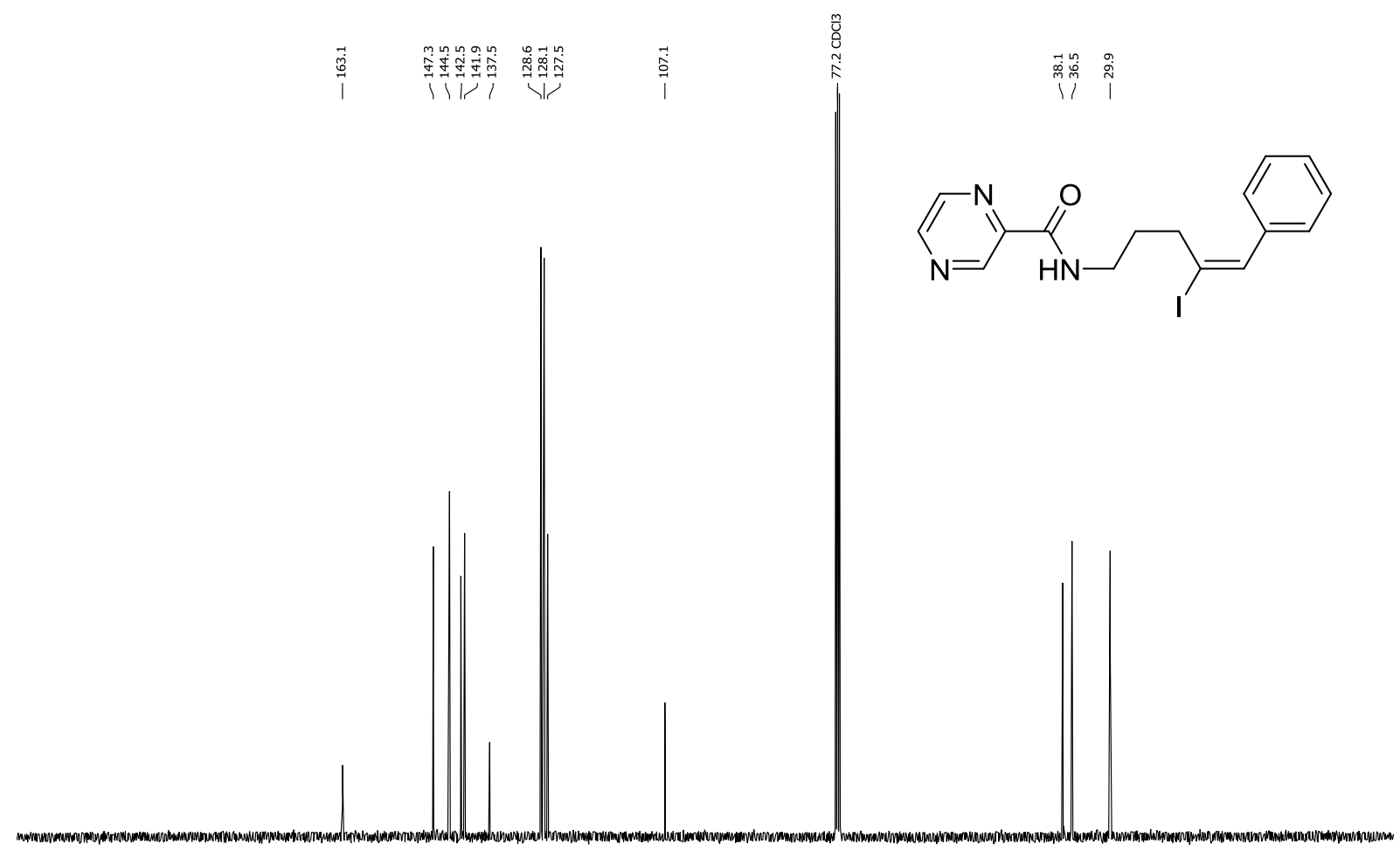

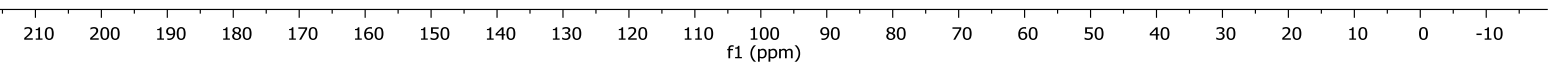


${ }^{1} \mathbf{H}$ NMR $\left(500 \mathrm{MHz}, \mathrm{CDCl}_{3}, 25^{\circ} \mathrm{C}\right)$ of $\mathbf{S 1 5}$

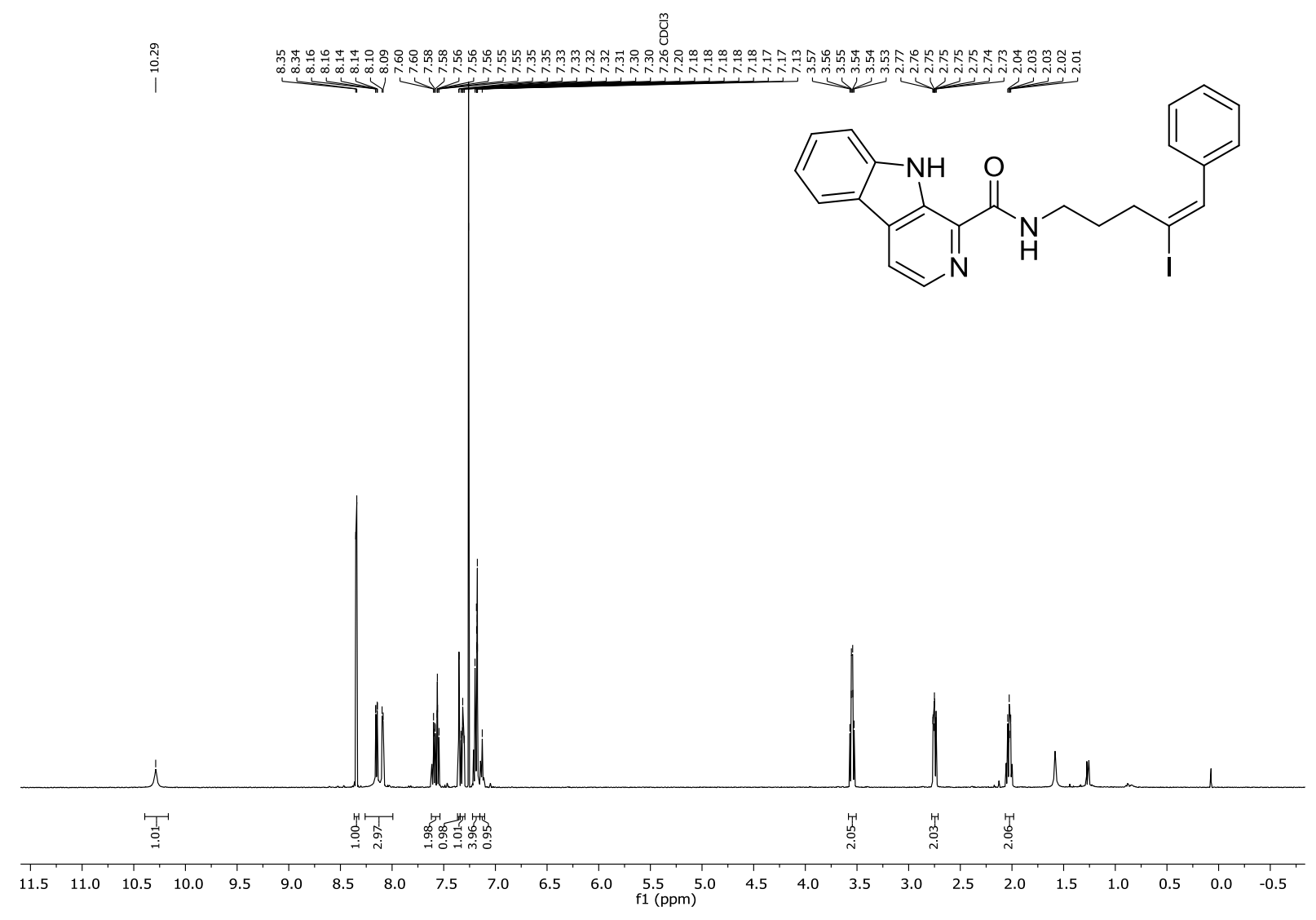

${ }^{13} \mathbf{C ~ N M R}\left(126 \mathrm{MHz}, \mathrm{CDCl}_{3}, 25^{\circ} \mathrm{C}\right)$ of $\mathbf{S 1 5}$

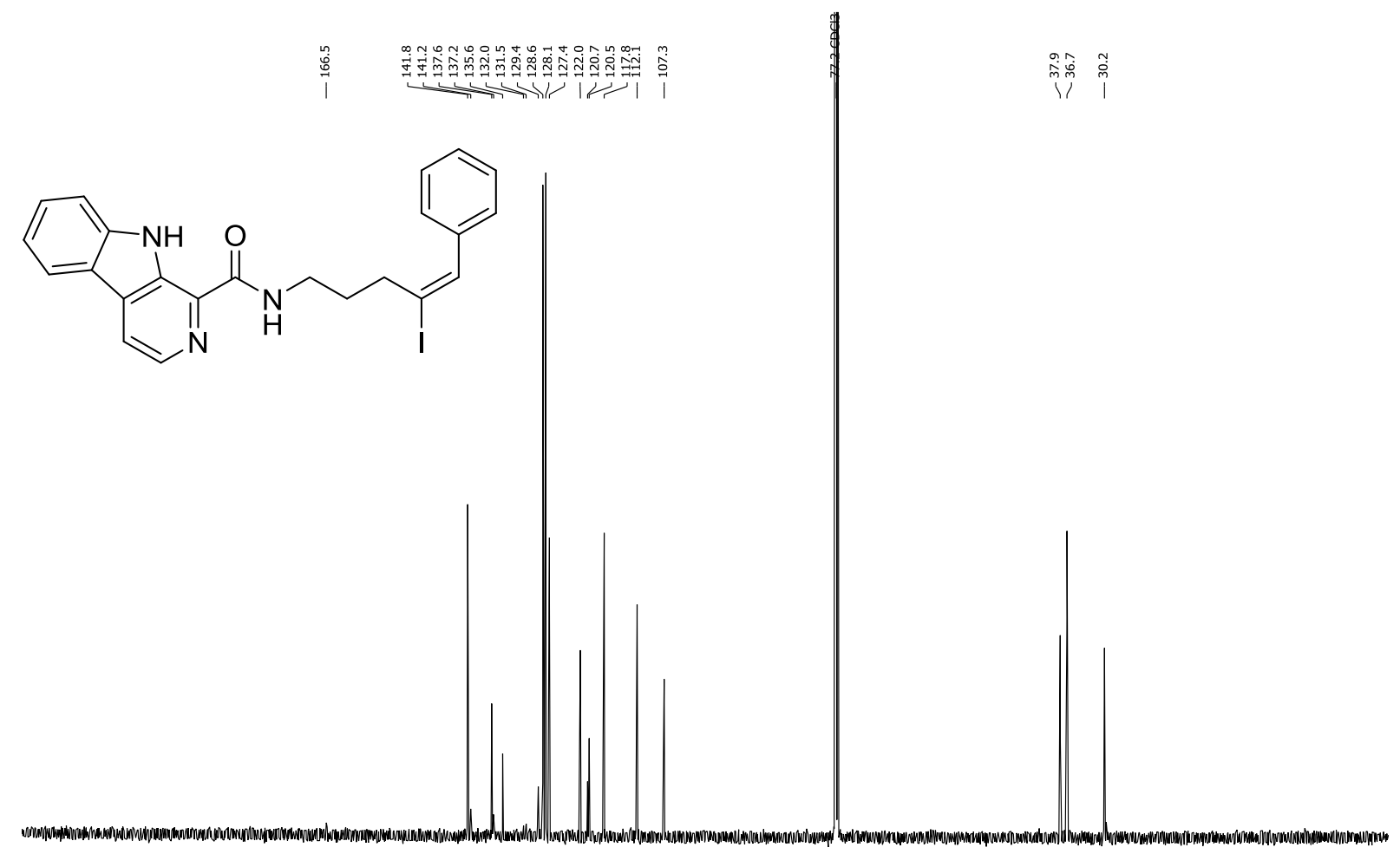

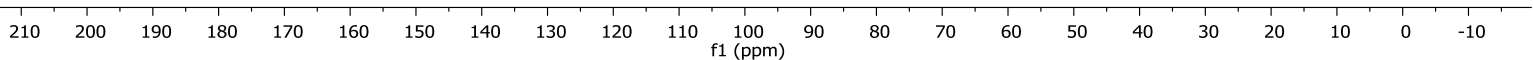


${ }^{\mathbf{1}} \mathbf{H} \mathbf{N M R}\left(400 \mathrm{MHz}, \mathrm{CDCl}_{3}, 25^{\circ} \mathrm{C}\right)$ of $\mathbf{S 1 6}$

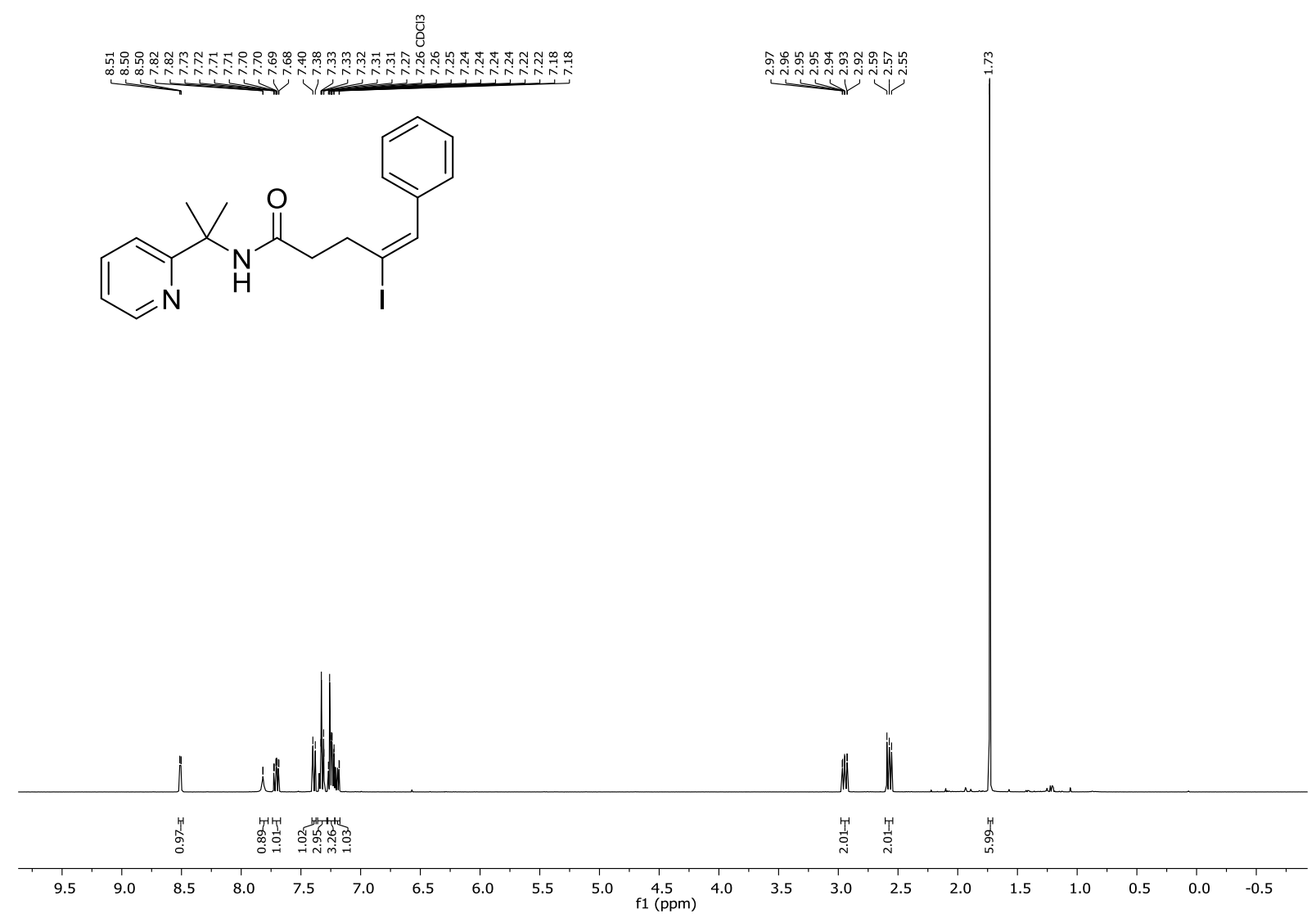

${ }^{13} \mathbf{C}$ NMR $\left(100 \mathrm{MHz}, \mathrm{CDCl}_{3}, 25^{\circ} \mathrm{C}\right)$ of $\mathbf{S 1 6}$

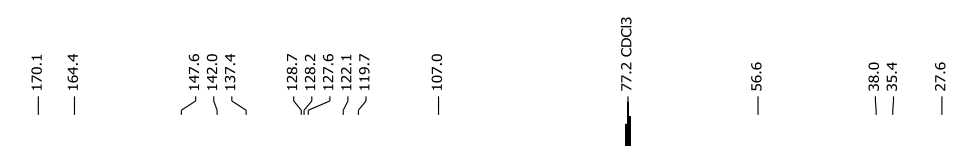<smiles>CC(C)(NC(=O)CC/C(I)=C\c1ccccc1)c1ccccn1</smiles>

\begin{tabular}{llllllllllllllllllllllllll}
\hline 230 & 220 & 210 & 200 & 190 & 180 & 170 & 160 & 150 & 140 & 130 & 120 & 110 & 100 & 90 & 80 & 70 & 60 & 50 & 40 & 30 & 20 & 10 & 0 & -10
\end{tabular} 
${ }^{1} \mathbf{H}$ NMR $\left(400 \mathrm{MHz}, \mathrm{CDCl}_{3}, 25^{\circ} \mathrm{C}\right)$ of $\mathbf{4}$<smiles>CC(C)(C)OC(=O)NCCC/C(I)=C\c1ccccc1</smiles>

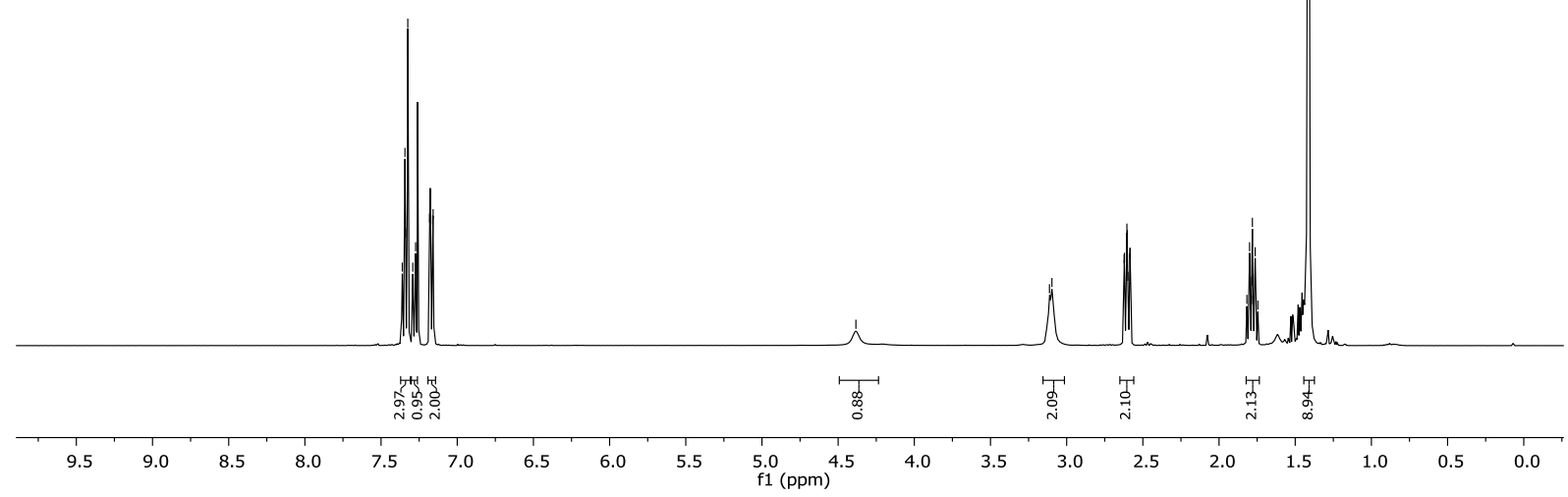

${ }^{13} \mathbf{C}$ NMR $\left(100 \mathrm{MHz}, \mathrm{CDCl}_{3}, 25{ }^{\circ} \mathrm{C}\right)$ of 4
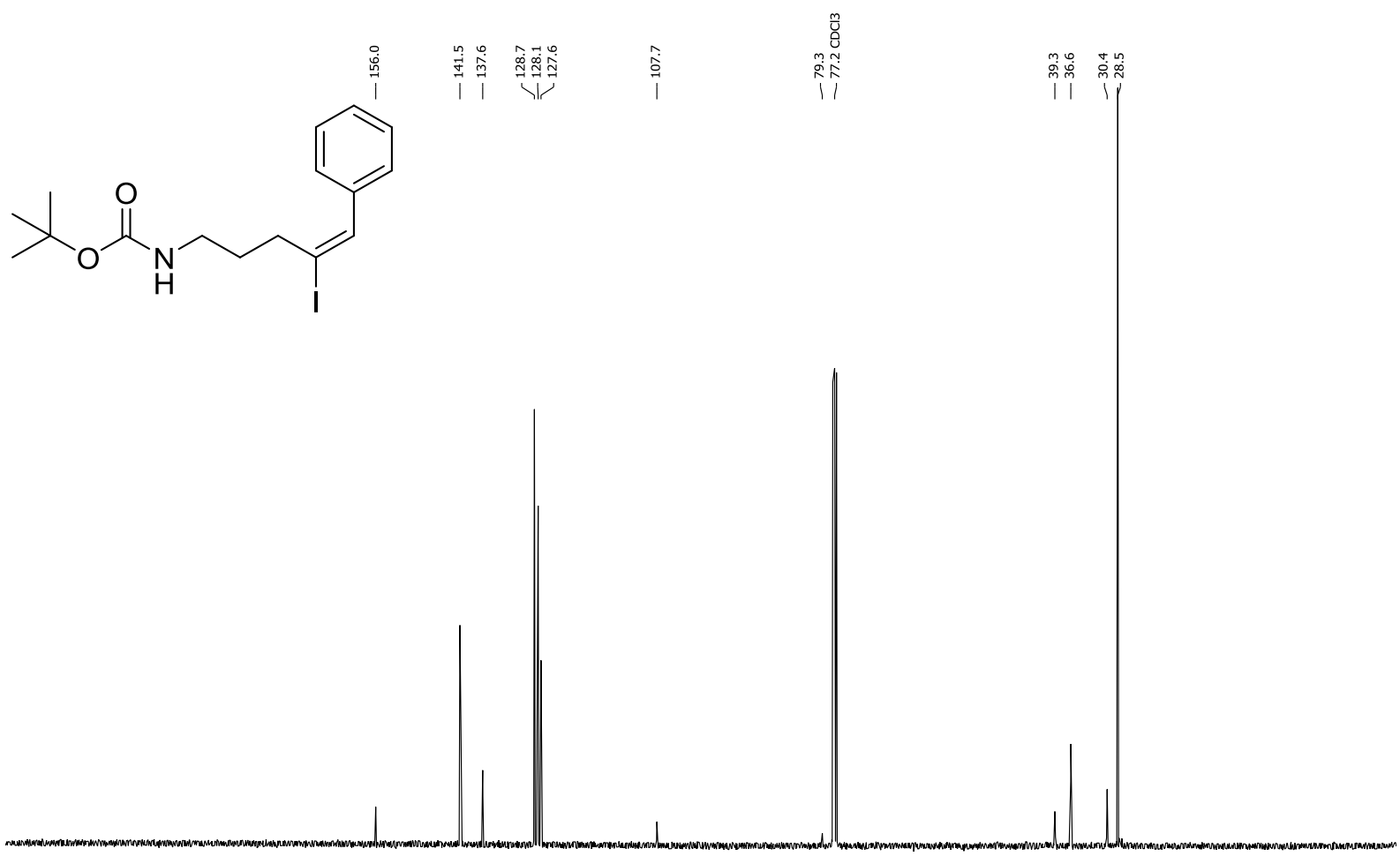

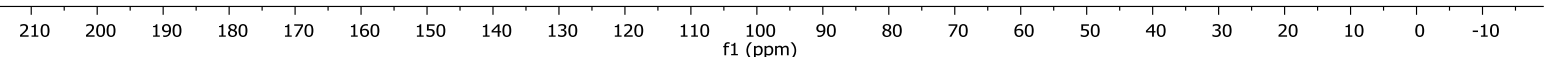


${ }^{1}$ H NMR $\left(400 \mathrm{MHz}, \mathrm{DMSO}-d_{6}, 25^{\circ} \mathrm{C}\right)$ of $\mathbf{5}$
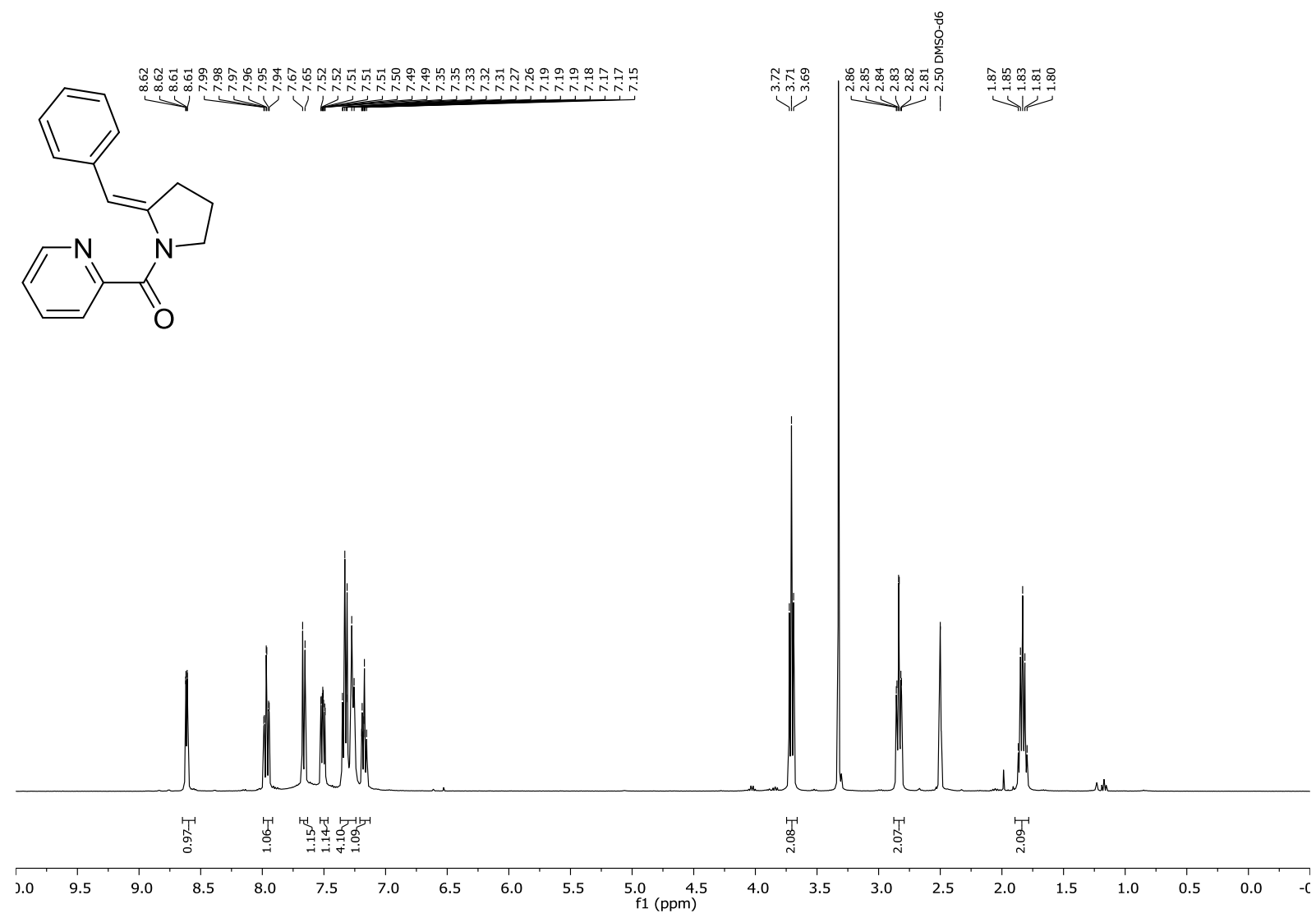

${ }^{13} \mathbf{C}$ NMR $\left(100 \mathrm{MHz}\right.$, DMSO- $\left.d_{6}, 25^{\circ} \mathrm{C}\right)$ of 5

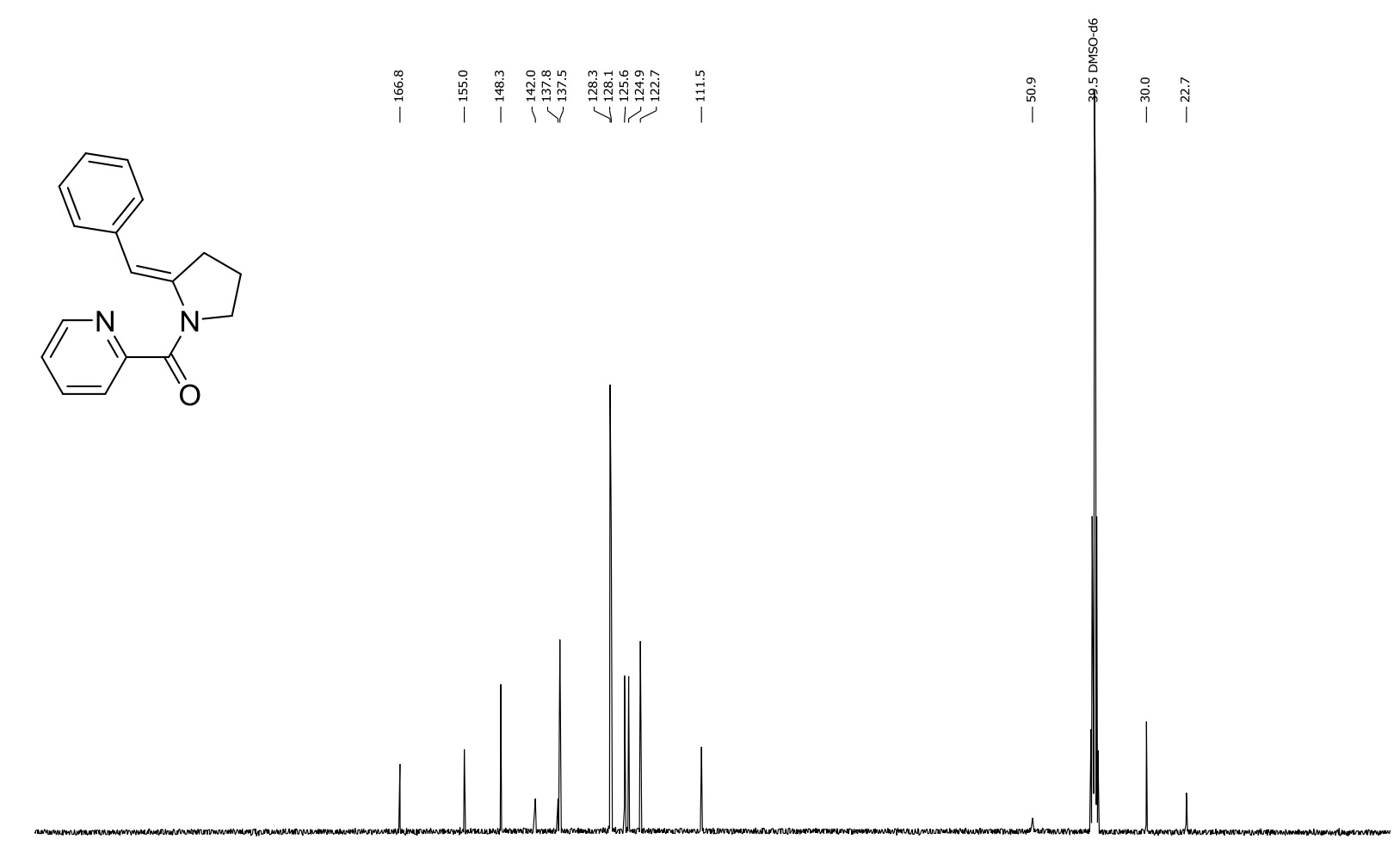

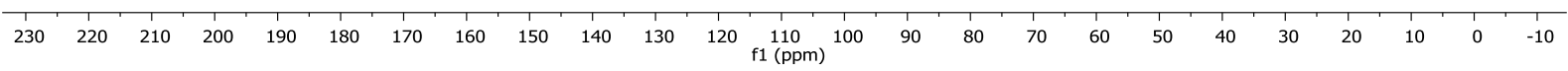


${ }^{1} \mathbf{H}$ NMR $\left(500 \mathrm{MHz}, \mathrm{DMSO}-d_{6}, 80{ }^{\circ} \mathrm{C}\right)$ of $\mathbf{5}$

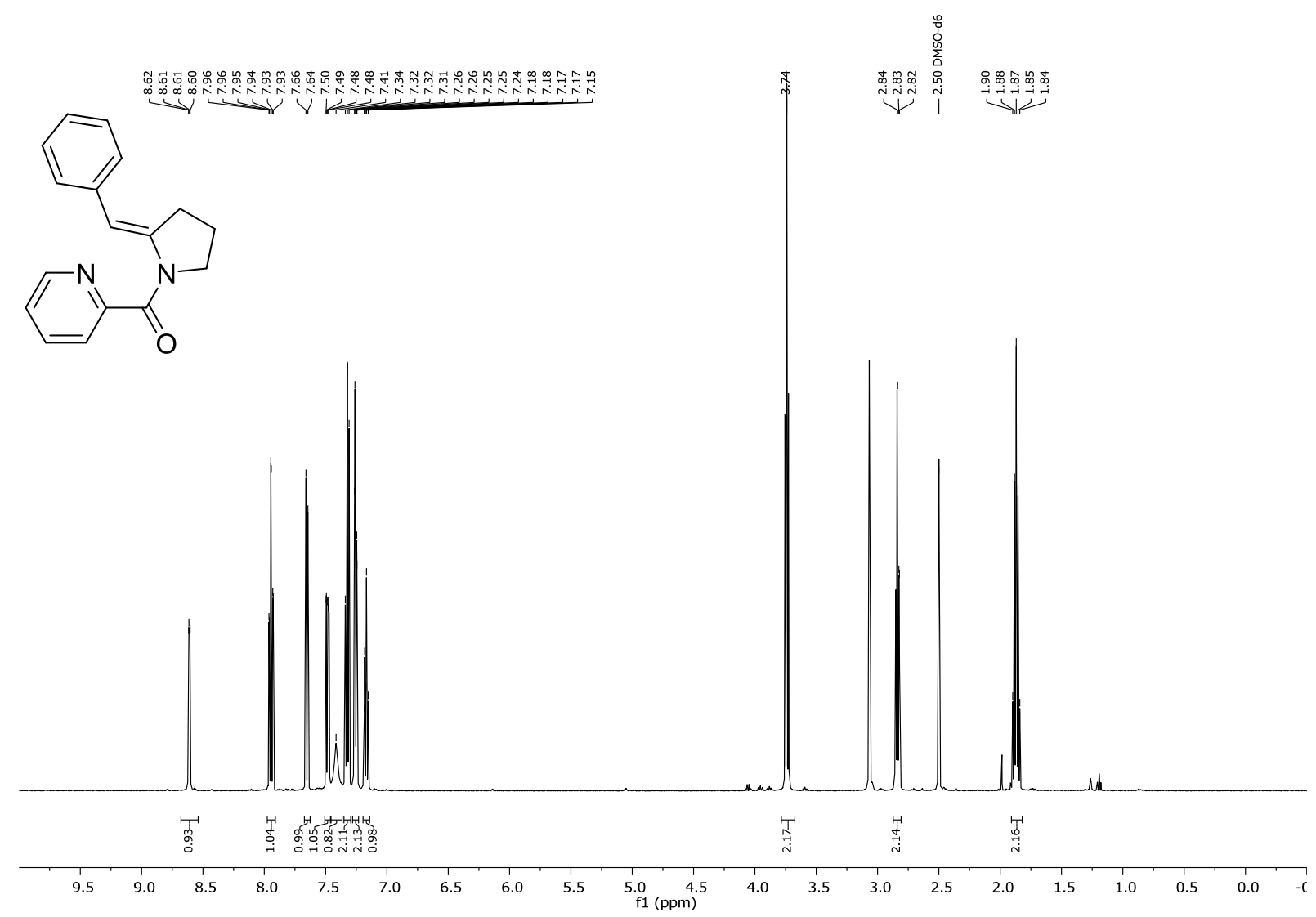

${ }^{13} \mathbf{C}$ NMR $\left(125 \mathrm{MHz}\right.$, DMSO- $\left.d_{6}, 80{ }^{\circ} \mathrm{C}\right)$ of 5
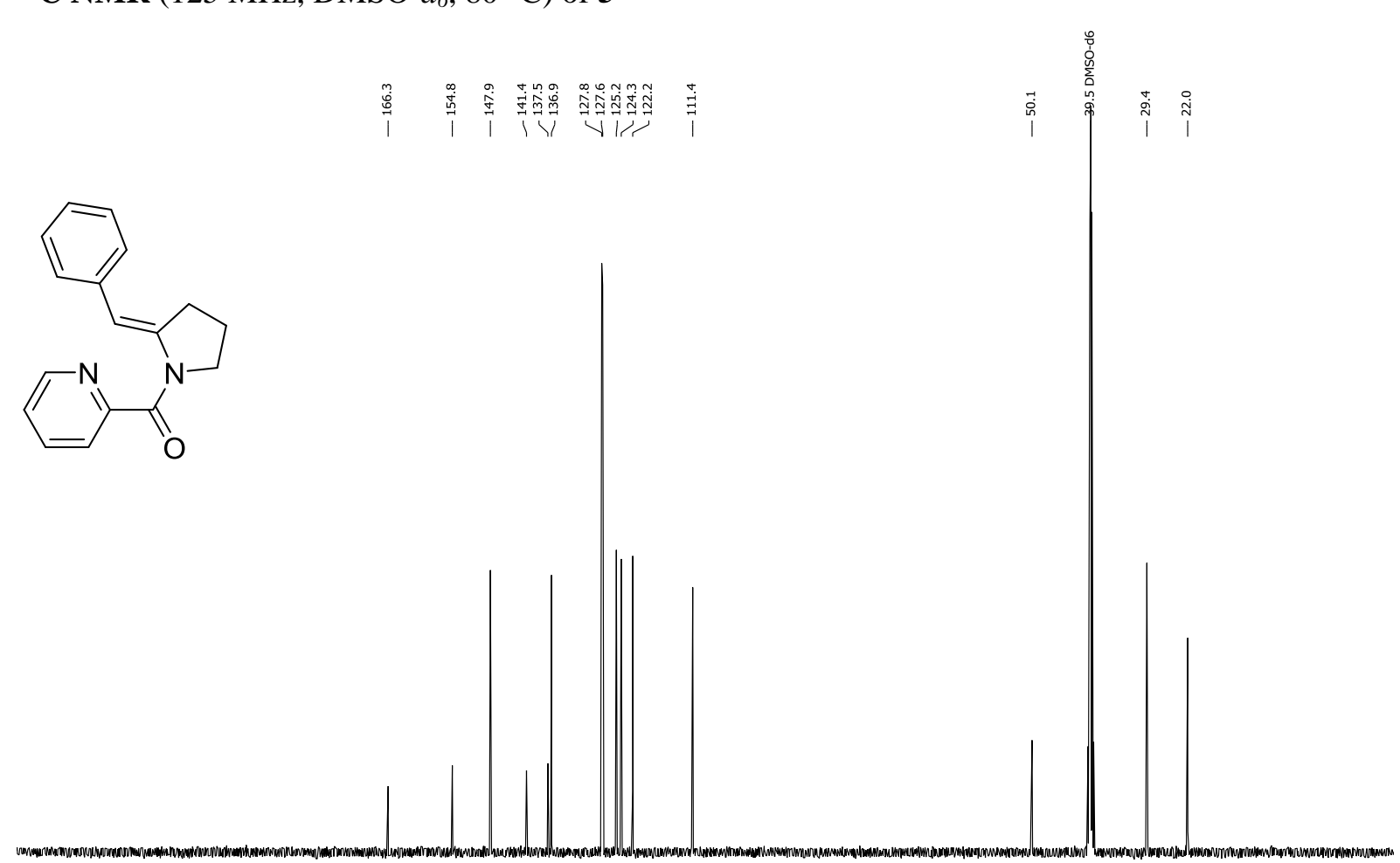

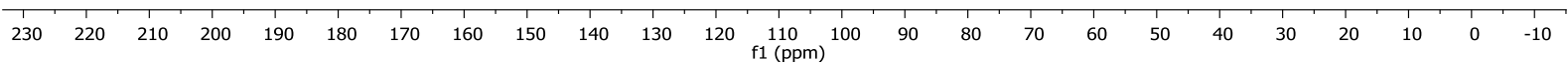


$\operatorname{NOESY}\left(500 \mathrm{MHz}, \mathrm{DMSO}-d_{6}, .25^{\circ} \mathrm{C}, \mathrm{t}_{\mathrm{m}}=500 \mathrm{~ms}\right)$ of 5

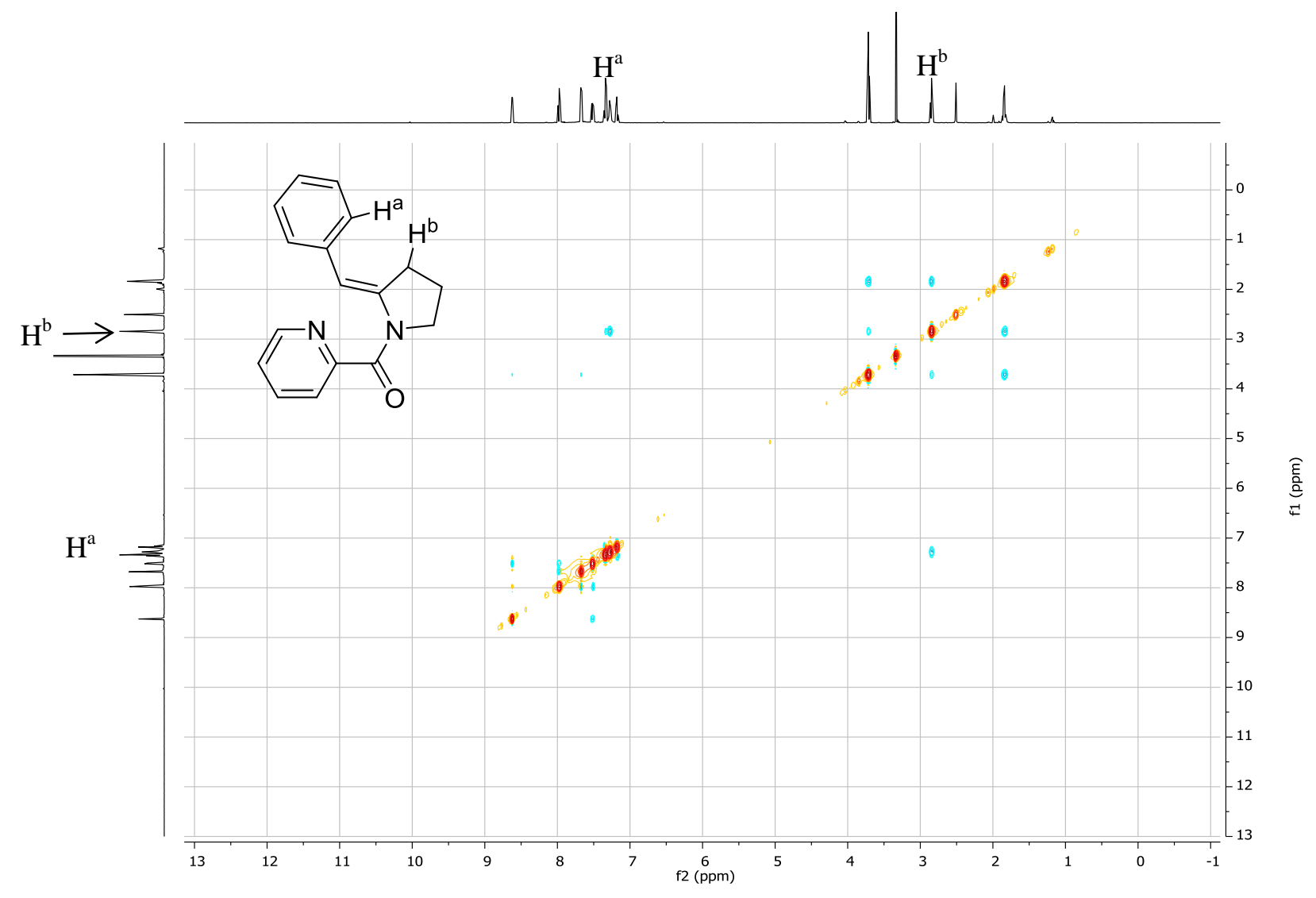


${ }^{1} \mathbf{H}$ NMR $\left(500 \mathrm{MHz}, \mathrm{CDCl}_{3}, 25^{\circ} \mathrm{C}\right)$ of 6

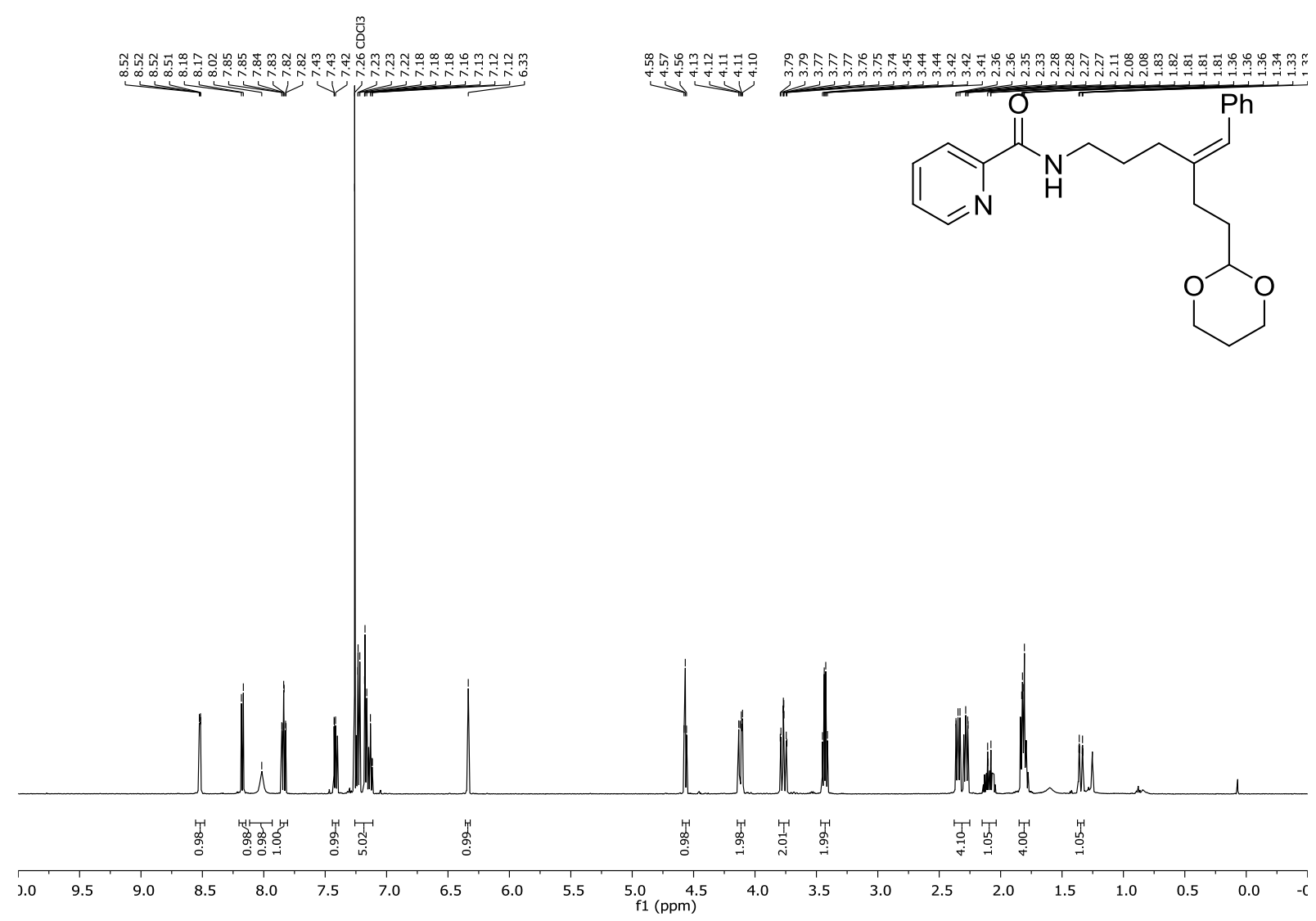

${ }^{13} \mathrm{C}$ NMR $\left(126 \mathrm{MHz}, \mathrm{CDCl}_{3}, 25^{\circ} \mathrm{C}\right)$ of 6

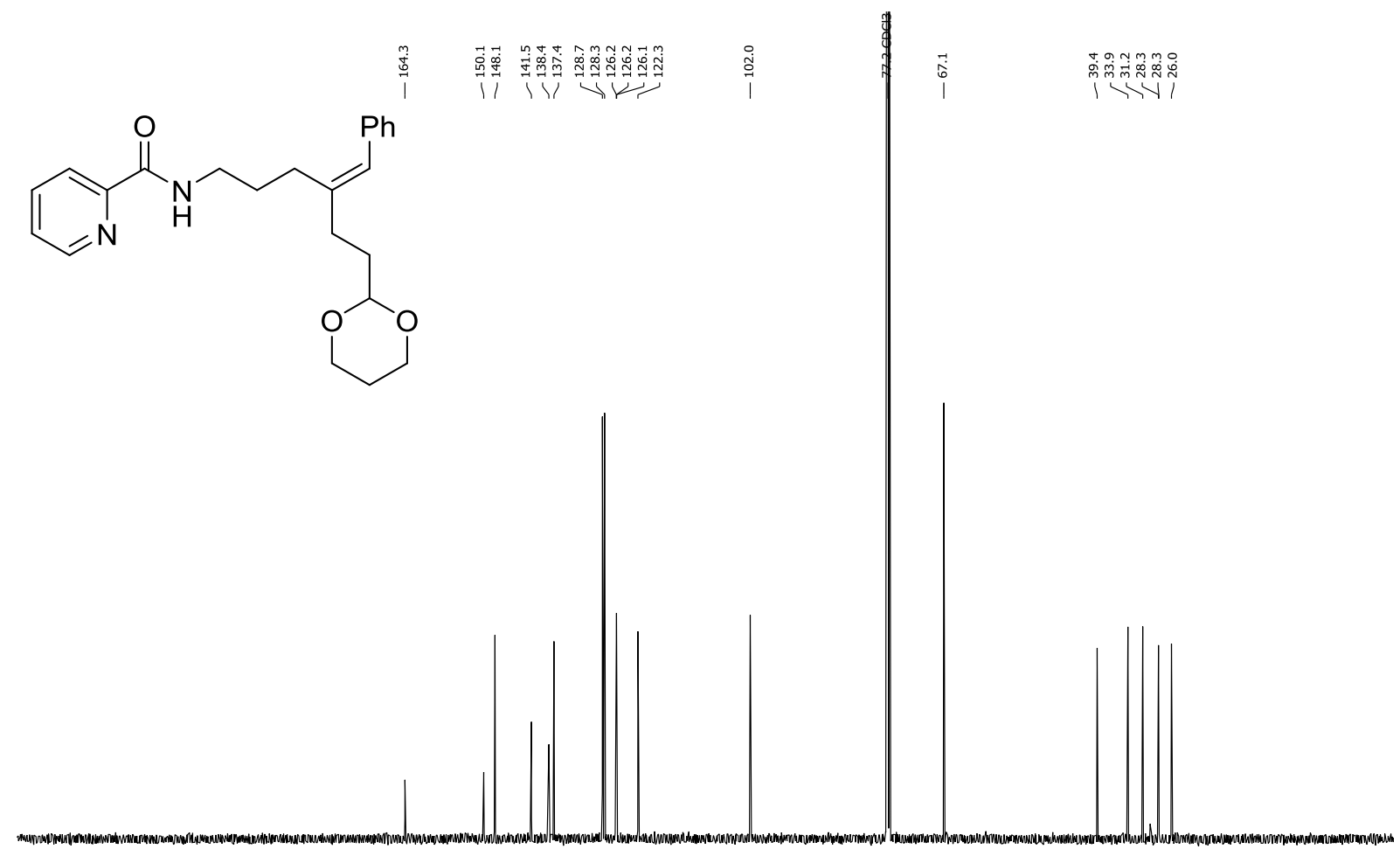

$\begin{array}{rllllllllllllllllllllllllll}130 & 220 & 210 & 200 & 190 & 180 & 170 & 160 & 150 & 140 & 130 & 120 & 110 & 100 & 90 & 80 & 70 & 60 & 50 & 40 & 30 & 20 & 10 & 0 & -10\end{array}$ 
NOESY $\left(500 \mathrm{MHz}, \mathrm{CDCl}_{3}, .25^{\circ} \mathrm{C}, \mathrm{t}_{\mathrm{m}}=500 \mathrm{~ms}\right)$ of 6
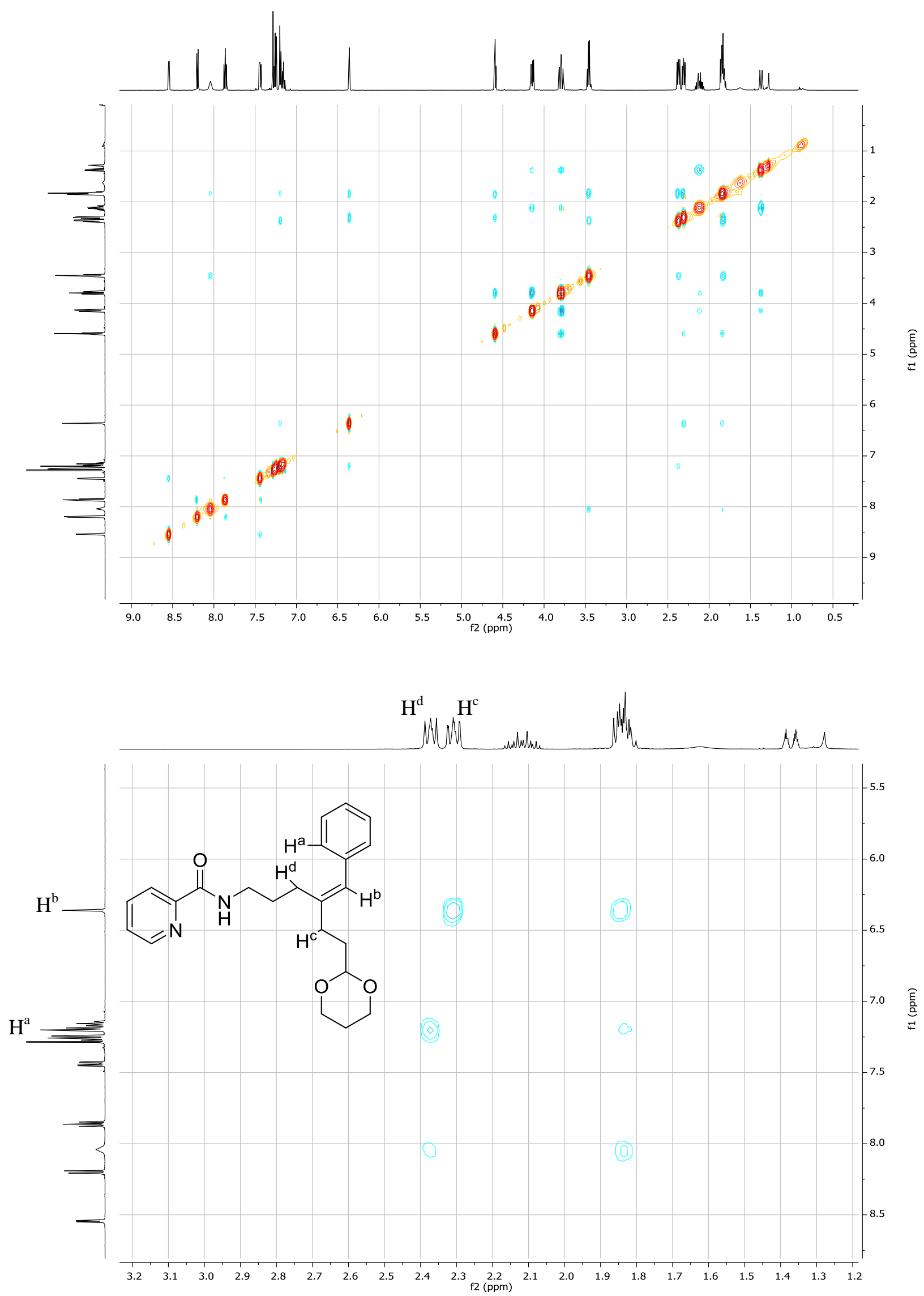
${ }^{1} \mathbf{H}$ NMR $\left(400 \mathrm{MHz}, \mathrm{CDCl}_{3}, 25^{\circ} \mathrm{C}\right)$ of 7

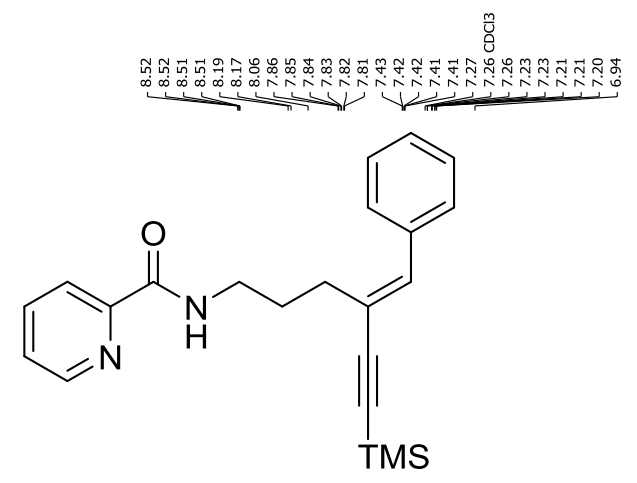

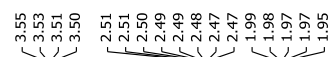
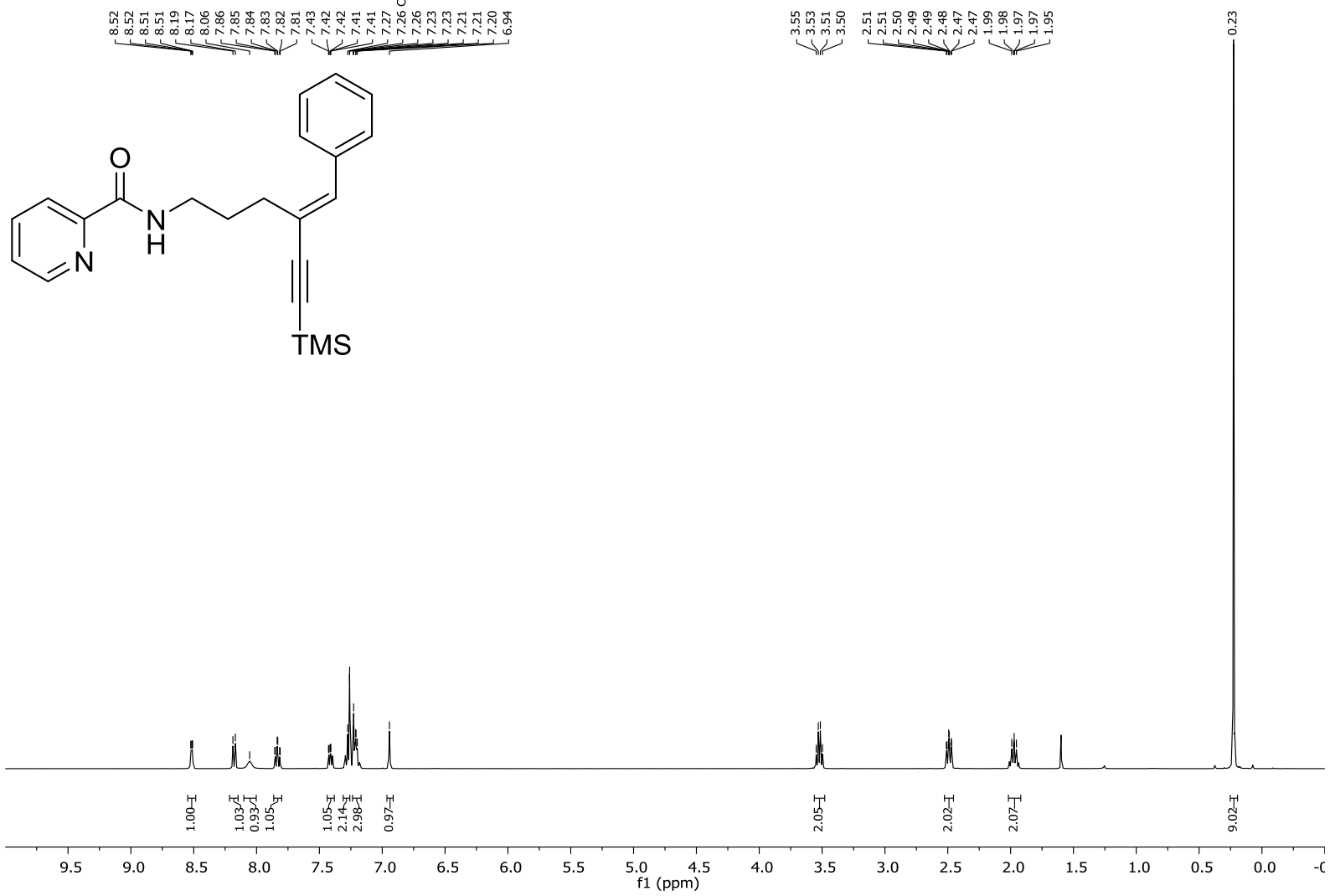

${ }^{13} \mathrm{C}$ NMR $\left(126 \mathrm{MHz}, \mathrm{CDCl}_{3}, 25^{\circ} \mathrm{C}\right)$ of 7

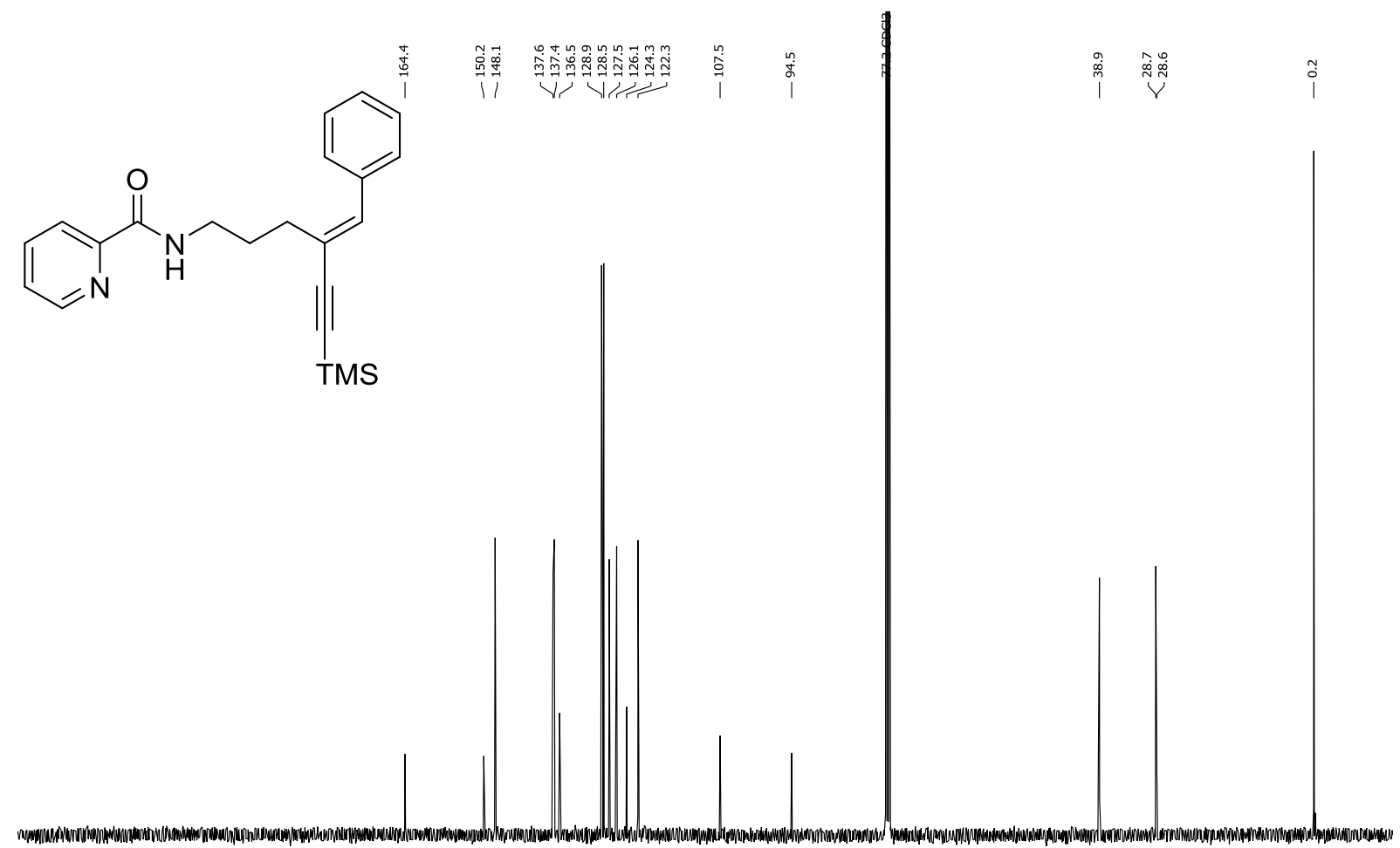

$\begin{array}{llllllllllllllllllllllllllllllll}230 & 220 & 210 & 200 & 190 & 180 & 170 & 160 & 150 & 140 & 130 & 120 & 110 & 100 & 90 & 80 & 70 & 60 & 50 & 40 & 30 & 20 & 10 & 0 & -10\end{array}$ 